\begin{tabular}{|l|l|}
\hline $\begin{array}{l}\text { 2. To: (Receiving Organization) } \\
\text { Distribution }\end{array}$ & $\begin{array}{l}\text { 3. From: coriginating Organization) } \\
\text { TWRS Projects/SST Retrieval }\end{array}$ \\
\hline 5. Proj-/Prog./Dept./Div.: D299/ & $\begin{array}{l}\text { 6. Design Authority/ Design Agent/Cog. } \\
\text { Engr: } \\
\text { W-320 TWRS/TCPN \# }\end{array}$ \\
\hline
\end{tabular}

8. Originator Remarks:

For approval and release of a new supporting document. This document has been generated to ensure retrievability of the Project W-320 "HVAC Calculations, Vol. 4".
11. Receiver Remarks:
11A. Design Baseline Document?
[] no

\section{Related EDT No.: \\ $\mathrm{n} / \mathrm{a}$}

7. Purchase Order Ho.:

$$
n / a
$$

9. Equip./Component Ho.:

$$
\mathrm{n} / \mathrm{a}
$$

10. System/Bldg./Facility: 241-C-106

12. Major Assm. Dwg. No.: $\mathrm{n} / \mathrm{a}$

13. Permit/Permit Application Ho.: $\mathrm{n} / \mathrm{a}$

14. Required Response Date:

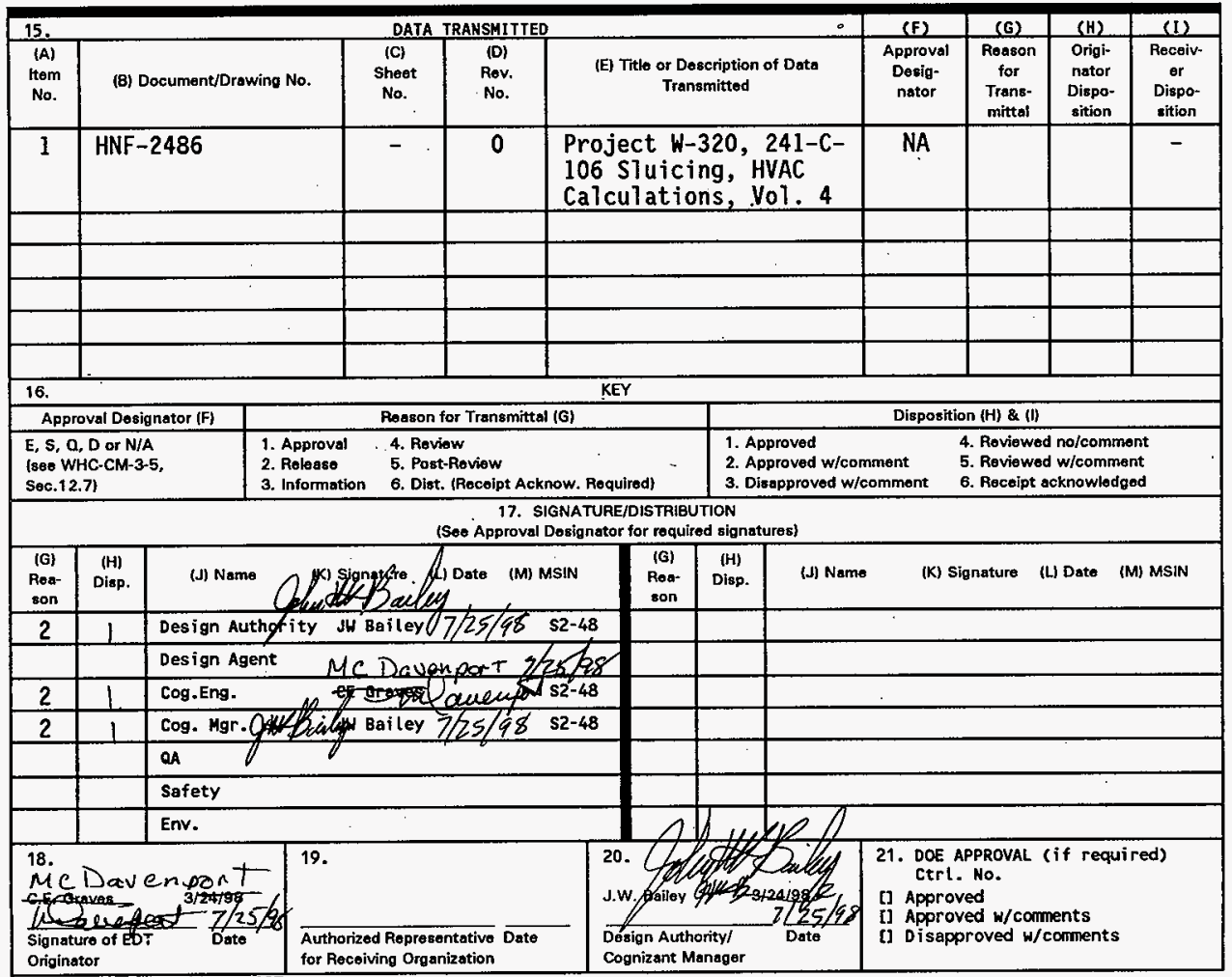




\section{Project W-320, 241-C-106 Sluicing HVAC Calculations, Vol. 4}

John W. Bailey

Numatec Hanford Co., Richland, WA 99352

U.S. Department of Energy Contract DE-AC09-96RL13200
EDT/ECN: $622244 \quad$ UC: 506
Org Code: 8C452 Charge Code: D2991/HANA0600
B\&R Code: EW3130010 Total Pages: 195

Key Words: W-320, Sluicing, Tank 241-C-106, Tank 241-AY-102, WRSS, calculations, HVAC.

Abstract: This supporting document has been prepared to make the FDNW calculations for Project $W-320$, readily retrievable.

Product cut sheets in this document have been reviewed and are not

Copyrighted.

TRADEMARK DISCLAIMER. Reference herein to any specific commercial product, process, or service by trade name, trademark, manufacturer, or otherwise, does not necessarily constitute or imply its endorsement, recommendation, or favoring by the United states Government or any agency thereof or its contractors or subcontractors.

Printed in the United States of America. To obtain copies of this document, contact: Document Control Services, P.0. Box 950, Mailstop H6-08, Richland WA 99352, Phone (50́9) 372-2420;

Fax (509) 376-4989.
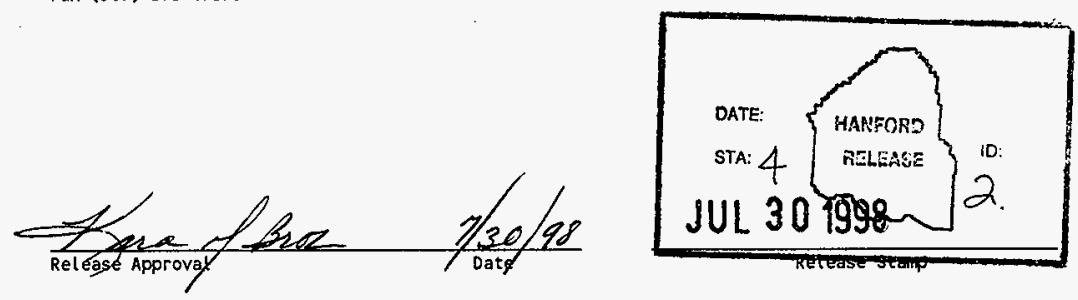

\section{Approved for Public Release}


HNF-2486, Rev. 0

\title{
Project W-320, 241-C-106 Sluicing HVAC Calculations, Vol. 4
}

\author{
TABLE OF CONTENTS
}

$\begin{array}{lll}\begin{array}{ll}\text { Calc. No. } \\ \text { W320-28-030 }\end{array} & \begin{array}{l}\text { Title } \\ \text { Cooling Load in Pump Pit 241-AY-102 }\end{array} & \begin{array}{l}\text { Page } \\ \mathrm{A}-\mathrm{i}\end{array} \\ \text { W320-28-031 } & \begin{array}{l}\text { Pressure Rel ief Seal Loop Design } \\ \text { Verification }\end{array} & \mathrm{B}-\mathrm{i} \\ \text { W320-28-032 } & \begin{array}{l}\text { Process Building Piping Stress Analysis } \\ \text { W320-28-033 }\end{array} & \begin{array}{l}\text { Process Building Duct Support } \\ \text { Stress Analysis }\end{array} \\ \text { W320-28-034 } & \begin{array}{l}\text { Exhaust Skid Maximum A17owable } \\ \text { Leakage Criteria }\end{array} & \text { E-i } \\ \text { W320-28-035 } & \begin{array}{l}\text { Recirculation Heater, N509 Duct } \\ \text { Requirements }\end{array} & \text { F-i }\end{array}$


HNF-2486, Rev, 0

W320-28-030

Cooling Load in Pump Pit 241-AY-102 


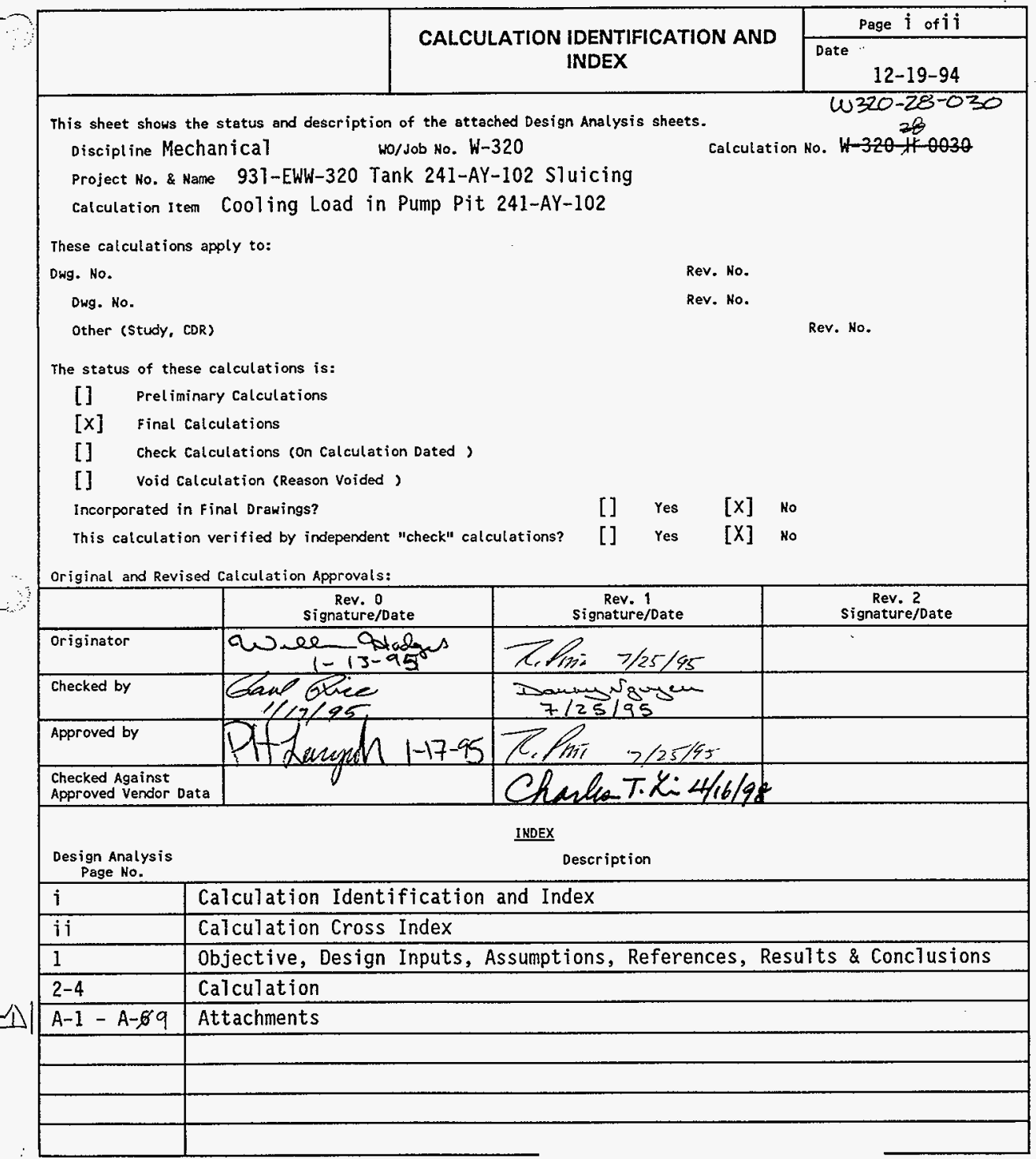


Client: WHC

Subject: COOLING LOAD ON PUMP PIT 241-AY-102

Location: 241-AY Tank Farm
Wo/Job No. W-320

Date 12-14-94 By William Hodges

checked $1 / 17 / 95$ By 6 . Piee

Revised $7 / 7 / 45$

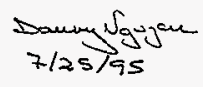

OBJECTIVE:

The purpose of the following calculations is to estimate the termperature of pump pit 241-AY-102 located at the AY tank farm and determine the cooling requirements.

DESIGN INPUTS:

1. The pump motor is variable speed rated at $125 \mathrm{hp}$. (normal operating) per Ref.(8).

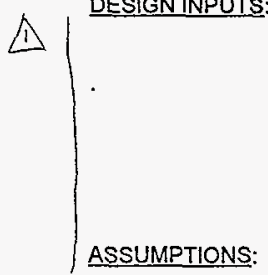

2. The pitambientesigntemperature is 120 of

3. The exterior walls are of concrete construction.

4. The cover block is $2 \mathrm{ft}$.

5. The pit demensions are as shown on drawing H-2-64314 Sht 1 Rev 1 . .

6. Maximum allowable pit temperature is $180^{\circ} \%$ per Ref.(7),

ASSUMPTIONS: 1.- Maximum-acoptablo air temperature is $410^{\circ}$ F with a salety factor of 10 -4.:

2. Outside air temperature is assumed to be $101^{\circ} \mathrm{F}$, inside air temperature is assumed to be $110^{\circ} \mathrm{F}$. The sol-air temp of the cover is assumed at $130^{\circ} \mathrm{F}$. (Ref 1 Table 1), therefore "delfa- $T^{\prime \prime}$ will be assumed to be $20^{\circ} \mathrm{F}$.

3. The temperature of the earth around the pit is $78.7^{\circ} \mathrm{F}$ (PNL data average value for August).

4. The pump is the only heat source in the pit..

\section{REFERENCES:}

1. ASHRAE Fundamentals, 1993

2. Genstrthetion gtide-Spec. (GS46400.sp-seetion 2.3.2)

3. $\mathrm{H}-2-64314$ Sht. 1

4. WHG-SOWA/320-FBCOOTREV:2

5. H-2-818693 Sht. 1 Rev. 0

6. tol $7 F 800-94054$

7. LOI $8 K 800-95.026$ - 8. LAWRENLE PLMP PERFORMAMLE CUT SHEET

RESULTS \& CONCLUSIONS

The final pit temperature without cooling is $192^{\circ} \mathrm{F}$.

The cooling required to maintain $110^{\circ} \mathrm{F}$. is $11,000 \mathrm{Btu} / \mathrm{hr}$.
9. Reliance motor cut SHTEeT

$$
12,000
$$

HNF-2486, Rev. 0 
Client: DEPARTMENT OF ENERGY

Subject: COOLING LOAD ON PUMP PIT 241-AY-102

Location: 241-AY Tank Farm
WO/Job No. W-320

Date 12-14-94 By William Hodges

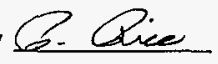

Revised

By

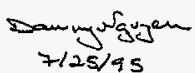

\section{CALCULATION}

\section{R-Values}

1. inside Air film

(REF. 1)

3 Concrete per inch thickness

$$
R_{\text {ia }}:=.68 \quad h^{\star} \mathrm{ft}^{2 \star} \mathrm{F} / \mathrm{Btu}
$$

$$
R_{c}:=0.075 \quad h^{*} \mathrm{ft}^{2 *} \mathrm{~F} / \mathrm{Btu}
$$

2. Cover Air film

4. Air Film floor

$$
\begin{aligned}
& R_{\text {ica }}:=.61 h^{\star} \mathrm{ft}^{2 \star} \mathrm{F} / \text { Btu } \\
& \mathrm{R}_{\text {oca }}:=.25
\end{aligned}
$$

$$
R_{f i}:=.92 \quad h^{\star} \mathrm{ft}^{2 *} \mathrm{~F} / \mathrm{Btu}
$$

\section{U-Valușes}

I ft. thick concrete construction

$$
U_{w 1}=1 / R
$$

$$
\begin{aligned}
& \mathrm{U}_{\mathrm{wl}}:=\frac{1}{\mathrm{R}_{\mathrm{ia}}+\mathrm{R}_{\mathrm{e}}} \cdot \frac{12}{12} \\
& U_{w l}=0.6329 \quad B t u / h r^{*} f^{2 *} F
\end{aligned}
$$

$2 \mathrm{ft}$. thick concrete cover

$$
\begin{aligned}
& U_{c 2}:=\frac{1}{R_{i c a}+R_{o c a}+R_{c}} \cdot \frac{24}{} \\
& U_{c 2}=0.3759 \quad \text { Btu/hr*tt }{ }^{* *} F
\end{aligned}
$$

$2 \mathrm{ft}$. thick concrete floor

$$
\begin{aligned}
& U_{f 2}:=\frac{1}{R_{f i}+R_{c}} \cdot \frac{24}{} \\
& U_{f 2}=0.3676 \quad B t u / h r^{*} f^{2 *}{ }^{*} F
\end{aligned}
$$

\section{AREAS}

Wall areas-Total area of the $1 \mathrm{ft}$ concrete wall is found to be $182 \mathrm{ft}^{2}$. (Ref. 3)

Total area of the cover and floor is found to be $48 \mathrm{ft}^{2}$ each.

From Newton's Law of Cooling:

$$
Q=U A(\text { delta- } T \text { ) }
$$

$Q$ is the heat loss, Btu/hr, $U$ is the heat transfer coefficient of the material, $A$ is the total surface area, and delta- $T$ is the desired temperature change. In this case: 
DESIGN ANALYSIS

Calc. No. W320-ל- -0030

Revision $\bullet$

Page 3 of 4

Client: WHC

WO/Job No. W-320

Subject: COOLING LOAD ON PUMP PIT 241-AY-102

Date 12-14-94 By William Hodges

Location: 241-AY Tank Farm
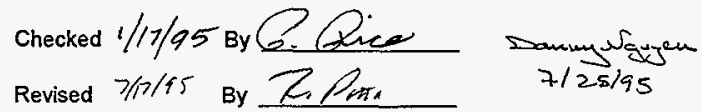

Heat produced by the motor due to ineficiecy of the motor. (Ref-2)

Efficiency $=95.4 \%$ - $425 \mathrm{hp}$-(Ref 5 )

$Q_{m}:=125.046 \cdot 746 \cdot 3.413 \cdot\left(\frac{1-.137}{.937}\right)$

$\begin{aligned} Q_{m}= & 1464 \cdot 10^{4} \mathrm{Btu} / \mathrm{hr} \\ & 16,259.5\end{aligned}$

$\frac{89 b h p}{.937}=94.98 \mathrm{ehp}$

bhp per Attachment 8

motor efficiency pir

At tochment

Surface area of the pump pit.

Area of the $1.5 \mathrm{ft}$. thick concrete wall.

$A_{1}:=182 \quad \mathrm{Ft}^{2}$

Area of the $2 \mathrm{ft}$. thick concrete floor.

$A_{\mathrm{f} 2}:=48 \quad \mathrm{Ft}^{2}$

Area of the Cover Block

$\mathrm{A}_{\mathbf{r}}:=48 \quad \mathrm{Ft}^{2}$

The final temperature in the pit due to the heat of the motor.

$Q_{m}=\left[U_{c 2} \cdot A_{r} \cdot\left(T_{1}-130\right)+U_{f 2} \cdot A_{f 2} \cdot\left(T_{1}-78.7\right)+U_{w 1} \cdot A_{1} \cdot\left(T_{1}-78.7\right)\right]$

$\mathrm{T}_{1}:=-1 \cdot \frac{\left(\mathrm{Q}_{\mathrm{m}}+130 \cdot-\mathrm{U}_{\mathrm{c} 2} \cdot \mathrm{A}_{\mathrm{r}}+78.7 \cdot \mathrm{U}_{\mathrm{f} 2} \cdot \mathrm{A}_{\mathrm{f} 2}\right.}{\left(-1 \cdot \mathrm{U}_{\mathrm{c} 2} \cdot \mathrm{A}_{\mathrm{r}}-1 \cdot \mathrm{U}_{\mathrm{f} 2} \cdot \mathrm{A}_{\mathrm{f} 2}-1 \cdot 18.7 \cdot \mathrm{U}_{\mathrm{w} 1}\right.} \cdot \frac{\left.\mathrm{U}_{\mathrm{w} 1}\right)}{\left.\mathrm{A}_{1}\right)}$

192.6

$\mathrm{T}_{1}=181.8552 \quad{ }^{\circ} \mathrm{F}$

The final estimated temperature of the Pump pit due to the heat of the motor

is estimated at $182^{\circ} \mathrm{F}$. Cooling is requised sinke it exceeds $180^{\circ} \mathrm{F}$ limit.

193

HNF-2486, Rev. 0

Page A-4 
Client: WHC

Subject: COOLING LOAD ON PUMP PIT 241-AY-102

Location: 241-AY Tank Farm
WO/Job No. W-320

Date 12-14-94 By William Hodges

checked 1/17/95 By D. Diee

Revised $7 / 2 / 45$ By Pr Plas.

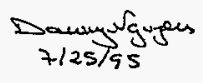

Cooling required to iower the temperature to $110^{\circ} \mathrm{F}$. (Ref-

Delta- $\mathrm{T} \quad \Delta \mathrm{T}:=110-78.7$

Heat transfer through the walls, floor and cover block.

Walls

$\mathrm{Q}_{1}:=\mathrm{U}_{\mathrm{wl}} \cdot \mathrm{A}_{1} \cdot \Delta \mathrm{T}$

$\mathrm{Q}_{1}=3.6054 \cdot 10^{3} \quad \mathrm{Btu} / \mathrm{hr}$

Floor

$Q_{2}:=U_{f 2} \cdot A_{12} \cdot \Delta T$

$\mathrm{Q}_{2}=552.3529$

Btu/hr

Cover Block

$Q_{\mathrm{r}}:=U_{\mathrm{c} 2} \cdot \mathrm{A}_{\mathrm{r}} \cdot(110-130)$

$Q_{r}=-360.9023$

Btu/hr

$$
Q:=\left[Q_{m}-\left(Q_{1}+Q_{2}+Q_{r}\right)\right]
$$

$$
Q=1,7411084 \cdot 10^{4} \quad \text { Btu/hr }
$$

The cooling required for the Pump Pit 241-AY-102 is 11,000 Btu/hr. 
Table 1 Sol-Air Temperatures $t_{\text {f }}$ for July $21,40^{\circ} \mathrm{N}$ Latitude

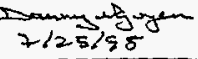

\begin{tabular}{|c|c|c|c|c|c|c|c|c|c|c|c|c|c|c|c|c|c|c|c|c|c|}
\hline & & & & & & & & & $=t_{0}$ & $+\alpha I_{t} / h_{0}$ & $a_{0} / h_{0}-$ & $\in \delta R$ & & & & & & & & & \\
\hline & Air & & & $\mathrm{ght} \mathrm{C}$ & lored & Surfac & e, $\alpha / h_{0}$ & $=0.1$ & & & & Air & & & ark Co & lored & Surface & $\alpha / h_{0}$ & $=0.3$ & & \\
\hline Time & $\begin{array}{c}\text { Temp., } \\
t_{0}\end{array}$ & $\mathbf{N}$ & NE & $\mathbf{E}$ & SE & $s$ & Sw & w & $\mathrm{NW}$ & HOR & Time & $\begin{array}{c}\text { Temp., } \\
\text { of }\end{array}$ & $\mathbf{N}$ & $\mathrm{NE}$ & E & SE & $\mathbf{s}$ & SW & W & NW & $\mathrm{HOR}$ \\
\hline 1 & 76 & 76 & 76 & 76 & 76 & 76 & 76 & 76 & 76 & 69 & 1 & 76 & 76 & 76 & 76 & 76 & 76 & 76 & 76 & 76 & 69 \\
\hline 2 & 76 & 76 & 76 & 76 & 76 & 76 & 76 & 76 & .76 & 69 & 2 & 76 & $76^{\circ}$ & 76 & 76 & 76 & 76 & 76 & 76 & 76 & 69 \\
\hline 3 & 75 & 75 & 75 & 75 & 75 & 75 & 75 & 75 & 75 & 68 & 3 & 75 & 75 & 75 & 75 & 75 & 75 & 75 & 75 & 75 & 68 \\
\hline 4 & 74 & 74 & 74 & 74 & 74 & 74 & 74 & 74 & 74 & 67 & 4 & 74 & 74 & 74 & 74 & 74 & 74 & 74 & 74 & 74 & 67 \\
\hline 5 & 24 & 74 & 74 & 74 & 74 & 74 & 74 & 74 & 74 & 67 & 5 & 74 & 74 & 75 & 75 & 74 & 74 & 74 & 74 & 74 & 67 \\
\hline 6 & 74 & 80 & 93 & 95 & 84 & 76 & 76 & 76 & 76 & 72 & 6 & 74 & 85 & 112 & 115 & 94 & 77 & 77 & 77 & 77 & 77 \\
\hline 7 & 75 & 80 & 99 & 106 & 94 & 78 & 78 & 78 & 78 & 81 & 7 & 75 & 84 & 124 & 136 & 113 & 81 & 81 & 81 & 81 & 94 \\
\hline 8 & 77 & 81 & 99 & 109 & 101 & 82 & 81 & 81 & 81 & 92 & 8 & 77 & 85 & 121 & 142 & 125 & 86 & 85 & 85 & 85 & 114 \\
\hline 9 & 80 & 85 & 96 & 109 & 106 & 88 & 85 & 85 & 85 & 102 & 9 & 80 & 90 & 112 & 138 & 131 & 96 & 89 & 89 & 89 & 131 \\
\hline 10 & 83 & 88 & 91 & 105 & 107 & 95 & 88 & 88 & .88 & 111 & 10 & 83 & 94 & 100 & 127 & 131 & 107 & 94 & 94 & 94 & 145 \\
\hline 11 & 87 & 93 & 93 & 99 & 106 & 102 & 93 & 93 & 93 & 118 & II & 87 & 98 & 99 & 111 & 125 & 118 & 100 & 98 & 98 & 156 \\
\hline 12 & 90 & 96 & 96 & 96 & 102 & 106 & 102 & 96 & 96 & 122 & 12 & 90 & 101 & 101 & 102 & 114 & 123 & 114 & 102 & 101 & 162 \\
\hline 13 & 93 & 99 & 99 & 99 & 99 & 108 & 112 & 105 & 99 & $124+6$ & 13 & 93 & 104 & 104 & 104 & 106 & 124 & 131 & 117 & 105 & 162 \\
\hline 14 & 94 & 99 & 99 & 99 & 99 & 106 & 118 & 116 & 102 & 122 & 14 & 94 & 105 & 105 & 105 & 105 & 118 & 142 & 138 & 111 & 156 \\
\hline 15 & $95+6$ & 100 & 100 & 100 & 100 & 103 & 121 & 124 & iII & 117 & 15 & 95 & 105 & 104 & 104 & 104 & ill & 146 & 153 & 127 & 146 \\
\hline 16 & 94 & 98 & 98 & 98 & 98 & 99 & i18 & 126 & 116 & 109 & 36 & 94 & 102 & 102 & 102 & 102 & 103 & 142 & 159 & 138 & 131 \\
\hline 17 & 93 & 98 & 96 & 96 & 96 & 96 & 112 & 124 & 117 & 99 & 17 & 93 & 102 & 99 & 99 & 99 & 99 & $13 !$ & 154 & 142 & 112 \\
\hline 18 & 91 & 97 & 93 & 93 & 93 & 93 & 101 & 112 & 110 & 89 & 18 & 91 & 102 & 94 & 94 & 94 & 94 & 111 & 132 & 129 & 94 \\
\hline 19 & 87 & 87 & 87 & 87 & 87 & 87 & 87 & 87 & 87 & 80 & 19 & 87 & 87 & 87 & 87 & 87 & 87 & 87 & 88 & 88 & 80 \\
\hline 20 & 85 & 85 & 85 & 85 & 85 & 85 & 85 & 85 & 85 & 78 & 20 & 85 & 85 & 85 & 85 & 85 & 85 & 85 & 85 & 85 & 78 \\
\hline 21 & 83 & 83 & 83 & 83 & 83 & 83 & 83 & 83 & 83 & 76 & 21 & 83 & 83 & 83 & 83 & 83 & 83 & 83 & 83 & 83 & 76 \\
\hline 22 & 81 & 81 & 81 & 81 & 81 & 81 & 81 & 81 & 81 & 74 & 22 & 81 & 81 & 81 & 81 & 81 & 81 & 81 & 81 & 81 & 74 \\
\hline 23 & 79 & 79 & 79 & 79 & 79 & 79 & 79 & 79 & 79 & 72 & 23 & 79 & 79 & 79 & 79 & 79 & 79 & 79 & 79 & 79 & 72 \\
\hline 24 & 77 & 77 & 77 & 77 & 77 & 77 & 77 & 77 & 77 & 70 & 24 & 77 & 77 & 77 & 77 & 77 & 77 & 77 & 77 & 77 & 70 \\
\hline $\mathrm{v}$ & 83 & 86 & 88 & 90 & 90 & 87 & 90 & 90 & 88 & 90 & Avg. & 83 & 89 & 94 & 99 & 97 & 93 & 97 & 99 & 94 & 104 \\
\hline
\end{tabular}

Note: Sol-air temperatures are calculated based on $\epsilon \delta / h_{0}=-7^{\circ} F$ for horizontal surfases and $0 . F$ for vertical surfaces.

Adjustmenis. Sol-air temperature cycles can be estimated for other dares and latitudes by using the data in Tables 12 through 18 , Chapter 27 . For any of the times, dates, and wall orientations listed in those tables, the value of $I_{t}$ is approximately $1.15 \times$ SHGF. However, the I.IS factor is approximate and only accounts for the solar energy excluded by a single sheet of ordinary window glass. For surfaces with other orientations or slope angles of other than $0^{\circ}$, and for more accurate estimates at incident angles above $50^{\circ}$ (particularly critical for southern exposures), the solar intensity can be found by the method outlined in Chapter 27 .

Average sol-air temperature. The average daily sol-air temperature $t_{e a}$ can be calculated for any of the situations covered by Tables 12 through 18 of Chapter 27:

$$
t_{* 0}=t_{o 0}+\alpha /\left[h_{o}\left(I_{D r} / 24\right)\right]-\left(\epsilon \Delta R / h_{o}\right)
$$

where $I_{D T}$ is the sum of two appropriate half-day totals of solar heat gain in $\mathrm{Btu} /\left(\mathrm{h} \cdot \mathrm{ft}^{2}\right)$. For example, the average sol-air temperature for a wall facing southeast at $40^{\circ} \mathrm{N}$ latitude on August 21 would be

$$
t_{e a}=t_{o q}+\alpha / h_{o}[1.15(956+205) / 24]
$$

The daily solar heat gain of double-strength sheet glass is $956+$ $20 \mathrm{Btu} /\left(\mathrm{h} \cdot \mathrm{ft}^{2}\right)$ in a southeast facade at this latitude and date (Table 16, Chapter 27); and $\epsilon \Delta R / h_{o}$ is assumed to be zero for this vertical surface.

Hourly air temperatures. The hourly air temperatures in Column 2, Table 1 are for a location with a design temperature of $95^{\circ} \mathrm{F}$ and a range of $21^{\circ} \mathrm{F}$. To compute corresponding temperatures for other locations, select a suitable design temperature from Column 6, Table 1 of Chapter 24 and note the outdoor daily range (Column 7). For each hour, take the percentage of the daily range indicated in Table 2 of this chapter and subtract from the design temperature.
Table 2 Percentage of Daily Range

\begin{tabular}{crrrrr}
\hline Time, h & $\sigma_{70}$ & Time, h & $\%$ & Time, $\mathrm{h}$ & $\sigma_{0}$ \\
\hline 1 & 87 & 9 & 71 & 17 & 10 \\
2 & 92 & 10 & 56 & 18 & 21 \\
3 & 96 & 11 & 39 & 19 & 34 \\
4 & 99 & 12 & 23 & 20 & 47 \\
5 & 100 & 13 & 11 & 21 & 58 \\
6 & 98 & 14 & 3 & 22 & $6 \%$ \\
7 & 93 & 15 & 0 & 23 & $7 t$ \\
8 & 84 & 16 & 3 & 24 & 8 \\
\hline
\end{tabular}

Example 1. Air temperature calculation. Calculate the summer dry-t temperature at $1200 \mathrm{~h}$ for Carson City, Nevada.

Solution: From Table 1, Chapter 24 , the daily range is $42^{\circ} \mathrm{F}$ the $1 \%_{0}$ design dry-bulb temperature is $93^{\circ} \mathrm{F}$. From Table 2 , the perc age of the daily range at 1200 hours is $23 \%$. Thus, the dry-bulb temf ture at $1200 h=$ Design dry-bulb - (Daily range $\times$ Percentage $)=$ $-[42(23 / 100)]=83.3^{\circ} \mathrm{F}$

Data limitations. The summer design temperatures in Tal Chapter 24, are based on the 4-month period, June through tember. (Canadian data are based on July only.) The outdoor range is the difference between the average daily maximum average daily minimum temperatures during the warmest $m$ More reliable results could be obtained by determining or est ing the shape of the temperature curve for typical hot days ? building site and considering each month separately. Peak ing load is often determined by solar heat gain through fenc tion; this peak may occur in winter months and/or at a time $c$ when outside air temperatuse is not at its peak.

\section{Heat Gain through Fenestration}

The sections that include Equations (30) through (41) in ter 27 describe one method used to calculate space coolin: resulting from heat transfer through fenestration. The sola gain profiles listed in Chapter 27 are for fenestration area 


\section{THERMAL AND WATER VAPOR TRANSMISSION DATA}

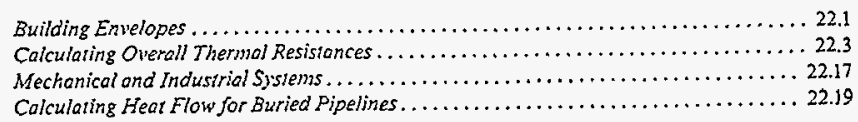

HIS chapter presents thermal and water vapor transmission data based on steady-state or equilibrium conditions. Chapter 3 covers heat transfer under transient or changing temperature conditions. Chapter 20 discusses selection of insulation materials and procedures for determining overall thermal resistances by simpliffed methods.

\section{BUILDING ENVETOPES}

\section{Thermal Transmission Data for Building Components}

The sieady-state thermal resistances (R-values) of building components (ualls, floors, windows, roof systems, etc) can be calculated from the thermal properties of the materials in the componemt; or the heat flow through the assembled component can be measured directly with laboratory equipment such as the guarded hot box (ASTM Siandard C 236) or the calibrated hot box (ASTMA Sionotard C 976).

Tables 1 through 6 list thermal values, which may be used to calculate thermal resistances of building walls, floors, and cejlings. The values show'n in these tables were developed under ideal conditions. In practice, overall thermal performance can be reduced sienificently by such factors as improper installation and shrink-

Table 1 Surface Conductances and Resistances for Air

\begin{tabular}{|c|c|c|c|c|c|c|c|}
\hline \multirow{3}{*}{$\begin{array}{l}\text { Position of } \\
\text { Surface }\end{array}$} & \multirow{3}{*}{$\begin{array}{l}\text { Direction of } \\
\text { Heat Flow }\end{array}$} & \multicolumn{6}{|c|}{ Surface Emiltance, $t$} \\
\hline & & \multicolumn{2}{|c|}{$\begin{array}{l}\text { Ton- } \\
\text { reflective } \\
\epsilon=0.90\end{array}$} & \multicolumn{4}{|c|}{$=0.20 \quad$ Reflective } \\
\hline & & $h_{i}$ & $R$ & $h_{1}$ & $R$ & $h_{i}$ & $R$ \\
\hline $\begin{array}{l}\text { STll AIR } \\
\text { Horizontal } \\
\text { Sloping-45 } \\
\text { Vertical } \\
\text { Sloping-45 } \\
\text { Horizonial }\end{array}$ & $\begin{array}{l}\text { Upward } \\
\text { Upward } \\
\text { Horizonial } \\
\text { Downuard } \\
\text { Downward }\end{array}$ & $\begin{array}{l}1.63 \\
1.60 \\
1.46 \\
1.32 \\
1.08\end{array}$ & $\begin{array}{l}0.61 \\
0.62 \\
0.68 \\
0.76 \\
0.92\end{array}$ & $\begin{array}{l}0.91 \\
0.88 \\
0.74 \\
0.60 \\
0.37\end{array}$ & $\begin{array}{l}1.10 \\
1.14 \\
1.35 \\
1.67 \\
2.70\end{array}$ & $\begin{array}{l}0.76 \\
0.73 \\
0.59 \\
0.45 \\
0.22\end{array}$ & $\begin{array}{l}1.32 \\
1.37 \\
1.70 \\
2.22 \\
4.55\end{array}$ \\
\hline MONINC & ny position) & $h_{0}$ & $R$ & $h_{0}$ & $R$ & $h_{0}$ & $R$ \\
\hline $\begin{array}{l}\text { 15-mph wind } \\
\text { (for winter) }\end{array}$ & Any & 6.00 & 0.17 & - & - & - & - \\
\hline $\begin{array}{l}\text { 7.5-mph Wind } \\
\text { (for summer) }\end{array}$ & Any & 4.00 & 0.25 & - & - & - & - \\
\hline
\end{tabular}

Noies

1. Surface conductance $h_{i}$ and $h_{0}$ measured in $\mathrm{Blu} / h \cdot \mathrm{f}^{2} \cdot{ }^{\circ} \mathrm{F}$; resistance $R$ in $\mathrm{F} \cdot \mathrm{ft}^{2} \cdot \mathrm{h} / \mathrm{Biv}$.

2. No susface has both an air space resistence raiue and 2 surface resistance value. 3. For ventilated aties or spaces abore ceilings under summer conditions (heat flow Coxin), see Table 5 .

4. Conductances ate for surfaces of the stated eminance facing virual blackbody surroundings at the same temperatute as the ambient air. values ate based on a surface-air iemperalure difference of $10^{\circ} \mathrm{F}$ and for su: face iempetatures of $70^{\circ} \mathrm{F}$.

1. Sec Chapler 3 for more detziled information, especielly Tables $\$$ and 6 , and see Figure 1 for additional dasa.

6. Condensate cen have a significant impact on surface tmillance (see Table 3).

The preparalion of this chapter is assigned to TC. 4.4. Thermal lnsulation and Moisture Relasders. age, settling, or compression of the insulation (Tye and Desjarlais 1983, Tye 1985, 1986).

Most values in these ables were oblained by accepted ASTM test methods described in ASTM Standaro's C 177 and C $\leq 18$ for materials and ASTM Siandards C 236 and C 976 for building envelope components. Because commercially available materials vary, not all values apply to specific products. (Previous editions of the hanobook can be consulted for data on materials no longer commercially available.)

The most accurate meihod of determining the overall thermal resistance for a combination of bujlding maierials assembled as a building envelope component is to test a representative sample by a hot box method. However, all combinations may not be conveniently or economically iested in this manner. For many simple consiructions, calculared R-values agree reasonably well with izlues determined by hot box measurement.

The performance of materials fabricated in the field is especially subject to the quality of workmanship during construction and installation. Good wortunanship becomes increasingly important as the insulation requirement becomes greater. Therefore, some engineers include aoditional insulation or other safeiy faciors based on experience in their design.

Figure 1 show's how convection alfects surface conductance of several materials. Other tests on smooth surfaces show that the average value of the convection part of conduclance decreases as the length of the surface increases.

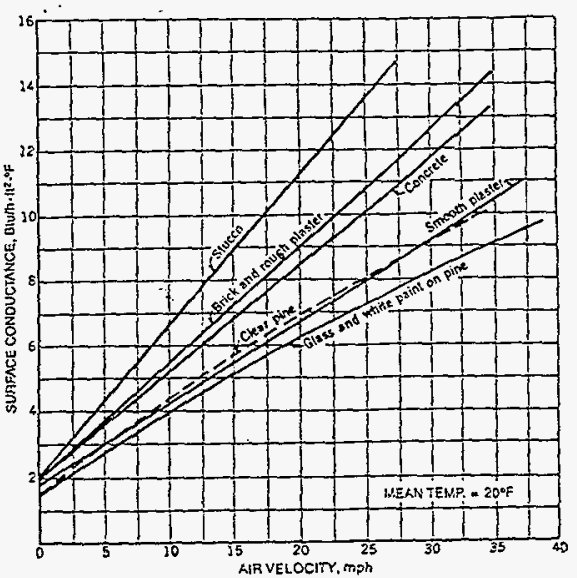

Fig. 1 Surface Conductance for Different 12-Inch-Square Surfaces as Affected by Air Movement 


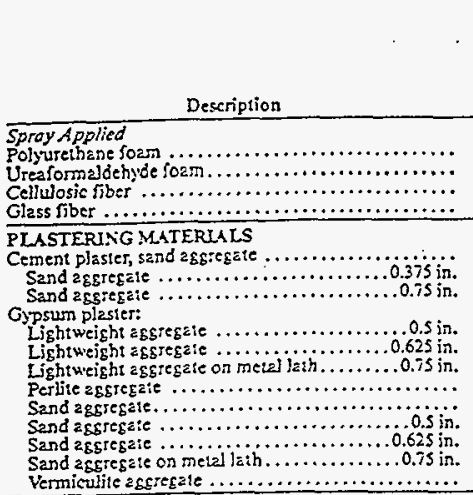

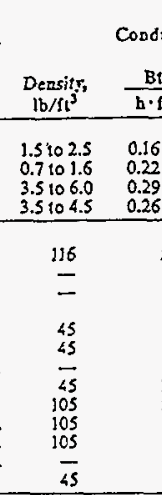

\section{Resistepce $R^{b}$}

Per Inch For Thickdess

Thicksess

$(1 / k)$

civitye Conoustance

1u-in $h \cdot 1 t^{2} \cdot 0 F$

$\frac{C}{b \cdot f t^{2} \cdot C F}$

${ }^{-} \mathrm{F} \cdot \mathrm{ft}^{2} \cdot \mathrm{h}$

Btu-ir.

Listed

(1/C)

FE: $\mathrm{II}^{2} \cdot \mathrm{h}$

6.25105 .56

$=$

$\overline{=}$

4.55103 .57

3.45102 .94 3.85103 .70

$\frac{B i a}{\mathrm{~B} \cdot \mathrm{CF}^{-F}}$

\section{MASONRY MATERIALS}

Concretes

Cemedi morias

Gypsum fiber comcicte.............

(1)

Lightweight assrtgaies including expendes st,de clay or slate; expanded slags; cinders; purice; vermiculite; also celluler concretes

Perlite expended

Sand and gravel or sione aggregate (oven died)

Sand and gravel or stone aggregate (rol dried) ........

SIDLNG MATERLALS (on Mat suriact)

Shingles

Asbestos-cement $\ldots \ldots \ldots \ldots \ldots \ldots \ldots \ldots \ldots \ldots \ldots$

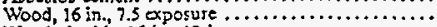

Wood, double, 16-in., 12-in. exposuze.............

Wood, plus insulating backer boasd, 0.3125 in. ....... Siding

Asbertos-cement, 0.25 in., lapped ..............

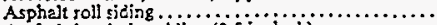

Asphalt insulating sioing $(0.5$ in. bed..$\ldots \ldots \ldots \ldots \ldots$

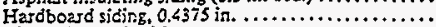

Wood, djop, 1 ty 8 in...........................

Wood, bevel, 0.5 ky $8 \mathrm{in}$, lapped .................

Wood, bevel, 0.75 by 10 in, lapped ..................

Wood, plywood, 0.375 in., lapped ................

Aluminum or Stetl, over sheathing

Hollow-backed........................

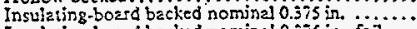

Insulating-bord backed nominal 0.375 in., foil

backed..................................

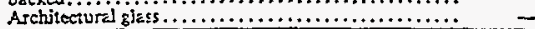

FOODS (12\% Moisture Conteni) him

Hardroods

Ozk................................ 41.21046 .8

Birch ............................... 42.6 to 45.4

Mapje .................................... 39.81044 .0

Ash ............................... 38.41041 .9

Sofiwoods

Southern Pine ....................... 55.61041 .2

Douglas Fir-Larch ........................ 33.5 to 36.3

Southers Cypress ........................ 31.4 to 32.1

Hers-Frr, Spruce-Pine-Fir.................. 24.5 10 31.4

West Coast woods, Cedars........................ 21.7 to 31.4

California Redwood ...................... 24.5 to 28.0

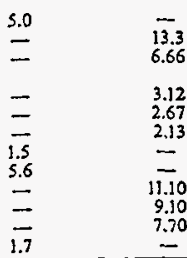

\subsection{0}

-

$\overrightarrow{0.08}$

0.15

0.20

0.20

6.66

$-$

0.32

2.67

2.13

11.10

9.10

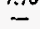

$=$
$\overline{0.67}$
0.18
$\overline{0}$
$\overline{0.59}$

0.32

0.47

$-$

0.32

0.20

0.11

0.13

.09

$\begin{array}{ll}\bar{z} & \\ = & \\ & \\ 0.08 & 0.20 \\ 0.15 & 0.20\end{array}$

\begin{tabular}{|c|c|c|}
\hline $0.20: 0.10$ & - & - \\
\hline 0.60 & - & 0.21 \\
\hline 0.18 to 0.09 & - & - \\
\hline 0.27 to 0.17 & - & 0.20 \\
\hline 0.40100 .29 & 一 & 0.20 \\
\hline 0.63100 .56 & - & - \\
\hline 1.08100 .90 & 一 & $\overrightarrow{3}$ \\
\hline 1.33101 .10 & $\rightarrow$ & 0.20 \\
\hline 1.59101 .20 & - & - \\
\hline 1.08 & - & 0.30 \\
\hline 1.41 & - & - \\
\hline 2.00 & - & 0.32 \\
\hline 0.13100 .06 & $\rightarrow$ & 0.38100 .22 \\
\hline 0.10100 .05 & $\rightarrow$ & 0.19100 .24 \\
\hline 0.20 & $\rightarrow$ & - \\
\hline
\end{tabular}

5.01010 .5

1.66

5.51011 .0

3.7105 .9

2.5 to 3.5

J.610 1.8

0.93 to 1.11

0.75100 .91

0.63100 .83

1.4101 .8

0.93

0.71

0.50

8.0 to 16.0

10.0 to 20.0 5.0 0.20
4.75

1.15
0.84

0.71

4.76

6.50

0.69

1.49

1.27

1.23

0.95

1.59

1.61

0.55

0 .

0.34

$\begin{array}{ll}= & 0.2 \\ = & 0.87 \\ & 1.19\end{array}$

0.21
0.87
1.19

1.40

- $\quad 0.21$

0.15

1.45

0.67

0.79

0.81

1.05

0.59

0.61
1.82

2.96

0.10

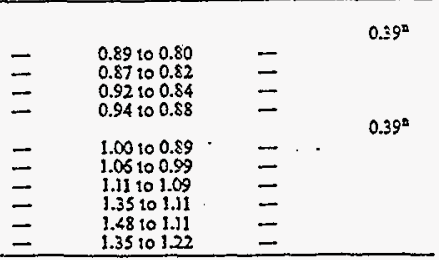

$0.5 i$

0.28

0.31

0.24

0.35

0.35

0.28

0.28

0.29

0.29

0.32

$$
-
$$

1.12101 .25

1.16 to 1.22

1.09101 .19

1.06101 .14

1.00101 .32

0.95 to 1.01

0.90100 .92

0.74100 .90

0.68100 .90

0.74100 .82

\begin{tabular}{l}
9 \\
4 \\
1 \\
92 \\
\hline
\end{tabular}


2.2.11.3 Equipment nameplates: Laminated plastic. 1/16-inch thick with white surface and black core, sized to meet legend requirements. Edges beveled and smooth. Engraved nomenclature sharp and clear. Engraved manufacturer's standard nameplates may be used if equal in quality and legibility.

2.2.11.3 Equipment nameplates: HPS I-2-7, sized to meet legend requirements. Engraved manufacturer's standard nameplates may be used if qqual in

2.3 quality and legibility.

2.3.1 Equipment Enclosures: NEMA ICS 6 Type [1], [3], [3RJ. [4], [12].

2.3.2 Motors: Provide motors with the following minimug nameplate efficiencies:
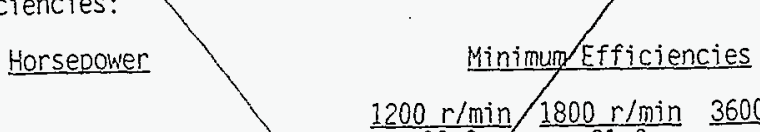

10
15
20
25
30
40
50
60
75
100
125
150
200
$200+$

2.3.3 Motor Contro] centers (MCC): [14-] [20-] inch deep enclosure for

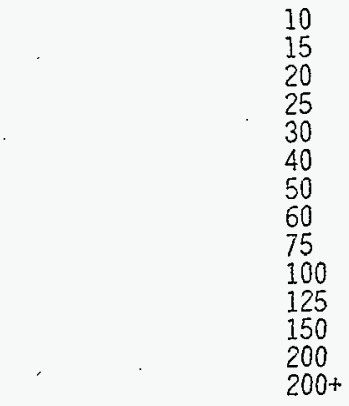
control equipment. assembled to provide dead-front unit, and meet the requirements of the Orawings. Incoming feeders sha 11 enter from [bottom] [top]. Size feeder terminal lugs to accept conductors specified.

2.3.3.1 Motor controllers: Horsepower rated with 2 NO and 2 NC auxiliary coptacts. Provide compartments with equipment shown on the Drawings.

2.3.3.2 Locate master terminal boards in [bottom] [top] sections and park with conductor numbers shown on the Drawings.

GS16400.SP

HNF-2486, Rev. 0

Page A-9

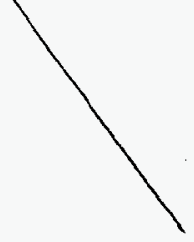

$<$ Spec No $>$ Rev 0 $10 / 26 / 94$ 


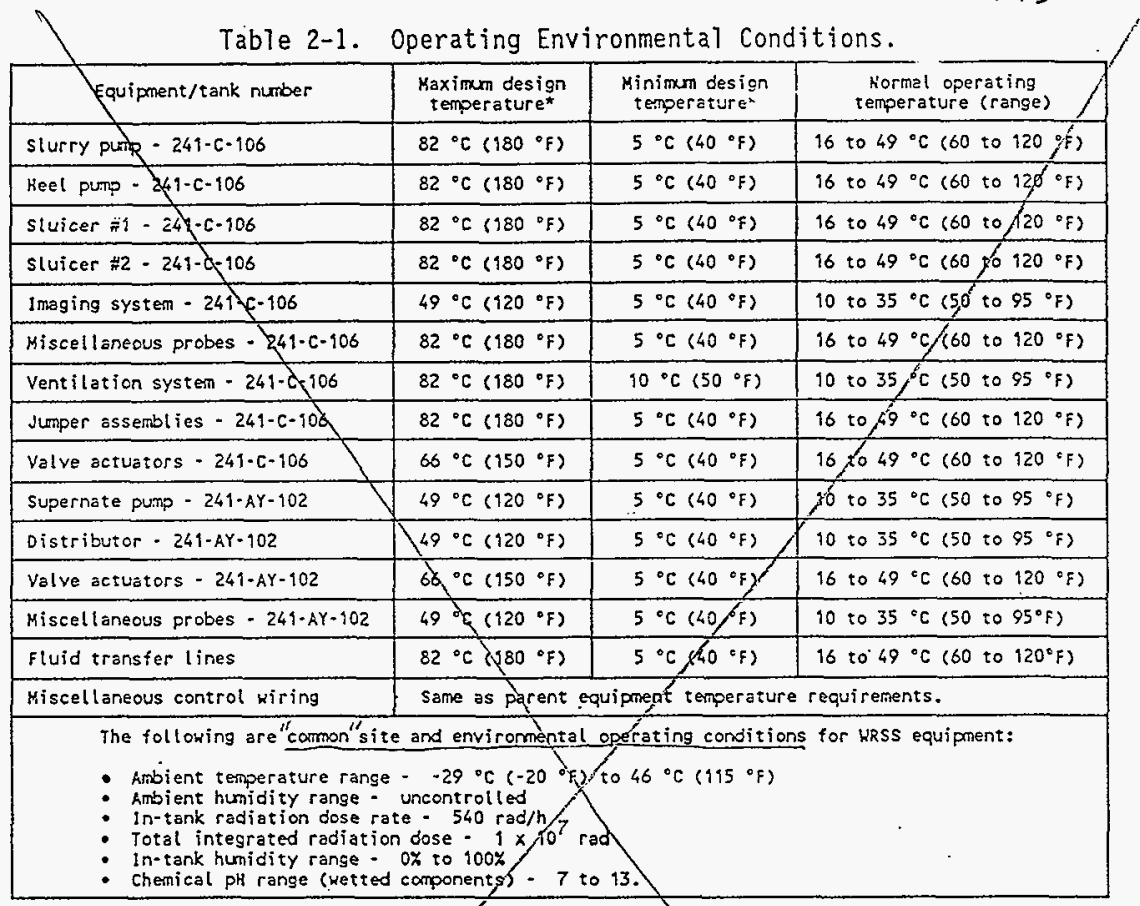

*Miniman and maximum design temperaturés are intended for purposes of structural design.

Note that tank 241-AY $102 \mathrm{pH}$ is maintained by $[\mathrm{OH}-1$ control requirements contained in the applicable operating specification documents. Tank 241-AY-102 supernatant shall be maintained within the [OH-] contro] Timits.

Information is unavailable on radiation levels within tank 241-AY-102. The level for tapk 241-C-106 shall be. used as a conservative estimate for design purposes.

\subsubsection{Operating Requirements.}

Equipment failure shall not preclude its removal. No modification to waste tanks (either single- or double-shell), risers, or pits shall be allowed to retrieve failed WRSS components or parent equipment.

HNF-2486, Rev. 0

Page A-10 


\section{3
3
3}
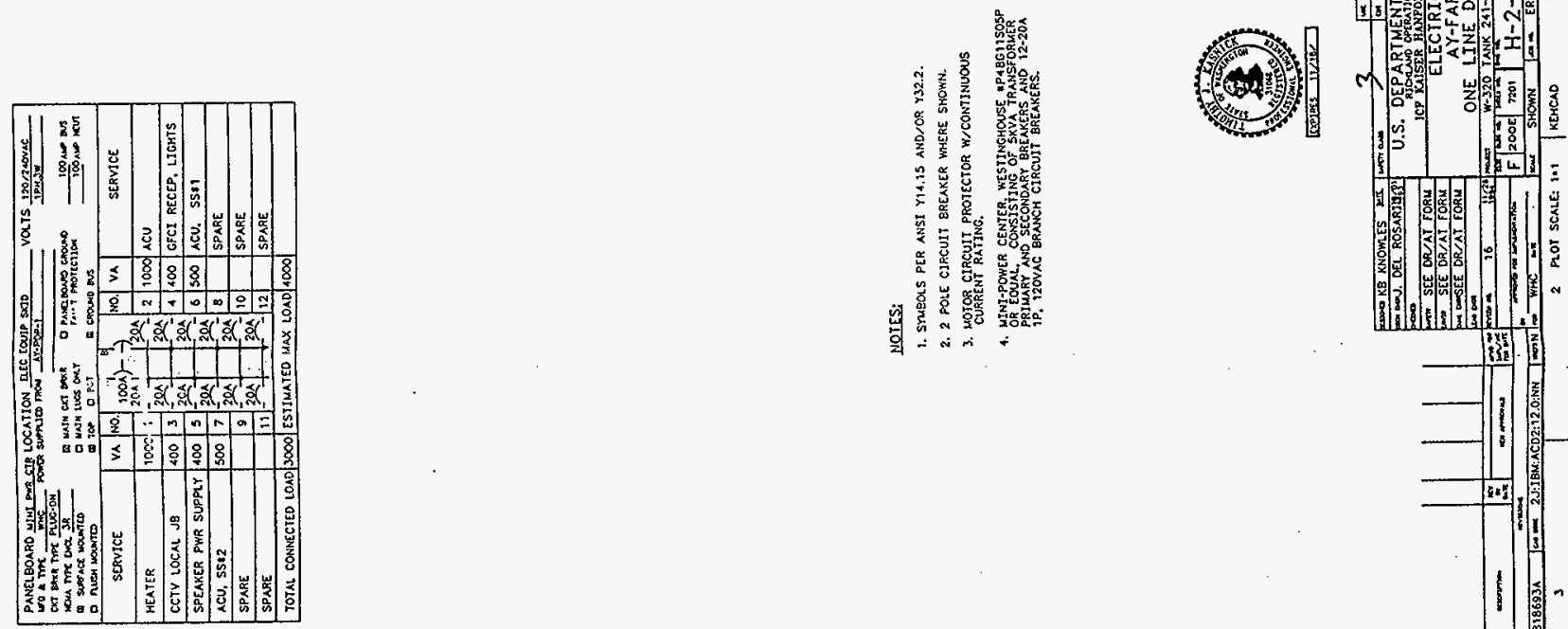

(n)

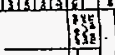

in

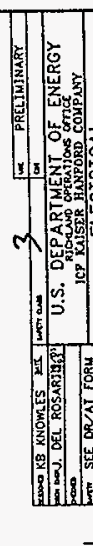

E\# गYด
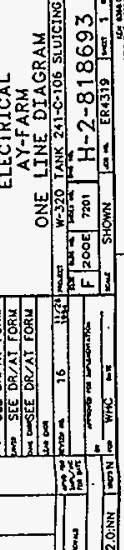

th

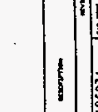

(1)
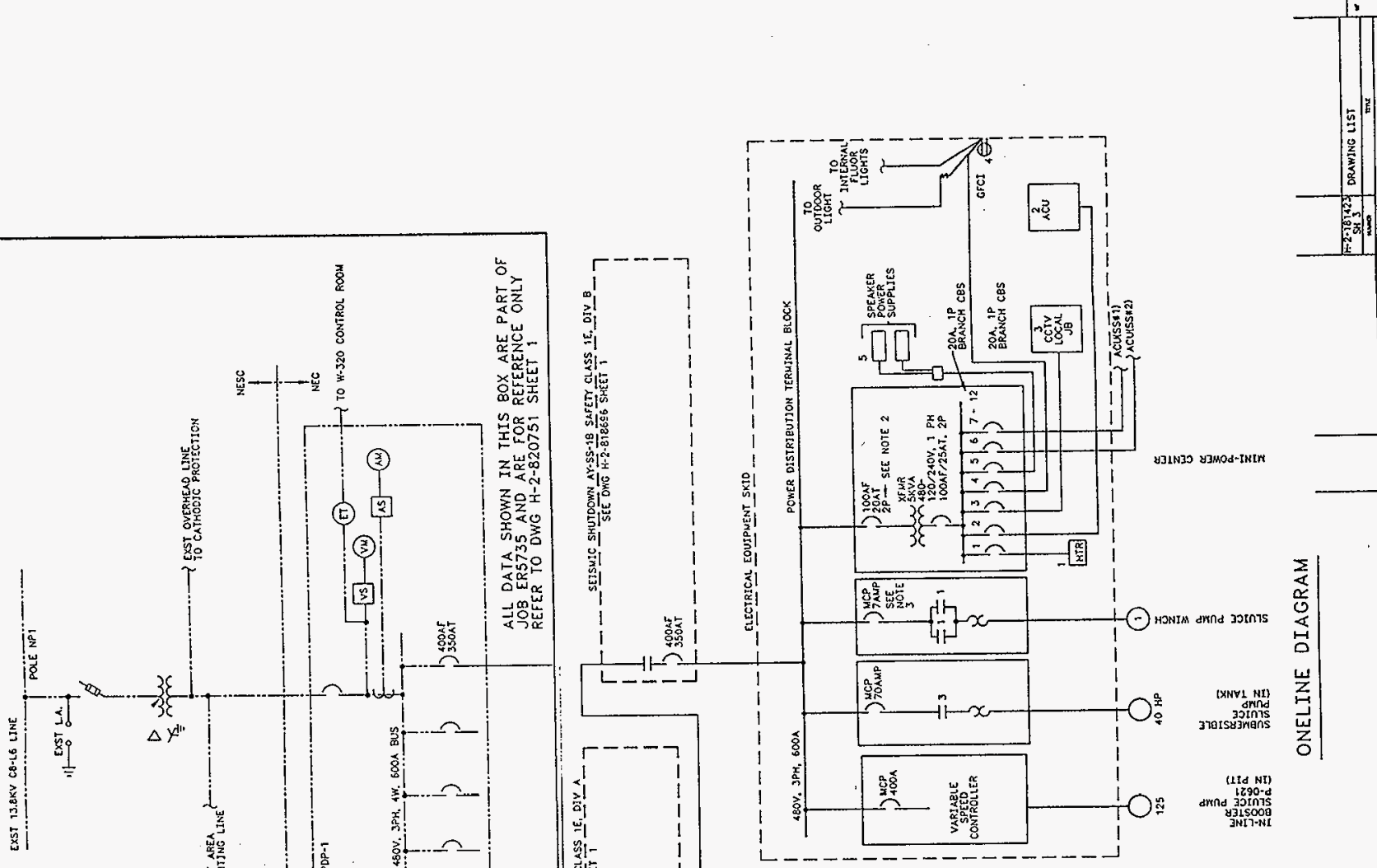

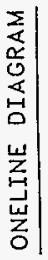
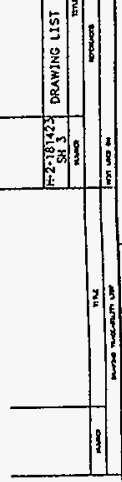

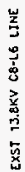
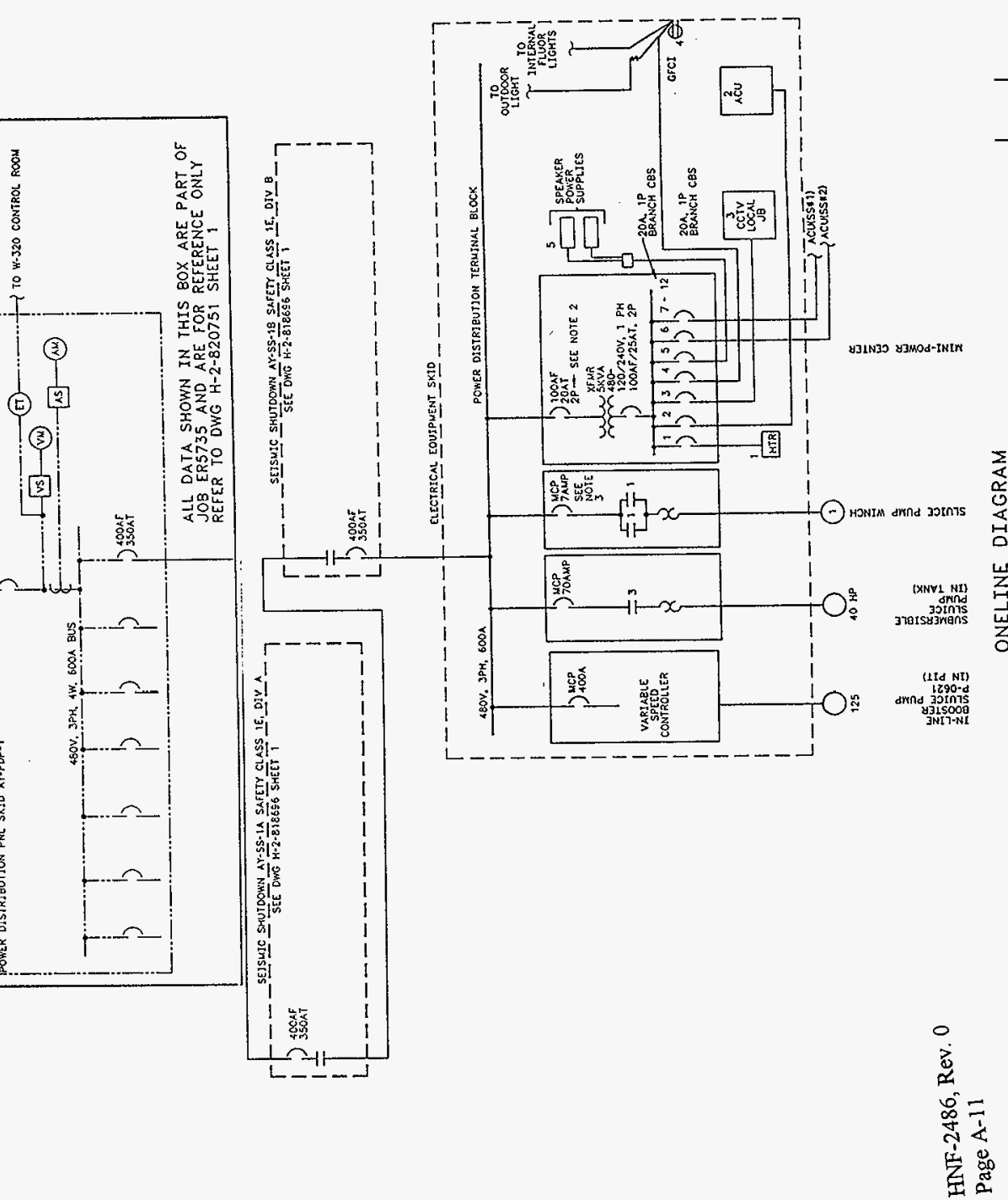
Date. December 13, 1994

Subject: PROJECT 93L-EWW-320, TANK 241-C-106 SLUICING, LETTER OF INSTRUCTION, DEFINITIVE DESIGN, ADDENDUM 7

To:

Reference:

\section{$53-07$}

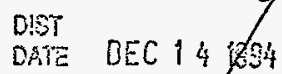

7F800-94-051

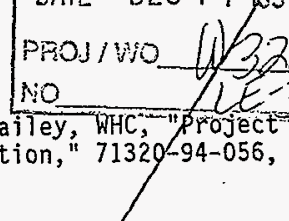

This Letter of Instruction (LOI) addendum provides design direction to ICF

$\mathrm{KH}$ on the following topics:

1. Characterization Changes.

2. H-14 Drawing Preparation ECC.

3. Pit operating temperature criteria clarification.

SCOPE

1. CHARACTERIZATION CHANGES - ICF KH is directed to utilize the revised waste characterization data contained in the reference letter, copy provided as Attachment 1 . This is the basis of definitive design pending issuance of a revision to the project FDC characterization appendix. If the characterization ata changes result in impacts to the project cost and/or schedule basejines, an appropriate ECC documenting the total project impacts of these changes is to be prepared and submitted to WHC.

2. H-14 DRAWING PREPARATION ECC - ICF KH is directed to prepare an ECC documenting the totai project cost and any schedule impacts associated with preparation of $\mathrm{H}-14$ Arawings. The $\mathrm{H}-14$ drawings are to be prepared in accordance with guidance contained in Attachment 2 for the purposes of the ECC estimate, ICF $\mathrm{KH}$ is to assume that the $\mathrm{H}-14$ drawings are prepared at the comptetion of the project during the as buiting process to avoid the need for any revisions.

3. PIT OPERATING TEMPERATURE CRITERIA CLARIFICATION - The following is provided as a klarification to the intended operating temperatuke limitations for equipment installed in the $A Y-102$ and $C-106$ tank pits. FDC paragraph 2.2.3, Table 2-1 normal operating temperature ranges ake intended to indicate the temperature of the process fluids to which wetted parts of the sluicing system will be exposed and are not to be used as pit environment criteria which must not be exceeded. ICF KH is digected to use a $120^{\circ} \mathrm{F}$ as the maximum allowable pit environment temperature. 
Westinghouse

Hanford Company

ATTACH MIENT

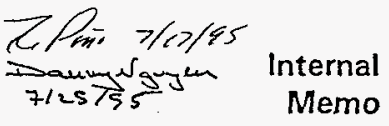

From: Single-Shell Tank Retrieval Projects

$8 \times 800-95-026$

Phone: $\quad 376-4772$ S2-48

Date: June 5, 1995

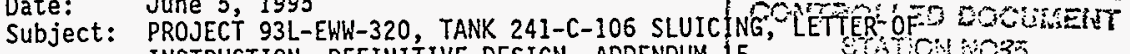
INSTRUCTION, DEFINITIVE DESIGN, ADDENDUM 15 ,

To: F. H. Bradshaw $\quad$ S2-47

cc: See Distribution List

Reference: 1) Letter, G. H. Sanders, RL, to C. A. Augustine, WHC, "TWRS Waste Retrieval Project $W-320$, Sluicing Tank C-106 Minimum Cost Approach," 95-RTI-042/9502160B, dated Apri1 27, 1995.

2) Letter, C. A. Augustine, WHC, to R. W. Bradshaw, ICF KH, "Project 93L-EWW-320, Tank 241-C-106 Sluicing, Letter of Instruction, Definitive Design, Addendum 14," 8K800-95-021, dated May 8, 1995.

This Letter of Instruction (LOI) addendum provides guidance from

Westinghouse Hanford Company on the following topics:

1. PRIOR LOI GUIDANCE MODIFICATION

2. FLUSHING REQUIREMENT GUIDANCE MODIFICATION

SCOPE

1. PRIOR LOI GUIDANCE MODIFICATION - The following provisions of previous project LoIs are rescinded or modified as indicated below in order to implement the minimized cost approach direction contained in references 1 and 2 . These LOI direction clarifications are being made to remove the potential for confusion in the intended scope for the rebaselined project.

A. Letter 71320-94-051, C. A. Augustine to F. W. Bradshaw, "PROJECT 93L-EWW-320, TANK 241-C-106 SLUICING, LETTER OF INSTRUCTION, DEFINITIVE DESIGN, ADDENDUM 7", dated December 13, 1994

Item 3. PIT OPERATING TEMPERATURE CRITERIA CLARIFICATION - The following is provided to clarify the intended operating temperature limitations for equipment installed in the $A Y-102$ and $C-106$ tank pits.

FDC paragraph 2.2.3 Table 2-1 normal operating temperature ranges are the temperatures of the process fluids to which wetted parts of the sluicing system will be exposed and are not to be used as pit environment criteria which must not be exceeded. ICF KH is directed to use 180 degrees $F$ as the maximum allowable pit environment temperature.

HNF-2486, Rev. 0

Page A-13 
Curve No.: $2 \times 3 \times 13$

LPI Ref:: 17482
Customer: WHC/Kaiser

User Name: WHC
Cust. Ref: W-78259-Z4

Item No: P-0621(Inline)

TDH

$\mathrm{Ft}$.

Description 2 in. T.S. Inline Slurry

2865 RPM
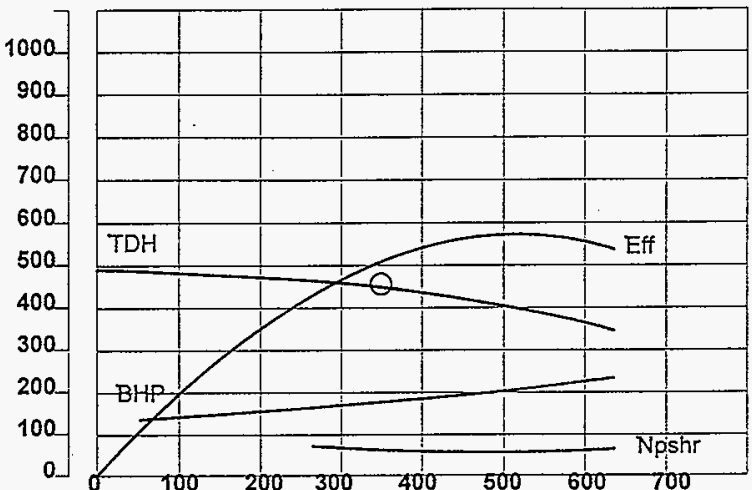

Impeller Dia. Rated: 13.00 in.

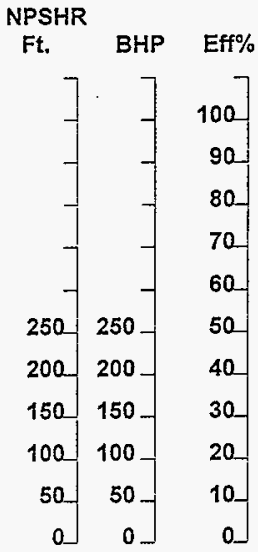

Serkice: Sluice

Sp.Gr.: 1.12

Visc.: $14.17 \mathrm{Cps}$

Max: 13.00 in.

Min: $9.00 \mathrm{in}$.

\begin{tabular}{|c|c|c|c|c|c|c|}
\hline \multirow[b]{2}{*}{$\begin{array}{l}\text { Flow: } \\
\text { head: } \\
\text { Eff: } \\
\text { Power: }\end{array}$} & \multirow{2}{*}{$\begin{array}{l}\text { Design } \\
350 \mathrm{gpm} \\
455 \mathrm{ft} \\
\frac{51 \%}{89.0 \mathrm{bnp}}\end{array}$} & \multicolumn{5}{|l|}{$\operatorname{Max}$} \\
\hline & & $\begin{array}{l}638 \mathrm{gpm} \\
503 \mathrm{ft} \\
57 \% \\
+15.8 \mathrm{bhp}\end{array}$ & $\begin{array}{l}\text { NS: } \\
\text { SS: } \\
\text { Suct Press: } \\
\text { NPSHA: }\end{array}$ & $\begin{array}{l}747 \\
6000 \\
.0 \text { Psi } \\
-\mathrm{ft}\end{array}$ & $\begin{array}{l}\text { NPSHR: } \\
\text { Solids: } \\
\text { Solid Size: } \\
\text { Max Soiid: }\end{array}$ & $\begin{array}{l}27 \mathrm{ft} \\
30 \% \\
.19 \text { in } \\
1 \text { in }\end{array}$ \\
\hline
\end{tabular}

HNF-2486, Rev. 0

Page A-14 
sommsis

ATTACHMENT 9

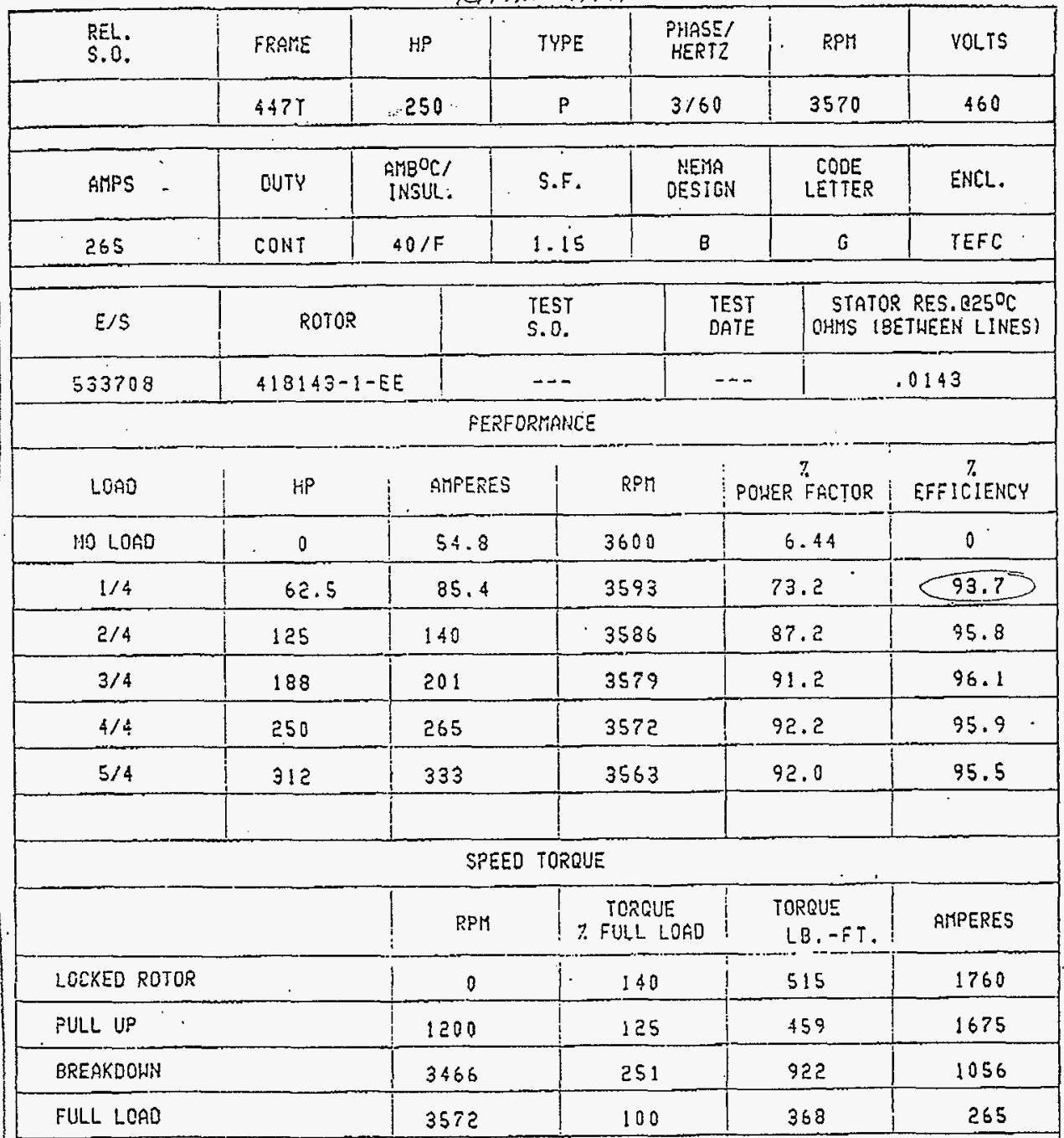

AMPERES SHOUR FOR 460. YOLT CONNECTIOH. IF OTHER VOLTAGE CONNECTIONS ARE RVAILABLE. THE AMPERES WILL YARY INVERSEY HITH THE RATED VOLTAGE

REMARKS: XE MOTOR-TYPICAL DATA-HEMP NOM. EFF. 95. 3 PCT. GUARANTEED MIH. EFF. 95.4 PCT.

HNF-2486, Rev. 0

Page A-15
DR. BY P. 3. GREENE A-C HOTOR E7416A-8-001

CK. EY. T. I. BYKU

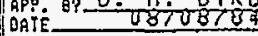
DATA ISSUE DATE O7/25/84

EK, ESTIMATE OF MOTOR EFFILIENCY, NOTE,

$\sim$ at Full load. 


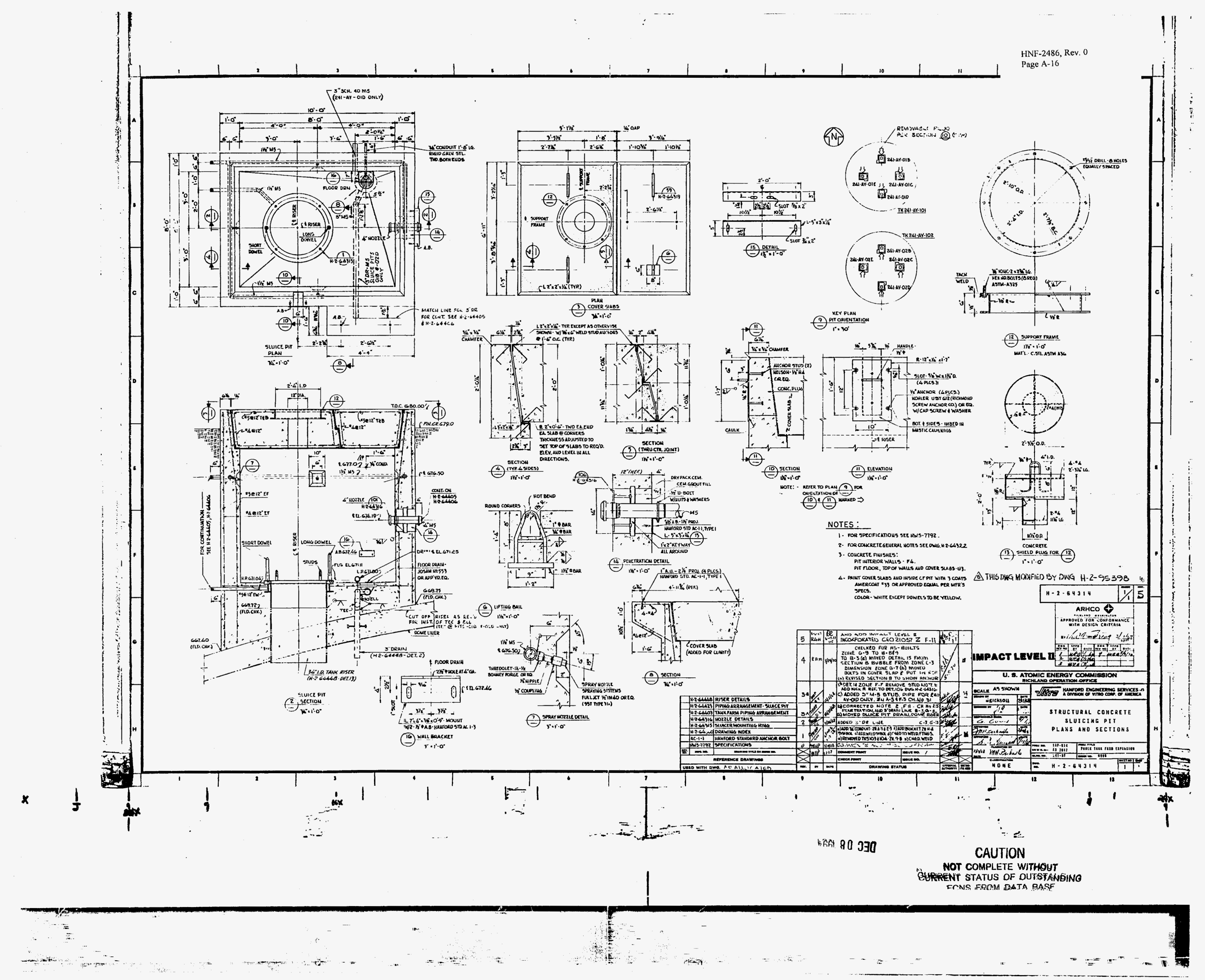


HNF-2486, Rev. 0

W320-28-031

Pressure Relief Seal Loop Design Verification 


\section{CALCULATION IDENTIFICATION AND INDEX}

This sheet shows the status and description of the attached Design Analysis sheets.

w320-28-031

Discipline 28/HVAC WO/Job No. ER4319

Calculation No.

Project No. \& Name W-320 Tank 241-C-106 Waste Retrieval (Sluicing)

Calculation Item Pressure Relief Seal Loop Design Verification

These calculations apply to: $\quad$ N320-28-016

Dwg. No. H-2-818477

Dwg. No. N/A

Other (Study, CDR)

Rev. No. 0

Rev. No. N/A

Rev. No.

The status of these calculations is:

[] Preliminary Calculations

[x] Final Calculations

[] Check Calculations (On Calculation Dated)

[] Void Calculation (Reason voided)

Incorporated in Final Drawings?

[] Yes $[\mathrm{x}]$ No

This calculation verified by independent "check" calculations? [] Yes $[X]$ No

Original and Revised Calculation Approvals:

\begin{tabular}{|c|c|c|c|}
\hline & $\begin{array}{c}\text { Rev. } 0 \\
\text { Signature/Date }\end{array}$ & $\begin{array}{c}\text { Rev. } 1 \\
\text { Signature/Date }\end{array}$ & $\begin{array}{c}\text { Rev. }{ }^{2} \\
\text { Signature/Date }\end{array}$ \\
\hline Originator & ELDalpiaz PHK $3-2845$ & & \\
\hline Checked by & 0 & & \\
\hline Approved by & & & \\
\hline $\begin{array}{l}\text { Checked Against } \\
\text { Approved Vendor } \\
\text { Data }\end{array}$ & & & \\
\hline
\end{tabular}

Design

Analysis

INDEX

Page No.

\begin{tabular}{|l|l|}
\hline$i$ & Calculation Identification and Index \\
\hline $1-5$ & Interoffice Memorandum E.L. Dalpiaz to C.B. Organ dated 3-17-95 \\
\hline A1-A28 & $\begin{array}{l}\text { Appendix A: Data used for design verification: H-2-818477 and W-320-C5 } \\
\text { section } 15507\end{array}$ \\
\hline
\end{tabular}

HNF-2486, Rev. 0

Page B-1 


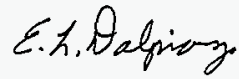

COFIES TO
A.W. Bjorkeda 7
$B 4-40$
P.H. Langowski
S3-07
S3-10

Document Control

308 NO.

sUBsect Design Verification Review - Project W-320

I have completed a design verification of the Safety Class 1 components of the pressure relief seal loop for Tank 241-C-106, project $W-320$. The verification followed "Design Review Method" as defined by ES-7, Revision 3, Section 6.2. The extent of this review was to verify the size of the pressure relief seal loop for Tank 241-C-105 is adequate to meet design requirements.

The following are my responses to the questions in section 6.2.1.4 of ES-7.

Were design input correctly selected?

The project's Functional Design criteria provided design basis for the pressure relief seal loop. This basis was followed and referred to in the design calculations for the design of the pressure relief seal loop.

Were al1 necessary conditions considered in the design?

The conditions listed in the calculations to determine the size of the seat loop appear adequate.

Are required assumptions adequately described; are sources identified and reasonable for the design?

Assumptions are jisted in the calculations and appear reasonable for the design of the pressure relief seal loop.

Where necessary, are the assumptions reverified when the design is complete?

The assumptions listed would not have to be reverified when the design is complete.

Was appropriate design method used?

Calculations which determined pipe pressure loss and fan characteristics referred to applicable ASHRAE references. Data for pipe dimensions used in the design of the seal loop was taken from applicable ASME/ANSI standards.

WORKING TOWARD ।

HNF-2486, Rev, 0

Page B-2
PLOYEE INVOLVEMENT

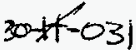

28 


\section{INTEROFFICE MEMORANDUM}

\section{Were design input correctly incorporated?}

Design input has been correctly incorporated except for the function described for the pressure relief loop seal in Table B-9 of the Safety Equipment List. The table states the loop seal "prevents under/over pressure of tank C-106". Design input has been established in the Functional Design Criteria for under pressure but not over pressure. The Functional Design Criteria and LOI clearly establish the seal loop is to function for under pressure in the tanks. Clarification for the function described in Table B-9 should be provided. "averl" will be taken out "n the SEL Is design output reasonable compared to design next revision por John C. Conneh 1s design output reasonable compared to design input? $3-27-95$ All calculation results reviewed appeared reasonable for the input criteria.

Are necessary design input and verification for interfacing organizations specified in design documents or in supporting procedures or instructions?

The "Draft" construction specification reviewed, and the unchecked drawing of the seal loop outline the functional design requirements, required material and fabrication, quality requirements, testing and submittals for verification.

Was the applicable Hanford Plan Standard (HPS) followed; if not, is documented approvat included from DOE-RL?

Hanford Plant Standard SDC.5.I Standard Design Criteria for Heating, Ventilating and Air Conditioning was followed. LOI memo JPH-95-003 gave direction to not require application of either Safety Class 1 seismic and tornado criteria for the design of the seal loops. Therefore Hanford Plant Standards governing these requirements were not included. Documented approval from DOE-RL was not included.

In addition, the following observations are made.

The seal loop is filled with a silicone fluid per drawing H-2-818477. The calculations should be revised to indicate differences between this fluid and water and the fluid's effect on the seal loop height. Also additional requirements may have to be added to the specifications to test the silicone fluid's properties. recesecy pronald recessery withall My conclusion is that the methods used and the results of the calculations show that the the design of the pressure relief seal $100 \mathrm{p}$ is adequate to protect Tank $241-C-106$ during under 6 pressure conditions as stated in the Functional Design Criteria. 


\section{INTEROFFICE MEMORANDUM}

The following documentation was used in this verification:

Drawing H-2-818477 Review No. 12, dated 1/31/95

Specification $W-320-C 5$ Section 15507, "Draft" dated $9 / 30 / 95$

Calculation W320- $/ 16$ Rev. 0 , dated $9 / 28 / 94$

Calculation W320-Hz-020 Rev. 0, dated 10/20/94

Functional Design Criteria WHC-SD-W320-FDC-001, Rev. 2, Page 2-4

Safety Equipment List for 24I-C-106 Waste Retrieval Project W-320, WHC-SD-WM-SEL-033

Rev. I, Table B-9 Page B-35

Letter of Instrucation (LOI) Rev. 8, Memo JPH-94-003, dated 7/11/94 
Project/Document Number $W-320$

Prepared By E.L. Dalplaz

verifier Name E.L. Dalpiaz

Verifier Name

$$
\text { (Print) }
$$

Verifier Name

(Print)

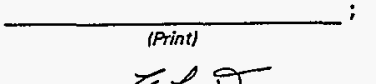

Initial $E h N$

Initial

Initial

Date

Formal Design Verification is determined to be necessary per the Design Verification Screening Criteria and was performed by the following method(s).

1. Alternate Calculation Method YES NO (Circle One)

Calculation Number

Revision

2. Testing Method

YES NO (Circle One)

Test Number

Test Title

3. Identical Method

YES NO (Circle One)

Document Number

Title

Revision

Date

4. Design Review Method Design Review Chairman (YES No (Circle One)

Finding or Attached Memo Report Number

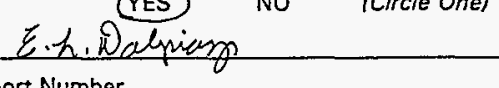

Date $3 / 17 / 95$

Formal design verification is complete and acceptable:

Design Verification Officer

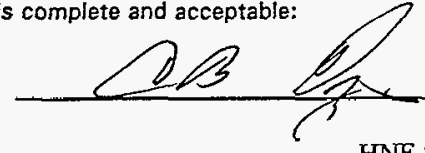

HNF-2486, Rev, 0 
TABLE B-Q. VENTILATION SYSTEM

FMEA SAFETY CLASS DESIONATION

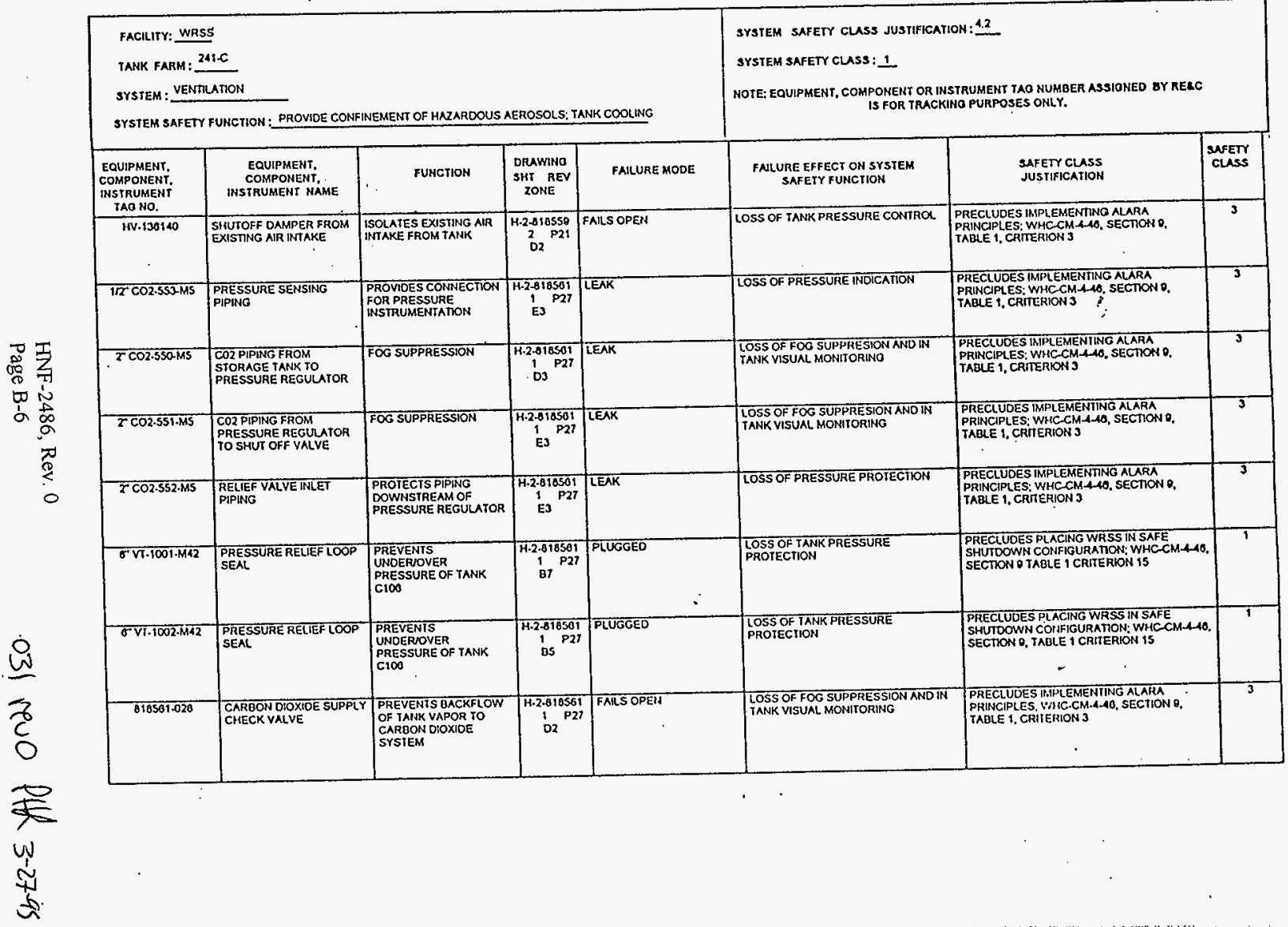




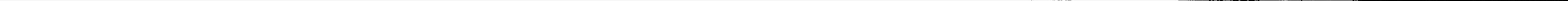




\section{APPENDIX A}

HNF-2486, Rev. 0

Page B-8

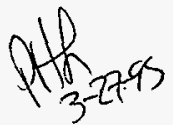


PART 1 - GENERAL

\subsection{REFERENCES}

1.1.1 The following documents, including others referenced therein, form part of this Section to the extent designated herein.

1.1.1.1 American National Standards Institute (ANSI)

A13.1-1981

Scheme for the Identification of Piping Systems

1.1.1.2 American Society of Mechanical Engineers (ASME)

B16.5-1992

Pipe Flanges and $\mathrm{Flanged} \mathrm{Fittings}$

B16.9-1993

Factory-Made Wrought Steel Buttwelding Fittings

B31.3-1993

Chemical Plant and Petroleum

Refinery Piping

NQA-1-19簿 89

Quality Assurance Program Requirements for Nuclear Facilities

1.1.1.3 American Society for Testing and Materials (ASTM)

A $53-93 a$

A $105-93 b$

A $182-92 a$

A $193-93 a$

A $194-93 a$

A $240-88 a$

A $276-89$
Pipe, Steel, Black and Hot-Dipped, Zinc-Coated Welded and Seamless

Forgings, Carbon Steel, for Piping Components

Forged or Rolled Alloy-Steel Pipe Flanges, Forged Fittings, and Valves and Parts for High-Temperature Service

Alloy-Steel and Stainless Steel Bolting Materials for High-Temperature Service

Carbon and A7loy Steel Nuts for Bolts for High-Pressure and High-Temperature Service

Heat-Resisting Chromium and ChromiumNickel Stainless Steel Plate, Sheet and Strip for Pressure Vessels

Stainless and Heat Resisting Steel Bars and Shapes

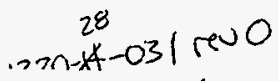



A $312-93$
A $403-93$
C $449-88$
C 547-77
E 84-91

1.1.1.4 American Welding Society (AWS)

01.3-89

1.1.1.2 Federal Specifications (FS)

HH-I-558

TT-S-00230
Seam7ess and Weided Austenitic

Stainless Steel Pipes

Wrought Austenitic Stainless Steel Piping Fittings

Mineral Fiber Hydraulic-Setting Thermal Insulating and Finishing Cement

Mineral Fiber Preformed Pipe Insulation

Test Method for Surface Burning

Characteristics of Building Materials

Welding Sheet Steel and Structures

Insulation, Blankets, Thermal (Minera] Fiber, Industrial Type)

Sealing Compound: Elastomeric Type. Single Component (For Caulking, Sealing and Glazing In Buildings and Other Structures)

1.1.1.4 Manufacturers Standardization Society of the Valve and Fittings Industry (MSS)

SP-89, 1991

Pipe Hangers and Supports - Fabrication and Installation Practices

1.1.1.6 Underwriters Laboratories (UL)

Building Materials Directory

1.2 SUBMITTALS

1.2.1 See Section 01300 for submittal procedures.

1.2.2 Approval Required

1.2.2.1 Approval data: Before delivery, submit information listed in the Approva 7 Data List in this Section.

1.2.2.2 Certified material test reports: Before delivery, submit legible reports, certified by responsibie manufacturer of materials used in fabrication of tubing, pipe, fittings, flanges, bolting, and weld rod for pipe codes and services listed below. Reports shali present results of chemical anatysis and physical tests specified in ASTM Codes and Standard Specifications for production lots and heats of materials. Submit separate reports for Pipe Code M-42. 
1.2.2.3 Traceability history: Before fabrication, submit a work plan detailing the proposed method of maintaining records of specific locations of each item covered by a report.

1.2.2.4 Material traceability (including heat/lot numbers) shall be maintained through fabrication for materials requiring Certified Material Test Reports.

1.2. Quality Assurance Programs: Before delivery, submit documentation for programs specified in 1.4.5.

\subsubsection{Approval Not Required}

1.2.3.1 Vendor information: Before installation, submit information listed in the Vendor Information List in this Section.

1.2.3.2 Certificates of conformance

a. With delivery, submit a legible certificate, certified by equipment manufacturer or supplier, stating that materials used in fabrication of the "Pressure Sea] Loops" (Pipe Code M-42. Service: Ventilation) meet requirements of the Drawings and this Section.

1.3 SYSTEM DESCRIPTION

1.3.1 Systems are stainless steel piping and duct for exhaust air of Tank C106 that contains radioactive contaminants. A short section of ventilation system is carbon steel pipe. The seal loop piping is Safety Class 1 and is stainless steel pipe.

1.3 .2 System por sources are show on the Drawings.

1.4 QUALITY ASSURANCE

1.4.1 Duct insulating materials shal1 be listed in the UL Building Materials Directory, and carry the UL mark.

1.4.2 Misrepresented Products: See Section 01400 for required measures to prevent the use of misrepresented products.

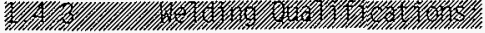

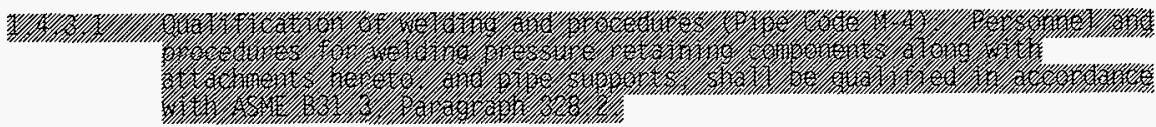

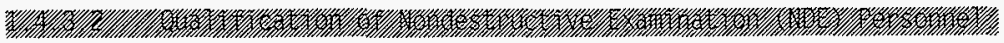

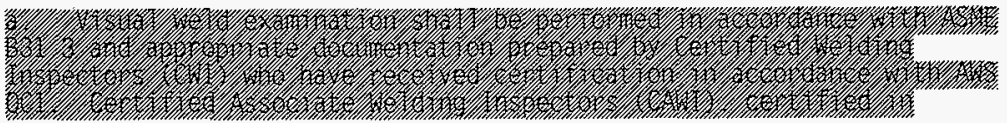



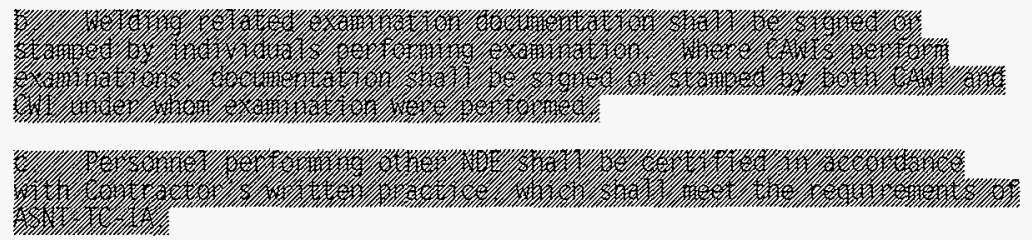

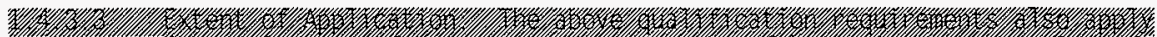

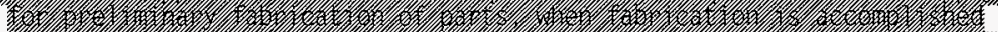

梠

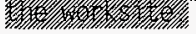

1.4.4 Deliverable Documentation: The following documents and records, required by this Section. shall be delivered to Construction Document Control (Buyer) in accordance with Section 01720.

Document

Welding Examination Documentation

Flushing/Mechanical cleaning Verification

Leak/Pressure Test Certification
Paragraph

3.2 .1 .6

3.2 .5 .1

$3.2 .1 .6 \mathrm{~b}$

1.4.5 Safety Related Products: Safety Class 1 products shall be procured from qualified suppliers having quality assurance programs meeting ASME NQA-1 basic requirements all sections and supplements.

1.4 DELIVERY, STORAGE, AND HANDLING

1.4.1 See Section 01610 for general requirements.

PART 2 - PRODUCTS

2.1 SUBSTITUTES

2.1.1 See Section 01630 for substitution approvals.

2.2 MATERIALS

2.2.1 Pipe and Fittings: See the Pipe Codes in this Section, and details on the Drawings.

2.2.1.1 When approved by the Field Engineer, integrally reinforced branch connections may be used on 2 -inch and larger welded main lines instead of buttwelding tees specified in the Pipe Codes.

HNF-2486, Rev. 0

W320C5.CPY

Page B-12

W-320-C5

Rev 0

09/30/94 
2.2.1.2 Material and weight sha71 be as specified in the Pipe Code for each piping system.

2.2.1.3 Close or butt nipples are not permitted, unless shown on the Drawings.

2.2.2 Pipe Joint Sealant (Lubricant) for Threaded Joints

2.2.2.1 For design temperatures up to $500^{\circ} \mathrm{F}$, use nonhardening teflon paste: Chesterton "Goldend" No. 7298, Federal Process Company "JC-30", or Lake Chemical Company "Slic-Tite".

2.2.3 Protective coating for piping in contact with earth.

2.2.3.1 Carbon steel piping: Field and Factory applied exterior protective coating; AWWA C213, fusion bonded epoxy coating of 14 Mils minimum thickness. Scotchkote $206 \mathrm{~N}$ or an approved substitute.

2.2.3 Ductwork Insulation: Provide uniform thickness for each duct size and piece of equipment.

2.2.3.1 Insulation and adhesive shall have UL fire hazard classifications of 25 maximum for flame spread and 50 maximum for smoke developed.

2.2.3.2 Insulation corrosive and moisture adsorption characteristics shall be in accordance with FS HH-I-558.

2.2.3.4 Insulation for exterior surfaces of outdoor duct and filter housing.

a. A $1 / 2$ monch thick glass fiberboard, minimum density, $6 \mathrm{~Tb} / \mathrm{ft}^{3}$; Manville No. 817 Spin-Glas board.

2.2.3.5 Weatherproofing: Foster "Sealfas" G.P.M. 35-00 mastic, with "Mast-AFab" reinforcing membrane.

2.2.3.6 ACCESSORIES: As specified below, or approved substitutes.

a. Mechanical Fasteners: Stick-Clips, Gemco insulation hangers with washers, type recommended by insulation manufacturer.

b. Cement: ASTM C449: Pabco Pabcote No. 127

2.2.4 Painting: See Section 09900.

2.2.5 Nonshrink Grout: Nonmetallic type: US Grout Corporation "Five Star Grout," Sika Corporation "Sika Grout 212." or Master Builders

"Masterflow 713.

2.2.6 Pipe Insulation

HNF-2486, Rev. 0

W320C5.CPY

Page B-13

$2795 \mathrm{~W}-320-\mathrm{C} 5$

$09 / 30 / 94$ 
2.2.6.2 Outdoor applications:

a. ASTM C 547 molded, sectiona 7 , rigid fiberglass pipe covering. Class 1 for temperatures up to $450^{\circ} \mathrm{F}$, and Class 2 for temperatures up to $650^{\circ} \mathrm{F}$.

b. Metal jacket: Provide circumferential seam closure with straps (bands), or tape with metal foil backing, and silicone sealant to provide weathertight joints, 0.016-inch minimum aluminum sheet.

c. Straps (Bands): Aluminum, with protective coating, 3/8-inch minimum wide and 0.005 -inch minimum thick.

2.2.6.3 Insulating cement for forming insulation covering over fittings and valves: ASTM C 449. Cements that contain asbestos are not acceptable.

2.2.6.5 Piping insulation schedule:

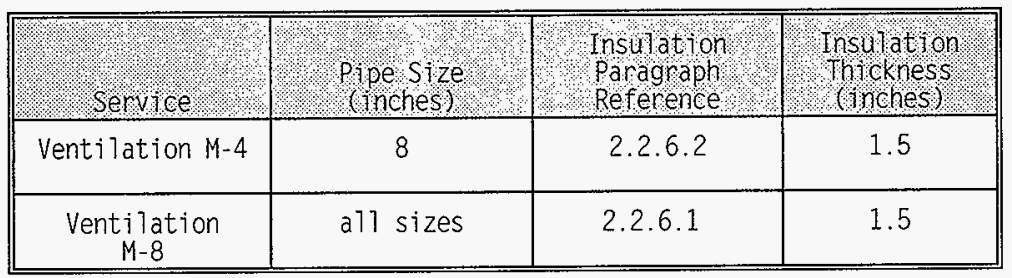


2.2.7 Pipe Supports: Standard, manufactured components as shown on the Drawings. Pipe supports and attachments welded to pressure containment boundaries may be noncertified material (note, does not apply to Safety Class 1), provided the material bears a type identifying mark, is suitable for welding, and compatible with the material to which it is attached.

2.2.9 Material Limitations for Stainless Steel Components:

2.2.9.1 Tape and adhesive leachable chloride content shall not exceed $15 \mathrm{ppm}$. and leachable fluoride content not to exceed $10 \mathrm{ppm}$. Use 3M Company preservation sealing tape No. 481,9 mil thick, or Kenda11 Company Polyken Division No. 226. Materials which contact stainless steel piping and components during fabrication and shipping shall not exceed these limits.

2.2.9.2 Grinding or machining of stainless steel shall be done only with tools which are allocated specifically for use on stainless steel. Use of grinding compounds containing halogens is strictly prohibited. Wire brushing of stainless steel shall be done with stainless steel brushes that are new or have not been used on material other than stainless steel.

2.2.9.3 Demineralized water used for cleaning or flushing shall be limited to a chloride content of $25 \mathrm{ppm}$ maximum. Cleaning solutions shall not contain halogenated compounds.

2.2.9 Penetration Sealants: FS TT-S-00230, Type II, Class A, 1-component polyurethane, nonsag type, light-colored

2.2.11 Identification Tags: 0.0478-inch (18-gage) minimum thickness stainless steel. Tag Attachment Wire: 20-gage, solid, stainless steel.

\subsection{EQUIPMENT}

2.3.1 Valves: Specified in Pipe Codes, unless shown otherwise on the Drawings.

2. 2.1.4 Butterfiy valves specified in Pipe Code M-8:

\begin{tabular}{|c|c|c|c|}
\hline Valve No. & $\begin{array}{l}\text { Size, } \\
\text { inches }\end{array}$ & Type-Keystone & Operator \\
\hline HV -13643 & 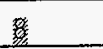 & 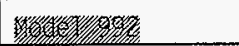 & 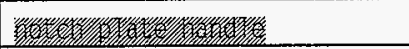 \\
\hline HV -136140 & 粼 & 增5 & 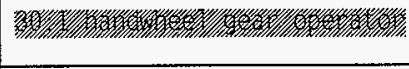 \\
\hline HV-136151 & 讋 & 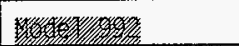 & 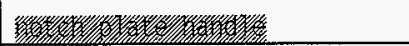 \\
\hline HV- 136150 & 缕喽 & & \\
\hline HV -136143 & 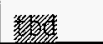 & & \\
\hline
\end{tabular}




\begin{tabular}{|l|l|l|l|}
\hline$H V-136144$ & 䍂楼 & & \\
\hline
\end{tabular}

\section{(S)}

PART 3 - EXECUTION

3.1 INSTALLATION

3.1.1 Equipment

3.1.1.1 Instal1 as shown on the Drawings and in accordance with the manufacturer's instructions.

3.1.1.2 Prime and paint in accordance with the Section 09900. Paint containing lead is not acceptable.

3.1.2 Piping

3.1.2.1 Fabricate and install pipe and tubing in accordance with ASME B31.3 (for normal service), the Drawings, and this Section.

3.1.2.2 Cut pipe and tubing using methods which result in clean, straight cuts. Do not use tubing cutters on tubing utilizing compression fittings.

3.1.2.3 Ream pipe and tubing to nominal inside diameter after cutting. Remove burrs from threads before assembly.

3.1.2.4 Tools marked for stainless steel shall be used on stainless steel only.

3.1.2.5 Bend pipe and tubing in accordance with ASME B31.3. and meet tolerances given in ASME B31.3. Use methods and equipment which produce bends free of wrinkles, bulges, or kinks. When wall thickness, diameter, and bend radius indicate possibility of wall collapse during bending, fill pipe or tubing with clean silica sand or fusible material similar to Cerrobend, manufactured by Cerro Corporation.

a. Application of heat to facilitate a pipe bend shall be brought to the attention of the responsible engineer for direction and disposition.

b. Cold springing pipe permitted only if specified on the Drawings.

3.1.2.6 Make joints in threaded piping systems with joint sealant specified. Apply sealant to male threads only.

3.1.2.7 Instal1 tubing fittings in accordance with the manufacturer's instructions.

3.1.2.8 Carbon steel embedded in concrete shall be bare.

3.1.2.9 Keep piping systems clean. Once fabrication has started, plug or cap ends of piping when installation is not in progress. Cap or plug 
openings in fabricated pipe spool assemblies until installation in the piping system. Leave ends of spare lines capped or blind flanged when installation has been completed.

3.1.2.10 Instal1 condensate piping with minimum slope of $1 / 4$ inch in feet in direction of flow. except where shown otherwise on the Drawings.

3.1.2.11 Install condensate piping free of low points or pockets in which condensate could collect, except at designated stations where traps are provided.

3.1.2.12 Make connections in top 180 degrees of condensate return line. Slope condensate vent lines down to condensate return line.

3.1.2.13 Provide vents at high points to purge air pockets and drain taps at low points to ensure complete drainage and drying in piping systems using water for cleaning and leak/pressure testing. Venting may be provided by loosening flanges having minimum of 4 bolts.

3.1.2.14 Pipe supports: Insta11 as shown on the Drawings, in accordance with manufacturer's instructions, and applicable recommended procedures of MSS SP-89.

3.1.3 Welding

3.1.3.1 Weld piping, attachments to pressure retaining components, and pipe supports in accordance with ASME B31.3.

3.1.3.1.1 Protect outdoor welding operation from rain and wind by using barriers to protect welder and weld joint.

3.1.3.1.2 Complete piping welds before tie-in welds to valve pits, risers, or fixed items.

3.1.3.1.3 Remove backing strips if used.

3.1.3.2 Weld duct per AWS D1.3.

3.1.4 Weld Identification

3.1.4.1 Prepare weld identification drawings, isometric and spool, which show relative positions of pressure containing welds and attachment welds to pressure retaining components.

3.1.4.2 Assign weld numbers to pressure containing welds and attachment welds to pressure retaining components as made. Record weld numbers on weld identification drawings as welds are made.

3.1.4.3 Place the welder identification symbol and weld number adjacent to welds upon completion. Place the identification symbol approximately every 3 feet on long seams or large weldments, using one of the marking methods specified in Section 01610. 
3.1.4.4 Do not reuse weld numbers. If a weld is completely replaced, assign a new number.

3.1.4.5 Show heat/lot numbers on weld identification drawings for materials requiring Certified Material Test Reports.

3.1.4.6 Maintain material traceability (including heat/lot numbers) through fabrication on weld identification drawings.

3.1.5 Perform NDE, flushing, leak/pressure testing, and disinfecting specified in 3.2 before coating application.

3.1.6 Piping Insulation

3.1.6.1 Preformed:

a. Apply after leak/pressure testing of line has been completed. Apply to pipe surfaces free of water, oil, dirt. loose scale, or other foreign material.

b. Abut sections and fi11 broken corners with insulating cement.

c. Apply molded, sectional, rigid fiberglass with factory applied nonmetaliic jacketing for indoor applications, with adhesive surface clean. Seal lap and end joints with factory applied pressure sensitive lap strip and factory furnished pressure sensitive butt strips.

e. Install outdoor metal jacketed insulation in accordance with manufacturer's recommendations. Position longitudinal joints to shed water. Cover circumferential joints with sealing compound, secure in place with specified straps (bands) or tape. Apply straps (bands) or tape at mid point of 3-foot insulation sections.

f. Insulate valves and fittings in pipe lines smaller than 4 inches with insulating cement. Apply cement in accordance with manufacturer's recommendations.

g. Insulate valves and fittings in 4 to 14 -inch pipe lines with sectional or block insulation of same material used on adjacent pipe, with same total thickness. Securely wire in place with 16-gage wire, and cover with 20 -gage, 1 by 1 -inch wire mesh, or glass fabric, stretched tightly over surface and fastened in place. Use specified tape on fiberglass, and 16-gage wire on calcium silicate, to secure insulation to 14 -inch and larger pipe. Top insulation with $1 / 2$ inch minimum thickness coating of insulating cement to present a smooth surface.

h. Mitered sections of straight jacketed pipe insulation may be used for fittings and elbows as an alternative to 3.1.6.1f and 3.1.6.1g. Cement mitered metal jacketed insulation joints with weatherproof seal and specified tape. Cement nonmetallic jacketed insulation joints with weatherproof seal and apply seal strips with lap adhesive. 
i. Insulate flanges with sectional or block insulation of same material used on pipe. Terminate insulation on pipe adjacent to flanges with bevel far enough from flanges to permit removal of flange bolts. Insulate flanges to a thickness of $1 / 2$ inch less than adjacent pipe covering to allow for $1 / 2$ inch cement finish. Flange insulation shall extend 2 inches over ends of pipe insulation. Wire flange insulation in place and cover with $1 / 2$ inch minimum thickness layer of insulating cement.

j. Wrap unjacketed insulation, for outdoor applications, applied to flanges, fittings, and valves with glass fabric over wet coat of weatherproof sealant lapping joints at least 2 inches. Apply second coat of weatherproof sealant, 1/8-inch wet thickness, after first coat has dried.

k. Seal penetrations of jacket for hanger rods and clamps for outdoor applications with weatherproof sealant.

3.1.7 Piping Identification: Identify exposed piping systems as to fluid carried and direction of flow as specified in Appendix A.

$3.1 .8 \quad$ DUCT

3.1.8.1 Fabricate duct in accordance with drawings and Duct Code D-3.

3.1 .8 .2 provide companion angle flanges at duct to-equipment joints, unless otherwise noted. Use Ethylene propylene-Ruber (EPR) gaskets, 1/16" thick.

3.1.8. At . Atah hangers and supports to primary building structure. Furnish-and install-secondary-steel structures where required to suppont duct on equipment.

3.1.8. Test as specified in 3.2.

\subsubsection{DUCT INSULATION}

3.1.9.1 Surfaces to receive insulation shall be clean. free of moisture, $0 i 1$, dirt, scale, rust, and other foreign material.

3.1.9.2 Insulation and finish materials shall be dry when installed.

3.1.9.3 Install a uniform thickness of insulation on each duct size.

3.1.9.4 Make installations continuous through sleeves and prepared openings, unless shown otherwise on the Drawings. Terminate insulation at fire dampers and flexible connections.

3.1.9.5 Score or groove insulation to fit contours of duct. Fill and seal scores, grooves, joints, and penetrations with insulating cement.

3.1.9.6 Where insulation abuts uninsulated surfaces, seal joints with mastic recommended by the insulation manufacturer.

HNF-2486, Rev. 0

W320C5.CPY

Page B-19

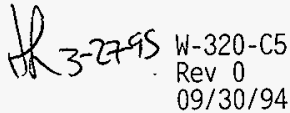


3.1.9.8 Outdoor Duct: Instal1 insulation of 1-1/2 inches on exterior surfaces (see 2.2.3.4). Wrap insulation with two $1 / 8$ inch wet coats of membrane reinforced mastic meeting the requirements of 2.2.3.5. Apply per manufacturers instructions.

3.1.9.9 Attach insulation to metal duct surfaces with adhered clips and washers of 2.2.3.6, and $100 \%$ coverage of insulation cement. Space clips 12 inches maximum on centers.

3.1.10 Identification Tag Features

3.1.10.1 Blanks: $3 / 4$ by 2 -inches (unless legends require longer tags), with a $1 / 8$-inch attachment wire hole $3 / 16$ inches in from one end.

3.1.10.2 Characters: Use 1/8-inch stamped or engraved characters.

3.1.10.3 Legends: Provide the following information, unless otherwise specified on the Drawings. Obtain information from the data sheets in this specification, or the Drawings.

a. ICF $\mathrm{KH}$ part number

3.1.10.4 Attach identification tags to valves, instruments and other equipment. as required, with twisted wire. Use enough wire to produce $\sim 1$-inch minimum installed lengths.

3.2 FIELD QUALITY CONTROL

3.2.1 Nondestructive Examination (NDE) of Welds for Piping

3.2.1.1 Perform NDE for each pipe code Tisted in the schedule in 3.2. Extent of required examination sha11 be per ASME B31.3, paragraph 341.4 .1 . Acceptance criteria for NDE sha17 be per ASME B31.3, paragraph 341.3 .

3.2.1.2 Visual examination: Perform in accordance with ASME B31.3. Paragraph 344.2 .

3.2.1.3 Radiographic examination (RT): Perform in accordance with ASME B31.3. Paragraph 344.5 .

a. Identify radiographic film with weld identification number and project or work order number assigned to work covered by this Section. In process examination per ASME B31.3, paragraph 344.7 may be substituted for Radiography.

b. Prepare radiographic examination reports as follows.

1) List each radiographic exposure location $(0-1,1-2, \ldots)$ individually on radiographic examination report.

2) Indicate location acceptability or rejectability and note discontinuities whether rejectable or not. 
3) When report includes radiographs of welds which have been repaired, indicate which welds are repair weids and how many times each weld has been repaired.

c. If additional welding is performed on weld area which has already been examined radiographically, this area is repair area. Identify subsequent radiographs by "R-1, R-2", etc.

3.2.1.4 Perform random examination of piping supports in accordance with ASME B31.3, Paragraph 344.2.

3.2.1.5 For Safety Class 1 systems (Pipe Code M-42), perform 100\% examination of piping suppports per ASME B31.3, paragraph 344.2 .

3.2.1.6 NDE documentation:

a. Document a11 examinations of pressure containment welds and welds to pressure containing components for piping systems on NDE/Weld Record Form KEH-0433. See Form KEH-0433 for instructions for recording weld identification drawings, weld numbers, welder identification, welding procedure numbers, weld filler material, visual examinations. nondestructive examinations, and for noting satisfactory completion of leak/pressure testing.

b. Required NDE shall be completed and documented before starting leak/pressure testing.

c. NDE/Weld Record information and weld identification drawings specified in 3.1.4 may be incorporated on a single format or traveler for a specific work package.

d. Document examination of pipe supports by recording on sketches or NDE/Weld Record form.

3.2.2 Nondestructive Examination (NDE) of welds for ducting.

3.2.2.1 Production welds sha71 be visually inspected per AWS D1.3.

3.2.2.2 Acceptance criteria for welds shall be per AWS D1.3.

3.2.2 Flushing/Testing Preparation: Obtain direction for disposal of waste flushing and testing water, prior to generation of wastes, from the Operating Contractor.

3.2.3 Cleaning and Flushing

3.2.3.1 After NDE is complete and before leak/pressure testing the system, or before connecting the system into existing systems, clean piping internal surfaces mechanically or by flushing as follows.

\begin{tabular}{||l|l|l|}
\hline \hline Service & Pipe Code & Cleaning Method \\
\hline
\end{tabular}

W320C5.CPY

HNF-2486, Rev, 0

Page B-21

$3-2755 \begin{aligned} & W-320-C 5 \\ & \text { Rev } 0 \\ & 09 / 30 / 94\end{aligned}$ 


\begin{tabular}{|l|l|l|}
\hline Ventilation & $M-4$ & $\begin{array}{l}\text { Pneumatic Flush or } \\
\text { paragraph 3.2.5 }\end{array}$ \\
\hline Ventilation & $M-8$ & $\begin{array}{l}\text { Pneumatic Flush or } \\
\text { paragraph 3.2.5 }\end{array}$ \\
\hline Pressure Relief & $M-42$ & $\begin{array}{l}\text { Pneumatic Flush or } \\
\text { paragraph 3.2.5 }\end{array}$ \\
\hline
\end{tabular}

3.2.3.2 Mechanical cleaning: Remove loose dirt, scale, and debris by brushing. wiping, high pressure water jetting, or other mechanical method approved by the Quality Control Inspector (Buyer).

a. When high pressure water jetting or other mechanical cleaning method utilizing water is used, and leak/pressure testing will not be performed immediately after cleaning, dry piping systems by blowing dry oil-free air or nitrogen through lines.

b. Dry until the relative humidity of discharge air is equal to that of inlet air. Use drain taps at low points of piping system to ensure complete drainage and drying. Cap lines or reconnect to system to maintain cleanliness. Note, humidity measurements from inlet and exhaust shall be converted to the same pressure to verify equality.

3.2.4 Pneumatic flushing:

a. Isolate instruments by valving, plugging, or disconnecting.

b. Blow lines clean with dry, oil-free air or nitrogen at 20 to $30 \mathrm{lb} / \mathrm{in}^{2}$ gage, until discharge air has no visible particulate matter, or for 5 minutes minimum.

c. If leak/pressure testing is not performed immediately after fiushing, cap lines or reconnect to system to maintain cleanliness.

3.2.5 Mechanical cleaning may be substituted for flushing if approved by the Quality Control Inspector (Buyer).

3.2.5.1 Provide documented evidence that flushing or mechanical cleaning has been accomplished as specified in this Section.

\subsubsection{Duct Cleaning}

3.2. 1 Provide necessary equipment, scaffolding, and materials for cleaning dutwork.

3.2.3 Remove debris from inside of new duct, then vacuum to remove small particles of rubbish and dust.

3.2.3 Replace duct section(s) removed for cleaning and those that have been damaged.

HNF-2486, Rev. 0

W320C5.CPY 


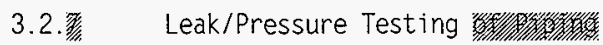

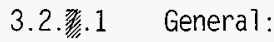

a. After completion of flushing, perform leak/pressure testing of pipe in accordance with ASME B31.3 and this Section. Use calibrated gages with ranges $1-1 / 2$ to 2 times the test pressure.

b. Document testing of each piping system on "Leak/Pressure Test Certification" Form KEH-1757. Use separate forms to describe and record each piping system. Under "Description" describe piping system in enough detail for correlation to weld identification drawings, shop fabrication drawings, and Project Drawings as applicable. For systems tested segmentally. indicate continuity in "Description" to assure that entire systems have been tested.

c. Pipe joints, fittings, and other potential leak sources to be tested shall be visible and accessible during tests.

d. Install one temporary relief valve during testing. Relief valve shall have a discharge capacity of $125 \%$ of capacity of the pressurizing device, and be set to operate at 1-1/3 maximum of the test pressure for hydrostatic testing and not higher than test pressure plus the lesser of $50 \mathrm{lb} / \mathrm{in}^{2}$ or $10 \%$ of the test pressure for pneumatic testing. Tag each relief valve used to show serial number, inspector, date, and pressure setting.

e. Install necessary restraining devices, before applying test pressure, to prevent distortion or displacement of piping.

f. Isolate instruments and other items which could be damaged by test pressures.

g. Continuously maintain test pressure for 10 minutes minimum. Examine weld joints and connections. Piping system, exclusive of possible localized instances at pump or valve packing, shall show no visual evidence of weeping or leaking.

h. Visually examine piping, weld joints and tubing joints, fittings, and other potential leak sources, including welds which attach wear plates, anchors, etc to piping systems, during testing. 
i. Complete testing of piping before field application of insulation or protective coating. Pipe with factory applied protective coating shall be considered as having been tested before protective coating application.

1) Pneumatic pressure testing:

a. Perform in accordance with ASME B31.3, Paragraph 345.5. Leaks may be detected by bubble method or equivalent method.

b. Perform testing with dry, oil-free air or nitrogen on tabulated piping systems.

\begin{tabular}{||l|l|}
\hline Service & Pipe code \\
\hline Ventilation & $M-4$ \\
\hline Ventilation & $M-8$ \\
\hline Ventilation & $M-42$ \\
\hline
\end{tabular}

3.2. 翟.2 Leak/Pressure Testing of Ductwork: Perform in the presence of the Quality Control Inspector (Buyer) unless otherwise instructed in writing.

a. Pressurize the duct to a pressure of - 1翟"W.G. With test boundary under continuous pressure, examine pressure boundaries duct for leaks.

b. Test duration sha7? be 15 minutes minimum, plus additional time necessary for finding leaks.

c. No leakage is allowed at - 13:"W.G.

d. Examine joints and other potential leak sources during testing. Leaks may be detected by bubble method or electronic sound detection. Repair detectable leaks and retest the system.

e. Document ductwork testing on "Leak/Pressure Test Certification," Form KEH-1757. 
3.2.8 Safety Related Product Inspections:

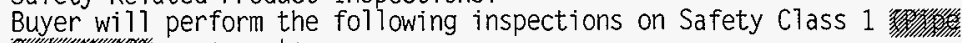

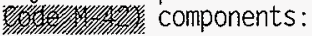

3.2.8.1 Types of inspection points are the following:

a. Receiving (R): Required for inspection of special fabricated items, equipment, or material scheduled to be delivered to the worksite, or other designated location, which require inspection upon arrival and before installation.

b. Construction inspection (H): Required for inspection of specific construction features, before further construction or installation is a) lowed to proceed.

3.2.8.2 Prior to welding, verify $(R)$ the internal diameters of the following components:

See DWG H-2-818477, HVAC PRESSURE RELIEF SEAL LOOP ASSEMBLY DETAILS for additional details.

Part 2, 6" sch 40 pipe

Part 3, 6" flange

Part 4, 6" elbow, short radius

Part 5, 6" bend, 180 deg short radius

Part 11, gasket

At each end of component, approximately $1 / 2$ inch into component, measure two internal diameters, approximately 90 degrees from each other. The gasket only requires two internal diameter measurements. The average internal diameter for each component sha11 be greater than 5.9 inches.

3.2.8.3 Following welding and prior to installation, verify $(\mathrm{H})$ that dimension "A" of the assemblery Detail $\mathrm{B}$. The wall thickness of pipe at dimension "A" may be measured prior to welding.

HNF-2486, Rev. 0

Page B-25

W320C5.CPY 
3.2.8.4 SCHEDULE FOR R, H, AND W POINTS

\begin{tabular}{|l|l|c|c|c|}
\hline $\begin{array}{l}\text { Section } \\
\text { Number }\end{array}$ & \multicolumn{1}{|c|}{ Subject } & Type & $\begin{array}{c}\text { Work- } \\
\text { site }\end{array}$ & Signature \\
\hline 15507 & $\begin{array}{l}\text { Part 2. } \\
\text { 6" sch 40 pipe }\end{array}$ & $R$ & $X$ & \\
\hline & $\begin{array}{l}\text { Part } 3 . \\
\text { 6" flange }\end{array}$ & $R$ & $X$ & \\
\hline & $\begin{array}{l}\text { Part 4, } \\
\text { 6" elbow, } \\
\text { short radius }\end{array}$ & $R$ & $X$ & \\
\hline & $\begin{array}{l}\text { Part 5, } \\
6 " \text { bend, } \\
\text { 180 deg short } \\
\text { radius }\end{array}$ & $R$ & $X$ & \\
\hline & \begin{tabular}{l} 
Part 11, gasket \\
\hline 15507
\end{tabular} & $R$ & $X$ & \\
\hline & $\begin{array}{l}\text { Assembly } \\
\text { Dimension "A" }\end{array}$ & $H$ & $X$ & \\
\hline & & & & \\
\hline
\end{tabular}


3.3 SCHEDULES

3.3.1 Schedule of Pipe Weld NDE/Testing

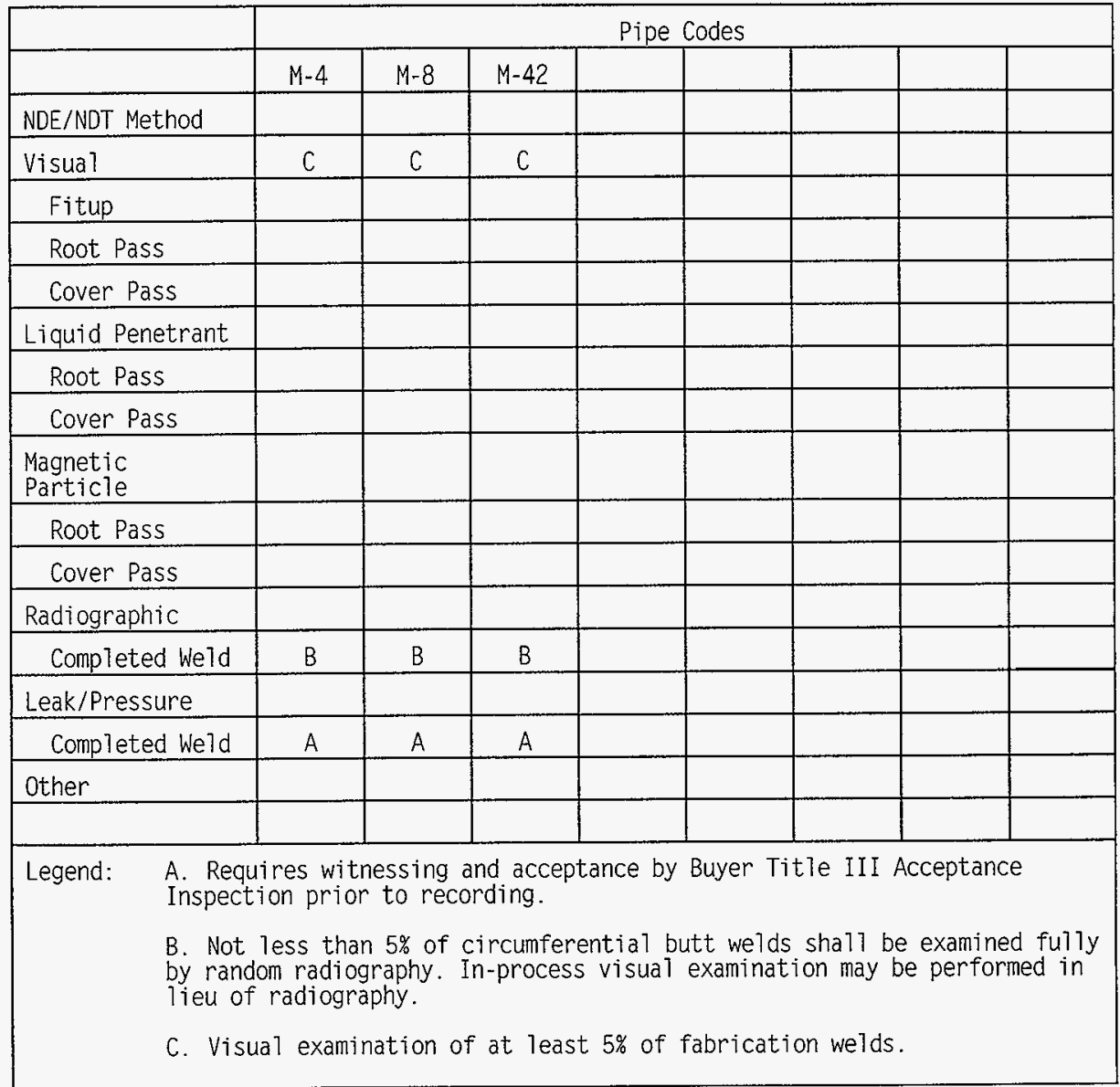




\begin{tabular}{|c|c|}
\hline $10.17 .0 \%$ & OUCT COOE D 3 (Sa fety Glass 3 ) \\
\hline servige & 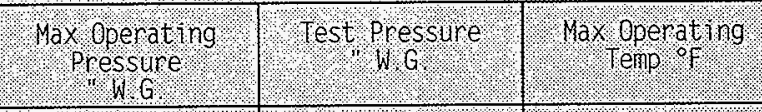 \\
\hline Vent trat on $(17)$ & 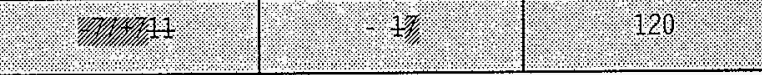 \\
\hline Duct Material & $\begin{array}{l}18 \text { GA. Stainless steel sheet, ASTM A } 240 \text {, Type } 304 L \text {. } \\
\text { Stainless steel shapes, ASTM A } 276 \text {, Type 304L. Slip- } \\
\text { on flanges: Light weight, stainless steel, Alaska } \\
\text { Copper and Brass SK-37, or an approved substitute. }\end{array}$ \\
\hline $\begin{array}{l}\text { Duct } \\
\text { Construction }\end{array}$ & $\begin{array}{l}\text { As shown on the Drawings and as specified in this } \\
\text { specification. }\end{array}$ \\
\hline Duct welding & $\begin{array}{l}\text { Butt type welding with full penetration weld on seams. } \\
\text { companion angle flanges shall be seal welded to duct. } \\
\text { or as noted on the Drawings. }\end{array}$ \\
\hline Duct Support & As specified on drawings \\
\hline Gaskets & $\begin{array}{l}\text { Ethylene Propylene Rubber (EPR), 1/16" thick. Use } \\
\text { full face gaskets with flat face flanges. Garlock } \\
\text { "Blue-Gard" style } 3700 \text {. }\end{array}$ \\
\hline Fast & $\begin{array}{l}\text { Bolts, ASTM A 193, Grade B8. Heavy hex nuts, ASTM A } \\
\text { 194, Grade 8F, UNC threads. }\end{array}$ \\
\hline
\end{tabular}




\begin{tabular}{|c|c|}
\hline $1.1 .1 \%$ & CoDE M 4 (Contaminated) (safety Class 3 ) \\
\hline (1.1. & 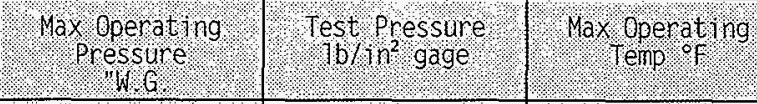 \\
\hline Ventivat ion & . \\
\hline Sizes & 8-inch \\
\hline Pipe & ASTM A 53, Type E or S, Grade B black steel \\
\hline Wal1 Thickness & Schedule 40 \\
\hline Fittings (1) & $\begin{array}{l}\text { ASTM A 234. Grade WPB, wrought stee1, buttwelding in } \\
\text { accordance with ASME B16.9. Wa11 thickness to match } \\
\text { pipe. }\end{array}$ \\
\hline Unions & None \\
\hline Flanges & $\begin{array}{l}\text { ASTM A 105. Class } 150 \text {, raised face, weld neck, in } \\
\text { accordance with ASME B16.5. Bore to match pipe ID. } \\
\text { Use slip-on for sizes } 8 \text {-inch and larger. Use flat } \\
\text { face where mating to flat face flanges. }\end{array}$ \\
\hline Bolting & $\begin{array}{l}\text { ASTM A 193, Grade B6, alloy steel studs, and ASTM A } \\
\text { 194, Grade 2, heavy hex nuts. }\end{array}$ \\
\hline Gaskets & $\begin{array}{l}\text { Ethylene Propylene Rubber (EPR), } 1 / 16 \text { " thick. Use } \\
\text { ful face gaskets with flat face flanges. Garlock } \\
\text { "Blue-Gard" style } 3700 \text {. }\end{array}$ \\
\hline
\end{tabular}




\begin{tabular}{|c|c|}
\hline \multicolumn{2}{|r|}{ PIPE CODE M-8 (Safety Class 3) } \\
\hline Service & 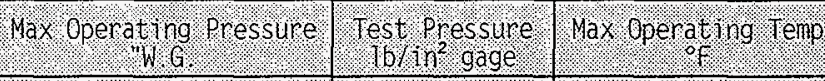 \\
\hline 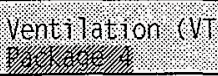 & (i. \\
\hline Sizes & All sizes \\
\hline Pipe & ASTM A 312, Grade TP 304L, seamless. \\
\hline Wa11 Thickness & Schedule $40 \mathrm{~S}$ \\
\hline Fittings & $\begin{array}{l}\text { ASTM A 403. Class WP } 304 \mathrm{~L} \text {, buttwelding in accordance with } \\
\text { ASME B16.9, wall thickness to match pipe. }\end{array}$ \\
\hline Flanges & $\begin{array}{l}\text { ASTM A } 182 \text {, Grade } F 304 L \text {, ClaSs } 150 \text {, forged, raised face, } \\
\text { weld neck in accordance with ASME B16.5. Bore to match } \\
\text { pipe ID. Use flat face where mating to flat face flanges. }\end{array}$ \\
\hline Bolting & $\begin{array}{l}\text { ASTM A 193, Grade B8, alloy steel studs, and ASTM A } 194 \text {, } \\
\text { Grade } 8 F \text {, heavy hex nuts. }\end{array}$ \\
\hline Gaskets & $\begin{array}{l}\text { Ethylene Propylene Rubber (EPR), 1/16" thick. Use full } \\
\text { face gaskets with flat face flanges. Garlock "Blue-Gard" } \\
\text { style } 3700 \text {. }\end{array}$ \\
\hline \multicolumn{2}{|l|}{ Vaives } \\
\hline $\mathrm{Ba} 77$ & \multirow{3}{*}{ 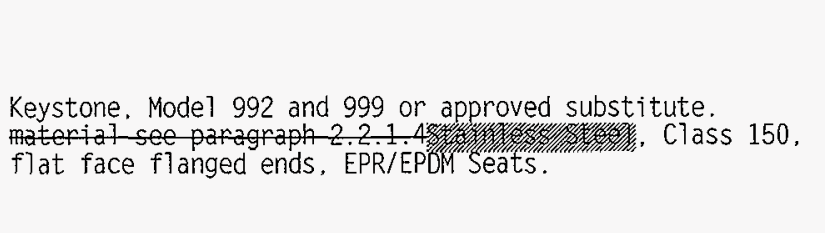 } \\
\hline Butterfiy & \\
\hline Expansion Joint & \\
\hline
\end{tabular}

HNF-2486, Rev. 0 


\begin{tabular}{|c|c|}
\hline \multicolumn{2}{|r|}{ PIPE CODE M-42 (Safety Class 1 ) } \\
\hline Service & $\begin{array}{l}\text { Max operating pressure } \\
\text { inches water gage }\end{array}$ \\
\hline 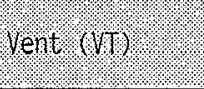 & 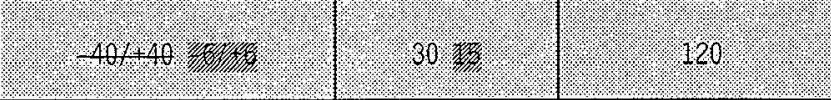 \\
\hline Size & 6-inch \\
\hline Pipe & ASTM A 312, Grade TP 304L, seamless. \\
\hline Fittings & $\begin{array}{l}\text { ASTM A } 403 \text {, Class WP } 304 \mathrm{~L} \text {, buttwelding in accordance with } \\
\text { ASME B16.9, wall thickness to match pipe. }\end{array}$ \\
\hline 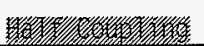 & 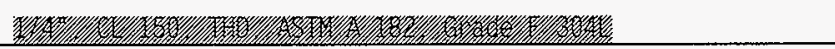 \\
\hline Flanges & $\begin{array}{l}\text { ASTM A } 182 \text {, Grade F } 304 L \text {, Class } 150 \text {, forged, raised face, } \\
\text { weld neck in accordance with ASME B16.5. Bore to match } \\
\text { pipe ID. Use flat face where mating to flat face flanges. }\end{array}$ \\
\hline Bolting & $\begin{array}{l}\text { ASTM A } 193 \text {, Grade B8, alloy steel studs, and ASTM A } 194 \text {, } \\
\text { Grade } 8 \mathrm{~F} \text {, heavy hex nuts. }\end{array}$ \\
\hline Gaskets & $\begin{array}{l}\text { Compressed fiber, nonasbestos, } 1 / 16 " \text { thick sheet: Garlock } \\
\text { "Blue-Gard" Style } 3000 \text {. Use ful1 face gaskets with flat } \\
\text { face flanges. }\end{array}$ \\
\hline Gauge & 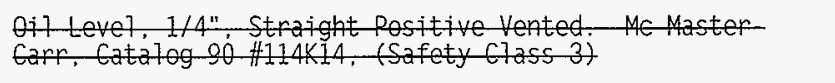 \\
\hline
\end{tabular}


1. GENERAL

The identification of piping systems shall be in accordance with the American Standard Scheme for the Identification of Piping Systems, ANSI A13.1, as supplemented herein.

2. LOCATION

a. Except as provided below, identification shall be located adjacent to outlets, valves, flanges, unions, changes-in-direction, where pipes pass through walls, floors, or ceilings, and along an uninterrupted length of pipe at maximum intervals of 50 feet. Each line or branch in a room shall have at least one identification.

b. Where a number of outlets, valves, flanges, unions, or changes of direction make identification at each item impracticable, they may be spaced at approximate 6 foot intervals, preferably adjacent to valves.

c. Legend shall be located on the pipe so that it can be read easily from the operator's normal viewing position. Labels shall be placed on the readily visible lower quadrant of overhead pipes, and on an upper quadrant of pipes below normal eye level. Above ceilings, labels shall be placed in locations most readily visible from access positions.

3. LEGEND

a. Positive identification of a piping system content shall be by lettered legend giving the name of the content in full or abbreviated form. Legends may also be as specified on drawings or in other specifications.

b. Abbreviation of words in the legend may be used only where unavoidable due to space limitations.

c. The legend shall include the nominal operating pressure for steam, compressed air, and when specifjed, the pressure or temperature for other materials.

d. An arrow indicating the direction of flow shall be placed near the legend on pipes normally having a flow in one direction only. The color and size of the arrow shall be consistent with the color and size of the legend letters.

e. Legend shall be located on or adjacent to the classification color band.

HNF-2486, Rev. 0

W320C5.CPY

Page B-34

Th $\begin{aligned} & W-320-C 5 \\ & \operatorname{Rev} 0\end{aligned}$ 
a. Legend and color classification may be accomplished by the use of approved labels conforming to this Standard and which are suitable for the temperature of the surface to which they are to be applied. Approved labels include the following.

\section{ALL-TEMPERATURE PIPE MARKERS}

W. H. Brady Company

727 West Glendale Avenue

Milwaukee, Wisconsin 53201

TEL-A-PIPES

Westline Products Division

Thomas \& Betts Company

220 South Rose Street

Los Angeles, California 90012

b. Single-word labels may be combined to form complete legends.

Individual-letter labels shall not be so combined.

c. Labels shall be installed after painting is complete.

\section{CLASSIFICATION COLOR}

a. When use of classification colors is specified, they shall conform to Table 1.

\begin{tabular}{|c|c|c|c|}
\hline \multicolumn{4}{|c|}{ Table 1} \\
\hline Glas st fica tion & Band or l l abel & legendirar row & 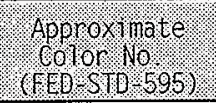 \\
\hline Dangerous & Yellow & Black (17038) & 13655 (yel1ow) \\
\hline 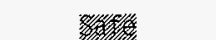 & 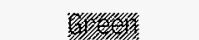 & 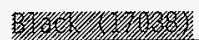 & 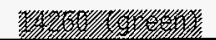 \\
\hline
\end{tabular}

b. Paint, if used, shall conform to FS TT-E-489, Class A, for synthetic gloss enamel.

c. Classification colors shall conform to Table 2 .

\begin{tabular}{|c|c|}
\hline \multicolumn{2}{|c|}{ Table 2. Classification Colors. } \\
\hline Materlall: & 1. \\
\hline Ventilation (VT) & Yellow \\
\hline 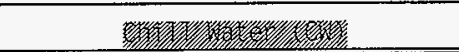 & 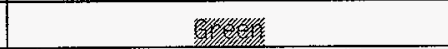 \\
\hline
\end{tabular}


HNF-2486, Rev. 0

W320-28-032

Process Building Piping Stress Analysis 
This shoot showe tho status and doscription of the sttachod Desion Analysis shoots.

Projoct No. \& Name W320, Tank 2HI-C-106 sluicing

calculation Itom Process Buildino Pipina stress Analysis

These calculation apply to:

Dwo. No. $\mathrm{H}-2-818480$

Rov. No.

Dwg. No.

Rov. No.

Other (Study, CDR)

Rov. No.

The status of these celculations is:

$\square$ Proliminary Calculations

Dinal Calculations

$\square$ Check Calculations (On Calcuitation Deted 1

$\square$ Void Calculstion (Reason Voided

$\begin{array}{lll}\text { Incorporatod in Finel Drswings? } & \square \text { Yos } & \square \text { No } \\ \text { This calculetion verified by independent "chock" celculations? } & \square \text { Yos }\end{array}$

Original and Revised Calculation Approvals:

\begin{tabular}{|c|c|c|c|}
\hline & $\begin{array}{c}\text { Rev. } 0 \\
\text { Signsture/Oate }\end{array}$ & $\begin{array}{c}\text { Rov. } 1 \\
\text { Signaturo/Date }\end{array}$ & $\begin{array}{c}\text { Rev. } 2 \\
\text { Signaturo/Dete }\end{array}$ \\
\hline Originator & Rodnad C. Cared oll z-23-25 & & \\
\hline Chocked by & 28195 & & \\
\hline Approved by & $1 / 95$ & & \\
\hline $\begin{array}{l}\text { Checked Against } \\
\text { Approved Vendor Oata }\end{array}$ & & & \\
\hline
\end{tabular}

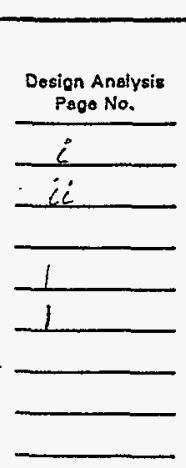

INDEX

Calculation Identification and Index
Calculation Cross Index

Sbiective, Criteria; Given Doto; Assumptions, Methods, References Colculotion Conclusions

Anpondix $\theta$

HNF-2486, Rev. 0

Page C-1 


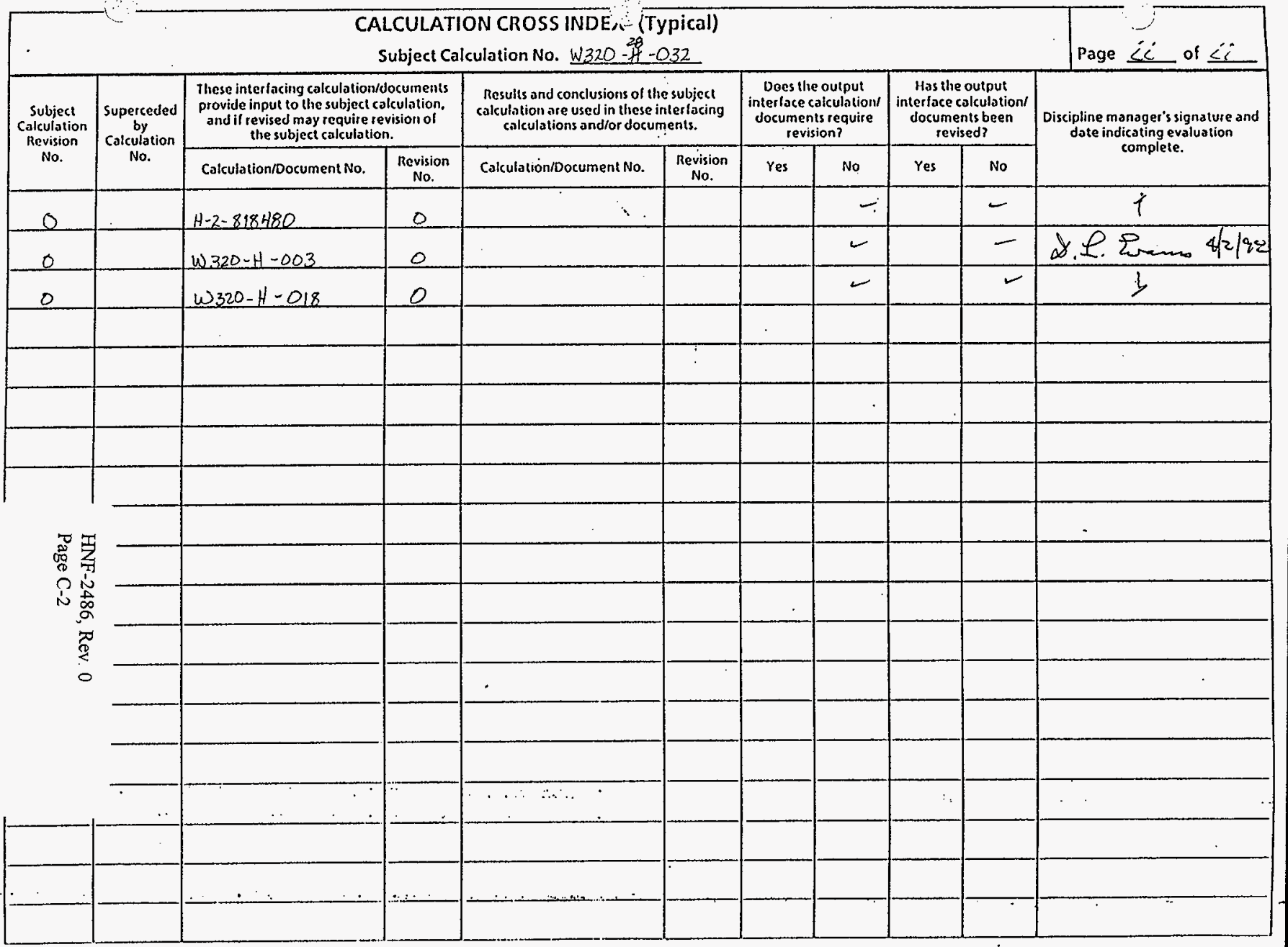


ICF Kaiser Hanford

\section{Company}

\section{Client WHC}

Subject Process Building Piping Stress Analysis

Location: Tank 241-C-106

\section{DESIGN ANALYSIS}

Calc. No. W320- $\stackrel{28}{28}-032$

Revision 0

Page No. 1 of 1

WO/Job No. ER4319N 320

Date 2-23-95

Checked

By Rodney C. Campbell

Revised

By

\section{OBJECTIVE:}

The objective of this calculation is to verify that the Process building HVAC piping meets ASME B31.3 code stress requirements.

\section{CRITERIA:}

1. Functional Design Criteria WHC-SD-W320-FDC-001, Rev. 2.

2. ASME B31.3-1993, Chemical Plant and Petroleum Refinery Piping

3. Standard Design Criteria 4.1, Rev. 12, Design Loads for Facilities.

4. DOE Order 6430.1A, General Design Criteria

5. W320-QAPP-001, Rev. 0, Project Specific Quality Assurance Plan.

\section{GIVEN DATA:}

Note: All the given data can be found in the criteria sources or references.

1. Ambient temperature: $50^{\circ} \mathrm{F}$.

2. Maximum temperature: $180^{\circ} \mathrm{F}$.

3. All ducting is safety class 3 .

4. All ducting is made from ASTM A312, type 304L, schedule 40, stainless steel pipe.

5. The horizontal components of the acceleration due to an earthquake are 0.12 .

6. The minimum corrosion allowance is .02 inches.

7. The pressure in the 8 " duct is -23.4 inches of water and the pressure in the 6 "line is -38.5 inches of water.

\section{ASSUMPTIONS:}

None

\section{METHODS:}

1. Autopipe+ Version 4.50

\section{REFERENCES:}

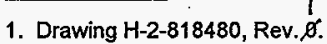

2. Tube Turn Weld-Neck Fittings.

3. Garlock Expansion Joints.

4. Keystone Super Seat Butterfly Valves.

5. W320

6. W320-F-018, Rev. 0

\section{CALCULATION:}

The Process building HVAC piping was analyzed in three separate sections labeled W32OH32A, W32OH32B, and W32OH32C. These three sections can be found on drawing H-2-818480. The Autopipe analysis for each section can be found in Appendix $A$.

\section{CONCLUSIONS:}

The three Process building HVAC piping sections analyzed meet the ASME B31.3 criteria. 
APPENDIX A

HNF-2486, Rev, 0

Page C-4 
H320H32A PROCESS BUIIDING PIPING STRESS AMALYSIS ICF KAISER HANFORD COMPANY 03/01/95 H320 TANK 241-C-106 SLUICING ER4319 AUTOPIPE+4.50 MODEL PACE

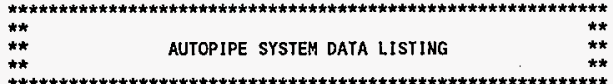

SYSTEM NAME : W32OH32A

PROJECT ID : PROCESS BUILDING PIPING STRESS ANALYSIS H320, TANK 241-C-106 SLUICING, ER4319

PREPARED BY :

RC CAMPBELL

CHECKED BY :

PIPING CODE : 831.3

AMBIENT TEMP. $(\operatorname{deg} F): 50.0$

COMPONENT LIBRARY : AUTOPIPE

MATERIAL LIBRARY : AUTOB313

MODEL REVISION NUMBER : 38

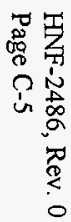

W320H32A PROCESS BUILDING PIPING STRESS ANALYSIS ICF KAISER HANFORD COMPAHY

ANALT ICF KAISER HAN MRD CONPANY DERE

POINT DATA LISTING

POINT

NAME TYPE $X$ $Y$ P $Z$ PIPE ID DESCRIPTION

AOD SEGERT (n)

Flex

0 10SCED40 wt $=35 \mathrm{tb}, \mathrm{Ar}=97.6 \mathrm{sq}$. in

Axial = 1200.0 Tors= RIGID

Y-Shear= RIGID Bend= RIGID

$\begin{array}{llrrr}\text { A01 } & \text { Valv } & 0 & 0.67 & 0 \\ & & & & \\ \text { A02 } & \begin{array}{l}\text { Run } \\ \text { Bund }\end{array} & 0 & 0.21 & 0 \\ \text { A03 } & 0 & 1.33 & 0 \\ & & & & \\ & & & & \\ \text { A04 } & \begin{array}{l}\text { Tee } \\ \text { Redu }\end{array} & 1.29 & 0.46 & 0 \\ \text { A05 } & \begin{array}{l}\text { Redu } \\ \text { Run }\end{array} & 0.58 & -0.09 & -0.17 \\ \text { A07 } & \text { Bend } & 0.75 & 0 & 0\end{array}$

Rating $=150$, Weight $=65 \mathrm{lb}$
Surface factor $=4.50$, S1F $=1.00$

Short Elbow, Radius $=10.00$ inch Bend angle change $=90.00$ deg

SIF : In $=3.41$, out $=2.84$

Long Elbow, Radius $=9.00$ inch Bend angle change $=90.00 \mathrm{deg}$

$\begin{array}{lllll}A 08 & \text { Run } & 0 & -0.94 & 0\end{array}$

$\begin{array}{lrrrr}* * * & \text { SEGMENT B } & & & \\ \text { AO4 } & \text { Tee } & 1.29 & 2.21 & 0 \\ \text { B01 } & \text { Bend } & 0 & -0.67 & 0.67\end{array}$

Long Elbow, Radius $=9.00$ inch Bend angle change $=45.00 \mathrm{deg}$

$\begin{array}{lllrr}\text { B02 } & \text { Run } & 0 & 0 & 0.31 \\ \text { B03 } & \text { Run } & 0 & -0.02 & 0.71\end{array}$

$\begin{array}{llll}\mathrm{B} 04 \text { Run } & 0 & -0.02 & 0.7 \\ & 0 & -0.02 & 1.72\end{array}$

Long Elbow, Radius $=9.00$ inch Bend angle change $=89.99$ deg

$\begin{array}{lllll}\text { B05 Run } & 2.62 & -0.05 & 0\end{array}$

$\begin{array}{llll}\text { B06 Tee } & 2.18 & -0.06 \\ \text { B07 Valv } & 0.47 & -0.01\end{array}$

B08 Run $0.18 \quad 0 \quad 0$

$\begin{array}{lllll}B 10 & \text { Bend } 0 & -1.82 & 0\end{array}$
Rating $=150$, Weight $=17 \mathrm{lb}$ Surface factor $=4.30$, SIF $=1.00$

Long Elbow, Radius $=9.00$ inch Bend angle change $=90.00$ deg

Long Elbow, Radius $=9.00$ inch Bend angle change $=90.00 \mathrm{deg}$ SIF - In $=2.27$, out $=1.89$ 

W320H32A PROCESS BUILDING PIPING STRESS ANALYSIS ICF KAISER HANFORD COMPANY

POINT DATA LISTING

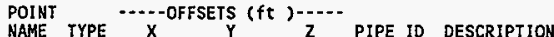

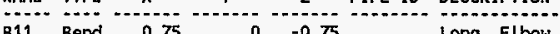

B11 Bend Long Elbow, Radius $=9.00$ inch SIF - In $=2.27$. $=45.00 \mathrm{deg}$

$\begin{array}{lllll}\text { B12 } & \text { Run } & 0 & 0 & -0.57 \\ \text { B13 Flex } & 0 & 0 & -0.25\end{array}$

Wt $=35 \mathrm{lb}, \mathrm{Ar}=40.6 \mathrm{sq}$. in Axial $=1360.0$ Tors= RIGID $\mathrm{Y}$-Shear= RIGID Bend= RIGID Z-Shear $=$ RIGID Bend $=$ RIGID

$\begin{array}{lllll}\text { B14 Run } & 0 & 0 & -0.50 \\ \text { B15 Bend } & 0 & 0 & -0.30\end{array}$

Long Elbow, Radius $=9.00$ inch Bend angle change $=45.00 \mathrm{deg}$

B16 Run $-0.22 \quad 0 \quad-0.22$

*** SEGMENT C

$\begin{array}{lllrr}\text { B06 } & \text { Tee } & 6.09 & 1.39 & 3.41 \\ \text { C01 } & \text { Bend } & 0.51 & 0 & -1.29\end{array}$

$\begin{array}{lllll}\text { c02 Valv } & 0.50 & 0.00 & 0.00\end{array}$

$\begin{array}{lllll}\text { C03 } & \text { Run } & 0.18 & 0 & 0 \\ \text { C04 } & \text { Run } & 0.02 & 0 & 0 \\ \text { C05 } & \text { Tee } & 0.45 & 0 & 0 \\ \text { C06 } & \text { Valv } & 0.45 & 0 & 0\end{array}$

$\begin{array}{lllll}\text { C07 } & \text { Run } & 0.18 & 0 & 0 \\ \text { Co8 } & \text { Bend } & 1.15 & 0 & 0\end{array}$

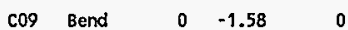

$\begin{array}{lllll}\text { c10 } & \text { Run } & 0 & 0 & 0.78 \\ \text { c11 } & \text { Tee } & 0 & 0 & 0.44 \\ \text { C12 } & \text { Run } & 0 & 0 & 0.47\end{array}$

*** SEGMENT D

$\begin{array}{llll}\text { C19 Tee } & 9.52 & -0.19 & 3.34 \\ 001 & 6 \text { SCHEO } 40\end{array}$

Long Elbow, Radius $=9.00$ inch

Bend angle change $=90.00 \mathrm{deg}$ Bend angle change $=68$. SIF - In $=2.97$, Out $=2.47$

Rating $=150$, Weight $=17 \mathrm{lb}$
Surface factor $=4.30$, sIF $=1.00$

Rating $=150$, Weight $=17 \mathrm{lb}$

Long Elbow, Radius $=9.00$ inch Bend angle change $=90.00 \mathrm{deg}$

Long Elbow, Radius $=9.00$ inch SIF - In $=2.27$, out $=1.89$
U320H32A PROCESS BUILDING PIPING STRESS ANALYSIS ICF KAISER HANFORD COMPAMY

03/01/95 H320, TANK 241-C-106 SLUICING ERG319 AUTOPIPE+4.50 MODEL PACE

A410-106 SLUICING, ER4319 AUTOPIPE+4.50 MODEL PAGE

POINT DATA LISTING

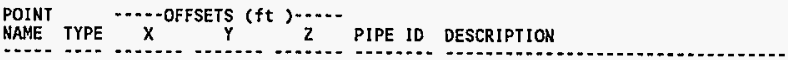

SIF - In $=2.27$, out $=1.89$

$\begin{array}{lllll}D 02 & \text { Run } & 0 & 0 & -1.36 \\ \text { D03 Flex } & 0 & 0 & -2.10\end{array} \quad W t=35 \mathrm{lb}, A r=40.6 \mathrm{sq}$. in

Axial $=$ ' 1360.0 Tors= RIGID

$Y$-Shear $=$ RIGID Bend $=$ RIGID

$\begin{array}{lllll}\text { D04 Run } & 0 & 0 & -0.50 \\ \text { D05 Bend } & 0 & 0 & -1.00 \quad \text { Long Elbow, Radius }=9.00 \text { inch }\end{array}$

Long Elbow, Radius $=9.00$ inch SIF - In $=2.27$, out $=1.89$

D06 Valv $0 \begin{array}{ccc}-0.75 & 0\end{array}$

D07 Run $0 \quad-0.18 \quad 0$

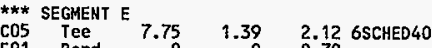

E01 Bend 0 Short Elbow, Radius $=6.00$ inch Surface factor $=4.30$, SIF $=1.00$

E02 Bend $1.14 \quad 0 \quad-1.14$

$\begin{array}{lllll}\text { E03 } & \text { Run } & 0.37 & 0 & 0 \\ \text { E04 } & \text { Tee } & 2.60 & 0 & 0\end{array}$

*** SEGMENT $F$, $11.85 \quad 1.39 \quad 0.206$ 6SCHED 40

E04 Tee 11.85

$\begin{array}{lllll}\text { F02 } & \text { Run } & 0 & 0 & -0.18 \\ \text { F03 } & \text { Run } & 0 & 0 & -0.88 \\ \text { F04 } & \text { Bend } & 0 & 0 & -1.00\end{array}$

F05 Bend $0 \quad 2.57 \quad 0$

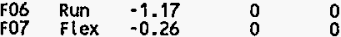
Bend angle change $=90.00 \mathrm{deg}$ Bend angle change $=45.03 \mathrm{deg}$

Short Elbow, Radius $=6.00$ inch Bend angle change $=44.97 \mathrm{deg}$

Rating $=150$, Weight $=17 \mathrm{lb}$

Surface factor $=4.30$, sif $=1.00$ SIF - In $=2.97$, Out $=2.47$

Rating $=150$, Weight $=17$ tb Surface factor $=4.30$, SIF $=1.00$

Long Elbow, Radius $=9.00$ inch SIF - In $=2.27$, out $=1.89$

Long Elbow, Radius $=9.00$ inch Bend angle change $=90.00 \mathrm{deg}$ $W t=35 \mathrm{lb}, A r=40.6 \mathrm{sq}$. in 
W320H32A PROCESS BUILDING PIPING STRESS ANALYSIS ICF KAISER HANFORD COMPANY 03/01/95 W320 TANK 241-C-106 SIUICIHG ER4319 AUTOPIPE+4 50 MOOEL PAGE

POINT DATA LISTING

POINT
NAME TYPE
$\ldots$

Y-Shear= RIGID Bend= RIGID

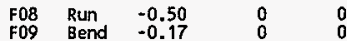

Short $\mathrm{Elbow}$, Radius $=6.00$ inch Bend angie change $=29.88$ deg

F10 Run $-0.17 \quad 0 \quad 0.10$

\#** SEGMENT $G$ Tee $11.85 \quad 1.39 \quad 0.20$ 6SCHED40

G01 Bend $000.97^{6 S C H E D 40}$ Short Elbow, Radius $=6.00$ inch Bend angle change $=90.00$ deg

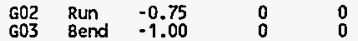

G04 Flex $0 \quad-1.45 \quad 0$

$\begin{array}{lllll}\text { G05 Run } & 0 & -0.50 & 0 \\ \text { G06 } & \text { Bend } & 0 & -0.50 & 0\end{array}$

607 Valv $\quad 0 \quad 0 \quad-0.65$

GOB Run $\quad 0 \quad 0 \quad-0.18$

Total weight of empty pipes : $1083 \mathrm{lb}$

Long Elbow, Radius $=9.00$ inch Bend angle change $=90.00 \mathrm{deg}$
SIF - in $=2.27$, out $=1.89$

$W t=35 \mathrm{lb}, A r=40.6 \mathrm{sq}$. in Axial $=$ 1360.0 Tors $=$ RIGID Y-Shear $=$ RIGID Bend= RIGID
Z-Shear= RIGID

Short Elbow, Radius $=6.00$ inch Bend angle change $=90.00$ deg

Rating $=150$, Weight $=17 \mathrm{lb}$ Rating $=150$, Weight $=17 \mathrm{Ib}$
Surface factor $=4.30$, SIF $=1.00$
$1320432 A$ PROCESS BUILDING PIPING STRESS ANALYSIS ICF KAISER 03/01/95 H320 TANK 241-C-106 SLUICING AMALSIS ICF KAISER HANFORD COAPANY

6

CONPONENT DATA L. ISTING

POINT ---COORDINATE(ft )--- DATA

NAME $X$ Y TYPE DESCRIPTION

W** SEGMENT A

A00 $\quad 0.00 \quad 0.00 \quad 0.00$ ANCHOR Rigid $\begin{aligned} & \text { Thermat movements : None } \\ & \text { A }\end{aligned}$

$\begin{array}{llll}A 01 & 0.00 & 0.67 & 0.00\end{array}$

0.00 FLANGE Weld neck
Rating $=150$, weight $=52$ ib

$\begin{array}{llll}A 03 & N \quad 0.00 & 1.38 & 0.00 \\ A 03 & 0.00 & 2.21 & 0.00\end{array}$

A03 M $\quad 0.24 \quad 1.97 \quad 0.00$ Y-STOP ID : A03 M1, Connected to Ground

$\mathrm{AO3} F \quad 0.83$

$\mathrm{AO4} \quad 1.29$

$\begin{array}{ll}A 05 & 1.75 \\ A 06 & 2.33\end{array}$

A07 N 2.34

A07 3.08

A07 F 3.08

$2.21 \quad 0.00$

$2.21 \quad 0.00$ TEE

B16.9 welding tee

SIF. In $=1.73$, out $=1.97$

$\begin{array}{rr}2.21 & 0.00\end{array}$

$2.12 \quad-0.17$

$2.12-0.17 \mathrm{~T}$

$1.37-0.17$

*** SEGMENT B

$\begin{array}{llll} & 2.21 & 0.00 \text { TEE } & \text { B16.9 welding tee } \\ \text { SIF }- \text { In }=1.73, \text { out }=1.97\end{array}$

$\begin{array}{llll}\text { B01 N } & 1.29 & 1.76 & 0.45 \\ \text { B01 } & 1.29 & 1.54 & 0.67\end{array}$

B01 F $\quad 1.29 \quad 1.54 \quad 0.98$

$\begin{array}{llll}\text { B02 } & 1.29 & 1.54 & 0.98 \\ & 1.54 & 0.98 \text { FLANGE Weld neck } & \text { SIF }=1.00\end{array}$

Rating $=150$, Weight $=24$ ib

FLANGE Weld neck SIF $=1.00$

$\begin{array}{lllll}\text { B03 } & 1.29 & 1.52 & 1.69 & \text { Rating }=150 \text {, Weight }=24 \mathrm{lb} \\ \text { B04 N } & 1.29 & 1.51 & 2.66 & \\ \text { B04 } & 1.29 & 1.50 & 3.41 \mathrm{TI} & \\ \text { B04 F } & 2.04 & 1.49 & 3.41 & \\ \text { B05 } & 3.91 & 1.45 & 3.41 \text { Y-STOP ID : B05 1, Connected to Ground }\end{array}$ Gap-Below $=0.00$, Above $=1.50$ inch Friction $=0.00$

B06 $6.09 \quad 1.39 \quad 3.41 \mathrm{TEE}$ Gaps set Weightless

SIF - in $=1.52$, Out $=1.69$

3.41 FLANGE Weld neck SIF $=1.00$

B08 $\quad 6.74 \quad 1.38 \quad 3.41$ FLANGE Weld neck

Rat $6.74 \quad 1.38=150$, weight $=24 \mathrm{ib}$

$\begin{array}{llll}\text { B09 N } & 6.74 & 1.38 & 3.41 \\ \text { B09 } & 7.49 & 1.38 & 3.41 \mathrm{TI} \\ \text { B09 F } & 7.49 & 0.63 & 3.41\end{array}$ 


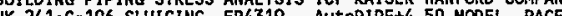
AUTOPIPEH 50 MODEL PAGE

COMPONENT DATA LISTING

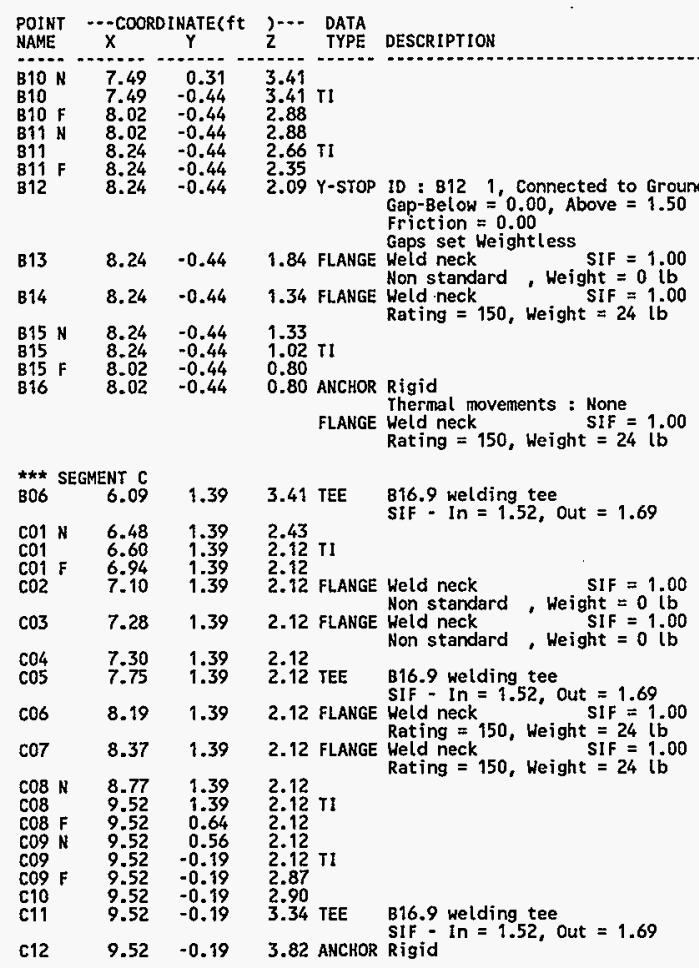

W32OH32A PROCESS BUILDING PIPING STRESS ANALYSIS ICF KAISER HANFORD COMPANY

03/01/95 H320, IANK 241-C-106 SLUICING, ER4319 AUTOPIPE+4.50 MOOEL PAGE 8

\section{COMPONENT DATA LISTING}

$\begin{aligned} & \text { POINT } \\ & \text { NAME }\end{aligned}$

\begin{tabular}{|c|c|c|c|c|}
\hline $\begin{array}{l}* * * \\
c 11\end{array}$ & $\begin{array}{r}\text { SEGMENT D } \\
9.52\end{array}$ & -0.19 & 3.34 TEE & B16.9 welding tee \\
\hline $\begin{array}{l}\text { Do1 } \\
\text { D01 }\end{array}$ & $\begin{array}{l}9.52 \\
9.52\end{array}$ & $\begin{array}{l}2.48 \\
3.23\end{array}$ & $\begin{array}{l}3.34 \\
3.34\end{array}$ & \\
\hline
\end{tabular}

D01 $\quad 9.52 \quad 3.23 \quad 3.34 \mathrm{TI}$

$\begin{array}{llllll}\text { DO1 F } & 9.52 & 3.23 & 2.59 & \\ D 02 & 9.52 & 3.23 & 1.98 & Y \text {-STOP ID : D02 } & 1, \text { Connected to Ground }\end{array}$ D02 $9.52 \quad 3.23 \quad 1.98$ Y-STOP ID : D02 1 , Connected to Ground Friction $=0.00$
D03 $\quad 9.52 \quad 3.23 \quad-0.13$ FLANGE Weid neck Heightless SIF $=1.00$
$\begin{array}{lllll}\text { D03 } & 9.52 & 3.23 & -0.13 \text { FLANGE Weld neck } & \text { SiF }=1.00 \\ \text { D04 } & 9.52 & 3.23 & -0.63 \text { FLANGE Weld neck }\end{array}$
Rating $=150$, Height $=24 \mathrm{lb}$

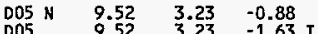

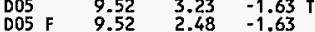

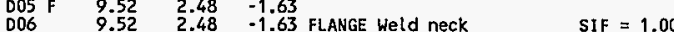

Rating $=150$, weight $=24 \mathrm{lb}$

$2.30-1.63$ ANCHOR Rigid

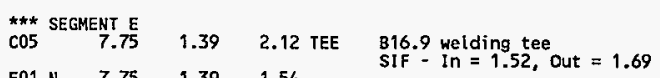

$\begin{array}{llll}\text { E01 N } & 7.75 & 1.39 & 1.54 \\ \text { E01 } & 7.75 & 1.39 & 1.33 \mathrm{TI} \\ \text { E01 F } & 7.89 & 1.39 & 1.19 \\ \text { E02 N } & 8.74 & 1.39 & 0.34 \\ \text { E02 } & 8.89 & 1.39 & 0.20 \mathrm{TI} \\ \text { E02 F } & 9.09 & 1.39 & 0.20 \\ \text { E03 } & 9.25 & 1.39 & 0.20\end{array}$

B16.9 welding tee
SIF - In $=1.52$, out $=1.69$

\begin{tabular}{|c|c|c|c|c|}
\hline $\begin{array}{l}* * * \text { SE } \\
\mathrm{EO4}\end{array}$ & $\begin{array}{l}\text { MENT } F \\
11.85\end{array}$ & 1.39 & 0.20 TEE & B16.9 welding tee \\
\hline F01 & 11.85 & 1.39 & -0.27 FLANGE & Weld neck \\
\hline F02 & 11.85 & 1.39 & -0.45 FLANGE & Weld neck \\
\hline $\begin{array}{l}\mathrm{FO3} \\
\mathrm{FO4} \\
\mathrm{FO4}\end{array}$ & $\begin{array}{l}11.85 \\
11.85\end{array}$ & $\begin{array}{l}1.39 \\
1.39 \\
1.30\end{array}$ & $\begin{array}{l}-1.33 \\
-1.59 \\
-2\end{array}$ & \\
\hline
\end{tabular}




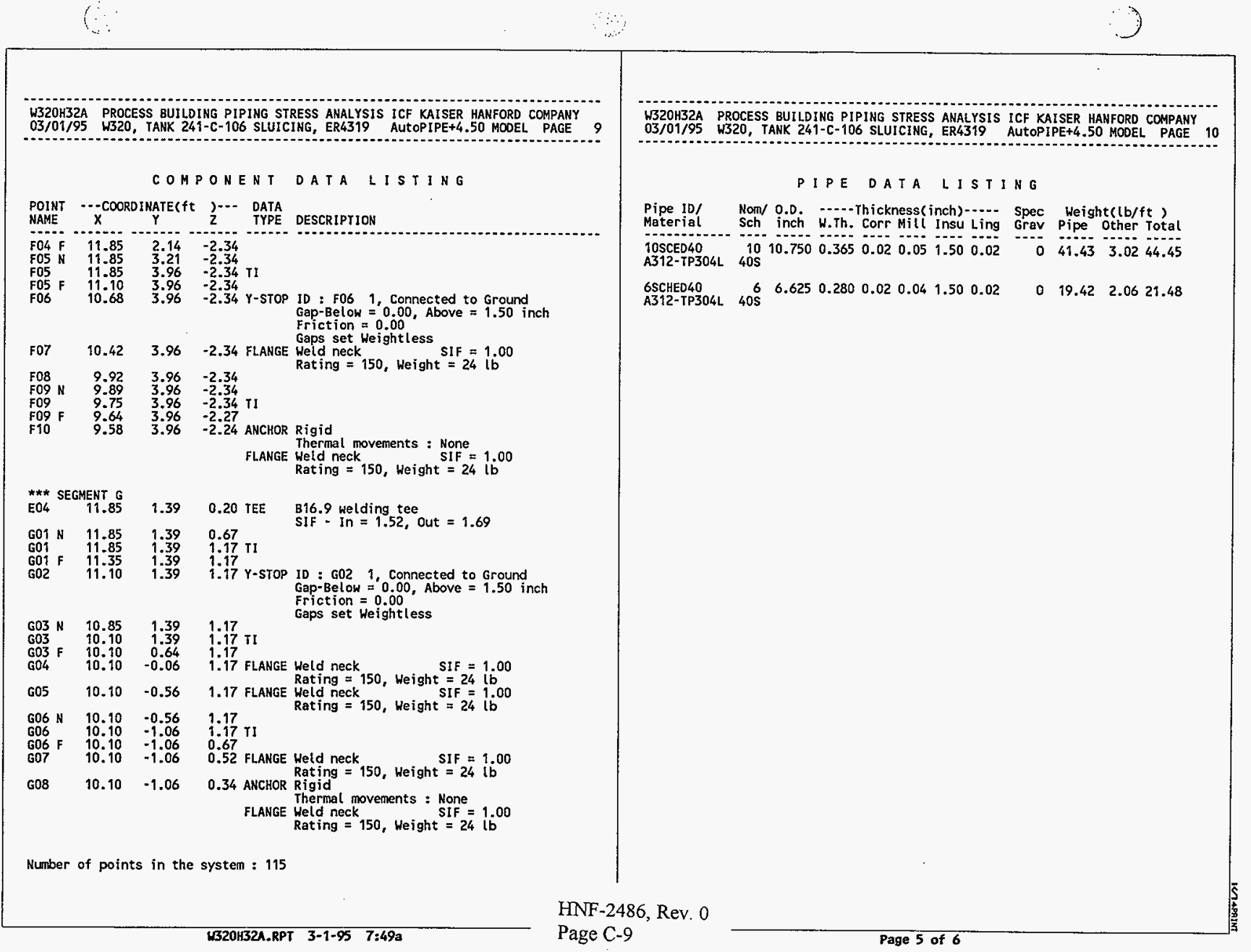


W320H32A PROCESS BUILDING PIPING STRESS ANALYSIS ICF KAISER HANFORD COMPANY O3/01/95 P320 TANK 241-C-106 SUUICING ER4319 AUTOPIPET4 50 MODEL PAGE

MATERIAL DATA LISTING

Material Density Pois. Temper. Modulus Expans. Allow. Name Pipe ID (b/cu.ft Ratio deg F E6 psi in/100ft psi

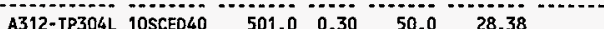
1.441316700 .0

\begin{tabular}{|c|c|c|c|c|}
\hline A312-TP304L 6SCHED40 & 501.0 & 0.3 & $\begin{array}{r}50.0 \\
180.0\end{array}$ & 28.38 \\
\hline
\end{tabular}

03/01/95 PROCESS BUILDING PIPING STRESS ANALYSIS ICF KAISER HANFORD

(1)

TEMPERATURE AND PRESSURE DATA

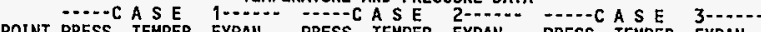

POINT PRESS. TEMPER EXPAN. PRESS. TEMPER EXPAN. PRESS. TEMPER EXPAN.

NAME psi deg $F$ in/100ft psi deg $F$ in/100ft psi deg $F$ in/100ft

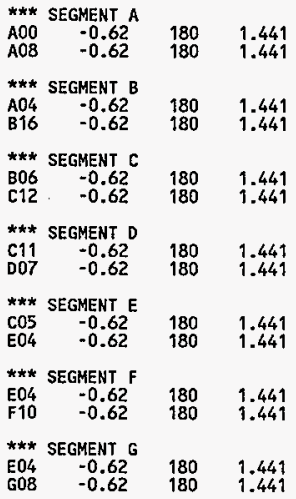


Point FG1 IF

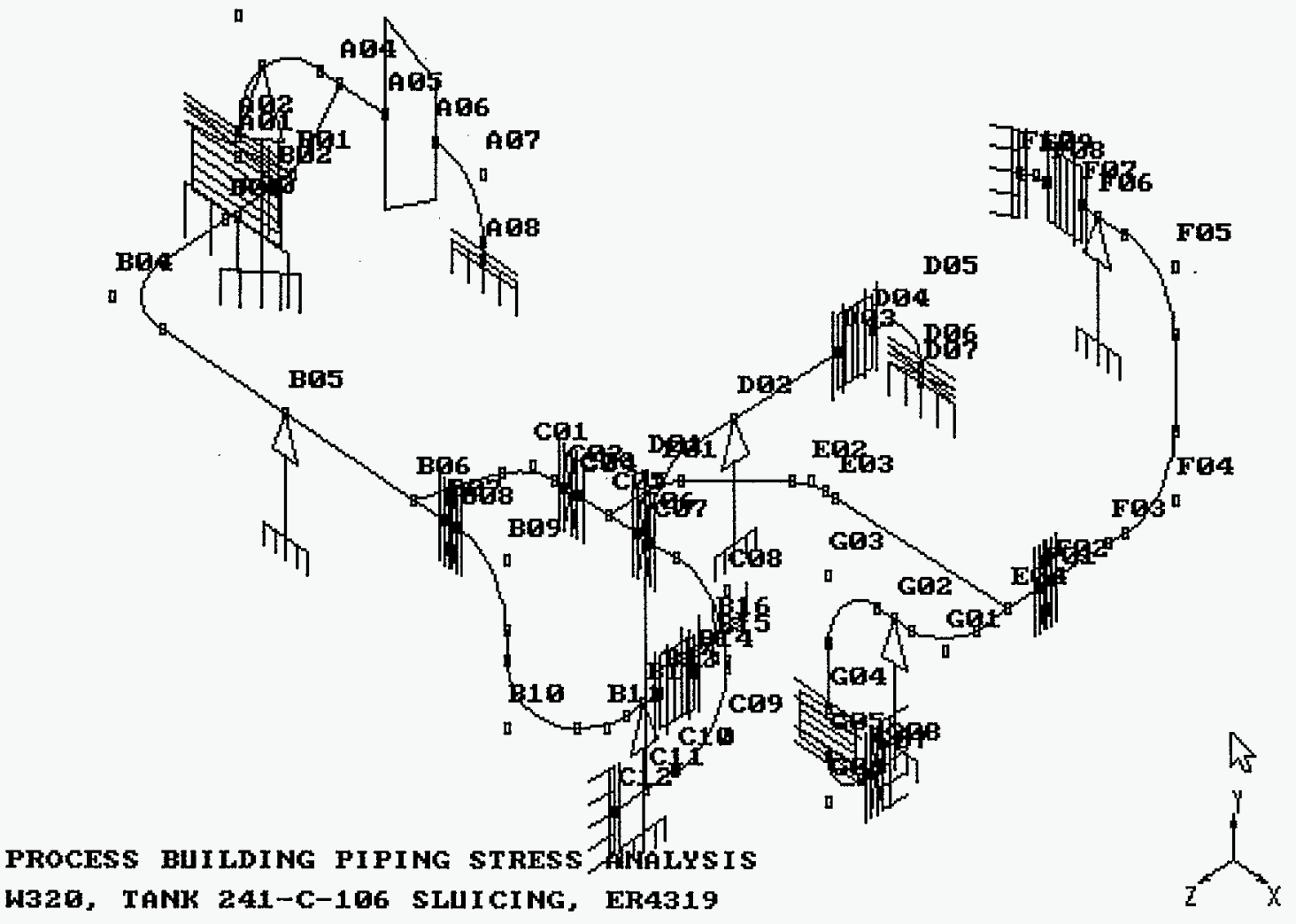




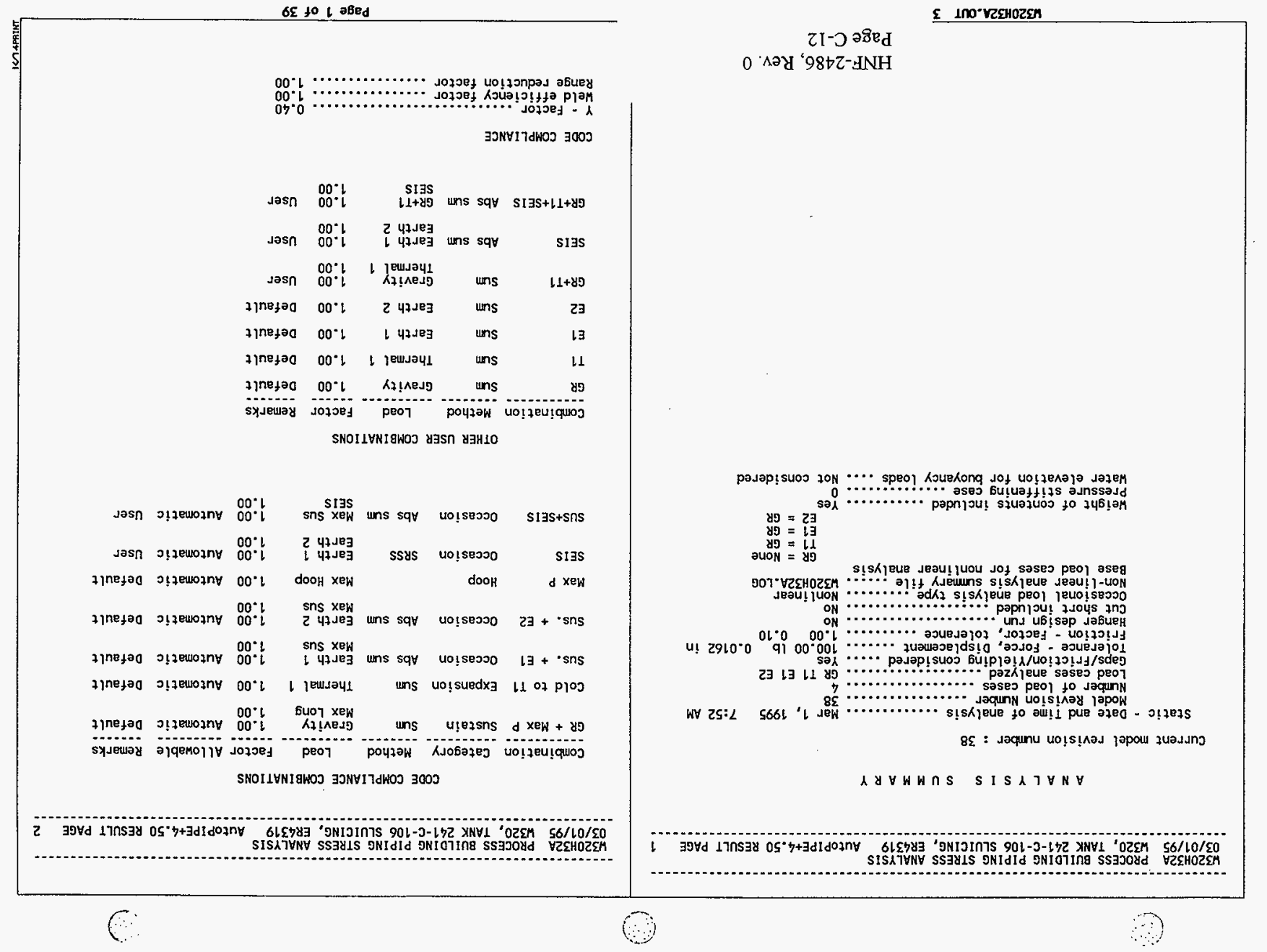


W32OH32A PROCESS BUILDING PIPING STRESS ANALYSIS

03 10195 WB20 TANK 241-C-106 SUUICING ER4319

AUTOPIPE+4.50 RESULT PAGE 3

Design Pressure Factor $\ldots \ldots \ldots \ldots \ldots \ldots 1.00$

Minimum stress ratio used in reports... 0.00

include corrosion in stress calcs.

include torsion in code stress ...........

include rigorous pressure ............. Not analyzed
WB20H32A PROCESS BUILDING PIPING STRESS ANALYSIS

03/01/95 W320, TANK 241-C-106 SLUICING, ER4319

AUTOPIPE+4.50 RESULT PAGE

EARTHQUAKE LOAD CASES

EARTHQUAKE LOAD CASES :

Number of load cases analysed : 2

Load case 1 - E'

$X$-Multiplier $=0.120 \quad$ Y-Multiplier $=0.000 \quad$ Z-Multiplier $=0.000$

Load case 2 - E2

X-Multiplier $=0.000 \quad Y$-Multiplier $=0.000 \quad Z$-Multiplier $=0.120$

HNF-2486, Rev. 0

Page C-13 


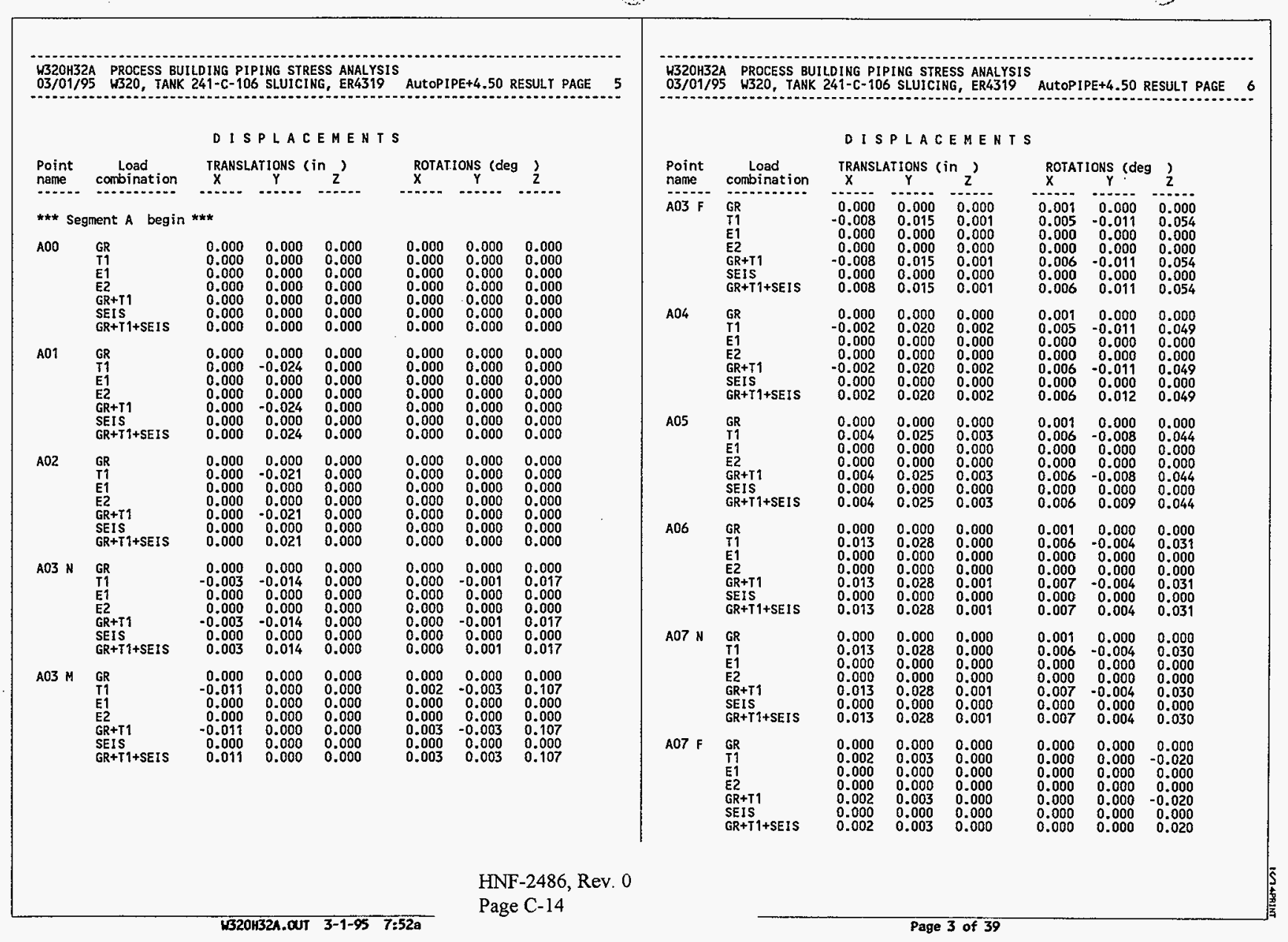




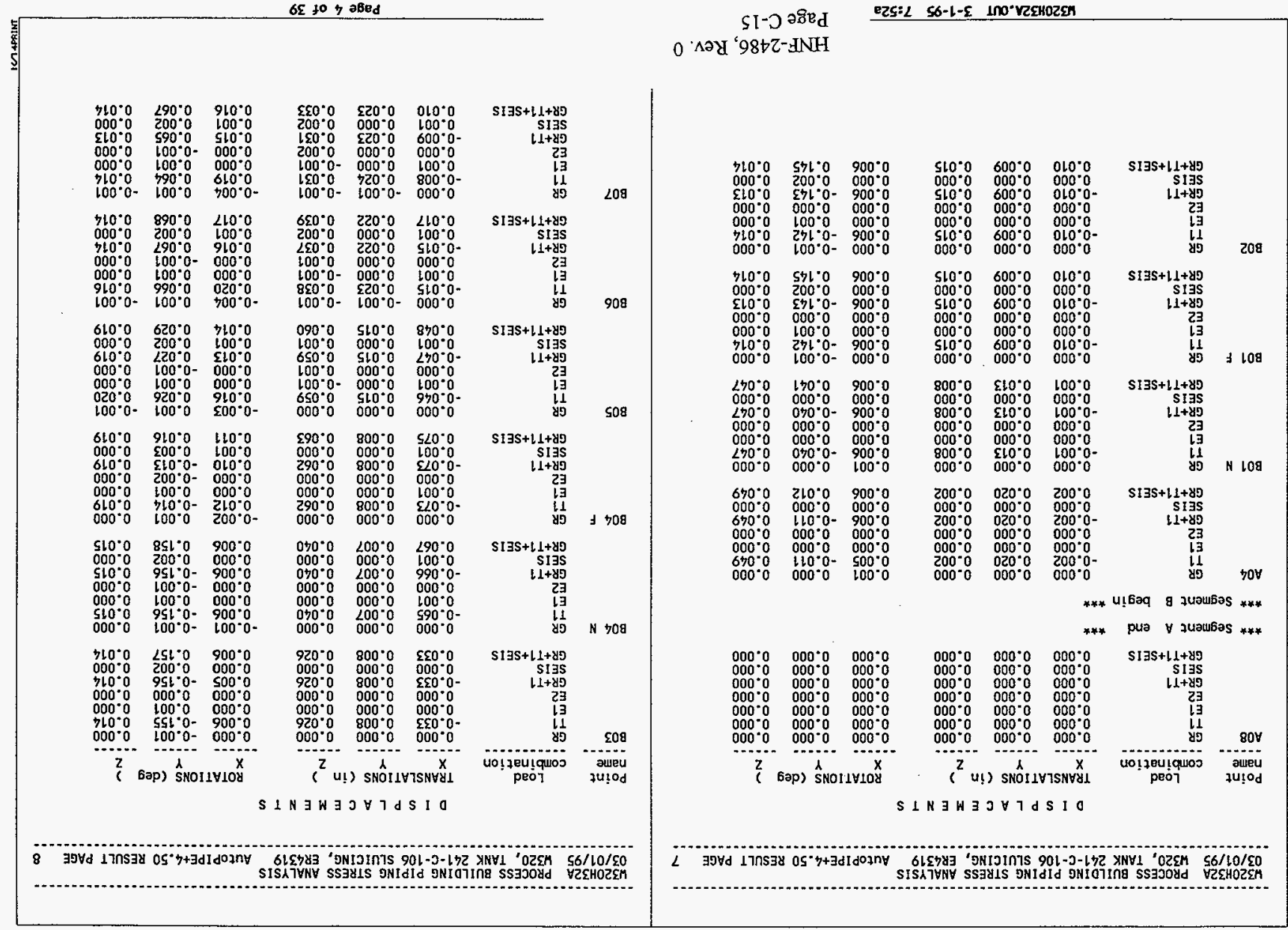


-

N $N$ icjujid

ะั

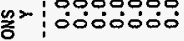

悹

$\infty$

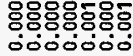

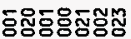

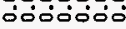

ธำธำำกิบี

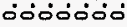

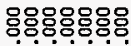

¿ȯoiòo

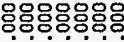

ócóíc

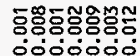

ธํํำำํํํำ

१ipợio

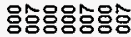

iojojodo

ㅇํㅇํํㅇํㅇํำ

íீல்

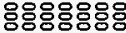

¿ீं0்0́

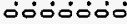

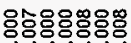

ถัชํํํํํํำ

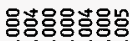

ó000ं00
ธํํㅇํำธํํำ

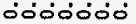

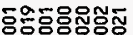

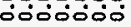

88880808

iciojo

웅잉용응

ல0்0ं0்

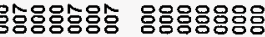

¿00ióio:

ióió

양융융응용융융

ஸீं0்0.

ㅇํㅇㅇํ요요

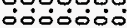

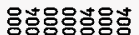

0000000

รำำㅇำ

0000000

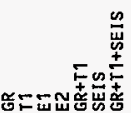

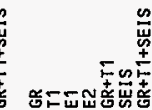

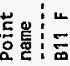

กับ

$\frac{m}{\infty}$

$\stackrel{\infty}{\infty}$
崖

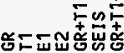

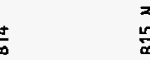

$\underset{\infty}{2}$

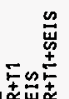

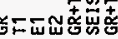

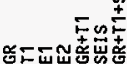

$\frac{12}{\operatorname{mon}}$

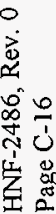

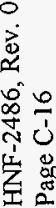

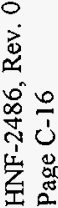

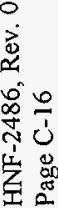

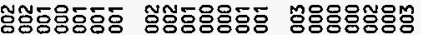

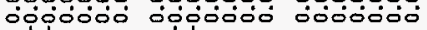

승용응응용응

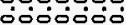

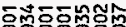
¿000000

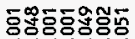

¿́0i்có

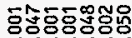

íóióí

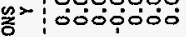

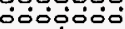

눙웅용용눙훙

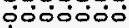

ㅎํํㅇํㅇํㅇํํ

icosió

옹ํํㅇํㅇํํㅇ

¿0ं0்0́.

m-oำㅇํㅇ

¿00000்

ธํํㅇㅎㅇ행ํㅇำ

ธ0ல0்000

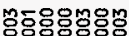

cócíció

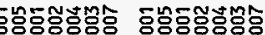

융둥응등응엉

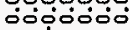

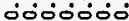

íciósó

드

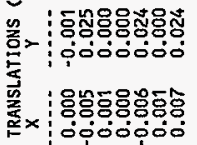

동융융유원

ஸ்0ंठ்0ं0்

홍영영영응

¡ं́ீíóí.

훙용응응

ió00்0.

응응응응웅음

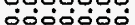

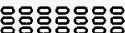

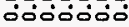

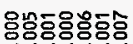

ิํํํํํํํํ

ํํํํํํํํํํํ

ㅇํำㅇํํํำ

ํํํํํㅇํㅇํㅇ

0000000 0000000

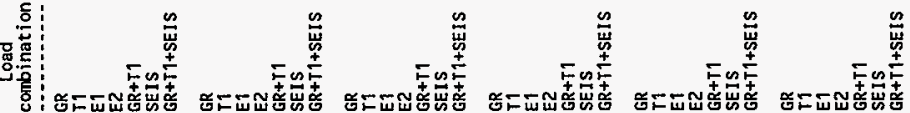

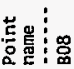

:

㟧

응

음

₹ 
W320H32A PROCESS BUILDING PIPING STRESS ANALYSIS

03/01/95 H320 TANK 241-C-106 SLUICING ER4319 AUTOPIPE+4.50 RESULT PAGE 11

DIS P LACEMENTS

\begin{tabular}{|c|c|c|c|c|c|c|c|}
\hline $\begin{array}{l}\text { Point } \\
\text { nane }\end{array}$ & $\begin{array}{l}\text { Load } \\
\text { combination }\end{array}$ & $\underset{\mathrm{X}}{\text { TRANS }}$ & TIONS & in $z^{\prime}$ & $\begin{array}{l}\text { ROTAT } \\
\mathrm{X}\end{array}$ & $\underset{Y}{\text { ONS }}$ & 2 \\
\hline 816 & $\begin{array}{l}\text { GR } \\
\text { T1 } \\
\text { E1 } \\
\text { E2 } \\
\text { GR+TI } \\
\text { SEIS } \\
\text { GR+T1+SEIS }\end{array}$ & $\begin{array}{l}0.000 \\
0.000 \\
0.000 \\
0.000 \\
0.000 \\
0.000 \\
0.000\end{array}$ & $\begin{array}{l}0.000 \\
0.000 \\
0.000 \\
0.000 \\
0.000 \\
0.000 \\
0.000\end{array}$ & $\begin{array}{l}0.000 \\
0.000 \\
0.000 \\
0.000 \\
0.000 \\
0.000 \\
0.000\end{array}$ & $\begin{array}{l}0.000 \\
0.000 \\
0.000 \\
0.000 \\
0.000 \\
0.000 \\
0.000\end{array}$ & $\begin{array}{l}0.000 \\
0.000 \\
0.000 \\
0.000 \\
0.000 \\
0.000 \\
0.000\end{array}$ & $\begin{array}{l}0.000 \\
0.000 \\
0.000 \\
0.000 \\
0.000 \\
0.000 \\
0.000\end{array}$ \\
\hline $\begin{array}{l}* * * \text { se } \\
* * * s e\end{array}$ & $\begin{array}{l}\text { ment } B \text { end } \\
\text { ment } C \text { begir }\end{array}$ & $\star \star \star \star *$ & & & & & \\
\hline B06 & $\begin{array}{l}\text { GR } \\
\text { TI } \\
\text { EI } \\
\text { E2 } \\
\text { GR+TI } \\
\text { SEIS } \\
\text { GR+T1+SEIS }\end{array}$ & $\begin{array}{r}0.000 \\
-0.015 \\
0.001 \\
0.000 \\
-0.015 \\
0.001 \\
0.017\end{array}$ & $\begin{array}{r}-0.001 \\
0.023 \\
0.000 \\
0.000 \\
0.022 \\
0.000 \\
0.022\end{array}$ & $\begin{array}{r}-0.001 \\
0.038 \\
-0.001 \\
0.001 \\
0.037 \\
0.002 \\
0.039\end{array}$ & $\begin{array}{r}-0.004 \\
0.020 \\
0.000 \\
0.000 \\
0.016 \\
0.001 \\
0.017\end{array}$ & $\begin{array}{r}0.001 \\
0.066 \\
0.001 \\
-0.001 \\
0.067 \\
0.002 \\
0.068\end{array}$ & $\begin{array}{r}-0.001 \\
0.016 \\
0.000 \\
0.000 \\
0.014 \\
0.000 \\
0.014\end{array}$ \\
\hline $\cot N$ & $\begin{array}{l}\text { GR } \\
\text { T1 } \\
\text { E1 } \\
\text { E2 } \\
\text { GR+T1 } \\
\text { SEIS } \\
\text { GR+I1+SEIS }\end{array}$ & $\begin{array}{r}-0.001 \\
-0.025 \\
0.001 \\
0.000 \\
-0.026 \\
0.001 \\
0.026\end{array}$ & $\begin{array}{r}-0.002 \\
0.029 \\
0.000 \\
0.000 \\
0.027 \\
0.000 \\
0.027\end{array}$ & $\begin{array}{r}-0.001 \\
0.017 \\
-0.001 \\
0.001 \\
0.017 \\
0.002 \\
0.019\end{array}$ & $\begin{array}{l}-0.005 \\
0.022 \\
0.000 \\
0.001 \\
0.017 \\
0.001 \\
0.019\end{array}$ & $\begin{array}{r}0.001 \\
0.077 \\
0.000 \\
-0.001 \\
0.077 \\
0.001 \\
0.078\end{array}$ & $\begin{array}{r}-0.001 \\
0.012 \\
0.000 \\
0.000 \\
0.011 \\
0.000 \\
0.011\end{array}$ \\
\hline $\operatorname{col} F$ & $\begin{array}{l}\text { GR } \\
\text { T1 } \\
\text { E1 } \\
\text { E2 } \\
\text { GR+T1 } \\
\text { SEIS } \\
\text { GR+T1+SEIS }\end{array}$ & $\begin{array}{r}-0.001 \\
-0.023 \\
0.001 \\
0.000 \\
-0.024 \\
0.001 \\
0.025\end{array}$ & $\begin{array}{r}-0.002 \\
0.030 \\
0.000 \\
0.000 \\
0.028 \\
0.000 \\
0.028\end{array}$ & $\begin{array}{r}-0.001 \\
0.006 \\
-0.001 \\
0.001 \\
0.005 \\
0.002 \\
0.008\end{array}$ & $\begin{array}{r}-0.004 \\
0.015 \\
0.000 \\
0.001 \\
0.011 \\
0.001 \\
0.012\end{array}$ & $\begin{array}{r}0.001 \\
0.055 \\
-0.001 \\
0.001 \\
0.055 \\
0.002 \\
0.058\end{array}$ & $\begin{array}{r}0.002 \\
-0.005 \\
0.000 \\
0.000 \\
-0.003 \\
0.000 \\
0.004\end{array}$ \\
\hline $\mathrm{CO2}$ & $\begin{array}{l}\text { GR } \\
\text { T1 } \\
\text { E1 } \\
\text { E2 } \\
\text { GR+T1 } \\
\text { SEIS } \\
\text { GR+T1+SEIS }\end{array}$ & $\begin{array}{r}-0.001 \\
-0.021 \\
0.001 \\
0.000 \\
-0.022 \\
0.001 \\
0.023\end{array}$ & $\begin{array}{r}-0.002 \\
0.030 \\
0.000 \\
0.000 \\
0.028 \\
0.000 \\
0.028\end{array}$ & $\begin{array}{r}-0.001 \\
0.004 \\
-0.001 \\
0.001 \\
0.003 \\
0.002 \\
0.006\end{array}$ & $\begin{array}{r}-0.004 \\
0.015 \\
0.000 \\
0.004 \\
0.017 \\
0.007 \\
0.012\end{array}$ & $\begin{array}{r}0.001 \\
0.053 \\
-0.001 \\
0.001 \\
0.054 \\
0.003 \\
0.057\end{array}$ & $\begin{array}{r}0.002 \\
-0.006 \\
0.000 \\
0.000 \\
-0.004 \\
0.000 \\
0.004\end{array}$ \\
\hline
\end{tabular}

HNF-2486, Rev. 0

Page C-17
4320H32A PROCESS BUILDING PIPING STRESS ANALYSIS

$03 / 01 / 95$ W320, TANK 247-C-106 SLUICING, ER4319

AutOPIPE+4.50 RESULT PAGE 12

DISPLACEMENTS

\begin{tabular}{|c|c|c|c|c|c|c|c|}
\hline $\begin{array}{l}\text { Point } \\
\text { name }\end{array}$ & $\begin{array}{l}\text { Load } \\
\text { combination }\end{array}$ & $\underset{X}{\text { TRANS }}$ & $\underset{Y}{T \text { IONS }}$ & $n_{z}{ }^{\prime}$ & $\begin{array}{l}\text { ROTA } \\
\mathrm{x}\end{array}$ & IONS Y $_{Y}$ & ? \\
\hline 03 & $\begin{array}{l}\text { GR } \\
\text { T1 } \\
\text { EI } \\
\text { E2 } \\
\text { GR+T1 } \\
\text { SEIS } \\
\text { GR+T1+SEIS }\end{array}$ & $\begin{array}{r}-0.001 \\
-0.018 \\
0.001 \\
0.000 \\
-0.019 \\
0.001 \\
0.020\end{array}$ & $\begin{array}{l}-0.002 \\
0.030 \\
0.000 \\
0.000 \\
0.028 \\
0.000 \\
0.028\end{array}$ & $\begin{array}{r}-0.001 \\
0.002 \\
-0.001 \\
0.001 \\
0.001 \\
0.002 \\
0.004\end{array}$ & $\begin{array}{l}-0.004 \\
0.015 \\
0.000 \\
0.001 \\
0.011 \\
0.001 \\
0.012\end{array}$ & $\begin{array}{r}0.001 \\
0.053 \\
-0.001 \\
0.001 \\
0.054 \\
0.003 \\
0.057\end{array}$ & $\begin{array}{r}0.002 \\
-0.006 \\
0.000 \\
0.000 \\
-0.004 \\
0.000 \\
0.004\end{array}$ \\
\hline $\mathrm{CO} 4$ & $\begin{array}{l}\text { GR } \\
\text { T1 } \\
E 1 \\
E 2 \\
\text { GR+T1 } \\
\text { SEIS } \\
\text { GR+T1+SEIS }\end{array}$ & $\begin{array}{r}-0.001 \\
-0.018 \\
0.001 \\
0.000 \\
-0.019 \\
0.001 \\
0.020\end{array}$ & $\begin{array}{r}-0.002 \\
0.030 \\
0.000 \\
0.000 \\
0.028 \\
0.000 \\
0.028\end{array}$ & $\begin{array}{r}-0.001 \\
0.002 \\
-0.001 \\
0.001 \\
0.001 \\
0.002 \\
0.003\end{array}$ & $\begin{array}{r}-0.004 \\
0.015 \\
0.000 \\
0.001 \\
0.011 \\
0.001 \\
0.012\end{array}$ & $\begin{array}{r}0.001 \\
0.053 \\
-0.001 \\
0.001 \\
0.054 \\
0.003 \\
0.057\end{array}$ & $\begin{array}{r}0.002 \\
-0.006 \\
0.000 \\
0.000 \\
-0.004 \\
0.000 \\
0.004\end{array}$ \\
\hline $\mathrm{C0} 5$ & $\begin{array}{l}\text { GR } \\
\text { T1 } \\
\text { E1 } \\
\text { E2 } \\
\text { GR+I1 } \\
\text { SEIS } \\
\text { GR+T }+ \text { SEIS }\end{array}$ & $\begin{array}{r}-0.001 \\
-0.012 \\
0.001 \\
0.000 \\
-0.012 \\
0.001 \\
0.013\end{array}$ & $\begin{array}{r}-0.002 \\
0.029 \\
0.000 \\
0.000 \\
0.027 \\
0.000 \\
0.028\end{array}$ & $\begin{array}{r}-0.001 \\
-0.003 \\
-0.001 \\
0.001 \\
-0.004 \\
0.002 \\
0.006\end{array}$ & $\begin{array}{r}-0.004 \\
0.015 \\
0.000 \\
0.001 \\
0.011 \\
0.001 \\
0.012\end{array}$ & $\begin{array}{r}0.001 \\
0.050 \\
-0.001 \\
0.001 \\
0.051 \\
0.003 \\
0.053\end{array}$ & $\begin{array}{r}0.002 \\
-0.009 \\
0.000 \\
0.000 \\
-0.006 \\
0.000 \\
0.007\end{array}$ \\
\hline $\mathrm{COS}$ & $\begin{array}{l}\text { GR } \\
\text { I1 } \\
\text { E1 } \\
\text { E2 } \\
\text { GR+I1 } \\
\text { SEIS } \\
\text { GR+I1+SEIS }\end{array}$ & $\begin{array}{r}-0.001 \\
-0.005 \\
0.001 \\
0.000 \\
-0.006 \\
0.001 \\
0.007\end{array}$ & $\begin{array}{r}-0.002 \\
0.029 \\
0.000 \\
0.000 \\
0.027 \\
0.000 \\
0.027\end{array}$ & $\begin{array}{r}-0.001 \\
-0.008 \\
-0.001 \\
0.001 \\
-0.009 \\
0.002 \\
0.010\end{array}$ & $\begin{array}{r}-0.004 \\
0.019 \\
0.000 \\
0.001 \\
0.015 \\
0.002 \\
0.016\end{array}$ & $\begin{array}{r}0.001 \\
0.050 \\
-0.001 \\
0.002 \\
0.051 \\
0.003 \\
0.054\end{array}$ & $\begin{array}{r}0.003 \\
-0.009 \\
0.000 \\
0.000 \\
-0.006 \\
0.001 \\
0.007\end{array}$ \\
\hline $\mathrm{CO}$ & $\begin{array}{l}\text { GR } \\
\text { I1 } \\
\text { E1 } \\
\text { E2 } \\
\text { GR+I1 } \\
\text { SEIS } \\
\text { GR+I1+SEIS }\end{array}$ & $\begin{array}{r}-0.001 \\
-0.003 \\
0.001 \\
0.000 \\
-0.003 \\
0.001 \\
0.004\end{array}$ & $\begin{array}{r}-0.002 \\
0.028 \\
0.000 \\
0.000 \\
0.027 \\
0.000 \\
0.027\end{array}$ & $\begin{array}{r}-0.001 \\
-0.009 \\
0.000 \\
0.001 \\
-0.011 \\
0.001 \\
0.012\end{array}$ & $\begin{array}{r}-0.004 \\
0.019 \\
0.000 \\
0.001 \\
0.015 \\
0.002 \\
0.016\end{array}$ & $\begin{array}{r}0.001 \\
0.050 \\
-0.001 \\
0.002 \\
0.051 \\
0.003 \\
0.054\end{array}$ & $\begin{array}{r}0.003 \\
-0.009 \\
0.000 \\
0.000 \\
-0.006 \\
0.001 \\
0.007\end{array}$ \\
\hline $8 \mathrm{~N}$ & $\begin{array}{l}\text { GR } \\
T 1 \\
\text { E1 } \\
\text { E2 } \\
\text { GR+T1 } \\
\text { SEIS } \\
\text { GR+T1+SEIS }\end{array}$ & $\begin{array}{r}-0.001 \\
0.003 \\
0.001 \\
0.000 \\
0.002 \\
0.001 \\
0.003\end{array}$ & $\begin{array}{r}-0.001 \\
0.028 \\
0.000 \\
0.000 \\
0.026 \\
0.000 \\
0.027\end{array}$ & $\begin{array}{r}-0.001 \\
-0.014 \\
0.000 \\
0.001 \\
-0.015 \\
0.001 \\
0.016\end{array}$ & $\begin{array}{r}-0.004 \\
0.023 \\
0.000 \\
0.002 \\
0.019 \\
0.002 \\
0.020\end{array}$ & $\begin{array}{r}0.001 \\
0.048 \\
-0.001 \\
0.002 \\
0.048 \\
0.003 \\
0.052\end{array}$ & $\begin{array}{r}0.003 \\
-0.011 \\
-0.001 \\
0.000 \\
-0.008 \\
0.001 \\
0.008\end{array}$ \\
\hline
\end{tabular}


W32OH32A PROCESS BUILDING PIPING STRESS ANALYSIS

$03 / 01 / 95$ W320 TANK $241-C-106$ SLUICING ER4319

AUtoPIPE 4.50 RESULT PAGE 13

D I S P L A CEMEN T S

\begin{tabular}{|c|c|c|c|c|c|c|c|}
\hline $\begin{array}{l}\text { Point } \\
\text { name }\end{array}$ & $\begin{array}{l}\text { Load } \\
\text { combination }\end{array}$ & $\underset{x}{\text { TRANSL }}$ & $\underset{Y}{\text { ATIONS }}$ & $z^{\prime}$ & $\mathrm{x}^{\text {ROTAT }}$ & ${ }_{Y}$ loNs & z \\
\hline $\cos F$ & $\begin{array}{l}\text { GR } \\
\text { I1 } \\
\text { E1 } \\
\text { E2 } \\
\text { GR+II } \\
\text { SEIS } \\
\text { GR+II+SEIS }\end{array}$ & $\begin{array}{l}0.000 \\
0.008 \\
0.001 \\
0.000 \\
0.008 \\
0.001 \\
0.008\end{array}$ & $\begin{array}{r}-0.001 \\
0.012 \\
0.000 \\
0.000 \\
0.012 \\
0.000 \\
0.012\end{array}$ & $\begin{array}{r}-0.001 \\
-0.022 \\
0.000 \\
0.000 \\
-0.023 \\
0.000 \\
0.023\end{array}$ & $\begin{array}{r}-0.004 \\
0.018 \\
0.000 \\
0.002 \\
0.014 \\
0.002 \\
0.017\end{array}$ & $\begin{array}{r}0.001 \\
0.010 \\
-0.002 \\
0.001 \\
0.011 \\
0.003 \\
0.014\end{array}$ & $\begin{array}{r}0.002 \\
-0.041 \\
-0.002 \\
0.000 \\
-0.039 \\
0.002 \\
0.041\end{array}$ \\
\hline $\mathrm{Cog} \mathrm{N}$ & $\begin{array}{l}\text { GR } \\
\text { T1 } \\
\text { E1 } \\
\text { EZ } \\
\text { GR+TI } \\
\text { SEIS } \\
\text { GR+TI+SEIS }\end{array}$ & $\begin{array}{l}0.000 \\
0.007 \\
0.001 \\
0.000 \\
0.007 \\
0.001 \\
0.007\end{array}$ & $\begin{array}{r}-0.001 \\
0.011 \\
0.000 \\
0.000 \\
0.011 \\
0.000 \\
0.011\end{array}$ & $\begin{array}{r}-0.001 \\
-0.022 \\
0.000 \\
0.000 \\
-0.023 \\
0.000 \\
0.023\end{array}$ & $\begin{array}{r}-0.004 \\
0.018 \\
0.000 \\
0.002 \\
0.014 \\
0.002 \\
0.017\end{array}$ & $\begin{array}{r}0.001 \\
0.008 \\
-0.002 \\
0.001 \\
0.009 \\
0.003 \\
0.012\end{array}$ & $\begin{array}{r}0.002 \\
-0.041 \\
-0.002 \\
0.000 \\
-0.039 \\
0.002 \\
0.041\end{array}$ \\
\hline $\cos \mathrm{F}$ & $\begin{array}{l}\text { GR } \\
\text { T1 } \\
\text { E1 } \\
\text { E2 } \\
\text { GR+T1 } \\
\text { SEIS } \\
\text { GR+T1+SEIS }\end{array}$ & $\begin{array}{l}0.000 \\
0.001 \\
0.000 \\
0.000 \\
0.001 \\
0.000 \\
0.001\end{array}$ & $\begin{array}{r}0.000 \\
-0.001 \\
0.000 \\
0.000 \\
-0.001 \\
0.000 \\
0.001\end{array}$ & $\begin{array}{r}0.000 \\
-0.014 \\
0.000 \\
0.000 \\
-0.014 \\
0.000 \\
0.014\end{array}$ & $\begin{array}{r}-0.002 \\
-0.001 \\
0.000 \\
0.000 \\
-0.003 \\
0.001 \\
0.004\end{array}$ & $\begin{array}{r}0.000 \\
-0.005 \\
-0.001 \\
0.000 \\
-0.005 \\
0.001 \\
0.006\end{array}$ & $\begin{array}{r}0.000 \\
-0.018 \\
-0.001 \\
0.000 \\
-0.017 \\
0.001 \\
0.018\end{array}$ \\
\hline C10 & $\begin{array}{l}\text { GR } \\
\text { T1 } \\
\text { E1 } \\
\text { E2 } \\
\text { GR+T1 } \\
\text { SEIS } \\
\text { GR+T1+SEIS }\end{array}$ & $\begin{array}{l}0.000 \\
0.001 \\
0.000 \\
0.000 \\
0.001 \\
0.000 \\
0.001\end{array}$ & $\begin{array}{r}0.000 \\
-0.001 \\
0.000 \\
0.000 \\
-0.001 \\
0.000 \\
0.001\end{array}$ & $\begin{array}{r}0.000 \\
-0.013 \\
0.000 \\
0.000 \\
-0.013 \\
0.000 \\
0.013\end{array}$ & $\begin{array}{r}-0.002 \\
-0.001 \\
0.000 \\
0.000 \\
-0.003 \\
0.001 \\
0.004\end{array}$ & $\begin{array}{r}0.000 \\
-0.005 \\
0.000 \\
0.000 \\
-0.005 \\
0.001 \\
0.006\end{array}$ & $\begin{array}{r}0.000 \\
-0.017 \\
-0.001 \\
0.000 \\
-0.017 \\
0.001 \\
0.017\end{array}$ \\
\hline$c 11$ & $\begin{array}{l}\text { GR } \\
T 1 \\
E 1 \\
E 2 \\
\text { GR+T1 } \\
\text { SEIS } \\
\text { GR+TI+SEIS }\end{array}$ & $\begin{array}{l}0.000 \\
0.000 \\
0.000 \\
0.000 \\
0.000 \\
0.000 \\
0.001\end{array}$ & $\begin{array}{l}0.000 \\
0.000 \\
0.000 \\
0.000 \\
0.000 \\
0.000 \\
0.000\end{array}$ & $\begin{array}{r}0.000 \\
-0.007 \\
0.000 \\
0.000 \\
-0.007 \\
0.000 \\
0.007\end{array}$ & $\begin{array}{r}-0.001 \\
-0.002 \\
0.000 \\
0.000 \\
-0.003 \\
0.000 \\
0.003\end{array}$ & $\begin{array}{r}0.000 \\
-0.004 \\
0.000 \\
0.000 \\
-0.004 \\
0.000 \\
0.004\end{array}$ & $\begin{array}{r}0.000 \\
-0.008 \\
0.000 \\
0.000 \\
-0.008 \\
0.000 \\
0.009\end{array}$ \\
\hline C12 & $\begin{array}{l}\text { GR } \\
\text { T1 } \\
\text { E1 } \\
\text { E2 } \\
\text { GR+T1 } \\
\text { SEIS } \\
\text { GR+T1+SEIS }\end{array}$ & $\begin{array}{l}0.000 \\
0.000 \\
0.000 \\
0.000 \\
0.000 \\
0.000 \\
0.000\end{array}$ & $\begin{array}{l}0.000 \\
0.000 \\
0.000 \\
0.000 \\
0.000 \\
0.000 \\
0.000\end{array}$ & $\begin{array}{l}0.000 \\
0.000 \\
0.000 \\
0.000 \\
0.000 \\
0.000 \\
0.000\end{array}$ & $\begin{array}{l}0.000 \\
0.000 \\
0.000 \\
0.000 \\
0.000 \\
0.000 \\
0.000\end{array}$ & $\begin{array}{l}0.000 \\
0.000 \\
0.000 \\
0.000 \\
0.000 \\
0.000 \\
0.000\end{array}$ & $\begin{array}{l}0.000 \\
0.000 \\
0.000 \\
0.000 \\
0.000 \\
0.000 \\
0.000\end{array}$ \\
\hline
\end{tabular}

*** Segment $C$ end $\star * \star *$
W32OH32A PROCESS BUILDING PIPING STRESS ANALYSIS

03/01/95 W320, TANK 241-C-106 SLUICING, ER4319 AUTOPIPE+4.50 RESULT PAGE 14

\section{ISP L ACEMENTS}

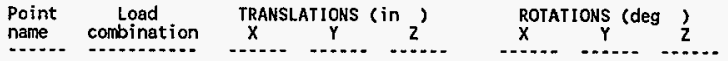

*** Segment $D$ begin $* * *$

C11

GR
T1
$E 1$
$E 2$
GR+T1
SEIS
GR+T1+SEIS

$0.000 \quad 0.000 \quad 0.000$ $\begin{array}{lll}0.000 & 0.000 & -0.007\end{array}$ $\begin{array}{rrr}0.000 & 0.000 & 0.000\end{array}$ $0.000 \quad 0.000 \quad 0.000$ $\begin{array}{lll}0.000 & 0.000 & -0.007\end{array}$

D01 N

GR
T1
E1

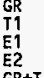

GR 2 T1

SEIS

0.001

$0.000 \quad 0.007$

D01 F

GR
T1
E1
E2
GR+TI
SEIS

$\begin{array}{lll}0.000 & 0.000 & 0.000\end{array}$

$\begin{array}{lll}0.004 & 0.038 & -0.009\end{array}$

$\begin{array}{lll}0.000 & 0.000 & 0.000\end{array}$

$\begin{array}{lll}0.000 & 0.000 & 0.000\end{array}$

$\begin{array}{llr}0.004 & 0.038 & -0.010 \\ 0.000 & 0.000 & 0.000\end{array}$

$\begin{array}{lll}0.005 & 0.038 & 0.010\end{array}$

GR+T1

GEIS

0.000

$0.000 \quad 0.000$

$\begin{array}{lll}0.004 & 0.044 & -0.023\end{array}$

$\begin{array}{lll}0.000 & 0.000 & 0.000\end{array}$

$\begin{array}{lll}0.000 & 0.000 & 0.000\end{array}$

$\begin{array}{lll}0.004 & 0.044 & -0.024\end{array}$

$\begin{array}{lll}0.000 & 0.000 & 0.000\end{array}$

D02

$\begin{array}{lrrr}\text { GR } & 0.000 & 0.000 & 0.000 \\ \text { TI } & 0.004 & 0.040 & -0.032 \\ \text { E1 } & 0.000 & 0.000 & 0.000 \\ \text { E2 } & 0.000 & 0.000 & 0.000 \\ \text { GR+T1 } & 0.004 & 0.040 & -0.032 \\ \text { SEIS } & 0.000 & 0.000 & 0.000 \\ \text { GR+T1+SEIS } & 0.004 & 0.040 & 0.033\end{array}$

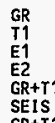

$\begin{array}{lll}0.000 & 0.000 & 0.000\end{array}$

$\begin{array}{lll}0.001 & 0.023 & -0.062\end{array}$

$\begin{array}{lll}0.000 & 0.000 & 0.000\end{array}$

$\begin{array}{lll}0.000 & 0.000 & 0.000\end{array}$

$\begin{array}{lll}0.001 & 0.023 & -0.063 \\ 0.000 & 0.000 & 0.000\end{array}$

$\begin{array}{lll}0.002 & 0.023 & 0.063\end{array}$ $\begin{array}{lll}-0.001 & 0.000 & 0.000\end{array}$

$\begin{array}{lll}-0.002 & -0.004 & -0.008\end{array}$

$\begin{array}{lll}0.000 & 0.000 & 0.000\end{array}$

$\begin{array}{lll}0.000 & 0.000 & 0.000\end{array}$

$\begin{array}{lll}-0.003 & -0.004 & -0.008\end{array}$

$\begin{array}{lll}0.000 & 0.000 & 0.000 \\ 0.003 & 0.004 & 0.009\end{array}$

$\begin{array}{llll}0.000 & 0.000 & 0.000\end{array}$

$\begin{array}{ccc}-0.009 & 0.000 & -0.006\end{array}$

$\begin{array}{lll}0.000 & 0.000 & -0.001\end{array}$

$\begin{array}{lll}0.001 & 0.000 & 0.000\end{array}$

$\begin{array}{rrr}-0.009 & 0.000 & -0.006\end{array}$

$\begin{array}{llll}0.001 & 0.000 & 0.001\end{array}$

$\begin{array}{lll}0.010 & 0.000 & 0.006\end{array}$

$\begin{array}{lll}0.000 & 0.000 & 0.000\end{array}$

$\begin{array}{llll}-0.037 & 0.005 & -0.003\end{array}$

$\begin{array}{lll}0.000 & 0.000 & -0.001\end{array}$

$\begin{array}{rrr}0.000 & 0.000 & 0.000\end{array}$

$0.037 \quad 0.005-0.003$

0.0370 .0050 .003

$\begin{array}{lll}0.000 & 0.000 & 0.000\end{array}$

$\begin{array}{lll}-0.038 & 0.005 & -0.003\end{array}$

$\begin{array}{lll}0.000 & 0.000 & -0.001\end{array}$

$\begin{array}{rrr}0.000 & 0.000 & 0.000 \\ -0.038 & 0.005 & -0.003\end{array}$

$\begin{array}{rrr}-0.038 & 0.005 & -0.003 \\ 0.000 & 0.000 & 0.001\end{array}$

$\begin{array}{lll}0.038 & 0.005 & 0.003\end{array}$

$\begin{array}{lll}0.000 & 0.000 \quad 0.000\end{array}$

$\begin{array}{lll}-0.036 & 0.005 & -0.003\end{array}$

$0.000 \quad 0.001 \quad-0.001$

$0.000 \quad 0.000 \quad 0.000$

$\begin{array}{lll}-0.036 & 0.005 & -0.003\end{array}$

$\begin{array}{lll}0.000 & 0.001 & 0.001 \\ 0.036 & 0.006 & 0.003\end{array}$
HNF-2486, Rev. 0

Page C-18 


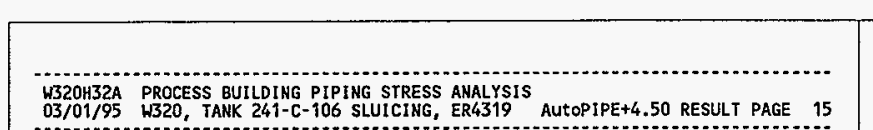

03/01/95 W320, TANK 241-C-106 SLUICING, ER4319 AUtOPIPE+4.50 RESULT PAGE 15

DIS PLA CEMEN TS

\begin{tabular}{|c|c|c|c|c|c|c|c|}
\hline Point & $\begin{array}{l}\text { Load } \\
\text { combination }\end{array}$ & $\underset{X}{\text { TRANSL }}$ & TIONS & $\left.\ln _{z}\right)$ & $\begin{array}{l}\text { ROTAT } \\
\mathrm{X}\end{array}$ & ONS (deg & ? \\
\hline D04 & $\begin{array}{l}\text { GR } \\
\text { T1 } \\
\text { Eq } \\
\text { E2 } \\
\text { GR+T1 } \\
\text { SEIS } \\
\text { GR+T1+SEIS }\end{array}$ & $\begin{array}{l}0.000 \\
0.001 \\
0.000 \\
0.000 \\
0.001 \\
0.000 \\
0.001\end{array}$ & $\begin{array}{l}0.000 \\
0.019 \\
0.000 \\
0.000 \\
0.019 \\
0.000 \\
0.019\end{array}$ & $\begin{array}{l}0.000 \\
0.012 \\
0.000 \\
0.000 \\
0.012 \\
0.000 \\
0.012\end{array}$ & $\begin{array}{r}0.000 \\
-0.036 \\
0.000 \\
0.000 \\
-0.036 \\
0.000 \\
0.036\end{array}$ & $\begin{array}{l}0.000 \\
0.005 \\
0.001 \\
0.000 \\
0.005 \\
0.001 \\
0.006\end{array}$ & $\begin{array}{r}0.000 \\
-0.003 \\
-0.001 \\
0.000 \\
-0.003 \\
0.001 \\
0.003\end{array}$ \\
\hline D05 N & $\begin{array}{l}\text { GR } \\
T 1 \\
\text { E1 } \\
\text { E2 } \\
\text { GR+T1 } \\
\text { SEIS } \\
\text { GR+T1+SEIS }\end{array}$ & $\begin{array}{l}0.000 \\
0.001 \\
0.000 \\
0.000 \\
0.001 \\
0.000 \\
0.001\end{array}$ & $\begin{array}{l}0.000 \\
0.017 \\
0.000 \\
0.000 \\
0.017 \\
0.000 \\
0.017\end{array}$ & $\begin{array}{l}0.000 \\
0.009 \\
0.000 \\
0.000 \\
0.009 \\
0.000 \\
0.009\end{array}$ & $\begin{array}{r}0.000 \\
-0.035 \\
0.000 \\
0.000 \\
-0.035 \\
0.000 \\
0.035\end{array}$ & $\begin{array}{l}0.000 \\
0.005 \\
0.001 \\
0.000 \\
0.005 \\
0.001 \\
0.005\end{array}$ & $\begin{array}{r}0.000 \\
-0.003 \\
-0.001 \\
0.000 \\
-0.003 \\
0.001 \\
0.003\end{array}$ \\
\hline D05 F & $\begin{array}{l}\text { GR } \\
\text { T1 } \\
\text { E1 } \\
\text { E2 } \\
\text { GR+T1 } \\
\text { SEIS } \\
\text { GR+T1+SEIS }\end{array}$ & $\begin{array}{l}0.000 \\
0.000 \\
0.000 \\
0.000 \\
0.000 \\
0.000 \\
0.000\end{array}$ & $\begin{array}{l}0.000 \\
0.003 \\
0.000 \\
0.000 \\
0.003 \\
0.000 \\
0.003\end{array}$ & $\begin{array}{l}0.000 \\
0.000 \\
0.000 \\
0.000 \\
0.000 \\
0.000 \\
0.000\end{array}$ & $\begin{array}{l}0.000 \\
0.000 \\
0.000 \\
0.000 \\
0.000 \\
0.000 \\
0.000\end{array}$ & $\begin{array}{l}0.000 \\
0.000 \\
0.000 \\
0.000 \\
0.000 \\
0.000 \\
0.000\end{array}$ & $\begin{array}{l}0.000 \\
0.000 \\
0.000 \\
0.000 \\
0.000 \\
0.000 \\
0.000\end{array}$ \\
\hline D06 & $\begin{array}{l}\text { GR } \\
\text { T1 } \\
\text { E1 } \\
\text { E2 } \\
\text { GR+T1 } \\
\text { SEIS } \\
\text { GR+T1+SEIS }\end{array}$ & $\begin{array}{l}0.000 \\
0.000 \\
0.000 \\
0.000 \\
0.000 \\
0.000 \\
0.000\end{array}$ & $\begin{array}{l}0.000 \\
0.003 \\
0.000 \\
0.000 \\
0.003 \\
0.000 \\
0.003\end{array}$ & $\begin{array}{l}0.000 \\
0.000 \\
0.000 \\
0.000 \\
0.000 \\
0.000 \\
0.000\end{array}$ & $\begin{array}{l}0.000 \\
0.000 \\
0.000 \\
0.000 \\
0.000 \\
0.000 \\
0.000\end{array}$ & $\begin{array}{l}0.000 \\
0.000 \\
0.000 \\
0.000 \\
0.000 \\
0.000 \\
0.000\end{array}$ & $\begin{array}{l}0.000 \\
0.000 \\
0.000 \\
0.000 \\
0.000 \\
0.000 \\
0.000\end{array}$ \\
\hline D07 & $\begin{array}{l}\text { GR } \\
T 1 \\
\text { E1 } \\
\text { E2 } \\
\text { GR+T1 } \\
\text { SEIS } \\
\text { GR+T1+SEIS }\end{array}$ & $\begin{array}{l}0.000 \\
0.000 \\
0.000 \\
0.000 \\
0.000 \\
0.000 \\
0.000\end{array}$ & $\begin{array}{l}0.000 \\
0.000 \\
0.000 \\
0.000 \\
0.000 \\
0.000 \\
0.000\end{array}$ & $\begin{array}{l}0.000 \\
0.000 \\
0.000 \\
0.000 \\
0.000 \\
0.000 \\
0.000\end{array}$ & $\begin{array}{l}0.000 \\
0.000 \\
0.000 \\
0.000 \\
0.000 \\
0.000 \\
0.000\end{array}$ & $\begin{array}{l}0.000 \\
0.000 \\
0.000 \\
0.000 \\
0.000 \\
0.000 \\
0.000\end{array}$ & $\begin{array}{l}0.000 \\
0.000 \\
0.000 \\
0.000 \\
0.000 \\
0.000 \\
0.000\end{array}$ \\
\hline
\end{tabular}

W320H32A PROCESS BUILDING PIPING STRESS ANALYSIS

$03 / 01 / 95$ W320, TANK 241-C-106 SLUICING, ER4319 AUtOPIPE+4.50 RESULT PAGE 16

D I S P I A CEMENTS

\begin{tabular}{|c|c|c|c|c|c|c|c|}
\hline $\begin{array}{l}\text { Point } \\
\text { name }\end{array}$ & $\begin{array}{c}\text { Load } \\
\text { combination }\end{array}$ & $\underset{X}{\text { TRANSL }}$ & ATIONS $\underset{Y}{6}$ & in $z^{\prime}$ & $\begin{array}{l}\text { ROTAI } \\
X\end{array}$ & IONS ${ }_{Y}$ (de & ? \\
\hline $\cos$ & $\begin{array}{l}\text { GR } \\
T 1 \\
E 1 \\
E 2 \\
\text { GR+T1 } \\
\text { SEIS } \\
\text { GR+T I+SEIS }\end{array}$ & $\begin{array}{r}-0.001 \\
-0.012 \\
0.001 \\
0.000 \\
-0.012 \\
0.001 \\
0.013\end{array}$ & $\begin{array}{r}-0.002 \\
0.029 \\
0.000 \\
0.000 \\
0.027 \\
0.000 \\
0.028\end{array}$ & $\begin{array}{r}-0.001 \\
-0.003 \\
-0.001 \\
0.001 \\
-0.004 \\
0.002 \\
0.006\end{array}$ & $\begin{array}{l}-0.004 \\
0.015 \\
0.000 \\
0.001 \\
0.011 \\
0.001 \\
0.012\end{array}$ & $\begin{array}{r}0.001 \\
0.050 \\
-0.001 \\
0.001 \\
0.051 \\
0.003 \\
0.053\end{array}$ & $\begin{array}{r}0.002 \\
-0.009 \\
0.000 \\
0.000 \\
-0.008 \\
0.000 \\
0.007\end{array}$ \\
\hline $\mathrm{E} 01 \mathrm{~N}$ & $\begin{array}{l}\text { GR } \\
\text { T1 } \\
\text { E1 } \\
\text { E2 } \\
\text { GR+T } 1 \\
\text { SEIS } \\
\text { GR+T 1+SEIS }\end{array}$ & $\begin{array}{r}-0.001 \\
-0.017 \\
0.001 \\
0.000 \\
-0.018 \\
0.001 \\
0.019\end{array}$ & $\begin{array}{l}-0.002 \\
0.031 \\
0.000 \\
0.000 \\
0.028 \\
0.001 \\
0.029\end{array}$ & $\begin{array}{r}-0.001 \\
-0.011 \\
-0.001 \\
0.001 \\
-0.012 \\
0.002 \\
0.014\end{array}$ & $\begin{array}{r}-0.004 \\
0.011 \\
0.000 \\
0.001 \\
0.007 \\
0.001 \\
0.008\end{array}$ & $\begin{array}{r}0.001 \\
0.043 \\
-0.001 \\
0.001 \\
0.044 \\
0.003 \\
0.047\end{array}$ & $\begin{array}{r}0.003 \\
-0.014 \\
0.000 \\
0.000 \\
-0.012 \\
0.000 \\
0.012\end{array}$ \\
\hline E01 F & $\begin{array}{l}\text { GR } \\
T 1 \\
\text { E1 } \\
\text { E2 } \\
\text { GR+TI } \\
\text { SEIS } \\
\text { GR+TI+SEIS }\end{array}$ & $\begin{array}{r}-0.001 \\
-0.017 \\
0.001 \\
0.000 \\
-0.018 \\
0.001 \\
0.019\end{array}$ & $\begin{array}{r}-0.003 \\
0.030 \\
0.000 \\
0.000 \\
0.027 \\
0.001 \\
0.028\end{array}$ & $\begin{array}{r}-0.001 \\
-0.016 \\
-0.001 \\
0.001 \\
-0.017 \\
0.002 \\
0.019\end{array}$ & $\begin{array}{r}-0.003 \\
-0.015 \\
0.000 \\
0.001 \\
-0.019 \\
0.001 \\
0.020\end{array}$ & $\begin{array}{r}0.000 \\
-0.005 \\
-0.001 \\
0.000 \\
-0.005 \\
0.002 \\
0.007\end{array}$ & $\begin{array}{r}0.003 \\
-0.028 \\
0.000 \\
0.000 \\
-0.025 \\
0.001 \\
0.026\end{array}$ \\
\hline$E 02 \mathrm{~N}$ & $\begin{array}{l}\text { GR } \\
\text { I1 } \\
\text { E1 } \\
\text { E2 } \\
\text { GR+I1 } \\
\text { SEIS } \\
\text { GR+T1+SEIS }\end{array}$ & $\begin{array}{r}-0.001 \\
-0.003 \\
0.001 \\
0.000 \\
-0.003 \\
0.002 \\
0.005\end{array}$ & $\begin{array}{r}-0.003 \\
0.021 \\
0.000 \\
0.000 \\
0.018 \\
0.001 \\
0.019\end{array}$ & $\begin{array}{r}-0.001 \\
-0.027 \\
0.000 \\
0.001 \\
-0.027 \\
0.002 \\
0.029\end{array}$ & $\begin{array}{r}-0.003 \\
-0.020 \\
0.000 \\
0.000 \\
-0.023 \\
0.009 \\
0.024\end{array}$ & $\begin{array}{r}0.000 \\
-0.018 \\
-0.001 \\
0.000 \\
-0.018 \\
0.002 \\
0.020\end{array}$ & $\begin{array}{r}0.003 \\
-0.035 \\
0.000 \\
0.000 \\
-0.032 \\
0.001 \\
0.032\end{array}$ \\
\hline $\mathrm{E} 02 \mathrm{~F}$ & $\begin{array}{l}\text { GR } \\
\text { T1 } \\
\text { E1 } \\
\text { E2 } \\
\text { GR+T1 } \\
\text { SEIS } \\
\text { GR+T1+SEIS }\end{array}$ & $\begin{array}{r}-0.001 \\
0.003 \\
0.001 \\
0.000 \\
0.003 \\
0.002 \\
0.004\end{array}$ & $\begin{array}{r}-0.003 \\
0.017 \\
0.000 \\
0.000 \\
0.015 \\
0.001 \\
0.015\end{array}$ & $\begin{array}{r}-0.001 \\
-0.026 \\
0.000 \\
0.001 \\
-0.027 \\
0.001 \\
0.028\end{array}$ & $\begin{array}{r}-0.003 \\
-0.027 \\
0.000 \\
0.000 \\
-0.029 \\
0.007 \\
0.030\end{array}$ & $\begin{array}{r}-0.001 \\
-0.053 \\
-0.001 \\
0.000 \\
-0.053 \\
0.001 \\
0.055\end{array}$ & $\begin{array}{r}0.004 \\
-0.050 \\
-0.001 \\
0.000 \\
-0.046 \\
0.001 \\
0.047\end{array}$ \\
\hline E03 & $\begin{array}{l}\text { GR } \\
T 1 \\
\text { E1 } \\
\text { E2 } \\
\text { GR+T1 } \\
\text { SEIS } \\
\text { GR+T1+SEIS }\end{array}$ & $\begin{array}{r}-0.001 \\
0.006 \\
0.001 \\
0.000 \\
0.005 \\
0.002 \\
0.007\end{array}$ & $\begin{array}{r}-0.002 \\
0.015 \\
0.000 \\
0.000 \\
0.013 \\
0.001 \\
0.014\end{array}$ & $\begin{array}{r}-0.001 \\
-0.024 \\
0.000 \\
0.001 \\
-0.025 \\
0.001 \\
0.026\end{array}$ & $\begin{array}{r}-0.003 \\
-0.027 \\
0.000 \\
0.000 \\
-0.030 \\
0.001 \\
0.030\end{array}$ & $\begin{array}{r}-0.001 \\
-0.054 \\
-0.001 \\
0.000 \\
-0.055 \\
0.001 \\
0.056\end{array}$ & $\begin{array}{r}0.004 \\
-0.051 \\
-0.001 \\
0.000 \\
-0.047 \\
0.001 \\
0.048\end{array}$ \\
\hline
\end{tabular}

HNF-2486, Rev, 0

Page C-19 


\begin{tabular}{|c|c|c|c|c|c|c|c|c|c|c|c|c|c|c|c|}
\hline \multicolumn{8}{|c|}{$\begin{array}{l}\text { U320H32A PROCESS BUILDING PIPING STRESS ANALYSIS } \\
03 / 01 / 95 \text { H320, TANK } 241-C-106 \text { SLUICING, ER4319 AUTOPIPE+4.50 RESULT PAGE } 17\end{array}$} & \multicolumn{8}{|c|}{$\begin{array}{l}\text { W32OH32A PROCESS BUILDING PIPING STRESS ANALYSIS } \\
03 / 01 / 95 \text { WB20, TANK } 241-6-106 \text { SLUICING, ER4319 AUTOPIPE+4.50 RESULT PA }\end{array}$} \\
\hline \multicolumn{8}{|c|}{ DISPLACEMENTS } & \multicolumn{8}{|c|}{ DISPLACEMENTS } \\
\hline $\begin{array}{l}\text { Point } \\
\text { name }\end{array}$ & $\begin{array}{l}\text { Load } \\
\text { combination }\end{array}$ & $\begin{array}{l}\text { TRANSL } \\
\mathrm{X}\end{array}$ & ATIONS ( & in $z^{\prime}$ & $\begin{array}{l}\text { ROTAT } \\
x\end{array}$ & lows (deg & $\begin{array}{l}3 \\
z\end{array}$ & $\begin{array}{l}\text { Point } \\
\text { name }\end{array}$ & $\begin{array}{l}\text { Load } \\
\text { conbinat ion }\end{array}$ & $\underset{\mathrm{X}}{\text { TRANSLA }}$ & ATIONS & ${ }^{\text {in }} z^{\prime}$ & $\begin{array}{l}\text { ROTATI } \\
\mathrm{X}\end{array}$ & IONS (deg & $\frac{z}{z}$ \\
\hline $\begin{array}{l}\vec{G} \\
\mathrm{G} \\
\mathrm{E} \\
\mathrm{E} \\
\mathrm{G} \\
\mathrm{S} \\
\mathrm{G}\end{array}$ & $\begin{array}{l}\text { GR } \\
\text { T1 } \\
\text { E1 } \\
\text { E2 } \\
\text { GR+T1 } \\
\text { SEIS } \\
\text { GR+T1+SEIS }\end{array}$ & $\begin{array}{r}-0.001 \\
0.043 \\
0.001 \\
0.000 \\
0.042 \\
0.002 \\
0.044\end{array}$ & $\begin{array}{r}0.000 \\
-0.013 \\
0.000 \\
0.000 \\
-0.013 \\
0.000 \\
0.013\end{array}$ & $\begin{array}{r}-0.001 \\
0.007 \\
0.000 \\
0.001 \\
0.007 \\
0.002 \\
0.008\end{array}$ & $\begin{array}{r}-0.002 \\
-0.035 \\
0.000 \\
0.000 \\
-0.036 \\
0.000 \\
0.037\end{array}$ & $\begin{array}{r}0.000 \\
-0.052 \\
-0.002 \\
0.000 \\
-0.052 \\
0.002 \\
0.054\end{array}$ & $\begin{array}{r}0.003 \\
-0.049 \\
-0.001 \\
0.000 \\
-0.045 \\
0.001 \\
0.047\end{array}$ & $\mathrm{~F} 04 \mathrm{~N}$ & $\begin{array}{l}\text { GR } \\
\text { T1 } \\
\text { E1 } \\
\text { E2 } \\
\text { GR+T1 } \\
\text { SEIS } \\
\text { GR+T1+SEIS }\end{array}$ & $\begin{array}{r}-0.001 \\
0.060 \\
0.002 \\
0.000 \\
0.059 \\
0.002 \\
0.062\end{array}$ & $\begin{array}{r}0.000 \\
-0.026 \\
0.000 \\
0.000 \\
-0.026 \\
0.000 \\
0.027\end{array}$ & $\begin{array}{r}-0.001 \\
-0.018 \\
0.000 \\
0.001 \\
-0.019 \\
0.002 \\
0.021\end{array}$ & $\begin{array}{r}0.000 \\
0.031 \\
0.000 \\
0.000 \\
-0.039 \\
0.000 \\
0.039\end{array}$ & $\begin{array}{r}0.000 \\
-0.038 \\
-0.002 \\
0.000 \\
-0.038 \\
0.002 \\
0.040\end{array}$ & $\begin{array}{r}0.002 \\
-0.038 \\
-0.001 \\
0.000 \\
-0.036 \\
0.001 \\
0.037\end{array}$ \\
\hline \multicolumn{5}{|c|}{$\begin{array}{l}* * * \text { Segment } \mathrm{E} \text { end } * * * \\
* * * \text { Segment } F \text { begin } * * *\end{array}$} & $\begin{array}{r}-0.002 \\
-0.035 \\
0.000 \\
0.000\end{array}$ & $\begin{array}{r}0.000 \\
-0.052 \\
-0.002 \\
0.000\end{array}$ & $\begin{array}{r}0.003 \\
-0.049 \\
-0.007 \\
0.000\end{array}$ & $\mathrm{FO} 4 \mathrm{~F}$ & $\begin{array}{l}\text { GR } \\
\text { T1 } \\
\text { E1 } \\
\text { E2 } \\
\text { GR+T1 } \\
\text { SEIS } \\
\text { GR+T1+SEIS }\end{array}$ & $\begin{array}{r}-0.001 \\
0.065 \\
0.002 \\
0.000 \\
0.064 \\
0.003 \\
0.066\end{array}$ & $\begin{array}{r}0.000 \\
-0.016 \\
0.000 \\
0.000 \\
-0.017 \\
0.000 \\
0.017\end{array}$ & $\begin{array}{r}0.000 \\
-0.027 \\
0.000 \\
0.001 \\
-0.027 \\
0.001 \\
0.029\end{array}$ & $\begin{array}{r}0.001 \\
0.035 \\
0.000 \\
0.001 \\
0.036 \\
0.002 \\
0.038\end{array}$ & $\begin{array}{r}0.000 \\
0.010 \\
-0.002 \\
-0.001 \\
0.010 \\
0.003 \\
0.012\end{array}$ & $\begin{array}{r}0.000 \\
0.001 \\
-0.001 \\
0.000 \\
0.002 \\
0.001 \\
0.003\end{array}$ \\
\hline E04 & $\begin{array}{l}G R+T 1 \\
\text { SEIS } \\
\text { GR+T1+SEIS }\end{array}$ & $\begin{array}{l}0.042 \\
0.002 \\
0.044\end{array}$ & $\begin{array}{r}-0.013 \\
0.000 \\
0.013\end{array}$ & $\begin{array}{l}0.007 \\
0.002 \\
0.008\end{array}$ & $\begin{array}{r}-0.036 \\
0.000 \\
0.037\end{array}$ & $\begin{array}{r}-0.052 \\
0.002 \\
0.054\end{array}$ & $\begin{array}{r}-0.045 \\
0.001 \\
0.047\end{array}$ & \multirow[t]{2}{*}{ F05 N } & \multirow{2}{*}{$\begin{array}{l}\text { GR } \\
T 1 \\
\text { E1 } \\
\text { E2 } \\
\text { GR+T1 } \\
\text { SEIS } \\
\text { GR+T1+SEIS }\end{array}$} & \multirow{2}{*}{$\begin{array}{r}-0.001 \\
0.064 \\
0.003 \\
0.000 \\
0.063 \\
0.003 \\
0.066\end{array}$} & \multirow{2}{*}{$\begin{array}{r}0.000 \\
-0.001 \\
0.000 \\
0.000 \\
-0.001 \\
0.000 \\
0.001\end{array}$} & \multirow{2}{*}{$\begin{array}{r}0.000 \\
-0.018 \\
0.000 \\
0.001 \\
-0.019 \\
0.001 \\
0.020\end{array}$} & \multirow{2}{*}{$\begin{array}{r}0.001 \\
0.037 \\
0.000 \\
-0.001 \\
0.038 \\
0.002 \\
0.039\end{array}$} & \multirow{2}{*}{$\begin{array}{r}0.000 \\
0.021 \\
-0.001 \\
-0.001 \\
0.021 \\
0.003 \\
0.024\end{array}$} & \multirow{2}{*}{$\begin{array}{r}0.000 \\
0.006 \\
-0.001 \\
0.000 \\
0.006 \\
0.001 \\
0.007\end{array}$} \\
\hline F01 & $\begin{array}{l}\text { GR } \\
\text { I1 } \\
\text { E1 } \\
\mathrm{E2}\end{array}$ & $\begin{array}{r}-0.001 \\
0.048 \\
0.002 \\
0.000\end{array}$ & $\begin{array}{r}0.000 \\
-0.016 \\
0.000 \\
0.000\end{array}$ & $\begin{array}{r}-0.001 \\
0.001 \\
0.000 \\
0.001\end{array}$ & $\begin{array}{r}-0.001 \\
-0.036 \\
0.000 \\
0.000\end{array}$ & $\begin{array}{r}0.000 \\
-0.047 \\
-0.002 \\
0.000\end{array}$ & $\begin{array}{r}0.003 \\
-0.046 \\
-0.001 \\
0.000\end{array}$ & & & & & & & & \\
\hline & $\begin{array}{l}G R+T 1 \\
\text { SEIS } \\
G R+I 1+S E I S\end{array}$ & $\begin{array}{l}0.047 \\
0.002 \\
0.049\end{array}$ & $\begin{array}{r}-0.017 \\
0.000 \\
0.017\end{array}$ & $\begin{array}{l}0.000 \\
0.002 \\
0.002\end{array}$ & $\begin{array}{r}-0.037 \\
0.000 \\
0.037\end{array}$ & $\begin{array}{r}-0.048 \\
0.002 \\
0.050\end{array}$ & $\begin{array}{r}-0.042 \\
0.001 \\
0.044\end{array}$ & \multirow[t]{2}{*}{ F05 F } & \multirow{2}{*}{$\begin{array}{l}\text { GR } \\
T 1 \\
\text { E1 } \\
\text { E2 } \\
\text { GR+T1 } \\
\text { SEIS } \\
\text { GR+T1+SEIS }\end{array}$} & \multirow{2}{*}{$\begin{array}{r}-0.001 \\
0.050 \\
0.003 \\
0.000 \\
0.050 \\
0.003 \\
0.053\end{array}$} & \multirow{2}{*}{$\begin{array}{l}0.000 \\
0.006 \\
0.000 \\
0.000 \\
0.006 \\
0.000 \\
0.006\end{array}$} & \multirow{2}{*}{$\begin{array}{r}0.000 \\
-0.010 \\
0.000 \\
0.000 \\
-0.010 \\
0.000 \\
0.010\end{array}$} & \multirow{2}{*}{$\begin{array}{l}0.000 \\
0.018 \\
0.000 \\
0.000 \\
0.019 \\
0.000 \\
0.019\end{array}$} & \multirow{2}{*}{$\begin{array}{r}0.000 \\
0.028 \\
-0.001 \\
-0.001 \\
0.028 \\
0.002 \\
0.030\end{array}$} & \multirow{2}{*}{$\begin{array}{r}-0.001 \\
0.024 \\
0.000 \\
0.000 \\
0.023 \\
0.000 \\
0.023\end{array}$} \\
\hline F02 & $\begin{array}{l}\text { GR } \\
\text { I1 } \\
\text { E1 } \\
E 2\end{array}$ & $\begin{array}{r}-0.001 \\
0.050 \\
0.002 \\
0.000\end{array}$ & $\begin{array}{r}0.000 \\
-0.018 \\
0.000 \\
0.000\end{array}$ & $\begin{array}{r}-0.001 \\
-0.002 \\
0.000 \\
0.001\end{array}$ & $\begin{array}{r}-0.001 \\
-0.036 \\
0.000 \\
0.000\end{array}$ & $\begin{array}{r}0.000 \\
-0.047 \\
-0.002 \\
0.000\end{array}$ & $\begin{array}{r}0.003 \\
-0.046 \\
-0.001 \\
0.000\end{array}$ & & & & & & & & \\
\hline & $\begin{array}{l}G R+T I \\
\text { SEIS } \\
G R+T\}+S E I S\end{array}$ & $\begin{array}{l}0.049 \\
0.002 \\
0.051\end{array}$ & $\begin{array}{r}-0.018 \\
0.000 \\
0.018\end{array}$ & $\begin{array}{r}-0.003 \\
0.002 \\
0.004\end{array}$ & $\begin{array}{r}-0.037 \\
0.000 \\
0.037\end{array}$ & $\begin{array}{r}-0.048 \\
0.002 \\
0.050\end{array}$ & $\begin{array}{r}-0.042 \\
0.001 \\
0.044\end{array}$ & \multirow[t]{2}{*}{ F06 } & \multirow{2}{*}{$\begin{array}{l}\text { GR } \\
\text { T1 } \\
\text { E1 } \\
\text { E2 } \\
\text { GR+TI } \\
\text { SEIS } \\
\text { GR+TI+SEIS }\end{array}$} & \multirow{2}{*}{$\begin{array}{r}-0.001 \\
0.044 \\
0.003 \\
0.000 \\
0.044 \\
0.003 \\
0.047\end{array}$} & \multirow{2}{*}{$\begin{array}{l}0.000 \\
0.004 \\
0.000 \\
0.000 \\
0.004 \\
0.000 \\
0.004\end{array}$} & \multirow{2}{*}{$\begin{array}{r}0.000 \\
-0.007 \\
0.000 \\
0.000 \\
-0.007 \\
0.000 \\
0.007\end{array}$} & \multirow{2}{*}{$\begin{array}{l}0.000 \\
0.013 \\
0.000 \\
0.000 \\
0.013 \\
0.000 \\
0.014\end{array}$} & \multirow{2}{*}{$\begin{array}{r}0.000 \\
0.028 \\
-0.001 \\
-0.001 \\
0.028 \\
0.002 \\
0.030\end{array}$} & \multirow{2}{*}{$\begin{array}{l}0.000 \\
0.023 \\
0.000 \\
0.000 \\
0.022 \\
0.000 \\
0.022\end{array}$} \\
\hline$F 03$ & $\begin{array}{l}\text { GR } \\
\text { T1 } \\
\text { E1 } \\
\text { E2 }\end{array}$ & $\begin{array}{r}-0.001 \\
0.058 \\
0.002 \\
0.000\end{array}$ & $\begin{array}{r}0.000 \\
-0.024 \\
0.000 \\
0.000\end{array}$ & $\begin{array}{r}-0.001 \\
-0.015 \\
0.000 \\
0.001\end{array}$ & $\begin{array}{r}0.000 \\
-0.033 \\
0.000 \\
0.000\end{array}$ & $\begin{array}{r}0.000 \\
-0.040 \\
-0.002 \\
0.000\end{array}$ & $\begin{array}{r}0.003 \\
-0.040 \\
-0.001 \\
0.000\end{array}$ & & & & & & & & \\
\hline & $\begin{array}{l}G R+T 1 \\
S E I S \\
G R+T 1+S E I S\end{array}$ & $\begin{array}{l}0.057 \\
0.002 \\
0.059\end{array}$ & $\begin{array}{r}-0.025 \\
0.000 \\
0.025\end{array}$ & $\begin{array}{r}-0.015 \\
0.002 \\
0.017\end{array}$ & $\begin{array}{r}-0.033 \\
0.000 \\
0.033\end{array}$ & $\begin{array}{r}-0.040 \\
0.002 \\
0.042\end{array}$ & $\begin{array}{r}-0.037 \\
0.001 \\
0.038\end{array}$ & $F 07$ & $\begin{array}{l}\text { GR } \\
Y 1 \\
E 1 \\
E 2 \\
G R+T 1 \\
\text { SEIS } \\
G R+T 1+S E I S\end{array}$ & $\begin{array}{r}-0.001 \\
0.041 \\
0.003 \\
0.000 \\
0.040 \\
0.003 \\
0.043\end{array}$ & $\begin{array}{l}0.000 \\
0.003 \\
0.000 \\
0.000 \\
0.003 \\
0.000 \\
0.003\end{array}$ & $\begin{array}{r}0.000 \\
-0.005 \\
0.000 \\
0.000 \\
-0.005 \\
0.000 \\
0.006\end{array}$ & $\begin{array}{l}0.000 \\
0.010 \\
0.000 \\
0.000 \\
0.010 \\
0.000 \\
0.010\end{array}$ & $\begin{array}{r}0.000 \\
0.027 \\
-0.001 \\
-0.001 \\
0.027 \\
0.002 \\
0.029\end{array}$ & $\begin{array}{l}0.000 \\
0.022 \\
0.000 \\
0.000 \\
0.021 \\
0.000 \\
0.021\end{array}$ \\
\hline
\end{tabular}

HNF-2486, Rev, 0

Page C-20

Page 9 of 39 

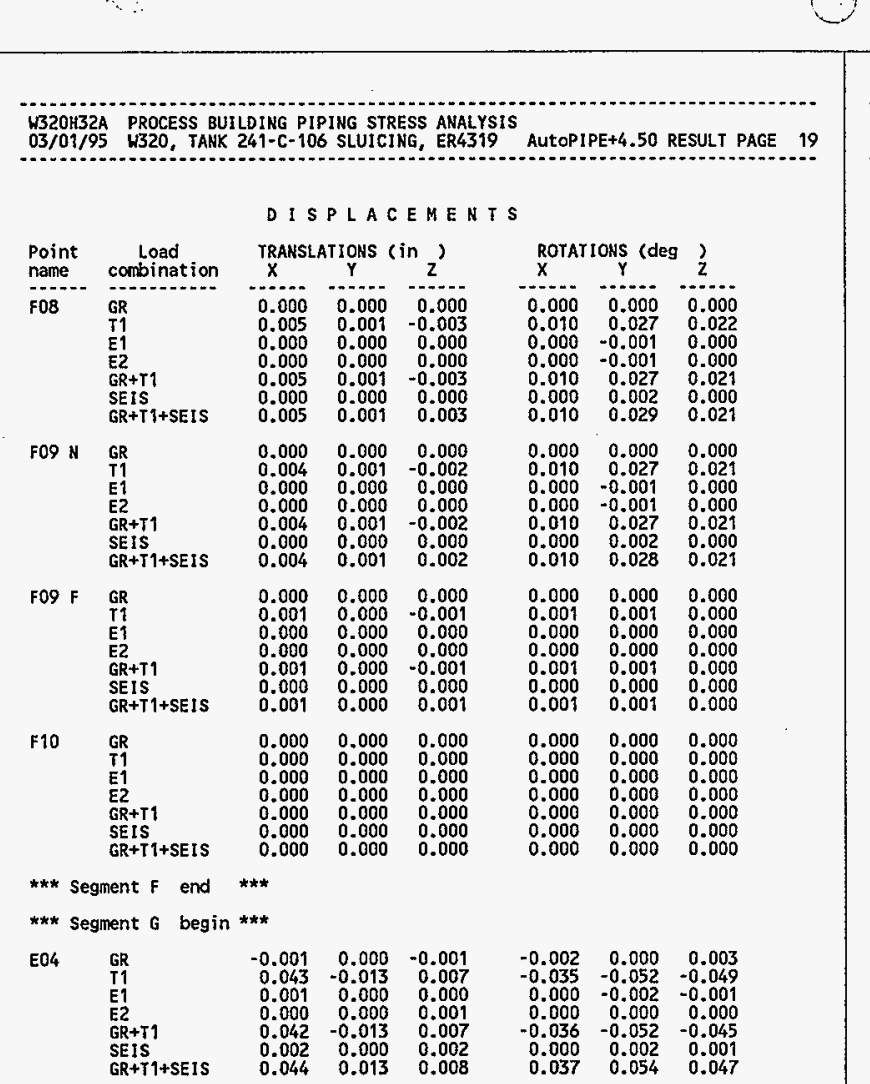

W32OH32A PROCESS BUILDING PIPING STRESS ANALYSIS

ERA319 AUtOPIPE+4 50 RESULT PAGE 20 DISPLACEMENTS

\begin{tabular}{|c|c|c|c|c|c|c|c|}
\hline $\begin{array}{l}\text { Point } \\
\text { name }\end{array}$ & $\begin{array}{l}\text { Load } \\
\text { combination }\end{array}$ & $\underset{\mathrm{X}}{\text { TRANSL }}$ & $\underset{Y}{T I O N S}$ & $z_{z}^{\prime}$ & $\begin{array}{l}\text { ROTAT } \\
\mathbf{x}\end{array}$ & Pis (de & $\begin{array}{l}\text { ? } \\
\text { Z }\end{array}$ \\
\hline GO1 N & $\begin{array}{l}\text { GR } \\
T 1 \\
E I \\
E 2 \\
\text { GR+TI } \\
\text { SEIS } \\
\text { GR+T }+ \text { +SEIS }\end{array}$ & $\begin{array}{r}-0.001 \\
0.038 \\
0.001 \\
0.000 \\
0.037 \\
0.002 \\
0.039\end{array}$ & $\begin{array}{r}0.000 \\
-0.009 \\
0.000 \\
0.000 \\
-0.009 \\
0.000 \\
0.010\end{array}$ & $\begin{array}{l}-0.001 \\
0.014 \\
0.000 \\
0.001 \\
0.013 \\
0.002 \\
0.015\end{array}$ & $\begin{array}{r}-0.002 \\
-0.034 \\
0.000 \\
0.000 \\
-0.036 \\
0.001 \\
0.036\end{array}$ & $\begin{array}{r}0.000 \\
-0.052 \\
-0.002 \\
0.000 \\
-0.052 \\
0.002 \\
0.054\end{array}$ & $\begin{array}{r}0.003 \\
-0.049 \\
-0.001 \\
0.000 \\
-0.046 \\
0.001 \\
0.047\end{array}$ \\
\hline $\mathrm{GO1} \mathrm{F}$ & $\begin{array}{l}\text { GR } \\
T 1 \\
\text { E1 } \\
E 2 \\
\text { GR+T1 } \\
\text { SEIS } \\
\text { GR+T1+SEIS }\end{array}$ & $\begin{array}{r}-0.001 \\
0.026 \\
0.001 \\
0.000 \\
0.025 \\
0.002 \\
0.026\end{array}$ & $\begin{array}{r}0.000 \\
-0.002 \\
0.000 \\
0.000 \\
-0.002 \\
0.000 \\
0.002\end{array}$ & $\begin{array}{r}-0.001 \\
0.017 \\
0.000 \\
0.001 \\
0.016 \\
0.001 \\
0.017\end{array}$ & $\begin{array}{r}-0.003 \\
-0.017 \\
0.000 \\
0.001 \\
-0.020 \\
0.002 \\
0.021\end{array}$ & $\begin{array}{r}0.000 \\
-0.035 \\
-0.001 \\
-0.001 \\
-0.035 \\
0.002 \\
0.037\end{array}$ & $\begin{array}{r}0.002 \\
-0.039 \\
-0.002 \\
0.001 \\
-0.036 \\
0.002 \\
0.039\end{array}$ \\
\hline $\mathrm{GO2}$ & $\begin{array}{l}\text { GR } \\
\text { T1 } \\
\text { E1 } \\
\text { E2 } \\
\text { GR+T1 } \\
\text { SEIS } \\
\text { GR+I1+SEIS }\end{array}$ & $\begin{array}{l}-0.001 \\
0.022 \\
0.001 \\
0.000 \\
0.021 \\
0.002 \\
0.023\end{array}$ & $\begin{array}{l}0.000 \\
0.000 \\
0.000 \\
0.000 \\
0.000 \\
0.000 \\
0.000\end{array}$ & $\begin{array}{r}-0.001 \\
0.015 \\
0.000 \\
0.001 \\
0.014 \\
0.001 \\
0.015\end{array}$ & $\begin{array}{r}-0.003 \\
-0.015 \\
0.000 \\
0.001 \\
-0.018 \\
0.002 \\
0.020\end{array}$ & $\begin{array}{r}0.000 \\
-0.034 \\
-0.001 \\
-0.001 \\
-0.034 \\
0.002 \\
0.036\end{array}$ & $\begin{array}{r}0.002 \\
-0.038 \\
-0.002 \\
0.001 \\
-0.036 \\
0.002 \\
0.038\end{array}$ \\
\hline $\cos \mathrm{N}$ & $\begin{array}{l}\text { GR } \\
\text { T1 } \\
\text { E1 } \\
\text { E2 } \\
\text { GR+T1 } \\
\text { SEIS } \\
\text { GR+T 1+SEIS }\end{array}$ & $\begin{array}{l}-0.001 \\
0.019 \\
0.001 \\
0.000 \\
0.018 \\
0.002 \\
0.019\end{array}$ & $\begin{array}{l}0.000 \\
0.002 \\
0.000 \\
0.000 \\
0.002 \\
0.000 \\
0.002\end{array}$ & $\begin{array}{r}-0.001 \\
0.013 \\
0.000 \\
0.001 \\
0.012 \\
0.001 \\
0.014\end{array}$ & $\begin{array}{r}-0.003 \\
-0.014 \\
0.001 \\
0.001 \\
-0.016 \\
0.002 \\
0.018\end{array}$ & $\begin{array}{r}0.000 \\
-0.033 \\
-0.001 \\
-0.001 \\
-0.033 \\
0.001 \\
0.035\end{array}$ & $\begin{array}{r}0.003 \\
-0.038 \\
-0.002 \\
0.001 \\
-0.035 \\
0.002 \\
0.038\end{array}$ \\
\hline $\mathrm{G03} F$ & $\begin{array}{l}\text { GR } \\
\text { T1 } \\
\text { E1 } \\
\text { E2 } \\
\text { GR+T1 } \\
\text { SEIS } \\
\text { GR+T1+SEIS }\end{array}$ & $\begin{array}{l}0.000 \\
0.004 \\
0.001 \\
0.000 \\
0.003 \\
0.001 \\
0.004\end{array}$ & $\begin{array}{r}-0.001 \\
-0.004 \\
0.000 \\
0.000 \\
-0.004 \\
0.001 \\
0.005\end{array}$ & $\begin{array}{l}0.000 \\
0.011 \\
0.000 \\
0.001 \\
0.010 \\
0.001 \\
0.011\end{array}$ & $\begin{array}{r}-0.001 \\
-0.006 \\
0.000 \\
0.002 \\
-0.007 \\
0.002 \\
0.009\end{array}$ & $\begin{array}{r}-0.001 \\
-0.017 \\
0.000 \\
-0.001 \\
-0.018 \\
0.001 \\
0.019\end{array}$ & $\begin{array}{r}0.002 \\
-0.017 \\
-0.002 \\
0.001 \\
-0.016 \\
0.003 \\
0.019\end{array}$ \\
\hline G04 & $\begin{array}{l}\text { GR } \\
\text { I1 } \\
\text { EI } \\
\text { E2 } \\
\text { GR+T1 } \\
\text { SEIS } \\
\text { GR+I1+SEIS }\end{array}$ & $\begin{array}{l}0.000 \\
0.001 \\
0.000 \\
0.000 \\
0.001 \\
0.001 \\
0.002\end{array}$ & $\begin{array}{r}-0.001 \\
-0.014 \\
0.000 \\
0.000 \\
-0.015 \\
0.001 \\
0.015\end{array}$ & $\begin{array}{l}0.000 \\
0.011 \\
0.000 \\
0.000 \\
0.011 \\
0.000 \\
0.012\end{array}$ & $\begin{array}{r}-0.001 \\
-0.004 \\
0.000 \\
0.002 \\
-0.005 \\
0.002 \\
0.007\end{array}$ & $\begin{array}{r}-0.001 \\
-0.012 \\
0.000 \\
-0.001 \\
-0.013 \\
0.001 \\
0.014\end{array}$ & $\begin{array}{r}0.001 \\
-0.014 \\
-0.002 \\
0.001 \\
-0.013 \\
0.003 \\
0.016\end{array}$ \\
\hline
\end{tabular}




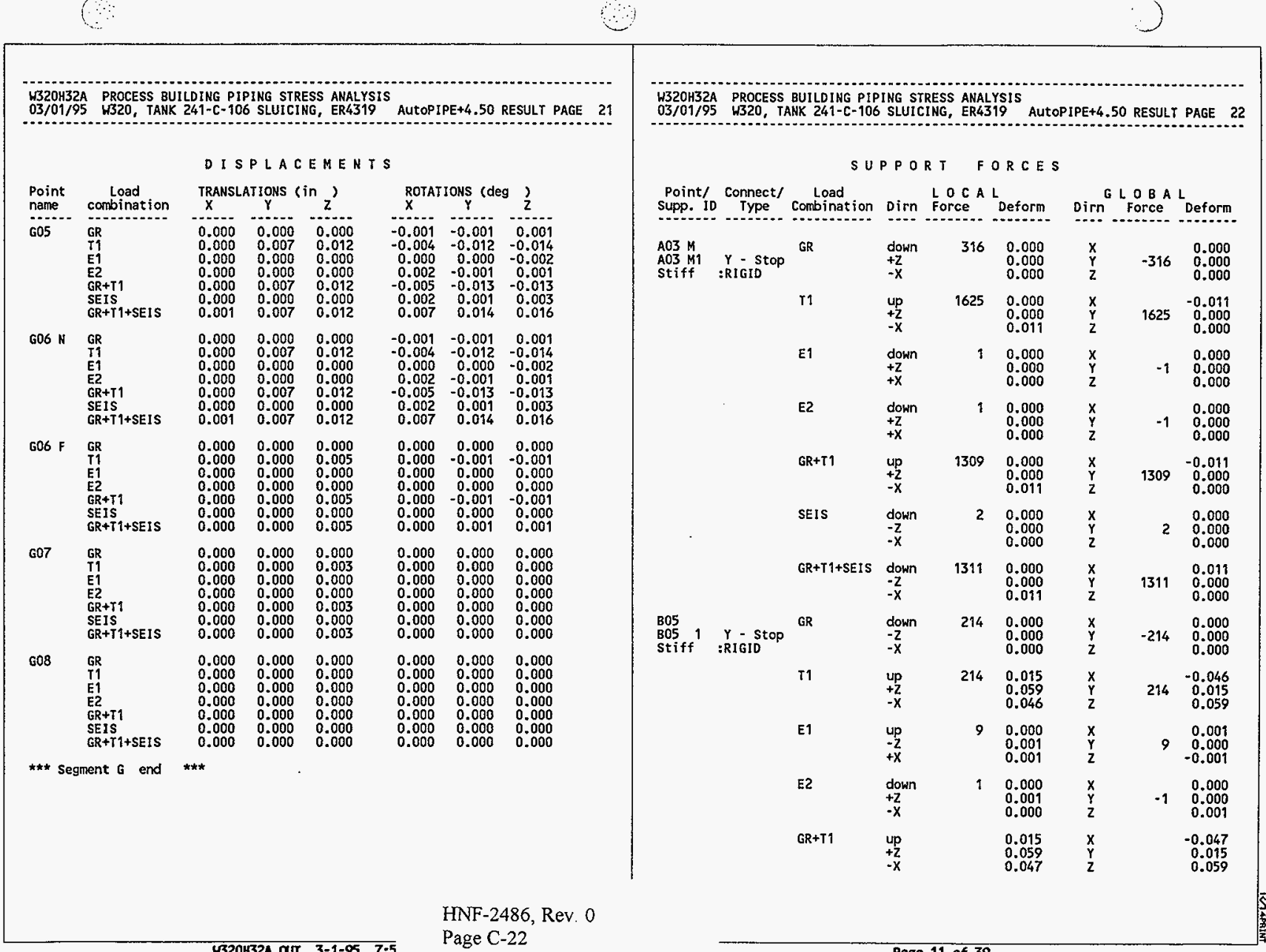


W32OH32A PROCESS BUILDING PIPING STRESS ANALYSIS

03/01/95 W320, TANK 241-C-106 SLUICING, ER4319 AutOPIPE+4.50 RESULT PAGE 23

SUPPORT FORCES

Point/ Connect/ Load

\begin{tabular}{|c|c|c|c|c|c|c|c|c|}
\hline \multirow{2}{*}{\multicolumn{2}{|c|}{ Supp. ID }} & $\begin{array}{l}\text { Combination } \\
\text { SEIS }\end{array}$ & \begin{tabular}{l} 
Dirn \\
\hdashline down \\
$-z$ \\
$-x$
\end{tabular} & $\begin{array}{r}\text { Force } \\
10\end{array}$ & $\begin{array}{l}\text { Deform } \\
0.000 \\
0.001 \\
0.001\end{array}$ & $\begin{array}{l}\text { Dirn } \\
X \\
Y \\
Z\end{array}$ & $\begin{array}{r}\text { Force } \\
10\end{array}$ & $\begin{array}{l}\text { Deform } \\
0.001 \\
0.000 \\
0.001\end{array}$ \\
\hline & & $G R+T 1+S E I S$ & $\begin{array}{l}\text { down } \\
-z \\
-x\end{array}$ & 10 & $\begin{array}{l}0.015 \\
0.060 \\
0.048\end{array}$ & $\begin{array}{l}X \\
y \\
z\end{array}$ & 10 & $\begin{array}{l}0.048 \\
0.015 \\
0.060\end{array}$ \\
\hline \multirow[t]{7}{*}{$\begin{array}{l}\text { B12 } \\
8121 \\
\text { stiff }\end{array}$} & $\begin{array}{l}\text { Y - Stop } \\
\text { :RIGID }\end{array}$ & GR & $\begin{array}{l}\text { down } \\
-z \\
+x\end{array}$ & 255 & $\begin{array}{l}0.000 \\
0.001 \\
0.000\end{array}$ & $\begin{array}{l}X \\
Y \\
Z\end{array}$ & -255 & $\begin{array}{r}0.000 \\
0.000 \\
-0.001\end{array}$ \\
\hline & & II & $\begin{array}{l}\text { down } \\
-z \\
+x\end{array}$ & $\begin{array}{l}35 \\
54 \\
51\end{array}$ & $\begin{array}{l}0.000 \\
0.008 \\
0.007\end{array}$ & $\begin{array}{l}X \\
Y \\
Z\end{array}$ & $\begin{array}{r}51 \\
-35 \\
-54\end{array}$ & $\begin{array}{r}0.007 \\
0.000 \\
-0.008\end{array}$ \\
\hline & & E1 & $\begin{array}{l}\text { down } \\
-2 \\
+x\end{array}$ & 4 & $\begin{array}{l}0.000 \\
0.001 \\
0.000\end{array}$ & $\begin{array}{l}X \\
Y \\
Z\end{array}$ & -4 & $\begin{array}{r}0.000 \\
0.000 \\
-0.001\end{array}$ \\
\hline & & $E 2$ & $\begin{array}{l}\text { down } \\
+z \\
-x\end{array}$ & 0 & $\begin{array}{l}0.000 \\
0.002 \\
0.000\end{array}$ & $\begin{array}{l}\mathbf{X} \\
\mathbf{Y} \\
\mathbf{Z}\end{array}$ & 0 & $\begin{array}{l}0.000 \\
0.000 \\
0.002\end{array}$ \\
\hline & & $G R+T 1$ & $\begin{array}{l}\text { down } \\
-z \\
+x\end{array}$ & $\begin{array}{r}291 \\
54 \\
51\end{array}$ & $\begin{array}{l}0.000 \\
0.009 \\
0.008\end{array}$ & $\begin{array}{l}X \\
Y \\
Z\end{array}$ & $\begin{array}{r}51 \\
-291 \\
-54\end{array}$ & $\begin{array}{r}0.008 \\
0.000 \\
-0.009\end{array}$ \\
\hline & & SEIS & $\begin{array}{l}\text { down } \\
-2 \\
-x\end{array}$ & 4 & $\begin{array}{l}0.000 \\
0.003 \\
0.000\end{array}$ & $\begin{array}{l}X \\
Y \\
Z\end{array}$ & 4 & $\begin{array}{l}0.000 \\
0.000 \\
0.003\end{array}$ \\
\hline & & $\mathrm{GR}+\mathrm{T} 1+\mathrm{SEIS}$ & $\begin{array}{l}\text { down } \\
-z \\
-x\end{array}$ & $\begin{array}{r}294 \\
54 \\
51\end{array}$ & $\begin{array}{l}0.000 \\
0.012 \\
0.008\end{array}$ & $\begin{array}{l}X \\
Y \\
Z\end{array}$ & $\begin{array}{r}51 \\
294 \\
54\end{array}$ & $\begin{array}{l}0.008 \\
0.000 \\
0.012\end{array}$ \\
\hline \multirow[t]{4}{*}{$\begin{array}{l}\text { Do2 } \\
0021 \\
\text { Stiff }\end{array}$} & $\begin{array}{l}Y=\text { Stop } \\
\text { :RIGID }\end{array}$ & GR & $\begin{array}{l}\text { down } \\
-z \\
-x\end{array}$ & 145 & $\begin{array}{l}0.000 \\
0.000 \\
0.000\end{array}$ & $\begin{array}{l}X \\
Y \\
Z\end{array}$ & -145 & $\begin{array}{l}0.000 \\
0.000 \\
0.000\end{array}$ \\
\hline & & $T 1$ & $\begin{array}{l}\text { up } \\
-z \\
+x\end{array}$ & 145 & $\begin{array}{l}0.040 \\
0.032 \\
0.004\end{array}$ & $\begin{array}{l}x \\
y \\
z\end{array}$ & 145 & $\begin{array}{r}0.004 \\
0.040 \\
-0.032\end{array}$ \\
\hline & & E1 & $\begin{array}{l}\text { down } \\
-z \\
+x\end{array}$ & 2 & $\begin{array}{l}0.000 \\
0.000 \\
0.000\end{array}$ & $\begin{array}{l}X \\
Y \\
Z\end{array}$ & -2 & $\begin{array}{l}0.000 \\
0.000 \\
0.000\end{array}$ \\
\hline & & E2 & up & 21 & 0.000 & $x$ & & 0.000 \\
\hline
\end{tabular}

W32OH32A PROCESS BUILDING PIPING STRESS ANALYSIS

D3/01/95 W30, TANK 241-E-106 SLUICING, ER4319 AUTOPIPE+4.50 RESULT PAGE 24

SUPPORT FORCES

Point/ Connect/ Load L OCA L G L O B A L

Supp. 10 Type Combination Dirn Force Deform Dirn Force Deform

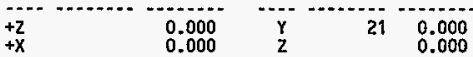

GR+T1 up $\quad 0.040 \quad Y \quad Y 004$

$\begin{array}{rrrr}-Z & 0.032 & Y & 0.040 \\ +X & 0.004 & Z & -0.032\end{array}$

SEIS down $23 \quad 0.000 \quad x \quad Y \quad r \quad 0.000$ $-2$ $0.000 \quad z \quad 0.000$

GR+T1+SEIS down $23 \quad 0.040 \quad X \quad 0.004$

$\begin{array}{llllll}\text { down } & 23 & 0.040 & X & & 0.004 \\ -Z & & 0.033 & Y & 23 & 0.040 \\ -X & & 0.004 & Z & & 0.033\end{array}$

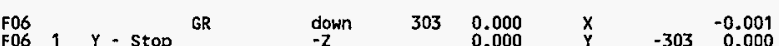

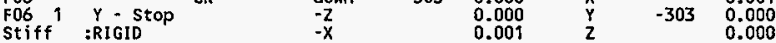

$\begin{array}{lllllll}\text { T1 } & \text { up } & 303 & 0.004 & X & 0.044\end{array}$

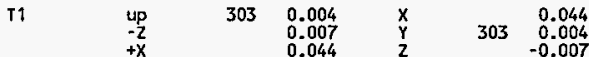

$\begin{array}{lllllll}E 1 & \text { down } & 13 & 0.000 & X & & 0.003 \\ +Z & & & 0.000 & Y & -13 & 0.000\end{array}$

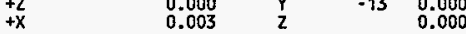

E2 down $4 \quad 0.000 \quad x \quad 0.000$

$\begin{array}{lllll}+Z & 4 & 0.000 & Y & 0.000 \\ -X & 0.000 & Y & -4 & 0.000\end{array}$

0.000

$G R+T 1$

0.004
0.007

0.007
0.044

0.044

0.004

$-0.007$

SEIS

down
$-z$
$-x$

$17 \quad 0.000$

0.000
0.003

0.003

$17 \quad 0.000$ 0.000

GR+T1+SEIS

dow
$-Z$
$-X$

$17 \quad 0.004$

0.007
0.047

0.047

$17 \quad 0.004$

0.007

G02
GO2 $1 Y$ - Stop
Stiff

$\begin{array}{lll}\text { down } & 224 \quad 0.000 \\ -Z & & 0.001\end{array}$

0.001
0.001

$-0.001$

$\begin{array}{ll}-224 & 0.000\end{array}$

0.001

$T 1$

$\begin{array}{rr}97 & 0.022 \\ -310 & 0.000\end{array}$ 
SUPPOR T FORCES

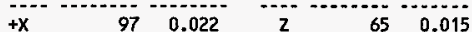

\begin{tabular}{|c|c|c|c|c|c|c|}
\hline E1 & $\begin{array}{l}\text { down } \\
+Z \\
+X\end{array}$ & 7 & $\begin{array}{l}0.000 \\
0.000 \\
0.001\end{array}$ & $\begin{array}{l}x \\
y \\
z\end{array}$ & -7 & $\begin{array}{l}0.001 \\
0.000 \\
0.000\end{array}$ \\
\hline E2 & $\begin{array}{l}\operatorname{up}_{+2} \\
-x\end{array}$ & 3 & $\begin{array}{l}0.000 \\
0.001 \\
0.000\end{array}$ & $\begin{array}{l}x \\
y \\
z\end{array}$ & 3 & $\begin{array}{l}0.000 \\
0.000 \\
0.001\end{array}$ \\
\hline$G R+T q$ & $\begin{array}{l}\text { down } \\
+Z \\
+X\end{array}$ & $\begin{array}{r}534 \\
65 \\
97\end{array}$ & $\begin{array}{l}0.000 \\
0.014 \\
0.021\end{array}$ & $\begin{array}{l}X \\
Y \\
Z\end{array}$ & $\begin{array}{r}97 \\
-534 \\
65\end{array}$ & $\begin{array}{l}0.021 \\
0.000 \\
0.014\end{array}$ \\
\hline SEIS & $\begin{array}{l}\text { down } \\
-Z \\
-X\end{array}$ & 10 & $\begin{array}{l}0.000 \\
0.001 \\
0.002\end{array}$ & $\begin{array}{l}X \\
y \\
z\end{array}$ & 10 & $\begin{array}{l}0.002 \\
0.000 \\
0.001\end{array}$ \\
\hline GR+T1+SEIS & $\begin{array}{l}\text { down } \\
-z \\
-x\end{array}$ & $\begin{array}{r}544 \\
65 \\
97\end{array}$ & $\begin{array}{l}0.000 \\
0.015 \\
0.023\end{array}$ & $\begin{array}{l}X \\
Y \\
Z\end{array}$ & $\begin{array}{r}97 \\
544 \\
65\end{array}$ & $\begin{array}{l}0.023 \\
0.000 \\
0.015\end{array}$ \\
\hline
\end{tabular}

RESTRAINT REACTIONS

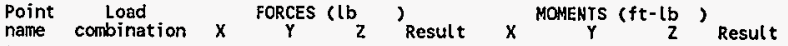
ESTRAINT REACTIONS

A00 Anchor

$\begin{array}{lrrrrrrrr}\text { Anchor } & -44 & -18 & -13 & 49 & 17 & 21 & 88 & 92 \\ \text { GR } & -21549 & -29 & -227 & 21550 & -349 & -489 & 42625 & 42629 \\ \text { T1 } & 49 & 0 & 15 & 51 & 31 & 1 & -67 & 73 \\ \text { E1 } & 3 & 0 & 50 & 50 & 66 & -15 & -6 & 68 \\ \text { E2 } & -21593 & -46 & -240 & 21594 & -331 & -469 & 42713 & 42716 \\ \text { GR+T1 } & 52 & 0 & 65 & 83 & 97 & 16 & 73 & 122 \\ \text { SEIS } & 54 & 96 & 78 & 78 \\ \text { GR+T1+SEIS } & 21645 & 46 & 305 & 21647 & 428 & 485 & 42785 & 42790\end{array}$

A03 M $Y$ - Stop

$\begin{array}{lrrrrlll}\text { GR } & 0 & -316 & 0 & 316 & 0 & 0 & 0 \\ \text { T1 } & 0 & 1625 & 0 & 1625 & 0 & 0 & 0 \\ \text { E1 } & 0 & -1 & 0 & 1 & 0 & 0 & 0 \\ \text { E2 } & 0 & -1 & 0 & 1 & 0 & 0 & 0 \\ \text { GR+T1 } & 0 & 1309 & 0 & 1309 & 0 & 0 & 0 \\ \text { SEIS } & 0 & 2 & 0 & 2 & 0 & 0 & 0 \\ \text { GR+T1+SEIS } & 0 & 1311 & 0 & 1311 & 0 & 0 & 0\end{array}$

A08

Anchor

GR

GR
I
E1
E2

GR $+T 1$

GR+T1

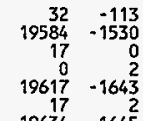

$\begin{array}{rr}9 & 118 \\ 63 & 19644 \\ -15 & 23 \\ 22 & 22 \\ 72 & 19686 \\ 37 & 41 \\ 109 & 19703\end{array}$

$\begin{array}{rrrr}18 & -2 & -2 & 18 \\ 221 & -129 & -12009 & 12012 \\ -8 & -5 & -8 & 12 \\ 11 & 7 & -1 & 13 \\ 239 & -131 & -12012 & 12015 \\ 19 & 12 & 99 & 24 \\ 257 & 143 & 12020 & 12024\end{array}$

B05

$Y$ - Stop

$\begin{array}{llr}\text { GR - Stop } & 0 & -214 \\ \text { T1 } & 0 & 214 \\ \text { E1 } & 0 & 9 \\ \text { E2 } & 0 & -1 \\ \text { GR+T1 } & 0 & 0 \\ \text { SEIS } & 0 & 10 \\ \text { GR+TI+SEIS } & 0 & 10\end{array}$

$$
\begin{array}{rr}
0 & 214 \\
0 & 214 \\
0 & 9 \\
0 & 1 \\
0 & 0 \\
0 & 10 \\
0 & 10
\end{array}
$$$$
\begin{aligned}
& 0 \\
& 0 \\
& 0 \\
& 0 \\
& 0 \\
& 0 \\
& 0
\end{aligned}
$$

812

$\begin{array}{lrrrr}Y \text { - Stop } & & & & \\ \text { GR } & 0 & -255 & 0 & 255 \\ T 1 & 51 & -35 & -54 & 83 \\ \text { E1 } & 0 & -4 & 0 & 4 \\ \text { E2 } & 0 & 0 & 0 & 0 \\ \text { GR+T1 } & 51 & -291 & -54 & 300 \\ \text { SEIS } & 0 & 4 & 0 & 4 \\ \text { GR+T1+SEIS } & 51 & 294 & 54 & 304\end{array}$


RESTRAINT REACTIONS

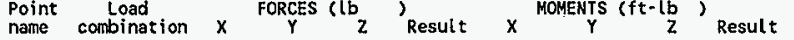
FORCES (Ib )

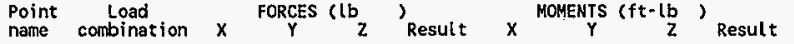

B16 Anchor $\begin{array}{lrrrr}\text { Anchor } & -2 & 1 & -1 & \\ \text { GR } & 93 & 27 & -26 & 100 \\ \text { I1 } & 31 & 7 & -2 & \\ \text { E1 } & -1 & 0 & 12 & 11 \\ \text { E2 } & 91 & 27 & -27 & 97 \\ \text { GR+T1 } & 32 & 7 & 14 & 36 \\ \text { SEES } & 123 & 35 & 41 & 134\end{array}$ GR+T $1+S E I S$

C12

Anchor

GR

T1
E1
E2
GR+T

GR+TI

GR+T I+SE IS

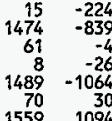

$\begin{array}{rr}14 & 225 \\ 937 & 1938 \\ -10 & 62 \\ 73 & 78 \\ 951 & 2062 \\ 83 & 112 \\ 1034 & 2167\end{array}$

$\begin{array}{rrr}2 & 26 & 13 \\ 100 & -10 & 507 \\ 32 & -7 & 39 \\ 12 & 0 & -12 \\ 99 & 16 & 520 \\ 36 & 7 & 51 \\ 134 & 23 & 571\end{array}$

$\begin{array}{rrr}13 & 91 & 96 \\ 507 & -9 & 507 \\ 39 & -14 & 42 \\ -12 & 0 & 12 \\ 520 & 83 & 526 \\ 51 & 14 & 53 \\ 571 & 97 & 579\end{array}$

D02

$Y$ - stop

$\begin{array}{lrr}Y=\text { Stop } & 0 & -145 \\ \text { GR } & 0 & 145 \\ \text { I1 } & 0 & -2 \\ \text { E1 } & 0 & 21 \\ \text { E2 } & 0 & 0 \\ \text { GR+II } & 0 & 23 \\ \text { SEIS } & 0 & 23 \\ \text { GR+T1+SEIS } & 0\end{array}$

D07

Anchor

GR

E1

GR+T1

SEIS

GR+T I+SEIS

$\begin{array}{rrr}0 & -127 & -1 \\ 45 & 144 & -101 \\ 24 & 0 & 0 \\ 0 & -2 & 14 \\ 45 & 16 & -102 \\ 24 & 2 & 14 \\ 69 & 19 & 116\end{array}$

$\begin{array}{lr}0 & 145 \\ 0 & 145 \\ 0 & 2 \\ 0 & 21 \\ 0 & 0 \\ 0 & 23 \\ 0 & 23\end{array}$

$\begin{array}{rr}-243 & -1 \\ -562 & -113 \\ -12 & -7 \\ 54 & 1 \\ -806 & -115 \\ 66 & 9 \\ 871 & 124\end{array}$

$\begin{array}{rrr}-14 & 24 & 245 \\ -1137 & -1306 & 1821 \\ -75 & -61 & 97 \\ 15 & -9 & 56 \\ -1152 & -1281 & 1902 \\ 90 & 70 & 131 \\ 1242 & 1352 & 2032\end{array}$

F06

$Y$ - Stop

GR

E1

GR+T 1

SEIS

$G R+T i+S E I S$

RESTRAINT REACTIONS

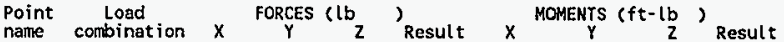

F10 Anchor

$\begin{array}{lrrrrrrrr}\text { GR } & -1 & 56 & -9 & 57 & 33 & 4 & 4 & 33 \\ \text { I1 } & 49 & 315 & -839 & 897 & 968 & 1128 & 694 & 1640 \\ \text { E1 } & 10 & 13 & 4 & 17 & -2 & -22 & 2 & 22 \\ \text { E2 } & 0 & 8 & 35 & 36 & -29 & -42 & 8 & 52 \\ \text { GR+T1 } & 48 & 371 & -848 & 927 & 1001 & 1132 & 698 & 1665 \\ \text { SEIS } & 10 & 21 & 39 & 45 & 31 & 64 & 10 & 71 \\ \text { GR+I1+SEIS } & 58 & 392 & 887 & 971 & 1032 & 1195 & 708 & 1731\end{array}$

G02

$Y$ - stop

$\begin{array}{lrrrr}\text { Y - Stop } & 0 & -224 & 0 & 224 \\ \text { GR } & 97 & -310 & 65 & 332 \\ \text { TI } & 0 & -7 & 0 & 7 \\ \text { EI } & 0 & 3 & 0 & 3 \\ \text { E2 } & 97 & -534 & 65 & 547 \\ \text { GR+T1 } & 0 & 10 & 0 & 10 \\ \text { SEIS } & 97 & 544 & 65 & 556\end{array}$

G08

Anchor

$\begin{array}{lrrrrrrrr}\text { Anchor } & 0 & -129 & 1 & 129 & 27 & -2 & 30 & 40 \\ \text { GR } & 155 & -29 & 181 & 240 & 7 & -445 & -602 & 749 \\ \text { T1 } & 50 & 1 & 8 & 50 & 7 & 7 & -64 & 64 \\ \text { E1 } & -11 & 0 & 36 & 37 & 27 & -16 & 19 & 36 \\ \text { E2 } & 154 & -157 & 182 & 286 & 34 & -447 & -572 & 727 \\ \text { GR+T1 } & 61 & 1 & 44 & 75 & 34 & 23 & 82 & 92 \\ \text { SEIS } & 215 & 158 & 226 & 350 & 67 & 470 & 655 & 809\end{array}$

HNF-2486, Rev. 0

Page C-25 


\section{W32OH32A PROCESS BUILOING PIPING STRESS ANALYSIS \\ AUTOPIPE+4.50 RESULT PAGE 29}

GLOBAL FORCES \& MOMENTS

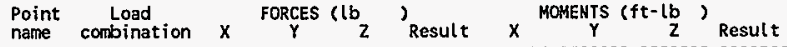

\section{*** Segment $A$ begin ***}

A00

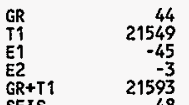

GR+T1

GR+T1+SEIS 21641

A01

GR
T1
E1
E2
GR+T1

GR+T1 21593

$\begin{array}{lr}\text { SEIS } & 48 \\ \text { GR+T1+SEIS } & 21641\end{array}$

$A 02$ - $G$

T1

E2

44
21549

-37
-3
21593

GR+T1 21593

GR+T1+SEIS 21633

$\mathrm{A02}$

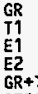

44
21549
-30

GR $R$ TR

GR+T1+SEIS 21627

A03 N

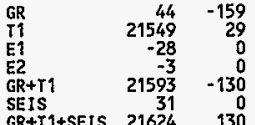

SEIS

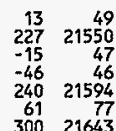

$300 \quad 21643$

$\begin{array}{rr}13 & 49 \\ 227 & 21550\end{array}$

$\begin{array}{ll}-15 & 47 \\ -46 & 46\end{array}$

$240 \quad 21594$

$300 \quad 21643$

$\begin{array}{rr}13 & 96 \\ 227 & 21550\end{array}$

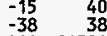

$240 \quad 21594$

$292 \quad 21635$

$\begin{array}{rr}13 & 144 \\ 227 & 21550\end{array}$

$-15{ }^{34}$

24021594

$286 \quad 21629$

$\begin{array}{rr}13 & 165 \\ 227 & 21550\end{array}$

$\begin{array}{ccc}-15 & 32 \\ -29 & 32\end{array}$

$240 \quad 21595$

$283 \quad 21626$

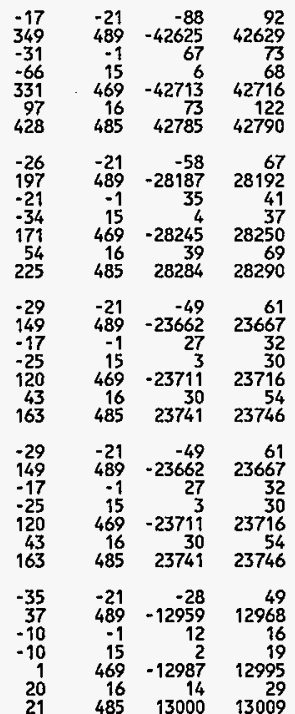

HNF-2486, Rev. 0

Page C-26
W320H32A PROCESS BUILDING PIPING STRESS ANALYSIS

03/01/95 W320, TANK 241-C-106 SLUICING, ER4319 AUTOPIPE+4.50 RESULT PAGE 30

GLOBAL FORCES \& MOMENTS

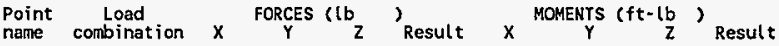

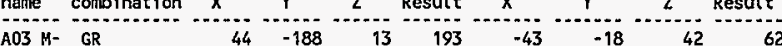

$\begin{array}{lrrrrrrrr}\text { GR } & 44 & -188 & 13 & 193 & -43 & -18 & 42 & 62 \\ \text { T1 } & 21549 & 29 & 227 & 21550 & -97 & 545 & -268 & 615 \\ \text { E1 } & -24 & 0 & -15 & 29 & -1 & -5 & -3 & 6 \\ \text { E2 } & -3 & 0 & -25 & 25 & 6 & 9 & 0 & 10 \\ \text { GR+I1 } & 21593 & -159 & 240 & 21595 & -140 & 527 & -226 & 590\end{array}$

$\begin{array}{lrrrrrrrr}E R+I 1 & 21593 & -159 & 240 & 21595 & -140 & 527 & -226 & 590 \\ \text { SRII } & 28 & 0 & 40 & 49 & 6 & 14 & 4 & 15 \\ \text { GR+T1+SEIS } & 21620 & 159 & 280 & 21623 & 146 & 541 & 230 & 605\end{array}$

$\mathrm{A03} \mathrm{M}+\mathrm{GR}-44 \quad 128-13 \quad 136$

$\begin{array}{rr}13 & 136 \\ 227 & 21609 \\ -15 & 29\end{array}$

$\begin{array}{lr}-15 & 29 \\ -25 & 25\end{array}$

$240 \quad 21644$

$\begin{array}{rr}40 & 49 \\ 280 & 21672\end{array}$

230

$\begin{array}{llr}-43 & -18 & 42 \\ -97 & 545 & -268 \\ -1 & -5 & -3\end{array}$

-1
-140
-96

59
527
14

-3
0
-226
4

SEIS 28 (15 2108

$\begin{array}{rr}13 & 109 \\ 227 & 21609\end{array}$

$\begin{array}{rr}-15 & 26 \\ -22 & 22\end{array}$

$\begin{array}{rr}-22 & 22 \\ 240 & 21646\end{array}$

$\begin{array}{rr}37 & 21644\end{array}$

A04 -

SEIS $244 \quad 147$

$13 \quad 91$

$\begin{array}{rr}-15 & 216 \\ -19 & 19\end{array}$

$240 \quad 21647$

$\begin{array}{rr}34 & 41 \\ 274 & 21670\end{array}$

$\begin{array}{rr}93 & 38 \\ 63 & 19644\end{array}$

$\begin{array}{rr}63 & 1964 \\ -15 & 16 \\ 10 & 11 \\ 72 & 19678 \\ 26 & 27\end{array}$

$\begin{array}{rr}26 & 27 \\ 98 & 19684\end{array}$

A05

$G R+T 1+S E I S \quad 21614 \quad 1519$

146

-46
-152

31
-198
-14

-198
14
213

213

541

230

-10
678
-14

-14
-5
668

19
687

-14
5932
-10

-1
5918

5929

78

665

11
-198

-21
-14
778

$\begin{array}{rr}14 & 35 \\ 213 & 812\end{array}$

6661
-10
-2
6606
12

6618

$\begin{array}{rr}0 & -24 \\ -104 & -3572\end{array}$

-62
5396

$T 1$
$E 1$
$E 2$

$\begin{array}{lrr}E 2 & 19617 & -1549\end{array}$

$\begin{array}{lll}S E I S & 6 \\ G R+T 1+S E 1 S \quad 19623 & 1550\end{array}$

$-7$

$\begin{array}{rr}-104 & -19 \\ -3596\end{array}$

15
119 $\quad 3636$

5334

$\begin{array}{rr}9 & 52 \\ 63 & 19644 \\ -15 & 17 \\ 13 & 13 \\ 72 & 19680 \\ 28 & 30 \\ 101 & 19688\end{array}$

GR
I1
E1

E1

SEIS $19584 \cdot-1530$

$\begin{array}{rr}0697 & -1569 \\ 1961 & \end{array}$

GR+T1+SEIS $19625 \quad 1571$ $\begin{array}{rr}0 & -19 \\ -104 & -3543\end{array}$

$\begin{array}{rr}-7 & -14 \\ -104 & -3562 \\ 15 & 28\end{array}$ $\begin{array}{rr}-49 & 53 \\ 6106 & 7060 \\ 4 & 17 \\ 2 & 16 \\ 6057 & 7027 \\ 6 & 32 \\ 6063 & 7047\end{array}$ 
W320H32A PROCESS BUILDING PIPING STRESS ANALYSIS

03/01/95 W320, TANK 241-C-106 SLUICING, ER4319 AUTOPIPE+4.50 RESULT PAGE 31

GLOBAL FORCES \& NOMENTS

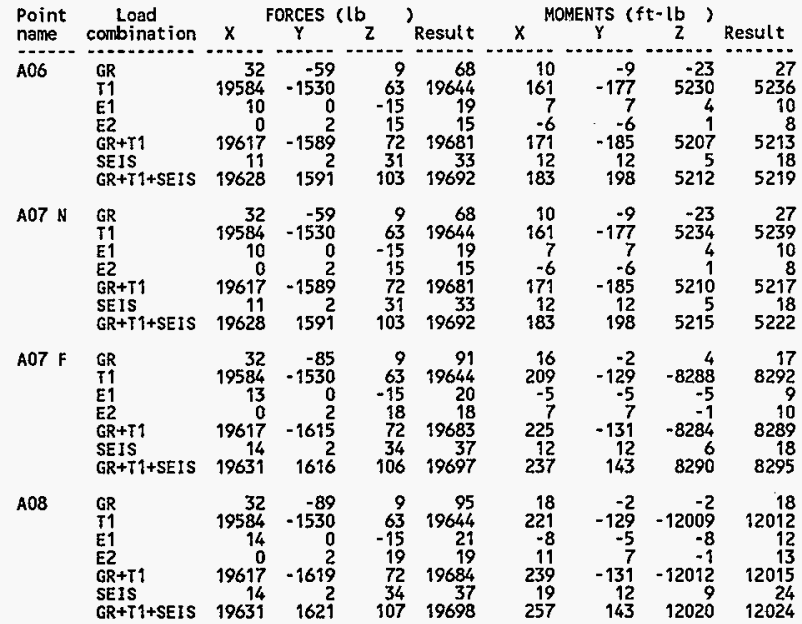

$* * *$ Segment $A$ end $* * *$

*** Segment B begin ***

A04

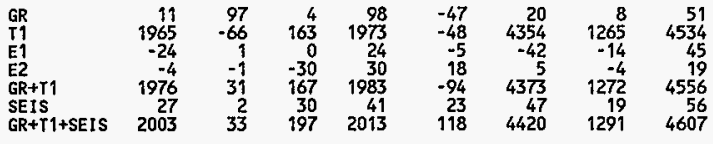

W32OH32A PROCESS BUILDING PIPING STRESS ANALYSIS

03/01/95 W320, TANK 241-C-106 SLUICING, ER4319 AUtOPIPE+4.50 RESULT PAGE 32

GLOBAL FORCES \& MOMENTS

$\begin{array}{l}\text { Point } \\ \text { name combination }\end{array}$ FORCES (lb $\left.{ }_{Z}\right)_{\text {Result }} \times{ }_{Y}^{\text {MOMENTS ( } f t-l b}{ }_{Z}$ ) Result

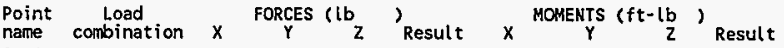

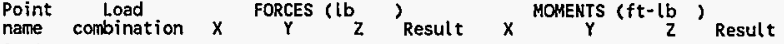

B01 N

$\begin{array}{lrrrr}\text { GR } & 11 & 83 & 4 & 84 \\ \text { T1 } & 1965 & -66 & 163 & 1973 \\ \text { E1 } & -22 & 1 & 0 & 22 \\ \text { E2 } & -4 & -1 & -28 & 28 \\ \text { GR+T1 } & 1976 & 17 & 167 & 1983 \\ \text { SEIS } & 26 & 2 & 28 & 38 \\ \text { GR+T1+SEIS } & 2002 & 19 & 196 & 2011\end{array}$

B01 F

GR+T1+SEIS

GR

T1
E2

E2

SEIS

GERTI+SEIS 2000

$\begin{array}{rr}11 & 71 \\ 1965 & -66\end{array}$

$\begin{array}{rr}4 & 72 \\ 163 & 1973\end{array}$

$\begin{array}{rr}0 & 21 \\ -27 & 27 \\ 167 & 1983\end{array}$

$\begin{array}{rr}167 & 1983 \\ 27 & 36 \\ 194 & 2010\end{array}$

$\mathrm{BO} 2$ - $\mathrm{G}$

$\mathrm{GR}$
$\mathrm{T} 1$
$\mathrm{E} 1$
$\mathrm{E} 2$

E2

SE1S

GR+T1+SEIS 200

$\mathrm{BO2}+$

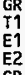

I1

GR+T1

SEIS

803

$G R$
T
E
E?

E2

GR+T1

GR+T1+SEIS

11

$163 \quad 1973$

$\begin{array}{rrrr}-21 & 1 & 0 & 21 \\ -4 & -1 & -27 & 27 \\ 1976 & 5 & 167 & 1983 \\ 24 & 2 & 27 & 36 \\ 2000 & 7 & 194 & 2010\end{array}$

$x$

....

$\begin{array}{llll}1993 & 61 & 187 & 2002\end{array}$

$804 N$

$\begin{array}{lrr}\text { GR } & 11 & -13 \\ \text { I1 } & 1965 & -66 \\ \text { E1 } & -11 & 1 \\ \text { E2 } & -4 & -1 \\ \text { GR+I1 } & 1976 & -79 \\ \text { SEIS } & 14 & 2 \\ \text { GR+I1+SEIS } & 1990 & 82\end{array}$

HNF-2486, Rev. 0

Page C-27 
H32OH32A PROCESS BUILDING PIPING STRESS ANALYYSIS

03/01/95 W320, TANK 241-C-106 SLUICING, ER4319 AUtOPIPE+4.50 RESULT PAGE 33

GLOBAL FORCES \& MOMENTS

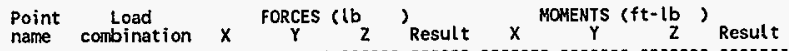
$29-15 \quad-22 \quad-30$

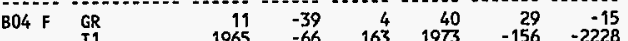
$\begin{array}{lrrrrrrrr}\text { T1 } & 1965 & -66 & 163 & 1973 & -156 & -2228 & -98 & 2236 \\ \text { E1 } & -8 & 1 & 0 & 8 & -1 & 8 & 0 & 9 \\ \text { E2 } & -4 & -1 & -13 & 14 & -5 & 7 & -1 & 8 \\ \text { GR+T1 } & 1976 & -105 & 167 & 1986 & -127 & -2244 & -76 & 2249\end{array}$

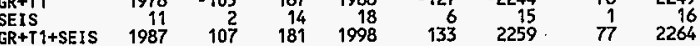

B05

GR
$T 1$
EI
ER
GR+

$\begin{array}{rr}11 & -79 \\ 1965 & -66 \\ -3 & 1 \\ -4 & -1\end{array}$

GR+T

$\begin{array}{rr}-4 & -1 \\ 1976 & -145\end{array}$

GR+T1+SEIS

B05

GR
T1
EI
E2
G

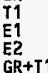

GR+T 1
SEIS

198

GR
EI
E2

$E 1$
$E 2$
$G R+T$

GR+TI
SE IS

$\begin{array}{rr}11 & 136 \\ 1965 & -280 \\ -3 & -8 \\ -4 & 0 \\ 1976 & -145\end{array}$

$\begin{array}{rr}6 & 8 \\ 1982 & 153\end{array}$

4.80

$80 \quad 29 \quad-8$

$\begin{array}{rr}0 & 3 \\ -9 & 9\end{array}$

$\begin{array}{rr}29 & -8 \\ -150 & -1923 \\ -1 & 9\end{array}$

$\begin{array}{rr}-5 & -14 \\ -121 & -1931\end{array}$

$\begin{array}{rrrr}167 & 1988 & -121 & -1931 \\ 9 & 11 & 6 & 23 \\ 176 & 1996 & 127 & 1953\end{array}$

gr

GR
TI
E2

$\mathrm{EL}+\mathrm{GR}$

SEIS

B07 -

GR
TI
ET
GR+TY
SEIS

$\begin{array}{rr}11 & 89 \\ 1965 & -280 \\ 3 & -8\end{array}$

$\begin{array}{rrr}-4 & -8 & 0\end{array}$

$1976-192$

4136

$\begin{array}{rr}29 & -8 \\ -150 & -1923 \\ -1 & 9 \\ -5 & -14\end{array}$

$\begin{array}{rrrr}-9 & 9 & -5 & -14 \\ 167 & 1988 & -121 & -1931 \\ 9 & 14 & 6 & 23 \\ 176 & 1996 & 127 & 1953\end{array}$

9914

$\begin{array}{rr}127 & 1953 \\ 29 & 2\end{array}$

$\begin{array}{rr}29 & 2 \\ -141 & -1567 \\ -1 & 10 \\ -6 & -26\end{array}$

$\begin{array}{rr}-6 & -26 \\ -111 & -1566\end{array}$

-3
167
3

1983200

$\begin{array}{rr}-1 & \\ -80 & 165 \\ -2 & 4 \\ -18 & 19 \\ -81 & 16 \\ 20 & 2 \\ 102 & 17\end{array}$

145

$143-10$

+T1+SEIS

SEIS $-2 \quad-11$

-11
-9
-20
-2

-1
-80
-2
-17
-81
19
100

-2
1
1
$131 \quad 134$

$\begin{array}{rr}1 & 15 \\ 87 & 1936\end{array}$

$\begin{array}{rr}3 & 24 \\ 90 & 1959\end{array}$

$131 \quad 134$

$\begin{array}{rr}131 & 134 \\ -44 & 1929 \\ -2 & 9\end{array}$

$\begin{array}{rr}1 & 15 \\ 87 & 1936\end{array}$

$\begin{array}{rr}3 & 24 \\ 90 & 1959\end{array}$

$.114 \quad 118$

449
16

$\begin{array}{rr}335 & 1605\end{array}$

$\begin{array}{rr}335 & 1605 \\ 17 & 40\end{array}$

3531644

-92
231
16

-1
139

139
18
157

-89
234
15

-1
145

145
16

12
383
31
408 $\begin{array}{rr}-44 & 1929 \\ -2 & 9\end{array}$
W320H32A PROCESS BUILDING PIPING STRESS ANALYSIS

$03 / 01 / 95$ W320, TANK $241-C-106$ SLUICING, ER4319 AUTOPIPE+4.50 RESULT PAGE 34

GLOBAL FORCES \& MOMENTS

Point Load FORCES (lb) MONENTS ( $f t-l b$ ) name combination $X \quad Y \quad Z$ Result $X \quad Y \quad Z$ Result

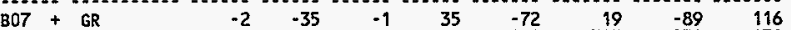
$\begin{array}{lrrrrrr}\text { GR } & -2 & -35 & -1 & 35 & -72 & 19 \\ \text { T1 } & 145 & -9 & -80 & 165 & 159 & 325 \\ \text { E1 } & 5 & 3 & -2 & 6 & 10 & -11 \\ \text { E2 } & -1 & 0 & -14 & 14 & 11 & 3 \\ \text { GR+T1 } & 143 & -44 & -81 & 170 & 87 & 344 \\ \text { SEIS } & 6 & 4 & 16 & 18 & 22 & 14 \\ \text { GR+T1+SEIS } & 148 & 48 & 97 & 184 & 109 & 358\end{array}$

$\mathrm{B08}-\mathrm{CR}$

GR
E1
E2

E1

GR+T 1

SEIS $T+T+S E I S$

$\begin{array}{rr}-2 & -53 \\ 145 & -9\end{array}$

$-1 \quad 54$

109

$-72$

-1
143
8

3
0
-62

-80
-2
-12
-81

8
12
176

10
11
87
22

808

GR

I1

ER+T1

SR+T

$\begin{array}{rrrrr}-2 & -77 & -1 & 78 & -72 \\ 145 & -9 & -80 & 165 & 159\end{array}$

95190

$109 \quad 342$

19
325
-11
3
344

$\begin{array}{rr}-89 & 1 \\ 234 & 43 \\ 15 & 2 \\ -1 & 1 \\ 145 & 38\end{array}$

$809 \mathrm{~N}$

GR

T1
E1
E2

$\mathrm{E} 2$

SEIS

GR+T\}+SEIS

$\begin{array}{rr}-1 & 0 \\ 143 & -86\end{array}$

$\begin{array}{ll}-2 & 11 \\ -9 & 18\end{array}$

$\begin{array}{rr}-81 & 185 \\ 11 & 16\end{array}$

11
87

22

$358 \quad 161$

$\begin{array}{lll}19 & -81 & 110\end{array}$

B09 F

GR

E1

GR+T1

SEIS

GR+T 1+SEIS

153

92200

342

19

$-11$

329

341

23

1
329
12
342

155
16
170

$\begin{array}{lll}310 & -81 & 110\end{array}$

$-11$

329
12

-1
155
16

$170 \quad 397$

$-81 \quad 110$

$235 \quad 420$

155 . 11

$\begin{array}{rr}16 & 29 \\ 170 & 397\end{array}$

B10 N G

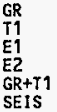

GR+T1

GR+T1+SEIS

18
250
-13
-5
268
18
28

$\begin{array}{rr}-14 & 76 \\ 134 & 300\end{array}$

$\begin{array}{rr}3 & 16 \\ -1 & 8\end{array}$

$119 \quad 295$

4
123 $\quad 314$

18

-14
87

$\pi$

$\begin{array}{rr}-2 & -110 \\ 145 & -9\end{array}$

$\begin{array}{rrr}-1 & 110 & -73 \\ -80 & 165 & 73\end{array}$

$\begin{array}{rrrr}-2 & 14 & 8 & -13\end{array}$

268

18
286 
GLOBAL FORCES \& MOMENTS

Point
name combination $X$
FORCES (lb
$Y$ ) Result $x$ MOMENTS (ft-lb ${ }_{Y}$ ) Result

B10 F GR

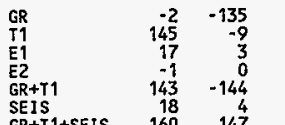

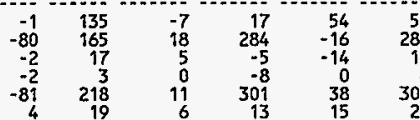

B11 N

$\begin{array}{rrrr}60 & 147 & 45 & 19\end{array}$

GR

E1

GR+T1

SEIS $\begin{array}{rrrr}-2 & -135 & -1 & 135 \\ 145 & -9 & -80 & 165\end{array}$

GR+T1+SEIS

$\begin{array}{rr}-1 & 0 \\ 143 & -144\end{array}$

B11 F

T1

E2

GR+T

$\begin{array}{llll}160 & 147 & 4 & 19\end{array}$

234

$\begin{array}{ll}-2 & -148\end{array}$

GR

E1

GR+T

SEIS

GR+T1+SEIS

$\begin{array}{ll}-80 & 165 \\ -2 & 19\end{array}$

$\begin{array}{rr}-1 & 0 \\ 143 & -156\end{array}$

$\begin{array}{rr}-1 & -81 \\ -3 & 227\end{array}$

GR

I1

E2

SEIS

GR+T1+SEIS

$162 \quad 160$

$84 \quad 243$

$\begin{array}{rrrrr}27 & 91 & 359 & 71 & 377 \\ 20 & 4 & 12 & 15 & 20 \\ 43 & 94 & 371 & 86 & 392\end{array}$

$\begin{array}{rrrr}-1 & 153 & 107 & \\ -80 & 165 & 25 & 38\end{array}$

$19 \quad 3$

$-2$

132

$\begin{array}{rr}85 & 138 \\ -14 & 38 \\ -15 & \end{array}$

$\begin{array}{rrr}8 & -15 & 18 \\ -9 & 0 & 9\end{array}$

$20 \quad-162$

$\begin{array}{rr}2 & 20 \\ 83 & 246\end{array}$

135

17
413

71
15
86

423
23
443

30

85
-14
-15

-2
-2

1
60
21

25
3
132
3
135

\begin{tabular}{c}
138 \\
382 \\
18 \\
423 \\
428 \\
\hline
\end{tabular}

B13 - GR

GR
E1
E2

GR+T1

SEIS

29

$\begin{array}{rr}97 & 8 \\ 100 & 18 \\ 21 & \\ 1 & \\ 156 & 100 \\ 22 & 10 \\ 174 & 101\end{array}$

396
17
413

15
86

15
404
13
-9
419
22

441

HNF-2486, Rev. 0

Page C-29

W32OH32A PROCESS BUILDING PIPING STRESS ANALYSIS

U320. TANK 241-C-106 SLUICING, ER4319 AUtOPIPE+4.50 RESULT PAGE 36

GLOBAL FORCES \& MOMENTS

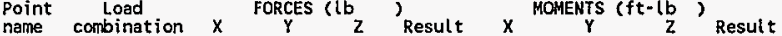

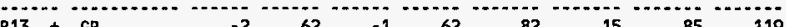

$\begin{array}{lrrrrrrrr}\text { GR } & -2 & 62 & -9 & 62 & 82 & 15 & 85 & 119 \\ \text { T1 } & 93 & 27 & -26 & 100 & 18 & 404 & -14 & 405 \\ \text { E1 } & 24 & 7 & -2 & 25 & 1 & 13 & -15 & 20 \\ \text { E2 } & -1 & 0 & 5 & 5 & 0 & -9 & 0 & \\ \text { GR+T1 } & 91 & 88 & -27 & 130 & 100 & 419 & 71 & 436 \\ \text { SEIS } & 25 & 7 & 6 & 27 & 1 & 22 & 15 & 27 \\ \text { GR+T1+SEIS } & 196 & 96 & 33 & 154 & 101 & 441 & 86 & 461\end{array}$

$B 14$ - GR

G1

T1
E1
E2

GR+T1

SEIS

$G R+T 1+S E I S$



1

$\mathrm{B14}+$

T1

GR+T1

SEIS

GR+T1+SE IS

$-2 \quad 38$

$\begin{array}{rr}-1 & 38 \\ -26 & 100 \\ -2 & 28\end{array}$

$\begin{array}{rr}-2 & 28 \\ -27 & 8 \\ 9 & 315 \\ 36 & 30\end{array}$

10

B15 N

GR
T1
E2

ER 2 TI

SEIS

GR+T1+SEIS

28

$\begin{array}{rr}9 & 30 \\ 36 & 143\end{array}$

$\begin{array}{rrrr}-2 & 37 & -1 & 37 \\ 93 & 27 & -26 & 100 \\ 27 & 7 & -2 & 28\end{array}$

0
64
7
71

$\begin{array}{rr}8 & 8 \\ -27 & 115 \\ 9 & 30\end{array}$

$B 15$ F

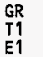

E1

$G R+T 1$

GEIS $1+$ SEIS

-2
93
28
9
29
12

B16

GR
II
EI
E2
GR+T
SEIS

E2

GR+T $\imath+S E I S$

*** Segment $B$ end *

$36 \quad 143$

25
100
29
9
108
32
139

26
-10
-7
0
16
7
23

85
-14
-15

24
-9

-9
464
34

-15
0
71
15

71
15
86

$\begin{array}{rrr}43 & 14 & 85 \\ 5 & 451 & -14\end{array}$

$\begin{array}{rrr}-3 & 451 & -14 \\ 0 & 24 & -15\end{array}$

48
3
50

464
34
498

0
71
15
86

$42 \quad 14 \quad 85$

$\begin{array}{rrr}5 & 14 & 85 \\ -3 & 451 & -14 \\ -3 & 24 & -15\end{array}$

-9
465
34

0
71
15
86

$\begin{array}{ll}15 & 473 \\ 86 & 509\end{array}$

13

$\begin{array}{cc}91 & 96 \\ -9 & 507\end{array}$

39
-12

519

$-14$

83
14

$\begin{array}{ll}14 & 526 \\ 97 & 579\end{array}$ $\begin{array}{rr}25 & -1 \\ 27 & -26 \\ 7 & -2 \\ 0 & \\ 51 & -27 \\ 7 & \\ 59 & \end{array}$ $\begin{array}{rr}-1 & 25 \\ -26 & 100 \\ -2 & 29 \\ 9 & 9 \\ -27 & 108 \\ 11 & 32 \\ 38 & 139\end{array}$
26
-10
-7
0
16
7
23

13
507
39
-12
520
51
57

$\begin{array}{rr}91 & 96 \\ -9 & 507 \\ -14 & 42 \\ 0 & 12 \\ 83 & 526 \\ 14 & 53 \\ 97 & 579\end{array}$

$\begin{array}{rr}85 & 119 \\ -14 & 405 \\ -15 & 20 \\ 0 & 9 \\ 71 & 436 \\ 15 & 27 \\ 86 & 461\end{array}$ 


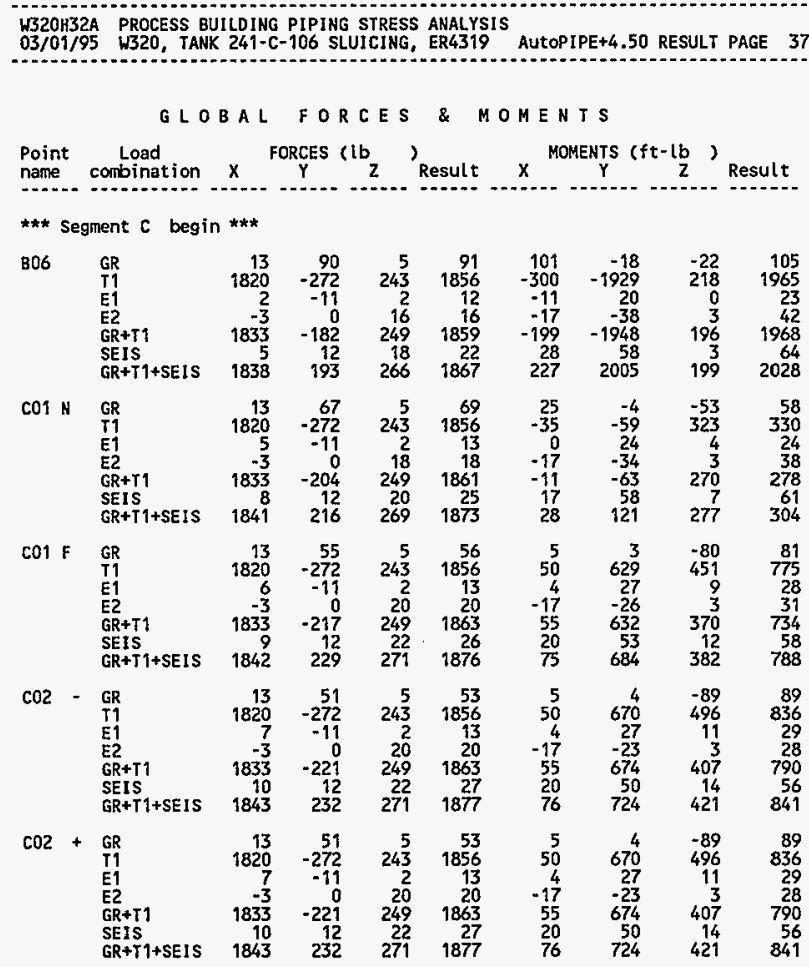

W320H32A PROCESS BUILDING PIPING STRESS ANALYSIS

03/01/95 W320, TANK 24i-C-106 SLUICING, ER4319 AUTOPIPE+4.50 RESULT PAGE 38

GLOBAL FORCES \& MOMENTS

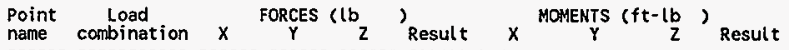

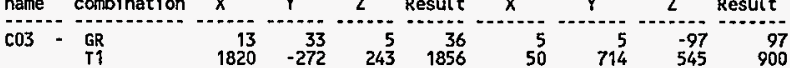

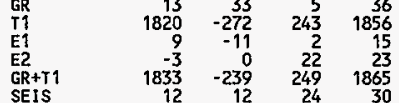

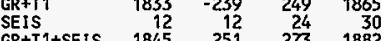

$\begin{array}{lllll}G R+T 1+S E I S & 1845 & 251 & 273 & 1882\end{array}$

$\mathrm{CO3}+\mathrm{GR}$

$\begin{array}{lrrrr}\text { GR } & 13 & 33 & 5 & 36 \\ \text { T1 } & 1820 & -272 & 243 & 1856 \\ \text { E1 } & 9 & -11 & 2 & 15 \\ \text { E2 } & -3 & 0 & 22 & 23 \\ \text { GR+T1 } & 1833 & -239 & 249 & 1865 \\ \text { SEIS } & 12 & 12 & 24 & 30 \\ \text { GR+T1+SE1S } & 1845 & 251 & 273 & 1882\end{array}$

C04

GR
T1
E1
ER
GR+T1 1 1845

$S E I S$
$G R+T 1+S E I S T$

$\cos$ -

GR
T1
E1
E2

T1
E1
GR+T

GR+T1

GR+T1+SEIS

$\begin{array}{rrrr}13 & 32 & 5 & 35 \\ 1820 & -272 & 243 & 1856\end{array}$

$\begin{array}{rr}-3 & 0 \\ 1833 & -239 \\ 12 & 12\end{array}$

$\begin{array}{rr}22 & 15 \\ 24 & 23 \\ 24 & 1865 \\ 24 & 30\end{array}$

50
4
-17
55
20

$\begin{array}{rr}4 & 27 \\ 17 & -19 \\ 55 & 719 \\ 20 & 46 \\ 76 & 765\end{array}$

545
13
3
449

3
449
16
465

5

75
714
27
790
74
76

$\cos +$

GR

T1
E1
$E 2$

GR+T1

GR+T

GR+T1+SEIS

$\begin{array}{rr}13 & 23 \\ 1820 & -272 \\ 10 & -11\end{array}$

$\begin{array}{rr}-3 & -11 \\ 1833 & -249\end{array}$

$\begin{array}{rr}1833 & -249 \\ 13 & 12\end{array}$

$273 \quad 1882$

$\begin{array}{rr}5 & 27 \\ 243 & 1856\end{array}$

$\begin{array}{rr}2 & 15 \\ 24 & 24 \\ 249 & 1867\end{array}$

$\begin{array}{rr}26 & 31 \\ 274 & 1885\end{array}$

$14 \quad-19$

$\begin{array}{rr}14 & -19 \\ 1520 & -550\end{array}$

$\begin{array}{rr}13 & 27 \\ 836 & 1820 \\ -10 & 30\end{array}$

$\begin{array}{rr}-10 & 30 \\ 30 & 31 \\ 849 & 1844\end{array}$

$\begin{array}{rrrr}1534 & -569 & 849 & 1844 \\ 36 & 12 & 40 & 55\end{array}$

C06 -

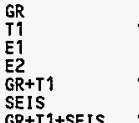

$\begin{array}{rr}13 & 35 \\ 836 & 1820 \\ -10 & 31 \\ 31 & 33 \\ 849 & 1847 \\ 41 & 57\end{array}$

GR+T + +SEIS 
H32OH32A PROCESS 8UILDING PIPING STRESS ANALYSIS

03/01/95 W320, TANK 241-C-106 SLUICING, ER4319 AUtOPIPE+4.50 RESULT PAGE 39

GLOBAL FORCES \& MOMENTS

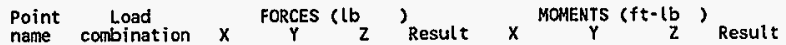

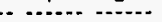

$\begin{array}{rrrr}-8 & -7 & -86 & 8 \\ -698 & 202 & 212 & 75 \\ 12 & 13 & 21 & 28 \\ -34 & -23 & -4 & 4 \\ -706 & 195 & 126 & 74 \\ 46 & 36 & 25 & 6\end{array}$

$G R+T$

$\begin{array}{rrrr}13 & 56 & -8 & -7 \\ 836 & 1820 & -698 & 202 \\ -10 & 34 & 12 & -13 \\ 34 & 35 & -34 & -23 \\ 849 & 1854 & -706 & 195\end{array}$

GR $\quad 14 \quad-71$

$\cot$

T1
E1
E2

$\mathrm{ER}+\mathrm{T1}$

\begin{tabular}{ll}
1534 & -621 \\
\hline & 42
\end{tabular}

C07

GR
T1
E1
E2

E1

GEIS

$\begin{array}{rrr}45 & 12 \\ \text { GR+T1+SEIS } \quad 1579 & 657\end{array}$

$\operatorname{COB~N}$

GR
T1
E1
E2

E1

GR+T 1

SEIS

$\cos \mathrm{F}$

GR
T1
E1
E2

E1

$G R+T 1$

GR+T1+SEIS

$\begin{array}{rr}14 & -103 \\ 1520 & -550\end{array}$

$\begin{array}{rr}38 & -6 \\ 8 & -6 \\ 1534 & -654 \\ 46 & 12 \\ 1580 & 665\end{array}$

8931911

$\begin{array}{rr}13 & 73 \\ 836 & 1820\end{array}$

$\begin{array}{rr}-10 & 36 \\ 36 & 37 \\ 849 & 1860\end{array}$

-8
-698
12

-8
-698
12
-34
-706

25
151

$\begin{array}{rr}-5 & -75 \\ 353 & 311\end{array}$

$\begin{array}{r}11 \\ -17 \\ \hline\end{array}$

-17
348
28
376

22
-3
236

236
25
261

$\begin{array}{rrr}-8 & -5 & -75 \\ -698 & 353 & 311 \\ 12 & 11 & 22\end{array}$

-34
-706

-344
-706
752

-17
348
28
376

-3
236
25

$898 \quad 1932$

752

$\begin{array}{rrr}0 & -36 & 36 \\ 685 & 530 & 1112 \\ 7 & 24 & 28\end{array}$

$836 \quad 1820$

$\begin{array}{rr}40 & 41 \\ 849 & 1871\end{array}$

$\begin{array}{rr}50 & 69 \\ 899 \quad 1936\end{array}$

-698
12
-34

-34
-706
46

$\begin{array}{rrr}7 & 24 & 28 \\ -1 & -1 & 34 \\ 685 & 494 & 1101\end{array}$

$\begin{array}{rrr}8 & 25 & 53 \\ 693 & 519 & 1147\end{array}$

14
$1520 \quad-550$

41
8
1534

$\begin{array}{rr}13 & 130 \\ 836 & 1820\end{array}$

752

$\begin{array}{rr}38 & 39 \\ -197 & 1328\end{array}$

312
-1

30
1322
30

30
1352

-1
-2
-159

4
163

30
1333
31

31
1364

$\operatorname{cog} N$

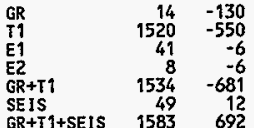

$13 \quad 132$

$-10 \quad 1820$
-43

$\begin{array}{rr}43 & 44 \\ 849 & 1881\end{array}$

$\begin{array}{rr}53 & 74 \\ 903 & 1950\end{array}$

$\begin{array}{rrrr}3 & 10 & 37 & 38 \\ -4 & 1312 & -320 & 1350 \\ 4 & -1 & -5 & 6 \\ 1 & 30 & -3 & 30 \\ 0 & 1322 & -283 & 1352 \\ 5 & 30 & 8 & 32 \\ 5 & 1352 & 291 & 1383\end{array}$

W320H32A PROCESS BUILDING PIPING STRESS ANALYSIS

$0301 / 95$ W320 TANK $241-\mathrm{C}-106$ SLUICING ER4319 AUTOPIPE+4.50 RESULT PAGE 40

GLOBAL FORCES \& MOHENTS

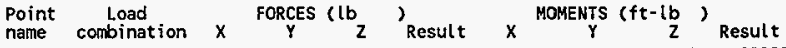

name combination $X$ -

$\begin{array}{rrrrrrrrrr}C 09 F & \text { GR } & 14 & -156 & 13 & 157 & -96 & 0 & 26 & 100 \\ & \text { T1 } & 1520 & -550 & 836 & 1820 & 210 & 172 & -1460 & 1485\end{array}$

E1

$\begin{array}{lrrrrr}E 2 & 8 & -6 & 46 & 47 & 30 \\ \text { GR+I1 } & 1534 & -706 & 849 & 1890 & 114 \\ \text { SEIS } & 52 & 12 & 56 & 78 & 38\end{array}$

$\begin{array}{lrrr}52 & 12 & 56 & 78\end{array}$

GR $14 \quad-156$

$906 \quad 1963$

C10

GR
T1
E1
E2

E2

GR+T

GR+T $1+S E I S$

$\begin{array}{rrrrr}14 & -156 & 13 & 158 & -102 \\ 1520 & -550 & 836 & 1820 & 192\end{array}$

$\begin{array}{rrrr}44 & -550 & 836 & 1820 \\ 8 & -6 & -10 & 46\end{array}$

$\begin{array}{rr}8 & -6 \\ 1534 & -707\end{array}$

$\begin{array}{rr}52 & 12 \\ 86 & 719\end{array}$

46
849
56

47
1891
78

$\begin{array}{llll}586 & 719 & 906 & 1963\end{array}$

C11 -

GR
T1
E1
E2

E2

SEIT

GR+T1+SEIS

$\begin{array}{rr}14 & -166 \\ 1520 & -550\end{array}$

$\begin{array}{rr}45 & -6 \\ 8 & -6 \\ 1534 & -716\end{array}$

$11+$

GR
T1
E1

E1

SEIS

GEIS + + + SEIS

$\begin{array}{rr}53 & 12 \\ 1587 & 728\end{array}$

$\begin{array}{rr}13 & 16 \\ 836 & 182 \\ -10 & 47\end{array}$

-10
47
849

849
58
907

49
1894
79

$\begin{array}{rr}15 & -190 \\ 1474 & -839\end{array}$

$\begin{array}{rr}14 & 191 \\ 937 & 1938 \\ -10 & 58\end{array}$

$\begin{array}{rrrr}8 & -26 & 69 & 74 \\ 1489 & -1029 & 951 & 2045\end{array}$

$\begin{array}{rrrr}1489 & -1029 & 951 & 2045\end{array}$

012

GR
T1
E1
E2

E2

SEIS

$2145 \quad 392$

172
-33
24
172
56

$\begin{array}{rr}-9 & 39 \\ -1434 & 949\end{array}$

-1434
45
1479

1449
82
1504

*** Segment $C$ end ***

*** Segnent $D$ begin ***

HNF-2486, Rev. 0

Page C-31 
W32OH32A PROCESS BUILDING PIPING STRESS ANALYSIS

\section{GLOBAL FORCES \& MOMENTS}

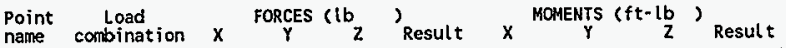

$\begin{array}{lllllllll}C 11 & \text { GR } & 0 & 24 & -1 & 24 & -22\end{array}$

$\begin{array}{lrrr}\text { GR } & 0 & 24 & -1 \\ T 1 & 45 & 289 & -101 \\ \text { E1 } & -12 & -1 & 0 \\ \text { E2 } & 0 & 20 & -21 \\ \text { GR+T1 } & 45 & 313 & -102 \\ \text { SEIS } & 12 & 21 & 21 \\ \text { GR+T1+SEIS } & 57 & 334 & 123\end{array}$

310
12

-22
114
-1
-39
93

GR+T1+SEIS

$001 \mathrm{~N}$

GR
T1
E1
E2

E2

GR+T1
SEIS

GR+T1+SEIS

$0 \quad-3$

D01 F

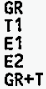

$G R+T 1$

SEIS

$\mathrm{DO2}-\mathrm{GR}$

GR
T1
E1
E2

E1

GR+T

SEIS

D02+

\begin{tabular}{l} 
GR \\
T1 \\
是 \\
E2 \\
\hline
\end{tabular}

GR+T1

GR+T1+SEIS

D03

GR
T1
E1
E2
Th

GR+T1

SEIS $\begin{array}{rr}-33 & -1 \\ -1 & -10\end{array}$

$\begin{array}{rr}-1 & 0 \\ 20 & -14 \\ 256 & -102 \\ 21 & 15\end{array}$

32

$33-20$

1020

9
104

36
37

1
-109
7

-33
2
0
-32
3
35

$-58$

-1
-101
0
-11

58
310
2
23

170

0
-76
-9
1

$\begin{array}{rr}4 & 1 \\ 261 & -76 \\ 4 & 10\end{array}$

$\begin{array}{rr}21 & 11 \\ 251 & 113\end{array}$

280

0
-48

$-10$

-48
10
58

$58 \quad 2 \quad 144$

$\begin{array}{rrr}0 & 1 & 57 \\ -48 & 1 & 81 \\ -10 & -1 & 10 \\ 1 & 0 & 8\end{array}$

-48
10

$\begin{array}{r}9 \\ 132 \\ \hline\end{array}$

$\begin{array}{rrrr}29 & -1 & 29 & -50 \\ 144 & -101 & 182 & -237\end{array}$

$\begin{array}{rrr}-1 & 1 & 50 \\ -4 & 1 & 242\end{array}$

$\begin{array}{rrrr}-2 & -4 & 5 & -4 \\ 172 & -102 & 205 & -287\end{array}$

-287
4
291

9
382
12
393

47
5
52
27
26
304
W32OH32A PROCESS BUILDING PIPING STRESS AMALYSIS

03/01/95 W320. TANK 241-C-106 SLUICING, ER4319 AUtOPIPE+4.50 RESULT PAGE 42

GLOBAL FORCES \& MOMENTS

Point Load FORCES (lb ) MOMENTS (ft-lb ) Result

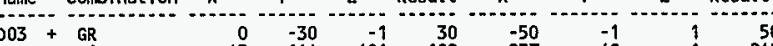

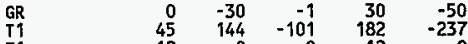

E1

E2

GEIS

GR+T 1+SEIS

12
45
12

-2
113
2
115

3
-102
3

12
3
159
13

0
-4
-287

-4
-287
4

-1
48
-5
0
47
5
5

DO4 -

GR
T1
E1
E2

$\begin{array}{lllll}0 & -30 & -1 & 30 & -44\end{array}$

$\begin{array}{rl}4 & E 2 \\ 291 & G R+T 1\end{array}$
E1

GR+T

GR+T1+SEIS

D04 +

GR
T1
E1
E2

E1

GR+T

GR+T 1+SEIS

D05 N

GR
T1
E 1
E2

E2

SEIS

GR+T1+SEIS

005

GR

E1

GR+T1

GEIS

D06

GR
T1
E1
E2
GR+T1

GR+T1+SE1S

$\begin{array}{rr}2 & 3 \\ 2 & -102\end{array}$

3
159
13

0
-3
-352

-352
3
-356

$\begin{array}{lll}-54 & -1 & -1\end{array}$

-44
-309
-3

15

15
0
45
15

0

$-10$

15
143
16

$-352$

356

$\begin{array}{rrrrr}0 & -60 & -1 & 60 & -30 \\ 45 & 144 & -101 & 182 & -345\end{array}$

45

45
61

$-24-102$

6

139
17
151

-3
-374
3

377

$\begin{array}{r}-85 \\ 144\end{array}-10$

0
-2

$\begin{array}{rr}-2 & -102 \\ 2 & -19\end{array}$

18

$\begin{array}{rr}-85 & -1 \\ 144 & -10\end{array}$
HNF-2486, Rev. 0

Page C-32 
H32OH32A PROCESS BUILDING PIPING STRESS AMALYSIS

03/01/95 W320, TANK 241-C-106 SLUICING, ER4319 AUtOPIPE+4.50 RESULT PAGE 43

GLOBAL FORCES \& MOMENTS

Point Load FORCES (lb ) MOMENTS (ft-tb ) name combination $X \quad Y \quad z$ Result $X \quad Y \quad z$ Result Dos

$\begin{array}{rrrr}22 & -1 & 1 & 22 \\ -528 & 116 & -33 & 542 \\ 0 & 16 & -94 & 21 \\ 5 & 0 & 0 & 5 \\ -507 & 114 & -33 & 520 \\ 5 & 17 & 14 & 22 \\ 512 & 131 & 47 & 530 \\ 22 & -1 & 1 & 22 \\ -547 & 116 & -42 & 560 \\ 0 & 16 & -18 & 24 \\ 7 & 0 & 0 & 7 \\ -525 & 114 & -41 & 539 \\ 7 & 17 & 18 & 26 \\ 532 & 131 & 59 & 551\end{array}$

«** Segment $D$ end ***

*** Segment $E$ begin ***

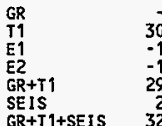

E01 $N$

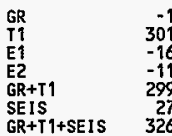

E01 F

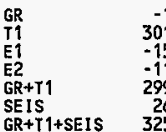

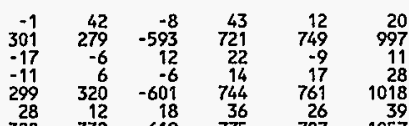

$\begin{array}{rr}26 & 39 \\ 787 & 1057\end{array}$

-8
589
-5
14
580
19
599

1170

22
1190
23

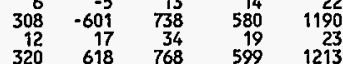

$\begin{array}{rr}-17 & 18 \\ 490 & 1190\end{array}$

-3
12

473

15
488

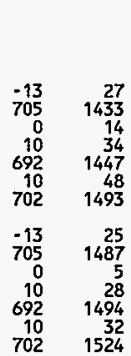

$\begin{array}{rr}-16 & 30 \\ 664 & 1448 \\ 1 & 4 \\ 9 & 23 \\ 648 & 1450 \\ 10 & 27 \\ 658 & 1476\end{array}$
W32OH32A PROCESS BUILDING PIPING STRESS ANALYSIS

W320, TANK 241-C-106 SLUICING, ER4319 AUtOPIPE+4.50 RESULT PAGE 44

GLOBAL FORCES \& MOMENTS

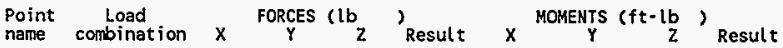

$\begin{array}{lrrrrrrrr}\text { GR } & -1 & -5 & -8 & 9 & -24 & 10 & -23 & 35 \\ \text { T1 } & 301 & 279 & -593 & 721 & 255 & 942 & 428 & 1060 \\ \text { E1 } & -12 & -6 & 12 & 18 & 2 & -3 & 6 & 10 \\ \text { E2 } & -11 & 6 & -1 & 12 & 7 & 6 & 4 & 106 \\ \text { GR+T1 } & 299 & 274 & -601 & 725 & 239 & 953 & 405 & 106 \\ \text { SEIS } & 23 & 12 & 13 & 29 & 8 & 10 & 10 & 16\end{array}$

$E 02 F$

GR
T1
E1
E2
GR+T
SEIS

GR+T1+SEIS

$\begin{array}{rrrr}-1 & -13 & -8 & 15 \\ -11 & 279 & -593 & 721 \\ -11 & -6 & 12 & 17 \\ 299 & 266 & 0 & 12 \\ 22 & 12 & 13 & 722 \\ 321 & 277 & 613 & 746\end{array}$

E03

GR

E1

$E 2$
GR+T1
SEIS

SEIS

$\begin{array}{rrr}-1 & -17 & -8 \\ -10 & 279 & -593 \\ -11 & -6 & 12\end{array}$

$\begin{array}{rr}6 & 6 \\ 12 & -60 \\ 274 & 13\end{array}$

18
721
17

$E 04$

GR

E2

SEIS

GR+T1+SEIS

$\begin{array}{rrr}-1 & -72 & -8 \\ 301 & 279 & -593 \\ -4 & -6 & 12\end{array}$

$-11$

*** Segment $E$ end

*** Segment $F$ begin ***

E04

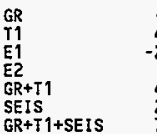

$\begin{array}{rrrr}-1 & 33 & -9 & 34 \\ 49 & 618 & -839 & 1043 \\ -24 & 9 & 4 & 24 \\ 0 & 3 & 1 & 4 \\ 48 & 651 & -848 & 1070 \\ 24 & 4 & 6 & 25\end{array}$
-23
214
2

6
191

8
199

$-23$

-23
214
2

6
191
8
199

-23
214
2

197

$\begin{array}{rr}191 & -872 \\ 8 & 48 \\ 199 & 920\end{array}$

HNF-2486, Rev. 0

Page C-33 
GLOBAL FORCES \& MOMENTS

Point Load

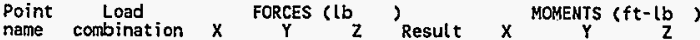

F01 - GR

E1

E2

SEIS

GR+T1+SEIS

$\mathrm{F01}+$

II

GR+T1

SEIS

F02

T1

2

$G R+T 1$

SEIS

GR+T1+SE1S

$\mathrm{FO2}+$

E1

ER

SEIS

GR+Ti+SEIS

F03

GR
I1
E 1
E2

$E 1$
$\varepsilon 2$
$G R+T 9$

GR+TI

SEIS

F04 N

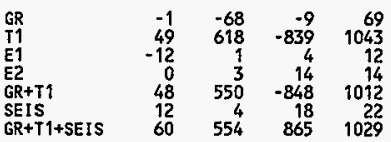
$\begin{array}{rrrrr}-1 & 23 & -9 & 24 & -121 \\ 49 & 618 & -839 & 1043 & \\ -23 & 1 & 4 & 23 & \\ 0 & 3 & 3 & 4 & \\ 48 & 640 & -848 & 1064 & -63 \\ 23 & 4 & 7 & 24 & \\ 70 & 644 & 854 & 1072 & \end{array}$ -

$\begin{array}{rrrr}121 & -14 & 47 & 130 \\ 58 & -872 & -499 & 1006 \\ 9 & 15 & 6 & 18 \\ 23 & 15 & -4 & 28 \\ -63 & -886 & -452 & 996 \\ 32 & 30 & 10 & 45 \\ 94 & 915 & 462 & 1030 \\ 121 & -14 & 47 & 130 \\ 58 & -872 & -499 & 1006 \\ 9 & 15 & 6 & 18 \\ 23 & 15 & -4 & 28 \\ -63 & -886 & -452 & 996 \\ 32 & 30 & 10 & 45 \\ 94 & 915 & 462 & 1030 \\ 119 & -14 & 47 & 129 \\ -53 & -863 & -499 & 998 \\ 8 & 11 & 6 & 15 \\ 23 & 15 & -4 & 28 \\ 172 & -877 & -452 & 1002 \\ 31 & 26 & 10 & 42 \\ 203 & 903 & 462 & 1035 \\ 119 & -14 & 47 & 129 \\ -53 & -863 & -499 & 998 \\ 8 & 11 & 6 & 15 \\ 23 & 15 & -4 & 28 \\ 172 & -877 & -452 & 1002 \\ 31 & 26 & 10 & 42 \\ 203 & 903 & 462 & 1035 \\ -72 & -15 & 47 & 87 \\ -599 & -820 & -499 & 1131 \\ 8 & -1 & 6 & 10 \\ 20 & 15 & -4 & 25 \\ 671 & -835 & -452 & 1163 \\ 28 & 16 & 10 & 33 \\ 699 & 850 & 462 & 1194\end{array}$
$\begin{array}{rr}47 & 74 \\ -499 & 1213 \\ 6 & 10 \\ -4 & 25 \\ -452 & 1241 \\ 10 & 34 \\ 462 & 1274\end{array}$
03/01/95 P320 TANK 241-C-106 SLUICING, ER4319

GLOBAL FORGES \& MOMENTS

Point Load FORCES (lb) MOMENTS ( $\mathrm{ft}-\mathrm{lb}$ )

name combination $X \quad Y \quad Z$ Result $X$ R $Y$ Result

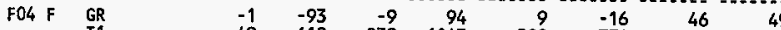

$\begin{array}{lrrrrrr}\text { GR } & -1 & -93 & -9 & 94 & 9 & -16 \\ \text { T1 } & 49 & 618 & -839 & 1043 & -589 & -77 \\ \text { E1 } & -9 & 1 & 4 & 10 & 4 & -12 \\ \text { E2 } & 0 & 3 & 17 & 17 & 5 & 15 \\ \text { GR+T1 } & 48 & 525 & -848 & 998 & -589 & -787 \\ \text { SEIS } & 9 & 4 & 21 & 23 & 9 & 26\end{array}$

$\begin{array}{rr}46 & 49 \\ -462 & 1075\end{array}$

GR+T

GR+T1+SEIS

F05 $N$

GR
T1
E1
E2

E2

GRIT

GR+T I+SEIS

$\begin{array}{llll}57 & 528 & 868 & 1018\end{array}$

F05 F

GR
E1
E2
GR+TI
SEIS

SEIS
GR+T1+SE IS

$\begin{array}{ll}-1 & -116 \\ 48 & 618\end{array}$

$\begin{array}{rr}-9 & 117\end{array}$

590

$-4 \quad 16$

$\begin{array}{rr}-4 & 16 \\ -47 & 1063\end{array}$

F06 -

GR
T1
E1
GR
GR+T
SE

E1
E2
GR+T1

GR+Y1+SEIS

$\begin{array}{rr}-6 & \\ 0 & \\ 48 & 502 \\ 6 & \end{array}$

$-8$

4
19
-848
23

77
20
96
25

18
307

$-16$

GR
T1
E1
E2

E1

SEIS

GR+T1+SEIS

F07 -

GR
T1
E1
E2
GR+
SE

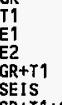

SEIS $\begin{array}{rrrr}-1 & -141 & -9 & 142 \\ 49 & 618 & -839 & 1043\end{array}$

$\begin{array}{rrrr}-3 & 1 & 4 & 5 \\ 0 & 3 & 22 & 23 \\ 48 & 476 & -848 & 973 \\ 3 & 4 & 26 & 27\end{array}$

$\begin{array}{rrr}51 & 480 & 26\end{array}$

$\begin{array}{rrrr}-1 & -150 & -9 & 151\end{array}$

$\begin{array}{llll}49 & 618 & -839 & 1043\end{array}$

46

23
-848
28
875

24
969
28
995

$\begin{array}{rrrr}-1 & 152 & -9 & 153\end{array}$

\begin{tabular}{rrrr}
-2 & 13 & -839 & 897 \\
0 & 8 & 23 & 14 \\
48 & 467 & -848 & 25 \\
\hline & 29 & 88 &
\end{tabular}

$\begin{array}{rrrr}48 & 467 & -848 & 969 \\ 2 & 21 & 28 & 35 \\ 50 & 488 & 875 & 1003\end{array}$

$35 \quad 961$

$\begin{array}{rrrr}-1 & 147 & -9 & 147 \\ 49 & 315 & -839 & 897 \\ -1 & 13 & 4 & 14 \\ 0 & 8 & 24 & 25 \\ 48 & 462 & -848 & 966 \\ 1 & 21 & 28 & 35 \\ 49 & 483 & 876 & 1001\end{array}$

HNF-2486, Rev. 0

Page C-34

Page 23 of 39 
W32OH32A PROCESS BUILDING PIPING STRESS ANALYSIS

AUTOPIPE +4.50 RESULT PAGE 47

GLOBAL FORCES \& MOMENTS

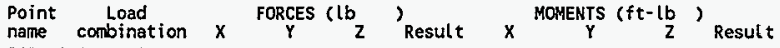

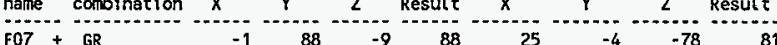

FO7 + GR

I1
E2

GR+T1

SEIS

$\begin{array}{rrr}-1 & 88 & -9 \\ 49 & 315 & -83 \\ 6 & 13 & 4 \\ 0 & 8 & 3 \\ 48 & 403 & -848 \\ 6 & 21 & 35 \\ 54 & 424 & 883\end{array}$

F08

68

E1

E2

GR+T1

GEIS

FO9 N

GR
T1
E1
E2

$G R+T 1$

SEIS

F09 F

GR

T1
E1
E2

GR+T1

SEIS

GR+T1+SEIS

$\$ 10$

GR
T1
E1
E2
GR+
SE1

GR+T1

GR+T +SEIS

-1
49
6
0
48
6
54

4

88
897
15
32
940
41
981

$\begin{array}{rr}25 & \\ 937 & 4 \\ -3 & - \\ -30 & \\ 961 & 4 \\ 33 & \end{array}$

$\begin{array}{rrr}-4 & -78 & 81 \\ 424 & 428 & 1114 \\ -17 & -9 & 20 \\ -17 & 1 & 34 \\ 421 & 351 & 1106\end{array}$

$\begin{array}{rrr}421 & 351 & 1106 \\ 34 & 10 & 48\end{array}$

$88 \quad-9$

$\begin{array}{rr}13 & 4 \\ 8 & 31 \\ 03 & -848 \\ 21 & 35\end{array}$

88
897

25
937
-3

$\begin{array}{lll}1 & -25 & 35\end{array}$

844
-19
-31

-31
844

844
51
895

-2
5
561
7

20
43
1397

33

$\begin{array}{rrr}-1 & 87 & -9 \\ 49 & 315 & -839\end{array}$

981

937

$\begin{array}{lll}1 & -22 & 33\end{array}$

-3
$-30 \quad-20$

961

33
994

-20
-32
875
52

-2
5
576
7

$\begin{array}{rr}5 & 44 \\ 7 & 1422\end{array}$

Segment $F$ end $* * *$

$* * *$ Segment $\mathrm{G}$ begin $* * *$

$3 \quad-1 \quad 30$

$\begin{array}{rrr}1080 & -1 & 30 \\ -21 & 676 & 1594 \\ -4 & 2 & 21\end{array}$

-21
-40
1083

1083

$675 \quad 1614$

1145

6841678

$\begin{array}{rr}4 & 33 \\ 694 & 1640\end{array}$

1132

1195
W320H32A PROCESS BUILDING PIPING STRESS ANALYSIS

03/01/95 W320, TANK 241-C-106 SLUICING, ER4399 AutoPIPE+4.50 RESULT PAGE 48

GLOBAL FORCES \& MOMENTS

Point Load $x$ FORCES (lb ) MONENTS ( $\mathrm{ft}-\mathrm{lb}$ )

name combination $X \quad Y \quad F$ Result $X \quad Y \quad Z$ Result

GR
T1
E1
E2
GR+T
SEIS

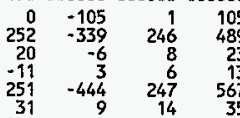

85
-134
-6
-19
-49
25

-1
37
7
36
4

G01 $N$

$\begin{array}{lrr}\text { GR } & 0 & -115 \\ \text { T1 } & 252 & -339 \\ \text { E1 } & 21 & -6 \\ \text { E2 } & -11 & 3 \\ \text { GR+T1 } & 251 & -454 \\ \text { SEIS } & 32 & 9\end{array}$

$1 \quad 115$

33
-293
-99

GR+T 1+SEIS

284

463

$\begin{array}{rr}246 & 489 \\ 8 & 24 \\ 7 & 13 \\ 247 & 575 \\ 15 & 37 \\ 262 & 603\end{array}$

-9
-18
-259
27
28

-81
-2

5
-82
8
89

5
618
-11

9199

$601 \mathrm{~F}$

GR
T1
E1
E2

$\begin{array}{ll}0 & -132\end{array}$

1132

-28
-462

GR+T

GR+T1+SEIS

-1
25
34

-6
-471
9

8
9
247
17

25
15
588

-462
-12
-16

-16
-490
29

G02

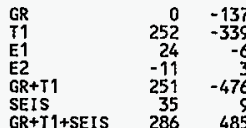

-137
-339
-6
3
-476
9
485

$1 \quad 137$

silo

-28
-462

246
8
10

10
247
18

26
15
593
40

-12
-16
-490

GR+T1+SEIS

$\mathrm{G02}$

GR
T1
E1
E2
GR+T1
SEIS
GR+T1+SEIS

86
-29
58

191
8
10
182
18
200

86

29
518

$518-416$

-1
-330
-17
7
$-33 ?$

24
355

-1
-391
-19

4
-393
24
416

$-19$

-8
-239
21

-1
-391
-19

GR+T1+SEIS

G03 N

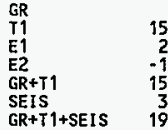

89
-29
1
0
52
1
53

18
18
19
201

181
8
11
182
19
201

HNF-2486, Rev. 0

Page C-35 
W320H32A PROCESS BUILDING PIPING STRESS ANALYSIS

AUtOPIPE +4.50 RESULT PAGE 48

GLOBAL FORCES \& MOMENTS

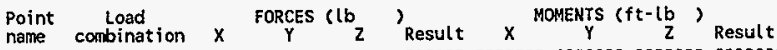

G03 F GR

11
E1
E2

GR+T1

SEIS

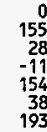

$\begin{array}{lll}56 & 1 & 56\end{array}$

$\begin{array}{rrrr}-27 & -2 & 29 & 40 \\ -326 & -572 & -338 & 74\end{array}$

GO4 - $G R$

T1
E1
E2

GR+T1

SEIS

$\mathrm{GO4}+\mathrm{GR}$

GR
E1
E2
GR+T1

SEIS

G05

GR
TI
EI
E2
GR+TI

SEIS

$G R+T 1+S E I S$

$\mathrm{G05}+\mathrm{G}$

GR
T1
E2
E2

$E 1$
E2
GR+TI

GR+T?

SEIS
GR+T1+SEIS

G06 N $\begin{array}{rr}27 & 182 \\ 1 & 22\end{array}$

$\begin{array}{rrr}0 & 41 & 1 \\ 155 & -29 & 181 \\ 29 & 1 & 8\end{array}$

$\begin{array}{rr}0 & 15 \\ 12 & 182\end{array}$

40

$12 \quad 206$

$\begin{array}{rrrr}0 & -18 & 1 & \\ 155 & -29 & 181 & 24 \\ 37 & 1 & 8 & \\ -11 & 0 & 22 & \\ 154 & -47 & 182 & 243 \\ 47 & 1 & 31 & \\ 202 & 48 & 213 & \end{array}$

202

-18
-29

18

$\begin{array}{rr}181 & 18 \\ 8 & 370\end{array}$

$\begin{array}{rrr}0 & 82 & 37 \\ -47 & 182 & 243\end{array}$

$\begin{array}{rrr}1 & 31 & 56 \\ 48 & 213 & 297\end{array}$

$\begin{array}{rrrr}0 & -42 & 1 & 42 \\ 155 & -29 & 181 & 240 \\ 39 & 1 & 8 & 40\end{array}$

$\begin{array}{rrr}0 & 25 & 28 \\ -71 & 182 & 249\end{array}$

$\begin{array}{rrrr}154 & -71 & 182 & 249 \\ 50 & 1 & 33 & 60 \\ 205 & 72 & 216 & 306\end{array}$ $\begin{array}{rrr}1 & 8 & 29 \\ 0 & 14 & 17\end{array}$
-353
13
366

-7
-575
35

0
-309

$315 \quad 778$

$\begin{array}{rr}-26 & -2 \\ -199 & -572\end{array}$

$\begin{array}{r}-1 \\ 3 \\ -225 \\ \hline\end{array}$

-7
-575
35

-575
35
609

$\begin{array}{rr}-26 & -2 \\ -199 & -572\end{array}$

$\begin{array}{rr}-199 & -572 \\ -1 & -28 \\ 3 & -7\end{array}$

$-225$

$229 \quad 609$

$\begin{array}{rr}-26 \\ -108 & -5 \\ -5 & -58\end{array}$

13
-134
17

15

$\begin{array}{rrrr}-26 & -2 & 30 & 39 \\ -108 & -572 & -525 & 784\end{array}$

$\begin{array}{rr}3 & -28 \\ -13 & -7\end{array}$

$\begin{array}{rr}-7 \\ -575 & -495\end{array}$

$\begin{array}{lll}35 & -495 & 770\end{array}$

$\begin{array}{rrrr}17 & 35 & 57 & 69\end{array}$

$\begin{array}{rrrr}-26 & -2 & 30 & 39 \\ -108 & -572 & -525 & 784\end{array}$

$\begin{array}{rr}3 & -28 \\ 13 & -7\end{array}$

133
-133
17

150

78

-44
13
-495

-495
57

$\begin{array}{rrr}35 & 57 & 69 \\ 609 & 552 & 836\end{array}$

GR
T1
E1
E2

GR+T

GR+T $1+S E 1 S$

$\begin{array}{rrrr}0 & -42 & 1 & 42 \\ 155 & -29 & 181 & 240 \\ 39 & 1 & 8 & 40 \\ -11 & 0 & 25 & 28 \\ 154 & -71 & 182 & 249 \\ 50 & 1 & 33 & 60 \\ 205 & 72 & 216 & 306\end{array}$

W32OH32A PROCESS BUILDING PIPING STRESS ANALYSIS

$03 / 01 / 95$ W320, TANK 241-C-106 SLUICING, ER4319 AUTOPIPE+4.50 RESULT PAGE 50

GLOBAL FORCES \& MOMENTS

Point Load FORCES (lb) MOMENTS ( $\mathrm{ft}-\mathrm{lb}$ )

name combination $X Y_{Z}$ Result $X$ M $Y$ Result

GO6 F GR

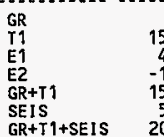

$\begin{array}{llll}0 & -59 & 1 & 59\end{array}$

$\begin{array}{rrr}1 & -2 & 30 \\ -3 & -495 & -602 \\ 7 & -7 & -64 \\ 27 & -13 & 19 \\ -2 & -498 & -572 \\ 34 & 20 & 82 \\ 35 & 517 & 655 \\ 10 & -2 & 30 \\ 1 & -473 & -602 \\ 7 & -1 & -64 \\ 27 & -14 & 19 \\ 11 & -475 & -572 \\ 34 & 15 & 82 \\ 45 & 490 & 655\end{array}$

3030

$\begin{array}{rrrr}154 & -88 & 182 & 255 \\ 52 & 1 & 36 & 63\end{array}$

$\begin{array}{lrrrr}G R+T 1+S E I S & 207 & 89 & 218 & 313\end{array}$

G07 - GR

T1

E2

SEIS

$\begin{array}{rr}0 & -62 \\ 155 & -29\end{array}$

GR+T 1+SEIS

G07 + GR

I1

E2

SEIS

$181 \quad 62$

$\begin{array}{rr}8 & 43 \\ 28 & 30 \\ 182 & 256\end{array}$

-11
154
53

-91
1

$\begin{array}{rr}36 & 64 \\ 218 & 314\end{array}$

.

$\begin{array}{rr}181 & 86 \\ 181\end{array}$

$\begin{array}{rr}0 & -86 \\ 155 & -29\end{array}$

45
-11

0
-115
1
116

8
31
182
39

45
32
265
68

$\begin{array}{rrr}10 & -2 & 30 \\ 1 & -473 & -602 \\ 7 & -1 & -64 \\ 27 & -14 & 19 \\ 11 & -475 & -572 \\ 34 & 15 & 82\end{array}$

608

GR

E1

GR+T

GR+T1+SEIS

326

$45 \quad 490$

$\begin{array}{lll}27 & -2 & 30\end{array}$

$\begin{array}{rrr}27 & -2 & -30 \\ 7 & -445 & -602 \\ 7 & 7 & -64 \\ 27 & -16 & 19 \\ 34 & -447 & -572 \\ 34 & 23 & 82 \\ 67 & 470 & 655\end{array}$

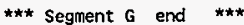

HNF-2486, Rev. 0

Page C-36

Page 25 of 39 
ASHE B31.3C (1992) CODE COMPLLANCE

(Moments in $\mathrm{ft}-\mathrm{lb}_{\mathrm{b}}$ ) (Stress in psi) Point Load In-Pl. Out-Pl. Torsion S.I.F Eq. Load code Code name combination Moment Moment Moment In Out no. type stress Allow.

\section{*** Segment $A$ begin ***}

$\mathrm{AO2}$

$\operatorname{Max} P$

$G R+\operatorname{Max} P$ Coid to T

Sus. + E1

SEIS

SUS+SEIS

A03 N- Max P

$\begin{array}{ll}\text { GR + Max P } & 35 \\ \text { Cold to TI } & 3 \\ \text { Sus. + E1 } & 4 \\ \text { SUS. + E2 } & 4 \\ \text { SEIS } & 1 \\ \text { SUS+SEIS } & 5\end{array}$

A03 $\mathrm{N}+\operatorname{Max} P$

$\begin{array}{lr}\text { GR + Max P } & 28 \\ \text { Cold to I1 } & 12959 \\ \text { SUS. + E1 } & 40 \\ \text { SUS. + E2 } & 29 \\ \text { SEIS } & 12 \\ \text { SUS+SEIS } & 40\end{array}$

A03 M Max P

$\begin{array}{lrr}\text { GR + Max P } & 42 & 18 \\ \text { Cold to T9 } & 268 & 454 \\ \text { SUs. + E1 } & 45 & 21 \\ \text { SUS. + E2 } & 42 & 20 \\ \text { SEIS } & 3 & 4 \\ \text { SUS+SEIS } & 45 & 22\end{array}$

AO3 F- Max P

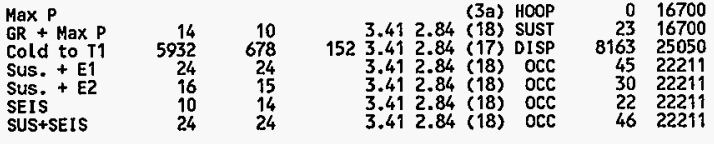

$\begin{array}{rr}0 & 16700 \\ 24 & 16700 \\ 9497 & 25050 \\ 37 & 22211 \\ 35 & 22211 \\ 17 & 22211 \\ 41 & 22211\end{array}$

(3a) HOOP

1.001 .00 (18) SUST

4891.001 .00 (17) DISP 1.001 .00 (18) OCC $1.00 \% .00$ (18) OCC

$0 \quad 16700$

$\begin{array}{rr}19 & 16700\end{array}$

2625050

$23 \quad 22211$

$\begin{array}{rr}8 & 22211 \\ 27 & 22211\end{array}$

(3a) HOOP

3.412 .84 (18) SUST

4893.412 .84 (17) DISP $3.412 .84(18)$ OCC 3.412 .84 (18) OCC $3.412 .84(18)$ OCC
3.412 .84 (18) OCC

(3a) HOOP

3173.412 .84 (18) SUST

73.412 .84 (17) DISP

$3.412 .84(18)$ OCC

3.412 .84 (18) OCC

3.412 .84 (18) OCC

o 16700

$\begin{array}{rr}58 & 16700 \\ 17753 & 25050\end{array}$

25050

71 22211

$\begin{array}{ll}25 & 22211 \\ 83 & 22211\end{array}$

0 16700

6416700
$647 \quad 25050$

70
72211

6722211

$\begin{array}{rr}6 & 22211 \\ 70 & 22211\end{array}$

- 16700

25050

22211

$46 \quad 22211$
$1.001 .00(18)$ OC
ASME B31.3c (1992) CODE COMPLIANCE

(Moments in $\mathrm{ft}$-lb) (Stress in psi )

point Load in-Pl. Out-PL. Torsion S.I.F Eq. Load Code Code name combination Moment Monent Moment In Out no. type stress Allow. A03 F+ Max P

$\begin{array}{lrr}\text { GR + Max P } & 14 & 10 \\ \text { Cold to T1 } & 5932 & 678 \\ \text { SUS. + E1 } & 24 & 24 \\ \text { SUS. + E2 } & 16 & 15 \\ \text { SEIS } & 10 & 14 \\ \text { SUS+SEIS } & 24 & 24\end{array}$

(3a) $400 P$ 0 16700 $1.001 .00(18)$ SUST 1.001 .00 (17) DISP $1.001 .00(18)$ OCC 1.001 .00 (18) OCC 1.001 .00 (18) OCC $\begin{array}{rr}7 & 16700 \\ 2397 & 25050\end{array}$ 239725050 $14 \quad 22211$ $\begin{array}{ll}9 & 22211 \\ 7 & 22211\end{array}$ $15 \quad 22211$

A04 - Max P $G R+\operatorname{Max} P$ Cold to $T$ Sus. + E1 Sus. + E2 SEIS

$\begin{array}{rr}36 & 47 \\ 4157 & 5263 \\ 43 & 63 \\ 45 & 53 \\ 12 & 24 \\ 47 & 66\end{array}$

(3a) HOOP 1.731 .97 (18) SUST 21.731 .97 (17) DISP 1.731 .97 (18) OCC $1.731 .97(18)$ OCC

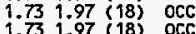

0 16700

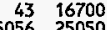
$\begin{array}{rr}056 & 25050 \\ 62 & 22211\end{array}$ $62 \quad 22211$ $54 \quad 22211$ $\begin{array}{ll}22 & 22211 \\ 65 & 22211\end{array}$

A04 + Max $P$

GR + Max P

Cold to $T$

Sus. + E1

Sus. + E2

SUS+SEIS

$\begin{array}{rr}27 & 61 \\ 6342 & 1290 \\ 39 & 79 \\ 43 & 73 \\ 20 & 22 \\ 47 & 82\end{array}$

HOOP

1.731 .97 (18) SUST 1.731 .97 (17) DISP 1.731 .97 (18) OCC $1.731 .97(18)$ OCC $1.731 .97(18)$ OCC
1.73
$1.97(18)$ OCC

o 16700

$\begin{array}{rr}54 & 16700 \\ 4509 & 25050\end{array}$

250925050

$\begin{array}{ll}72 & 22211 \\ 69 & 22211\end{array}$

$\begin{array}{ll}69 & 22211 \\ 23 & 22211\end{array}$

7722211

A05 Max P

GR + Max P

Cold to T1

Sus. + E1

Sus. + E2

SEIS
SUS+SEIS

$\begin{array}{rr}47 & 17 \\ 5830 & 3772 \\ 53 & 32 \\ 47 & 32 \\ 6 & 21 \\ 53 & 38\end{array}$

$\begin{array}{rr}1.00 & 1.00 \text { (3a) HOOP } \\ \text { (18) } & \text { sUST }\end{array}$ $\begin{array}{lll}1.00 & 1.00(17) & \text { DISP } \\ 1.00 & 1.00(18) & \text { OCC }\end{array}$ 1.001 .00 (18) OCC $1.001 .00(18)$
$1.001 .00(18)$

o 16700 $\begin{array}{rr}21 & 16700 \\ 2833 & 25050\end{array}$ 28 2221 2722211 $\begin{array}{rr}9 & 22211 \\ 30 & 22211\end{array}$

A06 Max P

$G R+\operatorname{Max} P$ Cold to T1 Sus. + E1 Sus. + E SEIS

SUS+SEIS

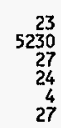
$61 \begin{array}{lll}1.00 & 1.00 \text { (3a) } & \text { HOOP } \\ 1.00 & 1.00 & \text { SUST } \\ \text { S17) } & \text { DISP }\end{array}$ 1.001 .00 (17) DISP $\begin{array}{lll}1.00 & 1.00(18) & \text { OCC } \\ 1.00 & 1.00(18) & \text { OCC }\end{array}$ $1.001 .00(18)$ OCC $\begin{array}{lll}1.001 .00(18) & \text { OCC } \\ 1.001 .00(18) & \text { OCC }\end{array}$

o 16700 $38 \quad 16700$ $\begin{array}{rr}396 & 25050 \\ 49 & 22211\end{array}$ 4922211 $\begin{array}{ll}46 & 22211\end{array}$ $\begin{array}{ll}14 & 22211 \\ 52 & 22211\end{array}$

A07 N- Max P

GR + Max $P$ Cold to T1 Sus. + E1 Sus. + E2 SUS+SEIS (3a) HOOP

1611.001 .00 (18) SUST $1.001 .00(17)$ DISP $1.001 .00(18)$ OCC $1.001 .00(18)$ OCC $1.001 .00(18)$
$1.001 .00(18)$ OCC 


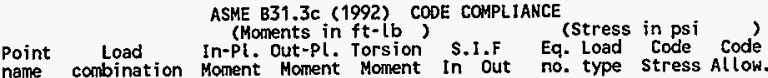
name combination Moment Moment Moment In Out no. type Stress Allaw.

A07 $\mathrm{N}+\mathrm{Max}$

$\begin{array}{lrr}\text { MaX P } & \\ \text { GR + Max P } & 23 & 9 \\ \text { Cold to T1 } & 5234 & 176 \\ \text { SUS. + E1 } & 27 & 15 \\ \text { SUS. + E2 } & 24 & 14 \\ \text { SEIS } & 4 & 9 \\ \text { SUS+SEIS } & 27 & 17\end{array}$

A07 F- Max P

$G R+\operatorname{Max} P$ Cold to T1 $8288 \quad 209$ with Sus; load margin Sus. + Et SUS. + E2 SUS+SEIS $\begin{array}{ll}9 & 21 \\ 5 & 23 \\ 5 & 9 \\ 9 & 25\end{array}$

A07 F+ Max P

$\mathrm{GR}+\operatorname{Max} \mathrm{P}$
Cold to $\mathrm{T1}$ Cold to 71 Sus. + E1
Sus. + E2 SEIS SUS+SEIS $\begin{array}{rr}16 & 4 \\ 209 & 8288 \\ 21 & 9 \\ 23 & 5 \\ 9 & 5 \\ 25 & 9\end{array}$ 27 (3a) HOOP 2.271 .89 (3a) SUS 2.271 .89 (18) OCC 2.271 .89 (18) OCC 2.271 .89 (18) OCC $\begin{array}{lll}2.27 & 1.89 & (18) \\ 2.27 & 1.89(18) & \text { OCC }\end{array}$ $\begin{array}{rr}0 & 16700 \\ 83 & 16700\end{array}$ 8316700 $10756 \quad 25050$ $\begin{array}{ll}09 & 22211 \\ 99 & 2211\end{array}$ 28 22211 11122211

(3a) HOOP 1292.271 .89 (18) SUS $\begin{array}{llll}2.27 & 1.89(17) & (18) & \text { OCC } \\ 2.27 & 1.89 \text { (18) OCC }\end{array}$ 2.271 .89 (18)
2.271 .89 (18) OCC 2.271 .89 (18)
2.271 .89 (18) OCC

016700 $\begin{array}{rl}48 & 16700 \\ 26530 & 25050 * *\end{array}$ $2653025050 *$ 71 6822211 $31 \quad 22211$ (3a) HOOP 1.001 .00 (18) SUST $1291.001 .00(17)$ DISP 1.001 .00 (18) OCC 1.001 .00 (18) OCC $1.001 .00(18)$ OCC 1.001 .00 (18) OCC

0 16700

1171225050

$36 \quad 22211$

$\begin{array}{ll}36 & 22211 \\ 15 & 22311\end{array}$

$40 \quad 22211$

A08

Max P

GR + Max $P$

Cold to

Sus. + E1

SEIS

SUS+SEIS

(3a) HOOP

1.001 .00 (18) SUST

1291.001 .00 (17) DISP 1.001 .00 (18) OCC 1.001 .00 (18) OCC

0 16700

$\begin{array}{rr}27 & 16700\end{array}$

$44 \quad 22219$

$\begin{array}{ll}43 & 22211\end{array}$

$\begin{array}{ll}23 & 22211 \\ 50 & 22211\end{array}$

*** Segment $\mathrm{A}$ end $* * *$

*** Segment $B$ begin $* * *$

A04 Max

GR + Max $P$

Cotd to T1 3973

Sus. + E

SEIS

SUS+SEIS
47
48
52
65
19
66 (3a) HOOP ${ }_{2184} 1.731 .97$ (18) SUST 1.731 .97 (18) OCC 1.731 .97 (18) OCC $1.731 .97(18)$ OCC $\begin{array}{rr}0 & 16700 \\ 107 & 16700 \\ 7483 & 25050 \\ 183 & 22211 \\ 146 & 22211 \\ 85 & 22211 \\ 192 & 22211\end{array}$

$03 / 01 / 85$

PIRESS ANALYSIS

AUtOPIPE+4.50 RESULT PAGE 54

ASME B31.3C (1992) CODE COMPLIANEE

Point Load In-PI (Stress in psi code' name combination Moment Moment Moment in Out no. type stress Allow.

B01 N- Max P

$\mathrm{GR}+\operatorname{Max} P$

Cold to T1

Sus. + E1

SEIS

SUS+SEIS

B01 $\mathrm{N}+\operatorname{Max} \mathrm{P}$

$\mathrm{GR}+\operatorname{Max} \mathrm{P}$

Cold to T1

Sus. + E1

SEIS + E

SUS+SEIS

B01 F- Max P

$\begin{array}{lrr}\text { GR + Max P } & 38 & 9 \\ \text { Cold to T1 } & 4 & 2428 \\ \text { Sus. + E1 } & 41 & 29 \\ \text { SUS. + E2 } & 39 & 17 \\ \text { SEIS } & 4 & 22 \\ \text { SUS+SEIS } & 42 & 31\end{array}$

12
2721
37
15
25
38 1.001 .00 (18) SUST 841.001 .00 (17) DISP 1.001 .00 (18) OCC 1.001 .00 (18) OCC 1.001 .00 (18) OCC

(3a) HOOP 2.271 .89 (18) SUST 2.271 .89 (17) DISP 2.271 .89 (18) OCC 2.271 .89 (18) OCC 2.271 .89 (18) OCC

(3a) HOOP 2.271 .89 (18) SUST 32.271 .89 (17) DISP 2.271 .89 (18) OCC $2.271 .89(18)$ OCC $2.271 .89(18)$ OCC
$2.279 .89(18)$ OCC

$\begin{array}{rr}0 & 16700 \\ 19 & 16700 \\ 4929 & 25050 \\ 58 & 22211 \\ 28 & 22211 \\ 39 & 22211 \\ 59 & 22211\end{array}$

016700 38
7927 $\quad 25700$

(5050

$\begin{array}{ll}11 & 22211 \\ 56 & 22211\end{array}$

$76 \quad 22219$

19422213

o 16700

13016700

647525050

18922211

22211

$195 \quad 22211$

B01 F+ Max P

GR + Max P
Cold to I1
Sus. + E1
Sus. + E2
SEIS
SUS+SEIS

(3a) HOOP

1.001 .00 (18) SUST 411.001 .00 (17) DISP $1.001 .00(18)$ OCC $1.001 .00(18)$ OCC $1.001 .00(18)$ OCC

0 16700 58
3430 343025050 8922211 $\begin{array}{ll}71 & 22211\end{array}$ $\begin{array}{ll}34 & 22211 \\ 92 & 22211\end{array}$

B02 GR + Max Cold to T1 Sus. + E1 Sus. + E2 SEIS

SUS+SEIS

Max $P$

$\mathrm{GR}+\operatorname{Max} \mathrm{P}$

Sus. + E1

Sus. + E2

SEIS

SUS+SE IS (3a) HоO 1.001 .00 (18) SUST

1091.001 .00 (17) DISP 1.001 .00 (18) OCC $1.001 .00(18)$ OCC $\begin{array}{ll}1.001 .00(18) & \text { OCC } \\ 1.001 .00(18) & \text { OCC }\end{array}$

$0 \quad 16700$

58
342700

342425050

722221

$34 \quad 22219$

(3a) $\mathrm{HOOP}$

$1.001 .00(48)$ SUST

921.001 .00 (17) DISP $1.001 .00(18)$ OCC $1.001 .00(18)$ OCC 1.001 .00 (18) OCC
016700

73
1450

145925050

892221

9122211

$\begin{array}{ll}24 & 22211 \\ 97 & 22211\end{array}$

HNF-2486, Rev. 0

Page C-38 
W320H32A PROCESS BUILDING PIPING STRESS ANALYSIS

HB20 TAUK 241-C-106 SLUICING ER4319 AUTOPIPE+4.50 RESULT PAGE 55

ASME B31.3C (1992) CODE COMPLIANCE

(Moments in $\mathrm{ft}$-lb) (Stress in psi )

Point Load In-PL. Out-PL. Torsion S.I.F Eq. Load Code Code

name combination Moment Moment Monent in Out no. type Stress Allow.

$804 \mathrm{~N}-$ Max P

$\mathrm{GR}+\operatorname{Max} P$

Cold to TI

Sus. + E1

SEIS

$\begin{array}{rr}46 & 10 \\ 110 & 879 \\ 48 & 11 \\ 50 & 25 \\ 4 & 15 \\ 50 & 25\end{array}$

0 16700

1.001 .00 (18) SUST

SUS+SEIS

$\mathrm{BO} 4 \mathrm{~N}+\mathrm{Max} \mathrm{P}$

GR + Max $P$

Cold to I1

Sus. + E

SUIS + E2

9
881
11
24
15

SUS+SE1S

Bal F- Max P Cold to It $2232 \quad 72 \quad 1142.271 .89$ (17) DISP

Sus. + E

SEIS

SUS+SEIS

2.271 .89 (18) OCC

$804 F+\operatorname{Max} P$

$\mathrm{GR}+\operatorname{Max} \mathrm{P}$

Sus. + EY

SEIS

SUS+SEIS

B05

$\operatorname{Max} P$

GR + Max P

Cold to $T 1$

Sus. + E1

SUS. +

SUS+SEIS

B06

$\begin{array}{lrr}\text { Max P } & & \\ \text { GR + Max P } & 4 & 114 \\ \text { Cold to T1 } & 1575 & 432 \\ \text { SUS. + E1 } & 13 & 130 \\ \text { SUS. + E2 } & 30 & 115 \\ \text { SEIS } & 28 & 16 \\ \text { SUS+SEIS } & 32 & 130\end{array}$

921.001 .00 (17) DISP

1.001 .00 (18) OCC

1.001 .00 (18) OCC

1.001 .00 (18) OCC

(3a) HOOP

2.271 .89 (18) SUST

$92 \begin{aligned} & 2.27 \\ & 2.27 \\ & 1.89 \\ & \text { (17) DISP }\end{aligned}$ 2.271 .89 (18) OCC $2.271 .89(18)$ OCC 2.271 .89 (18) OCC 271.89 (3a) HOOP 2.271 .89 (18) OCC $2.271 .89(18)$ OCC

(3a) HOOP

$\begin{array}{rr}71 & 16700 \\ 1258 & 25050\end{array}$

25050

$94 \quad 22211$

$23 \quad 22211$

13516700

$2832 \quad 25050$

14122211

$52 \quad 22211$

016700

8016700

$7146 \quad 25050$

$\begin{array}{ll}109 & 22211 \\ 103 & 22211\end{array}$

$\begin{array}{rl}37 & 22211 \\ 117 & 22211\end{array}$

$1.001 .00(18)$ SUST

0 16700

$40 \quad 16700$
$+3158 \quad 25050$

5222211

502211

$\begin{array}{ll}16 & 22211 \\ 56 & 22211\end{array}$

1.001 .00 (18) OCC

1.001 .00 (18) OCC

(3a) $\mathrm{HOO}$

1.001 .00 (18) SUST

71.001 .00 (17) DISP

$1.001 .00(18)$ OCC

$1.001 .00(18)$ OCC

$1.001 .00(18)$
$1.001 .00(18)$

(3a) 100

1.521 .69 (18) SUST

$971.52 \quad 1.69$ (17) DISP

1.521 .69 (18) OCC

1.521 .69 (18) OCC

1.521 .69 (18) OCC
0 16700

198
2725 25050

272525050

$\begin{array}{ll}211 & 22211 \\ 219 & 22211\end{array}$

25
22211

- 16700

$291 \quad 16700$

353525050

$337 \quad 22211$

$\begin{array}{ll}76 & 22211 \\ 366 & 22211\end{array}$
0 16700
W32OH32A PROCESS BUILDING PIPING STRESS ANALYSIS

UICING ER4319

AUtoPIPE+4.50 RESULT PAGE 56

ASME B31.3C (1992) CODE COMPLIANCE

(Moments in $\mathrm{ft}-\mathrm{lb}$ ) (Stress in psi )

Point Load In-Pl. Out-Pl. Torsion S.I.F Eq. Load code Code name combination Moment Moment Moment in Out no. type Stress Allow.

$\mathrm{BO6}+\mathrm{Max} \mathrm{P}$

GR + Max P

Sus. + E?

SEIS

SUS+SE IS

807 Max $P$

$\mathbf{G R}+\operatorname{Max} \mathbf{P}$

Cold to T1

Sus. + E1

SUS+SEIS

B08 Max $P$

$G R+\operatorname{Max} P$

Cold to T1

Sus. + E1

SEIS

SUS+SEIS

B09 N- Max P

$\mathrm{GR}+\operatorname{Max} \mathrm{P}$

Sus. + E1
Sus. + E2

SEIS

SUS+SEIS

$B 09 \mathrm{~N}+\operatorname{Max} \mathrm{P}$

$G R+\operatorname{Max} P$

Cold to T1

Sus. + El

SEIS

SUS+SEIS

809 F- Max P

$\mathrm{GR}+\operatorname{Max} \mathrm{P}$

Cold to T1

Sus. + E1

SUS+SE IS $\begin{array}{rr}20 & 91 \\ 363 & 235 \\ 30 & 108 \\ 31 & 93 \\ 16 & 16 \\ 35 & 108\end{array}$

1.521 .69 (18) HOOP
SUST

89
234
104
90
15
104

18

328

21

19

81
235
95

19
310
31
20
11

14
95

31

81
235
95
82
14
95

19
310
31

31
20
11

11
31

152 1.521 .69 (17) DISP 1.521 .69 (18) OCC 1.521 .69 (18) OCC 1.521 .69 (18) OCC

(3a) HOOP

1.001 .00 (18) SUST $1.001 .00 \mathrm{si7}$ DISP 1.001 .00 (18) OCC $1.001 .00(18)$ OCC 1.001 .00 (18) OCC D 16700 $237 \quad 16700$ 28522211 26522219 5522211

$0 \quad 16700$

$136 \quad 16700$

16422211

$142 \quad 22211$

$28 \quad 22211$

(3a) hOOP

1.001 .00 (18) SUST 1.001 .00 (17) DISP 1.001 .00 (18) OCC $.001 .00(18)$ OCC 1.001 .00 (18) OCC

(3a) 400

1591.001 .00 (18) SUS 1.001 .00 (17) 01S 1.001 .00 (18) OCC $.001 .00 \mathrm{c}(18)$ OCC $1.001 .00(18)$ OCC

(3a) HOOP 2.271 .89 (18) SUST $\begin{array}{rr}81 & 19 \\ 235 & 310 \\ 95 & 31\end{array}$ 19
310
31
20
11

158 .271 .89 (17) DISP 2.271 .89 (18) OCC $\begin{array}{lll}2.27 & 1.89 \text { (18) } & \text { OCC } \\ 2.27 & 1.89 \text { (18) OCC }\end{array}$

(3a) $400 \mathrm{P}$ 2.271 .89 (18) SUST 2.271 .89 (17) DISP 2.271 .89 (18) OCC 2.271 .89 (18) OCC 2.271 .89 (18) OCC o 16700

$125 \quad 16700$

$\begin{array}{ll}594 & 25050 \\ 153 & 22211\end{array}$

15322211

28
$153 \quad 22219$

0 16700

$125 \quad 16700$

59425050

12722211

2822211

0 16700

$1141 \quad 25050$

405

$286 \quad 22211$

$\begin{array}{rl}539 & 22211 \\ 22211\end{array}$

$\begin{array}{rr}0 & 16700 \\ 212 & 16700\end{array}$

$\begin{array}{ll}212 & 16700 \\ 615 & 25050\end{array}$

23022211

2292221

$\begin{array}{rr}32 & 22211 \\ 244 & 22211\end{array}$

HNF-2486, Rev. 0

Page C-39

Page 28 of 39 
ASME B31.3c (1992) CODE COHPLIANCE

(Moments in $\mathrm{ft}-\mathrm{lb}$ ) (Stress in psi) Point Load In-PI. Out-PI. Torsion S.I.F Eq. Load Code Code name combination Moment Moment Moment In Out no. type stress Allow. $B 09 \mathrm{~F}+\operatorname{Max} \mathrm{P}$

GR + Max P Cold to TI Sus. + E1 SEIS SUS+SEIS

$810 \mathrm{~N}-\operatorname{Max} \mathrm{P}$ $\mathrm{GR}+\operatorname{Max} \mathrm{P}$ Cold to TI Sus. + E1 SEIS

SUS+SEIS

$\begin{array}{rr}73 & 14 \\ 99 & 134 \\ 82 & 17 \\ 78 & 15 \\ 11 & 3 \\ 83 & 17\end{array}$

1.001 .00 (3) (13) HOOP 1.001 .00 (17) DISP 1.001 .00 (18) OCC .001 .00 (18) OCC $1.001 .00(18)$ OCC

$\begin{array}{ll}0 & 16700\end{array}$

$\begin{array}{rr}0 & 16700 \\ 112 & 16700\end{array}$

$424 \quad 25050$

$126 \quad 22211$

$\begin{array}{rr}120 & 22211 \\ 17 & 22211\end{array}$

128 22211

(3a) $\mathrm{HOOP}$

0. 16700 1.001 .00 (18) SUST

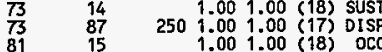
1.001 .00 (18) OCC $1.001 .00(18)$ OCC $1.001 .00(18)$ OCC

(3a) HOOP

$B 10 \mathrm{~N}+\operatorname{Max} P$

$G R+\operatorname{Max} P$

Cold to I1

Sus. + E1

SEIS

SUS+SEIS

B10 F- Max P

$\mathrm{GR}+\operatorname{Max} ?$

Cold to T1

Sus. + E1

SEIS

SUS+SEIS

2.271 .89 (18) SUSI 2.271 .89 (17) DISP 2.271 .89 (18) OCC 2.271 .89 (18) OCC 2.271 .89 (18) OCC

(3a) HOOP 2.271 .89 (18) SUST 242.271 .89 (17) DISP 2.271 .89 (18) OCC 2.271 .89 (18) OCC 2.271 .89 (18) OCC

B10 F+ Max P

$\mathrm{GR}+\operatorname{Max} \mathrm{P}$

Cold to $Y$ ?

Sus. + E1

SEIS

SUS+SEIS

(3a) $\mathrm{HOOP}$

1.001 .00 (18) SUST

B11 N- Max P

$G R+\operatorname{Max} P$

Cold to T1

Sus. + E

SEIS

SUS+SEIS
241.001 .00 (17) DISP

1.001 .00 (18) OCC 1.001 .00 (18) OCC 1.001 .00 (18) occ

(3a) HOOP

$$
\begin{array}{rr}
33 & 17 \\
1 & 284 \\
40 & 22 \\
34 & 24 \\
7 & 9 \\
40 & 26
\end{array}
$$

1.001 .00 (18) Sust

241.001 .00 (17) DISP $1.001 .00(18)$ OCC $1.001 .00(18)$ OCC 1.001 .00 (18) OCC
$26 \quad 22211$

$\begin{array}{rr}0 & 16700 \\ 247 & 16700\end{array}$

50825050

$267 \quad 22211$

$253 \quad 22211$

$\begin{array}{rr}28 & 22211 \\ 269 & 22211\end{array}$

0 16700

$124 \quad 16700$

$\begin{array}{ll}759 & 25050 \\ 151 & 22211\end{array}$

$145 \quad 22211$

$\begin{array}{rr}35 & 22211 \\ 158 & 22219\end{array}$

o 16700

$56 \quad 16700$

40325050

$17 \quad 22211$

$74 \quad 22211$

$\begin{array}{ll}0 & 16700 \\ 16 & 16700\end{array}$

$\begin{array}{rr}56 & 16700 \\ 403 & 25050\end{array}$

6922211

$68 \quad 22211$

$\begin{array}{ll}17 & 22211 \\ 74 & 22211\end{array}$
W320H32A PROCESS BUILDING PIPING STRESS ANALYSIS

03/01/95 W320, TANK 241-C-106 SLUICING, ER4319 AUtOPIPE+4.50 RESULT PAGE 58

ASME B31.3C (1992) CODE COMPLIANCE

(Moments in $\mathrm{ft}-\mathrm{lb}$ ) (Stress in psi)

Point Load In-PI. Out-Pl. Torsion S.I.F Eq. Load code code name combination Moment Moment Moment in out no. type stress Allow.

B11 $\mathrm{N}+\operatorname{Max}$

$G R+$ Max P

Cold to T1

Sus. + EI

SEIS

SUS+SEIS

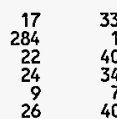

(3a) HOOP

.... -....

$\begin{array}{rr}0 & 16700 \\ 111 & 16700\end{array}$

B11 F- Max

$G R+$ Max $P$

Cold to $T$

Sus. + E1

SEIS

16
343
19
24
9
SUS+SEIS

$B 11 \mathrm{~F}+\operatorname{Max} P$

$G R+M a x P$

Cold to T1

Sus. + E2

SEIS

B12 $\operatorname{Max} P$

$\mathrm{GR}+$ Max P

(to 1

Sus. + E1

SEIS

SUS+SEIS

B13 Max P

GR + Max P

Sus. + E2

SEIS

SUS+SEIS

B14

Max $P$

$\mathrm{GR}+\operatorname{Max} \mathrm{P}$
Cold to TI

Sus. + EI

Sus. + E2

SUS+SEIS
91025050

13722211

$37 \quad 22211$

242.271 .89 (17) DISP 2.271 .89 (18) $\mathrm{OCC}$ 2.271 .89 (18) OCC

(3a) HOOP

2.271 .89 (18) SUST

142.271 .89 (17) DISP 2.271 .89 (18) OCC $2.271 .89(18)$ OCC 2.271 .89 (18) OCC

(3a) HOOP 1.001 .00 (18) SUST $141.001 .00(17)$ DISP 1.001 .00 (18) OCC 1.001 .00 (18) OCC 1.001 .00 (18) OCC

(3a) HOOP 1.001 .00 (18) SUST 41.001 .00 (17) DISP $1.001 .00(18)$ OCC $1.001 .00(18)$ OCC 1.001 .00 (18) OCC

(3a) HOOP $1.001 .00(18)$ SUST 41.001 .00 (17) DISP 1.007 .00 (18) OCC $1.001 .00(18)$ OCC $1.001 .00(18)$ OCC

(3a) HOOP 1.001 .00 (18) SUST 141.001 .00 (17) DISP 1.001 .00 (18) OCC $1.001 .00(18)$ OCC 1.001 .00 (18) OCC

016700 $201 \quad 16700$ $216 \quad 22211$ $230 \quad 2221$ $33 \quad 22211$

$0 \quad 16700$

$105 \quad 16700$

$485 \quad 25050$

113 22211 $15 \quad 22211$

$120 \quad 22211$

o 16700 16316700 53925050 $176 \quad 22211$ 176221 18222211

o 16700 $\begin{array}{ll}126 & 16700 \\ 572 & 25050\end{array}$ $\begin{array}{ll}146 & 22211\end{array}$ $139 \quad 22211$ $\begin{array}{ll}24 & 22211 \\ 150 & 22211\end{array}$

o 16700 $\begin{array}{rr}68 & 16700 \\ 637 & 25050\end{array}$ $104 \quad 22211$ 8222211 3922211 2.271 .89 (18)

HNF-2486, Rev. 0

Page C-40

Page 29 of 39 
ASNE $831.3 \mathrm{C}(1992)$ CODE COMPLIANCE
(Noments in $\mathrm{ft}-\mathrm{ib})$ (Stress in psi
Point Load In-PI. Out-Pl. Torsion S.I.F Eq. Load Code Code name combination Moment Moment Monent In Out no. type Stress Allow. B15 N- Max P

$\mathrm{GR}+\operatorname{Max} P$

Cold to $T$

Sus. + E1

Sus. + E2

SUS+SEIS

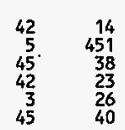
1.001 .00 (3a) HOOP 1.001 .00 (18) SUST 1.001 .00 (18) OCC 1.001 .00 (18) OCC 1.001 .00 (18) OCC 1.001 .00 (18) OCC

B15 N+ Max P

GR + Max $P$ Cold to $T 1$
Sus. + E1 SEIS

SUS+SEIS

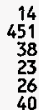

(3a) HOOP 2.271 .89 (18) SUST 2.271 .89 (17) DISP 2.271 .89 (18) OCC 2.271 .89 (18) OCC 2.271 .89 (18) OCC

B15 F- Max

$G R+\operatorname{Max} P$

Sus. $+\mathrm{E1}$

Sus. + E1

SEIS

SUS+SEIS

$815 \mathrm{~F}+\operatorname{Max} P$

GR + Max ?

Cold to $T$

Sus. + E1

SEIS

SUS+SEIS

B16

Max $P$

GR + Max P

cold to $T$

Sus. + E1

SEIS

SUS+SEIS

2.271 .89 (13) 18 SUST

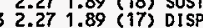
2.271 .89 (18) OCC 2.271 .89 (18) OCC 2.271 .89 (18) OCC 2.271 .89 (18) OCC
2.271 .89 (18) OCC

0716700 $\begin{array}{rr}67 & 16700 \\ 638 & 25050\end{array}$ 638
104
22211

8122211

$40 \quad 22211$

0 16700

$\begin{array}{rr}0 & 16700 \\ 129 & 16700\end{array}$

144525050

21322211

$162 \quad 22211$

21922211

o 16700

13916700

$275 \quad 22211$

18022211

14122211

28122211

(3a) HOOP

1.001 .00 (18) SUST

131.001 .00 (17) DISP 1.001 .00 (18) OCC 1.001 .00 (18) OCC

1.001 .00 (18) OCC

$\begin{array}{cc}0 & 16700 \\ 73 & 16700\end{array}$

$\begin{array}{rr}716 & 25050\end{array}$

$133 \quad 22211$

9122211

1352221

1.001 .00 (3a) HOOP

131.001 .00 (17) DISP

$1.001 .00(18)$ OCC

1.001 .00 (18) OCC

1.001 .00 (18) OCC $\begin{array}{cc}0 & 16700 \\ 73 & 16700\end{array}$

$716 \quad 25050$

13322211

$62 \quad 22211$

*** Segment $B$ end ***

*** Segment $\mathrm{C}$ begin ***

03/01/95 W320, TANK 244-C-106 SLUICING, ER4319

AutOPIPE+4.50 RESULT PAGE 60

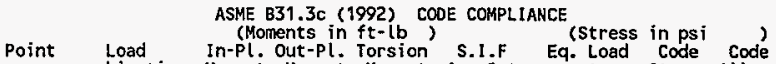

Point Load In-Pl. Out-Pl. Torsion S.I.F Eq. Load Code Code
name combination Moment Moment Moment In Out no. type Stress Allow.

BO6 Max $P$

$\begin{array}{lr}\text { Max P } & 17 \\ \text { GR + Max P } & 17 \\ \text { Cold to T1 } & 1933 \\ \text { SUs. + E1 } & 36 \\ \text { SUs. + E2 } & 55 \\ \text { SEIS } & 43 \\ \text { SUS+SEIS } & 59\end{array}$

$\begin{array}{rr}17 & 86 \\ 1933 & 155 \\ 36 & 97 \\ 55 & 100 \\ 43 & 18 \\ 59 & 104\end{array}$

(3a) HOOP

0 16700

131.521 .69 (18) SUST $1.521 .69(18)$ OCC 1.521 .69 (18) OCC

C01 N- Max P

$\mathrm{GR}+\operatorname{Max} \mathbf{P}$ Cold to T1 Sus. + E

SEIS

SUS+SEIS

3
86
5
18
15
18

$\operatorname{Cot} \mathrm{N}+\operatorname{Max} \mathrm{P}$

$\mathrm{GR}+\operatorname{Max} \mathbf{P}$ Cold to T1 Sus. + E

SEIS

SUS+SEIS

(3a) HOOP

1.001 .00 (18) SUST 1.001 .00 (18) OCC $1.001 .00(98)$ OCC $1.001 .00(18)$ Occ 1.001 .00 (18) OCC

$\begin{array}{rr}0 & 16700 \\ 8 & 16700 \\ 467 & 25050 \\ 44 & 22211 \\ 63 & 22211 \\ 67 & 22211 \\ 74 & 22211\end{array}$

CO1 F- Max P

GR + Max P Cold to T1 Sus. + E1

SEIS

SUS+SEIS

(3a) HOOP $\begin{array}{rrrrrr}4 & 3 & & 2.97 & 2.47 & \text { (3a) } \\ 60 & 86 & 313 & 2.97 & 2.47 & \text { HOOP } \\ \text { (17) } & \text { SUST }\end{array}$ 2.972 .47 (18) OCC 2.972 .47 (18) OCC 2.972 .47 (18) OCC
2.972 .47 (18) OCC

(3a) HOOP

2.972 .47 (18) SUST

$0 \begin{array}{lll}2.97 & 2.47 & (18) \\ 2.97 & \text { SUST }\end{array}$ 2.972 .47 (18) OCC 2.972 .47 (18) OCC $2.972 .47(18)$ OCC 2.972 .47 (18) OCC

(3a) HOOP

1.001 .00 (18) SUST GR + Max P Cold to T1 Sus. + E1 Sus. + E2

SEIS

SUS+SEIS

451
90
83
10
90

1.001 .00 (17) DISP 1.001 .00 (18) OCC 1.001 .00 (18) ocC

1.001 .00 (18) occ

1.001 .00 (18) OCC

$\mathrm{CO} 2 \operatorname{Max} \mathrm{P}$

$\mathrm{GR}+\operatorname{Hax} P$ Cold to T1 Sus. + E1

SEIS

SUS+SEIS (3a) HOOP

1.001 .00 ( 18 ) SUST

501.001 .00 (17) DISP

$1.001 .00(18)$ OCC

1.001 .00 (18) OCC

1.001 .00 (18) OCC

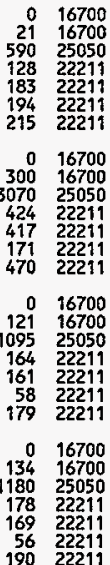

HNF-2486, Rev. 0

Page C-41 
W32OH32A PROCESS BUILDING PIPING STRESS ANALYSIS

03/01/95 W320, TANK 241-C-106 SLUICING, ER4319 AUtOPIPE+4.50 RESULT PAGE 61

ASHE B31.3C (1992) COOE COAPLIANCE

(Moments in $\mathrm{ft}-\mathrm{lb}$ ) (Stress in psi)

Point Load In-Pl. Out-Pl. Torsion S.I.F Eq. Load Code Code name combination Moment Moment Moment in out no. type stress Allow.

CO3 Max P

GR + Max $P$ Cold to T Sus. + E1 SUS: + E2 SUS+SEIS

$\begin{array}{rr}97 & \\ 545 & 714 \\ 110 & 32 \\ 99 & 24 \\ 14 & 33 \\ 110 & 38\end{array}$

(3a) $\mathrm{HOOP}$ 1.001 .00 (18) HOP 01.001 .00 (17) oIsP 1.001 .00 (18) OCC 1.001 .00 (18) OCC $1.001 .00(18)$
$1.001 .00(18)$ OCC 016700 $\begin{array}{rr}146 & 16700 \\ 1271 & 25050\end{array}$ 127125050 $\begin{array}{ll}192 & 22211 \\ 175 & 22211\end{array}$ $\begin{array}{rr}175 & 22211 \\ 54 & 2211\end{array}$ $\begin{array}{rr}54 & 22211 \\ 200 & 22211\end{array}$

CO4

lax $P$

$\mathrm{GR}+\operatorname{Max} P$ Cold to T1 Sus. + EI SEIS SUS+SEIS

97
550
111
100
14
111 1.001 .00 (13) HOOP 501.001 .00 (17) DISP $1.001 .00(18)$ OCC 1.001 .00 (18) 1.001 .00 (18) OCC

$0 \quad 16700$ $147 \quad 16700$ 19322211 $\begin{array}{ll}175 & 22211 \\ 175 & 2211\end{array}$ $54 \quad 2221$

$0 \quad 16700$ 1.521 .69 (3a) HOOP

$\operatorname{Cos}+\operatorname{Max} P$

$\mathrm{GR}+\operatorname{Max} P$

Sus. + E1

Sus. + E2

SEIS

SUS+SEIS

C06

Max P

GR + Hax P

Sus. + E1

Sus. + E2

SUS+SEIS

$\mathrm{C07}$

$\operatorname{Max} P$

$G R+\operatorname{Max} P$ Cold to I1 Sus. + E2 SEIS

SUS+SEIS 501.521 .69 (18) 1.521 .69 (18) 1521.69 (18) OCC 1.521 .69 (18) OCC 1.521 .69 (18) OCC

(3a) $\mathrm{HOOP}$

$\begin{array}{rr}7 & 109 \\ 827 & 672 \\ 36 & 128 \\ 16 & 112 \\ 30 & 19 \\ 37 & 128\end{array}$

$\begin{array}{rr}13 & 97 \\ 170 & 33 \\ 31 & 115 \\ 49 & 104 \\ 40 & 20 \\ 53 & 116\end{array}$
$88 \quad 1.521 .69$ (17) DISP 1.521 .69 (18) 1.521 .69 (18) OCC 1.521 .69 (18) OCC

(3a) HOOP

$\begin{array}{rr}86 & 7 \\ 212 & 202 \\ 107 & 20 \\ 91 & 30 \\ 21 & 26 \\ 108 & 33\end{array}$

1.001 .00 (18) SUST

6981.001 .00 (17) DISP $1.001 .00(18)$ OCC 1.001 .00 (18) OCC 1.001 .00 (18) OCC

(3a) HOOP 1.001 .00 (18) SUST 1.001 .00 (17) DISP $1.001 .00(18)$ OCC $1.001 .00(18)$
$1.001 .00(18)$
1.521 .69 (18) SUST

$\begin{array}{rr}75 & 5 \\ 311 & 353 \\ 97 & 16 \\ 78 & 21 \\ 22 & 20 \\ 97 & 25\end{array}$

$\begin{array}{rr}279 & 16700 \\ 2392 & 25050\end{array}$

36022211

30022219

83 22211

o 16700

$\begin{array}{rr}248 & 16700 \\ 1055 & 25050\end{array}$

31022211

$\begin{array}{ll}334 & 22211 \\ 105 & 22211\end{array}$

35422219

- 16700

$130 \quad 16700$

107025050

16722211

165122211

0. 16700

11316700

$150 \quad 22211$

$139 \quad 22211$

$\begin{array}{rr}45 & 22211 \\ 158 & 22211\end{array}$
18122211
W320H32A PROCESS BUILDING PIPING STRESS ANALYSIS

03/01/95 W320, TANK 241-C-106 SLUICING, ER4319 AUTOPIPE+4.50 RESULT PAGE 62

ASME B31.3c (1992) CODE COMPLIANCE
(Moments in ft-lb) (stress in psi s)
Point Load in-Pl. Out-pl. Torsion s.l.F Eq. Load Code code Point Load in-Pl. Out-Pl. Torsion S.I.F Eq. Load Code Code
name combination Moment Moment Moment in . Out no. type stress Allow. $\operatorname{Cos} N-\operatorname{Max} P$

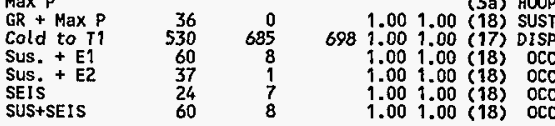

$\operatorname{Cos} N+\operatorname{Max} P$

GR + Max $P$ Cold to $T$ I Sus. + E1 SUIS + E 53 2.271 .89 (3a) Koop

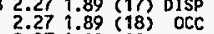
2.27 (18) OCC 2.271 .89 (18) OCC

$\operatorname{COB}$ F- Max P

$G R+\operatorname{Max} P$ Cold to T1

Sus. + E2

SUS+SEIS

$\operatorname{Cos} F+\operatorname{Max} P$

$\mathrm{GR}+\operatorname{Max} \mathrm{P}$ Cold to I1 Sus. + E1 SUIS + E

SUS+SEIS

$\operatorname{COS} \mathrm{N}-\operatorname{Max} \mathrm{P}$

GR + Max?

Cold to I1

Sus. + E1

SEIS

$\operatorname{Cog} N+\operatorname{Max} P$

$G R+\operatorname{Max} P$

Cold to $I 1$

Sus. + E1
Sus. $+E 2$

SEIS (3a) HOOP

2.271 .89 (18) SUST

$\begin{array}{lll}312 & 2.27 & 1.89 \\ \text { (17) DISP }\end{array}$ 2.271 .89 (18) OCC 2.271 .89 (18) OCC $2.271 .89(18)$ OCC

(3a) HOOP

13121.001 .00 (17) DISP 1.001 .00 (18) OCC 1.001 .00 (18) OCC $1.001 .00(18)$ OCC $1.001 .00(18)$ OCC

(3a) HOOP

13121.001 .00 (18) SUST 1.001 .00 (18) OCC 1.001 .00 (18) OCC $1.001 .00(18)$ OCC (3) $\mathrm{HOOP}$ 2.271 .89 (18) SUST $2.271 .89(18)$ OCC 2.271 .89 (18) OCC 2.271 .89 (18) OCC 2.271 .89 (17) OISP $\begin{array}{rr}0 & 16700 \\ 54 & 16700 \\ 1571 & 25050 \\ 92 & 22211 \\ 56 & 22211 \\ 38 & 22211 \\ 92 & 22211 \\ 0 & 16700 \\ 122 & 16700 \\ 2679 & 25050 \\ 207 & 22211 \\ 126 & 22211 \\ 85 & 22211 \\ 207 & 22211 \\ 0 & 16700 \\ 130 & 16700 \\ 1967 & 25050 \\ 144 & 22211 \\ 141 & 22211 \\ 18 & 22211 \\ 148 & 22211 \\ & \\ 0 & 16700 \\ 57 & 16700 \\ 1876 & 25050 \\ 65 & 22211 \\ 63 & 22211 \\ 9 & 22211 \\ 66 & 22211 \\ 0 & 16700 \\ 56 & 16700 \\ 1907 & 25050 \\ 65 & 22211 \\ 61 & 22211 \\ 10 & 22211 \\ 66 & 22211 \\ 0 & 16700 \\ 106 & 16700 \\ 2040 & 25050 \\ 124 & 22211 \\ 115 & 22211 \\ 21 & 22211 \\ 126 & 22211 \\ & \end{array}$

HNF-2486, Rev. 0

Page C-42 
W320H32A PROCESS BUILDING PIPING STRESS ANALYSIS

W3/01/95 W20, TANK 241-C-106 SLUICING, ER4319 AUTOPIPE+4.50 RESULT PAGE 63

ASME B31.3E (1992) CODE COMPLIANCE

(Moments in $\mathrm{ft}-\mathrm{ib}$ )
Point Load
name combination Moment Moment Moment In out no. type stress Allow.

name combination Moment Moment Moment In Out no. type Stress Allow.

COQ F- Max

GR + Max $P$

Cold to tr

Sus. + E1

SUS. + E2

SUS+SEIS

$\begin{array}{rr}96 & 0 \\ 210 & 172 \\ 105 & 33 \\ 126 & 24 \\ 31 & 40 \\ 127 & 41\end{array}$

(3a) HOOP

Max $P$

$\mathrm{GR}+$ Max P

Cold to $T$

Sus. + E1

SUS. + E

SUS+SEIS

96
210
105
126
31
127

172
33
24

14602.279 .89 (18) SUST 2.271 .89 (18) OCC 2.271 .89 (18) OCC 2.271 .89 (18) OCC 2.27
2.27
$1.89(18)$ OCC

$0 \quad 16700$

32916700

$\begin{array}{rr}2217 & 25050 \\ 427 & 22211\end{array}$

$\begin{array}{ll}427 & 22211 \\ 451 & 22211\end{array}$

$\begin{array}{ll}451 & 22211 \\ 155 & 22211\end{array}$

48522211

$\begin{array}{rr}0 & 16700 \\ 745 & 16700\end{array}$

1.001 .00 (18) HOA

14601001.00 (17) DISP 1.001 .00 (18) OCC .001 .00 (18) OCC (18) OCC 1.001 .00 (18) OCC

(3a) HOOP

C10 Max $P$

$\mathrm{GR}+\operatorname{Max} P$

Cold to TI

Sus. + E1

SEIS

SUS+SEIS

C11 - Max P

GR + Max P

Cold to I

Sus. + E1

SEIS

SUS+SEIS

C11 + Max P

GR + Max P

Cold to T1

Sus. + E1

SUS+SEIS

C12 Max P

GR + Max P

Cold to T1

Sus. + E1

Sus. +

SUS+SEIS

102
192
110
131
30

1.001 .00 (3a) HOOP

1.001 .00 (17) DISP

$1.001 .00(18)$ OCC

1.001 .00 (18) OCC

1.001 .00 (18) OCC

(3a) HOOP

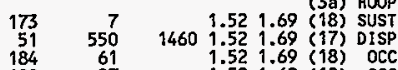
$1.521 .69(18)$ OCC 1.521 .69 (18) OCC

1.521 .69 (18) OCC

$196 \quad 22211$

$\begin{array}{ll}76 & 22211\end{array}$

$222 \quad 22211$

o 96700

15316700

208725050

$\begin{array}{ll}206 & 22211 \\ 210 & 22211\end{array}$

$78 \quad 22211$

16700

$396 \quad 16700$

$2448 \quad 25050$

$536 \quad 22211$

16122211

29
202

(3a) HOOP

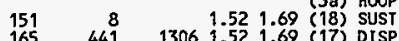

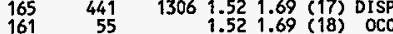
1.521 .69 (18) OCC 1.521 .69 (18) OCC

$\begin{array}{rr}0 & 16700 \\ 347 & 16700\end{array}$

215325050

46922211

$505 \quad 22211$

$\begin{array}{ll}200 & 22211 \\ 547 & 22211\end{array}$

(3a) HOOP

001.00 (3a) HOOP

(1) 16700

$\begin{array}{rr}368 & 16700 \\ 2572 & 25050\end{array}$

48122211

$452 \quad 22211$

$\begin{array}{ll}141 & 22211 \\ 509 & 22211\end{array}$ (18) $1.001 .00(18)$ OCC
U320H32A PROCESS BUILDING PIPING STRESS ANALYSIS

$03 / 01 / 95$ U320 TANK 241-0-106 SLUICING ER4319 AUTOPIPE+4.50 RESULT PAGE 64

ASME B31.3c (1992) CODE COHPLIANCE

Loint Load (Moments in ft-lb) (Stress in psi cole

name combination Moment Moment Moment in out no. type stress Allow.

Point conbination Moment Moment Moment In out ho. type stress Allow.

*** Segment $D$ begin ***

C11 Max P

GR + Max P Cold to T1 Sus. + E1

SEIS

SUS+SEIS

22
114
23
61
39
61

2
154
27
2
25
27

1.521 .69 (3a) HOOP

1.521 .69 (18) SUST

1.521 .69 (18) OCC

$\$ .521 .69(18)$ OCC

$1.521 .69(18)$ OCC
1.521 .69 (18) OCC

(3a) HOOP

1.001 .00 (3a) HOOP

GR + Max P

Cold to Ti
Sus. $+E$ I

Sus. + E2

SEIS

384
21
29
9
29

1
33
3
2
2

$1101.001 .00(17)$ DISP

1.001 .00 (18) OCC

1.001 .00 (18) OCC

1.001 .00 (18) OCC

2.271 .89 ( $3 a$ ) HOOP

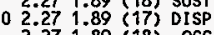
2.271 .89 (18) OCC 2.271 .89 (18) OCC $2.271 .89(18)$ OCC Sus. + E SEIS

2.27 (3a) HOOP

D01 F - Max P

$G R+\operatorname{Max} P$
Cold to T1

Sus. + E1

SUS. +

SUS+SEIS

D01 F+ Max P

$\mathrm{GR}+\operatorname{Max} \mathrm{P}$

Sus. + E1

Sus. + E

SUS+SEIS

12.271 .89 (17) DISP 2.271 .89 (18) OCC 2.271 .89 (18) OCC

$2.271 .89(18)$
$2.271 .89(18)$

(3a) HOOP

1.001 .00 (18) SUST

11.001 .00 (17) DISP 1.001 .00 (18) OCC 1.001 .00 (18) OCC

$1.001 .00(18)$ OCC

$\begin{array}{rr}0 & 16700 \\ 48 & 16700 \\ 450 & 25050 \\ 109 & 22211 \\ 134 & 22211 \\ 106 & 22211 \\ 154 & 22211\end{array}$

o 16700

3016700

56625050

$\begin{array}{ll}34 & 22211 \\ 43 & 22211\end{array}$

1422211

016700

\begin{tabular}{rr}
69 & 16700 \\
\hline & 75050
\end{tabular}

7622211

$98 \quad 22211$

3122211

$0 \quad 16700$

$\begin{array}{rr}59 & 16700 \\ 805 & 25050\end{array}$

8522211

$73 \quad 22211$

$29 \quad 22211$

o 16700

$\begin{array}{rr}26 & 16700 \\ 360 & 25050\end{array}$

$40 \quad 22211$

$32 \quad 22211$

4122213

segment $C$ end

$* * *$

HNF-2486, Rev. 0

Page C-43

Page 32 of 39 


\section{ASME B31.3C (1992) CODE COMPLIANCE}

(Monents in $\mathrm{ft}-\mathrm{lb}$ ) (Stress in psi)

Point Load In-Pl. Out-P!. Torsion S.I.F Eq. Load code code

name combination Moment Moment Moment in out no. type Stress Allow.

$D 02 \quad \operatorname{Max} P$

$G R+\operatorname{Max} P$

Cold to $\mathrm{J}$

Sus. + E1

Sus. + E2

SEIS

$\begin{array}{rr}57 & 0 \\ 66 & 48 \\ 58 & 10 \\ 65 & 1 \\ 8 & 10 \\ 65 & 10\end{array}$

(3a) HOOP

0 16700

1.001 .00 (18) SUST

11.001 .00 (17) DISP

1.001 .00 (18) OCC

1.001 .00 (18) OCC

$1.001 .00(18)$
$1.001 .00(18)$ OCC

D03

$\operatorname{Max} P$

$G R+\operatorname{Max} P$

Cold to TT

Sus. + EI

Sus. + E2

SUS+SEIS

$\begin{array}{rr}50 & 1 \\ 237 & 48 \\ 51 & 6 \\ 54 & 1 \\ 4 & 5 \\ 54 & 6\end{array}$

(3a) HOOP

1.001 .00 (18) SUST

11.001 .00 (17) DISP 1.00 1.00 (18) OCC 1.001 .00 (18) OCC 1.001 .00 (18) OCC

D04

$\operatorname{Max} P$

GR + Max P

Cold to $T$

Sus. + E1

SEIS

SUS+SEIS

D05 N- Max P

$\mathrm{GR}+\operatorname{Max} \mathrm{P}$

Cold to T1

Sus. + E1

SEIS

SUS+SEIS

D05 N+ Max P

$G R+M a x P$

Cold to I1

Sus. + E1

SUS: + E2

SUS+SEIS

D05 F- Max P

$\mathrm{GR}+\operatorname{Max} P$

Cold to T'

Sus. $+\varepsilon 1$

SEIS

SUS+SEIS

$$
\begin{array}{r}
44 \\
309 \\
44 \\
47 \\
3 \\
47
\end{array}
$$

1.001 .00 (3a) HOOP 11.001 .00 (18) SUST 1.001 .00 (18) OCC 1.001 .00 (18) OCC 1.001 .00 (18) OCC 1.001 .00 (18) OCC

(3a) HOOP

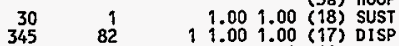
1.0001 .00 (18) OCC 1.001 .00 (18) OCC $1.001 .00(18)$ OCC

(3a) HOOP 2.271 .89 (18) SUST 2.271 .89 (17) DISP $2.271 .89(18)$ OCC 2.271 .89 (18) $\mathrm{OCC}$ $2.271 .89(18)$ OCC 2.271 .89 (3a) HOOP 2.271 .89 (17) DISP $2.271 .89(18)$ OCC 2.271 .89 (18) $\mathrm{OCC}$ $2.271 .89(18)$ OCC
$2.271 .89(18)$ OCC
$86 \quad 16700$

10122211

$98 \quad 2221$

10622211

0 16700

$\begin{array}{rr}0 & 16700 \\ 76 & 16700\end{array}$

34125050

832211

$\begin{array}{ll}82 & 22211 \\ 10 & 22211\end{array}$

$86 \quad 22211$

- 16700

$66 \quad 16700$

$\begin{array}{ll}66 & 22211 \\ 71 & 22211\end{array}$

5 22211

0 16700

$45 \quad 16700$

50025050

$\begin{array}{ll}50 & 22211 \\ 49 & 22211\end{array}$

722211

$0 \quad 16700$

$101 \quad 16700$

112425050

11022211

14
11522211

0
74 16700

$1700 \quad 25050$

11322211

9122211

$\begin{array}{rr}42 & 22211 \\ 116 & 22211\end{array}$
ASME B31.3C (1992) CODE COMPLIANCE

(Moments in $\mathrm{ft}-\mathrm{tb}$ ), (Stress in psi)

point Load In-Pl Out-Pl. Torsion S.I.F Eq. Load code code

name combination Moment Moment Moment In Out no. type stress Allow.

name combination Moment Monent Monent In out

D05 F+ Max P $G R+\operatorname{Max} P$ Sus. + E1

SUS.

SUS+SEIS

$\begin{array}{rr}22 & 1 \\ 528 & 33 \\ 22 & 14 \\ 27 & 1 \\ 5 & 14 \\ 27 & 14\end{array}$

1.009 .00 (18) SUST 1.001 .00 (18) SUST 1.001 .00 (18) OCC $1.001 .00(18)$ OCC 1.001 .00 (18) OCC

D06 Max P

$\mathrm{GR}+\operatorname{Max} \mathrm{P}$

Cold to TI

Sus. + E1

SUS, + E2

SUS+SEIS

22
528
22
27
5
27

22
528
22
27
5
27

$1.001 .00(18)$ OCC

(3a) HOOP

1.001 .00 (18) SUST

1161.001 .00 (17) DISP $1.001 .00(18)$ OCC 1.001 .00 (78) OCC .001 .00 (78) OCC $1.00 \% .00(18)$ OCC

$\begin{array}{rr}0 & 16700 \\ 33 & 16700 \\ 765 & 25050 \\ 53 & 22211 \\ 40 & 22211 \\ 22 & 22211\end{array}$

$\begin{array}{ll}22 & 22211 \\ 55 & 22211\end{array}$

**** segment $D$ end $* * *$

*** Segment $E$ begin ***

C05 Max $P$

GR + Max P Cold to $T 1$

Sus. + E1

SUS.

SUS+SEIS

E01 N- Max P

$\mathrm{GR}+\operatorname{Max} P$ Cold to T1

Sus. - E1

SEIS

SUS+SEIS

E01 $\mathrm{N}+\operatorname{Max} P$

$G R+$ Max $P$ Cold to $T 1$

Sus. + E1

SEIS

SUS+SEIS

20
997
31
49
30
51

12
749
21
29
19
31

8
589
14
22
15
23

20
1170

21
41
22

22

20
1170
21
41
22
41 (3a) HOOP

$\begin{array}{llll}705 & 1.52 & 1.69 \text { (18) } & \text { SUST } \\ 1.52 & 1.69 \text { (17) DISP }\end{array}$ 1.521 .69 (18) OCC 1.521 .69 (18) OCC 1.521 .69 (18) OCC

$\begin{array}{ll}0 & 16700 \\ 33 & 16700\end{array}$

3316700

5322211

$40 \quad 22211$

$22 \quad 22211$

(3a) HOOP 7051.001 .00 (18) sUST 1.001 .00 (18) OCC $1.001 .00(18)$ OCC $1.001 .00(18)$ OCC 1.001 .00 (18) OCC

(3a) HOOP 2.972 .47 (18) SUST 2.972 .47 (17) DISP $2.972 .47(18)$ OCC 2.972 .47 (18) OCC 2.972 .47 (18) OCC

HNF-2486, Rev. 0

Page C-44 
W320H32A PROCESS BUILOING PIPING SIRESS ANALYSIS

03/01/95 W320, TANK 241-C-106 SLUICING, ER4319 AUtOPIPE+4.50 RESULT PAGE 67

ASME B31.3C (1992) CODE COMPLIANCE

(Moments in $\mathrm{ft}-\mathrm{lb}$ ) (Stress in psi)

Point Load In-Pl, Out-pl. Torsion s.l.F Eq. Load code codé

Point Load In-Pl. Out-Pl. Torsion $\$ .1 . F$
name combination Moment Moment Moment ln Out no. type Stress Allow.

E01 F- Max

GR + Max

Cold to

Sus. + E1

SEIS

SUS+SEIS

$\begin{array}{rr}18 & 24 \\ 1190 & 816 \\ 20 & 25 \\ 35 & 39 \\ 17 & 15 \\ 36 & 39\end{array}$

2.972 .47 (3a) HOOP 2.972 .47 (18) OCC

E01 F+ Max P

$\mathrm{GR}+\operatorname{Max} \mathrm{P}$

Cold to T

Sus. + E1

SUS.

SUS+SEIS

1.001 .00 (18) SUST 2.001 .00 (17) DISP 1.001 .00 (18) OCC 1.001 .00 (18) OCC 1.001 .00 (18) OCC 1.001 .00 (18) OCC

(3a) HOOP

EO2 N- Max $P$

$\mathrm{GR}+\operatorname{Max} P$

Cold to $T$

Sus. + E1

SEIS

SUS+SEIS

E02 N+ Max P

$G R+\operatorname{Max} P$

Cold to $T$

Sus. + E1

SUS+SEIS

E02 F- $\operatorname{Max} P$

$\mathrm{GR}+\operatorname{Max} \mathrm{P}$

Cold to T1

Sus. + E1

SEIS

SUS +SEIS

$\begin{array}{rr}34 & 10 \\ 483 & 942 \\ 39 & 14 \\ 41 & 17 \\ 9 & 7 \\ 43 & 17\end{array}$

1.001 .00 (18) SUST
1.001 .00 (17) DISP

1.001 .00 (18) $00 \mathrm{CC}$

$1.001 .00(18)$ OCC

1.001 .00 (18) OCC

(3a) HOOP

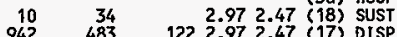

$\begin{array}{lllll}942 & 483 & 122 & 2.97 & 2.47 \\ 14 & 39 & 2.97 & 2.47 & (18) \text { OCC }\end{array}$ $2.972 .47(18)$ OCC 2.972 .47 (18) OCC 2.972 .47 (18) OCC

(3a) $\mathrm{HOOP}$

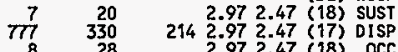
2.972 .47 (18) OCC 2.972 .47 (18) OCC 2.972 .47 (18) OCC

(3a) $\mathrm{HOOP}$

E02 F+ Max P

GR + Max P
Cold to T1

Sus. + E1

Sus. + E2

SEIS

SUS+SEIS

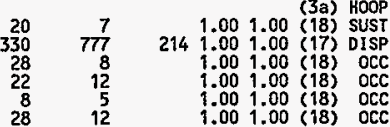

W32OH32A PROCESS BUILDING PIPING STRESS ANALYSIS

H320 TANK 241-C-106 SLUICING ER4319 AUTOPIPE+4.50 RESULT PAGE 68

ASME B31.3C (1992) CODE COHPLIANCE

(Moments in $\mathrm{ft}-\mathrm{lb})$ (Stress in psi
Point Load In-Pl. Out-pl. Torsion s.l.F Eq. Load code code

name combination Moment Moment Moment in Out no. type stress Allow.

-

o 16700

12016700

574825050

13222211

21522211

21622211

16700 4516700

4922211

7922211

$\begin{array}{ll}35 & 22211 \\ 80 & 22211\end{array}$

- 16700

$53 \quad 16700$

25050

$62 \quad 22211$

$\begin{array}{ll}68 & 22211 \\ 18 & 22211\end{array}$

0 16700

$133 \quad 16700$

430925050

$\begin{array}{ll}158 & 22211 \\ 174 & 22211\end{array}$

$\begin{array}{rr}174 & 22211 \\ 47 & 22211\end{array}$

47
181
22211

016700

81
3469
25050

$\begin{array}{rl}111 & 2211\end{array}$

$104 \quad 22211$

$118 \quad 22211$

Max

$\mathrm{GR}+\operatorname{Max} \mathrm{P}$

Cold to

Sus. + E

SEIS

SUS+SEIS

E04 Max P

$\mathrm{GR}+$ Max P

Cold to T1

Sus. + E1

SEIS

SUS+SEIS

$\begin{array}{rr}18 & 6 \\ 285 & 681 \\ 26 & 7 \\ 19 & 11 \\ 9 & 5 \\ 26 & 11\end{array}$

6
681
7
11
5
11

1.001 .00 (3a) HOOP

2141.001 .00 (17) DISP

$1.001 .00(18)$ OCC

1.001 .00 (78) OCC

$1.001 .00(98)$ OCC

(3a) HOOP

14
857 $98 \quad 214921.69$ (18) SUST

1.521 .69 (18) OCC

1.521 .69 (18) OCC

1.521 .69 (18) OCC

125

*** Segment $E$ end ***

*** Segment $\mathrm{F}$ begin $* * \star$

E04 Max $P$

$\mathrm{GR}+\operatorname{Max} \mathrm{P}$ Cold to T1

Sus. + E1
sus, + E2

SEIS

F01

SUS+SE IS

$\begin{array}{rr}0 & 16700 \\ 32 & 16700 \\ 1230 & 25050 \\ 44 & 22211 \\ 40 & 22211 \\ 14 & 22211 \\ 46 & 22211\end{array}$

$\operatorname{Max} P$

GR + Max P

Sus. + E1

Sus. + E2

SUS+SEIS

FO2

$\operatorname{Max} P$

$\mathrm{GR}+\operatorname{Max} \mathrm{P}$

Sus. + E1

SEIS

SUS+SEIS
13
895
39
29
30
43

108
348
117
133
26
134

134

121
58
129
144
25
146

14
872

29
29

21
35

14

14
863

25

29
19
33 (3a) HOOP

1.521 .69 (18) SUST $1.52, .69$ (18) DISP 1.521 .69 (18) OCC 1.521 .69 (18) OCC

1.521 .69 (18) OCC

(3a) HOOP

1.001 .00 (18) SUST

4991.001 .00 (17) DISP 1.001 .00 (18) OCC $1.001 .00(18)$ OCC 1.001 .00 (18) OCC 1.001 .00 (18) OCC

(3a) HOOP 1.001 .00 (18) SUST 4991.001 .00 (17) DISP 1.001 .00 (18) OCC 1.001 .00 (18) ocC 1.001 .00 (18) OCC $1.001 .00(18)$ OCC

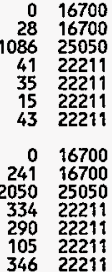

$\begin{array}{rr}0 & 16700 \\ 277 & 16700 \\ 2206 & 25050 \\ 339 & 22211 \\ 349 & 22211 \\ 95 & 22211 \\ 372 & 22211\end{array}$

o 16700 $183 \quad 16700$ 142125050 $209 \quad 22211$ 4922211 $232 \quad 22211$

o 16700 $\begin{array}{rr}181 & 16700 \\ 1410 & 25050\end{array}$ 20222211 22222211 $46 \quad 22211$ $227 \quad 22211$

HNF-2486, Rev. 0

Page C-45 
ASME B31.3c (1992) CODE CONPLIANCE (Moments in ft-lb)
Point Load in-Pl. Out-Pl. Torsion s.1.F Eq. Load Code Code name combination Moment Moment Moment In out no. type Stress Allow. F03 Max $P$ $\mathrm{GR}+\operatorname{Max} P$ Sus. + E1 Sus. + E1 SEIS + E2 SUS+SEIS

F04 N- Max P $G R+\operatorname{Max} P$ Cold to Th Sus. + E1 SEIS + E SUS+SEIS

FO4 $\mathrm{N}+\operatorname{Max} \mathrm{P}$ $\begin{array}{lrr}\text { GR + Max P } & 56 & 15 \\ \text { Cold to T1 } & 755 & 807 \\ \text { Sus. + E1 } & 63 & 19 \\ \text { SUS. + E2 } & 75 & 30 \\ \text { SEIS } & 21 & 15 \\ \text { SUS+SEIS } & 76 & 31\end{array}$

F04 F- Max P $G R+\operatorname{Max} P$ Cold to $T$ Sus. $+E 1$ SUS. + E2 SEIS SUS+SEIS

$\begin{array}{rr}72 & 95 \\ 599 & 820 \\ 80 & 16 \\ 92 & 30 \\ 21 & 15 \\ 93 & 30\end{array}$
(3a) HOOP 1.001 .00 (18) SUST 4991.001 .00 (17) DISP $\begin{array}{rr}0 & 16700 \\ 111 & 16700 \\ 1598 & 25050\end{array}$ $1.001 .00(18)$ OCC 1.001 .00 (18) OCC 1.001 .00 (18) 2322211 $\begin{array}{rr}148 & 22211 \\ 39 & 22211\end{array}$

(3a) HOOP 1.001 .00 (18) HOOP $\begin{array}{rrrr}56 & 15 & 4991.001 .00 \\ 755 & 807 & 1.001 .00(17) & \text { DISP }\end{array}$ 1.001 .00 (18) OCC 1.001 .00 (18) OCC $1.001 .00(18)$ occ (3a) HOOP 2.271 .89 (18) SUST 992.271 .89 (17) DISP 2.271 .89 (18) OCC 2.271 .89 (18) OCC $2.271 .89(18)$ OCC

\section{$0 \quad 16700$} 8716700 $1713 \quad 25050$ $\begin{array}{ll}100 & 22211 \\ 123 & 22211\end{array}$ $\begin{array}{ll}39 & 22211\end{array}$ $126 \quad 22211$

0
195
16700 $\begin{array}{rr}195 & 16700 \\ 3393 & 25050\end{array}$ 337325050 2233522211 8322211 27822211

\section{(3a) HOOP} $\begin{array}{llll}9 & 46 & 2.271 .89 \text { (18) SUST }\end{array}$ 12.271 .89 (17) DISP 2.271 .89 (18) OCC 2.271 .89 (18) OCC 2.271 .89 (18) OCC

o 16700 $134 \quad 16700$ 14925211 $155 \quad 22211$ $\begin{array}{rr}26 & 22211 \\ 159 & 22211\end{array}$

F04 F+ Max P

$G R+$ Max $P$ Cold to $T$ ? Sus. + E1 Sus. + E SEIS SUS+SEIS

\section{(3a) HOOP}

1.001 .00 (18) SUST 1.001 .00 (17) 015P 1.001 .00 (18) OCC 1.001 .00 (18) OCC $1.001 .00(18)$ OCC
1.001 .00 (18) OCC

$\begin{array}{rr}0 & 16700 \\ 70 & 16700\end{array}$

$\begin{array}{rr}70 & 16700 \\ 1518 & 25050\end{array}$

$1518 \quad 25050$

8022211

$\begin{array}{ll}12 & 22211 \\ 82 & 22211\end{array}$

(3a) HOOP

F05 N- Max P

$\mathrm{GR}+\operatorname{Max} \mathrm{P}$

sus. + E1

Sus. + E2

SEIS

SUS+SEIS
1.001 .00 (18) SUST

7711.001 .00 (17) DISP 1.001 .00 (18) OCC $1.001 .00(18)$ OCC $1.001 .00(18)$
1.00
$1.00(18)$ OCC

$\begin{array}{rr}0 & 16700 \\ 73 & 16700 \\ 1308 & 25050 \\ 87 & 22211 \\ 95 & 22211 \\ 26 & 22211 \\ 99 & 22211\end{array}$

ASME B31.3C (1992) CODE COMPLIANCE

(Moments in ft-lb '
Point Load In-Pl. Out-pl. Torsion s.1.F Eq. Load code code

name combination Moment Moment Moment in Out no. type stress Allow.

F05 $\mathrm{N}+\operatorname{Max} \mathrm{P}$

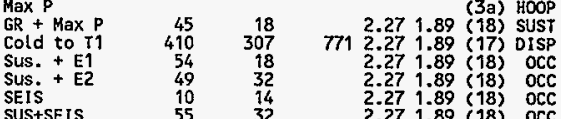

() 16700

16116700

89225050

20422211 2.271 .89 (18) OCC

(3a) $\mathrm{rOOP}$

F05 F- Max P

GR + Max P Cold to $T 1$

Sus. + E1

SEIS + E

$\begin{array}{lll}2.27 & 1.89 \text { (18) SUST } \\ 2.27 & 1.89 \text { (17) DISP }\end{array}$ 2.271 .89 (18) OCC 2.271 .89 (18) OCC 2.271 .89 (18) OCC

$214 \quad 22211$

F05 $\mathrm{F}+\operatorname{Max} \mathrm{P}$

GR + Hax P Cold to T1

Sus. + E1
Sus. + E2

SEIS

F06

$\operatorname{Max} P$

$\mathrm{GR}+\operatorname{Max} \mathrm{P}$

Cold to $T$ ?

Sus. + E1

SEIS

SUS+SEIS

55
90
68
57
13
68

0 16700

19116700

40525050

$250 \quad 22211$

19822211

6022211

(3a) KOOP

1.001 .00 (3a) KOOP

$\begin{array}{rr}0 & 16700 \\ 85 & 16700 \\ 1344 & 25050 \\ 113 & 22211 \\ 88 & 22211 \\ 29 & 22211 \\ 114 & 22211\end{array}$

9371.001 .00 (17) DISP 1.001 .00 (18) OCC

1.001 .00 (18) OCC

$1.001 .00(18)$ OCC

\section{(3a) HOOP}

9371.001 .00 (18) SUST 1.001 .00 (17) DISP 1.001 .00 (18) OCC 1.001 .00 (18) OCC

$\operatorname{Max} \mathbf{P}$

GR + Max P

Sus. + E1

SUS+SEIS

116
347
128
117
12
128

208
22
17
20

.00 1.00 (18) Occ

(3a) HOOP

$\operatorname{Max} P$

$\mathrm{GR}+$ Max ?

Cold to T1

Sus. + E1

SE1S

SUS+SEIS
9371.009 .00 (18) SUST 1.001 .00 (18) OCC $1.001 .00(18)$ OCC $1.001 .00(18)$
$1.001 .00(18)$ OCC

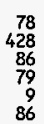

424
21
20
24
28

(3a) HOOP 1.001 .00 (18) OCC 1.001 .00 (18) OCC $1.001 .00(18)$ OCC
9371.001 .00 (18) SUST o 16700 17516700 $206 \quad 22211$ 19122211 35
210 22211

(0) 16700 19716700 157325050 146
142 22211 $\begin{array}{rr}39 & 22211 \\ 156 & 22211\end{array}$

o 16700 $\begin{array}{rr}37 & 16700 \\ 1963 & 25050\end{array}$ 6722211 8522211 $56 \quad 22211$

HNF-2486, Rev. 0

Page C-46

Page 35 of 39 
U320H32A PROCESS 8UILDING PIPING STRESS ANALYSIS

AUtOPIPE+4.50 RESULT PAGE 7

ASME B31.3C (1992) CODE COHPLIANCE

(Moments in $\mathrm{ft}-\mathrm{lb}$ ) (Stress in psi)

Point Load In-Pl. Out-pl. Torsion s.t.F Eq. Load code code Point Load
name combination Moment Moment Moment in Out no. type stress Allow. rog $\mathrm{H}-\mathrm{max}$ (

$\begin{array}{lrrr}\text { Max P } & & 1.001 .00(39) \text { HOOP } \\ \text { GR + Max P } & 22 & 1 & 937 \text { SUST } \\ \text { Cold to T1 } & 597 & 874 & 937.001 .00(17) \text { DISP } \\ \text { SUS.+E1 } & 23 & 21 & 1.001 .00(18) \text { OCC } \\ \text { SUS. + E2 } & 27 & 34 & 1.001 .00(18) \text { OCC } \\ \text { SEIS } & 6 & 38 & 1.001 .00(18) \text { OCC } \\ \text { SUS+SEIS } & 27 & 39 & 1.001 .00(18) \text { OCC }\end{array}$

FOS $\mathrm{N}+$ Max

$\mathrm{GR}+\operatorname{Max} P$

Cold to $T$

Sus. + E1

SEIS

SUS+SEIS

$\begin{array}{rr}1 & 22 \\ 874 & 597 \\ 21 & 23 \\ 34 & 27 \\ 38 & 6 \\ 39 & 27\end{array}$

2.972 .47 (18)

$937 \begin{aligned} & 2.97 \\ & 2.97 \\ & 2.47\end{aligned}$ (17) DISP 2.972 .47 (18) OCC

2.972 .47 (18) OCC

2.972 .47 (18) OCC

(3a) 4000

GR + Max $P$

Gold to $P 1$

Sus. + E!

Sus. +

SUS+SEIS

$\begin{array}{rr}3 & 15 \\ 1080 & 1063 \\ 24 & 15 \\ 44 & 23 \\ 46 & 8 \\ 49 & 23\end{array}$

2.972 .47 (18) SUST 2.972 .47 (17) D1SP 2.972 .47 (18) 2.972 .47 (18) OCC

2.972 .47 (18) OCC

F09 F+ Max P

GR + Max $P$

Cold to T

Sus. + E1

SEIS

SUS+SE1S

$\begin{array}{rr}15 & 3 \\ 1063 & 1080 \\ 15 & 24 \\ 23 & 44 \\ 8 & 46 \\ 23 & 49\end{array}$

F10

Max $P$

GR + Max P

sus. $+E$ ?

SUs. + E2

SEIS

SUS+SE IS

$\begin{array}{rr}20 & 4 \\ 1084 & 1128 \\ 21 & 25 \\ 28 & 46 \\ 8 & 47 \\ 28 & 51\end{array}$

Segment $F$ end $\star * *$

1.00 (3a) HOOP

$4941.001 .00(17)$ DISP

1.001 .00 (18) OCC

$1.001 .00(18)$ OCC

1.001 .00 (18) OCC

1.001 .00 (18) OCC

(3a) HOOP 1.001 .00 (18) SUST 4941.001 .00 (17) DISP $1.001 .00(18)$ OCC 1.001 .00 (18) OCC 1.001 .00 (18) OCC $\begin{array}{rr}0 & 16700 \\ 33 & 16700 \\ 1997 & 25050 \\ 62 & 22211 \\ 82 & 22211 \\ 58 & 22211 \\ 90 & 22211\end{array}$

o 16700

$81 \quad 16700$

44212505

$169 \quad 22211$

$\begin{array}{ll}228 & 22211 \\ 171 & 22211\end{array}$

$252 \quad 22211$

0 16700

$\begin{array}{rr}56 & 16700\end{array}$

15925050

$\begin{array}{ll}150 & 22211 \\ 239 & 22211\end{array}$

$206 \quad 22211$

$262 \quad 22211$

$\begin{array}{rr}0 & 16700 \\ 22 & 16700 \\ 2 & \end{array}$

225125050

8522211

7022211

0
31
16700

231725050

$63 \quad 22211$

$95 \quad 2221$

$\begin{array}{rr}72 & 22211 \\ 103 & 22211\end{array}$

$* * *$ segment $G$ begin $* * *$

HNF-2486, Rev. 0

Page C-47
W320H32A PROCESS BUILDING PIPING STRESS ANALYSIS

03/01/95 W320, TANK 241-C-106 SLUICING, ER4319 AUTOPIPE+4.50 RESULT PAGE 72

ASME B31.3C (1992) CODE COMPLIANCE

(Moments in $\mathrm{ft}-\mathrm{lb}$ )
Point Load In-Pl. Out-pl. Torsion in psi )

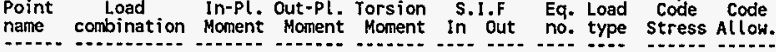

$\mathrm{E0} 4 \mathrm{Max} P$

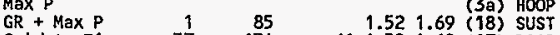
$\begin{array}{lrrrr}\text { GR + Max P } & 1 & 85 & 1.521 .69 \text { (18) SUST } \\ \text { Cold to T1 } & 37 & 134 & 611.521 .69 \text { (17) DISP }\end{array}$ $\begin{array}{lrrrrr}\text { Cold to T1 } & 37 & 134 & 611.521 .69(17) & \text { DISP } \\ \text { Sus. + E1 } & 8 & 91 & 1.521 .69(18) \text { OCC }\end{array}$

SUS. SUS+SEIS

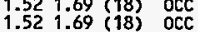
$1.521 .69(18)$ OCC 1.521 .69 (18) OCC $\begin{array}{rr}0 & 16700 \\ 216 & 16700\end{array}$ $\begin{array}{ll}216 & 16700 \\ 340 & 25050\end{array}$ 23922211 $264 \quad 22211$ $\begin{array}{rr}54 & 22211 \\ 269 & 22211\end{array}$

G01 N- Max P

$\begin{array}{lrr}\text { GR + Max P } & 33 & 1 \\ \text { Cold to I1 } & 293 & 81 \\ \text { SUs. + E1 } & 42 & 3 \\ \text { SUS. + E2 } & 51 & 6 \\ \text { SEIS } & 20 & 6 \\ \text { SUS+SEIS } & 53 & 7\end{array}$

(3a) HOOP

1.001 .00 (18) SUST

1.001 .00 (17) OISP 1.001 .00 (18) OCC 1.001 .00 (18) OCC 1.001 .00 (18) OCC

$0 \quad 16700$

$\begin{array}{rr}50 & 16700 \\ 437 & 25050\end{array}$

$64 \quad 22211$

$\begin{array}{ll}64 & 22211 \\ 22211\end{array}$

$\begin{array}{ll}31 & 22211 \\ 81 & 22211\end{array}$

G01 N+ Max P

$\mathrm{GR}+\operatorname{Max} \mathrm{P}$

Sus. + E1

SEIS

$\begin{array}{rr}1 & 33 \\ 81 & 293 \\ 3 & 42 \\ 6 & 51 \\ 6 & 20 \\ 7 & 53\end{array}$

(3a) hOOP

12.972 .47 (18) SUST 2.972 .47 (18) OCC 2.972 .47 (18) OCC 2.972 .47 (18) OCC 2.972 .47 (18) OCC

o 16700 $\begin{array}{rr}123 & 16700\end{array}$ 16022211 $193 \quad 22211$ $79 \quad 22211$

601 F- Max P

GR + Max P Cold to $T 1$ Sus. + E1 Sus.

SUS+SEIS

$\begin{array}{rr}1 & 12 \\ 330 & 109 \\ 18 & 27 \\ 8 & 21 \\ 19 & 17 \\ 20 & 29\end{array}$

(3a) HOOP 2.972 .47 (18) SUST (18) 2.972 .47 (18) OCC 2.972 .47 (18) OCC 2.972 .47 (18) OCC

(3a) HOOP

G01 F+ Max P

$\mathrm{GR}+\operatorname{Max} P$ Cold to $T 1$ Sus. + E1

Sus.

SUS+SEIS

12
109
27
21
17
29

1.001 .00 (18) SUST

4621.001 .00 (17) DISP $1.001 .00(18)$ OCC 1.001 .00 (18) OCC 1.001 .00 (18) 000

(3a) $\mathrm{HOOP}$

$602 \operatorname{Max} P$

$\mathrm{GR}+\operatorname{Max} \mathrm{P}$

Cold to Ty

Sus, $+E 1$

Sus. + E2

SUS +SEIS

1.001 .00 (18) SUST

46
193
5
15

46
193
5
5
15
61

1
391
21
5
20
21

4621.001 .00 (17) DISP 1.001 .00 (18) OCC 1.001 .00 (18) OCC

1.001 .00 (18) OCC

$0 \quad 16700$

$\begin{array}{rr}45 & 16700 \\ 1575 & 25050\end{array}$

157525050

$88 \quad 22211$

$104 \quad 22211$

$149 \quad 22211$

16700

$18 \quad 16700$

81625050

$34 \quad 22211$

$56 \quad 22211$

$0 \quad 16700$

$69 \quad 16700$

10425211

$82 \quad 22211$

$\begin{array}{rl}38 & 22211 \\ 106 & 22211\end{array}$ 
ASHE B31.3c (1992) CODE COMPLIANCE

(Moments in $\mathrm{ft}-\mathrm{lb}$ ) (Stress in psi) Point Load In-Pl. Out-Pl. Torsion s.I.F Eq. Load Code code name combination Moment Monent Moment in Out no. type stress Allow.

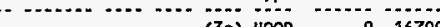
GR+ Max $P$ Cold to $\mathrm{YI}$ Sus. + E1 SEIS SUS+SEIS

$603 \mathrm{~N}+\operatorname{Max} \mathrm{P}$

$\mathrm{GR}+\operatorname{Max} \mathrm{P}$ Cold to T Sus. + EI SUS+SEIS

G03 F- Max P

$G R+\operatorname{Max} P$ Cold to TI Sus. + E1 SEIS + E2 SUS+SEIS

$603 \mathrm{~F}+\operatorname{Max} P$ $\mathrm{GR}+$ Max $P$
Cold to T1 Sus. + E1
Sus. + E2 SEIS SUS+SEIS

25
201
38
33
15
40

$$
\begin{array}{r}
437 \\
23 \\
3 \\
21 \\
23
\end{array}
$$

25
201
38
33
15
40

2
437
23
3
21
23
2.271 .89 (18) OCC

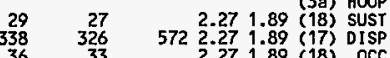

G04 Max $P$ GR + Max? Cold to $T 1$ Sus. + E1 Sus. + E2 SEIS SUS+SEIS

G05
GR + Max Cold to Sus. + E1 Sus, + E2 SEIS SUS+SEIS $\begin{array}{rr}27 & 29 \\ 326 & 338 \\ 33 & 36 \\ 34 & 30 \\ 9 & 6 \\ 36 & 36\end{array}$

26
199
27
29
3
30

30
447
56
37
28
57

26
108
29
39
14
40 1.001 .00 (18) SUST 1.001 .00 (18) SUS 1.001 .00 (18) OCC 1.001 .00 (18) OCC 1.00
1.00
1.00

2.271 .89 (3a) HOOP 622.271 .89 (17) DISP 2.271 .89 (18) OCC 2.271 .89 (18) OCC

(3a) HOOP 2.271 .89 (18) OCC 2.271 .89 (18) OCC 2.271 .89 (18) OCC

(3a) HOOP 1.001 .00 (18) SUST 21.001 .00 (17) DISP 1.001 .00 (18) OCC $1.001 .00(18)$ OCC 1.001 .00 (18) OCC

(3a) HOOP

$\begin{array}{rr}0 & 16700 \\ 37 & 16700\end{array}$ $\begin{array}{rr}37 & 16700 \\ 942 & 25050\end{array}$ $\begin{array}{ll}75 & 22211\end{array}$ 4922211 $40 \quad 22211$ 0 16700 $\begin{array}{rr}84 & 16700 \\ 1481 & 25050\end{array}$ 148125050 $112 \quad 22211$ $80 \quad 22211$ $165 \quad 22211$

\section{$0 \quad 16700$} $\begin{array}{rr}126 & 16700 \\ 1607 & 25050\end{array}$ $\begin{array}{rl}1607 & 25050 \\ 154 & 2221\end{array}$ $146 \quad 22211$ $\begin{array}{rr}34 & 22211 \\ 161 & 22211\end{array}$

\section{$0 \quad 16700$ $1046 \quad 16700$ 742221 $70 \quad 2221$ $17 \quad 22213$} 1.001 .00 (18) SUST 721.001 .00 (17) DISP 1.001 .00 (18) OCC $1.001 .00(18)$ OCC 1.001 .00 (18) OCC

(3a) HOOP 1.001 .00 (18) SUST 1.001 .00 (17) DISP $1.001 .00(18)$ OCC 1.001 .00 (18) OCC 1.001 .00 (18) OCC

\section{$0 \quad 16700$} 6016700 106425050 $\begin{array}{ll}99 & 22211\end{array}$ \begin{tabular}{ll}
72 & 22211 \\
\hline & 22211
\end{tabular} $\begin{array}{rr}42 & 22211 \\ 101 & 22211\end{array}$

016700 $\begin{array}{rr}59 & 16700 \\ 1107 & 25050\end{array}$ 110725050 $125 \quad 22211$ $\begin{array}{ll}88 & 22211 \\ 72 & 22211\end{array}$ $131 \quad 22211$
W320H32A PROCESS BUILDING PIPING STRESS ANALYSIS

03/01/95 W320, TANK 241-C-106 SLUICING, ER4319 AUtOPIPE+4.50 RESULT PAGE 74

ASME B31.3C (1992) CODE COMPLIANCE

Point Load In-Pl. Out-pl in forsion s.I.F Estress in psi ? name combination Monent Monent Moment in Out no. type stress Allow. G06 N- Max P 30 (3a) HOOP 0 16700

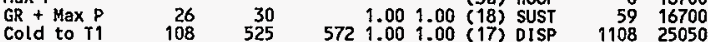
Sus. $+\varepsilon 1$ SEIS SUS+SEIS

$\mathrm{CO6} \mathrm{N}+\operatorname{Max} ?$

$G R+\operatorname{Max} P$ Cold to T1 Sus. + E1 Sus. + E2 SEIS

G06 F- Max P $\mathrm{GR}+\operatorname{Max} \mathrm{P}$ Cold to TI Sus. + E1 Sus. + E2 SEIS
SUS+SEIS

G06 F+ Max P $G R+\operatorname{Max} P$ Sus. + E2 SEIS $1.001 .00(18)$ OCC $1.001 .00(18)$ OCC $1.001 .00(18)$ OCC

2221 $88 \quad 22211$ $\begin{array}{rr}72 & 22211 \\ 131 & 22211\end{array}$

(3a) HOOP

$\begin{array}{rr}0 & 16700 \\ 160 & 16700\end{array}$ 2.972 .47 (18) SUST $2.972 .47(17)$ DISP 2.972 .47 (18) OCC 2.972 .47 (18) OCC 2.972 .47 (18) OCC $\begin{array}{rr}160 & 16700 \\ 2052 & 25050\end{array}$ $323 \quad 22211$ 18122211 34122211

(3a) HOOP 0 16700 2.972 .47 (18) SUST 2.972 .47 (17) DISP $2.972 .47(18)$ OCC 2.972 .47 (18) OCC 2.972 .47 (18) OCC 101670 192525050 52
138 22211 13422211 $6021.001 .00(17)$ OISP
607 Max $P$ $G R+\operatorname{Max} P$ Sus. + E1 Sus. + E2 SEIS
SUS+SEIS

*** Segment $G$ end $\star \star * *$
1.001 .00 (3a) HOOP

0 16700 1.001 .00 (18) OCC $1.001 .00(18)$ OCC $1.001 .00(18)$ OCC

110125050

$\begin{array}{ll}19 & 22211 \\ 48 & 22211\end{array}$

47.2211

(3a) HOOP $1.001 .00(18)$ SUST

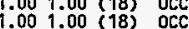
$1.001 .00(18)$ OCC $\begin{array}{rr}0 & 16700 \\ 15 & 16700 \\ 1081 & 25050 \\ 26 & 22211 \\ 61 & 22211 \\ 47 & 22211 \\ 62 & 22211\end{array}$ 21.001 .00 (17) DISP
HNF-2486, Rev. 0

Page C-48 


\section{W320H32A PROCESS 8UILDING PIPING STRESS ANALYSIS \\ PRO TANK 241-C-106 SLUICING ER4319 AUTOPIPE+4 50 RESULT PAGE 75}

\section{SYSTEM SUMMARY}

\section{Maximum displacements (in)}

$\begin{array}{ll}\text { Maximum X : } & 0.075 \\ \text { Maximum } Y & 0.044 \\ \text { Maximum Z } & 0.063 \\ \text { Max. total: } & 0.098\end{array}$
Point : $\mathrm{B04} F$ Point : 003

Point : $804 \mathrm{~F}$

Max, total:

(0.08

Maximun rotations (deg)

$\begin{array}{ll}\text { Maximum X } & \mathbf{0 . 0 3 9} \\ \text { Maximum } Y & \mathbf{0 . 1 5 8} \\ \text { Maximum } Z: & \mathbf{0 . 1 0 7}\end{array}$

Point : $F 05 \mathrm{~N}$ Point : $\mathrm{B04} \mathrm{N}$ Point : $\mathrm{A03} \mathrm{M}$ Point : $\mathrm{BO4}$ N

Max. total:

0.159

Lad COMb.: GR+T1+SEIS

Comb: I1

COMb: $G R+T 1+S E I S$

Load Comb.: GR+T1+SEIS

Load Comb.: GR+T1+SEIS

Load COmb. GR+T1+SEIS
Maximum restraint forces(lb)

$\begin{array}{lr}\text { Maximum } x: & 21645 \\ \text { Maximum } y: & 1645\end{array}$

Maximum 2: $\quad 1034$

Point : $A 00$

Point : $A 08$

Point : $\mathrm{C}_{12}$

Point : $\mathbf{A O O}$

Maximum restraint moments $(f t-l b)$

Maximun $X:$

Maximum $Y:$

Max total:

Point : F10

Point : $\mathrm{C12}$

Point : A0O

Point : AOO

Load Comb.: GR+T 1+SEIS

Load Comb.: GR+T1+SEIS

Load Comb.: GR+T1+SEIS

Load Conb.: GR+T1+SEIS

Load Comb.: GR+T1+SEIS

Load Comb.: GR+T1+SEIS

load Comb.: GR+T1+SEIS
W320H32A PROCESS BUILDING PIPING STRESS ANALYSIS

03/01/95 W320, TANK 241-C-106 SLUICING, ER4319 AUTOPIPE+4.50 RESULT PAGE 76

\section{SYSTEM SUMMARY}

Maximun pipe forces (lb)

Load Comb.: GR+T1+SEIS

$\begin{array}{lrl}\text { Maximum } X: & 21641 & \text { Point }: A 00 \\ \text { Maximum } y: & 1621 & \text { Point }: A 08 \\ \text { Maximum } Z: & 1031 & \text { Point }: C 12 \\ \text { Max_total }: & 21672 & \text { Point }: A 03\end{array}$

LOad Comb.: GR+T1+SEIS oad Comb.: GR+T1+SEIS Lad COmb. $G R+T 1+S E 1 S$ Load comb.: GR+T1+SEIS

Maximum pipe moments ( $f t-l b)$

$\begin{array}{lrl}\text { Maximum X: } & 1032 & \text { Point : F10 } \\ \text { Maximum Y } & 4420 & \text { Point : AO4 } \\ \text { Maximum Z : } & 42785 & \text { Point : A00 } \\ \text { Max. total: } & 42790 & \text { Point : A0O }\end{array}$

Load COMb.: GR+T1+SEIS Load Comb.: GR+T1+SEIS Load Comb.: GR+T1+SEIS Load Comb.: GR+ritSEIS

HNF-2486, Rev. 0

Page C-49 
W32OH32A PROCESS BUILDING PIPING STRESS ANALYSIS

03/01/95 W20, TANK 241-C-106 SLUICING, ER4319 AUTOPIPE+4.50 RESULT PAGE 77

\section{SY STEM SUMMARY}

Maximxm sustained stress

$$
\begin{array}{ll}
\text { Point } & : \text { ct1 } \\
\text { Stress psi } & : 396 \\
\text { Allowable psi } & \vdots 16700 \\
\text { Ratio } & : 0.02 \\
\text { Load combination } & : \mathrm{GR}+\text { Max P }
\end{array}
$$

Maximun displacement stress

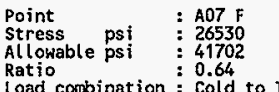

Maximum occasional stress

$$
\begin{aligned}
& \text { Point psi : } 111 \\
& \text { Allowable psi } \\
& \text { Ratio : :03 }
\end{aligned}
$$$$
\text { Load combination : SUS+SEIS }
$$

Maximum sustained stress ratio

$$
\begin{array}{ll}
\text { Point } & \text { : } 11 \\
\text { Stress psi } & 396 \\
\text { Allowable psi } & 16700 \\
\text { Ratio } & 0.02 \\
\text { Load combination } & \text { GR + Hax P }
\end{array}
$$

Maximum displacement stress ratio

$$
\begin{aligned}
& \text { Point : } \mathrm{A03} \mathrm{N} \\
& \text { Stress psi : } 17753 \\
& \text { Allowable psi : } 25050 \\
& \text { Ratio } \quad: 0.71
\end{aligned}
$$

Maximum occasional stress ratio

$$
\begin{array}{ll}
\text { Point } & \text { c11 } \\
\text { Stress psi } & 557 \\
\text { Allowable psi } & 22211 \\
\text { Ratio } & 0.03 \\
\text { Load combination } & \text { sus+SEIS }
\end{array}
$$

W320H32A PROCESS BUILDING PIPING STRESS ANALYSIS

03/01/95 W320, TAUK 241-C-106 SLUICING ER4319 AUTOPIPE+4.50 RESULT PAGE 78

SYSTEM SUMMARY

* * The system satisfies ASME B31.3 code requirements * * *

* * for the selected options
HNF-2486, Rev 0

Page C-50 


\begin{tabular}{|c|c|}
\hline ** & AUTOPIPE SYSTEM DATA LISTING \\
\hline
\end{tabular}

SYSTEM NAME : W32OH32B

PROJECT ID : PROCESS BUILDIHG PIPING STRESS ANALYSIS H320, TANK 241-CC-106 SLUICING

PREPARED BY :

RC CAMPBEIL

CHECKED BY :

PIPING CODE : B31.3

AMBIENT TEMP. ( $\operatorname{deg} F$ ) : $\mathbf{5 0 . 0}$

COMPONENT LIBRARY : AUTOPIPE

MATERIAL LIBRARY : AUTOB313

MODEL REVISION NUMBER : 4
W320H32B PROCESS BUILDING PIPING STRESS ANALYSIS ICF KATSER PROCESS BUILDING PIPING STRESS ANALYSIS ICF KAISER HANFORD COMPANY AUTOPIPE+4.50 HODEL PAGE

POINT DATA LISTING

POINT TYPE $\times$-OFFSETS (ft )

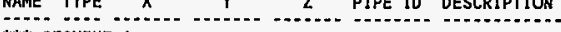

*** SEGMENT A 0 O 0 O

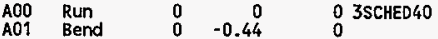

Short Elbow, Radius $=3.00$ inch Bend angle change $=90.00 \mathrm{deg}$

$\begin{array}{lllll}\text { A02 } & \text { Run } & 0 & 0 & 1.27 \\ \text { A03 } & \text { Bend } & 0 & 0 & 1.10 \quad \text { Short Elbow, Radius }=3.00 \text { inch }\end{array}$ Bend angle change $=90.00 \mathrm{deg}$

A04 Valv $-1.96 \quad 0 \quad 0 \quad$ Rating $=150$, weight $=119 \mathrm{lb}$

Surface factor $=4.00$, sif $=1.00$

$\begin{array}{lllll}\text { A05 } & \text { Run } & -1.00 & 0 & 0 \\ \text { A06 } & \text { Bend } & -0.25 & 0 & 0\end{array}$

A07 Run $\quad 0 \quad-0.38 \quad 0$

Short Elbow, Radius $=3,00$ inch Bend angle change $=90.00 \mathrm{deg}$ SIF - In $=2.33$, Out $=1.94$

Total weight of empty pipes : 39 tb

HNF-2486, Rev, 0

Page C-51 


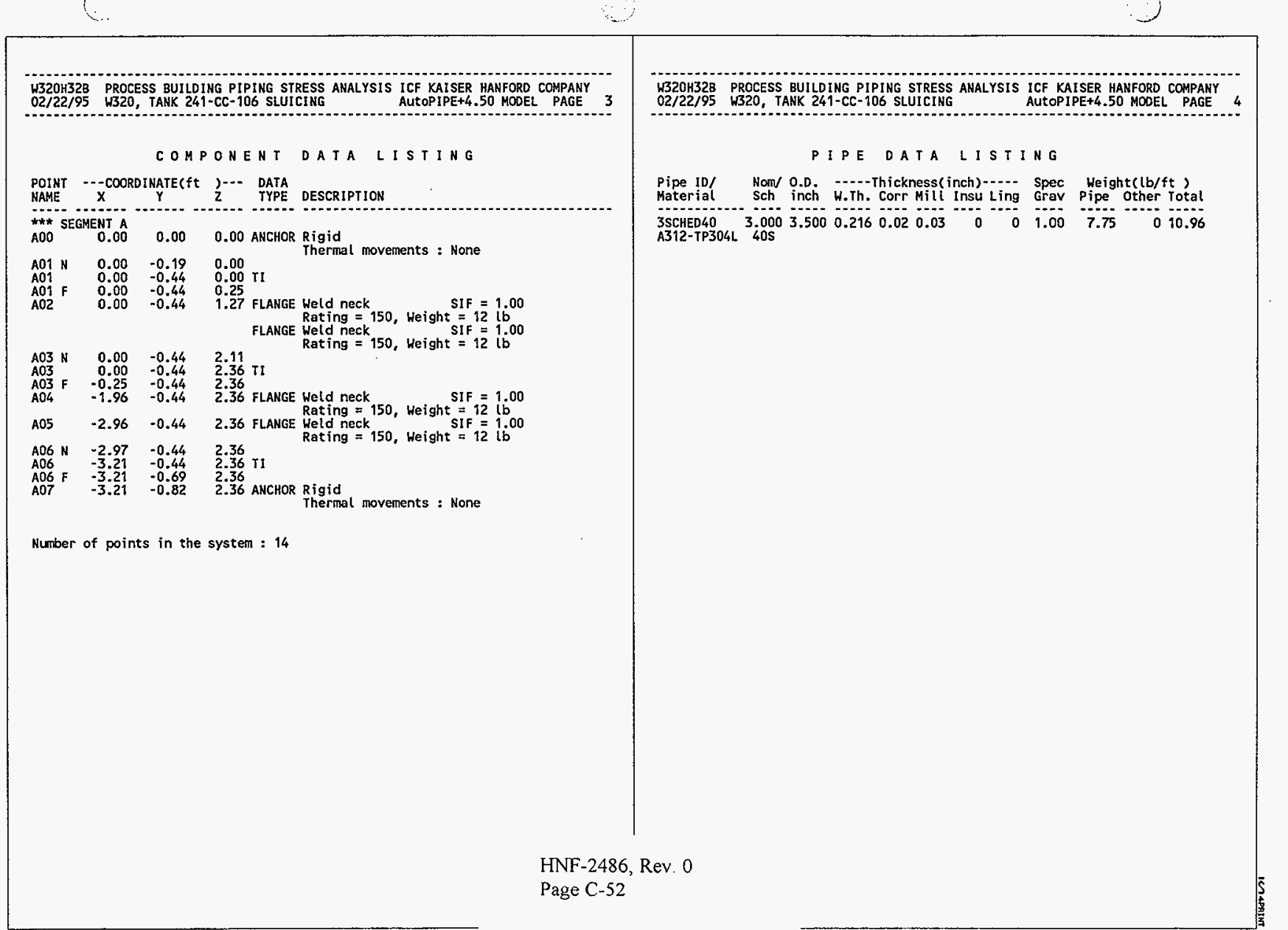




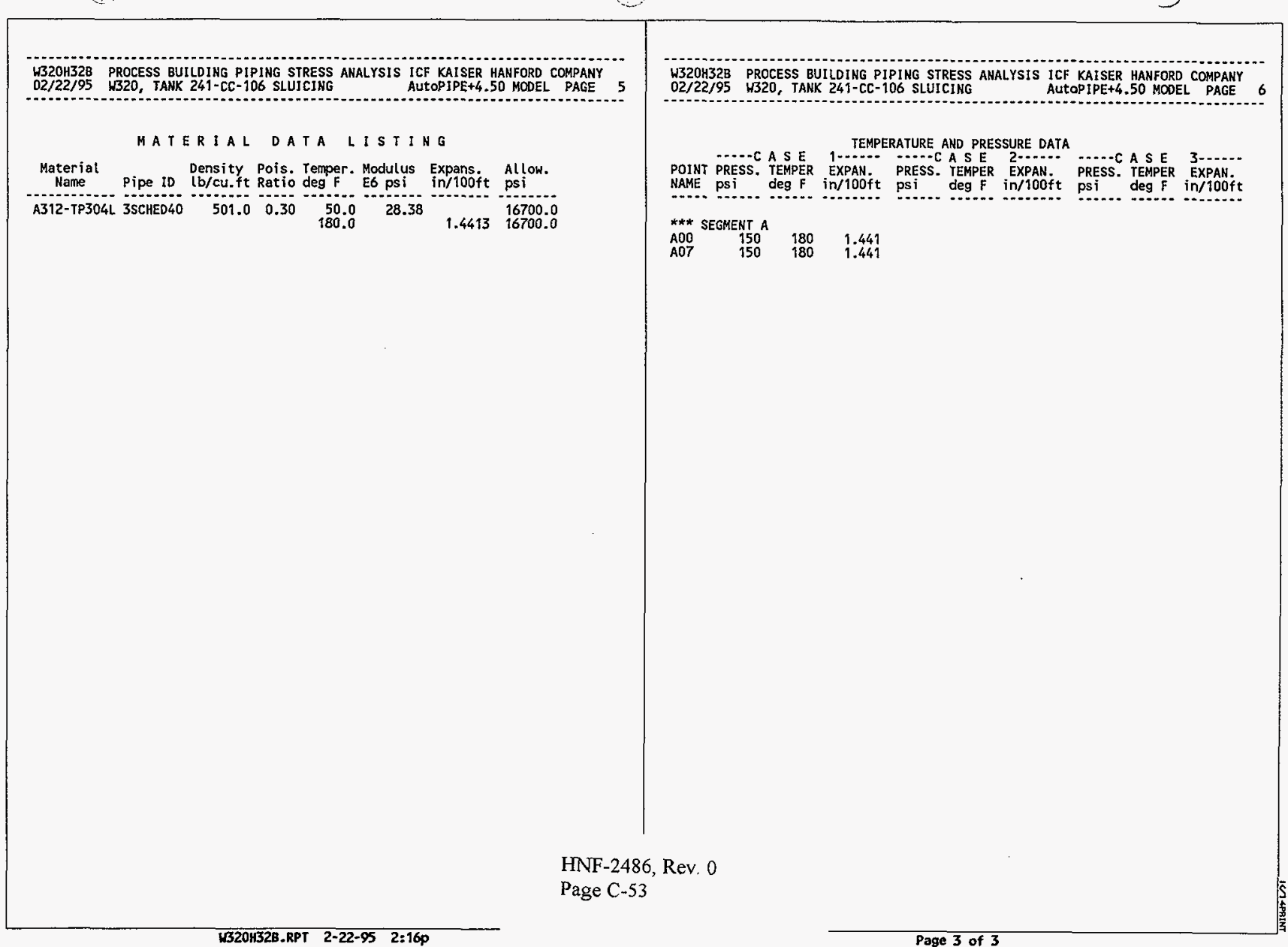



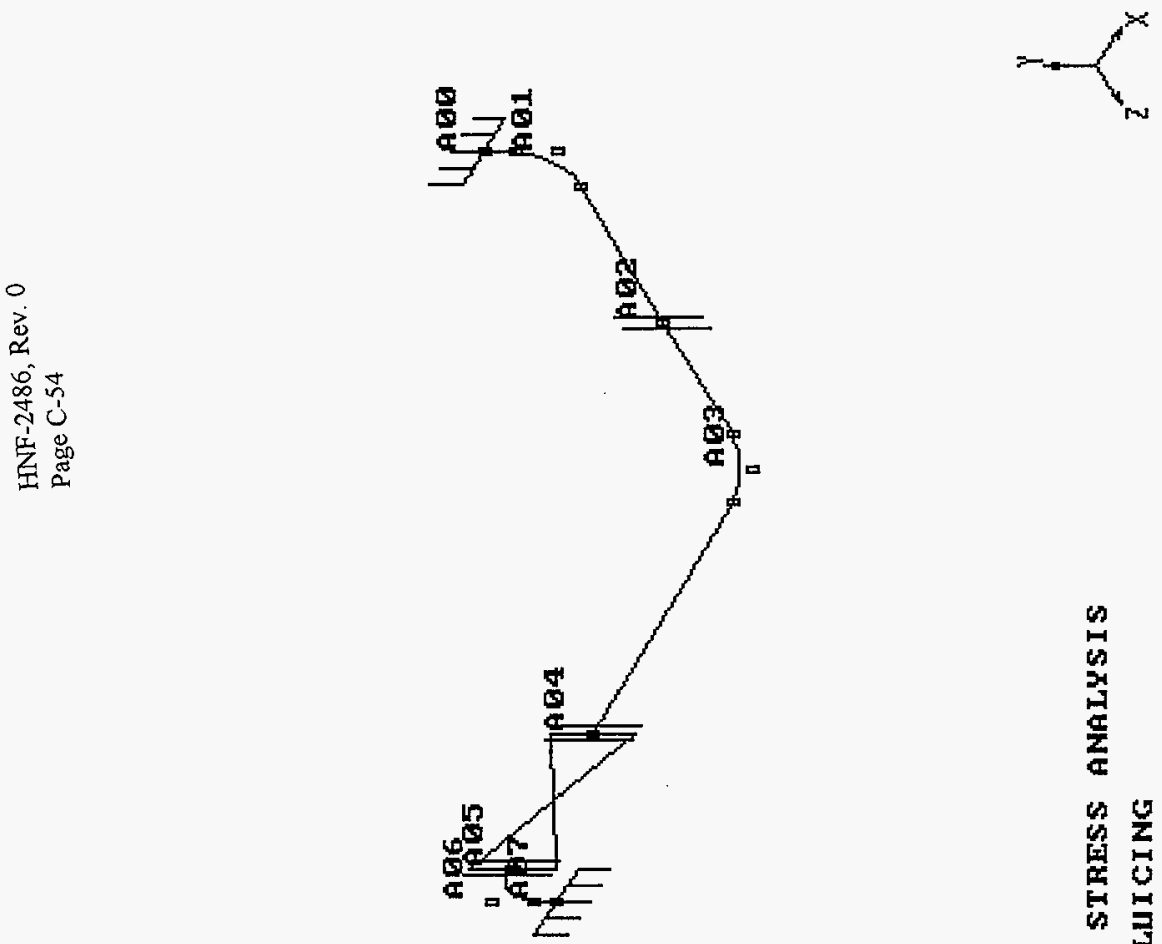

c

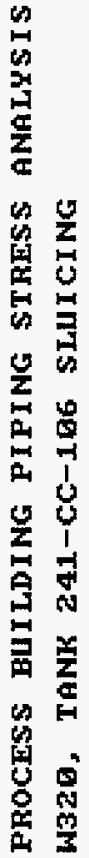


3320H32B PROCESS BUILDING PIPING STRESS ANALYSIS 02/22/95 W320. TAHK $241-C C-106$ SLUICIKG

\section{ANALYSIS SUHHARY}

Current model revision number : 4

Static - Date and Time of analysis ........... Feb 22, $1995 \quad 2: 48$ PM Model Revision Number

GR T1 E1 E2 P1

Gaps/Friction/Yielding considered ...... No

Hanger design run

Cut short included

Weight of contents included ........... Yes

Pressure stiffening case ...............

Water elevation for buoyancy loads .... Not considered
HNF-2486, Rev. 0

Page C -55
W320H32B PROCESS BUILDING PIPING STRESS ANALYSIS

CODE COMPLIANCE COMBINATIONS

\begin{tabular}{|c|c|c|c|c|c|c|}
\hline Combination & Category & Method & Load & Factor & Allowable & Remarks \\
\hline$G R+\operatorname{Max} P$ & Sustain & Sum & $\begin{array}{l}\text { Gravity } \\
\text { Max Long }\end{array}$ & $\begin{array}{l}1.00 \\
1.00\end{array}$ & Aut onatic & Default \\
\hline cold to I1 & Expansion & Sum & Thermat 1 & 1.00 & Automatic & Default \\
\hline Sus. $+E 1$ & Occasion & Abs sum & $\begin{array}{l}\text { Earth } 1 \\
\text { Max Sus }\end{array}$ & $\begin{array}{l}1.00 \\
1.00\end{array}$ & Automatic & Default \\
\hline Sus. + E2 & Dccasion & Abs sum & $\begin{array}{l}\text { Earth } 2 \\
\text { Max Sus }\end{array}$ & $\begin{array}{l}1.00 \\
1.00\end{array}$ & Automatic & Default \\
\hline $\operatorname{Max} P$ & Hoop & & Max Hoop & 1.00 & Automat ic & Default \\
\hline SEIS & Occasion & SRSS & $\begin{array}{l}\text { Earth } 1 \\
\text { Earth } 2\end{array}$ & $\begin{array}{l}1.00 \\
1.00\end{array}$ & Automatic & User \\
\hline SUS+SE1S & Occasion & Abs sum & $\begin{array}{l}\text { Max Sus } \\
\text { SEIS }\end{array}$ & $\begin{array}{l}1.00 \\
1.00\end{array}$ & Automatic & User \\
\hline
\end{tabular}

OTHER USER COMBINATIONS

\begin{tabular}{|c|c|c|c|c|}
\hline $\begin{array}{l}\text { Combination } \\
\text { GR }\end{array}$ & $\begin{array}{l}\text { Method } \\
\text { Sum }\end{array}$ & $\begin{array}{l}\text { Load } \\
\text { Gravity }\end{array}$ & \begin{tabular}{c} 
Factor \\
\hdashline 1.00
\end{tabular} & $\begin{array}{l}\text { Remarks } \\
\text { Defaut }\end{array}$ \\
\hline$T 1$ & Sum & Thermal 1 & 1.00 & Defau \\
\hline E1 & Sum & Earth 1 & 1.00 & Defau \\
\hline E2 & sum & Earth 2 & 1.00 & Defau \\
\hline P1 & sum & Press 1 & 1.00 & Defau \\
\hline SEIS & Abs sum & $\begin{array}{l}\text { Earth } 1 \\
\text { Earth } 2\end{array}$ & $\begin{array}{l}1.00 \\
1.00\end{array}$ & User \\
\hline$G R+T 1$ & sum & $\begin{array}{l}\text { Gravity } \\
\text { Thermal }\end{array}$ & $\begin{array}{l}1.00 \\
1.00\end{array}$ & User \\
\hline $\mathrm{GR}+\mathrm{T} 1+\mathrm{P} 1+\mathrm{S}$ & Abs sun & $\begin{array}{l}\text { GR+T1 } \\
\text { SEIS } \\
\text { Press } 1\end{array}$ & $\begin{array}{l}1.00 \\
1.00 \\
1.00\end{array}$ & User \\
\hline
\end{tabular}

CODE COMPLIAMCE 
W320H32B PROCESS BUILDING PIPING STRESS ANALYSIS

$02 / 22 / 95$ W320, TANK $241-C C-106$ SLUICING

AUtOPIPE+4.50 RESULT PAGE 3

$Y$ - Factor

\subsection{0}

Weld efficiency factor ................. 1.00

Range reduction factor $\ldots \ldots \ldots \ldots \ldots \ldots \ldots .1 .00$

Design Pressure factor $\ldots \ldots \ldots \ldots \ldots . . .1 \%$. 1.00

Include corrosion in stress calcs. ..... Y

Include torsion in code stress

Include axial force in code stress ..... N

Longitudinal pressure calculation ...... PD/4t

Include rigorous pressure
W32OH32B PROCESS BUILDING PIPING STRESS ANALYSIS

02/22/95 W320, TANK 241-CC-106 SLUICING

AUTOPIPE +4.50 RESULT PAGE

EARTHQUAKE LOAD CASES :

Number of load cases analysed : 2

Load case 1 - E1

$X$-Multiplier $=0.120 \quad$ Y-Multiplier= $0.000 \quad$ Z-Multiplier $=0.000$

Load case 2 - E2

X-Multiplier $=0.000 \quad Y$-Multiplier $=0.000 \quad$ Z-Multiplier $=0.120$

HNF-2486, Rev. 0

Page C -56 
W32OH32B PROCESS BUILOING PIPING STRESS ANALYSIS

02/22/95 W320, IANK 241-CC-106 SLUICING

AUTOPIPE+4.50 RESULT PAGE 5

DI S P L A C EMENTS

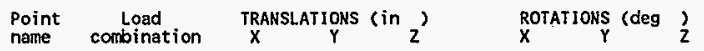

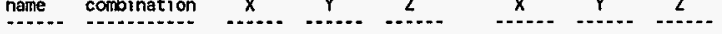

\section{*** Segment $A$ begin $* * *$}

\begin{tabular}{|c|c|c|c|c|c|c|c|}
\hline$A 00$ & $\begin{array}{l}\text { GR } \\
\text { I1 } \\
\text { E1 } \\
\text { E2 } \\
\text { P1 } \\
\text { SEIS } \\
\text { GR+I } \\
G R+T 1+P 1+S\end{array}$ & $\begin{array}{l}0.000 \\
0.000 \\
0.000 \\
0.000 \\
0.000 \\
0.000 \\
0.000 \\
0.000\end{array}$ & $\begin{array}{l}0.000 \\
0.000 \\
0.000 \\
0.000 \\
0.000 \\
0.000 \\
0.000 \\
0.000\end{array}$ & $\begin{array}{l}0.000 \\
0.000 \\
0.000 \\
0.000 \\
0.000 \\
0.000 \\
0.000 \\
0.000\end{array}$ & $\begin{array}{l}0.000 \\
0.000 \\
0.000 \\
0.000 \\
0.000 \\
0.000 \\
0.000 \\
0.000\end{array}$ & $\begin{array}{l}0.000 \\
0.000 \\
0.000 \\
0.000 \\
0.000 \\
0.000 \\
0.000 \\
0.000\end{array}$ & $\begin{array}{l}0.000 \\
0.000 \\
0.000 \\
0.000 \\
0.000 \\
0.000 \\
0.000 \\
0.000\end{array}$ \\
\hline$A 01 \mathrm{~N}$ & $\begin{array}{l}\text { GR } \\
\text { T1 } \\
\text { E1 } \\
\text { E2 } \\
\text { P1 } \\
\text { SEIS } \\
\text { GR+T1 } \\
\text { GR+r } 1+P 1+S\end{array}$ & $\begin{array}{l}0.000 \\
0.000 \\
0.000 \\
0.000 \\
0.000 \\
0.000 \\
0.000 \\
0.000\end{array}$ & $\begin{array}{r}0.000 \\
-0.003 \\
0.000 \\
0.000 \\
0.000 \\
0.000 \\
-0.003 \\
0.003\end{array}$ & $\begin{array}{l}0.000 \\
0.000 \\
0.000 \\
0.000 \\
0.000 \\
0.000 \\
0.000 \\
0.000\end{array}$ & $\begin{array}{l}0.001 \\
0.001 \\
0.000 \\
0.000 \\
0.000 \\
0.000 \\
0.002 \\
0.002\end{array}$ & $\begin{array}{l}0.000 \\
0.013 \\
0.000 \\
0.000 \\
0.000 \\
0.000 \\
0.013 \\
0.014\end{array}$ & $\begin{array}{l}0.000 \\
0.001 \\
0.000 \\
0.000 \\
0.000 \\
0.000 \\
0.001 \\
0.001\end{array}$ \\
\hline$A 01 \mathrm{~F}$ & $\begin{array}{l}\text { GR } \\
\text { T1 } \\
\text { E1 } \\
\text { E2 } \\
\text { P1 } \\
\text { SEIS } \\
\text { GR+T1 } \\
\text { GR+T1+P } 1+S\end{array}$ & $\begin{array}{l}0.000 \\
0.003 \\
0.000 \\
0.000 \\
0.000 \\
0.000 \\
0.003 \\
0.003\end{array}$ & $\begin{array}{r}-0.001 \\
-0.006 \\
0.000 \\
0.000 \\
0.000 \\
0.000 \\
-0.007 \\
0.007\end{array}$ & $\begin{array}{l}0.000 \\
0.004 \\
0.000 \\
0.000 \\
0.000 \\
0.000 \\
0.003 \\
0.003\end{array}$ & $\begin{array}{r}0.018 \\
-0.008 \\
0.000 \\
0.000 \\
0.000 \\
0.000 \\
0.010 \\
0.010\end{array}$ & $\begin{array}{l}0.000 \\
0.086 \\
0.000 \\
0.000 \\
0.000 \\
0.000 \\
0.087 \\
0.087\end{array}$ & $\begin{array}{r}-0.002 \\
0.028 \\
0.000 \\
0.000 \\
0.000 \\
0.000 \\
0.026 \\
0.026\end{array}$ \\
\hline 102 & $\begin{array}{l}\text { GR } \\
T 1 \\
\text { E1 } \\
\text { E2 } \\
\text { P1 } \\
\text { SEIS } \\
\text { GR+T1 } \\
\text { GR+T1+P } \hat{q}+S\end{array}$ & $\begin{array}{l}0.000 \\
0.025 \\
0.000 \\
0.000 \\
0.000 \\
0.000 \\
0.025 \\
0.026\end{array}$ & $\begin{array}{r}-0.005 \\
-0.004 \\
0.000 \\
0.000 \\
0.000 \\
0.000 \\
-0.009 \\
0.009\end{array}$ & $\begin{array}{l}0.000 \\
0.018 \\
0.000 \\
0.000 \\
0.000 \\
0.000 \\
0.018 \\
0.018\end{array}$ & $\begin{array}{r}0.020 \\
-0.010 \\
0.000 \\
0.000 \\
0.000 \\
0.000 \\
0.011 \\
0.011\end{array}$ & $\begin{array}{l}0.001 \\
0.107 \\
0.000 \\
0.000 \\
0.000 \\
0.000 \\
0.108 \\
0.108\end{array}$ & $\begin{array}{r}-0.004 \\
0.014 \\
0.000 \\
0.000 \\
0.000 \\
0.000 \\
0.010 \\
0.010\end{array}$ \\
\hline $03 \mathrm{~N}$ & $\begin{array}{l}\text { GR } \\
T \text { I } \\
\text { E } \\
\text { EL } \\
P \text { P } \\
\text { SEIS } \\
\text { GR+T1 } \\
\text { GR+T } 1+P 1+S\end{array}$ & $\begin{array}{l}0.000 \\
0.044 \\
0.000 \\
0.000 \\
0.000 \\
0.000 \\
0.044 \\
0.044\end{array}$ & $\begin{array}{r}-0.008 \\
-0.003 \\
0.000 \\
0.000 \\
0.000 \\
0.000 \\
-0.011 \\
0.011\end{array}$ & $\begin{array}{l}0.000 \\
0.030 \\
0.000 \\
0.000 \\
0.000 \\
0.000 \\
0.030 \\
0.030\end{array}$ & $\begin{array}{r}0.019 \\
-0.006 \\
0.000 \\
0.000 \\
0.000 \\
0.000 \\
0.013 \\
0.013\end{array}$ & $\begin{array}{l}0.001 \\
0.089 \\
0.000 \\
0.000 \\
0.000 \\
0.000 \\
0.091 \\
0.091\end{array}$ & $\begin{array}{r}-0.006 \\
0.002 \\
0.000 \\
0.000 \\
0.000 \\
0.000 \\
-0.003 \\
0.004\end{array}$ \\
\hline
\end{tabular}

W32OH32B PROCESS BUILDING PIPING STRESS ANALYSIS

02/22/95 W320, TANK 241-CC-106 SLUICIMG

AUtoPIPE+4.50 RESUIT PAGE

DISPLACEMENTS

\begin{tabular}{|c|c|c|c|c|c|c|c|}
\hline $\begin{array}{l}\text { Point } \\
\text { name }\end{array}$ & $\begin{array}{l}\text { Load } \\
\text { combination }\end{array}$ & $\underset{X}{\text { TRANSL }}$ & ATIONS & $z^{\prime}$ & $x^{\text {ROTAT }}$ & ONS (des & $z$ \\
\hline $\mathrm{A} 03 \mathrm{~F}$ & $\begin{array}{l}\text { GR } \\
\text { T1 } \\
\text { E1 } \\
\text { E2 } \\
\text { P1 } \\
\text { SEIS } \\
\text { GR+T1 } \\
\text { GR+T1+P1+S }\end{array}$ & $\begin{array}{l}0.000 \\
0.043 \\
0.000 \\
0.000 \\
0.000 \\
0.000 \\
0.043 \\
0.043\end{array}$ & $\begin{array}{r}-0.009 \\
-0.002 \\
0.000 \\
0.000 \\
0.000 \\
0.000 \\
-0.011 \\
0.011\end{array}$ & $\begin{array}{l}0.000 \\
0.035 \\
0.000 \\
0.000 \\
0.000 \\
0.000 \\
0.034 \\
0.035\end{array}$ & $\begin{array}{r}0.012 \\
-0.001 \\
0.000 \\
0.000 \\
0.000 \\
0.000 \\
0.012 \\
0.012\end{array}$ & $\begin{array}{r}0.001 \\
-0.029 \\
0.000 \\
0.000 \\
0.000 \\
0.000 \\
-0.028 \\
0.028\end{array}$ & $\begin{array}{r}-0.011 \\
-0.008 \\
0.000 \\
0.000 \\
0.000 \\
0.000 \\
-0.019 \\
0.019\end{array}$ \\
\hline A04 & $\begin{array}{l}\text { GR } \\
\text { T1 } \\
\text { E1 } \\
\text { E2 } \\
\text { P1 } \\
\text { SEIS } \\
\text { GR+T1 } \\
\text { GR+T1+P1+S }\end{array}$ & $\begin{array}{l}0.000 \\
0.018 \\
0.000 \\
0.000 \\
0.000 \\
0.000 \\
0.018 \\
0.019\end{array}$ & $\begin{array}{r}-0.004 \\
0.002 \\
0.000 \\
0.000 \\
0.000 \\
0.000 \\
-0.002 \\
0.002\end{array}$ & $\begin{array}{l}0.000 \\
0.015 \\
0.000 \\
0.000 \\
0.000 \\
0.000 \\
0.015 \\
0.015\end{array}$ & $\begin{array}{l}0.005 \\
0.019 \\
0.000 \\
0.000 \\
0.000 \\
0.000 \\
0.023 \\
0.024\end{array}$ & $\begin{array}{r}0.000 \\
-0.062 \\
0.000 \\
0.000 \\
0.000 \\
0.000 \\
-0.062 \\
0.062\end{array}$ & $\begin{array}{r}-0.016 \\
-0.014 \\
0.000 \\
0.000 \\
0.000 \\
0.000 \\
-0.030 \\
0.030\end{array}$ \\
\hline A05 & $\begin{array}{l}\text { GR } \\
\text { T1 } \\
\text { E1 } \\
\text { E2 } \\
\text { P1 } \\
\text { SE1S } \\
\text { GR+T1 } \\
\text { GR+T1+P1+S }\end{array}$ & $\begin{array}{l}0.000 \\
0.004 \\
0.000 \\
0.000 \\
0.000 \\
0.000 \\
0.004 \\
0.004\end{array}$ & $\begin{array}{r}-0.001 \\
0.005 \\
0.000 \\
0.000 \\
0.000 \\
0.000 \\
0.004 \\
0.005\end{array}$ & $\begin{array}{l}0.000 \\
0.002 \\
0.000 \\
0.000 \\
0.000 \\
0.000 \\
0.002 \\
0.002\end{array}$ & $\begin{array}{l}0.005 \\
0.019 \\
0.000 \\
0.000 \\
0.000 \\
0.000 \\
0.023 \\
0.024\end{array}$ & $\begin{array}{r}0.000 \\
-0.062 \\
0.000 \\
0.000 \\
0.000 \\
0.000 \\
-0.061 \\
0.062\end{array}$ & $\begin{array}{r}-0.016 \\
-0.014 \\
0.000 \\
0.000 \\
0.000 \\
0.000 \\
-0.030 \\
0.030\end{array}$ \\
\hline $\mathrm{A} 06 \mathrm{~N}$ & $\begin{array}{l}\text { GR } \\
\text { T1 } \\
\text { E1 } \\
\text { E2 } \\
\text { P1 } \\
\text { SEIS } \\
\text { GR+T1 } \\
\text { GR+T1+P1+S }\end{array}$ & $\begin{array}{l}0.000 \\
0.004 \\
0.000 \\
0.000 \\
0.000 \\
0.000 \\
0.004 \\
0.004\end{array}$ & $\begin{array}{r}-0.001 \\
0.005 \\
0.000 \\
0.000 \\
0.000 \\
0.000 \\
0.004 \\
0.005\end{array}$ & $\begin{array}{l}0.000 \\
0.002 \\
0.000 \\
0.000 \\
0.000 \\
0.000 \\
0.002 \\
0.002\end{array}$ & $\begin{array}{l}0.005 \\
0.019 \\
0.000 \\
0.000 \\
0.000 \\
0.000 \\
0.023 \\
0.024\end{array}$ & $\begin{array}{r}0.000 \\
-0.062 \\
0.000 \\
0.000 \\
0.000 \\
0.000 \\
-0.061 \\
0.062\end{array}$ & $\begin{array}{r}-0.016 \\
-0.014 \\
0.000 \\
0.000 \\
0.000 \\
0.000 \\
-0.030 \\
0.030\end{array}$ \\
\hline$A 06 \mathrm{~F}$ & $\begin{array}{l}\text { GR } \\
\text { T1 } \\
\text { E1 } \\
\text { E2 } \\
\text { P1 } \\
\text { SEIS } \\
\text { GR+T1 } \\
\text { GR+T1+P1+S }\end{array}$ & $\begin{array}{l}0.000 \\
0.000 \\
0.000 \\
0.000 \\
0.000 \\
0.000 \\
0.000 \\
0.000\end{array}$ & $\begin{array}{l}0.000 \\
0.002 \\
0.000 \\
0.000 \\
0.000 \\
0.000 \\
0.002 \\
0.002\end{array}$ & $\begin{array}{l}0.000 \\
0.000 \\
0.000 \\
0.000 \\
0.000 \\
0.000 \\
0.000 \\
0.000\end{array}$ & $\begin{array}{l}0.000 \\
0.000 \\
0.000 \\
0.000 \\
0.000 \\
0.000 \\
0.000 \\
0.001\end{array}$ & $\begin{array}{r}0.000 \\
-0.007 \\
0.000 \\
0.000 \\
0.000 \\
0.000 \\
-0.006 \\
0.007\end{array}$ & $\begin{array}{l}-0.001 \\
0.001 \\
0.000 \\
0.000 \\
0.000 \\
0.000 \\
0.000 \\
0.000\end{array}$ \\
\hline
\end{tabular}

HNF-2486, Rev. 0

Page C-57 


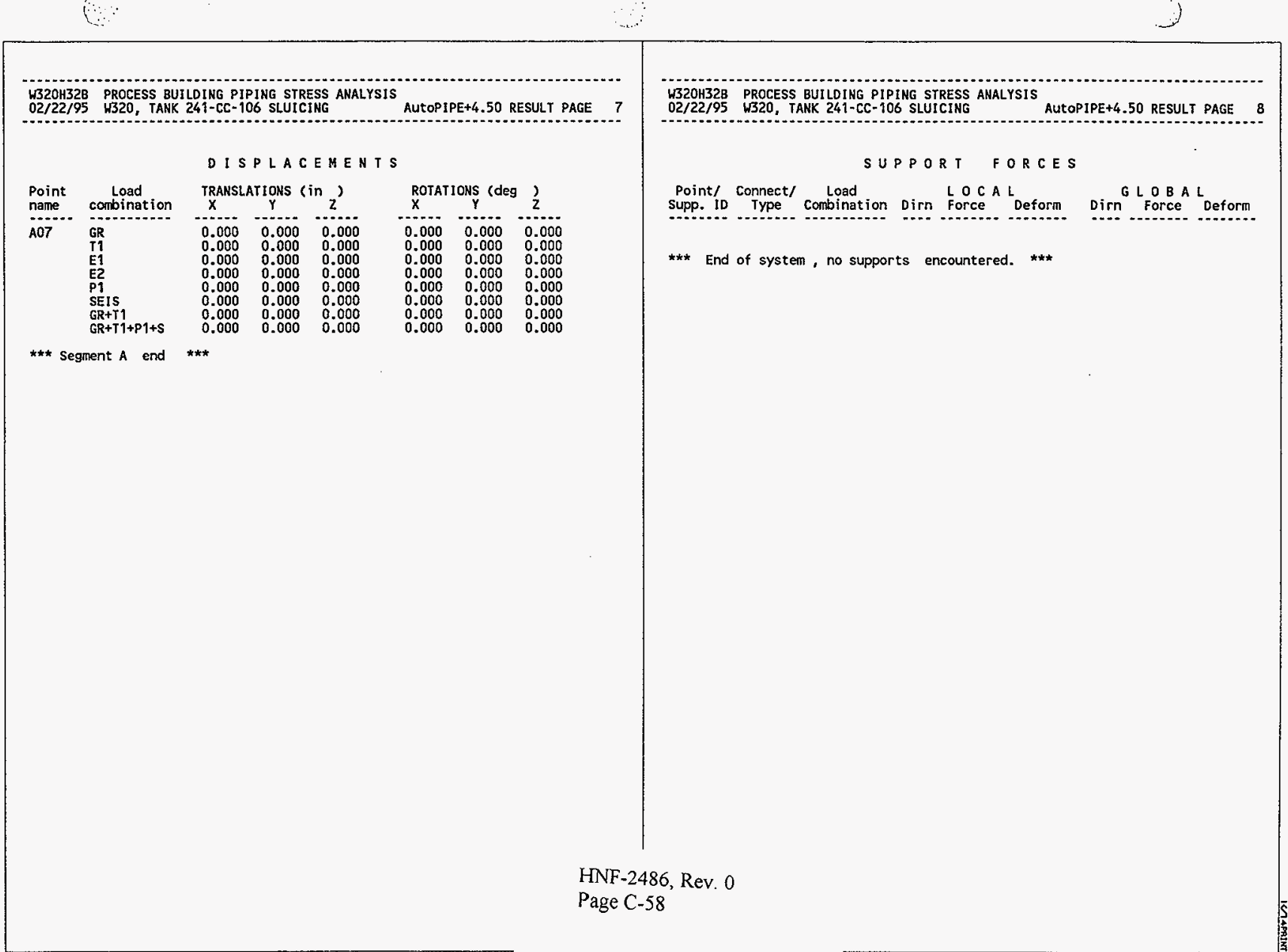


W320H32B PROCESS BUILDING PIPING STRESS AMALYSIS

$02 / 22 / 95$ H320, TANK 241-CC-106 SLUICING

RESTRAINT REACTIONS

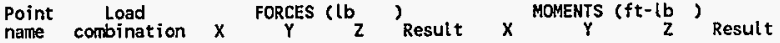

A00 Anchor

$\begin{array}{lrrrrrrrr}\text { Anchor } & 3 & -73 & 6 & 73 & 69 & 9 & -14 & 71 \\ \text { GR } & 459 & 66 & -295 & 550 & 63 & 561 & 92 & 572 \\ \text { I1 } & 4 & 0 & 0 & 4 & 1 & 1 & 1 & 2 \\ \text { E1 } & -1 & 0 & 12 & 12 & -4 & 0 & 0 & 4 \\ \text { E2 } & 4 & 0 & -1 & 1 & 0 & 1 & 0 & 1 \\ \text { P1 } & 4 & 0 & 12 & 13 & 5 & 1 & 1 & 5 \\ \text { SEIS } & 463 & -7 & -289 & 546 & 132 & 570 & 77 & 590 \\ \text { GR+T1 } & 465 \\ \text { GR+T1+P1+S } & 468 & 7 & 302 & 557 & 137 & 572 & 79 & 594\end{array}$

A07

$\begin{array}{lrrrrrrrr}\text { Anchor } & -3 & -151 & -6 & 151 & 34 & 17 & -72 & 81 \\ \text { GR } & -459 & -66 & 295 & 550 & 22 & -423 & 71 & 430 \\ \text { T3 } & 23 & 0 & 0 & 23 & 0 & 0 & -7 & 7 \\ \text { E1 } & 1 & 0 & 15 & 15 & 5 & -3 & 0 & 6 \\ \text { E2 } & -1 & 0 & 1 & 1 & 0 & -2 & 0 & 2 \\ \text { P1 } & 24 & 0 & 15 & 29 & 5 & 3 & 7 & 9 \\ \text { SEIS } & -463 & -217 & 289 & 587 & 55 & -406 & -1 & 410 \\ \text { GR+T1 } & 488 & 218 & 306 & 616 & 61 & 419 & 8 & 416\end{array}$

W320H32B PROCESS BUILDING PIPING STRESS ANALYSIS

02/22/95 W320, TANK 241-CC-106 SLUICING

AUTOPIPE+4.50 RESULT PAGE 10

GLOBAL FORCES\& MOMENTS

Point Load FORCES (lb)

MOMENTS ( $f t-l b$ )

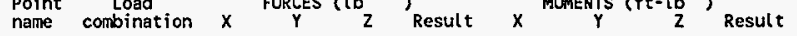

*** Segment $A$ begin $* * *$

A00

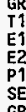

GR
$\mathrm{T} 1$
$\mathrm{E} 1$
$\mathrm{E2}$

P1

SEIS

$\mathrm{GR}+\mathrm{T} 1+\mathrm{P} 1+\mathrm{S}$

A01 N

GR
TI
E1
E2
P1
SEIS
GR+II

GR+T $1+P 1+S$

A01 F

GR
I1
E1
E2
P1

SEIS

$G R+T 1$
$G R+T 1+P I+S$

A02

GR
I1
E1
E2
PI

SEIS

$G R+T 1$

$\mathrm{AO2}$

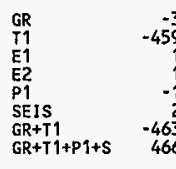

$\begin{array}{rrr}-3 & 73 & -6 \\ -459 & -66 & 295 \\ -4 & 0 & 0 \\ 1 & 0 & -12 \\ -1 & 0 & 1 \\ 4 & 0 & 12 \\ -463 & 7 & 289 \\ 468 & 7 & 302\end{array}$

$\begin{array}{rr}73 & -60 \\ 550 & -63 \\ 4 & - \\ 12 & \\ 1 & \\ 13 & \\ 546 & -13 \\ 557 & 13\end{array}$

$\begin{array}{rr}-69 & -9 \\ -63 & -561 \\ -1 & -1 \\ 4 & 0 \\ 0 & -1 \\ 5 & 1 \\ -132 & -570 \\ 137 & 572\end{array}$

$\begin{array}{rrrr}-3 & 71 & -6 & 71 \\ -459 & -66 & 295 & 550\end{array}$

$\begin{array}{rr}-459 & -66 \\ -3 & 0\end{array}$

0
-11

12

4
-463
468

5

11

1
12
546

$\begin{array}{lr}-70 & -9 \\ -7 & -561\end{array}$

-1
2
0
3
-77
80

-1
0
-1
1
-570
572

$\begin{array}{rr}-3 & 66 \\ -459 & -66\end{array}$

-6
295
-1

1
11
289

67

-55
50

-4 i

$\begin{array}{ll}289 & 546 \\ 301 & 556\end{array}$

$\begin{array}{rr}-6 & 55 \\ 295 & 550 \\ 0 & 1 \\ -9 & 10\end{array}$

-9
1

10
289

1
10
546
554

-17
-17
-1
-1

$\begin{array}{rr}-6 & 33 \\ 295 & 550 \\ 0 & 1 \\ -7 & 7 \\ 1 & 1 \\ 7 & 7 \\ 289 & 547 \\ 297 & 554\end{array}$

-17
-1
-1
-10
11 $\begin{array}{rr}-6 & 73 \\ 295 & 550 \\ 0 & 4 \\ -12 & 12 \\ 1 & 1 \\ 12 & 13 \\ 289 & 546 \\ 302 & 557\end{array}$
-8
-446
0
0

-1
0
-454

$\begin{array}{rr}14 & 7 \\ -92 & 57 \\ -1 & \\ 0 & \\ 0 & \\ 1 & \\ -77 & 59 \\ 79 & 59\end{array}$

$\begin{array}{rr}15 & 72 \\ -5 & 56\end{array}$

$\begin{array}{rr}-1 & 1 \\ 0 & 2\end{array}$

$\begin{array}{rr}1 & 1 \\ 10 & 575\end{array}$

11578

$\begin{array}{rr}16 & 57 \\ 110 & 462 \\ 0 & 1\end{array}$

0
0
0

126
126

471
472

$-4 \quad 16 \quad 18$

$\begin{array}{rr}-4 & 16 \\ 22 & 1100 \\ 2 & 0 \\ -1 & 0 \\ 0 & 0 \\ 3 & 0 \\ 18 & 126 \\ 21 & 126 \\ -4 & 16 \\ 22 & 110 \\ 2 & 0 \\ -1 & 0 \\ 0 & 0 \\ 3 & 0 \\ 18 & 126 \\ 21 & 126 \\ & \end{array}$ 
W320H328 PROCESS BUILDING PIPING STRESS ANALYSIS

02/22/95 W320, TANK 241-CC-106 SLUICING

AUTOPIPE+4.50 RESULT PAGE 11

GLOBAL FORCES \& MOMENTS

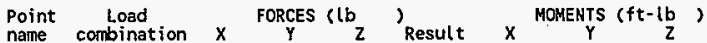

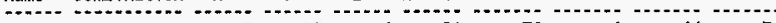

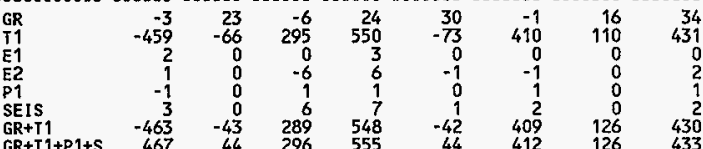

$\mathrm{A} 03 \mathrm{~F}$

GR
T1
E1
E2
P1 SEIS $G R+T 1$ -463
468

A04.

GR

PI SEIS $G R+T 1$
$G R+T 1+P 1+S$

A04

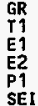

PEIS GR+T?

$G R+T\{+P 1+S$

A05

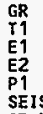

$T 1$
E1
E2
P1

SEIS

$\mathrm{GR}+\mathrm{T}\}+P 1+S$

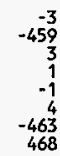

$\begin{array}{rrr}19 & -6 & 20 \\ -66 & 295 & 550\end{array}$

$\begin{array}{rrr}-66 & 295 & 5 \\ 0 & 0 & \\ 0 & -5 & \end{array}$

0
0
-47

28

$\begin{array}{rrrr}-3 & 0 & -6 & 7 \\ -459 & -66 & 295 & 550 \\ 5 & 0 & 0 & 5 \\ 1 & 0 & -3 & 3 \\ -1 & 0 & 1 & 1 \\ 6 & 0 & 3 & 7 \\ -463 & -66 & 289 & 550 \\ 470 & 67 & 293 & 558\end{array}$

$\begin{array}{ll}-3 & -12 \\ -459 & -66\end{array}$

-4
7
-1
-1
-463
471

0
0
-78
78

$\begin{array}{rr}-6 & 95 \\ 295 & 550 \\ -1 & \end{array}$

$\begin{array}{rr}1 & \\ 2 & \\ 289 & 551\end{array}$

$\begin{array}{rr}-3 & -1 \\ -459 & -66 \\ 21 & \\ -1 & \end{array}$

-1
22

-463
486

-6
295
0
13
1
13
289
304 $\begin{array}{rr}36 & 1 \\ -89 & 451 \\ 0 & 0 \\ -1 & 0 \\ 0 & 1 \\ 1 & 1 \\ -54 & 452 \\ 55 & 454\end{array}$

$\begin{array}{rrr}11 & 21 & 42 \\ 451 & 93 & 469\end{array}$

$\begin{array}{rr}36 & 10 \\ -89 & -54 \\ -0 & 0\end{array}$

-1
0
1
-54

-54
55

36
-89
0

-1
0

1
-54
55

-54
0
7
-1
-4
-44
5 $\begin{array}{rr}36 & 16 \\ -89 & -349\end{array}$

$\begin{array}{rr}-89 & -349 \\ -1 & 0 \\ -1 & 1\end{array}$

$\begin{array}{rr}0 & -2 \\ 1 & 1 \\ -54 & -333 \\ 55 & 336\end{array}$ 469
471

$\begin{array}{rr}37 & 52 \\ -20 & 107\end{array}$

0

17
18

7
71

1
0
0

1
17

$\begin{array}{lr}-36 & 53 \\ -86 & 37\end{array}$

2

0
2
-122
124

2
359
362
W32OH32B PROCESS BUILOING PIPING STRESS ANALYSIS

W2/22/95 W320, TANK 241-CC-106 SLUICING

AUTOPIPE +4.50 RESULT PAGE 12

GLOBAL FORCES \& MOMENTS

Point Load FORCES (lb ) MOMENTS (ft-lb)

name combination $X \quad Y \quad Z$ Result $X \quad Y \quad Z$ Result n05

$\begin{array}{rrrr}36 & 16 & -36 & 53 \\ -89 & -349 & -86 & 371 \\ 0 & 0 & 2 & 2 \\ -1 & 1 & 0 & 1 \\ 0 & -2 & 0 & 2 \\ 1 & 1 & 2 & 2 \\ -54 & -333 & -122 & 359 \\ 55 & 336 & 124 & 362 \\ 36 & 16 & -36 & 54 \\ -89 & -350 & -86 & 371 \\ 0 & 0 & 2 & 2 \\ -1 & 1 & 0 & 1 \\ 0 & -2 & 0 & 2 \\ 1 & 1 & 2 & 2 \\ -54 & -334 & -122 & 360 \\ 55 & 337 & 125 & 363 \\ 34 & 17 & -72 & 82 \\ -16 & -423 & 12 & 424 \\ 0 & 0 & -4 & 4 \\ 3 & -3 & 0 & 4 \\ 0 & -2 & 0 & 2 \\ 3 & 3 & 4 & 6 \\ 18 & -406 & -60 & 411 \\ 22 & 411 & 65 & 417 \\ 34 & 17 & -72 & 81 \\ 22 & -423 & 71 & 430 \\ 0 & 0 & -7 & 7 \\ 5 & -3 & 0 & 6 \\ 0 & -2 & 0 & 2 \\ 5 & 3 & 7 & 9 \\ 55 & -406 & -1 & 410 \\ 61 & 411 & 8 & 416 \\ & & & \end{array}$

$\begin{array}{rrrr}23 & 0 & 0 & 23 \\ 1 & 0 & 15 & 15 \\ -1 & 0 & 1 & 1 \\ 23 & 0 & 15 & 28 \\ 463 & -211 & 289 & 585\end{array}$

$\begin{array}{rr}23 & 0 \\ -463 & -211 \\ 487 & 212\end{array}$

$\begin{array}{ll}289 \\ 305 & 585\end{array}$

$\begin{array}{rr}-3 & -149 \\ -459 & -66 \\ 23 & 0\end{array}$

$-7$

.215

$-6$

$\begin{array}{rr}-6 & 150 \\ 295 & 550 \\ 0 & 23\end{array}$

15
15

15
289

289
306

-151
-66
0

0
0
-217

$\begin{array}{rr}-6 & 151 \\ 295 & 550 \\ 0 & 23 \\ 15 & 15\end{array}$

15
1
15

$306 \quad 587$

145
50
23
15
1
28
585
613
1
2
2
359 62 2 4
4
2
6
11
17
81
30
7
6
2
9
10
16
*** Segment $A$ end ***

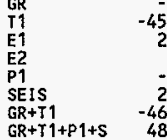

A06 N

GR
T1
E1
E2
P1
SEIS
GR+

$37 \quad 52$

GR+T1

GR+Ti+P $1+S$

A06 F $G$

GR
E1
E2
P1

SE1S

GR+T $1+P 1+S$

A07

GR
T1
E1
E2

PI

SEIS.

GR+T

53

1 $\begin{array}{rrr}-3 & -145 & \\ -459 & -66 & 2 \\ 23 & 0 & \\ 1 & 0 & \\ -1 & 0 & \\ 23 & 0 & \\ 463 & -211 & 289 \\ 487 & 212 & 305\end{array}$

$\begin{array}{rr}-6 & 145 \\ 295 & 55 \\ 0 & 23 \\ 15 & \\ 1 & \\ 15 & \\ 289 & 58 \\ 305 & 6\end{array}$

$\begin{array}{rr}-3 & -145 \\ -459 & -66\end{array}$

$\begin{array}{rr}-6 & 145 \\ 295 & 550\end{array}$
HNF-2486, Rev. 0

Page C-60 
02/22/95 H320. TANK 241-CC-106 SLUICING

ASME B31.3C (1992) CODE COMPLIANCE

(Moments in $\mathrm{ft}-\mathrm{lb}$ ) (Stress in psi)

Point Load In-Pl. Out-PL. Torsion S.I.F Eq. Load code Code

Point Load
name combination Moment Moment Moment in out no. type stress Allow.

(1)

\section{*** Segment $A$ begin ***}

$\mathrm{AOO}$

Max $P$
GR + Max $P$
Cold to T1
SUs. + E1
SUS. + E2
SEIS
SUS+SEIS

A01 N- Max P

Cold to T1

Sus. + E1

SEIS + E2

SUS+SEIS

A01 $N+\operatorname{Max} P$

$\mathrm{GR}+\operatorname{Max} \mathrm{P}$

Cold to

Sus. + E1

SUIS. +

SUS+SEIS

A01 F- $\operatorname{Max} P$

$\begin{array}{lrr}\text { GR }+ \text { Max } P & 55 & 8 \\ \text { Cold to T1 } & 50 & 446 \\ \text { SUS. }+ \text { E1 } & 55 & 8 \\ \text { SUS. }+ \text { E2 } & 55 & 8 \\ \text { SEIS } & 1 & 0 \\ \text { SUS+SEIS } & 56 & 8\end{array}$

A01 $F+\operatorname{Max} P$

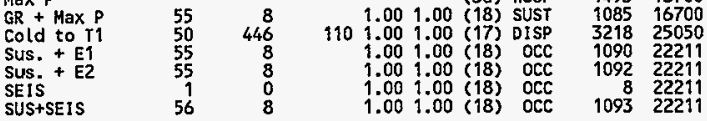

HNF-2486, Rev. 0

Page C-61

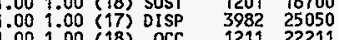
1.001 .00 (18) OCC 123222211 $1.001 .00(18)$ OCC $1.001 .00(18)$ OCC

(3a) 1.001 .00 (18) SUST 11.001 .00 (17) DISP 1.001 .00 (18) OCC 1.001 .00 (18) OCC 1.001 .00 (18) OCC

(3a) HOOP 2.331 .94 (18) SUST 12.331 .94 (17) DISP $\begin{array}{llll}2.33 & 1.94 & (18) & \text { OCC } \\ 2.33 & 1.94(18) & \text { OCC }\end{array}$ 2.331 .94 (18) OCC

(3a) 100 2.331 .94 (18) SUSY $2.331 .94(18)$ OCC 2.331 .94 (18) OCC 2.339 .94 (18) OCC $\begin{array}{ll}1234 & 22211 \\ 22211\end{array}$ $1493 \quad 16700$ 121016700 390625050 122522211 122622211

149316700 192016700 193322211 $1954 \quad 22211$ $1956 \quad 22211$

149316700 $1634 \quad 16700$ 612925050 164922211 $\begin{array}{rr}18 & 22211 \\ 1653 & 22211\end{array}$

149316700

16700 22211 09322211
AUTOPIPE+4.50 RESULT PAGE 13

W320H32B PROCESS BUILDING PIPING STRESS ANALYSIS

02/22/95 W320, TANK 241-CC-106 SLUICING

AutOPIPE+4.50 RESULT PAGE 14

ASME B31.3C (1992) CODE COMPLIANCE

(Moments in $\mathrm{ft}$-lb) (Stress in psi )

point Load In-pl. Out-pl. Torsion S.I.F Eq. Load Code Code

name combination Moment Moment Moment in out no. type stress Allow.

A02

GR + Max P

Cold to Th

Sus. $+\mathrm{E} 1$

Sus. + E2

SUS+SEIS

17
A03 N- Max P GR + Max P Cold to T? Sus. + E1 SEIS + SUS+SEIS

A03 N+Max P

$\begin{array}{lrr}\text { GR + Max P } & 1 & 30 \\ \text { Cold to T1 } & 410 & 73 \\ \text { Sus. + E1 } & 2 & 31 \\ \text { Sus. + E2 } & 3 & 31 \\ \text { SEIS } & 1 & 3 \\ \text { SUS+SEIS } & 3 & 31\end{array}$

A03 F- Max P GR + Max P Cold to T1 Sus. + E1 SEIS SUS+SE IS

A03 F+ Max P $G R+\operatorname{Max} P$ Cold to $T 1$ Sus. + E1 Sus. + E SEIS SUS+SEIS

A04 $\operatorname{Max} P$ $G R+\operatorname{Max} P$ Cold to Ti Sus. + El Sus. + SUS+SEIS
1.001 .00 (18) SUST 1.001 .00 (17) DISP $1.001 .00(18)$ OCC $1.001 .00(18)$ OCC 1.001 .00 (18) OCC

(3a) HOOP 1.001 .00 (18) SUST 1.001 .00 (17) DISP $1.001 .00(18)$ OCC $\begin{array}{lll}1.00 & 1.00(18) & \text { OCC } \\ 1.00 & 1.00(18) & \text { OCC }\end{array}$

(3a) HOOP 2.331 .94 (18) SUST 2.331 .94 (17) DISP 2.331 .94 (18) OCC 2.331 .94 (18) OCC

(3a) HOOP 2.331 .94 (18) SUST 2.331 .94 (17) DISP 2.331 .94 (18) OCC 2.331 .94 (18) OCC

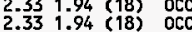

(3a) HOOP 1.001 .00 (18) SUST 891.001 .00 (17) DISP 1.001 .00 (18) OCC 1.001 .00 (18) OCC $\begin{array}{lll}1.001 .00(18) & \text { OCC } \\ 1.001 .00(18) & \text { OCC }\end{array}$

734
78700 78825050 $748 \quad 22211$ 162221 $750 \quad 22211$

149316700 90016700 299925050 902 22211 $12 \quad 22211$ $912 \quad 22211$

$1493 \quad 16700$ 111616700 676425050 $1122 \quad 2211$ 11432221 114322211

$1493 \quad 16700$ $\begin{array}{rr}977 & 16700 \\ 7448 & 25050\end{array}$ $7448 \quad 25050$ 986 2221 98722211

$1493 \quad 16700$ 82816700 326825050 8302221 83022211 $\begin{array}{ll}4 & 22211 \\ 832 & 22211\end{array}$ $\begin{array}{rr}1.00 & 1.00 \text { (3a) HOOP } \\ \text { (18) SUST } & \text { SU }\end{array}$ $891.001 .00(17)$ DISP 1.001 .00 (18) OCC $1.001 .00(18)$ OCC $1.001 .00(18)$ OCC
$1.001 .00(18)$ OCC
$1493 \quad 16700$ 95716700 96522211 $1006 \quad 2221$ $1007 \quad 22211$ 
W32OH32B PROCESS BUILDING PIPIHG STRESS ANALYSIS

02/22/95 W320, TANK 241-CC-106 SLUICIKG

AUTOPIPE+4.50 RESULT PAGE 15

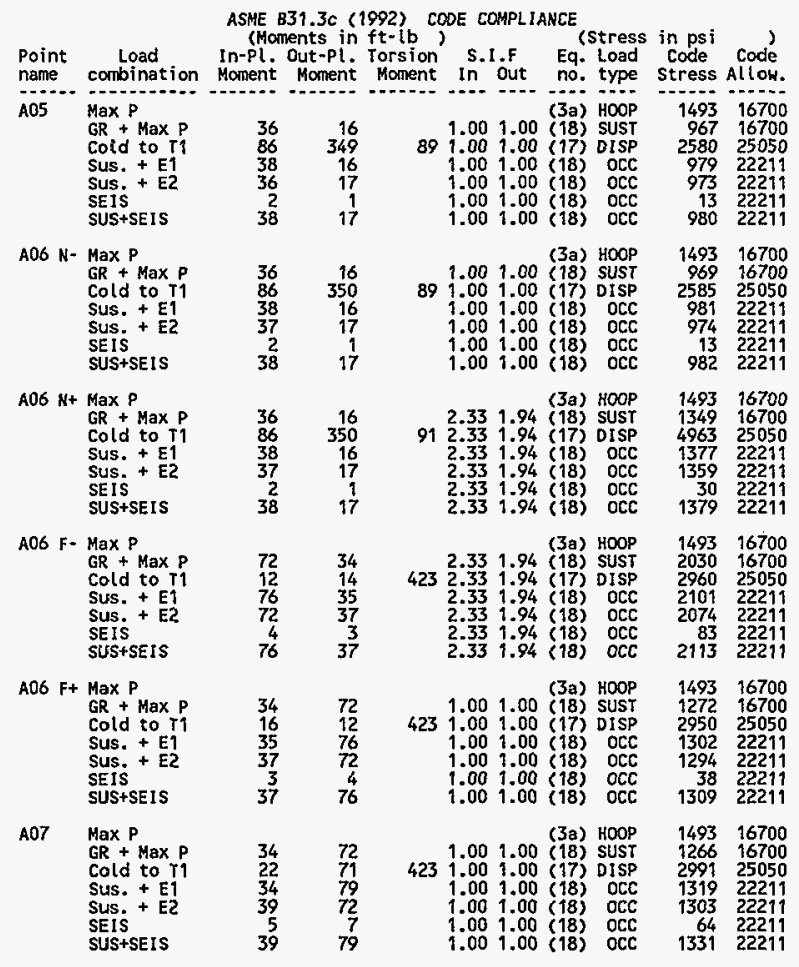

W320H32B PROCESS BUILDING PIPING STRESS ANALYSIS

02/22/95 W320, TANK 241-CC-106 SLUICING

AUtOPIPE+4.50 RESULT PAGE 16
Maximum displacenents (in)

$\begin{array}{ll}\text { Maximum X: } & 0.044 \\ \text { Maximum Y : } & 0.011 \\ \text { Maximum Z : } & 0.035 \\ \text { Max. total : } & 0.056\end{array}$

Maximum Z:- 0.035

Maximum rotations (deg)

$\begin{array}{ll}\text { Maximum X : } & 0.024 \\ \text { Maximum Y : } & 0.108 \\ \text { Maximum Z : } & 0.030 \\ \text { Max. total: } & 0.109\end{array}$

Max. total:

Maximum restraint forces(lb)

$\begin{array}{lll}\text { Maximum } x: & 488 & \text { Point : A07 } \\ \text { Maximum } y: & 218 & \text { Point : A07 } \\ \text { Maximum Z : } & 306 & \text { Point :A07 } \\ \text { Max. total: } & 616 & \text { Point : A07 }\end{array}$

Maximun restraint moments $(f t-l b)$

$\begin{array}{lr}\text { Maximum } x: & 137 \\ \text { Maximum } y: & 572 \\ \text { Maximum } z: & 92 \\ \text { Max. total: } & 594\end{array}$

Point : $\mathrm{A03} \mathrm{N}$ Point : $A 03$ F Point : $\mathrm{A03} F$

Point : A06 N Point : $\mathrm{AO2}$

Point : $\mathrm{AO}^{4}$
Point : $\mathrm{A02}$
Point : $A 00$ Point : AOO Point : $A 00$ Point : AOO
Load Comb.: GR+Ti+P $\}+S$ Load Comb.: GR+T1+P $1+S$ Load Comb.: $G R+T 1+P 1+S$

Laad Comb.: GR+TI+PI+S Load Comb.: $G R+T 1+P 1+S$

Load Comb.: $\mathrm{GR}+\mathrm{T} 1+\mathrm{P} 1+\mathrm{S}$

Load Comb.: GR+Ti+P $1+S$ Load Comb.: $G R+T i+P \uparrow+S$ Load Comb.: GR+T1+P1+S

Load Comb.: GR+T1+P1+S

Load Comb.: $\mathrm{GR}+\mathrm{T}\}+\mathrm{P}\}+\mathrm{S}$ Load Comb.: GR+T1+P1+S Load Comb.: T1 Load Comb.: GR+r1+P1+S

*** Segment $A$ end

HNF-2486, Rev. 0

Page C-62 


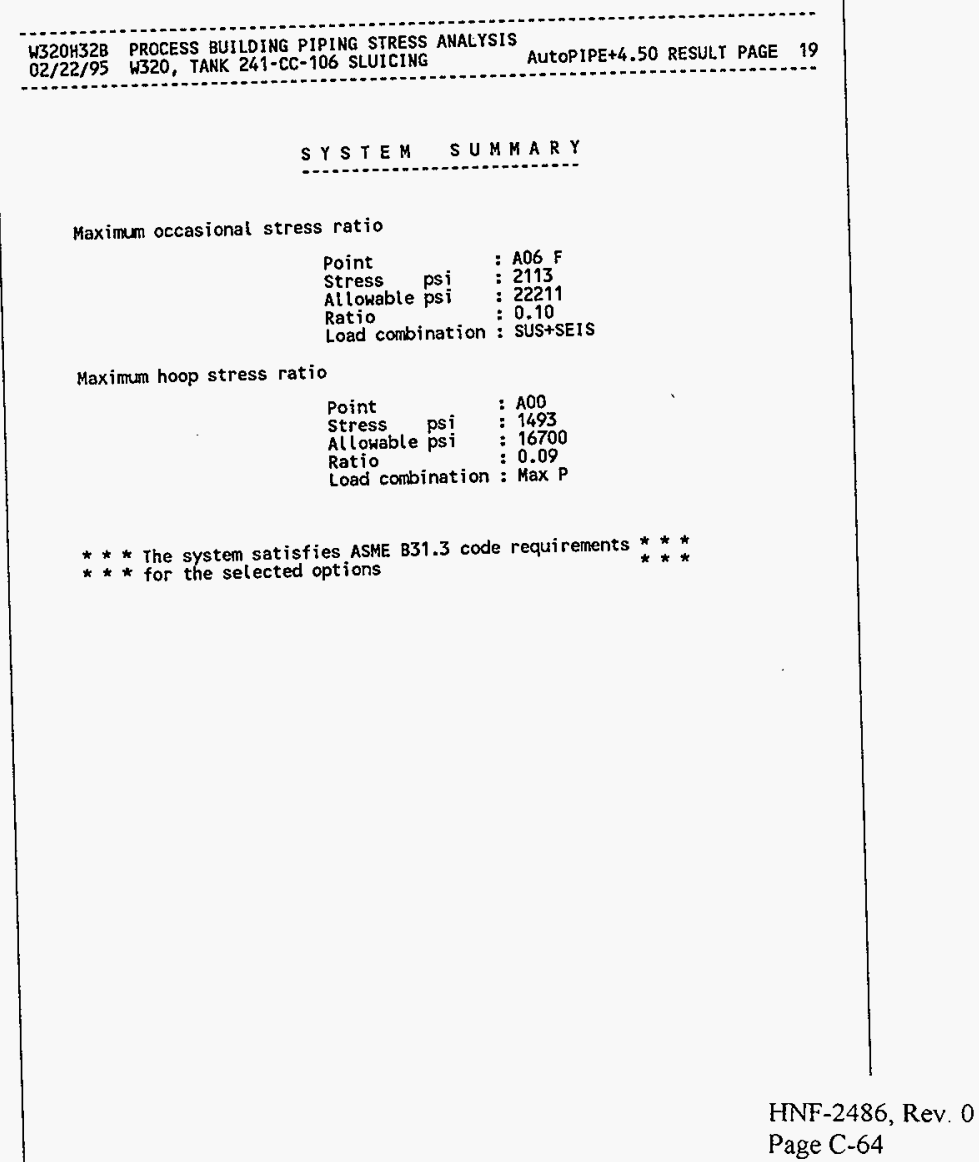


H320H32C PROCESS BUILOING PIPING STRESS ANALYSIS ICF KAISER HANFORD COMPANY $02 / 22 / 95$ W320. TANK 241-C-106, ER4319 H-2-818480 AUTOPIPE+4.50 MOOEL PAGE 02

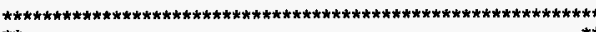 \\ $\star \star *$ \\ ** \\ AUTOPIPE SYSTEM DATA LISTING \\ $* *$}

SYSTEM NAME : $\mathbf{W} 320 \mathrm{H} 32 \mathrm{C}$ PROJECT ID : PROCESS BUILDING PIPING STRESS ANALYSIS
W320, TANK 241-C-106, ER4319 $\mathrm{H}-2-818480$

PREPARED BY :

RC CANPBELL

CHECKED BY :

PIPING CODE : $B 31.3$

AMBIENT TEMP. $(\operatorname{deg} F): 50.0$

COMPONENT LIBRARY : AUTOPIPE

MATERIAL LIBRARY : AUTOB313

MODEL REVISION NUMBER : 20
W32OH32C PROCESS BUILDING PIPING SIRESS ANALYSIS ICF KAISER HANFORD CONPANY 02/22/95 W320, TANK 241-C-106, ER4319 H-2-818480 AUTOPIPE+4.50 MOOEL PAGE

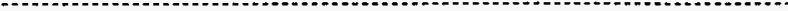

POINT DATA LISTING

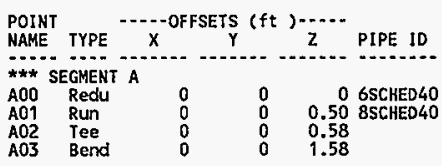

Long Elbow, Radius $=12.00$ inch Bend angle change $=90.00 \mathrm{deg}$

$\begin{array}{lllll}\text { A04 } & \text { Redu } & -1.33 & 0 & 0 \\ \text { A05 } & \text { Run } & -1.33 & 0 & 0 \\ \text { A06 } & \text { Redu } & -1.00 & 0 & 0 \\ \text { A07 } & \text { Run } & -1.33 & 0 & 0 \\ \text { A08 } & \text { Bend } & -1.33 & 0 & 0\end{array}$

A08 Bend -1.3

Long Elbow, Radius $=12.00$ inch Bend angle change $=90.00$ deg

$\begin{array}{lllll}\text { A09 } & \text { Run } & 0 & 0 & -1.32 \\ \text { A10 } & \text { Run } & 0 & 0 & -3.34 \\ \text { A11 } & \text { Flex } & 0 & 0 & -0.50\end{array}$

$W t=35 \mathrm{tb}, A r=74.4 \mathrm{sq}$. in Axial $=1040.0$ rors= RIGI $\mathrm{Y}$-shear= RIGID Bend= RIGID Z-Shear= RIGID $\quad$ Bend= RIGIO

$\begin{array}{lcrrr}\text { A12 Run } & 0 & 0 & -0.50 \\ \text { *** SEGMENT B } & & & \\ \text { A02 } & \begin{array}{c}\text { Tee } \\ 801\end{array} & 0 & 0 & 1.08 \text { BSCHED } 40 \\ \text { Bend } & 0 & 1.25 & 0\end{array}$

Long Elbow, Radius $=12.00$ inch Bend angle change $=90.00 \mathrm{deg}$
SIF - in $=2.44$, Out $=2.03$

Total weight of empty pipes : 457 tb

HNF-2486, Rev. 0

Page C-65 
W320H32C PROCESS BUILDING PIPING STRESS ANALYSIS ICF KAISER HANFORD COMPANY 02/22/95 H320, TAHK 241-C-106, ER4319 H-2-818480 AUTOPIPE+4.50 MODEL PAGE

COMPONENT DATA LISTING

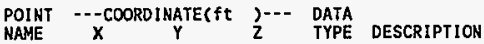

*** SEGMENT A

$\begin{array}{cccc}\text { AOO } \quad 0.00 & 0.00 \quad 0.00 \text { ANCHOR Rigid } \\ \text { Thermal movenents : None } & \text { SIF } \\ \text { FLANGE Weld neck } & \end{array}$

$01 \quad$ Rating $=150$, Weight $=24 \mathrm{ib}$

$\begin{array}{lllll}\text { A01 } & 0.00 & 0.00 & 0.50 & \\ \text { A02 } & 0.00 & 0.00 & 1.08 \text { TEE } & B 16.9 \text { welding tee }\end{array}$

SIF - In $=1.63$, Out $=1.84$

$\begin{array}{llll}\mathrm{A} 03 \mathrm{~N} & 0.00 & 0.00 & 1.67 \\ \mathrm{~A} 03 & 0.00 & 0.00 & 2.67\end{array}$

$\begin{array}{llll}\mathrm{A} 03 & 0.00 & 0.00 & 2.67 \\ \mathrm{~A} 03 & -1.00 & 0.00 & 2.67\end{array}$

$\begin{array}{llll}\text { AO4 } & -1.33 & 0.00 & 2.67 \\ & & \text { FLANGE Weld neck } & \text { SIF }=1.00\end{array}$

Rating $=150$, Weight $=39 \mathrm{lb}$

FLANGE Weld neck SIF $=1.00$

A05 $-2.66 \quad 0.00 \quad 2.67$ ANCHOR Rating $=150$, weight $=39$ tb

2.67 ANCHOR Rigid movements : None

$\begin{array}{llll}\text { A06 } & -3.66 \quad 0.00 \quad 2.67 \text { ANCHOR Rigid }\end{array}$

A07 $\quad-5.00 \quad 0.00 \quad 2.67$ FLANGE Weld neck

theck 150 , weight $=39$ lib

FLANGE Weld neck $S: F=1.00$ $\begin{array}{lllll}\text { A08 N } & -5.33 & 0.00 & 2.67 & \text { Rating }=150 \text {, Weight }=39 \mathrm{lb} \\ \text { A08 } & -6.33 & 0.00 & 2.67 \mathrm{TI} & \\ \text { A08 F } & -6.33 & 0.00 & 1.67 & \\ \text { A09 } & -6.33 & 0.00 & 1.34 & \\ \text { A10 } & -6.33 & 0.00 & -2.00 \mathrm{Y}-\mathrm{STOP} \text { ID : A10 1, Connected to Ground } \\ \text { Gap-Below }=0.00, \text { Above }=1.50 \text { inch }\end{array}$ Friction $=0.00$

Gaps set Weightless

A11 $-6.33 \quad 0.00 \quad-2.50$ FLANGE Weld neck $\quad$ SIF $=1.00$

A12 $-6.33 \quad 0.00-3.00$ ANCHOR Rigid $=150$, weight $=39 \mathrm{lb}$

Thermal movements : None

FLANGE Weld neck
Rating $=150$, weight $=39 \mathrm{li}$ (b

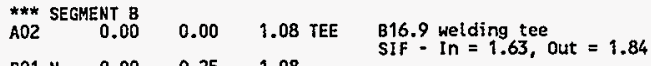

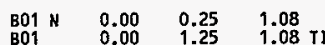

B01 F $0.00 \quad 1.25$

$\begin{array}{lllll}\mathrm{B} 02 & 0.00 & 1.25 & 2.08 & \text { Y-STOP ID : } 802 \quad 1 \text {, Connected to Ground }\end{array}$ Gap-Below $=0,00$, Above $=1.50$ inch Friction $=0.00$

B03

$0.00 \quad 1.25$
Gaps set Heightless

3.08 FLANGE Weld neck SIF $=1.00$

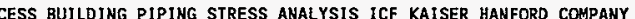
O2/22/95 W320, TANK 241-C-106, ER4319 H-2-818480 AUTOPIPE 44 .50 MODEL PAGE

COMPONENT DATA LISTING

POINT ---COORDINATE(ft, $)--$ DATA

NAME $X \quad Y \quad z$ TYPE DESCRIPTION Rating $=150$, Weight $=39 \mathrm{lb}$

Number of points in the system : 23

HNF-2486, Rev. 0

Page C-66 


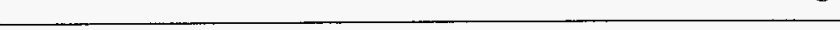
W320H32C PROCESS BUILDING PIPIHG STRESS ANALYSIS ICF KAISER HANFORD COHPANY
$02 / 22 / 95$
W320, TANK $24 \%-\mathrm{C}-106$, ER4319 H-2-818480 AUTOPIPE+4.50 MODEL PAGE 5

02/22/95 W320, TANK 241-C-106, ER4319 H-2-81B4BO AUTOPIPE+4.50 MODEL PAGE 5

\section{PI PE DATA L I ST I N G}

Pipe ID/ Nom/ O.D. ...--Thickness(inch)-...- Spec Weight(lb/ft ) Material Sch inch W.Th. Corr Mill Insuling Grav Pipe other Total

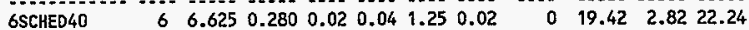
A312-TP304L $40 \mathrm{~S}$
8SCHED 40
$\begin{array}{lllllll}8 & 8.625 & 0.322 & 0.02 & 0.04 & 1.25 & 0.02\end{array}$
0 $\quad 29.22 \quad 4.13 \quad 33.36$ A312-TP304L 40
HEATER
NS $29.940 \quad 0.134 \quad 0.02 \quad 0.01 \quad 1.25 \quad 0.02 \quad 0 \quad 43.82 \quad 14.48 \quad 58.30$
$0 \quad 43.8214 .4858 .30$

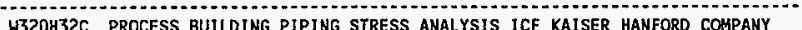

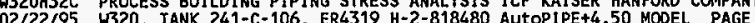
02722/95 W320, JANK $241-C-106$, ER4319 H-2-818480 AUtOP1PE+4.50 MODEL PAGE

\section{MATERIAL DATA LISTING}

Material Density Pois. Temper. Modulus Expans. Allow. Name Pipe 10 lb/cu.ft Ratio deg F E6 psi in/100ft psi $\begin{array}{llllll}\text { A312-TP304L 6SCHED } 40 & 501.0 & 0.30 & 50.0 & 28.38 & 16700.0\end{array}$

$\begin{array}{llllll} & & 180.0 & 1.4413 & 16700.0\end{array}$

$\begin{array}{lrrrrr}\text { A312-TP304L 8SCHED } 40 \quad 501.0 & 0.30 & 50.0 & 28.38 & 16700.0\end{array}$

$\begin{array}{lllrlrr}\text { A312-TP304L HEATER } \quad 501.0 & 0.30 & 50.0 & 28.38 & 16700.0\end{array}$




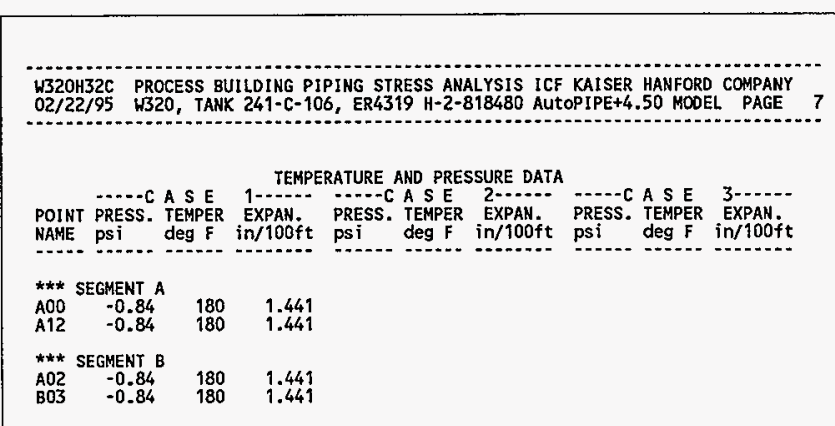



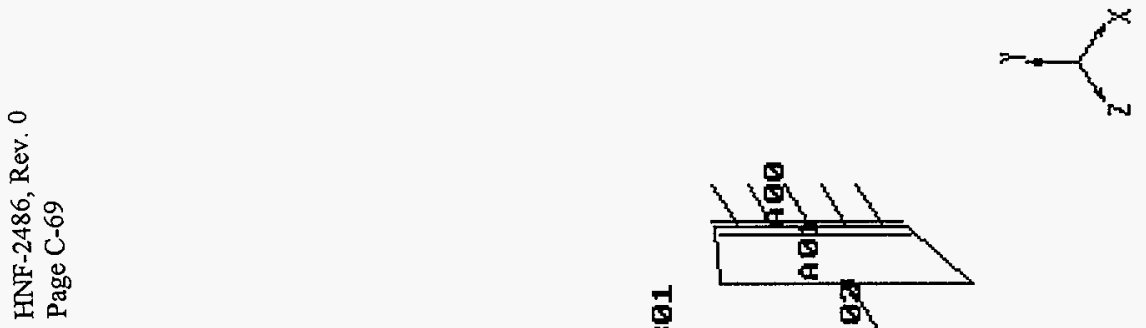

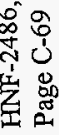
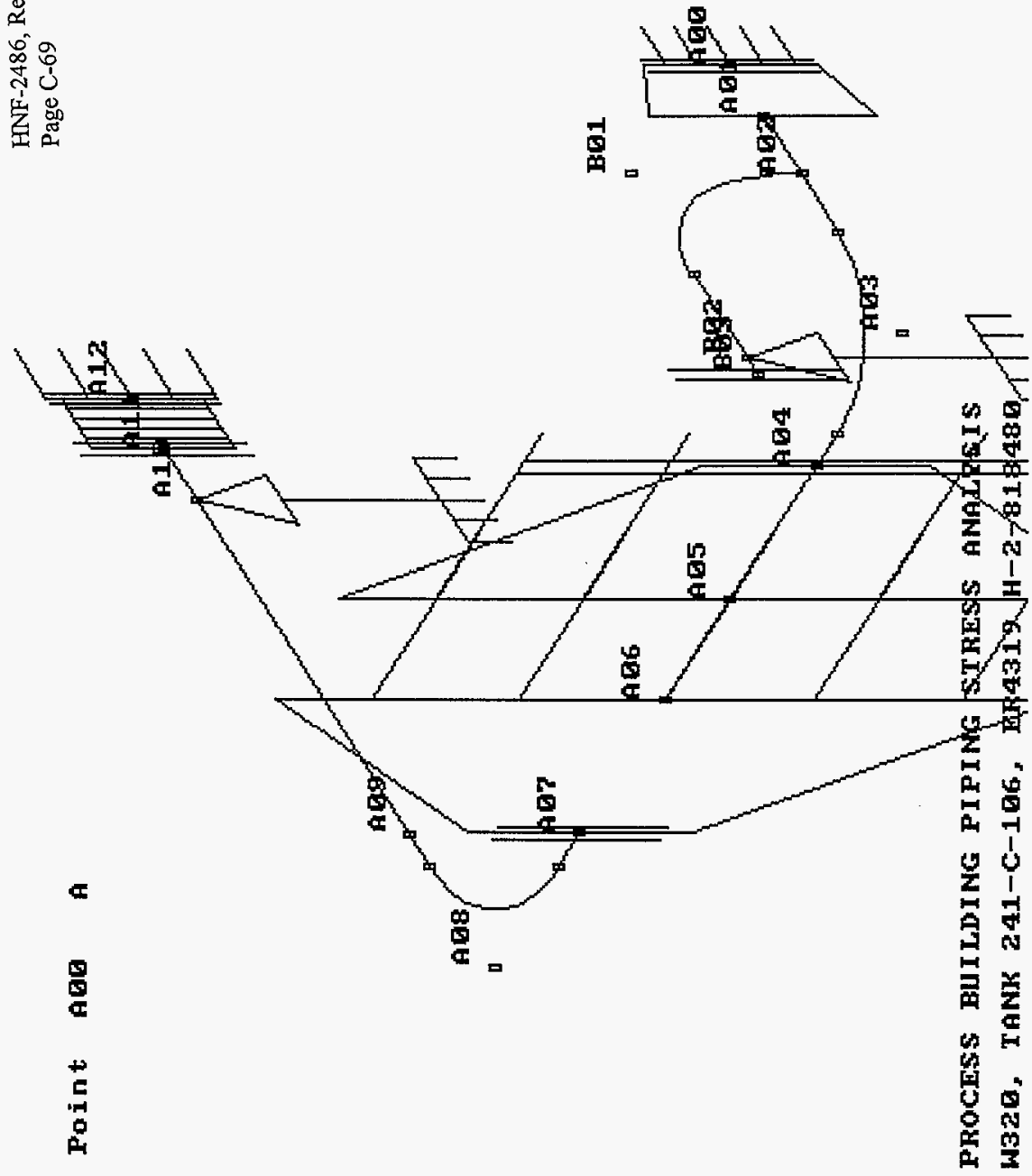
32OH32C PROCESS BUILOING PIPING STRESS ANALYSIS

2/22/95 H320, TANK 241-C-106, ER4319 H-2-818480 AUtOPIPE+4.50 RESULT PAGE

\section{A NALYSIS SUMMARY}

Current model revision number : 20

Static - Date and Time of analysis .......... feb 22, $1995 \quad 2: 48$ PM Model Revision Number .................20

Load cases analyzed .................... GR T1 E1 E2

Load cases analyielding considered ..... Yes

Tolerance - Force, Displacement ........ 100.00 b 0.0162 in

Tolerance - Force, Displacement ......... 100 00 ib 10

Hanger design run, tolerance........... No

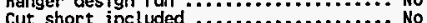

Docasional loed analysis type............. Nonl inear

occasional load analysis type $\ldots \ldots \ldots \ldots$....... Nontinear

Non- load cases for nont inear analysis

$$
\begin{aligned}
& \mathrm{GR}=\text { None } \\
& \mathrm{T} 1=\mathrm{GR} \\
& \mathrm{E} 1=\mathrm{GR} \\
& \mathrm{E2}=\mathrm{GR}
\end{aligned}
$$

Weight of contents included Yes

Pressure stiffening case

Hater elevation for buoyancy ioads ..... Not considered
HNF-2486, Rev. 0

Page $\mathrm{C}-70$
W320H32C PROCESS BUILDING PIPING STRESS ANALYSIS

\begin{tabular}{|c|c|c|c|c|c|c|}
\hline $\begin{array}{l}\text { Combination } \\
\mathbf{G R}+\operatorname{Max} \mathbf{P}\end{array}$ & $\begin{array}{l}\text { Category } \\
\text { Sustain }\end{array}$ & $\begin{array}{l}\text { Nethod } \\
\text { Sum }\end{array}$ & $\begin{array}{l}\text { Load } \\
\text { Gravity } \\
\text { Max Long }\end{array}$ & $\begin{array}{l}\text { Factor } \\
1.00 \\
1.00\end{array}$ & $\begin{array}{l}\text { Allowable } \\
\text { Automatic }\end{array}$ & $\begin{array}{l}\text { Remarks } \\
\text { Default }\end{array}$ \\
\hline Cold to $\mathrm{TI}$ & Expansion & sum & Thermal 1 & 1.00 & Automatic & Defaut \\
\hline Sus. + E1 & Occasion & Abs sum & $\begin{array}{l}\text { Earth } 1 \\
\text { Max Sus }\end{array}$ & $\begin{array}{l}1.00 \\
1.00\end{array}$ & Automatic & Default \\
\hline Sus. + E2 & Occasion & Abs sum & $\begin{array}{l}\text { Earth } 2 \\
\text { Max Sus }\end{array}$ & $\begin{array}{l}1.00 \\
1.00\end{array}$ & Automatic & Default \\
\hline $\operatorname{Max} P$ & Hoop & & Max Hoop & 1.00 & Automatic & Default \\
\hline SEIS & Occasion & Abs sum & $\begin{array}{l}\text { Earth } 1 \\
\text { Earth } 2\end{array}$ & $\begin{array}{l}1.00 \\
1.00\end{array}$ & Autonat ic & User \\
\hline SUS+SEIS & Occasion & Abs sum & $\begin{array}{l}\text { Max SUS } \\
\text { SEIS }\end{array}$ & $\begin{array}{l}1.00 \\
1.00\end{array}$ & Automatic & User \\
\hline
\end{tabular}
W2/22/95 WB20, TANK 241-C-106, ER4319 H-2-818480 AUTOPIPE+4.50 RESULT PAGE 2

CODE COMPLIANCE COMBINATIONS

\begin{tabular}{|c|c|c|c|c|}
\hline Combination & Method & Load & Factor & Remarks \\
\hline GR & Sum & Gravity & 1.00 & Default \\
\hline$T 1$ & Sum & Thermal 1 & 1.00 & Default \\
\hline E1 & Sum & Earth 1 & 1.00 & Defau \\
\hline E2 & Sum & Earth 2 & 1.00 & Defaul \\
\hline SEIS & Abs sum & $\begin{array}{l}\text { Earth } 1 \\
\text { Earth } 2\end{array}$ & $\begin{array}{l}1.00 \\
1.00\end{array}$ & User \\
\hline$G R+T 1$ & Sum & $\begin{array}{l}\text { Gravity } \\
\text { Thermal } 1\end{array}$ & $\begin{array}{l}1.00 \\
1.00\end{array}$ & User \\
\hline GR+T1+SEIS & Abs sum & $\begin{array}{l}\text { GR+I } \\
\text { SEIS }\end{array}$ & $\begin{array}{l}1.00 \\
1.00\end{array}$ & User \\
\hline
\end{tabular}

\section{OTHER USER COMBINATIONS}

COOE COMPLIANCE

$Y$ - Factor ........................ 0.40 Weld efficiency factor $\ldots \ldots \ldots \ldots \ldots \ldots \ldots 1.00$

Range reduction factor $\ldots \ldots \ldots \ldots \ldots \ldots . .1 .00$ 
W320H32C PROCESS BUILDING PIPING STRESS ANALYSIS

$02 / 22195$ W320, TANK 241-C-106, ER4319 H-2-818480 AUtOPIPE+4.50 RESULT PAGE

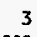

Design Pressure Factor

............. 1.00

include corrosion in stress calcs.

Include torsion in code stress .......

include axial force in code stress......

longitudinal pressure calculation ...... PD/4t

Include rigorous pressure .............. Not analyzed
W32OH32C PROCESS BUILDING PIPING STRESS ANALYSIS

02/22/95 W320, TANK 241-C-106, ER4319 H-2-818480 AUTOPIPE+4.50 RESULT PAGE

EARTHQUAKE LOAD CASES :

Number of load cases anatysed : 2

Load case 1 - E1

X-Multiplier $=0.120 \quad$ Y-Multiplier $=0.000 \quad$ Z-Multiplier $=0.000$

Load case 2 - E2

$x$-Multiplier $=0.000 \quad$ Y-Multiplier $=0.000 \quad$ Z-Multiplierz 0.120

HNF-2486, Rev. 0

Page C-71 


\begin{tabular}{|c|c|c|c|c|c|c|c|}
\hline \multirow{2}{*}{\multicolumn{8}{|c|}{$\begin{array}{l}\text { W320H32C PROCESS BUILDING PIPING STRESS ANALYSIS } \\
\text { O2/22/95 W320, TANK } 241-C-106, \text { ER } 4319 \mathrm{H}-2-818480 \\
\text { DI S P L A C EMEN T S }\end{array}$}} \\
\hline & & & & & & & \\
\hline $\begin{array}{l}\text { Point } \\
\text { name } \\
\text { - }\end{array}$ & $\begin{array}{l}\text { Load } \\
\text { combination }\end{array}$ & \multicolumn{3}{|c|}{ TRANSLATIONS (in $z_{Y}^{2}$} & \multicolumn{2}{|c|}{$\begin{array}{l}\text { ROTATIONS (deg } \\
\mathrm{X}\end{array}$} & $\begin{array}{l}? \\
z\end{array}$ \\
\hline \multicolumn{8}{|c|}{$\star * * *$ Segment A begin $* \star *$} \\
\hline $\mathrm{A} 00$ & $\begin{array}{l}\text { GR } \\
\text { T1 } \\
\text { E1 } \\
\text { E2 } \\
\text { SEIS } \\
\text { GR+T1 } \\
\text { GR+T1+SEIS }\end{array}$ & $\begin{array}{l}0.000 \\
0.000 \\
0.000 \\
0.000 \\
0.000 \\
0.000 \\
0.000\end{array}$ & $\begin{array}{l}0.000 \\
0.000 \\
0.000 \\
0.000 \\
0.000 \\
0.000 \\
0.000\end{array}$ & $\begin{array}{l}0.000 \\
0.000 \\
0.000 \\
0.000 \\
0.000 \\
0.000 \\
0.000\end{array}$ & $\begin{array}{l}0.000 \\
0.000 \\
0.000 \\
0.000 \\
0.000 \\
0.000 \\
0.000\end{array}$ & $\begin{array}{l}0.000 \\
0.000 \\
0.000 \\
0.000 \\
0.000 \\
0.000 \\
0.000\end{array}$ & $\begin{array}{l}0.000 \\
0.000 \\
0.000 \\
0.000 \\
0.000 \\
0.000 \\
0.000\end{array}$ \\
\hline$A 01$ & $\begin{array}{l}\text { GR } \\
\text { T1 } \\
\text { E1 } \\
\text { E2 } \\
\text { SEIS } \\
\text { GR+T1 } \\
\text { GR+T1+SEIS }\end{array}$ & $\begin{array}{l}0.000 \\
0.007 \\
0.000 \\
0.000 \\
0.000 \\
0.007 \\
0.007\end{array}$ & $\begin{array}{l}0.000 \\
0.000 \\
0.000 \\
0.000 \\
0.000 \\
0.000 \\
0.000\end{array}$ & $\begin{array}{l}0.000 \\
0.006 \\
0.000 \\
0.000 \\
0.000 \\
0.006 \\
0.006\end{array}$ & $\begin{array}{l}0.000 \\
0.000 \\
0.000 \\
0.000 \\
0.000 \\
0.001 \\
0.001\end{array}$ & $\begin{array}{l}0.000 \\
0.071 \\
0.000 \\
0.000 \\
0.000 \\
0.071 \\
0.071\end{array}$ & $\begin{array}{l}0.000 \\
0.000 \\
0.000 \\
0.000 \\
0.000 \\
0.000 \\
0.000\end{array}$ \\
\hline $\mathrm{A} 02$ & $\begin{array}{l}\text { GR } \\
\text { T1 } \\
\text { E1 } \\
\text { E2 } \\
\text { SEIS } \\
\text { GR+T1 } \\
\text { GR+T1+SEIS }\end{array}$ & $\begin{array}{l}0.000 \\
0.021 \\
0.000 \\
0.000 \\
0.000 \\
0.021 \\
0.021\end{array}$ & $\begin{array}{l}0.000 \\
0.000 \\
0.000 \\
0.000 \\
0.000 \\
0.000 \\
0.000\end{array}$ & $\begin{array}{l}0.000 \\
0.014 \\
0.000 \\
0.000 \\
0.000 \\
0.014 \\
0.014\end{array}$ & $\begin{array}{l}0.000 \\
0.001 \\
0.000 \\
0.000 \\
0.000 \\
0.001 \\
0.001\end{array}$ & $\begin{array}{l}0.000 \\
0.101 \\
0.000 \\
0.000 \\
0.000 \\
0.101 \\
0.101\end{array}$ & $\begin{array}{l}0.000 \\
0.000 \\
0.000 \\
0.000 \\
0.000 \\
0.000 \\
0.000\end{array}$ \\
\hline$A 03 N$ & $\begin{array}{l}\text { GR } \\
\text { T1 } \\
\text { E1 } \\
\text { E2 } \\
\text { SEIS } \\
\text { GR+T1 } \\
\text { GR+T1+SEIS }\end{array}$ & $\begin{array}{l}0.000 \\
0.036 \\
0.000 \\
0.000 \\
0.000 \\
0.036 \\
0.036\end{array}$ & $\begin{array}{l}0.000 \\
0.000 \\
0.000 \\
0.000 \\
0.000 \\
0.000 \\
0.000\end{array}$ & $\begin{array}{l}0.000 \\
0.021 \\
0.000 \\
0.000 \\
0.000 \\
0.021 \\
0.021\end{array}$ & $\begin{array}{l}0.000 \\
0.000 \\
0.000 \\
0.000 \\
0.000 \\
0.001 \\
0.001\end{array}$ & $\begin{array}{l}0.000 \\
0.106 \\
0.000 \\
0.000 \\
0.000 \\
0.106 \\
0.106\end{array}$ & $\begin{array}{l}0.000 \\
0.000 \\
0.000 \\
0.000 \\
0.000 \\
0.000 \\
0.000\end{array}$ \\
\hline A03 F & $\begin{array}{l}\text { GR } \\
\text { T1 } \\
\text { E1 } \\
\text { E2 } \\
\text { SEIS } \\
\text { GR+T1 } \\
\text { GR+T1+SEIS }\end{array}$ & $\begin{array}{l}0.000 \\
0.023 \\
0.000 \\
0.000 \\
0.000 \\
0.023 \\
0.023\end{array}$ & $\begin{array}{l}0.000 \\
0.000 \\
0.000 \\
0.000 \\
0.000 \\
0.000 \\
0.000\end{array}$ & $\begin{array}{l}0.000 \\
0.017 \\
0.000 \\
0.000 \\
0.000 \\
0.017 \\
0.017\end{array}$ & $\begin{array}{l}0.000 \\
0.000 \\
0.000 \\
0.000 \\
0.000 \\
0.000 \\
0.000\end{array}$ & $\begin{array}{r}0.000 \\
-0.048 \\
0.000 \\
0.000 \\
0.000 \\
-0.048 \\
0.048\end{array}$ & $\begin{array}{l}0.000 \\
0.000 \\
0.000 \\
0.000 \\
0.000 \\
0.000 \\
0.000\end{array}$ \\
\hline
\end{tabular}

HNF-2486, Rev. 0

Page C-72

ज320H32c.ôT 2-2 
pua 8 quatr6as ***

\begin{tabular}{|c|c|c|}
\hline $\begin{array}{l}200^{\circ} 0 \\
000^{\circ} 0 \\
000^{\circ} 0 \\
000^{\circ} 0 \\
000^{-} 0 \\
000^{\circ} 0 \\
000^{\circ} 0\end{array}$ & $\begin{array}{l}201.0 \\
101^{\circ}=0 \\
000 \cdot 0 \\
000^{\circ} 0 \\
000^{\circ} 0 \\
100^{\circ} 0 \\
000^{\circ} 0\end{array}$ & $\begin{array}{l}900^{\circ} 0 \\
900^{\circ} 0 \\
000^{\circ} 0 \\
000^{\circ} 0 \\
000^{\circ} 0 \\
900^{\circ} 0 \\
000^{\circ} 0\end{array}$ \\
\hline $\begin{array}{l}100^{\circ} 0 \\
000^{\circ} 0 \\
000^{\circ} 0 \\
000^{\circ} 0 \\
000^{\circ} 0 \\
000^{\circ} 0 \\
000^{\circ} 0\end{array}$ & $\begin{array}{l}201 \cdot 0 \\
101 \cdot 0 \\
000 \cdot 0 \\
000 \cdot 0 \\
000 \cdot 0 \\
101-0 \\
000^{\circ} 0\end{array}$ & $\begin{array}{l}900^{\circ} 0 \\
900^{\circ} 0 \\
000^{\circ} 0 \\
000^{\circ} 0 \\
000^{\circ} 0 \\
900^{\circ} 0 \\
000^{\circ} 0\end{array}$ \\
\hline $\begin{array}{c}100^{*} 0 \\
000^{\circ}=0 \\
000^{\circ}-0 \\
000^{-} 0 \\
000^{\circ} 0 \\
000^{-} 0 \\
000^{\circ} 0\end{array}$ & $\begin{array}{l}201: 0 \\
101 \cdot 0 \\
000 \cdot 0 \\
000 \cdot 0 \\
000 \cdot 0 \\
101 \cdot 0 \\
000.0\end{array}$ & $\begin{array}{l}900^{\circ} 0 \\
900^{\circ} 0 \\
000^{\circ} 0 \\
000^{\circ} 0 \\
000^{\circ} 0 \\
900^{\circ} 0 \\
000^{\circ} 0\end{array}$ \\
\hline $\begin{array}{c}000^{*} 0 \\
000^{\circ} 0 \\
000^{\circ} 0 \\
000^{\circ} 0 \\
000^{\circ} 0 \\
000^{*} 0 \\
000^{\circ} 0\end{array}$ & $\begin{array}{l}101 \cdot 0 \\
101 \cdot 0 \\
000 \cdot 0 \\
000.0 \\
000 \cdot 0 \\
101 \cdot 0 \\
000^{\circ} 0\end{array}$ & $\begin{array}{l}100^{\circ} 0 \\
100^{\circ} 0 \\
000^{\circ} 0 \\
000^{\circ} 0 \\
000^{\circ} 0 \\
100^{\circ} 0 \\
000^{\circ} 0\end{array}$ \\
\hline$z$ & $\lambda$ & $x$ \\
\hline
\end{tabular}

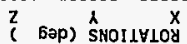

\begin{tabular}{|c|c|c|}
\hline $\begin{array}{l}\sum \neq 0^{\circ} 0 \\
\sum \neq 0^{\circ} 0 \\
000^{\circ} 0 \\
000^{\circ} 0 \\
000^{\circ} 0 \\
\sum \neq 0^{\circ} 0 \\
000^{\circ} 0\end{array}$ & $\begin{array}{l}910^{\circ} 0 \\
910^{\circ} 0 \\
000 \cdot 0 \\
000^{\circ} 0 \\
000^{\circ} 0 \\
910^{\circ} 0 \\
000^{\circ} 0\end{array}$ & $\begin{array}{l}\$ 90^{\circ} 0 \\
\Sigma 90^{\circ} 0 \\
000^{\circ} 0 \\
000^{\circ} 0 \\
000^{\circ} 0 \\
\Sigma 90^{\circ} 0 \\
000^{\circ} 0\end{array}$ \\
\hline $\begin{array}{l}l \neq 0^{\circ} 0 \\
1 \neq 0^{\circ} 0 \\
000^{\circ} 0 \\
000^{\circ} 0 \\
000^{\circ} 0 \\
l \neq 0^{\circ} 0\end{array}$ & $\begin{array}{l}910 \\
910 \\
000 \\
000 \\
000 \\
910 \\
000\end{array}$ & $\begin{array}{l}090^{\circ} 0 \\
650^{\circ} 0 \\
000^{\circ} 0 \\
000^{\circ} 0 \\
000^{\circ} 0 \\
650^{\circ} 0 \\
000^{\circ} 0\end{array}$ \\
\hline $\begin{array}{l}620^{\circ} \\
000^{\circ} \\
000^{\circ} \\
000^{\circ} \\
60^{\circ}\end{array}$ & $\begin{array}{l}210 \\
2 \neq 0 \\
000 \\
000 \\
000 \\
210 \\
000\end{array}$ & \\
\hline $\begin{array}{l}+10^{\circ} \\
+10^{\circ} \\
000^{\circ} \\
000^{\circ} \\
000^{\circ} \\
+10^{\circ} \\
000^{\circ}\end{array}$ & $\begin{array}{l}\angle 00^{\circ} 0 \\
\varepsilon 00^{\circ} 0 \\
000^{\circ} \\
000^{\circ} 0 \\
000^{\circ} 0 \\
700^{\circ} 0 \\
000^{\circ} 0\end{array}$ & \\
\hline
\end{tabular}

$S \perp N \exists K \exists \partial \forall T$ I

\section{$S 1 \exists S+1++89$}

$11+49$

23

l.

508

SI $3 S+11+\not 19$

$1+49$
sigs

5135
23
13

13
11
89

208

$2132+i 1+82$

SI
IJS

SIJS

11

$\pm 108$

$2 I 3 S+h+29$

$1+89$
Sijs

23

就 N 108

Uo!łeutquos aljeu peulquos glued
peoj zu!od

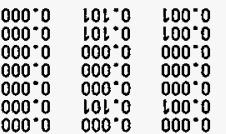

$\begin{array}{lll}000^{\circ} 0 & 100^{\circ} 0 & 100^{\circ} 0 \\ 000 & 000^{\circ} 0 & 000^{\circ} 0\end{array}$

$000^{\circ}$

000

$000^{\circ} 0$

$000 \%$
$000 \%$
$000 \%$

$000^{\circ} 0$

$000^{\circ}$

$000^{\circ}$

$000^{\circ} 0$

$000^{\circ} 0$

$000^{\circ} 0$

$000 \div 0$

$000^{\circ} 0$

$000^{\circ} 0$

$000^{\circ} 0$

$000 \%$

$000^{\circ} 0$

$000^{\circ} 0$

$000^{\circ} 0$

$000^{\circ}$

$$
\text { ? }
$$$$
\begin{aligned}
& 000 \\
& 000 \\
& 00 \\
& 000 \\
& 000 \\
& 000 \\
& 000 \\
& 0000 \\
& 000 \\
& 000 \\
& 000 \\
& 000 \\
& 000 \\
& 000 \\
& 212 \\
& 120 \\
& 000 \\
& 000 \\
& 000 \\
& 110
\end{aligned}
$$

$000^{\circ} 0 \quad 000^{\circ} 0$

$000.0 \quad 000 * 0$

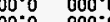

$000^{\circ} 00$

$000^{\circ} 0$ 000.0

$000^{\circ} 0$

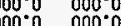

0000.0

000.000000

$\begin{array}{ll}000^{\circ} 0 & 000^{\circ} 0 \\ 000.0 & 000^{\circ} 0\end{array}$

นL०० $000 \%$

000.0000

$000^{\circ} 000^{\circ} 0$

$000^{\circ} 0000^{\circ}$

$870^{\circ} 0 \quad 000^{\circ} 0$

$870^{\circ} 0-000^{\circ} 0$

$000^{\circ} 0000^{\circ} 0$

$000^{\circ} 0000$

$870^{\circ} 0.000^{\circ} 0$

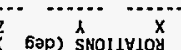

$\begin{array}{lll}210^{\circ} 0 & 000^{\circ} 0 & L 200^{\circ} \\ 710^{\circ} 0 & 000^{\circ} 0 & 120^{\circ} 0 \\ 000^{\circ} 0 & 000^{\circ} 0 & 000^{\circ} 0 \\ 000^{\circ} 0 & 000^{\circ} 0 & 000^{\circ} 0 \\ 000^{\circ} 0 & 000^{\circ} 0 & 000^{\circ} 0 \\ 710^{\circ} 0 & 000^{\circ} 0 & 1200^{\circ} \\ 000^{\circ} 0 & 000^{\circ} 0 & 000^{\circ} 0\end{array}$

SIIS $+1+1+80$

bI+85

$2 \exists$

13

ZOY

*** U!6วa 8 zuaw6อS ***

*** puə $\forall$ zuəw6as $* * *$

\begin{tabular}{|c|c|c|c|}
\hline $\begin{array}{l}000=0 \\
000^{\circ} 0 \\
000^{\circ} 0 \\
000^{\circ} 0 \\
000^{\circ} 0 \\
000^{\circ} 0\end{array}$ & $\begin{array}{l}000^{\circ} 0 \\
000^{\circ} 0 \\
000^{\circ} 0 \\
000^{\circ} 0 \\
000^{\circ} 0 \\
000^{\circ} 0\end{array}$ & $\begin{array}{l}000^{\circ} 0 \\
000^{\circ} 0 \\
000^{\circ} 0 \\
000^{\circ} 0 \\
000^{\circ} 0 \\
000^{\circ} 0\end{array}$ & 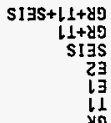 \\
\hline
\end{tabular}

Ziv

$080^{\circ} 0 \quad 000.0 \quad 000^{\circ} 0 \quad$ sias+lit.

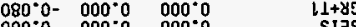

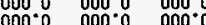

$000.0 \quad 000.0 \quad 000.0$

$080^{\circ} 0-000 \% 00000$

$000^{\circ} 0000^{\circ} 0 \quad 000^{\circ} 0$

$210^{\circ} 0 \quad 000^{\circ} 0 \quad 100^{\circ} 0$

$000^{\circ} 000^{\circ} 0000$

$000^{\circ} 000^{\circ} 0 \quad 000^{-0}$

$000^{\circ} \quad 000^{\circ} 0 \quad 000^{\circ} 0$

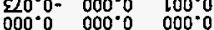

$520^{\circ} 0 \quad 000^{\circ} 0 \quad \angle 20^{\circ} 0$

$520^{\circ} 0-000^{\circ} 0 \quad \angle 20^{\circ} 0$ -

$000^{\circ} 0000^{\circ} 000000$

$000 \% 000^{\circ} 0 \quad 000^{\circ} 0$

$\mathrm{SZO} 0^{\circ} 0^{-} 000^{\circ} 0 \quad \angle 20^{\circ} 0$ -

$\begin{array}{ccc}0 & 000^{\circ} 0 & 000^{\circ} 0\end{array}$

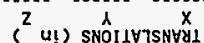

Uotzeutciuos

604

peoj jutod 
320H32C PROCESS BUILING PIPING STRESS ANALYSIS

SUPPORT FORCES

Point/ Connect/ Load LO C A L Supp. 10 Iype Combination Dirn Force Deform Dirn Force Deform

\begin{tabular}{|c|c|c|c|c|c|c|c|c|}
\hline \multirow[t]{7}{*}{$\begin{array}{l}\text { A10 } \\
\text { A10 } \\
\text { Stiff }\end{array}$} & $\begin{array}{l}\text { Y - Stop } \\
\text { : RIGID }\end{array}$ & GR & $\begin{array}{l}\text { down } \\
-z \\
-x\end{array}$ & 159 & $\begin{array}{l}0.000 \\
0.000 \\
0.000\end{array}$ & $\begin{array}{l}x \\
y \\
Z\end{array}$ & -159 & $\begin{array}{l}0.000 \\
0.000 \\
0.000\end{array}$ \\
\hline & & 11 & $\begin{array}{l}\text { up } \\
-Z \\
-\mathrm{X}\end{array}$ & $\begin{array}{r}0 \\
25 \\
0\end{array}$ & $\begin{array}{l}0.000 \\
0.073 \\
0.001\end{array}$ & $\begin{array}{l}X \\
Y \\
Z\end{array}$ & $\begin{array}{r}0 \\
0 \\
-25\end{array}$ & $\begin{array}{r}-0.001 \\
0.000 \\
-0.073\end{array}$ \\
\hline & & E1 & $\begin{array}{l}\text { down } \\
-z \\
+x\end{array}$ & 0 & $\begin{array}{l}0.000 \\
0.000 \\
0.000\end{array}$ & $\begin{array}{l}X \\
Y \\
Z\end{array}$ & 0 & $\begin{array}{l}0.000 \\
0.000 \\
0.000\end{array}$ \\
\hline & & E2 & $\begin{array}{l}\text { down } \\
+z \\
-x\end{array}$ & 0 & $\begin{array}{l}0.000 \\
0.000 \\
0.000\end{array}$ & $\begin{array}{l}X \\
Y \\
Z\end{array}$ & 0 & $\begin{array}{l}0.000 \\
0.000 \\
0.000\end{array}$ \\
\hline & & SEIS & $\begin{array}{l}\text { down } \\
-z \\
-x\end{array}$ & 0 & $\begin{array}{l}0.000 \\
0.000 \\
0.000\end{array}$ & $\begin{array}{l}X \\
Y \\
Z\end{array}$ & $\mathbf{0}$ & $\begin{array}{l}0.000 \\
0.000 \\
0.000\end{array}$ \\
\hline & & $\mathrm{GR}+\mathrm{T}\}$ & $\begin{array}{l}\text { down } \\
-Z \\
-x\end{array}$ & $\begin{array}{r}159 \\
25 \\
0\end{array}$ & $\begin{array}{l}0.000 \\
0.073 \\
0.001\end{array}$ & $\begin{array}{l}X \\
Y \\
Z\end{array}$ & $\begin{array}{r}0 \\
-159 \\
-25\end{array}$ & $\begin{array}{r}-0.001 \\
0.000 \\
-0.073\end{array}$ \\
\hline & & $G R+T 1+S E I S$ & $\begin{array}{l}\text { down } \\
-z \\
-x\end{array}$ & $\begin{array}{r}159 \\
25 \\
0\end{array}$ & $\begin{array}{l}0.000 \\
0.073 \\
0.001\end{array}$ & $\begin{array}{l}X \\
y \\
Z\end{array}$ & $\begin{array}{r}0 \\
159 \\
25\end{array}$ & $\begin{array}{l}0.001 \\
0.000 \\
0.073\end{array}$ \\
\hline \multirow[t]{5}{*}{$\begin{array}{l}802 \\
802 \\
\text { stiff }\end{array}$} & $\begin{array}{l}\text { Y - Stop } \\
\text { :RIGID }\end{array}$ & GR & $\begin{array}{l}\text { down } \\
+Z \\
+x\end{array}$ & 83 & $\begin{array}{l}0.000 \\
0.000 \\
0.000\end{array}$ & $\begin{array}{l}X \\
Y \\
Z\end{array}$ & -83 & $\begin{array}{l}0.000 \\
0.000 \\
0.000\end{array}$ \\
\hline & & $T 1$ & $\begin{array}{l}\text { up } \\
+2 \\
+\mathrm{X}\end{array}$ & 83 & $\begin{array}{l}0.016 \\
0.041 \\
0.059\end{array}$ & $\begin{array}{l}x \\
y \\
z\end{array}$ & 83 & $\begin{array}{l}0.059 \\
0.016 \\
0.041\end{array}$ \\
\hline & & E1 & $\begin{array}{l}\text { down } \\
+2 \\
+x\end{array}$ & 0 & $\begin{array}{l}0.000 \\
0.000 \\
0.000\end{array}$ & $\begin{array}{l}X \\
Y \\
Z\end{array}$ & 0 & $\begin{array}{l}0.000 \\
0.000 \\
0.000\end{array}$ \\
\hline & & E2 & $\begin{array}{l}\text { down } \\
+z \\
-x\end{array}$ & 4 & $\begin{array}{l}0.000 \\
0.000 \\
0.000\end{array}$ & $\begin{array}{l}X \\
Y \\
Z\end{array}$ & -4 & $\begin{array}{l}0.000 \\
0.000 \\
0.000\end{array}$ \\
\hline & & SEIS & $\begin{array}{l}\text { down } \\
-z \\
-x\end{array}$ & 4 & $\begin{array}{l}0.000 \\
0.000 \\
0.000\end{array}$ & $\begin{array}{l}X \\
Y \\
Z\end{array}$ & 4 & $\begin{array}{l}0.000 \\
0.000 \\
0.000\end{array}$ \\
\hline
\end{tabular}

HNF-2486, Rev. 0

Page C-74
W32OH32C PROCESS BUILDING PIPING SIRESS ANALYSIS

$02 / 22 / 95$ W320, TANK 241-C-106, ER4319 H-2-818480 AUtOPIPE+4.50 RESULT PAGE 10

SUPPORT FORCES

Point/ Connect/ Load LOCA L GLO 8 A L

Supp. ID Type Combination Dirn Force Deform Dirn Force Deform

Supp. I0 Type Combination 0 -... \begin{tabular}{lllll}
$\mathrm{GR}+\mathrm{T1}$ & up & 0.016 & $\mathrm{X}$ & \\
\hline
\end{tabular}

$\begin{array}{cccc}+Z & 0.041 & Y & 0.016 \\ +X & 0.059 & Z & 0.041\end{array}$

$\begin{array}{lllllll}G R+T 1+S E I S & \text { down } & 4 & 0.016 & X & & 0.060 \\ & -Z & & 0.041 & Y & 4 & 0.016 \\ & -X & & 0.060 & Z & & 0.041\end{array}$ 


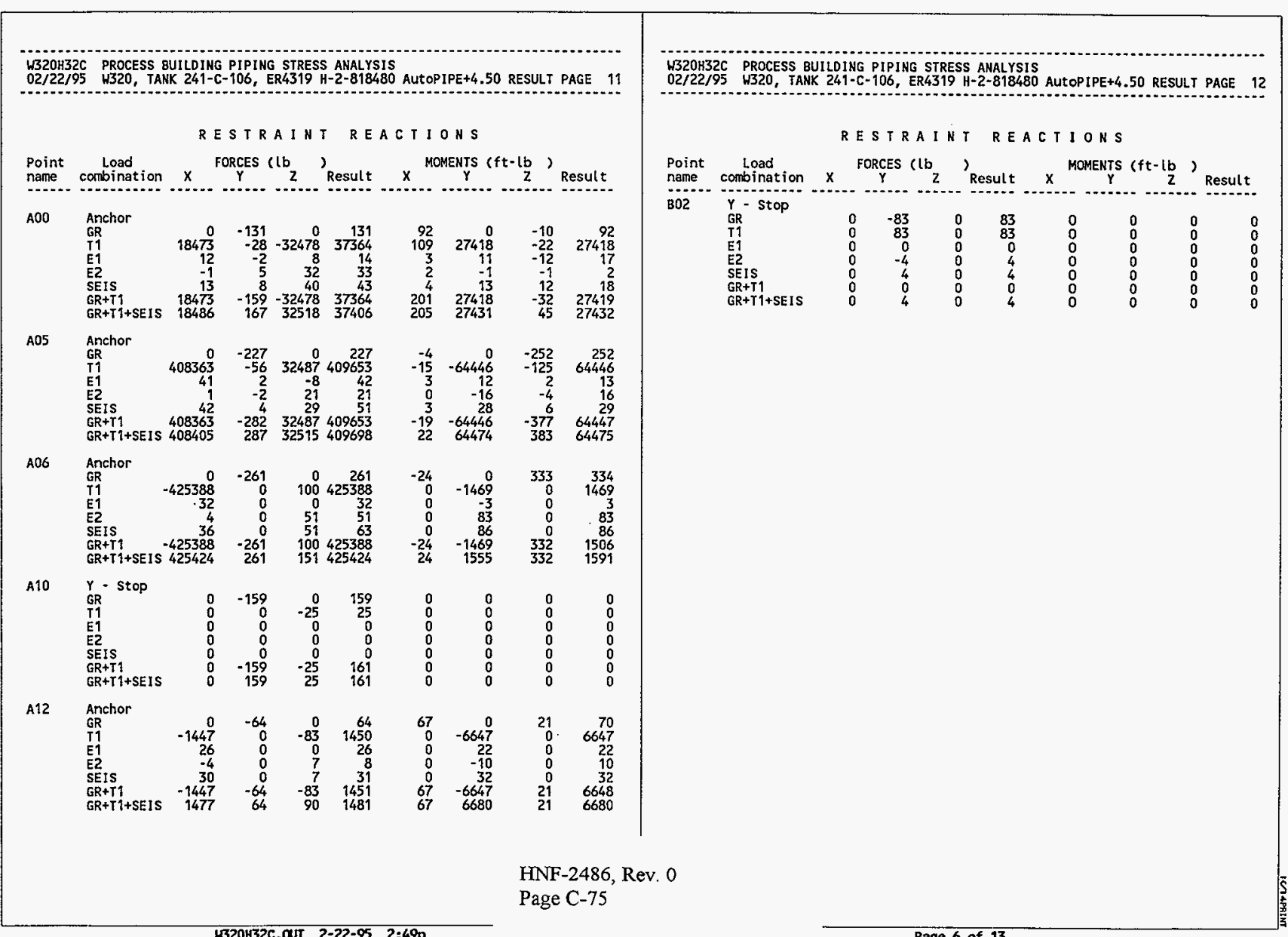


W32OH32C PROCESS BUILDING PIPING STRESS ANALYSIS

02/22/95 W320, TANK 241-C-106, ER4319 $\mathrm{H}-2-818480$ AUTOPIPE+4.50 RESULT PAGE 13

GLOBAL FORCES \& MOMENTS

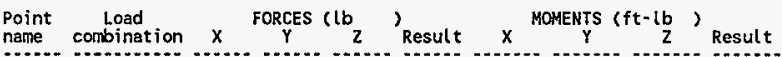

\section{*** Segment $A$ begin ***}

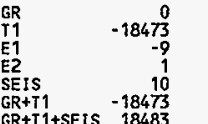

A01

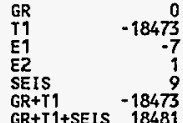

$\begin{array}{rrr}94 & 0 & 94 \\ 28 & 32478 & 37364\end{array}$

$\begin{array}{rrr}2 & -8 & 11 \\ -5 & -28 & 28 \\ 8 & 36 & 37\end{array}$

$121132478 \quad 37364$

GR+T1+SEIS 18481

A02.

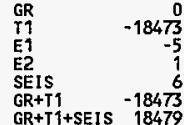

$\begin{array}{rrr}74 & 0 & 74 \\ 28 & 32478 & 37364 \\ 2 & -8 & 10\end{array}$

$\begin{array}{lll}2 & -8 & 10 \\ -5 & -25 & 26\end{array}$

$\begin{array}{rrr}8 & 33 & 35 \\ 102 & 32478 & 37364\end{array}$

$\begin{array}{lr}\text { GR+T1 } & -18473 \\ \text { GR+Ti+SEIS } & 18479\end{array}$

A02

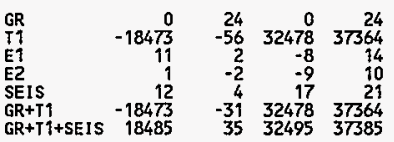

A03

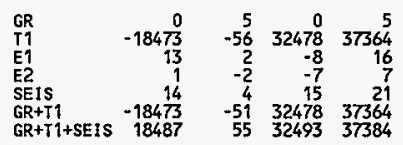

HNF-2486, Rev, 0

Page C-76 $-137-18182$ $\begin{array}{rr}12 & 16 \\ 32 & 18183 \\ 45 & 18191\end{array}$

$\begin{array}{rr}7 & 0 \\ -79 & -7406\end{array}$

$\begin{array}{rr}-79 & -7406 \\ 0 & -4 \\ -7 & 0 \\ 8 & 4 \\ -72 & -7406\end{array}$

$\begin{array}{rr}10 & 12 \\ 22 & 7407\end{array}$

$\begin{array}{rr}8 & 4 \\ -72 & -7406 \\ 79 & 7410\end{array}$

$\begin{array}{rr}12 & 12 \\ 12 & 75 \\ 32 & 7407\end{array}$

$\begin{array}{lr}12 & 15 \\ 32 & 7407 \\ 45 & 7419\end{array}$

$\begin{array}{rr}2 & 0 \\ 73 & -7406\end{array}$

$\begin{array}{rr}10 & 10 \\ 22 & 7407\end{array}$

$\begin{array}{rr}2 & 0 \\ 3 & 14 \\ 75 & -7406\end{array}$

$\begin{array}{rr}-5 & 15 \\ 1 & 2 \\ 5 & 15\end{array}$

$\begin{array}{rr}5 & 15 \\ 32 & 7407 \\ 38 & 7421\end{array}$

$\begin{array}{rr}10 & 0 \\ 41 & 3369\end{array}$

$\begin{array}{rr}10 & 14 \\ 22 & 3370\end{array}$

\begin{tabular}{rr}
1 & 7 \\
1 & -1 \\
2 & 8 \\
51 & 3369 \\
\hline & 3377
\end{tabular}

8
2
10
3370
3378
W320H32C PROCESS BUILDING PIPING STRESS ANALYSIS

02/22/95 W320. TAHK 241-C-106, ER4319 $4-2-818480$ AUTOPIPE+4.50 RESULT PAGE 14

GLOBAL FORCES \& MOMENTS

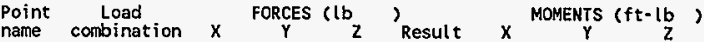

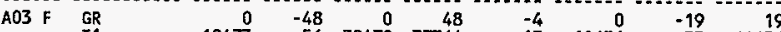
$\begin{array}{lrrrrrrrr}\text { GR } & 0 & -48 & 0 & 48 & -4 & 0 & -19 & \\ \text { T1 } & -18473 & -56 & 32478 & 37364 & -15 & -10636 & -33 & 10636 \\ \text { E1 } & 20 & 2 & -8 & 21 & 3 & -1 & -2 & \\ \text { E2 } & 1 & -2 & -1 & 2 & 0 & 1 & -1 & \\ \text { SEIS } & 21 & 4 & 9 & 23 & 3 & 2 & 3 & \\ \text { GR+T1 } & -18473 & -103 & 32478 & 37364 & -18 & -10636 & -52 & 10636 \\ \text { GR T1 } & & 1843 & 107 & 3248 & 3738 & 22 & & 5\end{array}$ $\begin{array}{lrrrrrrrr}\text { GR+T1 } & -18473 & -103 & 32478 & 37364 & -18 & -10636 & -52 & 10636 \\ \text { GR+T1+SEIS } & 18493 & 107 & 32487 & 37382 & 22 & 10638 & 55 & 10638\end{array}$

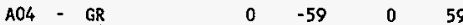
$\begin{array}{lrrrr}\text { GR } & -18473 & -56 & 32478 & 37364 \\ \text { E1 } & 21 & 2 & -8 & 22 \\ \text { E2 } & 1 & -2 & 1 & 2 \\ \text { SEIS } & 22 & 4 & 8 & 24 \\ \text { GR+T1 } & -18473 & -114 & 32478 & 37364 \\ \text { GR+T1+SEIS } & 18495 & 118 & 32487 & 37382\end{array}$ $\begin{array}{rrrr}-4 & -314 & -36 & 37 \\ -15 & -21462 & -52 & 21462\end{array}$

$\begin{array}{rrr}0 & 1 \\ 3 & 3\end{array}$ $\begin{array}{rr}-18 & -21462\end{array}$ GR $0-137 \quad 0 \quad 137$ $\begin{array}{rr}-4 & -45 \\ -15 & -2146 ?\end{array}$ $\begin{array}{lrrrr}\text { GR } & 0 & -137 & 0 & 137 \\ \text { T1 } & -18473 & -56 & 32478 & 37364 \\ \text { E1 } & 30 & 2 & -8 & 31 \\ \text { E2 } & 1 & -2 & 10 & 10 \\ \text { SEIS } & 31 & 4 & 18 & 36 \\ \text { GR+T1 } & -18473 & -192 & 32478 & 37364\end{array}$

A05 -

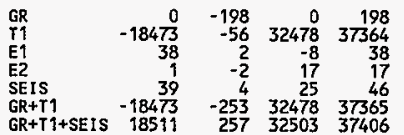

$\begin{array}{rr}3 & 18 \\ -18 & -21462\end{array}$

$-1$ 3
-88

$-88 \quad 21463$

21463

$\mathrm{A04}+$

A05

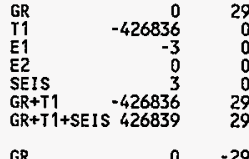

$\begin{array}{rr}0 & 29 \\ -8 & 426836\end{array}$

0
-3
3

$-8426836$

12426839

$\begin{array}{rr}-4 & 0 \\ -15 & -64658 \\ 3 & 12 \\ 0 & -17 \\ 3 & 29 \\ -18 & -64658 \\ 22 & 64687\end{array}$

-1
-2
-3

3
-88
-21463

21463
21466

A06 -

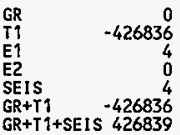

$\begin{array}{rr}0 & 29 \\ -8 & 426836\end{array}$

4

4

4
-826836

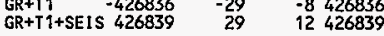

$\begin{array}{rr}-259 & 259 \\ -126 & 64658\end{array}$

$-126 \quad 64658$

$\begin{array}{rr}-4 & 17 \\ 6 & 30\end{array}$

$\begin{array}{rr}384 & 64659 \\ 390 & 64689\end{array}$

$\begin{array}{rrrr}0 & 0 & -7 & 7 \\ 0 & -213 & 0 & 213 \\ 0 & 0 & 0 & 0 \\ 0 & -1 & 0 & 1 \\ 0 & 1 & 0 & 1 \\ 0 & -213 & -7 & 213 \\ 0 & 214 & 7 & 214 \\ 0 & 0 & -7 & 7 \\ 0 & -204 & 0 & 204 \\ 0 & 0 & 0 & 0 \\ 0 & -1 & 0 & 1 \\ 0 & 1 & 0 & 1 \\ 0 & -204 & -7 & 204 \\ 0 & -205 & 7 & 205\end{array}$


B32OH32C PROCESS BUILDING PIPING STRESS ANALYSIS

AUTOPIPE +4.50 RESULT PAGE 15

GLOBAL FORCES \& MOMENTS

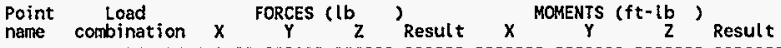

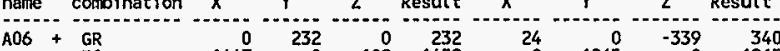

$\begin{array}{lrrrr}\text { GR } & 0 & 232 & 0 & 232 \\ \text { T1 } & -1447 & 0 & -109 & 1452 \\ \text { E1 } & -29 & 0 & 0 & 29 \\ \text { E2 } & -44 & 0 & -48 & 48 \\ \text { SEIS } & 33 & 0 & 48 & 58 \\ \text { GR+T1 } & -1447 & 232 & -109 & 1470 \\ \text { GR+T1+SEIS } & 1480 & 232 & 156 & 1507\end{array}$

A07 -

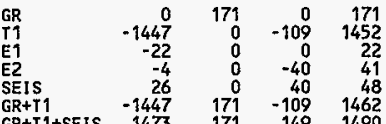

$\mathrm{A} 07+$

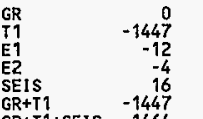

$\begin{array}{rrrr}-1447 & 93 & -109 & 1455 \\ \text { GR+T1+SEIS } & 1464 & 93 & 140\end{array}$

A08 N

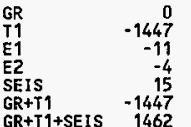

A08 F

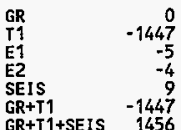

A09

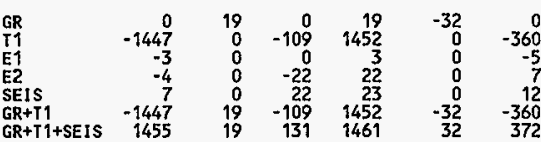

$\begin{array}{lrrrrrr}\text { GR+T1 } & -1447 & 19 & -109 & 1452 & -32 & -36 \\ \text { GR+T1+SEIS } & 1455 & 19 & 131 & 1461 & 32 & 372\end{array}$ $\begin{array}{rr}0 & 1410 \\ 0 & 3 \\ 0 & 25 \\ 0 & 28 \\ -71 & 1412\end{array}$ $\begin{array}{rr}-71 & 1412 \\ 71 & 1440\end{array}$ $\begin{array}{lllll}93 & 140 & 1473 & 24 & 1438\end{array}$ $\begin{array}{rr}-42 & 48 \\ 0 & 1446\end{array}$ 18
1446 $\begin{array}{rrr}0 & -30 & 30 \\ 0 & 30 & 33 \\ 82 & -109 & 1454 \\ 82 & 138 & 1471\end{array}$ $\begin{array}{rr}-42 & 1447 \\ 42 & 1465\end{array}$ $138 \quad 1471$

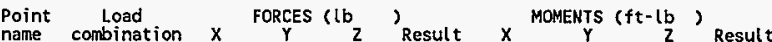

LOBAL FORCES

MOMENTS

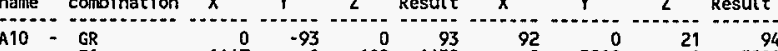
$\begin{array}{lrrrr}\text { GR } & 0 & -93 & 0 & 93 \\ \text { I1 } & -1447 & 0 & -109 & 1452 \\ \text { E1 } & 10 & 0 & 0 & 10 \\ \text { E2 } & -4 & 0 & -9 & 10 \\ \text { SEIS } & 14 & 0 & 9 & 17 \\ \text { GR+11 } & -1447 & -93 & -109 & 1455\end{array}$ $-1447$ $\begin{array}{rrr}93 & -109 & 1455\end{array}$

A10 $\begin{array}{lrrrr}\text { GR } & 0 & 66 & 0 & 66 \\ \text { I1 } & -1447 & 0 & -83 & 1450 \\ \text { E1 } & 10 & 0 & 0 & 10 \\ \text { E2 } & -4 & 0 & -9 & 10 \\ \text { SE1S } & 14 & 0 & 9 & 17 \\ \text { GR+r1 } & -1447 & 66 & -83 & 1451\end{array}$ $-71 \quad 75$ $\begin{array}{rr}0 & 1410 \\ 0 & 3\end{array}$ $\begin{array}{rr}0 & 25 \\ -71 & 28\end{array}$ $\begin{array}{rr}0 & 1446 \\ 0 & 15 \\ 0 & 18 \\ -42 & 1447\end{array}$

$\begin{array}{rr}21 & 32 \\ 0 & 107 \\ 0 & 4 \\ 0 & 8 \\ 0 & 12 \\ 21 & 112 \\ 21 & 123 \\ 21 & 38 \\ 0 & 360 \\ 0 & 5 \\ 0 & 7 \\ 0 & 12 \\ 21 & 362 \\ 21 & 374\end{array}$ $\begin{array}{lrr}\text { GR } & 0 & 49 \\ \text { I1 } & -1447 & \\ \text { E1 } & 12 & \\ \text { E2 } & -4 & \\ \text { SEIS } & 16 & \\ \text { GR+T1 } & -1447 & 49 \\ \text { GR+T1+SEIS } & 1463 & 49\end{array}$ $\begin{array}{rr}0 & 49 \\ -83 & 1450\end{array}$

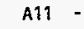

$\star \star *$ Segment B begin $* * *$

HNF-2486, Rev. 0

Page C-77 
W320H32C PROCESS BUILDING PIPING STRESS ANALYSIS

02/22/95 W320, TANK 241-C-106, ER4319 H-2-818480 AUtOPIPE +4.50 RESULT PAGE 17

GLOBAL FORCES\& MOMENTS

\begin{tabular}{|c|c|c|c|c|c|c|c|c|c|}
\hline $\begin{array}{l}\text { Point } \\
\text { name }\end{array}$ & Load & \multicolumn{3}{|c|}{ FORCES ( $\mathrm{b}$} & & \multicolumn{3}{|c|}{ MOMENTS ( $\mathrm{ft}$-lb } & Result \\
\hline A02 & $\begin{array}{l}\text { GR } \\
\text { T1 } \\
\text { E1 } \\
\text { E2 } \\
\text { SEIS } \\
\text { GR+T } 1 \text { GEIS } \\
\text { GR+T }++ \text { SEIS }\end{array}$ & $\begin{array}{r}0 \\
0 \\
-16 \\
0 \\
16 \\
0 \\
16\end{array}$ & $\begin{array}{r}50 \\
83 \\
0 \\
-4 \\
4 \\
133 \\
137\end{array}$ & $\begin{array}{r}0 \\
0 \\
0 \\
-16 \\
16 \\
0 \\
16\end{array}$ & $\begin{array}{r}50 \\
83 \\
16 \\
16 \\
23 \\
133 \\
139\end{array}$ & $\begin{array}{r}6 \\
-153 \\
0 \\
-10 \\
10 \\
-147 \\
157\end{array}$ & $\begin{array}{r}0 \\
0 \\
-18 \\
0 \\
18 \\
0 \\
18\end{array}$ & $\begin{array}{r}0 \\
0 \\
17 \\
0 \\
17 \\
0 \\
17\end{array}$ & $\begin{array}{r}6 \\
153 \\
24 \\
10 \\
26 \\
147 \\
159\end{array}$ \\
\hline $\mathrm{BO} ? \mathrm{~N}$ & $\begin{array}{l}\text { GR } \\
\text { T1 } \\
E 11 \\
\text { E2 } \\
\text { SEIS } \\
\text { GR+T1 } \\
\text { GR+T1+SEIS }\end{array}$ & $\begin{array}{r}0 \\
0 \\
-15 \\
0 \\
15 \\
0 \\
15\end{array}$ & $\begin{array}{r}41 \\
83 \\
0 \\
-4 \\
4 \\
125 \\
129\end{array}$ & $\begin{array}{r}0 \\
0 \\
0 \\
-15 \\
15 \\
0 \\
15\end{array}$ & $\begin{array}{r}41 \\
83 \\
15 \\
15 \\
22 \\
125 \\
130\end{array}$ & $\begin{array}{r}6 \\
-153 \\
0 \\
-6 \\
6 \\
-147 \\
153\end{array}$ & $\begin{array}{r}0 \\
0 \\
-18 \\
0 \\
18 \\
0 \\
18\end{array}$ & $\begin{array}{r}0 \\
0 \\
13 \\
0 \\
13 \\
0 \\
13\end{array}$ & $\begin{array}{r}6 \\
153 \\
22 \\
6 \\
23 \\
147 \\
155\end{array}$ \\
\hline B01 F & $\begin{array}{l}\text { GR } \\
\text { T1 } \\
\text { E1 } \\
\text { E2 } \\
\text { SEIS } \\
\text { GR+T1 } \\
\text { GR+T1+SEIS }\end{array}$ & $\begin{array}{r}0 \\
0 \\
-9 \\
0 \\
9 \\
0 \\
9\end{array}$ & $\begin{array}{r}-11 \\
83 \\
0 \\
-4 \\
4 \\
72 \\
76\end{array}$ & $\begin{array}{r}0 \\
0 \\
0 \\
-9 \\
9 \\
0 \\
9\end{array}$ & $\begin{array}{r}11 \\
83 \\
9 \\
9 \\
13 \\
72 \\
77\end{array}$ & $\begin{array}{r}14 \\
-69 \\
0 \\
3 \\
3 \\
-56 \\
59\end{array}$ & $\begin{array}{r}0 \\
0 \\
-7 \\
0 \\
7 \\
0 \\
7\end{array}$ & $\begin{array}{l}0 \\
0 \\
0 \\
0 \\
0 \\
0 \\
0\end{array}$ & $\begin{array}{r}14 \\
69 \\
7 \\
3 \\
7 \\
56 \\
59\end{array}$ \\
\hline B02 & $\begin{array}{l}-\mathrm{GR} \\
\mathrm{T} 1 \\
\mathrm{E} 1 \\
\mathrm{E} 2 \\
\mathrm{SE} 1 \mathrm{~S} \\
\mathrm{GR}+\mathrm{T} 1 \\
\mathrm{GR}+\mathrm{T} 1+\mathrm{SE} I \mathrm{~S}\end{array}$ & $\begin{array}{r}0 \\
0 \\
-5 \\
0 \\
5 \\
0 \\
5\end{array}$ & $\begin{array}{r}-39 \\
83 \\
0 \\
-4 \\
4 \\
45 \\
49\end{array}$ & $\begin{array}{r}0 \\
0 \\
0 \\
-5 \\
5 \\
0 \\
5\end{array}$ & $\begin{array}{r}39 \\
83 \\
5 \\
7 \\
9 \\
45 \\
49\end{array}$ & $\begin{array}{r}-7 \\
0 \\
0 \\
0 \\
0 \\
-7 \\
-7\end{array}$ & $\begin{array}{r}0 \\
0 \\
-1 \\
0 \\
1 \\
0 \\
1\end{array}$ & $\begin{array}{l}0 \\
0 \\
0 \\
0 \\
0 \\
0 \\
0\end{array}$ & $\begin{array}{l}7 \\
0 \\
1 \\
0 \\
1 \\
7 \\
7\end{array}$ \\
\hline $\mathrm{B} 02$ & $\begin{array}{l}\text { + GR } \\
T 1 \\
\text { E1 } \\
\text { E2 } \\
\text { SEIS } \\
\text { GR+T1 } \\
\text { GR+T } 1+\text { SEIS }\end{array}$ & $\begin{array}{r}0 \\
0 \\
-5 \\
0 \\
5 \\
0 \\
5\end{array}$ & $\begin{array}{r}45 \\
0 \\
0 \\
0 \\
0 \\
45 \\
45\end{array}$ & $\begin{array}{r}0 \\
0 \\
0 \\
-5 \\
5 \\
0 \\
5\end{array}$ & $\begin{array}{r}45 \\
0 \\
5 \\
5 \\
8 \\
45 \\
45\end{array}$ & $\begin{array}{r}-7 \\
0 \\
0 \\
0 \\
0 \\
-7 \\
7\end{array}$ & $\begin{array}{r}0 \\
\mathbf{0} \\
-1 \\
0 \\
1 \\
0 \\
0 \\
1\end{array}$ & $\begin{array}{l}0 \\
0 \\
0 \\
0 \\
0 \\
0 \\
0\end{array}$ & $\begin{array}{l}7 \\
0 \\
1 \\
0 \\
1 \\
7 \\
7\end{array}$ \\
\hline B03 & $\begin{array}{l}\text { GR } \\
\text { T1 } \\
\text { E1 } \\
\text { E2 } \\
\text { SEIS } \\
\text { GR+T1 } \\
\text { GR+TI+SEIS }\end{array}$ & $\begin{array}{r}0 \\
0 \\
-5 \\
0 \\
5 \\
0 \\
5\end{array}$ & $\begin{array}{r}39 \\
0 \\
0 \\
0 \\
0 \\
39 \\
39\end{array}$ & $\begin{array}{r}0 \\
0 \\
0 \\
-5 \\
5 \\
0 \\
5\end{array}$ & $\begin{array}{r}39 \\
0 \\
5 \\
5 \\
7 \\
39 \\
40\end{array}$ & $\begin{array}{l}0 \\
0 \\
0 \\
0 \\
0 \\
0 \\
0\end{array}$ & $\begin{array}{l}0 \\
0 \\
0 \\
0 \\
0 \\
0 \\
0\end{array}$ & $\begin{array}{l}0 \\
0 \\
0 \\
0 \\
0 \\
0 \\
0\end{array}$ & $\begin{array}{l}0 \\
0 \\
0 \\
0 \\
0 \\
0 \\
0\end{array}$ \\
\hline
\end{tabular}

*** Segment $B$ end $\star * \star *$

W32OH32C PROCESS BUILDING PIPING STRESS ANALYSIS

$02 / 22 / 95$ W320, TANK $241-C-106$, ER4319 H-2-818480 AUTOPIPE+4.50 RESULT PAGE 18

\section{ASME 831.3C (1992) CODE COMPLIANCE}

(Monents in ft-lb)
Point Load In-pl. Out-Pl. Torsion s.t.F Eq. Load Code code name combination Moment Moment Moment in out no. type stress Allow.

\section{$\star \star \star$ Segment A begin $* * *$}

AOO Max

$\begin{array}{lcr}\text { Max P } & \\ \text { GR + Max P } & 92 & 0 \\ \text { Cold to T1 } & 109 & 27418 \\ \text { With SUs. load margin } & \\ \text { Sus. + E1 } & 94 & 11 \\ \text { SUS. + E2 } & 93 & 1 \\ \text { SEIS } & 4 & 13 \\ \text { SUS+SEIS } & 96 & 13\end{array}$

A01

Max $P$ GR + Max P
Cold to T' Sus. + E1 SUIS. + E2 SUS+SEIS

A02 - Max P GR + Max P Cold to $T$ Sus. + E1 SEIS SUS+SEIS

$\mathrm{A02}+\operatorname{Max} P$ $\mathrm{GR}+\operatorname{Max} P$ Cold to $T$ Sus. + E1 SEIS SUS+SE IS

AO3 N- Max P GR + Max P
Cold to T? Sus. + El Sus. + E2 SEIS SUS+SEIS
HNF-2486, Rev. 0

Page C-78
1.001 .00 (3a) HOOP 221.001 .00 (18) SUST 1.001 .00 (17)
(17) 1.001 .00 (18) OCC 1.001 .00 (18) OCC $1.001 .00(18)$
$1.001 .00(18)$

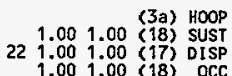

$\begin{array}{rr}41 & 0 \\ 95 & 18182 \\ 43 & 7 \\ 46 & 1 \\ 6 & 8 \\ 47 & 8\end{array}$
.00 1.00 (18) OCC 1.001 .00 (18) OCC 1.001 .00 (18) OCC $\begin{array}{rr}7 & 0 \\ 79 & 7406 \\ 8 & 4 \\ 15 & 0 \\ 8 & 4 \\ 15 & 4\end{array}$

1.631 .84 (18) $22 \begin{aligned} & 1.631 .84 \text { (18) SUST } \\ & 1.631 .84 \text { (17) DISP }\end{aligned}$ 1.631 .84 (18) OCC 1.631 .84 (18) OCC 1.631 .84 (18) OCC

(3a) HOOP $\begin{array}{rrrr}2 & 0 & 1.639 .84 \text { (18) SUST } \\ 73 & 7406 & 22 \\ 3 & 14 & & 1.63939 .84 \text { (17) DISP } \\ 4 & & & 1.84 \text { (18) OCC }\end{array}$ 1.631 .84 (18) OCC 1.631 .84 (18) OCC 1.631 .84 (18) OCC

$\begin{array}{rl}0 & 16700 \\ 138 & 16700 \\ 38728 & 25050^{* *} \\ 38728 & 41612 \\ 156 & 22211 \\ 141 & 22211 \\ 20 & 22211 \\ 158 & 22211 \\ 0 & 16700 \\ 31 & 16700 \\ 12980 & 25050 \\ 37 & 22211 \\ 35 & 22211 \\ 7 & 22211 \\ 39 & 22211 \\ 0 & 16700 \\ 9 & 16700 \\ 9745 & 25050 \\ 14 & 22211 \\ 18 & 22211 \\ 11 & 22211 \\ 20 & 22211 \\ 0 & 16700 \\ 2 & 16700 \\ 9745 & 25050 \\ 22 & 22211 \\ 5 & 22211 \\ 20 & 22211 \\ 22 & 22211 \\ & \end{array}$
1.001 .00 (3a) HOOP 0 16700 221.001 .00 (17) DISP 1.001 .00 (18) OCC 1.001 .00 (18) OCC 1.001 .00 (18) OCC 
W32OH32C PROCESS BUILDING PIPING STRESS ANALYSIS

$02 / 22 / 95$ W320. TANK 241-C-106, ER4319 $\mathrm{H}-2-818480$ AUTOPIPE+4.50 RESULT PAGE 19

ASHE B31.3c (1992) CODE COMPLIANCE

(Moments in ft-ib)' Loint Load In-Pl. Out-pl. Torsion s.1.F Eq. Load code code
(Stress in psi

name combination Moment Moment Moment in Out no. type stress Allow.

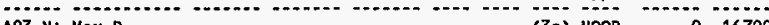

$G R+\operatorname{Max} P$ Cold to 11

Sus. + E1

SUS. + E2

0
3369
7
1
8
8

10
41
11
12
2
12

$2.442 .03(18)$ SUST 2.442 .03 (18) 2.442 .03 (18) $2.442 .03(18)$ OCC 2.442 .03 (18) 2.442 .03 (18) OCC

A03 F- Max P $\begin{array}{rr}0 & 19 \\ 10636 & 33 \\ 1 & 21 \\ 1 & 20 \\ 2 & 3 \\ 2 & 22\end{array}$

2.442 .03 (18) SUST

$5 \begin{aligned} & 2.44 \\ & 2.44 \\ & 2.03 \\ & (17) \text { DISP }\end{aligned}$

2.442 .03 (18) OCC

2.442 .03 (18) OCC

2.442 .03 (18) OCC

$2.442 .03(18)$ OCC

$\mathrm{A} 03 \mathrm{~F}+\operatorname{Max} P$

$G R+\operatorname{Max} P$

Sus. + E1

Sus. + E

SUS+SEIS

$\begin{array}{rr}19 & 0 \\ 33 & 10636 \\ 21 & 1 \\ 20 & 1 \\ 3 & 2 \\ 22 & 2\end{array}$

AO4 Max P

GR + Max P

Cold to $T$ I

Sus. + E1

SEIS

SUS+SEIS

A05 - Max P

$\mathrm{GR}+\operatorname{Max} ?$

Sus. $t E 1$

Sus. + E2

SEIS

SUS+SEIS

A05 + Max $P$

GR + Max P

Sus. + Ei

Sus. + E2

SEIS

SUS+SEIS
1.001 .00 (18) SUST

151.001 .00 (17) DISP $1.001 .00(18)$ OCC

1.001 .00 (18) OCC

$1.001 .00(18) \quad 0 C C$

(3a) HOOP

1.001 .00 (18) SUST

151.001 .00 (17) DISP

1.001 .00 (18) occ

1.001 .00 (18) OCC

1.001 .00 (18)

(3a) HOOP

1.001 .00 (18) SUST

$1.001 .00(18)$ OCC

1.001 .00 (18) OCC

$1.001 .00(18)$ OCC

(3a) HOOP

1.001 .00 (18) SUST

01.001 .00 (17) DISP

$1.001 .00(18)$ OCC

$\begin{array}{llll}1.001 .00(18) & \text { OCC } \\ 1.001 .00(18) & \text { OCC }\end{array}$
$1.001 .00(18)$ OCC

W320H32C PROCESS BUILDING PIPING STRESS ANALYSIS

U320. TANK 241-C-106, ER4319 $4-2-818480$ AUTOPIPE+4.50 RESULT PAGE 20

name combination

.

(3a) HOOP

(3a) HOOP $\begin{array}{rr}0 & 16700 \\ 16 & 16700\end{array}$

586625050

2922211

$18 \quad 22211$

$\begin{array}{ll}14 & 22211\end{array}$

o 16700

$29 \quad 16700$

$32 \quad 22211$

$31 \quad 22211$

622211

0 16700

$14 \quad 16700$

$16 \quad 22211$

3 22211

$\begin{array}{rr}3 & 22211 \\ 17 & 22211\end{array}$

$\begin{array}{rr}0 & 16700 \\ 27 & 16700\end{array}$

$15322 \quad 25050$

$29 \quad 22211$

$\begin{array}{ll}31 & 22211 \\ & 22211\end{array}$

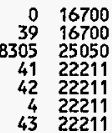

0 16700

$\begin{array}{rr}1 & 16700 \\ 7 & 25050\end{array}$

22211

122211

$\begin{array}{ll}0 & 22211 \\ 1 & 22211\end{array}$
A06 - Max P

GR + Max P

Cold to T1

Sus. + E1

SEIS

SUS+SEIS

$A 06+\operatorname{Max} P$

GR + Max P

Cold to T

Sus. + E1

SEIS

SUS+SEIS

A07 $\quad \operatorname{Max} P$

$\mathrm{GR}+\operatorname{Max} P$

Sus. + E1

SEIS

SUS+SEIS

A08 N- Max P

$\mathrm{GR}+$ Max P

Sus. + E1
Sus. + E2

SUS+SEIS

A08 $\mathrm{N}+\operatorname{Max} \mathrm{P}$

$G R+\operatorname{Max} P$

Cold to $I 1$

Sus. + E1

SEIS

SUS+SEIS

A08 F- Max P

GR + Max P

Sus. + E1

Sus. + E1
Sus. + E2

SEIS

SUS+SE $1 S$

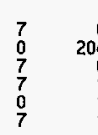

$\begin{array}{rr}339 & 0 \\ 0 & 1265 \\ 339 & 3 \\ 339 & 84 \\ 0 & 87 \\ 339 & 87\end{array}$

79

$\begin{array}{rr}71 & 0 \\ 0 & 1410 \\ 71 & 3 \\ 71 & 25 \\ 0 & 28 \\ 71 & 28\end{array}$

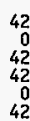

42
0
42
42
0
42

140
1446
3
15
18
18

0
1446
3
15
18
18

42
0
42
42
0
42

HNF-2486, Rev. 0

Page C-79
1.001 .00 (3a) HOOP

1.001 .00 (18) SUST

1.001 .00 (17) DISP

1.001 .00 (18) OCC

1.001 .00 (18) OCC

1.001 .00 (18) occ

(3a) HOOP

1.001 .00 (18) SUST

1.001 .00 (17) DISP

.001 .00 (18) OCC

.001 .00 (18) OCC

1.001 .00 (18) OCC

(3a) HOOP

1.001 .00 (18) SUST

01.001 .00 (17) DISP

1.001 .00 (18) OCC

1.001 .00 (18) OCC

1.001 .00 (18) OCC

(3a) HOOP

1.001 .00 (18) SUST

.00 1.00 (17) DIS 1.001 .00 (18) OCC 1.001 .00 (18) OCC

(3a) HOOP

2.442 .03 (18) SUST

02.442 .03 (17) DISP 2.442 .03 (18) OCC 2.442 .03 (18) OCC 2.442 .03 (18) OCC

(3a) $\mathrm{HOOP}$

2.442 .03 (18) SUST 2.442 .03 (17) DISP $2.442 .03(18)$ OCC 2.442 .03 (18) 2.442 .03 (18) OCC o 16700

16700

122211

12221

0 2221

- 16700

$51 \quad 16700$

$162 \quad 25050$

$52 \quad 22211$

1322211

99700

$54 \quad 16700$

25050

$\begin{array}{ll}56 & 22211\end{array}$

$\begin{array}{ll}73 & 22211 \\ 21 & 22211\end{array}$

o 16700

$\begin{array}{rr}32 & 16700\end{array}$

$34 \quad 22211$

$43 \quad 22211$

$45 \quad 22219$

0 16700

64
16700

$70 \quad 22211$

$92 \quad 2221$

$34 \quad 22211$

$0 \quad 16700$ $\begin{array}{rr}0 & 16700 \\ 38 & 16700\end{array}$ 18725050

$44 \quad 22211$

53 22211

$60 \quad 22211$ 
W32OH32C PROCESS BUILDING PIPING STRESS ANALYSIS

02/22/95 W320, TANK 241-C-106, ER4319 H-2-818480 AUtOPIPE+4.50 RESULT PAGE 21

\section{ASME $831.3 \mathrm{C}$ (1992) CODE COMPLIANCE}

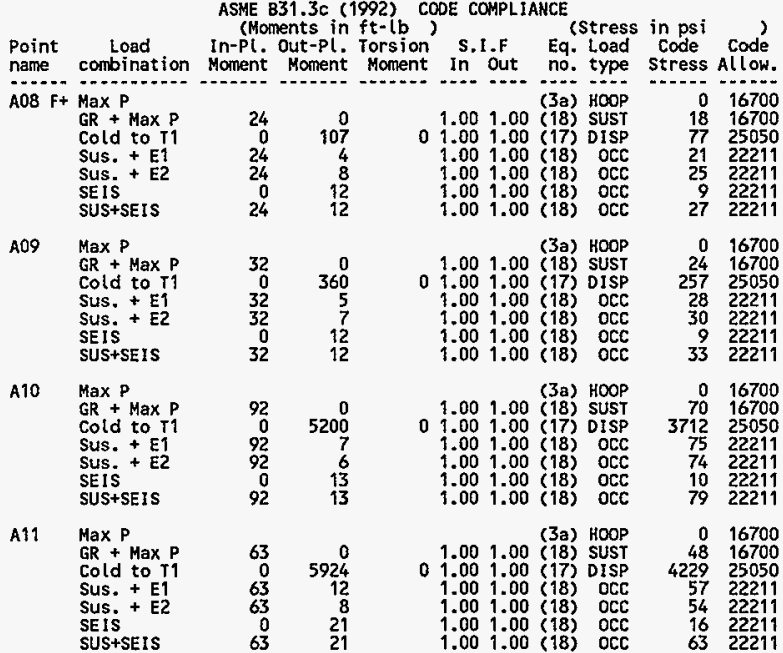

*** Segment B begin ***

A02 Max $P$

\begin{tabular}{|c|c|c|c|c|c|}
\hline $\begin{array}{l}\text { GR + Max P } \\
\text { Cold to TY } \\
\text { Sus. + E1 } \\
\text { Sus, + E2 } \\
\text { SEIS } \\
\text { SUS+SEIS }\end{array}$ & $\begin{array}{r}65 \\
153 \\
6 \\
15 \\
10 \\
16\end{array}$ & $\begin{array}{r}0 \\
0 \\
17 \\
0 \\
17 \\
17\end{array}$ & $\begin{array}{lll}1.63 & 1.84 \\
0 & 1.63 & 1.84 \\
1.63 & 1.84 \\
1.63 & 1.84 \\
1.63 & 1.84 \\
1.63 & 1.84\end{array}$ & $\begin{array}{l}(18) \\
(17) \\
(18) \\
(18) \\
(18) \\
(18)\end{array}$ & $\begin{array}{l}\text { SUS } \\
\text { DIS } \\
\text { OC } \\
\text { OC } \\
\text { Od } \\
\text { OC }\end{array}$ \\
\hline
\end{tabular}

HNF-2486, Rev. 0

Page C-80
U320H32C PROCESS BUILDING PIPING STRESS ANALYSIS

02/22/95 W320, TANK 241-C-106, ER4319 H-2-818480 AUTOPIPE+4.50 RESULT PAGE 22

ASME 831.3C (1992) CODE COMPLIANCE

(Moments in $\mathrm{ft}-\mathrm{lb}$ )

Point Load In-Pl. Out-Pl. Torsion S.I.F Eq. Load Code code name combination Moment Moment Moment in Out no. type Stress Allow. B01 H- Max P

$\begin{array}{lrr}\text { GR }+ \text { Max } P & 6 & 0 \\ \text { Cold to } T 1 & 153 & 0 \\ \text { SUs. }+ \text { E1 } & 6 & 13 \\ \text { SUS. }+ \text { E2 } & 11 & 0 \\ \text { SEIS } & 6 & 13 \\ \text { SUS+SEIS } & 12 & 13\end{array}$

0
0
13
0
13
13

0
0
13
0
13
13 (3a) HOOP 1.001 .00 (18) SUST 1.001 .00 (17) DISP $1.001 .00(18)$ OCC $1.001 .00(18)$ OCC $1.001 .00(18)$ DCC (0) 16700 $\begin{array}{rr}4 & 16700 \\ 109 & 25050\end{array}$ 1422211 (3a) HOOP 2.442 .03 (18) SUST 2.442 .03 (17) DISP $2.442 .03(18)$ OCC 2.442 .03 (18) OCC 2.442 .03 (18) OCC $\begin{array}{ll}11 & 22211 \\ 15 & 22211\end{array}$

B01 $N+$ Max P

$\begin{array}{lrr}\text { GR }+ \text { Max P } & 6 & 0 \\ \text { Cold to T1 } & 153 & 0 \\ \text { SUS. }+E 1 & 6 & 13 \\ \text { SUS. }+E 2 & 19 & 0 \\ \text { SEIS } & 6 & 13 \\ \text { SUS+SEIS } & 12 & 13\end{array}$

B01 F- Max P $G R+\operatorname{Max} P$ Sus. + E1 SEIS SUS+SEIS

14
69
14
17
3
17

(3a) HOOP 2.442 .03 (18) SUST 2.442 .03 (17) DISP 2.442 .03 (18) OCC 2.442 .03 (18) OCC 2.442 .03 (18) OCC 2.442 .03 (18) OCC

o 16700 $\begin{array}{rr}0 & 16700 \\ 10 & 16700\end{array}$ $266 \quad 25050$ $30 \quad 22211$ $23 \quad 22211$ $33 \quad 22211$

B01 F+ Max P GR + Max P Cold to $T$ Sus. + E1 SEIS SUS+SEIS (3a) HOOP 1.001 .00 (18) SUST 1.001 .00 (17) DISP 1.001 .00 (18) OCC 1.001 .00 (18) OCC 1.001 .00 (18) OCC 1.001 .00 (18) OCC

อ 16700 $25 \quad 16700$ 2125050 $31 \quad 22211$ 3722211

B02 Max P (3a) HOOP GR + Max P Sus. $+\mathrm{E} 1$ Sus. + E2 SEIS SUS+SEIS

B03 Max P

\begin{tabular}{|c|c|c|c|}
\hline $\begin{array}{l}\text { MaX P } \\
\text { GR + Max P } \\
\text { Cold to T1 } \\
\text { Sus. + E1 } \\
\text { Sus. + E2 } \\
\text { SEIS } \\
\text { SUS+SEIS }\end{array}$ & $\begin{array}{l}0 \\
0 \\
0 \\
0 \\
0 \\
0\end{array}$ & $\begin{array}{l}0 \\
0 \\
0 \\
0 \\
0 \\
0\end{array}$ & 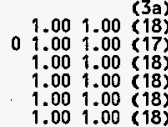 \\
\hline
\end{tabular}

$0 \quad 16700$

1016700

5025050

$\begin{array}{rr}13 & 22211 \\ 6 & 22211\end{array}$ $16 \quad 22211$

1.001 .00 (18) SUS 1.001 .00 (17) DISP 1.001 .00 (18) 1.001 .00 (18) OCC 1.001 .00 (18) OCC

0 16700 16700 5 2221 22211

0 16700 o 16700 o 25050 o 22211 2221 SUS+SEIS

1.001 .00 (18) OCC 


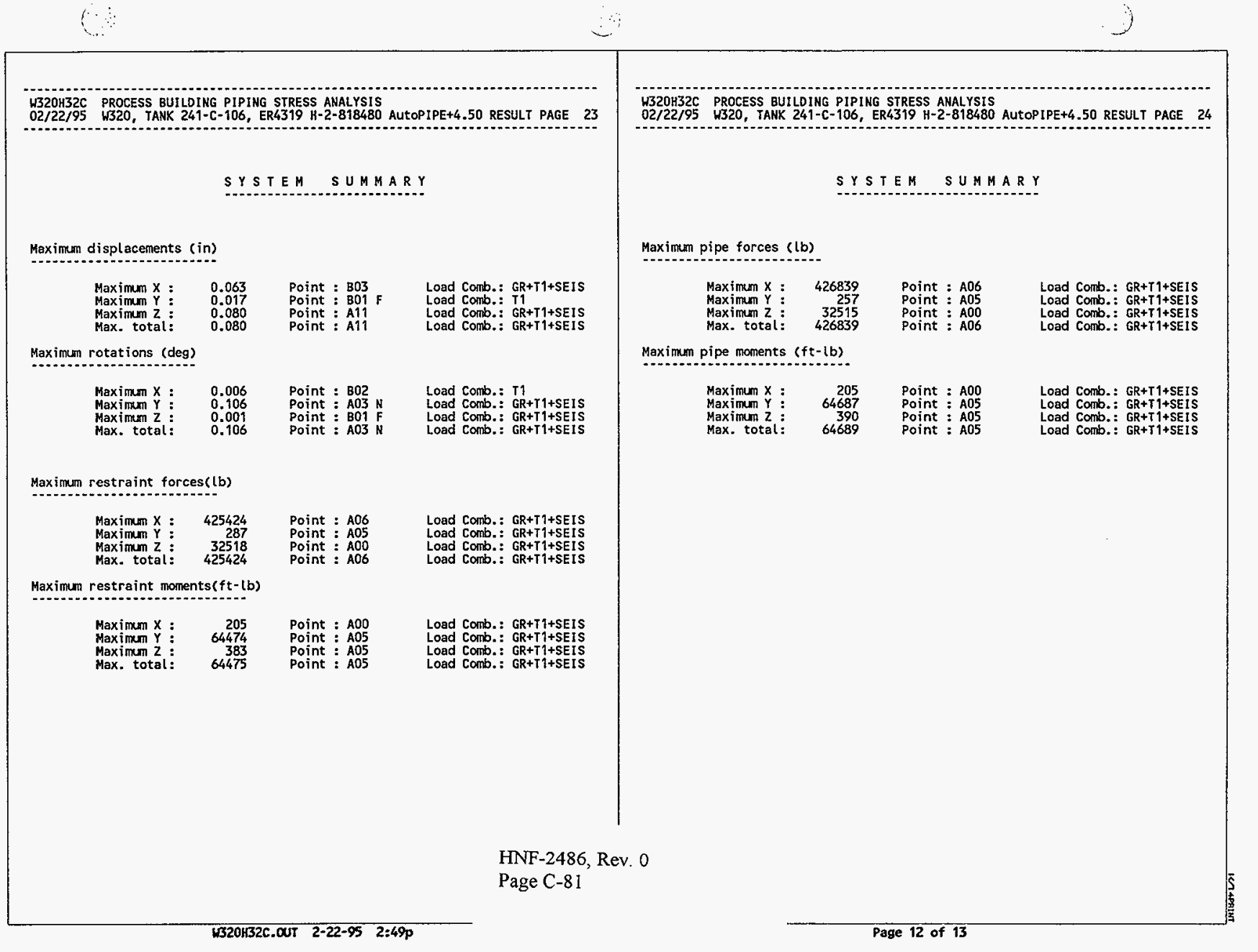


32OH32C PROCESS BUILDING PIPING STRESS ANALYSIS

106 TR4319 $\mathrm{H-2}-818480$ AUTOPIPE+4.50 RESULT PAGE

\section{SYSTEM SUMMARY}

Maximum sustained stress

$$
\begin{array}{ll}
\text { Point } & : A 00 \\
\text { Stress psi } & 138 \\
\text { Aliowable psi } & \vdots 16700 \\
\text { Ratio } & 0.01 \\
\text { Load combination } & \text { GR }+ \text { Max } P
\end{array}
$$

Maximum displacement stress

$$
\begin{array}{ll}
\text { Point } & : \text { A00 } \\
\text { Stress psi } & : 38728 \\
\text { Allowable psi } & : 41612 \\
\text { Ratio } & : 0.93
\end{array}
$$$$
\text { Load combination : Cold to } \mathrm{I}
$$

Maximum occasional stress

$$
\begin{array}{ll}
\text { Point } & \text { A00 } \\
\text { Stress psi } & : 158 \\
\text { Allowable psi } & \vdots 22211 \\
\text { Ratio } & : 0.01 \\
\text { Load combination } & \text { sus+SEIS }
\end{array}
$$

Maximum sustained stress ratio

$$
\begin{array}{ll}
\text { Point } & : A 00 \\
\text { Stress psi } & 138 \\
\text { Aliowable psi } & \vdots 16700 \\
\text { Ratio } & : 0.01 \\
\text { Load combination } & \text { GR + Max P }
\end{array}
$$

Maximum displacement stress ratio

$$
\begin{array}{ll}
\text { Point } & \text { A00 } \\
\text { Stress psi } & 38728 \\
\text { Allowable psi } & \vdots 41612 \\
\text { Ratio } & 0.93 \\
\text { Load combination } & \text { Cold to II }
\end{array}
$$

Maximum occasional stress ratio

$$
\begin{array}{ll}
\text { Point } & \text { A00 } \\
\text { Stress psi } & 158 \\
\text { Allowable ps } 1 & \vdots 22211 \\
\text { Ratio } & 0.01 \\
\text { Load combination } & \text { SUS+SEIS }
\end{array}
$$

1320H32C PROCESS BUILDING PIPTNG STRESS AMAYYSIS

W320, TANK 241-C-106, ER4319 H-2-818480 AutOP1PE+4.50 RESULT PAGE 26

\section{SYSTEM SUMMAR Y} * * The system satisfies ASHE B31.3 code requirements *** for the selected options 


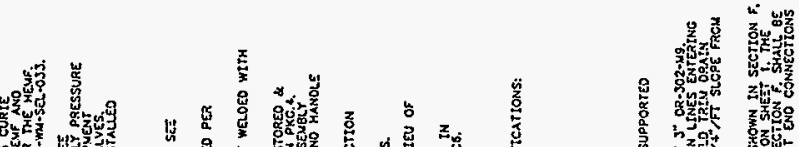

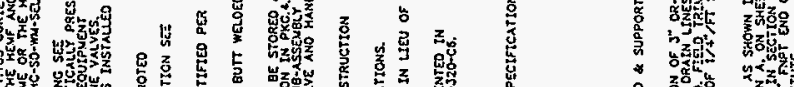

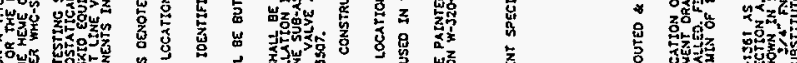

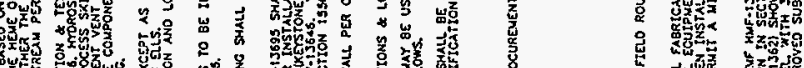

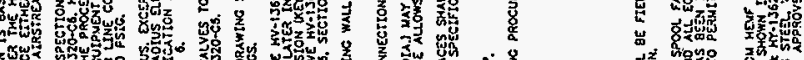

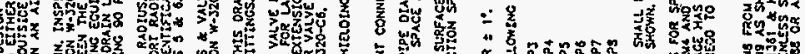

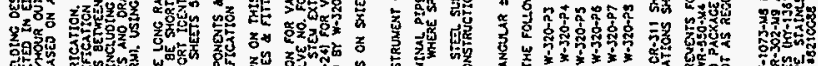

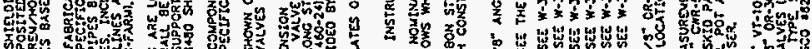

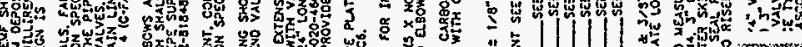

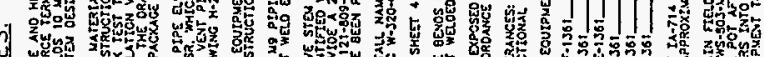

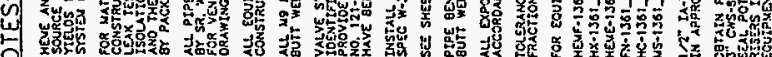
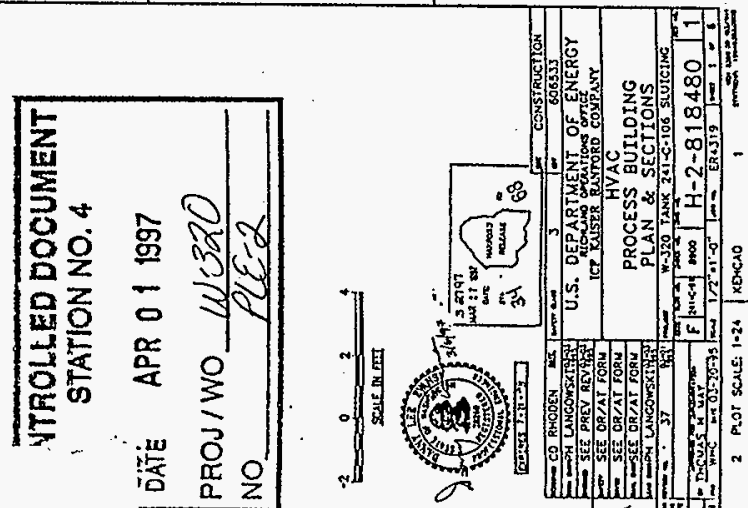

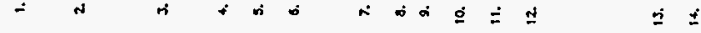
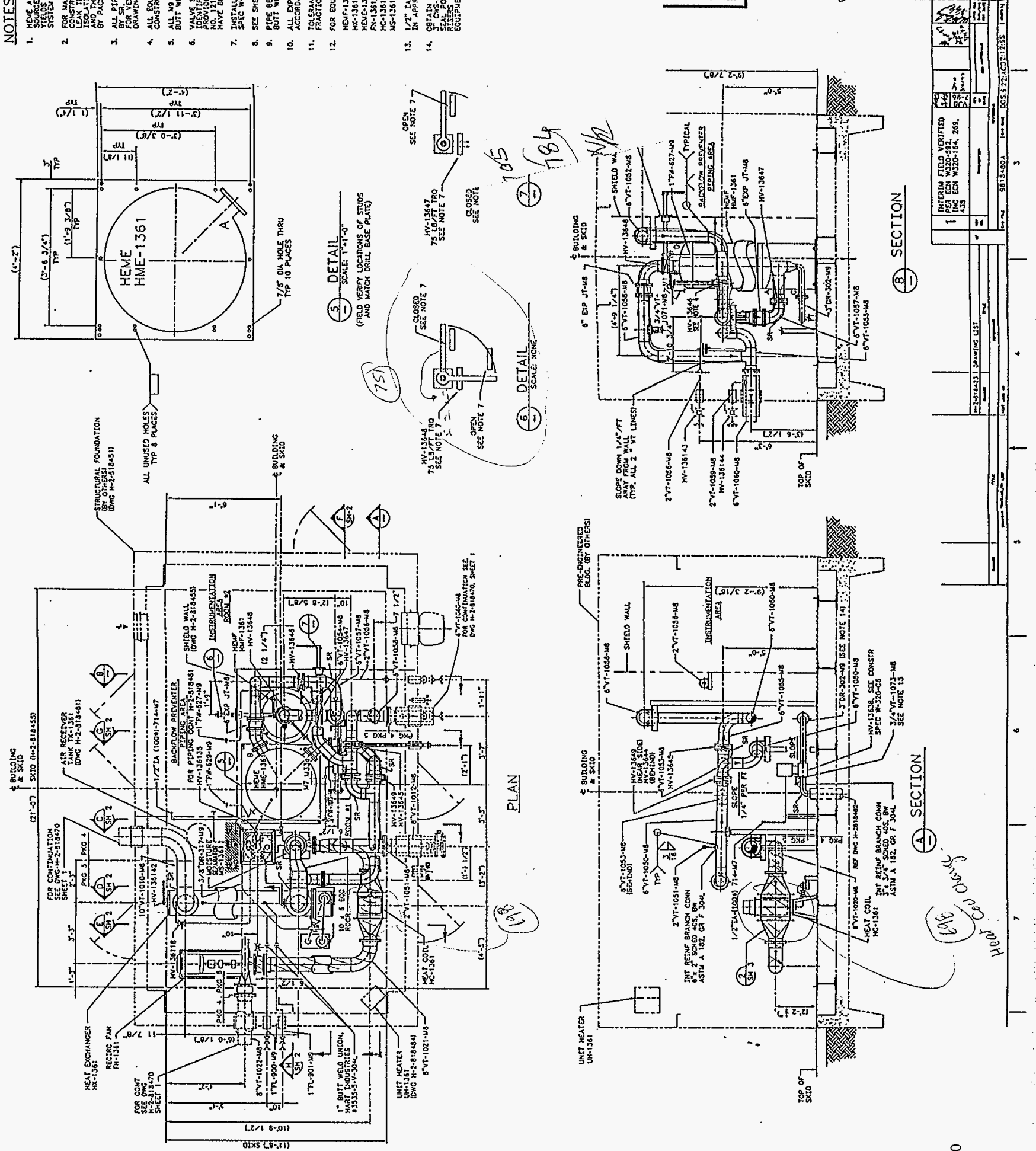


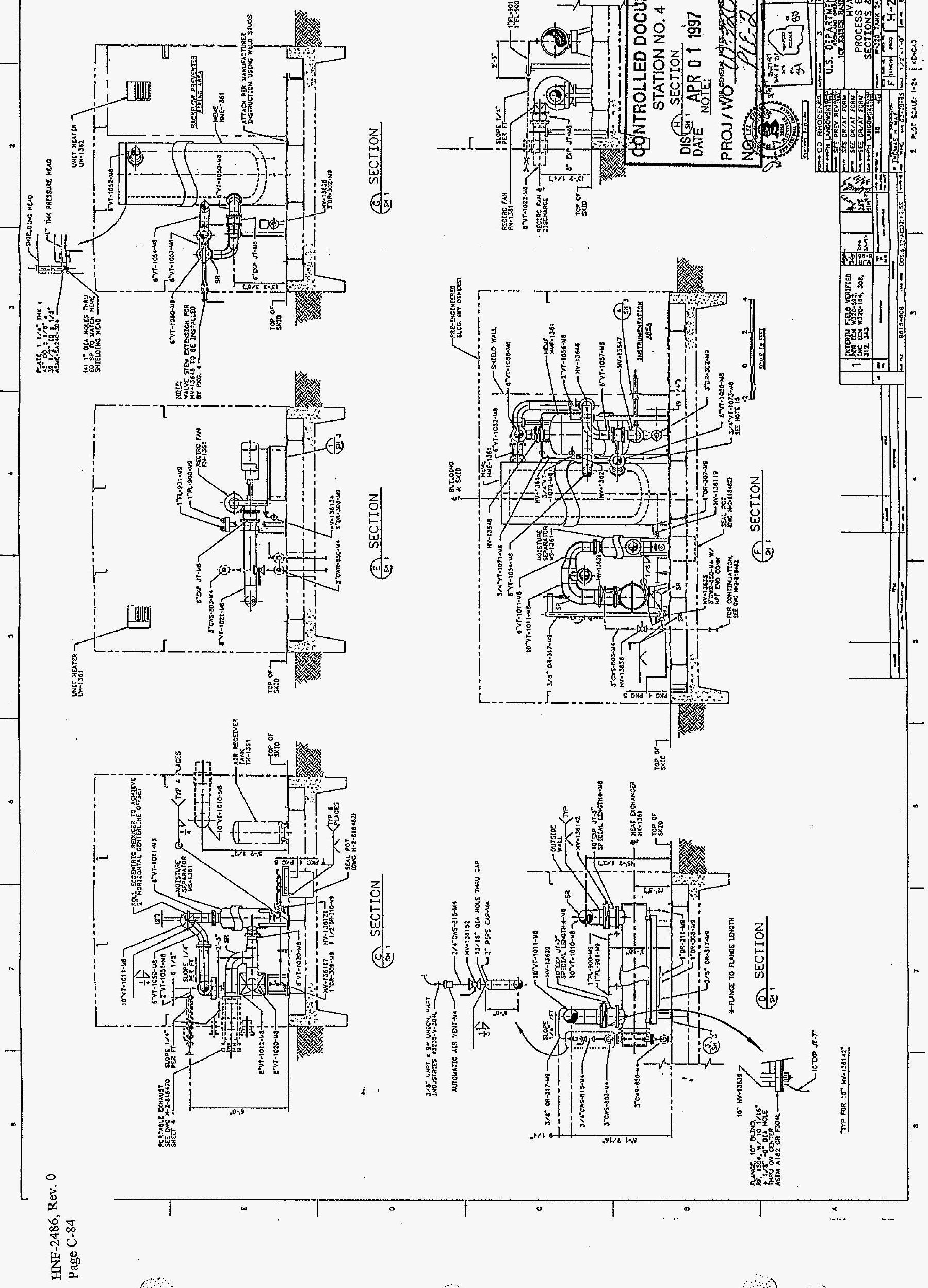




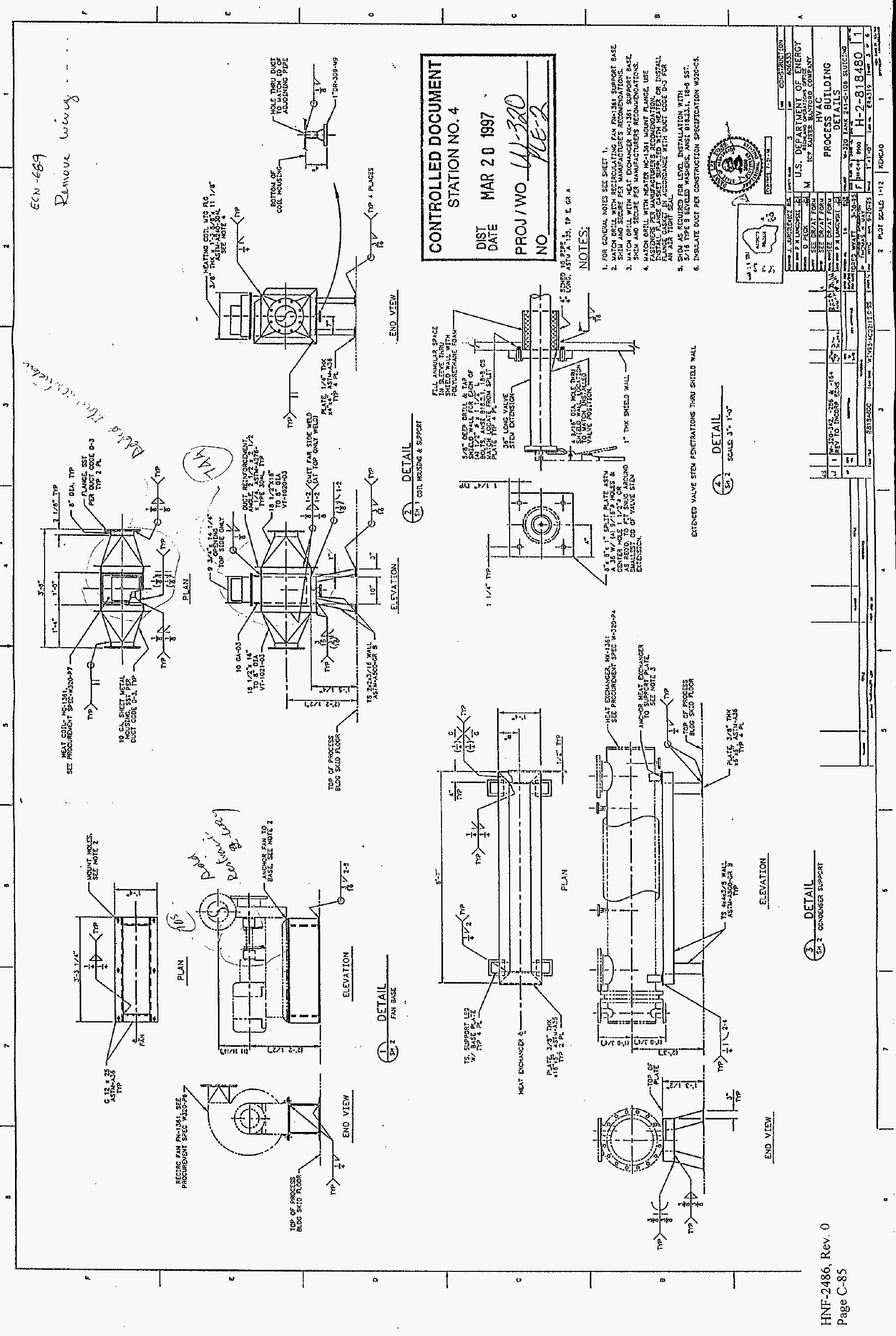



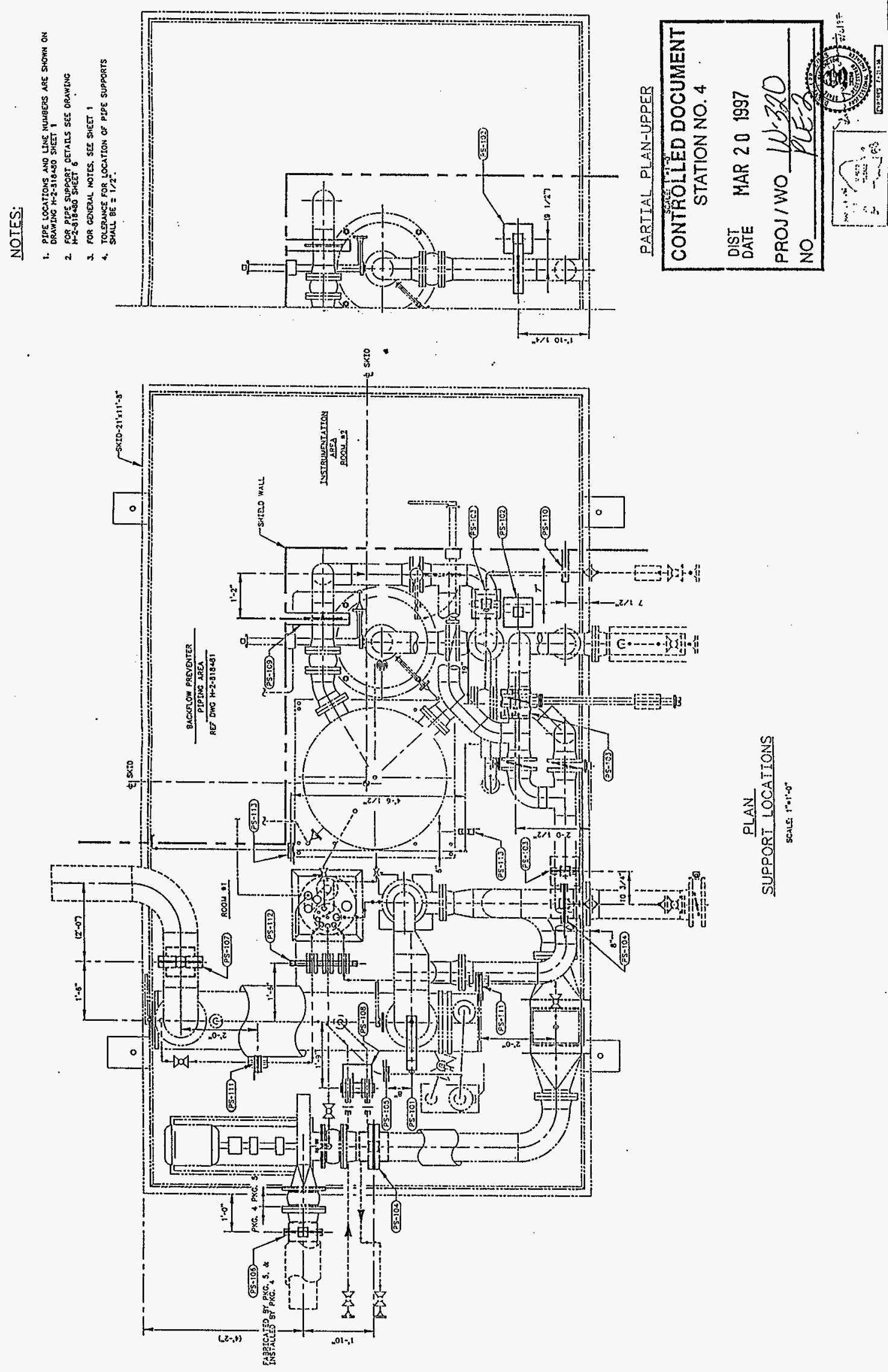

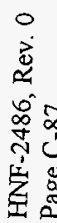


HNF-2486, Rev. 0

W320-28-033

Process Building Duct Support

Stress Analysis

$$
D-\mathbf{i}
$$


KAISER ENGINEERS

HANFDRD

This shoet shows the etatus and description of the etiached Desion Analysis shoets.

Discipline $28-H V A C$ WolJob No. ER4319/W320

Projoct No: \& Namo w320, Tank $241-c-106$ S/uicina

calculation tiem Process Building Duct support stress Anol/sis

These calculetions apply to:

Dwo. No. $H-2-818480$

Rev. No. 0

Dwg. No.

Rov. No.

Other (StudY, CDR)

The status of these calculations is:

$\square$ Preliminary Calculations

区] Fina: Calculations

DChack Calculations fon Calculation DBtod

$-1$

Void Calculation Roeson Voided

Incorporated in Finsl Drawings?

Q Yos $\square$ No

This calculation verified by independent "chock" caleuletions?

$\square$ Yes $\quad \square$ No

Original and Revised Calculation Approvals:

\begin{tabular}{|c|c|c|c|}
\hline & $\begin{array}{c}\text { Rev. } 0 \\
\text { Signature/Oete }\end{array}$ & $\begin{array}{c}\text { Rev. } 1 \\
\text { Signetureroate }\end{array}$ & $\begin{array}{c}\text { Rev. } 2 \\
\text { Signaturo/Dato }\end{array}$ \\
\hline Originstor & Rosues C. Complell $3-1-95$ & & \\
\hline Checked by & & & \\
\hline Approved by & & & \\
\hline $\begin{array}{l}\text { Checked Agsinet } \\
\text { Approved Vendor Date }\end{array}$ & & & \\
\hline
\end{tabular}

INDEX

Design Analysis

Pago No.

\section{Description}

i Calculation Identification and Index

$i c$ Calculation Cross Index

$\frac{1}{18}$

Obiective, Criteria, Given Dota; Assumotions, Methods, References

Colculation

Conclusions

Appendix A

HNF-2486, Rev. 0

Page D- ]
Rev. No. $\omega 320-28-033$ 28 Calculation No. $54320=17-032$ 
HNF-2486, Rev. 0

Page D-3

ICF Kaiser Hanford

Company

DESIGN ANALYSIS

Calc. No. W320- $28-033$

Revision 0

Page No. 1 of 18

Client WHC

Subject Process Building Duct Support Stress Analysis

Location: Tank 241-C-106
WO/Job No. ER4319M320

Date 3/1/95 By Rodney C. Campbell

Checked \&187/95 By N. Jiplo

Revised

By

\section{OBJECTIVE:}

The objective of this calculation is to verify that the Process Building HVAC ducting supports meet ASME B31.3 code stress requirements.

\section{CRITERIA:}

1. Functional Design Criteria WHC-SD-W320-FDC-001, Rev. 2.

2. ASME B31.3-1993, Chemical Plant and Petroleum Refinery Piping

\section{GIVEN DATA:}

1. Minimum design temperature: $50^{\circ} \mathrm{F}$.

2. Maximum design temperature: $180^{\circ} \mathrm{F}$.

3. All supports are made from ASTM A36, Grade B carbon steel or ASTM A 500 Grade B carbon steel.

4. All ducting is safety class 3 .

5. Ail plates are made from ASTM A36 steel.

6. Maximum allowable stress of ASTM A36 steel is 17,800 psi.

7. Yield stress of ASTM A500 steel is $42,000 \mathrm{psi}$.

\section{ASSUMPTIONS:}

1. All supports were analyzed as $Y$-Stops due to the fact that the restraining bolt is on the outside of the insulation and its coverin making it impossible to control the pipe. The Y-Stop can only carry a load in the vertical downward direction. Horizontal loads will come due to friction between the support and the pipe.

\section{METHODS:}

Hand calculations using Mathcad 5.0.

\section{REFERENCES:}

1

1. Drawing H-2-818480, Rev. 6 .

2. Calculation $\mathbf{W} 320-\mathrm{H}-32$, Process Building Pipe Stress.

4. AISC Manual of Steel Construction, Ninth Edition.

5. Blodgett, Design of Welded Structures

8. ASTM A 500-93 Specification for Cold-Formed Welded and Seamless Carbon Steel Structural Tubing in Rounds and Shapes.

9. Grinnell Pipe Hanger Handbook.

\section{CALCULATION:}

Maximum allowable stress of $A 36$ carbon steel at $120^{\circ} \mathrm{F}$ is; $\quad F_{36}:=17800 \cdot \mathrm{psi}$

Yield stress of $A 500$, Grade $B$ carbon steel at $120^{\circ}$ is: $\quad F_{\mathbf{y 5 0 0}}:=42000 \cdot \mathrm{psi} \quad$ Ref. 8

Young's modulus $E$ is: $\quad E:=29 \cdot 10^{6} \cdot$ psi $\quad$ Ref. 4

There are five types of pipe supports used in the Process Building. The worst load on each type of support will be used to verify that the support is adequate. 
Client WHC

Subject Process Building Duct Support Stress Analysis

Location: Tank 241-C-106
WO/Job No. ER4319N320

Date $3 / 1 / 95$

By Rodney C. Campbell

Checked $3 / 1 / 95$ By N.NUPLO

Revised

By

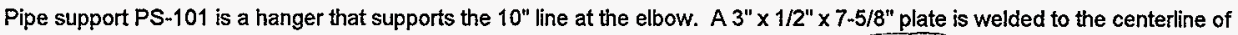
the pipe. A clevis and rod connect to the plate and is supported by a $3^{\prime \prime} \times 3-1 / 2$ " $\times 1 / 4^{\prime \prime}$ steelangle. The steel angle is welded $t$ a. $3^{\prime \prime} \times 3^{\prime \prime} \times 3 / 16^{\prime \prime}$ tube. The tube is welded to a baseplate that is welded to the floor.

The toads on the hanger are:

$\mathbf{F}_{\mathbf{h a}}:=\mathbf{0} \cdot \mathbf{l b f} \quad$ First horizontal load on hanger rod. Ref. 2.

$\mathbf{F}_{\mathbf{h b}}$ := 0. lbf Second horizontal load on hanger rod. Ref. 2.

$F_{\mathbf{~} 1}:=1311-$ lbf Vertical load on hanger rod. Ref. 2.

$F_{\text {v2 }}:=8.02 \cdot$ lbf Weight of Angle. Ref. 4.

$\mathbf{F}_{\mathbf{v}}:=\mathbf{F}_{\mathbf{v 1}}+\mathbf{F}_{\mathbf{v} 2}$ Total vertical load.

The distance from the support to the load is: $\mathbf{L}_{\mathbf{a}}:=\mathbf{1 2}$ in Ref. 1

The length of the column is: $L_{p s 101}:=7.1875 . \mathrm{ft} \quad$ Ref. 1

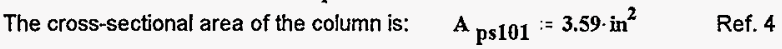

The section modulus of the $4 " \times 4^{\prime \prime} \times 1 / 4 "$ féctangular tube is: $\quad S_{\text {ps101 }}:=4.11 \cdot$ in $^{3} \quad$ Ref. 4

Governing radius of gyration of the column, $r$ is: $\quad r:=1.51 \cdot$ in $\quad$ Ref. 4

The section modulus of the $3^{\prime \prime} \times 3^{\prime \prime} \times 3 / 16^{\prime \prime}$ tube steel is: $s_{a}:=1.73 \cdot$ in $^{3} \quad$ Ref. 4

A. Verify that the tube steel is adequate to carry the load.

The tube steel can be analyzed as a cantilever with a concentrated load at any point.

$M:=F_{\text {v1 }} \cdot L_{a} \quad M=15732 \cdot 1 b f$ in

$\mathbf{f}_{\mathbf{b}}:=\frac{\mathbf{M}}{\mathbf{S}_{\mathbf{a}}}$

$f_{b}=9093.64 \cdot p s i \quad$ Calculated bending stress

$f_{b}<F_{36}=1$ The calculated bending stress is less than the allowable. The tube steel will not fail.

B. Determine if the combined stresses on support PS-101 satisfy the requirements of the AISC manual. The combined stresses are determined per section 1.6, pg 5.26, Ref. 4.

Determine the allowable load in compression $F_{\text {a500 }}$.

Determine if $C_{c}$ is less than or greater than $\mathrm{kl} / \mathrm{r}$.

$C_{c}:=\sqrt{\frac{2 \cdot \pi^{2} \cdot E}{F y 500}} \quad C_{c}=116.75$

$K:=2 \quad$ Ref. 4, Table C1.8.1

HNF-2486, Rev. 0

Page D-4

$\frac{K \cdot L \text { ps101 }}{r}=114.24$

Because the $\mathrm{k} / \mathrm{r}$ is less than $\mathrm{C}_{\mathrm{c}}, \mathrm{F}_{\mathrm{a} 500}$ (allowable compression stress) is determined from the following formula. 


\section{Company}

Client WHC

Subject Process Building Duct Support Stress Analysis

Location: Tank 241-C-106
DESIGN ANALYSIS

Calc. No. W320-K -033

Revision 0

Page No. 3 of 18

WO/Job No. ER4319N 320
Date $3 / 1 / 95$
By Rodney C. Campbell
Checked $3 / 1 / 95$
Revised
By N. JUPLO
By

$$
F_{2500}:=\frac{\left[1-\left.\frac{\left(\frac{K \cdot L_{p s 101}}{r}\right)^{2}}{2 \cdot C_{c}{ }^{2}}\right|_{y 500}\right.}{\left(\frac{5}{3}\right)+\frac{3 \cdot\left(\frac{K \cdot L_{p s 101}}{r}\right)}{8 \cdot C_{c}}-\frac{\left(\frac{K \cdot L_{p s 101}}{r}\right)^{3}}{8 \cdot C_{c}^{3}}}
$$

$F_{\text {a500 }}=11423.03 \cdot p s i \quad$ Allowable compression stress. Ref. 4 .

Determine if $f_{a} / F_{a}$ is less than 0.15 .

$$
\begin{aligned}
& f_{a}:=\frac{F_{v}}{A_{p s 101}} \quad f_{a}=367.42 \cdot p s i \\
& \frac{f_{a}}{F_{2500}}=0.0322
\end{aligned}
$$

Because $f_{a} / F_{a}$ is less than 0.15, formula 1.6-2 may be used.

Determine the allowable bending stress, $F_{\mathrm{b} 500}$.

$F_{b 500}:=.60 \cdot F_{y 500} \quad F_{b 500}=25200 \cdot p s i$

Determine bending stress, $f_{b}$.

$$
\begin{array}{lr}
M:=F_{v} \cdot L_{a} & M=15828.24 \cdot \text { lbf in } \\
f_{b}:=\frac{M}{S_{p s 101}} & f_{b}=3851.15 \cdot p s i
\end{array}
$$

Verify that the stresses meet the requirements of 1.6-2.

$\frac{f_{a}}{F_{a 500}}+\frac{f_{b}}{F_{b 500}}=0.185$

The stresses meet the requirements of section 1.6-2, by being less than or equal to one.

C. Verify that the welds on support PS-101 are adequate to carry the load.

Calculate the required size of the fillet weld holding the tube steel to the tube steel.

The drawing shows a 1/4" fillet weld holding the tube steel to the tube steel.

$$
\begin{array}{ll}
\omega:=.25 \cdot \text { in } & \text { Size of fillet weld. } \\
\mathbf{l}:=4 \cdot 3 \cdot \mathrm{in} & \text { Length of weld. All around tube steel. } \\
\tau:=9600 \cdot \mathrm{psi} & \text { Allowable shear stress. Ref. } 5, \text { Chapter } 7, \text { Table } 6 . \\
\mathbf{b}:=3 \cdot \mathrm{in} & \text { Horizontal length of weld on tube steel. } \\
\mathbf{d}:=3 \cdot \mathrm{in} & \text { Vertical length of weld on tube steel. }
\end{array}
$$

$$
f_{t}:=\frac{F v}{I} \quad f_{t}=109.92 \cdot \frac{\text { lbf }}{\text { in }} \quad \text { Shear load on weld. }
$$


Client WHC

Subject Process Building Duct Support Stress Analysis

Location: Tank 241-C-106
WO/Job No. ER4319N320
Date $3 / 1 / 95$
By Rodney C. Campbell
Checked $3 / 1 / 95$
Revised
By N. NUPLO
By

$$
\begin{aligned}
& S_{w}:=\mathbf{b} \cdot \mathbf{d}+\frac{d^{2}}{3} \quad \text { Ref. } 5, \text { Table } 5 . \\
& f_{b}:=\frac{M}{S_{w}} \quad f_{b}=1319.02 \cdot \frac{\text { lbf }}{\text { in }} \quad \text { Bending load on weld. } \\
& f_{r}:=\sqrt{f_{t}{ }^{2}+f_{b}{ }^{2}} \quad f_{r}=1323.59 \cdot \frac{\text { lbf }}{\text { in }} \quad \text { Resultant load on weld. } \\
& { }^{\omega_{\mathbf{r}}}:=\frac{\mathbf{f}_{\mathbf{r}}}{\tau \cdot .707} \quad \text { Required size of fillet weld. Ref. } 5, \text { Chapter } 7 . \\
& { }^{\infty} \mathrm{r}=0.195 \cdot \text { in Calculated fillet weld required to carry the load. }
\end{aligned}
$$

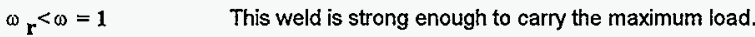

Calculate the required size of the throat of the fillet weld holding the plate to the elbow.

The plate dimensions are $3^{\prime \prime} \times 1 / 2^{\prime \prime} \times 7-5 / 8^{\prime \prime}$ and it is cut to conform to the outer radius of a $10^{\prime \prime}$, short radius elbow. For the calculation, the increase in length of the weld due to the curvature of the elbow will not be considered. The plate is welded $t$ the elbow with a $1 / 4$ " fillet weld.

$\omega:=.25 \cdot$ in $\quad$ Throat of weld.

$1:=2 \cdot(3 \cdot \mathrm{in}+.5 \cdot \mathrm{in}) \quad$ Length of weld.

$\tau:=9600 \cdot$ psi $\quad$ Allowable shear stress. Ref. 5 , Chapter 7, Table 6.

$$
\begin{aligned}
& f_{t}:=\frac{F_{v}}{l} \quad f_{t}=188.43 \cdot \frac{\mathrm{lbf}}{\text { in }} \\
& f_{x a}:=\frac{F_{h a}}{l} \quad f_{x a}=0 \cdot \frac{\text { bf }}{\text { in }} \\
& f_{x b}:=\frac{F_{h b}}{l} \quad f_{x b}=0 \cdot \frac{\text { bbf }}{\text { in }} \\
& f_{r}:=\sqrt{f_{t}^{2}+f_{x a}^{2}+f_{x b}^{2}} \quad f_{r}=188.43 \cdot \frac{\mathrm{bf} f}{\text { in }}
\end{aligned}
$$

$\omega_{\mathbf{r}}:=\frac{\mathbf{f}_{\mathbf{r}}}{\tau \cdot 707} \quad$ Required size of fillet weld. Ref. 5, Chapter 7.

${ }^{\omega_{r}}=0.0278 \cdot$ in Calculated size of fillet weld required to carry the load. PNF-2486,

${ }^{\omega} r^{<\omega}=1 \quad$ This weld is strong enough to carry the maximum load.

Calculate the required size of the fillet weld holding the tube steel to the plate on the floor.

The plate on the floor is $6^{\prime \prime} \times 6^{\prime \prime} \times 1 / 2^{\prime \prime} . A 3 / 16^{\prime \prime}$ fillet weld all around holds the tube steel to the plate. 
Client WHC

Subject Process Building Duct Support Stress Analysis

Location: Tank 241-C-106
WO/Job No. ER4319N 320

Date 3/1/95

By Rodney C. Campbell

Checked 3/1/95 By M. JUPLD

Revised

$$
\begin{aligned}
& \tau:=9600 \cdot \text { psi } \quad \text { Allowable shear stress. Ref. 5, Chapter 7, Table } 6 . \\
& b:=4 \cdot \text { in } \\
& d:=4 \cdot \text { in } \\
& \text { I }:=\mathbf{2} \cdot \mathbf{b}+\mathbf{2} \cdot \mathbf{d} \\
& f_{t}:=\frac{F v}{l} \quad f_{t}=82.44 \cdot \frac{1 b f}{\text { in }} \\
& S_{w}:=b \cdot d+\frac{d^{2}}{3} \\
& M=15828.24 \cdot \mathrm{lb} f \cdot \text { in } \\
& f_{b}:=\frac{M}{S_{w}} \quad f_{b}=741.95 \cdot \frac{\text { lbf }}{\text { in }} \\
& f_{r}:=\sqrt{f_{b}^{2}+f_{t}^{2}} \quad f_{r}=746.51 \cdot \frac{l b f}{\text { in }} \\
& \omega_{\mathbf{r}}:=\frac{\mathbf{f}_{\mathbf{r}}}{\tau \cdot .707} \quad \text { Required size of fillet weld. Ref. 5, Chapter } 7 . \\
& { }^{\infty} r=0.11 \cdot \text { in Calculated size of fillet weld required to carry the load. } \\
& { }^{\omega}{ }_{r}<\omega=1 \quad \text { This weld is strong enough to carry the maximum load. }
\end{aligned}
$$

Page No. 5 of 18 
Client WHC

Subject Process Building Duct Support Stress Analysis

Location: Tank 241-C-106
WO/Job No. ER4319N320

Date 3/1/95 By Rodney C. Campbell

Checked $3 / 1 / 95$ By N. JUPLO

Revised

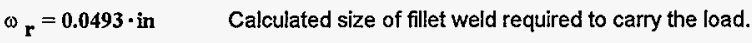

${ }^{\omega} \mathbf{r}^{<\omega}=1$ This weld is strong enough to carry the maximum load.

D. Verify that the threaded rod, clevis and pin on support PS-10? are adequate to carry the load.

From reference 9 , the allowable on each of these pieces is $1810 \mathrm{lbf}$. They are adequate to carry the load.

E. Verify the stresses in the plate do not exceed the allowables.
$A:=3 \cdot$ in $\cdot 3 \cdot$ in Area that force is acting upon.
$h:=\mathbf{l} \cdot$ in
b : $=3 \cdot$ in $+4 \cdot 1 \cdot$ in
Effective width of plate, $D+4 T$.
$\mathrm{S}:=\frac{\mathbf{b} \cdot \mathbf{h}^{2}}{6}$

The stress generated in the base plate is:

$$
\begin{aligned}
& \sigma:=\frac{F_{\mathbf{V}}}{A}+\frac{M}{S} \\
& \sigma=13713.62 \cdot p s i
\end{aligned}
$$

The stress of 13,696 psi is less than the allowable stress of 17,800 psi. The plate thickness is acceptable.

2. Analyze the pipe support PS-102 (loads from Autopipe analysis W32OH32A).

Pipe support PS-102 is a $3^{\prime \prime} \times 3-1 / 2$ " $\times 1 / 4^{\prime \prime}$ angle welded to a $3^{\prime \prime} \times 3$ " $\times 3 / 16^{\prime \prime}$ tube. The pipe rests on the angle.

The loads on the support are:

$F_{\text {ha }}:=\mathbf{0}$ - tbf First horizontal load on support. Ref. 2.

$\mathbf{F}_{\mathbf{h b}}:=\mathbf{0} \cdot \mathbf{l b f} \quad$ Second horizontal load on support. Ref. 2.

$F_{\text {v1 }}:=23 \cdot$ lbf Vertical load on support. Ref. 2.

$\mathbf{F}_{\mathbf{v 2}}:=\mathbf{0} \cdot \mathbf{l b f} \quad$ Weight of Angle. Ref. 4.

$\mathbf{F}_{\mathbf{v}}:=\mathbf{F}_{\mathbf{v} 1}+\mathbf{F}_{\mathbf{v} 2}$ Total vertical load.

The length of the column is: $L_{\text {ps102 }}:=6.9 \cdot \mathrm{ft} \quad$ Ref. 1

The distance from the support to the load is: $\mathrm{L}_{\mathbf{a}}:=8 \cdot \mathrm{in}$

Ref. 1

The cross-sectional area of the column is: $A_{\text {ps102 }}:=2.02 \cdot \mathrm{in}^{2}$

The section modulus of the $3^{\prime \prime} \times 3^{\prime \prime} \times 3 / 16^{\prime \prime}$ rectangular tube is:

Governing radius of gyration of the column, $r$ is: $\quad r:=1.13 \cdot$ in

$2 \quad$ Ref. 4

The section modulus of the $3^{\prime \prime} \times 3-1 / 2 " \times 1 / 4$ " (with bending about the $3-1 / 2$ " leg) angle is:

Ref. 4

Ref. 4

$$
S_{a}:=.776 \cdot \mathrm{in}^{3}
$$

Ref. 4 
Client WHC

Subject Process Building Duct Support Stress Analysis

Location: Tank 241-C-106
WO/Job No. ER4319N 320

Date 3/1/95 By Rodney C. Campbell

Checked $3 / 1 / 45$ By N. JUPLO

Revised

By

A. Verify that the angle is adequate to carry the load.

The angle can be analyzed as a cantilever with a concentrated load at any point.

$M:=F_{v r} \cdot L_{a} \quad M=184 \cdot b_{b} \cdot$ in

$\mathbf{f}_{\mathbf{b}}:=\frac{\mathbf{M}}{\mathbf{S}_{\mathbf{a}}}$

$\mathbf{f}_{b}=\mathbf{2 3 7 . 1 1} \cdot \mathbf{p s i} \quad$ Calculated bending stress

$f_{b}<F_{36}=1$ The calculated bending stress is less than the allowable. The angle will not fail.

B. Determine if the combined stresses on support PS-102 satisfy the requirements of the AlSC manual. The combined stresses are determined per section 1.6, pg 5.26, Ref. 4 .

Determine the allowable load in compression $\mathrm{F}_{\mathrm{a} 500}$.

Determine if $C_{c}$ is less than or greater than $\mathrm{k} / \mathrm{r}$.

$C_{c}:=\sqrt{\frac{2 \cdot \pi^{2} \cdot E}{F y 500}} \quad C_{c}=116.75$

$\mathrm{K}:=2 \quad$ Ref. 4 , Table C1.8.1

$\frac{K \cdot L \text { ps102 }}{r}=146.55$

Because the $\mathrm{Kl} / \mathrm{r}$ is greater than $\mathrm{C}_{\mathrm{c}}, \mathrm{F}_{\mathrm{a} 500}$ (allowable compression stress) is determined from the following formula.

$F_{2500}:=\frac{12 \cdot \pi^{2} \cdot E}{23 \cdot\left(\frac{K \cdot L_{p s 102}}{r}\right)^{2}}$

$F_{\text {a500 }}=6953.24 \cdot \mathrm{psi} \quad$ Allowable compression stress. Ref. 4.

Determine if $f_{\mathrm{a}} / \mathrm{F}_{\mathrm{a}}$ is less than 0.15 .

$f_{a}:=\frac{F_{v}}{A_{\text {ps102 }}} \quad f_{a}=11.39 \cdot p s i$

$\frac{f_{\mathrm{a}}}{\mathrm{F}_{\mathrm{a500}}}=0.0016$

Because $f_{a} / F_{a}$ is less than 0.15 , formula $1.6-2$ may be used.

Determine the allowable bending stress, $F_{b 500}$.

$F_{b 500}:=.60 \cdot F_{y 500} \quad F_{b 500}=25200 \cdot p s i$

HNF-2486, Rev. 0

Determine bending stress, $f_{b}$.

Page D-9

$M:=F_{\mathbf{v}} \cdot L_{\text {a }} \quad M=184 \cdot \mathbf{l b f} \cdot$ in 
Client WHC

Subject Process Building Duct Support Stress Analysis

Location: Tank 241-C-106
WO/Job No. ER4319/W320

Date $3 / 1 / 95$

By Rodney C. Campbell

Checked 3/1/95 By N. JUPLO

By

$f_{b}:=\frac{M}{S_{p s 102}} \quad f_{b}=106.36 \cdot p s i$

Verify that the stresses meet the requirements of 1.6-2.

$\frac{f_{a}}{F_{a 500}}+\frac{f_{b}}{F_{b 500}}=0.0059$

The stresses meet the requirements of section $1.6-2$, by being less than or equal to one.

C. Verify that the welds on support PS-102 are adequate to carry the load.

Calculate the required size of the fillet weld holding the angle to the tube steel.

The drawing shows a $3 / 16$ " fillet weld holding the angle to the tube steel.

$\omega:=\mathbf{. 1 8 7 5} \cdot$ in Size of fillet weld.

$I:=2 \cdot 3 \cdot i n+3.5 \cdot$ in $\quad$ Length of weld. Inside and on top of the angle.

$\tau:=9600 \cdot$ psi $\quad$ Allowable shear stress. Ref. 5, Chapter 7, Table 6.

b := 3. in Horizontal length of weld on angle.

d : = 3.5. in Vertical length of weld on angle.

$f_{t}:=\frac{F_{v}}{l} \quad f_{t}=2.42 \cdot \frac{l b f}{\text { in }} \quad$ Shear load on weld.

$S_{w}:=\frac{d^{2}}{6} \cdot \frac{(4 \cdot b+d)}{(2 \cdot b+d)} \quad$ Ref. 5 , Table 5.

$\mathrm{M}=184 \cdot \mathrm{lbf} \cdot$ in

$f_{b}:=\frac{M}{S_{w}} \quad f_{b}=55.24 \cdot \frac{\text { Ibf }}{\text { in }} \quad$ Bending load on weld.

$f_{r}:=\sqrt{f_{t}^{2}+f_{b}^{2}} \quad f_{r}=55.29 \cdot \frac{\text { lbf }}{\text { in }} \quad$ Resultant load on weld.

$\begin{array}{ll}{ }^{\omega} \mathbf{r}:=\frac{\mathbf{f}}{\tau} & \text { Required fillet weld. Ref. 5, Chapter } 7 . \\ { }^{\omega} \mathbf{r}=\mathbf{0 . 0 0 5 8} \cdot \text { in } & \text { Calculated fillet weld required to carry the load. }\end{array}$

HNF-2486, Rev. 0

Page D-10

${ }^{\omega} r_{r}<\omega=1 \quad$ This weld is strong enough to carry the maximum load.

Calculate the required size of the fillet weld holding the pipe to the plate.

The plate on the floor is $9^{\prime \prime} \times 9^{\prime \prime} \times 1 / 2$ ". A 3/16" fillet weld all around holds the tube steel to the plate.

$\omega:=.1875 \cdot$ in Size of weld.

$\tau:=9600$. psi $\quad$ Allowable shear stress. Ref. 5, Chapter 7, Table 6. 
Client WHC

Subject Process Building Duct Support Stress Analysis

Location: Tank 241-C-106
WO/Job No. ER4319/W320

Date 3/1/95

Checked $3 / 1 / 95$

Revised
By Rodney C. Campbell

By N. JUPLO

By

$$
\begin{aligned}
& \text { b : }=\mathbf{3} \cdot \text { in } \\
& f_{t}=1.92 \cdot \frac{\mathrm{lbf}}{\mathrm{in}}
\end{aligned}
$$

$\omega_{\mathbf{r}}:=\frac{\mathbf{f} \mathbf{r}}{\tau \cdot .707}$

Required size of fillet weld. Ref. 5, Chapter 7.

${ }^{\infty}{ }_{\mathbf{r}}=\mathbf{0 . 0 0 2 3} \cdot$ in $\quad$ Calculated size of fillet weld required to carry the load.

$\omega_{r}<\omega=1 \quad$ This weld is strong enough to carry the maximum load.

Calculate the required size of the fillet weld holding the plate to the floor.

The plate on the floor is 9 " $\times 9$ " $\times 1 / 2^{\prime \prime}$. A $3 / 16^{\prime \prime}$ fillet weld all around holds the plate to the floor.

$\omega:=.1875 \cdot$ in $\quad$ Throat of weld.

$\tau:=9600 \cdot$ psi $\quad$ Allowable shear stress. Ref. 5, Chapter 7, Table 6.

b : $=9 \cdot$ in

d $:=9 \cdot$ in

$1:=\mathbf{2} \cdot \mathbf{b}+\mathbf{2} \cdot \mathbf{d}$

$f_{t}:=\frac{F v}{l} \quad f_{t}=0.64 \cdot \frac{\mathrm{lbf}}{\text { in }}$

$S_{w}:=b \cdot d+\frac{d^{2}}{3}$

$M=184 \cdot 1 b f \cdot$ in

$\mathbf{f}_{\mathbf{b}}:=\frac{\mathbf{M}}{\mathbf{S}_{\mathbf{w}}}$

$$
f_{b}=1.7 \cdot \frac{1 b f}{\text { in }}
$$

$\mathbf{f}_{\mathbf{r}}:=\sqrt{\mathbf{f}_{\mathbf{b}}^{2}+\mathbf{f}_{\mathbf{t}}^{2}}$

$$
f_{r}=1.82 \cdot \frac{\text { lbf }}{\text { in }}
$$

HNF-2486, Rev. 0

$\omega^{\omega}:=\frac{\mathbf{f}_{\boldsymbol{r}}}{\tau \cdot .707} \quad$ Required size of fillet weld. Ref. 5, Chapter 7.

$\omega_{r}=\mathbf{0 . 0 0 0 3} \cdot$ in Calculated size of fillet weld required to carry the load. 
Client WHC

Subject Process Building Duct Support Stress Analysis

Location: Tank 241-C-106
WOIJob No. ER4319N320

Date 3/1/95 By Rodney C. Campbell

Checked 3/1495 By N. SUPLO

Revised

D. Verify the stresses in the plate do not exceed the allowables.
$A:=\mathbf{3} \cdot \mathrm{in} \cdot \mathbf{3} \cdot$ in Area that force is acting upon.
$\mathbf{h}:=\mathbf{. 5} \cdot$ in
$b:=3 \cdot$ in $+4 \cdot .5 \cdot$ in
Effective width of plate, $D+4 T$.
$S:=\frac{b \cdot h^{2}}{6}$

The stress generated in the base plate is:

$$
\begin{aligned}
& \sigma:=\frac{F \mathbf{v}}{A}+\frac{M}{S} \\
& \sigma=885.76 \cdot \mathrm{psi}
\end{aligned}
$$

The stress of $1156 \mathrm{psi}$ is less than the allowable stress of 17,800 psi. The plate thickness is acceptable.

\section{Analyze the pipe support PS-103 (loads from Autopipe analysis $\mathrm{W} 32 \mathrm{OH} 32 \mathrm{~A}$ ).}

Pipe support PS-103 is a 3-1/2" $\times 3-1 / 2^{\prime \prime} \times 1 / 4^{\prime \prime}$ angle welded to the top of a $3^{\prime \prime} \times 3^{\prime \prime} \times 3 / 16^{\prime \prime}$ tube. The tube column is welded to a plate that is welded to the floor. Pipe support PS-103 is used in three locations. The longest column and the greatest load will be used to verify that it is adequate to carry the loads imposed.

The highest loads on the support are:

$\mathrm{F}_{\text {ha }}:=97 \cdot$ lbf First horizontal load on support. Ref. 2.

$F_{h b}:=65 \cdot l b f \quad$ Second horizontal load on support. Ref. 2.

$\mathbf{F}_{\mathbf{v}}:=\mathbf{5 4 4} \cdot \mathbf{l b f}$ Vertical load on support. Ref. 2.

The length of the longest column is: $L_{\text {ps } 103}:=5.08 \cdot \mathrm{ft}$

The cross-sectional area of the column is: $\quad A_{\text {ps103 }}:=2.02 \cdot \mathrm{in}^{2} \quad$ Ref. 4

The section modulus of the $3^{\prime \prime} \times 3^{\prime \prime} \times 3 / 16^{\prime \prime}$ rectangular tube is: $S_{p s 103}:=1.73 \cdot$ in $^{3} \quad$ Ref. 4

Governing radius of gyration of the column, $r$ is: $\quad r:=1.13$ in $\quad$ Ref. 4

A. Determine if the combined stresses on support PS-103 satisfy the requirements of the AISC manual, The combined stresses are determined per section 1.6, pg 5.26, Ref. 4.

Determine the allowable load in compression $F_{\text {a500. }}$.

Determine if $C_{c}$ is less than or greater than $\mathrm{kl} / \mathrm{r}$.

$C_{c}:=\sqrt{\frac{2 \cdot \pi^{2} \cdot E}{F y 500}} \quad C_{c}=116.75$

$\mathrm{K}:=\mathbf{2} \quad$ Ref. 4 , Table C1.8.1

HNF-2486, Rev. 0

Page D-12 


\section{Company}

Client WHC

Subject Process Building Duct Support Stress Analysis

Location: Tank 241-C-106
DESIGN ANALYSIS

Calc. No. W320-H-033

Revision 0

Page No. 11 of 18

WO/Job No. ER4319/W320

Date $3 / 1 / 95 \quad$ By Rodney C. Campbell

Checked 3/1/95 By N. JUFLO

Revised

$\frac{K \cdot L_{p s 103}}{r}=107.89$

Because the $\mathrm{Kl} / \mathrm{r}$ is smaller than $\mathrm{C}_{\mathrm{c}}, \mathrm{F}_{\mathrm{a} 500}$ (allowable compression stress) is determined from the following formula.

$F_{a 500}:=\frac{\left[1-\frac{\left(\frac{K \cdot L_{p s 103}}{r}\right)^{2}}{2 \cdot C_{c}^{2}} F_{y 500}\right.}{\left(\frac{5}{3}\right)+\frac{3 \cdot\left(\frac{K \cdot L_{p s 103}}{r}\right)}{8 \cdot C_{c}}-\frac{\left.K \cdot L_{p s 103}\right)^{3}}{r}}$

$\mathrm{F}_{\text {a500 }}=12568.73 \cdot \mathrm{psi} \quad$ Allowable compression stress. Ref. 4 .

Determine if $f_{\mathrm{a}} / F_{\mathrm{a}}$ is less than 0.15 .

$f_{a}:=\frac{F_{v}}{A_{p s 103}} \quad f_{a}=269.31 \cdot p s i$

$\frac{f_{a}}{F_{\text {a500 }}}=0.0214$

Because $f_{\mathrm{a}} / \mathrm{F}_{\mathrm{a}}$ is less than 0.15 , formula $1.6-2$ may be used.

Determine the allowable bending stress, $\mathbf{F}_{\mathrm{b} 500}$.

$F_{b 500}:=.60 \cdot F_{y 500} \quad F_{b 500}=25200 \cdot p s i$

Determine bending stress, $f_{b}$.

$M:=L_{p s 103} \sqrt{F_{h a}{ }^{2}+F_{h b}{ }^{2}} \quad M=7117.98 \cdot l b f \cdot$ in

$f_{b}:=\frac{M}{s_{p s 103}} \quad f_{b}=4114.44 \cdot p s i$

Verify that the stresses meet the requirements of 1.6-2.

HNF-2486, Rev. 0

$\frac{f_{a}}{F_{a 500}}+\frac{f_{b}}{F_{b 500}}=0.1847$

Page D-13

The stresses meet the requirements of section 1.6-2, by being less than or equal to one.

B. Verify that the welds on support PS-103 are adequate to carry the load.

Calculate the required size of the fillet weld holding the angle to the tube steel.

The weld holding the angle to the tube steel is a flare-bevel weld with a $3 / 16$ " fillet on top of that, both sides of the tube steel.

$\omega:=.1875$ in $\quad$ Size of weld. 


\section{Company}

Client WHC

Subject Process Building Duct Support Stress Analysis

Location: Tank 241-C-106
Calc. No. W320-tt-033

Revision 0

Page No. 12 of 18

DESIGN ANALYSIS

WO/Job No. ER4319/W320

Date 3/1/95 By Rodney C. Campbell

Checked 3/1/95 By N. SLPLO

Revised

By

$\tau:=9600 \cdot$ psi $\quad$ Allowable shear stress. Ref. 5, Chapter 7, Table 6.

$1:=2 \cdot 2.5 \cdot$ in Length of weld.

$\mathrm{f}_{\mathrm{t}}:=\frac{\mathrm{F}_{\mathrm{v}}}{\mathrm{l}} \quad \mathrm{f}_{\mathrm{t}}=108.8 \cdot \frac{\mathrm{lbf}}{\text { in }}$

$f_{x a}:=\frac{F_{h a}}{l} \quad f_{x a}=19.4 \cdot \frac{\mathrm{lbf}}{\text { in }}$

$f_{x b}:=\frac{F_{h b}}{l} \quad f_{x b}=13 \cdot \frac{l b f}{\text { in }}$

$f_{r}:=\sqrt{f_{t}^{2}+f_{x a}^{2}+f_{x b}^{2}} \quad f_{r}=111.28 \cdot \frac{l b f}{\text { in }}$

${ }^{\omega_{\mathbf{r}}}:=\frac{\mathbf{f} \mathbf{r}}{\tau \cdot .707} \quad$ Required size of fillet weld. Ref. 5, Chapter 7.

${ }^{\omega_{r}}=\mathbf{0 . 0 1 6 4}$ in Calculated size of fillet weld required to carry the load.

${ }^{\omega} r^{<\omega}=1 \quad$ This weld is strong enough to carry the maximum load.

Calculate the required size of the fillet weld holding the tube steel to the plate.

The plate on the floor is 9 " $\times 9^{\prime \prime} \times 1 / 2 "$. A $3 / 16^{\prime \prime}$ fillet weld all around holds the tube steel to the plate.

$\omega:=.1875$. in Size of weld.

$\tau:=9600 \cdot$ psi $\quad$ Allowable shear stress. Ref. 5, Chapter 7, Table 6.

b := 3. in

d $:=3 \cdot$ in

$F_{h}:=\sqrt{F_{h a}^{2}+F_{h b}^{2}}$

$\mathbf{M}:={ }_{\mathbf{h}}{ }^{\mathrm{L}}$ ps103

$S_{w}:=b \cdot d+\frac{d^{2}}{3}$

$\mathbf{l}: \mathbf{=} \mathbf{4} \cdot \mathbf{b}$

$f_{t}:=\frac{F v}{l} \quad f_{t}=45.33 \cdot \frac{\mathrm{lbf}}{\text { in }}$

$f_{b}:=\frac{M}{S_{w}} \quad f_{b}=593.16 \cdot \frac{\mathrm{lbf}}{\text { in }}$

HNF-2486, Rev. 0

$f_{r}:=\sqrt{f_{t}{ }^{2}+f_{b}^{2}} \quad f_{r}=594.89 \cdot \frac{\mathrm{lbf}}{\mathrm{in}}$

Page D-14

${ }^{\omega_{r}}:=\frac{f_{r}}{\tau \cdot .707} \quad$ Required size of fillet weld. Ref. 5, Chapter 7. 
ICF Kaiser Hanford

\section{Company}

Client WHC

Subject Process Building Duct Support Stress Analysis

Location: Tank 241-C-106
DESIGN ANALYSIS

alc. No. W320-HK-033

Revision 0

Page No. 13 of 18
WO/Job No. ER4319N320

Date $3 / 1 / 95$

Checked $3 / 1 / 95$

Revised
By Rodney C. Campbell

By N. JUPLO

$\omega_{r}=\mathbf{0 . 0 8 7 6} \cdot$ in Calculated size of fillet weld required to carry the load.

${ }^{\omega} \mathbf{r}<\omega=1 \quad$ This weld is strong enough to carry the maximum load.

Calculate the required size of the fillet weld holding the plate to the floor.

The plate on the floor is 9" $\times 9^{\prime \prime} \times 1 / 2^{\prime \prime}$. A $3 / 16^{\prime \prime}$ fillet weld all around holds the plate to the floor.

$\omega:=.1875 \cdot$ in $\quad$ Throat of weld.

$\tau:=9600 \cdot \mathrm{psi} \quad$ Allowable shear stress. Ref. 5, Chapter 7, Table 6.

b : $:=9 \cdot$ in

d $:=9 \cdot$ in

$1:=2 \cdot b+2 \cdot d$

$f_{t}:=\frac{F v}{l} \quad f_{t}=15.11 \cdot \frac{l b f}{\text { in }}$

$S_{w}:=b \cdot d+\frac{d^{2}}{3}$

$M=7117.98 \cdot \mathrm{lbf} \cdot$ in

$f_{b}:=\frac{M}{S_{w}} \quad f_{b}=65.91 \cdot \frac{\mathrm{lbf}}{\mathrm{in}}$

$f_{r}:=\sqrt{f_{t}{ }^{2}+f_{b}^{2}} \quad f_{r}=67.62 \cdot \frac{\mathrm{lbf}}{\mathrm{in}}$

${ }^{\omega} \mathbf{r}:=\frac{\mathbf{f}_{\mathbf{r}}}{\tau \cdot .707} \quad$ Required size of fillet weld to carry load in tension. Ref. 5, Chapter 7.

${ }^{\infty} \mathbf{r}=\mathbf{0 . 0 1} \cdot$ in $\quad$ Calculated size of fillet weld required to carry the load.

$\omega_{r}<\omega=1 \quad$ This weld is strong enough to carry the maximum load.

C. Verify the stresses in the plate do not exceed the allowables.

$M:=L_{p s 103} \sqrt{F_{h a}^{2}+F_{h b}^{2}}$

$\mathrm{M}=593.16 \cdot \mathrm{ft} \cdot \mathrm{Ibf}$

$h:=.75 \cdot$ in

$b:=3 \cdot$ in $+4 \cdot .75 \cdot$ in $\quad$ Effective width of plate, $D+4 T$.

S : $=\frac{b \cdot h^{2}}{6}$

$\sigma:=\frac{\mathbf{M}}{\mathbf{S}}+\frac{\mathbf{F}_{\mathbf{v}}}{\mathbf{A}_{\text {ps103 }}}$

HNF-2486, Rev. 0

$\sigma=12923.49 \cdot \mathrm{psi}$ 


\section{Company}

Client WHC

Subject Process Building Duct Support Stress Analysis

Location: Tank 241-C-106

\section{DESIGN ANALYSIS}

WO/Job No. ER4319N320

Date 3/1/95 By Rodney C. Campbell

Checked $3 / 1 / 95$

Revised

Revision 0

Page No. 14 of 18

The stress of $13,137 \mathrm{psi}$ is less than the allowable stress of 17,800 psi. The plate thickness is acceptable.

4. Analyze the pipe support PS-104 (loads from Autopipe analysis $\mathrm{W} 32 \mathrm{OH} 32 \mathrm{C}$ ).

By N, SUPLO

By

Pipe support PS-104 is a 3-1/2" $\times 3-1 / 2$ " $\times 1 / 4^{\prime \prime}$ angle welded to the top of a $3^{\prime \prime} \times 3^{\prime \prime} \times 3 / 16^{\prime \prime}$ tube. The tube column is welded to a plate that is welded to the floor. Pipe support PS-104 is used in two locations. The longest column and the highest load will be used to verify that it is adequate to carry the loads imposed.

The highest loads on the support are:

$F_{\text {ha }}:=25 \cdot$ lbf First horizontal load on support. Ref. 2.

$\mathbf{F}_{\mathbf{h b}}:=\mathbf{0} \cdot \mathbf{l b f}$ Second horizontal load on support. Ref. 2.

$F_{\mathbf{v}}:=159 \cdot \mathbf{l b f}$ Vertical load on support. Ref. 2.

The length of the longest column is: $\mathrm{L}_{\text {ps104 }}:=3.5 \cdot \mathrm{ft}$

The cross-sectional area of the column is: A $A_{\text {ps104 }}:=2.02 \cdot \mathrm{in}^{2} \quad$ Ref. 4

The section modulus of the $3^{\prime \prime} \times 3^{\prime \prime} \times 3 / 16^{\prime \prime}$ rectangular tube is: $S_{\text {ps104 }}:=1.73 \cdot \mathrm{in}^{3} \quad$ Ref. 4

Governing radius of gyration of the column, $r$ is: $\quad r:=1.13$ in $\quad$ Ref. 4

A. Determine if the combined stresses on support PS-104 satisfy the requirements of the AISC manual. The combined stresses are determined per section 1.6, pg 5.26, Ref. 4.

Determine the allowable load in compression $\mathrm{F}_{\text {a500 }}$.

Determine if $\mathrm{C}_{\mathrm{c}}$ is less than or greater than $\mathrm{kl} / \mathrm{r}$.

$C_{c}:=\sqrt{\frac{2 \cdot \pi^{2} \cdot E}{F y 500}} \quad C_{c}=116.75$

$\mathrm{K}:=2 \quad$ Ref, 4 , Table C1.8.1

$\frac{K \cdot L \text { ps104 }}{r}=74.34$

Because the $\mathrm{Kl} / \mathrm{r}$ is less than $\mathrm{C}_{\mathrm{c}}, \mathrm{F}_{\mathrm{a} 500}$ (allowable compression stress) is determined from the following formula.

$$
F_{2500}:=\frac{\left[1-\frac{\left(\frac{K \cdot L_{p s 103}}{r}\right)^{2}}{2 \cdot C_{c}{ }^{2}}\right]_{y 500}}{\left(\frac{5}{3}\right)+\frac{3 \cdot\left(\frac{K \cdot L_{p s 103}}{r}\right)}{8 \cdot C_{c}}-\frac{\left(\frac{K \cdot L_{p s 103}}{r}\right)^{3}}{8 \cdot C_{c}^{3}}}
$$

Determine if $\mathrm{f}_{\mathrm{a}} / \mathrm{F}_{\mathrm{a}}$ is less than 0.15 .
$F_{2500}=12568.73 \cdot p s i$ Allowable compression stress. Ref. 4 .

HNF-2486, Rev. 0

Page D-16

$f_{a}:=\frac{F_{v}}{A_{p s 104}} \quad f_{a}=78.71 \cdot p s i$ 


\section{Company}

Client WHC

Subject Process Building Duct Support Stress Analysis

Location: Tank 241-C-106

\section{DESIGN ANALYSIS}

Calc. No. W320-H-033

Revision 0

Page No. 15 of 18
WO/Job No. ER4319NW320

Date $3 / 1 / 95$

Checked $3 \lambda / 95$

Revised
By Rodney C. Campbell

By N. JwPLO

$\frac{f_{a}}{F_{\text {a500 }}}=0.0063$

Because $f_{a} / F_{a}$ is less than 0.15 , formula $1.6-2$ may be used.

Determine the allowable bending stress, $F_{b 500}$.

$$
F_{b 500}:=.60 \cdot F_{y 500} \quad F_{b 500}=25200 \cdot p s i
$$

Determine bending stress, $f_{b}$.

$$
\begin{aligned}
& M:=L_{p s 104} \sqrt{F_{h a}^{2}+F_{h b}^{2}} \quad M=1050 \cdot l b f \cdot i n \\
& f_{b}:=\frac{M}{S_{p s 104}} \quad f_{b}=606.94 \cdot p s i
\end{aligned}
$$

Verify that the stresses meet the requirements of 1.6-2.

$$
\frac{f_{a}}{F_{a 500}}+\frac{f_{b}}{F_{b 500}}=0.0303
$$

The stresses meet the requirements of section $1.6-2$, by being less than or equal to one.

B. Verify that the welds on support PS-104 are adequate to carry the load.

Calculate the required size of the fillet weld holding the angle to the tube steel.

The weld holding the angle to the tube steel is a flare-bevel weld with a 3/16" fillet on top of that, both sides of the tube steel.

$$
\begin{aligned}
& \omega:=.1875 \text { in } \text { Size of weld. } \\
& \tau:=9600 \cdot \text { psi } \quad \text { Allowable shear stress. Ref. 5, Chapter 7, Table } 6 . \\
& \mathbf{l}:=\mathbf{2} \cdot \mathbf{2 . 5} \cdot \text { in . Length of weld. } \\
& f_{t}:=\frac{F v}{l} \quad f_{t}=31.8 \cdot \frac{l b f}{\text { in }} \\
& f_{x a}:=\frac{F_{h a}}{l} \quad f_{x a}=5 \cdot \frac{l b f}{\text { in }} \\
& f_{x b}:=\frac{F_{h b}}{l} \quad f_{x b}=0 \cdot \frac{\text { lbf }}{\text { in }} \\
& f_{r}:=\sqrt{f_{t}{ }^{2}+f_{x a}^{2}+f_{x b}^{2}} \quad f_{r}=32.19 \cdot \frac{\text { lbf }}{\text { in }} \quad \text { HNF-2486, Rev. } 0 \\
& \omega_{\mathbf{r}}:=\frac{\mathbf{f}_{\mathbf{r}}}{\tau \cdot .707} \quad \text { Required size of fillet weld. Ref. 5, Chapter } 7 . \\
& \omega_{r}=0.0047 \cdot \text { in Calculated size of fillet weld required to carry the load. } \\
& \omega_{r}<\omega=1 \quad \text { This weld is strong enough to carry the maximum load. }
\end{aligned}
$$


Client WHC

Subject Process Building Duct Support Stress Analysis

Location: Tank 241-C-106
WO/Job No. ER4319N320

Date 3/1/95

Checked $3 / 1 / 95$

Revised
By Rodney C. Campbell

By N. JUFLO

By

Calculate the required size of the fillet weld holding the tube steel to the plate.

The plate on the floor is 9 " $\times 9 " \times 1 / 2$ ". A $3 / 16^{\prime \prime}$ fillet weld all around holds the tube steel to the plate.

$$
\begin{aligned}
& \omega:=.1875 \text { in } \text { Size of weld. } \\
& \tau:=9600 \cdot \mathrm{psi} \quad \text { Allowable shear stress. Ref. 5, Chapter 7, Table } 6 . \\
& \text { b : }=\mathbf{3} \cdot \text { in } \\
& d:=3 \cdot \text { in } \\
& F_{h}:=\sqrt{F_{h a}{ }^{2}+F_{h b}{ }^{2}} \\
& \mathbf{M}:=\mathbf{F}_{\mathbf{h}} \cdot \mathbf{L} \mathbf{p s 1 0 4} \\
& S_{w}:=b \cdot d+\frac{d^{2}}{3} \\
& \text { I : }=4 \cdot b \\
& f_{t}:=\frac{F_{v}}{l} \quad f_{t}=13.25 \cdot \frac{l b f}{\text { in }} \\
& f_{b}:=\frac{M}{S_{w}} \quad f_{b}=87.5 \cdot \frac{\text { lbf }}{\text { in }} \\
& f_{r}:=\sqrt{f_{t}^{2}+f_{b}^{2}} \quad f_{r}=88.5 \cdot \frac{l b f}{\text { in }} \\
& { }^{\omega_{r}}:=\frac{\mathbf{f}_{\mathbf{r}}}{\tau \cdot .707} \quad \text { Required size of fillet weld. Ref. } 5 \text {, Chapter } 7 . \\
& { }^{\omega} \mathbf{r}=\mathbf{0 . 0 1 3} \cdot \text { in Calculated size of fillet weld required to carry the load. } \\
& { }^{\omega}{ }_{r}<\omega=1 \quad \text { This weld is strong enough to carry the maximum load. }
\end{aligned}
$$

Calculate the required size of the fillet weld holding the plate to the floor.

The plate on the floor is $9^{\prime \prime} \times 9 " \times 1 / 2 "$. A $3 / 16^{\prime \prime}$ fillet weld all around holds the plate to the floor.

$$
\begin{aligned}
& j^{\omega}:=\mathbf{. 1 8 7 5} \cdot \text { in } \quad \text { Throat of weld. } \\
& \tau:=9600 \cdot \text { psi } \quad \text { Allowable shear stress. Ref. 5, Chapter 7, Table } 6 . \\
& b:=9 \cdot \text { in } \\
& d:=9 \cdot \text { in } \\
& I:=\mathbf{2} \cdot \mathbf{b}+\mathbf{2} \cdot \mathbf{d} \\
& f_{t}:=\frac{F}{l} \quad f_{t}=4.42 \cdot \frac{\text { lbf }}{\text { in }} \\
& S_{w}:=b \cdot d+\frac{d^{2}}{3} \\
& M=1050 \cdot \text { lbf } \cdot \text { in }
\end{aligned}
$$

HNF-2486, Rev. 0

Page D-18 
Client WHC

Subject Process Building Duct Support Stress Analysis

Location: Tank 241-C-106
WO/JOb No. ER4319N320

Date $3 / 1 / 95$

Checked 3/1/97

Revised

$$
\begin{aligned}
& f_{b}:=\frac{M}{S_{w}} \quad f_{b}=9.72 \cdot \frac{\text { lbf }}{\text { in }} \\
& f_{r}:=\sqrt{f_{t}^{2}+f_{b}^{2}} \quad f_{r}=10.68 \cdot \frac{l b f}{\text { in }} \\
& { }^{\omega} \mathbf{r}:=\frac{\mathbf{f}_{\mathbf{r}}}{\tau \cdot .707} \quad \text { Required size of fillet weld. Ref. } 5 \text {, Chapter } 7 . \\
& \omega_{r}=0.0016 \cdot \text { in Calculated size of fillet weld required to carry the load. } \\
& { }^{\omega} \mathbf{r}^{<\omega}=\mathbf{1} \text { This weld is strong enough to carry the maximum load. }
\end{aligned}
$$

C. Verify the stresses in the plate do not exceed the allowables.

$$
\begin{aligned}
& M:=L_{p s 104} \cdot \sqrt{F_{h a}{ }^{2}+F_{h b}{ }^{2}} \\
& M=87.5 \cdot f t \cdot l b f \\
& h:=.5 \cdot \text { in } \\
& b:=3 \cdot \text { in }+4 \cdot .5 \cdot \text { in } \quad \text { Effective width of plate, D + 4T. } \\
& S:=\frac{b \cdot h^{2}}{6} \\
& \sigma:=\frac{M}{S}+\frac{F}{A_{p s 104}} \\
& \sigma=5118.71 \cdot p s i
\end{aligned}
$$

The stress of $5,119 \mathrm{psi}$ is less than the allowable stress of 17,800 psi. The plate thickness is acceptable.

5. Analyze the pipe support PS-109 (loads from Autopipe analysis W320H32A).

Pipe support PS-109 is a $3-1 / 2 " \times 3-1 / 2 " \times 1 / 4^{\prime \prime}$ angle welded to the shieid wall.

The loads on the support are:

$\mathbf{F}_{\text {ha }}:=\mathbf{0} \cdot \mathbf{l b f} \quad$ First horizontal load on support. Ref. 2.

$\mathbf{F}_{\mathbf{h b}}:=\mathbf{0} \cdot \mathbf{l b f}$ Second horizontal load on support. Ref. 2.

$\mathbf{F}_{\mathbf{v}}:=\mathbf{1 7} \cdot \mathbf{l b f} \quad$ Vertical load on support. Ref. 2.

HNF-2486, Rev. 0

Page D-19

Distance from the load to the support is: $L_{p s 109}:=1 \cdot f t$

The section modulus of the 3-1/2" $\times 3-1 / 2^{\prime \prime} \times 1 / 4^{\prime \prime}$ angle is: $s_{a}:=.794 \cdot$ in $^{3} \quad$ Ref. 4

A. Verify that the angle is adequate to carry the load.

The angle can be analyzed as a cantilever with a concentrated load at any point. 
Client WHC

Subject Process Building Duct Support Stress Analysis

Location: Tank 241-C-106
DESIGN ANALYSIS

Page No. 18 of 18
WO/Job No. ER4319NW320

Date 3/1/95 By Rodney C. Campbell

Checked $3 / 1 / 95$ By N. WUPLO

Revised By

$M:=F_{v} \cdot \mathrm{L}_{\text {ps109 }} \quad M=204 \cdot \mathrm{lbf} \cdot$ in

$\mathbf{f}_{\mathbf{b}}:=\frac{\mathbf{M}}{\mathbf{S}_{\mathbf{a}}}$

$f_{b}=256.93 \cdot p s i \quad$ Calculated bending stress

$\mathbf{f}_{b}<F_{36}=1$ The calculated bending stress is less than the allowable. The angle will not fail.

B. Verify that the weld on support PS-109 is adequate to carry the load.

Calculate the required size of the fillet weld holding the angle to the shielding wall.

The drawing shows a $3 / 16$ " fillet weld holding the angle to the shielding wall.

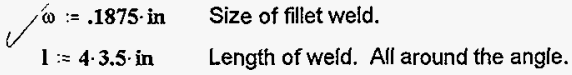

$\tau:=9600 \cdot$ psi $\quad$ Allowable shear stress. Ref. 5, Chapter 7, Table 6.

b : $=3 \cdot$ in Horizontal length of weld on angle.

d : $=3.5 \cdot$ in Vertical length of weid on angle.

$f_{t}:=\frac{F}{i} \quad f_{t}=1.21 \cdot \frac{\text { Ibf }}{\text { in }} \quad$ Shear load on weld.

$S_{w}:=\frac{d^{2}}{6} \cdot \frac{(4 \cdot b+d)}{(2 \cdot b+d)} \quad$ Ref. 5 , Table 5.

$f_{b}:=\frac{M}{S_{w}} \quad f_{b}=61.24 \cdot \frac{\text { lbf }}{\text { in }} \quad$ Bending load on weld.

$f_{r}:=\sqrt{f_{t}{ }^{2}+f_{b}^{2}} \quad f_{r}=61.25 \cdot \frac{l b f}{\text { in }} \quad$ Resultant load on weld.

${ }^{\infty} \mathbf{r}:=\frac{\mathbf{f}_{\mathbf{r}}}{\tau \cdot . \mathbf{7 0 7}} \quad$ Required fillet weld. Ref. 5, Chapter 7.

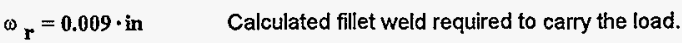

${ }^{\infty} \mathbf{r}<\omega=1$ This weld is strong enough to carry the maximum load.

\section{CONCLUSIONS:}

The Process Building HVAC duct supports are adequate as designed to carry the loads calculated to be imposed upon them.

HNF-2486, Rev. 0

Page D-20 


\section{APPENDIX A}

HNF-2486, Rev. 0

Page D-21 
W32OR32A PROCESS BUILDING PIPING STRESS AWALYSIS

33/01/95 U320, TAUK 241-C-100 SLUICING ER/319 AUTOPIPE+4.50 RESULT PAGE 2

DISPLACE HENTS

\begin{tabular}{|c|c|c|c|c|c|c|c|}
\hline \multirow{2}{*}{$\begin{array}{l}\text { Point } \\
\text { name } \\
-\ldots . .\end{array}$} & \multirow{2}{*}{$\begin{array}{l}\text { Lond } \\
\text { combination }\end{array}$} & \multirow{2}{*}{\multicolumn{3}{|c|}{ TRARSLATIONS (in ${ }_{Y}$ ) }} & \multicolumn{2}{|c|}{ ROTATIONS (deg } & \multirow{2}{*}{ z } \\
\hline & & & & & $x$ & \% & \\
\hline & $\begin{array}{l}\text { GR } \\
\text { I1 } \\
E 1 \\
E 2 \\
\text { GR+T1 } \\
\text { SEIS } \\
\text { GR+TI+SEIS }\end{array}$ & $\begin{array}{l}0.000 \\
0.000 \\
0.000 \\
0.000 \\
0.000 \\
0.000 \\
0.001\end{array}$ & $\begin{array}{l}0.000 \\
0.007 \\
0.000 \\
0.000 \\
0.007 \\
0.000 \\
0.007\end{array}$ & $\begin{array}{l}0.000 \\
0.012 \\
0.000 \\
0.000 \\
0.012 \\
0.000 \\
0.012\end{array}$ & $\begin{array}{r}-0.001 \\
-0.004 \\
0.000 \\
0.002 \\
-0.005 \\
0.002 \\
0.007\end{array}$ & $\begin{array}{r}-0.001 \\
-0.012 \\
0.000 \\
-0.001 \\
-0.013 \\
0.001 \\
0.014\end{array}$ & $\begin{array}{r}0.001 \\
-0.014 \\
-0.002 \\
0.001 \\
-0.013 \\
0.003 \\
0.016\end{array}$ \\
\hline G06 N & $\begin{array}{l}\text { GR } \\
T 1 \\
E 1 \\
E 2 \\
\text { GR+T1 } \\
\text { SEIS } \\
\text { GR+T1+SEIS }\end{array}$ & $\begin{array}{l}0.000 \\
0.000 \\
0.000 \\
1.000 \\
1.000 \\
0.000 \\
0.001\end{array}$ & $\begin{array}{l}0.000 \\
0.007 \\
0.000 \\
0.000 \\
0.007 \\
0.000 \\
0.007\end{array}$ & $\begin{array}{l}0.000 \\
0.012 \\
0.000 \\
0.000 \\
0.012 \\
0.000 \\
0.012\end{array}$ & $\begin{array}{r}-0.001 \\
-0.004 \\
0.000 \\
0.002 \\
-0.005 \\
0.002 \\
0.007\end{array}$ & $\begin{array}{r}-0.001 \\
-0.012 \\
0.000 \\
-0.001 \\
-0.013 \\
0.001 \\
0.014\end{array}$ & $\begin{array}{r}0.001 \\
-0.014 \\
-0.002 \\
0.001 \\
-0.013 \\
0.003 \\
0.016\end{array}$ \\
\hline $606 F$ & $\begin{array}{l}\text { GR } \\
T 1 \\
E 1 \\
E 2 \\
G R+T 1 \\
\text { SEIS } \\
\text { GR+TI+SEIS }\end{array}$ & $\begin{array}{l}0.000 \\
0.000 \\
0.000 \\
0.000 \\
0.000 \\
0.000 \\
0.000\end{array}$ & $\begin{array}{l}0.000 \\
0.000 \\
0.000 \\
0.000 \\
0.000 \\
0.000 \\
0.000\end{array}$ & $\begin{array}{l}0.000 \\
0.005 \\
0.000 \\
0.000 \\
0.005 \\
0.000 \\
0.005\end{array}$ & $\begin{array}{l}0.000 \\
0.000 \\
0.000 \\
0.000 \\
0.000 \\
0.000 \\
0.000\end{array}$ & $\begin{array}{r}0.000 \\
-0.001 \\
0.000 \\
0.000 \\
-0.001 \\
0.000 \\
0.001\end{array}$ & $\begin{array}{r}0.000 \\
-0.001 \\
0.000 \\
0.000 \\
-0.001 \\
0.000 \\
0.001\end{array}$ \\
\hline 5,07 & $\begin{array}{l}\text { GR } \\
\text { T1 } \\
E 1 \\
E 2 \\
\text { GR+T1 } \\
S E \text { IS } \\
\text { GR+T1+SEIS }\end{array}$ & $\begin{array}{l}0.000 \\
0.000 \\
0.000 \\
0.000 \\
0.000 \\
0.000 \\
0.000\end{array}$ & $\begin{array}{l}0.000 \\
0.000 \\
0.000 \\
0.000 \\
0.000 \\
0.000 \\
0.000\end{array}$ & $\begin{array}{l}0.000 \\
0.003 \\
0.000 \\
0.000 \\
0.003 \\
0.000 \\
0.003\end{array}$ & $\begin{array}{l}0.000 \\
0.000 \\
0.000 \\
0.000 \\
0.000 \\
0.000 \\
0.000\end{array}$ & $\begin{array}{l}0.000 \\
0.000 \\
0.000 \\
0.000 \\
0.000 \\
0.000 \\
0.000\end{array}$ & $\begin{array}{l}0.000 \\
0.000 \\
0.000 \\
0.000 \\
0.000 \\
0.000 \\
0.000\end{array}$ \\
\hline G08 & $\begin{array}{l}\text { GR } \\
T 1 \\
E 1 \\
E 2 \\
G R+I 1 \\
\text { SEIS } \\
\text { GR+T1+SEIS }\end{array}$ & $\begin{array}{l}0.000 \\
0.000 \\
0.000 \\
0.000 \\
0.000 \\
0.000 \\
0.000\end{array}$ & $\begin{array}{l}0.000 \\
0.000 \\
0.000 \\
0.000 \\
0.000 \\
0.000 \\
0.000\end{array}$ & $\begin{array}{l}0.000 \\
0.000 \\
0.000 \\
0.000 \\
0.000 \\
0.000 \\
0.000\end{array}$ & $\begin{array}{l}0.000 \\
0.000 \\
0.000 \\
0.000 \\
0.000 \\
0.000 \\
0.000\end{array}$ & $\begin{array}{l}0.000 \\
0.000 \\
0.000 \\
0.000 \\
0.000 \\
0.000 \\
0.000\end{array}$ & $\begin{array}{l}0.000 \\
0.000 \\
0.000 \\
0.000 \\
0.000 \\
0.000 \\
0.000\end{array}$ \\
\hline
\end{tabular}

$\star * *$ Segment $G$ end $* * * *$
W32OH32A PROCESS BUILDING PIPING STRESS ANALYSIS

03/01/95 W320, TANK $241-C-106$ SLUICING, ER4319 AUTOPIPE+4.50 RESULT PAGE 22 SUPPORT FORLES

Point/ Connect/ Load LOCAL GLOBAL Supp. ID Type Conbination Dirn Force Deform Dirn Force Deform

\begin{tabular}{|c|c|c|c|c|c|c|c|c|}
\hline \multirow{7}{*}{$\begin{array}{l}A 03 \mathrm{M} \\
A 03 \mathrm{M1} \\
\text { Stiff } \\
P S-101\end{array}$} & $\begin{array}{l}Y-S t o p \\
\text { :RIGID }\end{array}$ & GR & $\begin{array}{l}\text { down } \\
+z \\
-x\end{array}$ & 316 & $\begin{array}{l}0.000 \\
0.000 \\
0.000\end{array}$ & $\begin{array}{l}X \\
y \\
Z\end{array}$ & -316 & $\begin{array}{l}0.000 \\
0.000 \\
0.000\end{array}$ \\
\hline & & $\mathrm{T} 1$ & $\begin{array}{l}u_{p} \\
+2 \\
-x\end{array}$ & 1625 & $\begin{array}{l}0.000 \\
0.000 \\
0.011\end{array}$ & $\begin{array}{l}X \\
Y \\
Z\end{array}$ & 1625 & $\begin{array}{r}-0.011 \\
0.000 \\
0.000\end{array}$ \\
\hline & & E1 & $\begin{array}{l}\text { down } \\
+7 \\
+x\end{array}$ & 1 & $\begin{array}{l}0.000 \\
0.000 \\
0.000\end{array}$ & $\begin{array}{l}X \\
Y \\
Z\end{array}$ & -1 & $\begin{array}{l}0.000 \\
0.000 \\
0.000\end{array}$ \\
\hline & & $\mathrm{E} 2$ & $\begin{array}{l}\text { down } \\
+2 \\
+x\end{array}$ & 1 & $\begin{array}{l}0.000 \\
0.000 \\
0.000\end{array}$ & $\begin{array}{l}X \\
Y \\
Z\end{array}$ & -1 & $\begin{array}{l}0.000 \\
0.000 \\
0.000\end{array}$ \\
\hline & & $G R+Y 1$ & $\begin{array}{l}\text { up } \\
+2 \\
-x\end{array}$ & 1309 & $\begin{array}{l}0.000 \\
0.000 \\
0.011\end{array}$ & $\begin{array}{l}X \\
Y \\
Z\end{array}$ & 1309 & $\begin{array}{r}-0.011 \\
0.000 \\
0.000\end{array}$ \\
\hline & & SEIS & $\begin{array}{l}\text { down } \\
-z \\
-x\end{array}$ & 2 & $\begin{array}{l}0.000 \\
0.000 \\
0.000\end{array}$ & $\begin{array}{l}X \\
Y \\
Z\end{array}$ & 2 & $\begin{array}{l}0.000 \\
0.000 \\
0.000\end{array}$ \\
\hline & & $G R+T 1+S E I S$ & $\begin{array}{l}\text { down } \\
-z \\
-x\end{array}$ & 1311 & $\begin{array}{l}0.000 \\
0.000 \\
0.011\end{array}$ & $\begin{array}{l}X \\
Y \\
Z\end{array}$ & 1311 & $\begin{array}{l}0.011 \\
0.000 \\
0.000\end{array}$ \\
\hline $\begin{array}{l}805 \\
805 \\
\text { stiff }\end{array}$ & $\begin{array}{l}Y=\text { Stop } \\
: R I G I D\end{array}$ & $G R$ & $\begin{array}{l}\text { down } \\
-z \\
-x\end{array}$ & 214 & $\begin{array}{l}0.000 \\
0.000 \\
0.000\end{array}$ & $\begin{array}{l}X \\
Y \\
Z\end{array}$ & -214 & $\begin{array}{l}0.000 \\
0.000 \\
0.000\end{array}$ \\
\hline \multirow{4}{*}{\multicolumn{2}{|c|}{$P S-103$}} & T1 & $\begin{array}{l}\text { up } \\
+Z \\
-x\end{array}$ & 214 & $\begin{array}{l}0.015 \\
0.059 \\
0.046\end{array}$ & $\begin{array}{l}x \\
y \\
z\end{array}$ & 214 & $\begin{array}{r}-0.046 \\
0.015 \\
0.059\end{array}$ \\
\hline & & E1 & $\begin{array}{l}\text { up } \\
-Z \\
+X\end{array}$ & 9 & $\begin{array}{l}0.000 \\
0.001 \\
0.001\end{array}$ & $\begin{array}{l}X \\
Y \\
Z\end{array}$ & 9 & $\begin{array}{r}0.001 \\
0.000 \\
-0.001\end{array}$ \\
\hline & & E2 & $\begin{array}{l}\text { down } \\
+Z \\
-X\end{array}$ & 1 & $\begin{array}{l}0.000 \\
0.001 \\
0.000\end{array}$ & $\begin{array}{l}x \\
y \\
z\end{array}$ & -1 & $\begin{array}{l}0.000 \\
0.000 \\
0.001\end{array}$ \\
\hline & & $G R+T 1$ & $\begin{array}{l}\text { up } \\
+Z \\
-x\end{array}$ & & $\begin{array}{l}0.015 \\
0.059 \\
0.047\end{array}$ & $\begin{array}{l}X \\
Y \\
Z\end{array}$ & & $\begin{array}{r}-0.047 \\
0.015 \\
0.059\end{array}$ \\
\hline
\end{tabular}

HNF-2486, Rev. 0

Page D-22 
W320H32A PROCESS BUILDING PIPING STRESS ANALYSIS

$03 / 01 / 95$ W320, TANK 241-C-106 SLUICING, ER4319 AUTOPIPE+4.50 RESULT PAGE 23

\section{SUPPORT FORCES}

Supp. ID Type Combination Dirn Force Deform SEIS $\begin{array}{lll}\text { down } & \text { 10 } & 0.000 \\ -Z & & 0.001\end{array}$ $\begin{array}{ll}-2 & 0.001 \\ -x & 0.001\end{array}$

GR+TH+SE IS

$$
\begin{aligned}
& \text { down } \\
& -2 \\
& -x
\end{aligned}
$$

B12
B12 1 S Stop
Stiff : RIGIO

PS-103

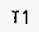

E1

$E 2$

$G R+I 1$

$+2$

SEIS

down
-2
$+x$

$10 \quad 0.015$ 0.060
0.048

do

$1 \quad \begin{aligned} & -2 \\ & -2\end{aligned}$

down
$-z$
$+x$

deiwn
-7
$+x$

down

+2
$-x$

$255 \quad 0.000$
0.001 0.000

$\begin{array}{ll}35 & 0.000 \\ 54 & 0.008 \\ 51 & 0.007\end{array}$

$4 \quad 0.000$

0.000 0.000

$0 \quad 0.000$
0.002 0.000

$\begin{array}{lrr}\text { down } & 291 & 0.000 \\ -z & 54 & 0.009\end{array}$

0.003

\section{clown}

$-2$

$4 \quad 0.000$

0.003
0.000

$G R+T 1+S E I S$ down

-2
$-x$

D02
D02 1
Stiff

PS -102

down

down
$-z$
$-x$

$294 \quad 0.000$

$54 \quad 0.012$

$\begin{array}{ll}145 & 0.000\end{array}$

0.000

0.000

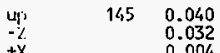

down
-2
$+\mathrm{X}$

$2 \quad 0.000$

0.000
0.000

E2

$21 \quad 0.000$
$G L O B A L$

Dirn Force Deform

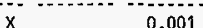

$10 \quad 0.000$
0.001

$10 \quad 0.048$

$\begin{array}{ll}0 & 0.015 \\ 0.060\end{array}$

0.000

$\begin{array}{ll}-255 & 0.000 \\ -0.001\end{array}$

$\begin{array}{ll}51 & 0.007\end{array}$

$\begin{array}{lr}-35 & 0.000 \\ -54 & -0.008\end{array}$

0.000
$-4 \quad 0.000$

$\begin{array}{lr}-4 & 0.000 \\ -0.001\end{array}$

0.000

0.000
0.002

$\begin{array}{rr}51 & 0.008 \\ -291 & 0.000\end{array}$

$\begin{array}{rr}-299 & 0.000 \\ -54 & -0.009\end{array}$

$\begin{array}{r}0.000 \\ 4 \quad 0.000 \\ \hline\end{array}$

0.000
0.003

$\begin{array}{rr}51 & 0.008 \\ 294 & 0.000\end{array}$

$54 \quad 0.012$

$.145 \quad 0.000$

0.000

0.004

$145 \quad 0.040$

0.000

0.000

0.000
W32OH32A PROCESS BUILDING PIPING STRESS ANALYSIS

3/01/95 W320, TANK 241-C-106 SLUICING, ER4319 AUTOPIPE+4.50 RESULT PAGE 24

SUPPORT FORCES

Point/ Connect/ Load LOCAL GLOBAL

Supp. ID Type Combination Dirn Force Deform Dirn Force Deform $\begin{array}{lllll}+Z & 0.000 & Y & 21 & 0.000 \\ +X & 0.000 & Z & & 0.000\end{array}$

$G R+11$ up $\quad 0.040$

0.040
0.032 0.004

SEIS -2
$+X$

0.000

$\begin{array}{lll}\text { down } & 23 & 0.000 \\ -2 & & 0.000 \\ -x & & 0.000\end{array}$

GR+T1+SEIS down $23 \quad 0.040$

0.040
0.033
0.004

FO6
Fo6 1 Y - Stop
Stiff

PS -109

T1

$T 1$

-2
$-x$

GR

$$
\begin{aligned}
& -x \\
& -2 \\
& -2
\end{aligned}
$$

303

0.000

0.000
0.000
0.001

$-x$

30

0.004
0.007

$\begin{array}{clll}E 1 & \text { down } & 13 & 0.000 \\ +Z & & & 0.000 \\ & +X & & 0.003\end{array}$

E2

down
$+z$
$-x$

$4 \quad 0.000$

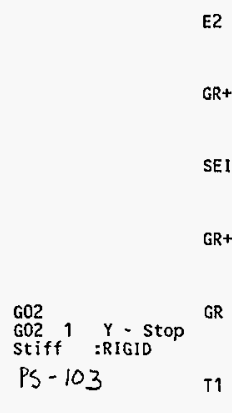

HNF-2486, Rev. 0

Page D-23
0.000

0.004

0.007

SEIS

down
$-z$
$-x$

17

0.000

0.000

R+T1+SEIS

dow
$-Z$
$-X$

0.007
0.047

$\begin{array}{lll}\text { down } & 224 & 0.000 \\ -Z & & 0.001 \\ -X & & 0.001\end{array}$

down $\quad 310 \quad 0.000$
0.004

0.040
-0.032

0.000

$23 \quad 0.000$
0.000

0.004

$\begin{array}{ll}23 & 0.040 \\ 0.033\end{array}$

$-0.001$

0.000
0.000

0.044

0.004

$-0.007$

$\begin{array}{rr} & 0.003 \\ -13 \quad 0.000\end{array}$

0.000
0.000

0.000

$-4 \quad 0.000$

0.044

0.004

$17 \quad 0.003$

0.000

0.047

$17 \quad 0.004$

$-0.001$

$-224 \quad 0.000$

$-0.001$

$\begin{array}{rr}97 & 0.022 \\ -310 & 0.000\end{array}$

- 
W32OH32A PROCESS BUILDING PIPIUG STRESS ANALYSIS

03/01/95 H320, TANK 241-C-106 SLUICHUG ER/319 AUTOPIPE+4.50 RESULT PAGE 25

SUPPORT FORCES

Point/ Connect/ Load LOC A L Deform Dirn L O B A L Deform

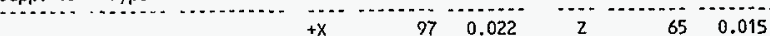

\begin{tabular}{|c|c|c|c|c|c|c|}
\hline E1 & $\begin{array}{l}\text { down } \\
+2 \\
+x\end{array}$ & 7 & $\begin{array}{l}0.000 \\
0.000 \\
0.001\end{array}$ & $\begin{array}{l}x \\
y \\
z\end{array}$ & -7 & $\begin{array}{l}0.001 \\
0.000 \\
0.000\end{array}$ \\
\hline$E 2$ & $\begin{array}{l}\text { up } \\
+z \\
-x\end{array}$ & 3 & $\begin{array}{l}0.000 \\
0.001 \\
0.000\end{array}$ & $\begin{array}{l}X \\
y \\
z\end{array}$ & 3 & $\begin{array}{l}0.000 \\
0.000 \\
0.001\end{array}$ \\
\hline$G R+\Gamma 1$ & $\begin{array}{l}\text { down } \\
+2 \\
+x\end{array}$ & $\begin{array}{r}534 \\
65 \\
97\end{array}$ & $\begin{array}{l}0.000 \\
0.014 \\
0.021\end{array}$ & $\begin{array}{l}X \\
y \\
Z\end{array}$ & $\begin{array}{r}97 \\
-534 \\
65\end{array}$ & $\begin{array}{l}0.021 \\
0.000 \\
0.014\end{array}$ \\
\hline SEIS & $\begin{array}{l}\text { down } \\
-z \\
-x\end{array}$ & 10 & $\begin{array}{l}0.000 \\
0.001 \\
0.002\end{array}$ & $\begin{array}{l}X \\
Y \\
Z\end{array}$ & 10 & $\begin{array}{l}0.002 \\
0.000 \\
0.001\end{array}$ \\
\hline$G R+T H+S E I S$ & $\begin{array}{l}\text { down } \\
-2 \\
-x\end{array}$ & $\begin{array}{r}544 \\
65 \\
87\end{array}$ & $\begin{array}{l}0.000 \\
0.015 \\
0.023\end{array}$ & $\begin{array}{l}X \\
Y \\
Z\end{array}$ & $\begin{array}{r}97 \\
544 \\
65\end{array}$ & $\begin{array}{l}0.023 \\
0.000 \\
0.015\end{array}$ \\
\hline
\end{tabular}

W32OH32A PROCESS BUILDING PIPING STRESS ANALYSIS

03/01/95 W320, TANK 241-C-106 SLUICING, ER4319 AUTOPIPE+4.50 RESULT PAGE 26

\section{RESTRAINT REA C T I ON S}

Point Load $x$ FORCES (lb ${ }_{Y}$ ) Result $x$ MOMENTS (ft-lb ) Result

A00 Anchor

$$
\text { GR }
$$

$\begin{array}{lr}\text { Anchor } & \\ \text { GR } & -44 \\ \text { T1 } & -21549 \\ \text { E1 } & 49 \\ \text { E2 } & 3 \\ \text { GR+T1 } & -21593 \\ \text { SEIS } & 52\end{array}$

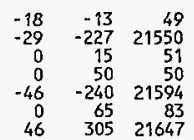

17
-349
31
66
-331
97
428

21
-489
1
-15
-469
16
485

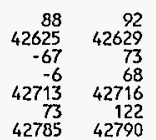

A03 M Y - Stop

PS-pol

$\begin{array}{lrr}Y-\text { Stop } & 0 & -316 \\ \text { GR } & 0 & 1625 \\ \text { T1 } & 0 & -1 \\ \text { E1 } & 0 & -7 \\ \text { E2 } & 0 & 1309 \\ \text { GR+T1 } & 0 & 2 \\ \text { SEIS } & 0 & 1311\end{array}$

$\begin{array}{rr}0 & 316 \\ 0 & 1625 \\ 0 & 1 \\ 0 & 1 \\ 0 & 1309 \\ 0 & 2 \\ 0 & 1311\end{array}$

A08

\section{Anchor}

Anc
GR
T1
E1

E1

$\begin{array}{rr}32 & -113 \\ 19584 & -1530\end{array}$

GR+T1

$\begin{array}{rr}17 & 0 \\ 0 & 2 \\ 19617 & -1643\end{array}$

SEIS $19617-1643$

GR+T1+SEIS $19634 \quad 1645$

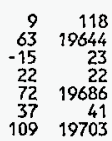

18
221
-8
11
239
19
257

305
$33-103$

$Y-$
GR
I1
E1

E2

SEIS

GR+T $1+S E$ IS

$\begin{array}{rrrr}0 & -214 & 0 & 214 \\ 0 & 214 & 0 & 214 \\ 0 & 9 & 0 & 9 \\ 0 & -1 & 0 & 1 \\ 0 & 0 & 0 & 0 \\ 0 & 10 & 0 & 10 \\ 0 & 10 & 0 & 10\end{array}$

B12

$Y$ - stop

PS -103

$\begin{array}{lrr}\text { GR } & 0 & -255 \\ \text { T1 } & 51 & -35 \\ \text { E1 } & 0 & -4 \\ \text { E2 } & 0 & 0 \\ \text { GR+T1 } & 51 & -291 \\ \text { SEIS } & 0 & 4 \\ \text { GR+T 1+SEIS } & 51 & 294\end{array}$

$\begin{array}{rrr}-2 & -2 & 18 \\ -129 & -12009 & 12012 \\ -5 & -8 & 12 \\ 7 & -1 & 13 \\ -131 & -12012 & 12015 \\ 12 & 9 & 24 \\ 143 & 12020 & 12024\end{array}$

0
0
0
0
0
0
0

0
0
0
0
0
0
0

$\begin{array}{rr}0 & 0 \\ 0 & 0 \\ 0 & 0 \\ 0 & 0 \\ 0 & 0 \\ 0 & 0 \\ 0 & 0 \\ & \\ -2 & 18 \\ 09 & 12012 \\ -8 & 12 \\ -1 & 13 \\ 12 & 12015 \\ 9 & 24 \\ 20 & 12024\end{array}$

$\begin{array}{lll}0 & 0 & 0 \\ 0 & 0 & 0 \\ 0 & 0 & 0 \\ 0 & 0 & 0 \\ 0 & 0 & 0 \\ 0 & 0 & 0 \\ 0 & 0 & 0 \\ & & \\ 0 & 0 & 0 \\ 0 & 0 & 0 \\ 0 & 0 & 0 \\ 0 & 0 & 0 \\ 0 & 0 & 0 \\ 0 & 0 & 0 \\ 0 & 0 & 0\end{array}$
0
0
0
0
0
0
0

HNF-2486, Rev. 0

Page D-24 
W320H32A PROCESS BUILDIHI PIPING ITRESS ANALYSIS

03/01/95 W320, TANK 241 r:-106 SLUICING, ER 3319 AUTOPIPE+4.50 RESULT PAGE

\section{RESTRAINTTREACTIONS}

point Load FORCES (lb) MOMENTS ( $\mathrm{ft}-\mathrm{lb}$ ) name combination $X \quad Y \quad Z$ Result $X$ Y

B16 Anchor

GR

II

E?

GR+1 1

GR+I1+SF: IS

$$
\begin{array}{rrrr}
-2 & 1 & -1 & 2 \\
93 & 27 & -26 & 100 \\
31 & 7 & -2 & 32 \\
-1 & 0 & 12 & 12 \\
91 & 27 & -27 & 99 \\
32 & 7 & 14 & 36 \\
123 & 31 & 41 & 134
\end{array}
$$

$\begin{array}{rr}2 & 26 \\ 100 & -10 \\ 32 & -7 \\ 12 & 0 \\ 99 & 16 \\ 36 & 7 \\ 134 & 23\end{array}$

$\begin{array}{rr}26 & 13 \\ -10 & 507 \\ -7 & 39 \\ 0 & -12 \\ 16 & 520 \\ 7 & 51 \\ 23 & 571\end{array}$

$\begin{array}{rr}91 & 96 \\ -9 & 507 \\ -14 & 42 \\ 0 & 12 \\ 83 & 526 \\ 14 & 53 \\ 97 & 579\end{array}$

$\mathrm{c} 12$

Anchor

GR

E1

ER+T1

SEIS

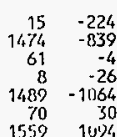

$\begin{array}{rr}14 & 225 \\ 937 & 1938 \\ -10 & 62 \\ 75 & 78 \\ 251 & 2062 \\ 83 & 112\end{array}$

-245
-562
-806
871

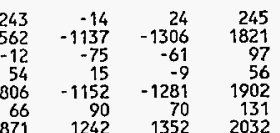

00 $Y$. Stop

GR

E1

$\mathrm{G} R+[1$

$S E I S$
$G R+I 1+S E I S$

$$
\begin{array}{rr}
0 & -145 \\
0 & 145 \\
0 & -2 \\
0 & 21 \\
0 & 0 \\
0 & 23 \\
0 & 23
\end{array}
$$$$
\begin{array}{rlr}
145 & 0 & 145 \\
145 & 0 & 145 \\
-2 & 1 & 2 \\
21 & \because & 21 \\
0 & 11 & 0 \\
23 & 0 & 23 \\
23 & 0 & 23
\end{array}
$$

145
145
2
21
0
23
23

$\begin{array}{ll}0 & 0 \\ 0 & 0 \\ 0 & 0 \\ 0 & 0 \\ 0 & 0 \\ 0 & 0 \\ 0 & 0\end{array}$

$\begin{array}{ll}0 & 0 \\ 0 & 0 \\ 0 & 0 \\ 0 & 0 \\ 0 & 0 \\ 0 & 0 \\ 0 & 0\end{array}$

$\begin{array}{ll}0 & 0 \\ 0 & 0 \\ 0 & 0 \\ 0 & 0 \\ 0 & 0 \\ 0 & 0 \\ 0 & 0\end{array}$

Anclior

GR

I1 1

E2

$\mathrm{GR+T}$

SE IS

45

FO6 Y - Stop

PS-109

$\begin{array}{rrrrrrrr}0 & -127 & -1 & 127 & 22 & -1 & 1 & 22 \\ 1,5 & 144 & -101 & 182 & -547 & 116 & -42 & 560 \\ 24 & 0 & 0 & 24 & 0 & 16 & -18 & 24 \\ 0 & -2 & 14 & 14 & 7 & 0 & 0 & 7 \\ 45 & 16 & -102 & 113 & -525 & 114 & -41 & 539 \\ 24 & 2 & 14 & 28 & 7 & 17 & 18 & 26 \\ 69 & 19 & 116 & 136 & 532 & 131 & 59 & 551\end{array}$

W320132A PROCESS BUILDING PIPING STRESS ANALYSIS

W320, AUTOPIPE+4.50 RESULY PAGE 28

AUTOPIPE +4.50 RESULY PAGE 28

RESTRAINT REACTIONS

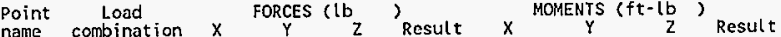

F10 Anchor

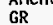

GR

E1

E2

GR+T

GR+T1-SEIS

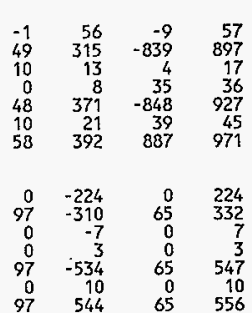

33
968
-2
-29
1001
31
1032

4
1128
-22
-42
1132
64

4
694
2
8
698
10
708

$\mathrm{GO2} Y$ - Stop

PS. 103

$\begin{array}{lrr}\text { GR Stop } & 0 & -224 \\ \text { T1 } & 97 & -310 \\ \text { E1 } & 0 & -7 \\ \text { E2 } & 0 & 3 \\ \text { GR+T1 } & 97 & -534 \\ \text { SEIS } & 0 & 10 \\ \text { GR+I1+SEIS } & 97 & 544\end{array}$

0
0
0
0
0
0
0

$\begin{array}{lll}0 & 0 & 0 \\ 0 & 0 & 0 \\ 0 & 0 & 0 \\ 0 & 0 & 0 \\ 0 & 0 & 0 \\ 0 & 0 & 0 \\ 0 & 0 & 0\end{array}$

GOB

Anchor

GR

E1

$\mathrm{ER}+\mathrm{T}$

SEIS

GR+T1+SEIS 
W320H32C PROCESS BUILDING PIPING STRESS ANALYSIS

02/22/95 W320, TANK 241-C-106, ER4319 H-2-818480 AUtOPIPE+4.50 RESULT PAGE

\begin{tabular}{|c|c|c|c|c|c|c|c|c|c|}
\hline \multirow{3}{*}{$\begin{array}{l}\text { Point/ } \\
\text { Supp. ID } \\
\text { A10 } \\
\text { A10 } 1 \\
\text { Stiff }\end{array}$} & \multicolumn{6}{|c|}{ SUPPORT } & \\
\hline & \multirow{2}{*}{$\begin{array}{l}\begin{array}{c}\text { Connect/ } \\
\text { Type }\end{array} \\
\text { Y - Stop } \\
\text { :RIGID }\end{array}$} & \multirow{2}{*}{$\begin{array}{l}\text { Load } \\
\text { Combination } \\
\text { GR }\end{array}$} & \multicolumn{3}{|c|}{ Dirn Force } & \multirow{2}{*}{$\begin{array}{c}\text { L Deform } \\
\text { D.000 } \\
0.000 \\
0.000\end{array}$} & \multicolumn{2}{|c|}{$\begin{array}{c}\text { G } \angle O B A \\
\text { Dirn Force }\end{array}$} & \multirow{2}{*}{$\begin{array}{l}\text { L Deform } \\
\\
0.000 \\
0.000 \\
0.000\end{array}$} \\
\hline & & & $\begin{array}{l}\text { down } \\
-2 \\
-x\end{array}$ & 15 & 159 & & $\begin{array}{l}x \\
y \\
z\end{array}$ & -159 & \\
\hline \multirow[t]{6}{*}{$p 5-104$} & & $T 1$ & $\begin{array}{l}\text { up } \\
-2 \\
-x\end{array}$ & & $\begin{array}{r}0 \\
25 \\
0\end{array}$ & $\begin{array}{l}0.000 \\
0.073 \\
0.001\end{array}$ & $\begin{array}{l}X \\
Y \\
Z\end{array}$ & $\begin{array}{r}0 \\
0 \\
-25\end{array}$ & $\begin{array}{r}-0.001 \\
0.000 \\
-0.073\end{array}$ \\
\hline & & E1 & $\begin{array}{l}\text { down } \\
-z \\
+x\end{array}$ & & 0 & $\begin{array}{l}0.000 \\
0.000 \\
0.000\end{array}$ & $\begin{array}{l}X \\
Y \\
Z\end{array}$ & 0 & $\begin{array}{l}0.000 \\
0.000 \\
0.000\end{array}$ \\
\hline & & $\mathrm{E} 2$ & $\begin{array}{l}\text { down } \\
+z \\
-x\end{array}$ & & 0 & $\begin{array}{l}0.000 \\
0.000 \\
0.000\end{array}$ & $\begin{array}{l}X \\
Y \\
z\end{array}$ & 0 & $\begin{array}{l}0.000 \\
0.000 \\
0.000\end{array}$ \\
\hline & & SEIS & $\begin{array}{l}\text { down } \\
-2 \\
-x\end{array}$ & & 0 & $\begin{array}{l}0.000 \\
0.000 \\
0.000\end{array}$ & $\begin{array}{l}X \\
Y \\
Z\end{array}$ & 0 & $\begin{array}{l}0.000 \\
0.000 \\
0.000\end{array}$ \\
\hline & & $G R+T 1$ & $\begin{array}{l}\text { down } \\
-z \\
-x\end{array}$ & & $\begin{array}{r}59 \\
25 \\
0\end{array}$ & $\begin{array}{l}0.000 \\
0.073 \\
0.001\end{array}$ & $\begin{array}{l}\mathbf{X} \\
\mathbf{Y} \\
\mathbf{Z}\end{array}$ & $\begin{array}{r}0 \\
-159 \\
-25\end{array}$ & $\begin{array}{r}-0.001 \\
0.000 \\
-0.073\end{array}$ \\
\hline & & $G R+I 1+S E I S$ & $\begin{array}{l}\text { down } \\
-z \\
-x\end{array}$ & & $\begin{array}{r}59 \\
25 \\
0\end{array}$ & $\begin{array}{l}0.000 \\
0.073 \\
0.001\end{array}$ & $\begin{array}{l}x \\
y \\
z\end{array}$ & $\begin{array}{r}0 \\
159 \\
25\end{array}$ & $\begin{array}{l}0.001 \\
0.000 \\
0.073\end{array}$ \\
\hline $\begin{array}{l}\mathrm{BO2} \\
\mathrm{BO2} 1 \\
\text { Stiff }\end{array}$ & $\begin{array}{l}Y \text { S Stop } \\
: \text { RIGID }\end{array}$ & GR & $\begin{array}{l}\text { down } \\
+2 \\
+x\end{array}$ & & 83 & $\begin{array}{l}0.000 \\
0.000 \\
0.000\end{array}$ & $\begin{array}{l}\mathrm{x} \\
\mathrm{y} \\
\mathrm{z}\end{array}$ & -83 & $\begin{array}{l}0.000 \\
0.000 \\
0.000\end{array}$ \\
\hline \multirow[t]{2}{*}{ PS-104 } & & T1 & $\begin{array}{l}\operatorname{up}_{+2} \\
+x\end{array}$ & & 83 & $\begin{array}{l}0.016 \\
0.041 \\
0.059\end{array}$ & $\begin{array}{l}X \\
Y \\
Z\end{array}$ & 83 & $\begin{array}{l}0.059 \\
0.016 \\
0.041\end{array}$ \\
\hline & & E1 & $\begin{array}{l}\text { down } \\
+2 \\
+x\end{array}$ & & 0 & $\begin{array}{l}0.000 \\
0.000 \\
0.000\end{array}$ & $\begin{array}{l}x \\
y \\
z\end{array}$ & 0 & $\begin{array}{l}0.000 \\
0.000 \\
0.000\end{array}$ \\
\hline \multirow[t]{2}{*}{. } & & E2 & $\begin{array}{l}\text { down } \\
+Z \\
-x\end{array}$ & & 4 & $\begin{array}{l}0.000 \\
0.000 \\
0.000\end{array}$ & $\begin{array}{l}x \\
y \\
z\end{array}$ & -4 & $\begin{array}{l}0.000 \\
0.000 \\
0.000\end{array}$ \\
\hline & & SEIS & $\begin{array}{l}\text { down } \\
-z \\
-x\end{array}$ & & 4 & $\begin{array}{l}0.000 \\
0.000 \\
0.000\end{array}$ & $\begin{array}{l}X \\
Y \\
Z\end{array}$ & 4 & $\begin{array}{l}0.000 \\
0.000 \\
0.000\end{array}$ \\
\hline
\end{tabular}

W32OH32C PROCESS BUILDING PIPING STRESS AMALYSIS

ALYSIS AUTOPIPE +4.50 RESULT PAGE $02 / 22 / 95$ W320, TANK 241-C-106, ER4319

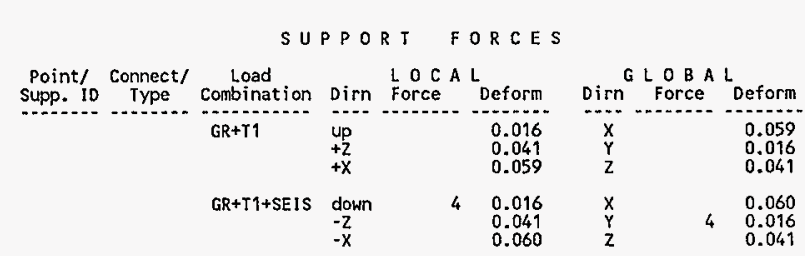

HNF-2486, Rev. 0

Page D-26 


\section{H32OH32C PROCESS BUILDING PIPING STRESS ANALYSIS}

H320, TANK 241-C- 106 , ER4319 H-2-818480 AUTOPIPE+4.50 RESULI PAGE 11

RESTRAINT REACTIONS

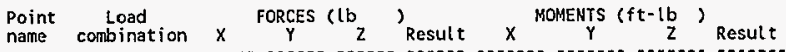

AOD Anchor

\begin{tabular}{|c|c|c|c|c|c|c|c|c|}
\hline $\begin{array}{l}\text { Anchor } \\
\text { GR } \\
\text { I1 } \\
\text { E1 } \\
\text { E2 } \\
\text { SEIS } \\
\text { GR+T1 } \\
\text { GR+T1+SE IS }\end{array}$ & $\begin{array}{r}0 \\
18473 \\
12 \\
-1 \\
13 \\
18473 \\
18486\end{array}$ & $\begin{array}{r}-131 \\
-28 \\
-2 \\
5 \\
8 \\
-159 \\
167\end{array}$ & $\begin{array}{r}0 \\
-32478 \\
8 \\
32 \\
40 \\
-32478\end{array}$ & $\begin{array}{r}131 \\
37364 \\
14 \\
33 \\
43 \\
37364 \\
37406\end{array}$ & $\begin{array}{r}92 \\
109 \\
3 \\
2 \\
4 \\
201 \\
205\end{array}$ & $\begin{array}{r}0 \\
27418 \\
11 \\
-1 \\
13 \\
27418 \\
2743\end{array}$ & $\begin{array}{r}-10 \\
-22 \\
-12 \\
-1 \\
12 \\
-32 \\
45\end{array}$ & $\begin{array}{r}92 \\
27418 \\
17 \\
2 \\
18 \\
27419 \\
27432\end{array}$ \\
\hline
\end{tabular}

A05

Anchor

GR

T1

E1

SEIS $\begin{array}{lr}\text { SEIS } & 42 \\ \text { GR+T1 } & 408363 \\ \text { GR+T1+SEIS } & 408405\end{array}$

A06

Anchor

GR

I1

E2

SEIS

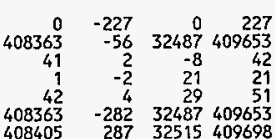

$\begin{array}{rr}-4 & 0 \\ -15 & -64446 \\ 3 & 12 \\ 0 & -16 \\ 3 & 28 \\ -19 & -64446 \\ 22 & 64474\end{array}$

$\begin{array}{lr}-252 & 252 \\ -125 & 64446\end{array}$

$\begin{array}{rr}2 & 6446 \\ -4 & 13\end{array}$

$\begin{array}{rr}-4 & 16 \\ -6 & 29 \\ -377 & 64447\end{array}$

$\begin{array}{rrrr}0 & -261 & 0 & 261 \\ -425388 & 0 & 100 & 425388\end{array}$

$\begin{array}{rrr}0 & 0 & 32 \\ 0 & 51 & 51\end{array}$

$\begin{array}{rrr}0 & 51 & 63 \\ -261 & 100425388\end{array}$

$\begin{array}{rr}100 & 425388 \\ 151 & 425424\end{array}$

$\begin{array}{rr}-24 & 0 \\ 0 & -1469 \\ 0 & -3 \\ 0 & 83 \\ 0 & 86 \\ -24 & -1469 \\ 24 & 1555\end{array}$

$\begin{array}{rr}333 & 33 \\ 0 & 1469\end{array}$

\begin{tabular}{rr}
0 & 83 \\
0 & 86 \\
\hline & 1506
\end{tabular}

A10 $Y$ - Stop

BS-ROH II

I1
E1
E2

SEIS

$G R+T 1$

$G R+T i+S E I S$

$\begin{array}{rrrr}0 & -159 & 0 & 159 \\ 0 & 0 & -25 & 25 \\ 0 & 0 & 0 & 0 \\ 0 & 0 & 0 & 0 \\ 0 & 0 & 0 & 0 \\ 0 & -159 & -25 & 161 \\ 0 & 159 & 25 & 161\end{array}$

A12
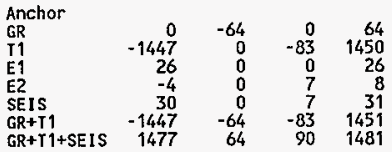

W320H32C PROCESS BUILDING PIPING STRESS ANALYSIS

\section{RESTRAINT REACTIONS}

Point
name $\begin{aligned} & \text { combination } \\ & \text { comb }\end{aligned}$

name combination $X{ }_{Y} Z_{Z}$ Result $X{ }_{Y}^{\text {NOMENT }} Z$ Result

$802 \quad Y$ - Stop

HNF-2486, Rev. 0

Page D-27 


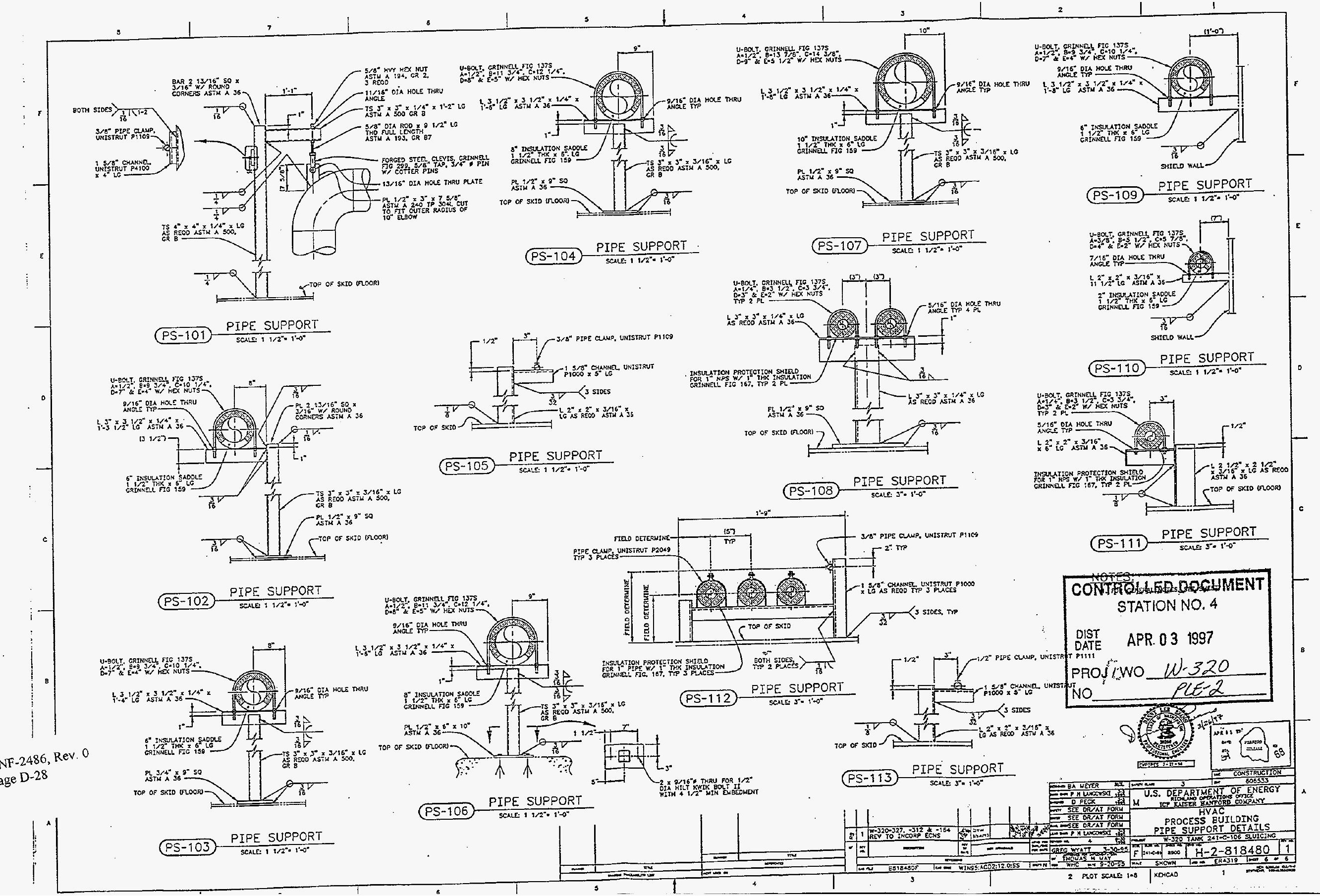




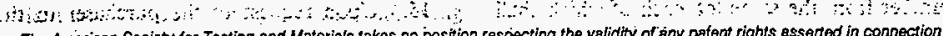

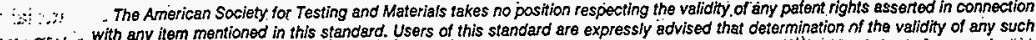

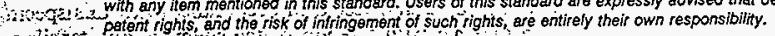

is : This stendard is subjoct to revision at any time by the responsible technical committoe and must be reviewed every fhvo yaars and if not revised, either reapproved or withorawt. Your comments are invited enther for revision of this standard or for additional standarc's . and should be addressed to ASTM Headquarters. Your comments will seceive careful consideration al a meeting of the responsible tochnical committee, which you may attend. If you feol that your comments have not received a fair hoaring you should make your views known to the ASTM Committes on Stendards; 1916 Race St., Philadelphija, PA 19103.

2. Refere 
the original number from the same lot, each of which shall conform to the requirement specified, or the tubing represented by the test is subject to rejection.

13.2 In case of failure on retest to meet the requirements of Sections 8 and 9, the manufacturer may elect to retreat, rework, or otherwise eliminate the condition responsible for failure to meet the specified requirements. Thereafter, the material remaining from the lot originally represented may be tested and shall comply witn ail requirements of this specification.

\section{Test Methods}

14.1 The tension specimens required by this specification shall conform to those described in the latest issue of Test Methods and Definitions A.370, Annex A 2 on Tubular Products.

14.2 The tension test specimen shall be taken longitudinally from a section of the finished tubing, at a location at least $90^{\circ}$ from the weld in the case of welded tubing, and shall not be flattened between gage marks. If desired, the tension tests may be made on the full section of the tubing; otherwise, a longitudinal strip-test specimen as prescribed in Test Methods and Definitions A 370 Supplementary Requirements II shall be used. The specimens shall have all burrs removed and shall not contain surface imperfections which would interfere with proper determination of the tensile properties of the metal.

14.3 The yield strength corresponding to a permanent offset of $0.2 \%$ of the gage length of the specimen or to a total extension of $0.5 \%$ of the gage length under load shall be determined.

\section{Inspection}

15.1 All tubing shall be subject to an inspection at place of manufacture to assure conformance to the requirements of this specification.

15.2 All tubing shall be free from defects and shall have a workmanlike finish.

15.2.1 Surface imperfections shall be classed as Afforts when their depth reduces the remaining wall thickness to less than $90 \%$ of the specified nominal wall. Defects having a depth not in excess of $331 / 3 \%$ of the wall thickness may be repaired by welding, subject to the following conditions:

15.2.1.1 The defect shall be completely removed by chipping or grinding to sound metal,

15.2.1.2 The repair weld shall be made using a lowhydrogen welding process, and

15.2.1.3 The projecting weld metal shall be removed to produce a workmanlike finish.

15.2.2 Surface imperfections such as handling marks, light die or roll marks, or shallow pits are not considered defects providing the imperfections are removable within the 4. minimum wall permitted. The removal of such surface imperfections is not required. Welded tubing shall be free of protruding metal on the outside surface of the weld seam.

15.3 The ends of structural tubing, unless otherwise specified, shall be finished square cut and the burr held to a minimum. The burr can be removed on the outside diameter, inside diameter, or both, as a supplementary requirement. When burrs are to be removed, it shall be specified on * the purchase order.
15.4 Upon request of the purchaser in the contract or order, a manufacturer's certification that the material was manufactured and tested in accordance with this specification together with a report of the chemical and tensile tests shall be furnished.

\section{Rejection}

16.1 Each length of tubing received from the manufacturer may be inspected by the purchaser and, if it does not meet the requirements of this specification based on the inspection and test method as outlined in the specification, the length may be rejected and the manufacturer shall be notified. Disposition of rejected tubing shall be a matter of agreement between the manufacturer and the purchaser.

16.2 Tubing found in fabrication or in installation to be unsuitable for the intended use, under the scope and requirements of this specification, may be set aside and the manufacturer notified. Such tubing shall be subject to mutual investigation as to the nature and severity of the deficiency and the forming or installation, or both, conditions involved. Disposition shall be a matter for agreement.

\section{Product Marking}

17.1 Except as noted in 17.2, each length of structural tubing shall be legibly marked to show the following information: manufacturer's name, brand, or trademark; the specification number; and grade letter.

17.2 For structural tubing having a largest dimension of 4 in. $(101.6 \mathrm{~mm})$ or less, the information listed in 17.1 may be marked on a tag securely attached to each bundle.

17.3 Bar Coding -In addition to the requirements in 17.1 and 17.2, bar coding is acceptable as a supplementary identification method. Bar coding should be consistent with the Automotive Industry Action Group (AIAG) standard prepared by the Primary Metals Subcommittee of the AIAG Bar Code Project Team.

\section{Packing, Marking, and Loading}

18.1 When specified in the order, contract, etc., packing, marking, and loading shall be in accordance with those procedures of Practices A 700 .

\section{Government Procurement}

19.1 When specified in the contract, material shall be preserved, packaged and packed in accordance with the requirements of MIL-STD 163 . The applicable levels shall be as specified in the contract. Marking for shipment of such materials shall be in accordance with Federal Std. No. 123 for civil agencies and MIL-STD 129 or Federal Std. No. 183 if continuous marking is required, for military agencies.

19.2 Inspection-Unless otherwise specified in the contract, the producer is responsible for the performance of all inspection and test requirements specified herein. Except as otherwise specified in the contract, the producer may use his own, or any other suitable facilities for performing the inspection and test requirements specified heroin, unless otherwise disapproved by the purchaser in the contract or purchase order. The purchaser shall have the right to perform any of the inspections and tests set forth in this specification where deemed necessary to ensure that the material conforms to the prescribed requirements. 
unsound material that is revealed during the entire flattening test shall be cause for rejection.

9.4 Surface imperfections not found in the test specimen vefore flattening, but revealed during the first step of the flattening test, shall be juoged in accordaiisi with Section 14 .

9.5 When low $D$-to-t ratio tubulars are tested, because the strain imposed due to geometry is unreasonably high on the inside surface at the 6 and 12 o'clock locations, cracks at these locations shall not be cause for rejection if the $D$-to- $t$ râtio is less than 10 .

\section{Permissible Variations in Dimensions}

\subsection{Outside Dimensions:}

10.1.1 Round Structural Tubing-The outside diameter shall not vary more than $\pm 0.5 \%$ rounded to the nearest 0.005 in. $(0.13 \mathrm{~mm})$, of the nominal outside diameter size specified, for nominal outside diameters 1.900 in. (48.26 $\mathrm{mm}$ ) and smaller, and plus and minus $0.75 \%$ rounded to the nearest 0.005 in. of the nominal outside diameter for nominal outside diameters $2.00 \mathrm{in}$. $(50.8 \mathrm{~mm})$ and larger. The outside diameter measurements shall be made at positions at least 2 in. $(50.8 \mathrm{~mm})$ from either end of the tubing.

10.1.2 Square and Rectangular Structural Tubing-The specified dimensions, measured across the flats at positions at least 2 in. ( $50.8 \mathrm{~mm}$ ) from either end of the tubing and including an allowance for convexity or concavity, shall not exceed the plus and minus tolerances shown in Table 3 .

10.2 Wall Thickness-The minimum wall thickness at any point of measurement on the tubing shall be not more an $10 \%$ less than the nominal wall thickness specified. ihe maximum wail thickness, excluding the weld seam of welded tubing, shall be not more than $10 \%$ greater than the nominal wall thickness specified. The wall thickness on square and rectangular tubing is to be measured at the center of the flat.

10.3 Length-Structural tubing is normally produced in random mill lengths $5 \mathrm{ft}(1.5 \mathrm{~m})$ and over, in multiple lengths, and in specified mill lengths. Refer to Section 3. When specified mill lengths are ordered, the length tolerance shall be in accordance with Table 4 .

10.4" Straightness-The permissible variation for straightness of structural tubing shall be $1 / 8$ in. times the

TABLE 3 Outside Dimension Tolerances for Square and Rectangular Structural Tubing

\begin{tabular}{|c|c|}
\hline $\begin{array}{l}\text { Outside Large Flat Dimension, } \\
\text { in. (mm) }\end{array}$ & $\begin{array}{l}\text { Large Flat Dimension } \\
\text { Tolerance, }{ }^{\wedge} \text { plus } \\
\text { and minus, } \\
\text { in. (mm) }\end{array}$ \\
\hline $\begin{array}{l}21 / 2(63.5) \text { or under } \\
\text { Over } 21 / 2 \text { to } 31 / 2(63.5 \text { to } 88.9) \text {, ind } \\
\text { Over } 31 / 2 \text { to } 51 / 2(88.9 \text { to } 139.7) \text {, incl } \\
\text { Over } 51 / 2(139.7)\end{array}$ & $\begin{array}{l}0.020(0.51) \\
0.025(0.64) \\
0.030(0.76) \\
0.01 \text { times large flat } \\
\quad \text { dimension }\end{array}$ \\
\hline
\end{tabular}

i Tolerances include allowance for convexity or concavity. For rectangular tubing having a ratio of outside large to small flat dimension less than 1.5 , and for square tubing, the tolerance on small fiat dimension shall be identical to the large flat dimension tolerance. For rectangular tubing having a ratio of outside large to mall fat dimension in the range of 1.5 to 3.0 inclusive, the tolerance on small flat mension shall be 1.5 times the large flat dimension tolerance. For rectangular fubing having a ratio of outside large to small flat dimension greater than 3.0 , the tolerance on small fiat dimension shall be 2.0 times the large flat dimension tolerance.
TABLE 4 Specified Mill Length Tolerances for Structural Tubing

\begin{tabular}{|c|c|c|c|c|}
\hline \multirow[t]{2}{*}{$\cdots$} & \multicolumn{2}{|c|}{$\begin{array}{c}22 \mathrm{ft}(6.7 \mathrm{~m}) \\
\text { and Under }\end{array}$} & \multicolumn{2}{|c|}{$\begin{array}{l}\text { Over } 22 \text { to } 44 \\
\text { ft }(6.7 \text { to } \\
\{3.4 \mathrm{~m}) \text {, ind }\end{array}$} \\
\hline & Over & Under & Over & Undet \\
\hline $\begin{array}{l}\text { Length tolerance for specified } \\
\text { mill length, in. (mm) }\end{array}$ & $\begin{array}{l}1 / 2 \\
(12.7)\end{array}$ & $\begin{array}{l}1 / 4 \\
(6.4)\end{array}$ & $\begin{array}{l}3 / 4 \\
(19.0)\end{array}$ & $\begin{array}{l}1 / 4 \\
(6.4)\end{array}$ \\
\hline
\end{tabular}

number of feet (10.4 mm times the number of metres) of total length divided by 5 .

10.5 Squareness of Sides-For square or rectangular structural tubing, adjacent sides may deviate from $90^{\circ}$ by a tolerance of $\pm 2^{\circ} \max$.

10.6 Radius of Corners-For square or rectangular structural tubing, the radius of any outside corner of the section shall not exceed three times the specified wall thickness.

10.7 Twist-The tolerances for twist or variation with respect to axial alignment of the section, for square and rectangular structural tubing, shall be as shown in Table 5. Twist is measured either by holding down one end of a square or rectangular tube on a flat surface plate with the bottom side of the tube parallel to the surface plate and noting the difference in height above the surface plate of the two comers at the opposite end of the bottom side of the tube or by measuring this difference on the heavier sections by a suitable measuring device. The difference in the height of the corners shall not exceed the values in Table 5 . Twist measurements are not to be taken within $2 \mathrm{in}$. $(50 \mathrm{~mm})$ of either end of the product.

\section{Special Shape Structural Tubing}

11.1 The dimensions and tolerances of special shape structural tubing are available by inquiry and negotiation with the manufacturer.

\section{Number of Tests}

12.1 One tension test as specified in Section 14 shall be made from a length of tubing representing each lot.

12.2 The flattening test, as specified in Section 9 shall be made on one length of round tubing from each lot.

12.3 The term "lot" applies to all tubes of the same nominal size and wall thickness which are produced from the same heat of steel.

\section{Retests}

13.1 If the results of the mechanical tests representing any lot do not conform to a requirement as specified in Sections 8 and 9 , retests may be made on additional tubing of double

TABLE 5 . Twist Tolerances for Square and Rectangular Structural Tubing

\begin{tabular}{|c|c|c|c|c|c|c|}
\hline & \multirow[t]{2}{*}{$\begin{array}{l}\text { Specified Dimension of } \\
\text { Longest Side, in. ( } \mathrm{mm} \text { ) }\end{array}$} & \multicolumn{4}{|c|}{$\begin{array}{c}\text { Maximum Twist in } \\
\text { the First } 3 \mathrm{ft}(1 \mathrm{~m}) \\
\text { and in each } \\
\text { additional } 3 \text { it }\end{array}$} & \\
\hline & & & + in. & $\therefore i$ & $\mathrm{~mm}$ & \\
\hline & $\begin{array}{l}11 / 2(38.1) \text { and under } \\
\text { Over } 11 / 2 \text { to } 23 / 2(38.1 \text { to } 63.5) \text {, ind } \\
\text { Over } 21 / 2 \text { to } 4(63.5 \text { to } 101.6) \text {, ind } \\
\text { Over } 4 \text { to } 6(101.6 \text { to } 152.4) \text {, ind } \\
\text { Over } 6 \text { to } 8(152.4 \text { to } 203.2) \text {, ind } \\
\text { Over } 8(203)\end{array}$ & . & $\begin{array}{l}0.050 \\
0.062 \\
0.075 \\
0.087 \\
0.100 \\
0.112\end{array}$ & . & $\begin{array}{r}1.39 \\
1.72 \\
2.09 \\
2.42 \\
2.78 \\
3.11\end{array}$ & \\
\hline
\end{tabular}

the origin: conform $t$ sented by 13.2 In of Section sework, or failure to material $\mathrm{r}$ be tested specificati

\section{Test l}

$14.1 \mathrm{TH}$ shall conf Methods Products.

I4.2 TI nally fron least $90^{\circ} \mathrm{fi}$ not be fla tests may otherwise, Test Met! quiremen: burns rem which wc tensile prs

$14.3 \mathrm{~T}$ ! offset of 0 extension determine

15. Inspe

$15.1 \mathrm{~A}$ manufact this specil

$15.2 \mathrm{~A}$ workman

15.2.1

when thei than 90 \% depth no1 repaired I

15.2.1.' ping or g:

15.2.1: hydrogen

15.2.1.' produce :

15.2.2 light die sofonte a: minimun imperfect protrudin

$15.3 \mathrm{~T}$ specified, minimun eter, insic ment. W] the purct: 
TABLE 1 Chemical Requirements

\begin{tabular}{|c|c|c|c|c|}
\hline \multirow{3}{*}{ Element } & \multicolumn{4}{|c|}{ Composition, $\%$} \\
\hline & \multicolumn{2}{|c|}{ Grades $A, B$ and $D$} & \multicolumn{2}{|c|}{ Grade C } \\
\hline & $\begin{array}{c}\text { Heat } \\
\text { Analysis }\end{array}$ & $\begin{array}{l}\text { Product } \\
\text { Analysis }\end{array}$ & $\begin{array}{c}\text { Heat } \\
\text { Analysis }\end{array}$ & $\begin{array}{l}\text { Product } \\
\text { Analysis }\end{array}$ \\
\hline Carbon, max & 0.26 & .0 .30 & 0.23 & 0.27 \\
\hline Manganese, $\max$ & $\cdots$ & $\therefore$ & 1.35 & 1.40 \\
\hline Phosphorus, max & 0.035 & 0.045 & 0.035 & 0.045 \\
\hline Sultur, max & 0.035 & 0.045 & 0.035 & 0.045 \\
\hline $\begin{array}{l}\text { copper, when copper steel is } \\
\text { specified, min }\end{array}$ & 0.20 & 0.18 & 0.20 & 0.18 \\
\hline
\end{tabular}

steel by בal butt Eness in of the

sistance ff inside

$\mathrm{dd}$ as is $\exists$ to the by 5.4 . nimum $\Rightarrow 1 / 2 h$

in Eacturer. ring the pouring of the heat. The chemical composition thus determined shall conform to the requirements specified in Table 1 for heat analysis.

\section{Product Analysis}

7.1 An analysis may be made by the purchaser from finished tubing manufactured in accordance with this specification, or an analysis may be made from flat-rolled stock from which the welded tubing is manufactured. When product analyses are made, two sample lengths from a lot of each 500 lengths or fraction thereof shall be selected. Methods and practices relating to chemical analysis shall be in accordance with Test Methods, Practices and Terminology A 751. The chemical composition thus determined shall conform to the requirements specified in Table 1 for product analysis.

7.2 In the event the chemical composition of one of the sample lengths does not conform to the requirements shown in Table 1 for product analysis, an analysis of two additional lengths selected from the same lot shall be made, each of which shall conform to the requirements shown in Table 1 for product analysis, or the lot is subject to rejection.

\section{Tensile Requirements}

8.1 The material, as represented by the test specimen, shall conform to the requirements as to tensile properties prescribed in Table 2.

\section{Flattening Test}

9.1 The flattening test shall be made on round structural tubing. A flattening test is not required for shaped structural tubing.

9.2 For welded round structural tubing, a specirien at least $4 \mathrm{in} .(102 \mathrm{~mm})$ in length shall be flattened cold between parallel plates in three steps, with the weld located at $90^{\circ}$ from the line of direction of force. During the first step, which is a test for ductility of the weld, no cracks or breaks on the inside or outside surfaces shall occur until the distance between the plates is less than two thirds of the original outside diameter of the tubing. As a second step, the flattening shall be continued. During the second step, which is a test for ductility exclusive of the weld, no cracks or breaks on the inside or outside surfaces, except as provided for in 9.5, shall occur until the distance between the plates is less than one half of the original outside diameter of the tubing but is not less than five times the wall thickness of the tubing. During the third step, which is a test for soundness, the flattening shall be continued until the specimen breaks or the opposite walls of the tubing meet. Evidence of laminated
TABLE 2 Tensile Requirements

\begin{tabular}{|c|c|c|c|c|}
\hline \multicolumn{3}{|c|}{ Round Structural Tubing } & & \multirow{2}{*}{ Grade D } \\
\hline & Grade A & Grade B & Grade C & \\
\hline $\begin{array}{l}\text { Tensile strength, min, psi (MPa) } \\
\text { Yield strength, min, psi (MPa) } \\
\text { Elongation in } 2 \text { in. }(50.8 \mathrm{~mm}) \text {. } \\
\text { min, \%^ }\end{array}$ & $\begin{array}{l}45000 \\
(310) \\
33000 \\
(228) \\
25^{9}\end{array}$ & $\begin{array}{r}58000 \\
\quad(400) \\
42000 \\
(290) \\
23^{\circ}\end{array}$ & $\begin{array}{r}62000 \\
(427) \\
46000 \\
(317) \\
210\end{array}$ & $\begin{array}{l}58000 \\
\because(400) \\
36000 \\
(250) \\
23^{\circ}\end{array}$ \\
\hline \multicolumn{5}{|c|}{ Shaped Structural Tubing } \\
\hline & Grade A & Grade B & Grade C & Grade D \\
\hline $\begin{array}{l}\text { Tensile strength, min, psi (MPa) } \\
\text { Yield strength, min, psi (MPa) } \\
\text { Elongation in } 2 \text { in. (50.8 mm), } \\
\text { min. }\end{array}$ & $\begin{array}{r}45000 \\
(310) \\
39000 \\
(269) \\
25^{8}\end{array}$ & $\begin{array}{r}58000 \\
(400) \\
46000 \\
(317) \\
23^{c}\end{array}$ & $\begin{array}{r}62000 \\
(427) \\
50000 \\
(345) \\
210\end{array}$ & $\begin{array}{r}58000 \\
(400) \\
36000 \\
(250) \\
23^{c} \\
:\end{array}$ \\
\hline
\end{tabular}

A The minimum elongation values specified apply only to testing pertomed prior to shipment because of the possibility of strain aging. Tests performed after shioment may show values below those stated.

${ }^{s}$ Applies to specified wall thicknesses 0.120 in. $(3.05 \mathrm{~mm})$ and over. For wall thicknesses under $0.120 \mathrm{in}$., the minimum elongation shall be calculated by the formula: percent elongation in 2 in, $=56 t+17.5$.

$c$ Applies to specified wall thicknesses 0.180 in. $(4.57 \mathrm{~mm})$ and over. For wall thicknesses under 0.180 in., the minimum elongation shall be calculated by the formula: percent elongation in 2 in. $=6 t t+12$.

- Applies to specified wall thicknesses $0.120 \mathrm{in}$. $(3.05 \mathrm{~mm})$ and over. For lighter wall thicknesses, elongation shall be by agreement with the manufacturer.

NoTE-The following table gives calculated minimum values for longitudinal strip tests:

\begin{tabular}{ccc}
\hline \multirow{2}{*}{ Wall thickness, in. (mm) } & \multicolumn{2}{c}{ Elongation in 2 in. (50.8 mm), min, $\delta$} \\
\cline { 2 - 3 } & Grade A & Grade 8 \\
\hline $0.180(4.57)$ & $\ldots$ & 23 \\
$0.165(4.19)$ & $\cdots$ & 22 \\
$0.148(3.76)$ & $\ldots$ & 21 \\
$0.134(3.40)$ & 25 & 20 \\
$0.120(3.05)$ & 23.5 & 19.5 \\
$0.109(2.77)$ & 23 & 19 \\
$0.095(2.41)$ & 22 & 18 \\
$0.083(2.11)$ & 21 & 17 \\
$0.065(1.65)$ & 20 & 16 \\
$0.049(1.24)$ & 19.5 & 15 \\
$0.035(0.89)$ & & 14 \\
\hline
\end{tabular}

or unsound material or of incomplete weld that is revealed during the entire flattening test shall be cause for rejection.

9.3 For seamless round structural tubing $23 / 8$ in. $(60.3$ $\mathrm{mm}$ ) outside diameter and larger, a section not less than $21 / 2$ in. $(63.5 \mathrm{~mm})$ in length shall be flattened cold between parallel plates in two steps. During the first step, which is a test for ductility no cracks or breaks on the inside or outside surfaces, except as provided for in 9.5 , shall occur until the distance between the plates is less than the value of " $\mathrm{H}$ " calculated by the following equation:

$$
H=(1+e) t /(e+t / D)
$$

where:

$H=$ distance between flattening plates, in.,

$e=$ deformation per unit length (constant for a given grade of steel, 0.09 for Grade A, 0.07 for Grade B, and 0.06 for Grade C),

$t=$ nominal wall thickness of tubing, in., and

$D=$ actual outside diameter of tubing, in.

During the second step, which is a test for soundness, the flattening shall be continued until the specimen breaks or the opposite walls of the tubing meet. Evidence of laminated or 


\title{
Cold-Formed Welded and Seamless Carbon Steel Structural Tubing in Rounds and Shapes ${ }^{1}$
}

\begin{abstract}
This standard is issued under the fixed designation A 500; the number inmediately following the designation indicates the year of original adoption or, in the case of revision, the year of last revision. A nursber in parentheses indicates the year of last reapproval. A superscript epsilon (o) indicates an editorial change since the last revision or reapproval.

This specification has been approved for use by agencies of the Department of Defense. Consult the DoD Index of Specifications and Slandiards for the specific year of issue which has been adopted by the Depanment of Deferse.
\end{abstract}

\section{Scope}

1.1 This specification covers cold-formed welded and seamless carbon steel round, square, rectangular, or special shape structural tubing for welded, riveted, or bolted construction of bridges and buildings, and for general structural purposes.

1.2 This tubing is produced in both welded and seamless sizes with a maximum periphery of $64 \mathrm{in} .(1626 \mathrm{~mm})$ and a maximum wall of $0.625 \mathrm{in} .(15.88 \mathrm{~mm})$. Grade $D$ requires heat treatment.

Note 1-Products manufactured to this specification may not be suitable for those applications such as dynamically loaded elements in welded structures, etc., where low-temperature notch-toughness properties ray be important.

1.3 The values stated in inch-pound units are to be rded as the standard.

\section{Referenced Documents}

\subsection{ASTM Standards:}

A 370 Test Methods and Definitions for Mechanica: Testing of Steel Products ${ }^{2}$

A 700 Practices for Packaging, Marking, and Loading Methods for Steel Products for Domestic Shipment ${ }^{3}$

A 751 Test Methods, Practices, and Terminology for Chemical Analysis of Steel Products ${ }^{2}$

\subsection{Military Standards:}

MIL-STD-129 Marking for Shipment and Storage 4

MIL-STD-163 Steel Mill Products, Preparation for Shipment and Storage 4

2.3 Federal Standards:

Fed. Std. No. 123 Marking for Shipment ${ }^{4}$

Fed. Std. No. 183 Continuous Identification Marking of Iron and Steel Products ${ }^{4}$

\section{Ordering Information}

3.1 Orders for material under this specification should include the following, as required, to describe the desired material adequately.

1 This specification is under the jurisdiction of A STM Committee A-I on Steel, Stainiess Stecl, and Related Alloys and is the direct responsibility of Subcomminee ${ }^{4} 01.09$ on Steel Pipe.

Current edition approved July 15, 1993. Published September 1993. Originally of blished as A $500-64$. Last previous edition A $500-92$

2 Anntual Book of ASTM Stondards, Vol 01.03

3 Ammual Boak of ASTM Standards, Vol 01.05 .

- Available from Standardization Documents Order Desk, BJd\& 4 Section D, 700 Robbins Ave., Philadelphia, PA $19111-5094$, Ant NPODS.

\subsubsection{Quantity (feet or number of lengths),}

3.1.2 Name of material (cold-formed tubing);

3.1.3 Method of manufacture (seamless or welded),

3.1.4 Grade (Table 1 and 2),

3.1.5 Size (outside diameter and nominal wall thickness for round tubing and the outside dimensions and nominal wall thickness for square and rectangular tubing),

3.1.6 Length (specific or random, see 10.3),

3.1.7 End condition (see 15.3),

3.1.8 Burr removal (see 15.3),

3.1.9 Certification (see 15.4),

3.1.10 ASTM specification number,

3.1.11 End use, and

3.1.12 Special requirements.

\section{Process}

4.1 The steel shall be made by one or more of the following processes: open-hearth, basic-oxygen, or electricfurnace.

4.2 Steel may be cast in ingots or may be strand cast.

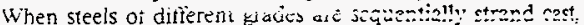
identification of the resultant transituvid isicicial is Itiquizes. The producer shall remove the transition material by any established procedure that positively separates the grades.

\section{Manufacture}

5.1 The tubing shall be made by a seamless or welding process.

5.2 Welded tubing shall be made from flat-rolled steel by the electric-resistance welding process. The longitudinal butt joint of welded tubing shall be welded across its thickness in such a manner that the structural design strength of the tubing section is assured.

5.2.1 Structural tubing welded by electric-resistance methods is normally furnished without removal of inside flash.

S.3 The tubing may be stress relieved or annealed as is considered necessary by the manufacturer to conform to the reouirements of this specification, except as required by 5.4 .

5.4 Grade $D$ tubing shall be heat treated at a minimum temperature of $1100^{\circ} \mathrm{F}$ for $1 \mathrm{~h} / \mathrm{in}$. of thickness, $1 / 2 \mathrm{~b}$ minimum.

\section{Heat Analysis}

6.1 An analysis of each heat of open-hearth, basic-oxygen;: $:$ or electric-furnace steel shall be made by the manufacturer. This analysis shall be made from a test ingot taken during the
Carbon, max

Manganese, $\max$

Phosphorus, max

Sulfur, max

copper, when cop specified, min

:

pouring of th mined shall cr for heat analy

7. Product $\mathbf{A}$

$7.1 \mathrm{An}$ an finished tubir fication, or a from which product analy each 500 le Methods and in accordanc nology A 75: shail conforn product anal

7.2 In the sample lengt in Table 1 fo lengths selec which shall for product

Q. Tamsile R

8.1 The I shall confor prescribed if

9. Elattenin 9.1 The f] tubing. A fla tubing.

9.2 For $v$ least 4 in. (1) parallel plat from the lit which is a $t$ on the insidt between the outside dia: lattening sh; is a test for breaks on tl for in 9.5 , 1 less than of tubing but $i$ tubing. Dur the flattenin the opposite 
Lonemeisur jadord

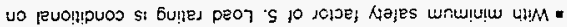

\begin{tabular}{|c|c|c|c|c|}
\hline $\begin{array}{l}088 \\
091\end{array}$ & $\begin{array}{r}\varepsilon \\
z, \tau \\
\end{array}$ & $\begin{array}{l}91 / 62 \\
21 / 62\end{array}$ & $\begin{array}{l}\$ \\
\forall \\
\end{array}$ & $\begin{array}{l}8 / \varepsilon \\
8 \cdot s \\
\end{array}$ \\
\hline 079 & 2,12 & $91 / 22$ & $8^{\prime} z / \varepsilon$ & $z_{i}$ \\
\hline $05 \varepsilon$ & $z i z$ & $z$ & $8^{2} \cdot \xi \varepsilon$ & $\varepsilon_{\hat{\tau}}$ \\
\hline 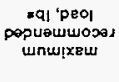 & $\begin{array}{c}\text { U! 'a } \\
\text { PEQ J4? pOS } \\
\text { PJEPUE! }\end{array}$ & $\begin{array}{l}\text { U!'0 } \\
\text { pвasul } \\
\text { majos } \\
\text { 40еos }\end{array}$ & 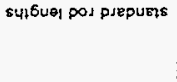 & $\begin{array}{l}\forall \\
\text { oz!s } \\
\text { pod }\end{array}$ \\
\hline
\end{tabular}

speoj - syiburl peəduł pdepuels

पाtбua! pos

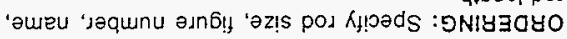

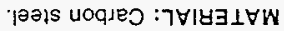

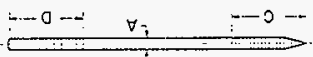

:pua alisoddo uo papeadut әuाчวеu

乙๖レ 6 ! spol Majos yoeos

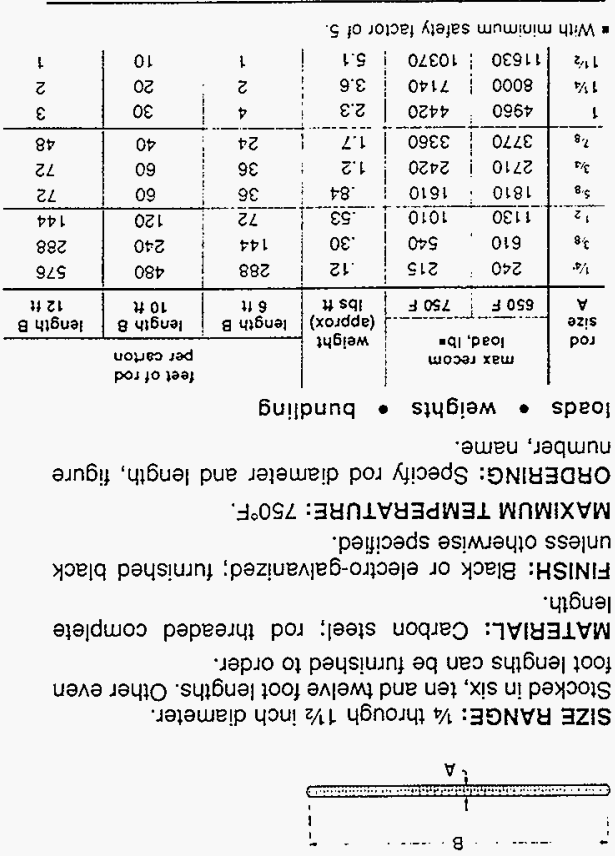

9७レ・6!ł :peəduł snonu!̣uos

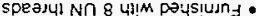

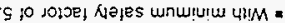
-

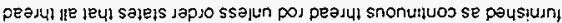

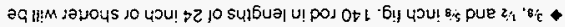

\begin{tabular}{|c|c|c|c|}
\hline SI & SS8EL & 06828 & - tक \\
\hline 5 & $\varepsilon 6+\varepsilon 9$ & $0821 L$ & $-z_{.1} \varepsilon$ \\
\hline$\varepsilon !$ & \$068 & 08509 & 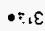 \\
\hline 21 & S80st & 08909 & $\varepsilon$ \\
\hline 11 & $990 \angle \varepsilon$ & $089 ! t$ & $5 / 2 Z$ \\
\hline Ol & $0886 z$ & OOSEE & 2,12 \\
\hline 6 & $09 z \triangleright z$ & $00 z \angle Z$ & $5 / 2$ \\
\hline 8 & $09+81$ & $00 \angle 0 z$ & 2 \\
\hline$L$ & $000 t 1$ & $00 \angle 9+$ & $\nabla / \epsilon !$ \\
\hline 9 & $0 \angle E O L$ & 08911 & $2 / 1$ \\
\hline$S$ & $0 \nabla \$ L$ & 0008 & pit \\
\hline$t$ & $0 z 0 \%$ & 0967 & 1 \\
\hline Z1 & $09 \varepsilon \varepsilon$ & $0 \angle \angle E$ & $\theta_{i}$ \\
\hline$\varepsilon$ & $0 Z \nabla Z$ & $0 L \angle Z$ & $7 \%$ \\
\hline $2 / 12$ & $019 !$ & 0.81 & 8 \\
\hline$z / 12$ & 0101 & OE! I & $z_{1}$ \\
\hline$z / A Z$ & $0+5$ & 019 & 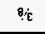 \\
\hline \multirow{2}{*}{ 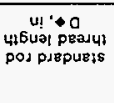 } & $s: 0 s \angle$ & \pm 059 & \multirow{2}{*}{$\begin{array}{c}\forall \\
\text { az!s } \\
\text { pos }\end{array}$} \\
\hline & \multicolumn{2}{|c|}{ 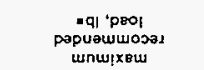 } & \\
\hline
\end{tabular}

s4tбuә peәduł pdepuets - speol

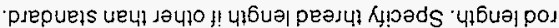

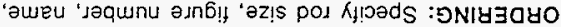
$\exists: 0 G L: \exists \forall \cap \perp \forall 43 d W \exists \perp$ WกWIX $\forall W$

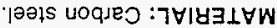

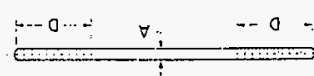

$\varepsilon \varsigma 2 \cdot 6 !$ :speəג41 pueч-нә| pue -146!ג 0เ। 6 !! :speәлчł рuеप-746!1 spuә uдoq popeəd41 spod pәреәдч1 әи!чэеш 


\section{forged steel clevis}

fig. 299
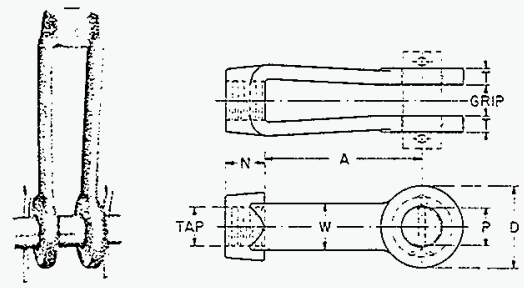

MAXIMUM RECOMMENDED LOAD: $95,400 \mathrm{lbs}$.

MATERIAL: Forged steel.

SERVICE: For use on high temperature piping installation.

APPROVALS: Complies with Federal Specification WW-H-171E (Type 14) and Manufacturers Standardization Society SP-69 (Type 14).

FEATURES:

- Complies fully with the code for pressure piping.

- Supports loads equal to the full limitation of the hanger rod.

- Available with pin and cotter pins, if required.

ORDERING: Specify rod size, figure number, name. If pin and cotter pins are required, specify "with pin." if other than standard combination of clevis size and rod tapping size is required, specify clevis number, special rod tapping size, pin size, grip.

loads - weights - dimensions (inches)

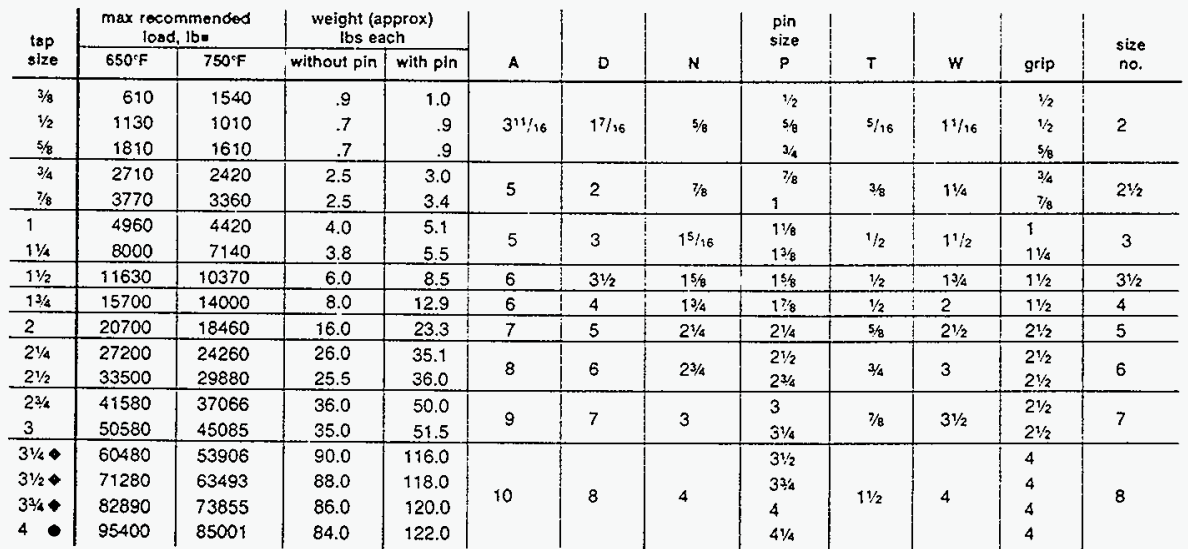

- Based on the allowable stresses shown in the ANSI Code for Pressure Piping.

- Furnished with 8 UN series threads. 
HNF-2486, Rev. 0

Grinnell

Page D-36

\section{standard U-bolt}

fig. 137

special U-bolt (non-standard dimensions)

fig. $137 \mathrm{~S}^{\circ}$
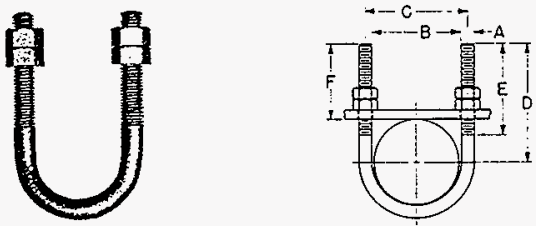

plastic coated: fig. $137 \mathrm{C}$

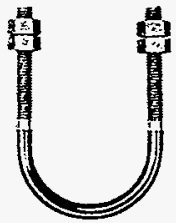

SIZE RANGE: $1 / 2$ to 36 inch pipe.

U-bolts

MATERIAL: Carbon steel U-bolt and four finished hex nuts.

FINISH: Black or galvanized; furnished black unless otherwise specified.

SERVICE: Recommended for support, or guide of heavy toads; often employed in power and process plant service.

MAXIMUM TEMPERATURE: $750^{\circ} \mathrm{F}$.

APPROVALS: Complies with Federal Specification WW-H-171E (Type 24) and Manufacturers Standardization Society SP-69 (Type 24).

ORDERING FIG. 137: Specify rod size $x$ pipe size (as $5 / 8 \times 6)$, figure number, name. U-bolt will be furnished with longer tangents $D$ or with longer threads $E$ if so required and ordered. If hex nuts are not required, specify "without hex nuts."

ORDERING FIG. 137S: Specify figure number, name, material specification, dimensions $A, B, C, D$ and $E$, and "with hex nuts" or "without hex nuts."

SPECIAL NOTE: When furnished hot-dip galvanized, oversize hex nuts must be used.

fig. 137C coated U-bolt

SIZ.E RANGE: $1 / 2$ to 8 inch pipe.

MATERIAL: Carbon steel U-bolt and four finished hex nuts. Formed portion of the U-bolt is plastic coated.

SERVICE: Recommended for support, anchor or guide for glass, copper, brass and aluminum pipe.

MAXIMUM TEMPERATURE: $225^{\circ} \mathrm{F}$.

ORDERING: Specify rod size $\times$ pipe size (as $3 / 8 \times 2$ ), figure number, name. If hex nuts are not required, specify "without hex nuts."

load - weights - packaging - dimensions (inches)

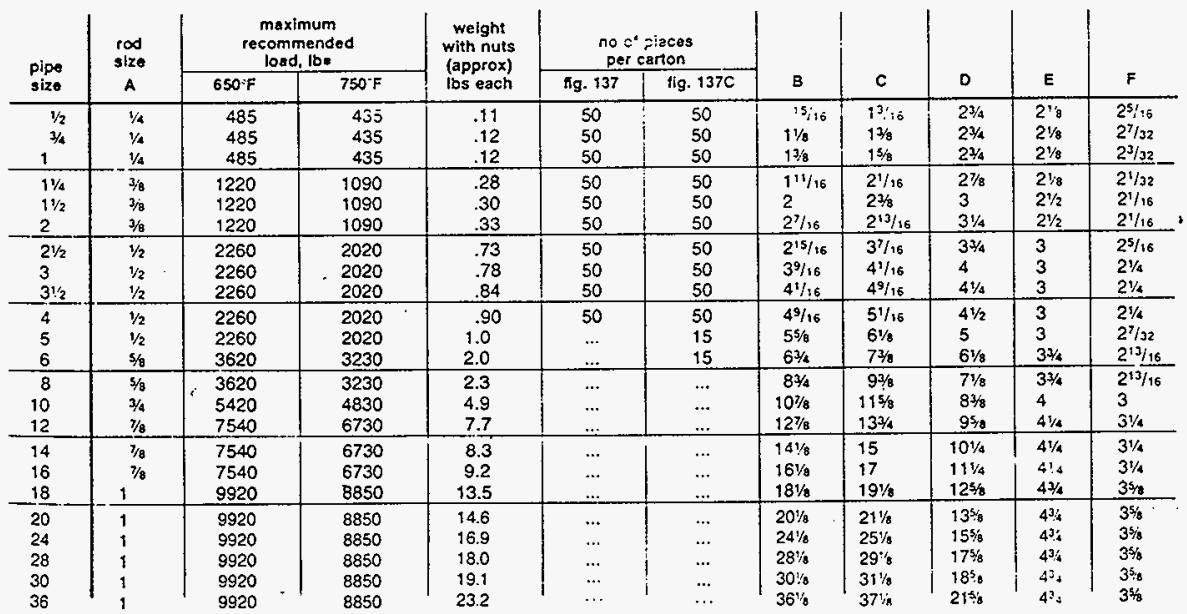


HNF-2486, Rev. 0

W320-28-034

Exhaust Skid Maximum Allowable Leakage Criteria

E- $\mathbf{i}$ 


\section{CALCULATION IDENTIFICATION AND INDEX}

Page $i$ of $i$

Date

3-15-95

This sheet shows the status and description of the attached Design Analysis sheets.

Discipline 28/HVAC wo/Job No. ER4319

Calculation No. W20 134

Project No. \& Name W-320 Tank 241-C-106 Waste Retrieval

Calculation Item Exhaust Skid Maximum A7lowable Leakage Criteria

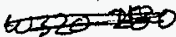

These calculations apply to:

Dwg. No. N/A

Rev. No. N/A

Dwg. No. N/A

Rev. No. N/A

Other (Study, COR) Procurement Specifications:

preliminary

\section{W-320-P1 Exhaust Skid}

Rev. No. 0

The status of these calculations is:

[] Preliminary Calculations

[x] Final Calculations

[] Check Calculations (On Calculation Dated)

[] Void Calculation (Reason Voided)

Incorporated in Final Drawings?

[] Yes [X] No

This calculation verified by independent "check" calculations? [] Yes [X]

No

Original and Revised Calculation Approvals:

\begin{tabular}{|c|c|c|c|}
\hline & $\begin{array}{c}\text { Rev. } 0 \\
\text { Signature/0ate }\end{array}$ & $\begin{array}{c}\text { Rev. } 1 \\
\text { Signature/Date }\end{array}$ & $\begin{array}{c}\text { Rev. } 2 \\
\text { Signature/Date }\end{array}$ \\
\hline Originator & & & \\
\hline Checked by & $C / \lim 3 / 20$ & & \\
\hline Approved by & 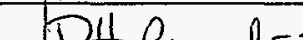 & & \\
\hline $\begin{array}{l}\text { Checked Aga } \\
\text { Approved Ver } \\
\text { Data }\end{array}$ & Charle T.K: & & \\
\hline $\begin{array}{l}\text { Design } \\
\text { Analysis } \\
\text { Page No. }\end{array}$ & & Description & \\
\hline$i$ & Calculation Identific & ndex & \\
\hline 1 & Objective, Design Inp & ations & \\
\hline 2 & Findings \& Conclusion & & \\
\hline$\overline{A 1-A 2}$ & Appendix A: ASME N50 & Figure B-5 & \\
\hline
\end{tabular}

HNF-2486, Rev. 0

KEH 0378.00 (06/92) KEF072

Page E-I 


\section{DESIGN ANALYSIS}

Client WHC

Subject Exhaust Skid Maximum Allowable Leakage Criteria

Location 241-C/200 East
Wo/Job No. ER4319

Date 3-15-95

checked $3 / 20 / 95$

Revised

\subsection{OBJECTIVE}

The objective of this calculation is to determine the maximum allowable leakage from the Exhaust Skid per ASME N509 for input to procurement specification W320-P1.

\subsection{DESIGN INPUTS}

\subsection{CRITERIA AND SOURCE}

DOE General Order 6430.1A

Functional Design Criteria WHC-SD-W320-FDC-001, rev. 2, 1/18/94

\subsection{GIVEN DATA}

1. Non-ESF (Engineered Safety Feature) per W320-P1.

2. 180 to $360 \mathrm{scfm}$ exhaust flow rate per W320-P1.

\subsection{ASSUMPTIONS}

none.

\subsection{METHOOS}

Hand calculations.

\subsection{REFERENCES}

1. W-320-P1 "Procurement Specification, Exhaust Skid Ventilation Air Cleanup

Trains", rev. 0

2. ASME N509-1989 "Nuclear Power Plant Air-Cleaning Units and Components"

\subsection{CALCULATIONS}

Maximum allowable leakage of the Exhaust Skid is determined by following the ASME N509 (ref. 1) Appendix B information included in Appendix A of this calculation. To use Table B-3, the leakage class of the Exhaust Skid must first be determined.

The Leakage class of the Exhaust Skid is determined by Figure B-5. Scheme \#10. In scheme \#10, the contaminated space is the Tank 241-C-106, the interspace is the "clean" outdoors of the Tank Farm, and the protected space is also the "clean" outdoors of the Tank Farm. From Note 4, with a clean interspace and exhaust piping which is always under negative pressure for all modes of operation. the piping between the Tank and the Exhaust Skid may be leakage Class II. Figure B-5 lists the Air Cleaning Unit and the Fan as leakage Class II.

Therefore, leakage Class II, non-ESF (see given data, ref. 2) criteria will be used for Table B-3. Per Table B-3, at leakage Class II and non-ESF, a total of $6 \%$ of the rated flow is the maximum allowable leakage. Per note 1 of Tabie $B-3$, this leakage rate is applicable at operating pressure. In note 4 of Table B-3, the housing surface area to total surface area ratio is assumed to approximate the Exhaust Skid with the Exhaust Skid test sections considered as duct. Note 2 of Table B-3 gives the formula to determine the allowable leakage. 
Calc. No. W320- $甘=034$

Revision 0

\section{DESIGN ANALYSIS}

Client WHC

Subject Exhaust Skid Maximum Allowable Leakage Criteria

Location 241-C/200 East
W0/Job No. ER4319

Date $3-15-95$

Checked

Revised

Page No. 2 of 2

The first component of the formula goes to unity as the surface area of the duct section (a) and the surface area of the total system ductwork per leakage class (A) are the same as when the system considered is the Exhaust Skid only. The B31.3 piping between the Tank and the Exhaust Skid does not fall under the guidelines of N509. For our purposes, the N509 system is the Exhaust Skid only. Therefore, the maximum allowable leakage rate is the product of the Table B-3 value $(P)$ and the system rated flow (Q).

For a value of $P=0.06$ and $Q=180 \mathrm{scfm}$, the maximum allowable leakage rate is 10.8 scfm. For a value of $P=0.06$ and $Q=360 \mathrm{scfm}$, the maximum allowable leakage rate is 21.6 scfm.

\subsection{FINDINGS \& CONCLUSIONS}

The Exhaust Skid maximum allowable leakage rate as specified in W320-P1 shall be $10 \mathrm{scfm}$ at $-50^{\prime \prime} \mathrm{w} . \mathrm{g}$. The $10 \mathrm{scfm}$ correlates to the maximum allowable at the minimum 180 scfm airflow rate. The $-50^{\prime \prime} \mathrm{w} . \mathrm{g}$. is the maximum approximate pressure at a deadheaded exhaust fan inlet and is consistent with the remainder of the Project documentation.

Vendor experience with the E17 is \& Watts air cleanup train for $W-030$ with a maximum allowable leakage rate of $10 \mathrm{scfm}$ at $-32^{\prime \prime}$. w.g. showed an actual leakage rate of approximately $2 \mathrm{scfm}$ (including the fan shaft seal). The $W-030$ unit is a similarly sized unit to the $W-320$ Exhaust Skid. Therefore, the $10 \mathrm{scfm}$ criteria on the $W-320$ unit should not prove unobtainable.

HNF-2486, Rev. 0

Page E-3 
APPENDIX A

HNF-2486, Rev. 0

Page E-4

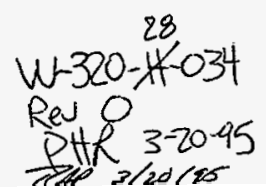




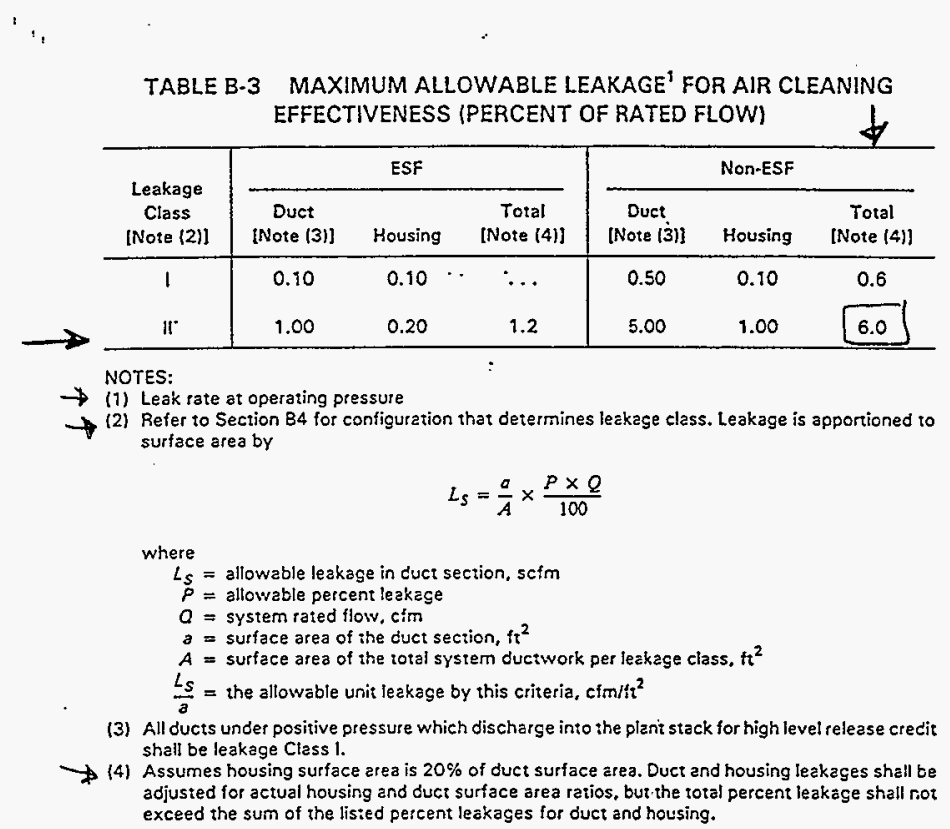

on ANSI/ASQC Z1.4 or other equivalent standard; however, this is not mandatory.

\section{B4 NUCLEAR AIR TREATMENT SYSTEM CON- FIGURATIONS AND LEAKAGE CLASSES}

A nuclear air treatment system can be defined schematically in terms of three spaces and two components.

The three spaces (refer to para. 3 definitions) may be either exterior or interior and are:

(a) the contaminated space $C-106$

(b) the protected space the outdooss

(c) the interspace the outdoors

(I) contaminated

(2) clean

$$
\text { clean }
$$

The two components are:

(d) fan

(e) air-cleaning unit

All three of the above spaces represent possible locations for the different parts of the nuclear air treatment system. The contaminated and protected spaces atso include the points of system origin and termina- tion, respectively. The interspace refers to all other spaces - contaminated or clean - where the nuclear air treatment system or its parts may be located.

Examples of contaminated space/interspace/ protected space arrangements are:

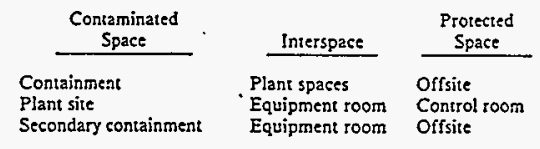

For recirculating systems, the contaminated space and protected space merge into one "contaminated and protected space."

Leakage Classes I and II have been assigned to the various sections of each nuclear air treatment system to represent the qualitative effect of leakage on the nuclear air treatment system function. Thus, Leakage Class II classification indicates that due to system configurations and location a higher leak rate may be allowable. Conversely, a Leak Class I classification indicates a more stringent leak rate is required.

Leakage Classes are noted on Figs. B-5, B-6, and B-7.

HNF-2486, Rev. 0

Page E-5 


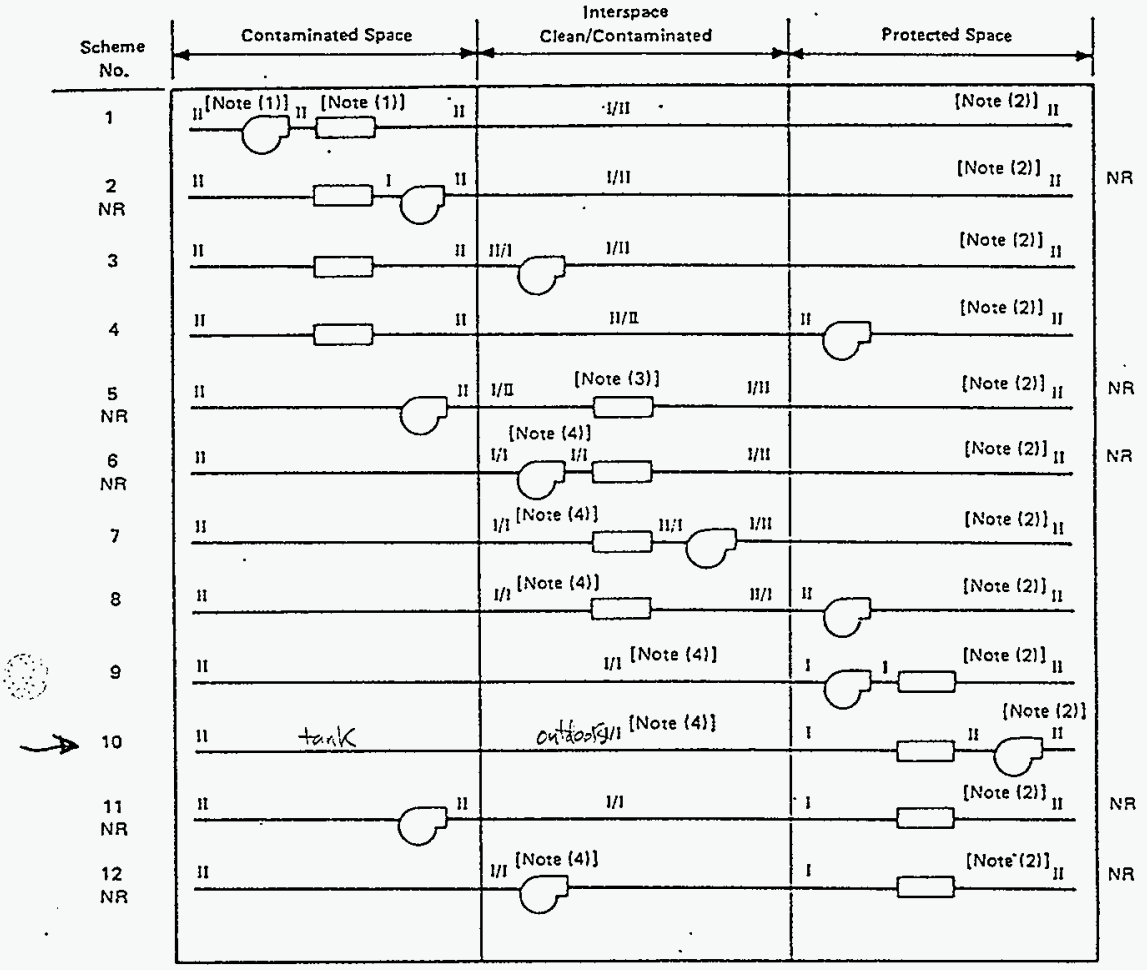

NOTES:

(1) Symbois -
NR - Not Recommended

(2) All ducts under positive pressure which discharge into the plant stack for high level release credit shall be leakage Class I.

(3) Space classification is based on the relative concentration of the space with respect to the duct le.g., Contaminated interspace means concentration within space is greater than duct or housing at that point). Thus, as duct concenzration changes due to filtration, the soace classification will ehange in a given atea.

(4) Noted duct section which pass through a Clean Inter space and which are under a neative pressure for all modes of operation may be leakage Class if.

FIG. B-5 SINGLE PASS AIR CLEANING SYSTEM CONFIGURATIONS

HNF-2486, Rev. 0

Page E-6 
HNF-2486, Rev. 0

W320-28-035

Recirculation Heater, N509 Duct Requirements 


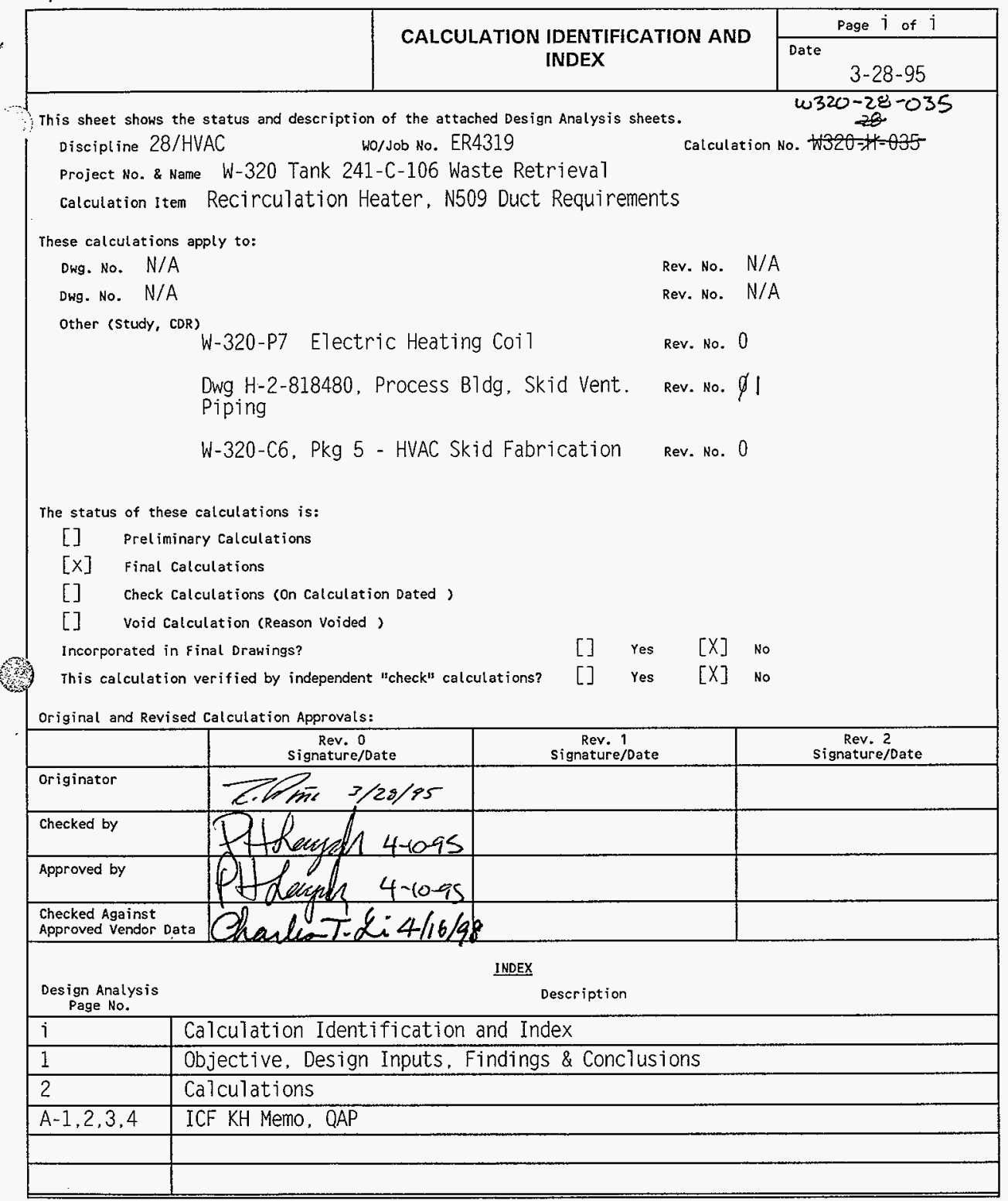


KAISER ENGINEERS

\section{HANFORD}

Client WHC

Subject Recirculation Heater, N509 Duct Requirements

Location: 241-C/200E
DESIGN ANALYSIS

Calc. No. W320- -035

Revision 0

Page No. 1 of 2

WO/Job No. ER4319

$\begin{array}{lll}\text { Date } & 3 / 28 / 95 & \text { By R.Pina } \\ \text { Checked } 4 \text { Ho- } 45 & \text { By PHid } \\ \text { Revised } & \text { By }\end{array}$

\subsection{OBJECTIVE}

To verify the Recirculation Heater Duct Housing is constructed in accordance with the requirements of ASME N509 and ASME N510, as applicable.

\subsection{CRITERIA}

DOE General Order $6430.1 \mathrm{~A}$

Functional Design Criteria, WHC-SD-W320-FDC-001, Rev. 2, 1/18/94

Nuclear Power Plant Air-Cleaning Units and Components, ASME N509-1989

Testing of Nuclear Air Treatment Systems, ASME N510-1989

\subsection{GIVEN DATA}

Normal Operating Pressure

\subsection{ASSUMPTION}

See text for assumptions.

5.0 REFERENCES 28

1) Calc W320-H-022, Seal Pot Sizing For Leak Detection, Rev. 0

2) ERDA 76-21,Nuclear Air Cleaning Handbook, 1970

3) Calc W320- - h

4) W-320-P7, Electric Heating Coil, Rev. 0

5) Dwg H-2-818480, Process Building, Skid Ventilation Piping, Rev. 0

6) W-320-C6, Tank 241-C-106 Sluicing, Pkg 5 - HVAC Skid Fabrication, Rev. 0

7) Rectangular Industrial Duct Construction Standards, SMACNA, 1980

\subsection{FINDINGS AND CONCLUSIONS}

The requirements for the fabrication, inspection and testing of the recirculation heater and heater duct housing comply with the applicable requirements of ASME N509 and ASME N510. References (4), (5) and (6) contain the details for duct construction and heater selection. The applicable ASME N509/N510 requirements have been verified satisfactory in this calculation. See Appendix A of this calculation for clarification of the applicable N509 technical requirements.

\subsection{CALCULATIONS}

Requirement of N509, para

Structural Load Requirements, 4.5

Normal Operating Pressure, 4.6.3

Leak Pressure Test, $4.6 .4,4.6 .5 .3$ \& 4.14
Resolution

n/a since not an Engineered Safety Feature (ESF)

- 19.75. in.WG Satistied, see given data

$-20 \cdot$ in.WG

Satisfied, based on Table 5.5, Ref (2), process off-gas, operating mode $B$, Zone III which indicates Level 5 . From Table 5.6, zero leakage up to 20 in. WG. See Ref. (6) 


\section{KAISER ENGINEERS HANFORD}

Client WHC

Subject Recirculation Heater, N509 Duct Requirements

Location: 241-C/200E
DESIGN ANALYSIS

WO/Job No. ER4319

Date 3/28/95 By R.Pina

Checked 4-6-45 By Piff

Revised
Calc. No. W320-H-035

Revision 0

Page No. 2 of 2

\subsection{CALCULATIONS cont.}

Requirement of N509, para

Maximum Design Pressure, 4.6.5

Structurai Integrity Test, 4.6.6.1

$(1.25 \times-40$ in WG)

Heater Selection, 5.5

Heater meets 4.5

Replaceable

Not mounted to Adsorber Frame

Face velocity exceed manuf. min.

Heater Stage based on calcs, 5.5.1

Heater Overtemperture Protection, 5.5.1

Visual Inspection, 5.5.1.7, ASME N510

Drawings, 5.5.2

ESF Heaters meet IEEE 323 \& $344,5.5 .3$

Documentation, 5.5 .4

Structural Requirements, 5.10.3

Duct Materials, 5.10.4, 5.10.6

Housing Leak \& Structural Test, 6, 6.2.2, ASME N510

Duct Welding, 5.10.5, 7.3

NQA-1, 8.1

Air Heater Performance, 14, 14.7

ASME N510

\section{Resolution}

-40 -in.WG

$-50 \cdot$ in. WG

$\mathrm{n} / \mathrm{a}$

Satisfied

$\mathrm{n} / \mathrm{a}$

Satisfied

Satisfied, see Ref. (3)

Satisfied, see Ref. (4)

Satisfied, see Ref. (4) \& Ref. (6)

Satisfied, see Ref. (5)

n/a, non-ESF

Satisfied, see Ref. (4)

Design based on Ref. (7) \& (2) duct construction tables

Satisfied, see Ref (6) \& Ref. (5), duct thickness \& stiffners based on Table 5.3 \& 5.4 of ERDA 76-21. Materials Selected: reinforcement 2-1/2 × 2-1/2 $\times 1 / 4$, duct 10GA, ASTM A240, TY 304L

Satisfied, see Ref. (4) and (6). Since no leakage is allowed, the pressure decay and the constant pressure methods are not utilized. The specified test will verify that no leakage has occured at the test pressure.

Satisfied, see Ref. (6)

Satisfied by WHC input to Ref. (4)

Satisfied, see Ref. (4)

HNF-2486, Rev. 0

Page F-3 
DON'T SAY IT --- Write It!

TO: David Shrimpton, Roland Niemuth
DATE: August 30, 1994

cc: Rick Bradshaw, Dan Evans, Ray Pina, Paul Rice, Luther Smith

SUBJECT: W-320 QAP-826, rev. 1

\section{INTRODUCTION \& BACKGROUND}

I have been operating on Project W-320 for the last ten months under Quality Assurance Plan (QAP) \#826, rev. 0 signed 10/26/93 by Mr. Shrimpton. I recently received your revision \#1 to the QAP \# 826 signed $8 / 25 / 94$ by Mr. Shrimpton and wish to inform you of my interpretation of your change. In addition, Mr. Shrimpton is asked to provide further feedback on questions stated herein.

Section 1 item 3.c. of QAP rev. 1 now states that the following is applicable to the W-320 Project:

"ASME N509. Procurement, installation shall be performed and completed based on approved design document and technical requirements of ASME N509. Quality program requirements shall be imposed using a graded approach. (DOE 6430.1A, section 1550-2.5.5 invokes ASME N509 when design includes HEPA filters.)"

My interpretation of this statement is that the technical requirements of N509 which apply to HEPA filter assemblies (as is stated in the 6430.1A paragraph referenced) are to be implemented on Project W-320. Quality requirements are to be implemented by using a graded approach for the safety class 3 HEPA filter assemblies. As discussed in the interpretation below, the implementation of these technical requirements is not a task which should be achieved with a simple reference to N509.

\section{DISCUSSION}

In addition to it's requirements for HEPA filter assemblies, N509 also includes many technical design requirements for instrumentation, fans, heating coils, etc. "HEPA filter assemblies" are the exact words used by the 6430.1A paragraph referenced on the QAP. "HEPA filter assemblies" are interpreted to consist of "HEPA filters" and "HEPA filter housings". It is assumed that for Project W-320 only the HEPA filter assemblies will comply with ASME N509. The WHC should clarify their intentions on this issue (Action: Mr. Shrimpton).

Section 2 of N509 states that: "ANSI/ASME AG-1-1988 contains code requirements for nuclear air and gas treatment equipment. These code requirements may be substituted for the requirements listed herein." Please note that many of the specific design requirements of AG-1 were included in the Project W-320 design media, particularly procurement

HNF-2486, Rev. 0

$54-3000-101(12 / 92)$ GEF014
Page F-4
W320 - H-O 35

REV. $O$

$A-1$ 
specifications P3, P4, P5, P6, P7, and P8. The entire AG-1 document was not imposed in these specifications, but specific design requirements derived from AG-1 were included.

For Project W-320, the ASME N509 technical requirements for HEPA filters will be shown in the drawing notes with a simple statement: "HEPA Filters: In accordance with HPS-158-M, Hydrogen Fluoride- and Caustic-Resistant Nuclear Grade HEPA Filters, size x, stainless steel case, pleated fiberglass-aromatic polyamide media with coated aluminum separators, and flat strip gasket. Filter units will be provided by the Government and furnished separately."

The HEPA filter housings defined in N509 are for lighted, walk-in type, open-face housings as typically found in a large nuclear power plant air-cleaning unit. Project W-320 will use bag-in/bag-out side-access HEPA filter bousings. There are more design details different than there are similar between the two types of HEPA filter housings.

W-320-P1 Exhaust Skid Procurement Specification will only convey the individual technical requirements for housing the HEPA filters so as not to confuse the vendor with a sweeping reference to N509. The statement in the P1 spec reads: "The ACT shall meet the design requirements of ASME N509 (non-ESF) as detailed in the following paragraphs...". It is simpler and less confusing to the vendor to only list the few attributes of N509 that apply to HEPA filter housings than to implement the entire N509 and include a long list of exceptions. There will be no direct requirement in Project W-320 design documents to implement N509 in it's entirety unless specifically requested by WHC (Action: Mr. Shrimpton).

\section{SUMMARY}

There will be no direct referenced requirement to design to N509 in it's entirety in any of the Project W-320 documentation even though it is now imposed by QAP rev. 1. Mr. Rice concurs with my interpretation of the QAP. I wanted to give you this heads up on my interpretation of your QAP before you reviewed the design documents.

I am proceeding with the above interpretation of the QAP. Mr. Shrimpton please provide additional information or feedback to actions as requested above.

Please contact me with questions. We can gather to discuss the issue at your convenience.

c: |wpwin|w320c106/w320_85.dsi 
(4)

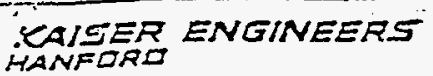

QUALTY ASSURANCE PLAN

Prove 1 of 2

\begin{tabular}{|c|c|c|c|}
\hline $\begin{array}{l}\text { Proimer No. } \\
\text { W- } 320\end{array}$ & $\begin{array}{l}\text { Work order } \mathrm{Na} \\
E R-4319\end{array}$ & $\begin{array}{r}\text { Content } \mathrm{Na} \\
\mathrm{N} / \mathrm{A}\end{array}$ & $\begin{array}{r}\text { Q4 Plan No. } \\
826\end{array}$ \\
\hline
\end{tabular}

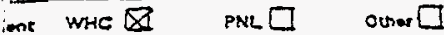

Proinesw.o. Cemeription

$$
\text { TANK 241-C-106 SLUENG }
$$

XEH Afxigment Inctudex: CD $A C D$ $00 \longleftarrow$ cF CM $\angle A B \perp$ Procuremene $L$ Other Section 1

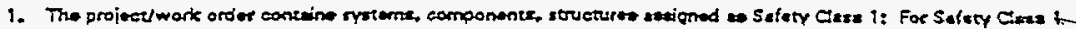

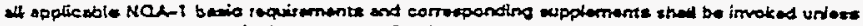
exespoion is documonted in Supplamentul Section.

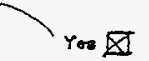

No $\square$

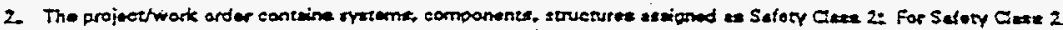

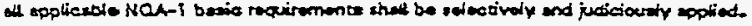

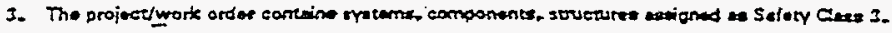

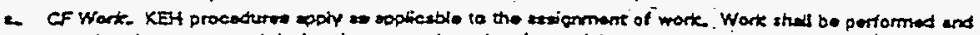

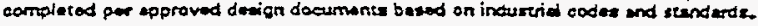

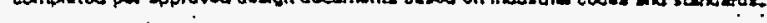

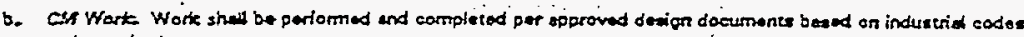
and sunderde.

c. ASMENSOS. Proeurement, inateiledon shall be porformed and completed based an approved devign

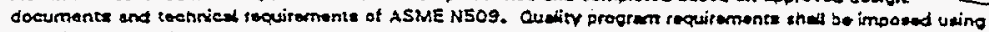
- oroded sporadech.

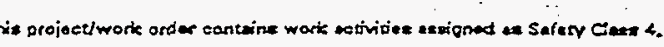

a. CF Work XEH procedures oply apelicsile to the senignment of worte. Worte shall be performad and completed por approvod dovign documents based on secopted commeriad gresticse.

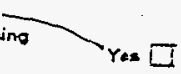

Nas No $\triangle$

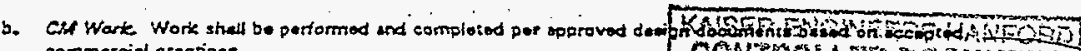
commercial practicos.

ミ. This work is W-DOE Parr $\mathrm{B}$ pertixit work. CONTMOERE DOEUHER STATON NO. 2

1. Oudity Engineoring foview of Sefety Can 1 a 2 requidions is roquired. $5: \because 1,1: 2$ 2321903 CNEEE $5-2$ 3

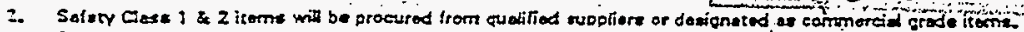

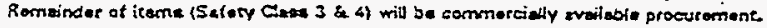

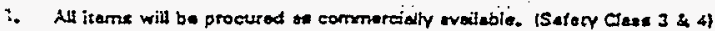

\section{- KeH QC recaiving inspoction ie requirod for Safery Clase 1 andlot 2 it ame endior for the itome listed on une} supplertental racuirement: thoot

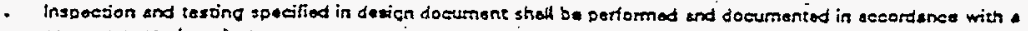
procesx control pecespe.

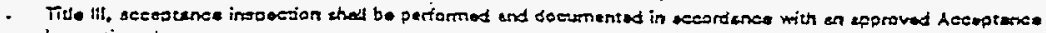
inspecion plan.

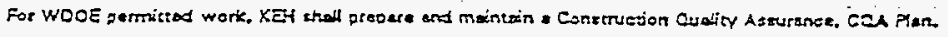

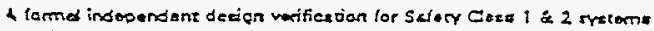
eal be pertormed by:

$$
\begin{aligned}
& \text { other } \square \text { XEH } 8 \\
& \text { Genoral focitit } \square
\end{aligned}
$$

The focility dowsification Der DCE Oroin 9430.14 ix:

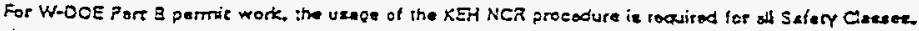

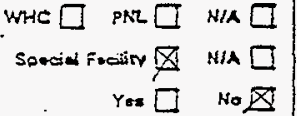

HNF-2486, Rev. 0 W320-H-035
Page F.6

$K E+19 \times 5.01[17 / 97)$ 


$$
\therefore
$$

Pogo 2 of 2

KAISER ENGINEERS

QUALITY REQUIREMENT DOCUMENTS

QA Plan No.

Section III

1. Functional Design Criteria (FDC): $\omega H C-5 D-\omega-320-F D C-001$

2. Conceptual Design Report (CDR): N/A

826

3. Project Specific Quality Assurance Project Plan (OAPP): WHC - SD-W320-QAPP-DOI

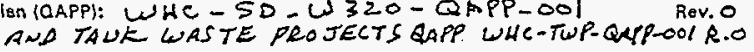

4. Safety Evaluation documents): NOT ISSUED

5. Letters) of Instruction (LOW): $93527579355663,9357233,9358855, \mathrm{kH}$.

Rev. 0

NSA $\square$

Rev.

NRA

NRA

Rev.

NRA

nev.

6. Supplemental Design Criteria (SOC):

Rev. N/A $\square$

7. Technical Data Checklist (TDC): Not iss u CD

Rev. N/A

8. Other: (PSEL) WUL-SD-WM-SEL-033.

LETTER REACT WHC-SD-WH-ES-234, REV .Z

SUPPLEMENTAL REQUIREMENTS

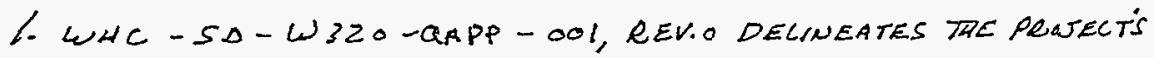
SAFETY Classes and types of inspection for Each SYSTEM, Component, AND STRUCTURE.

2. Quality Control Receiving InSPEction IS Required for all

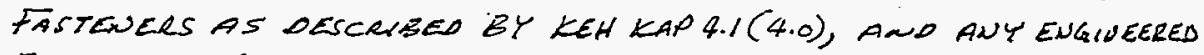

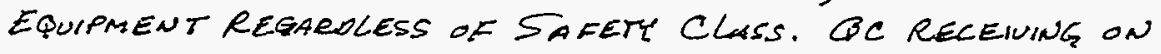

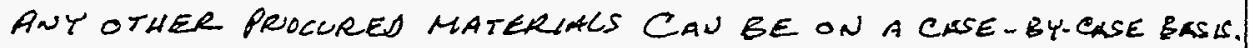

3. SEe QAP No. 828 for the 3 Equipment Containers.

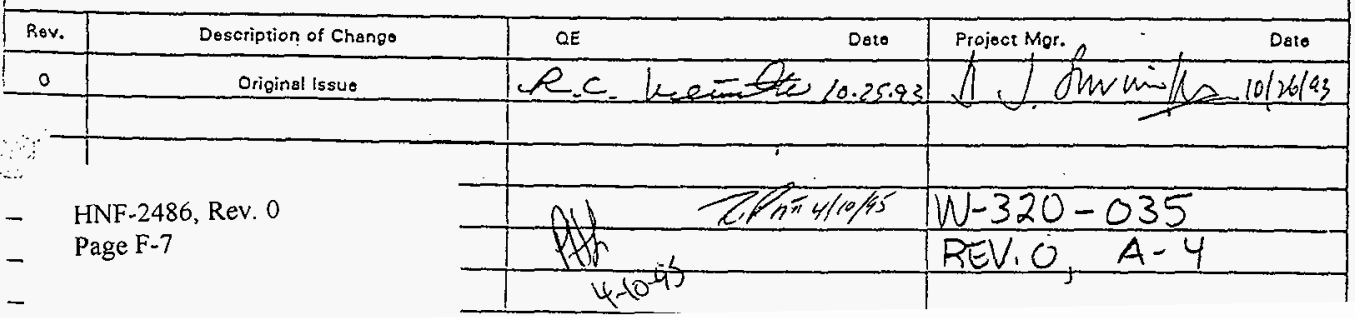




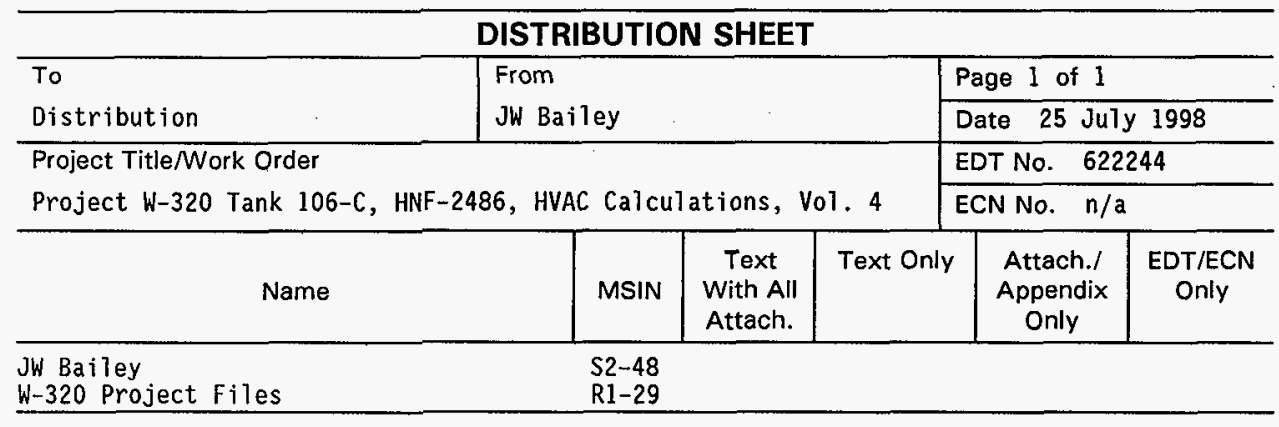

